FORSCHUNGSERGEBNISSE DER WIRTSCHAFTSUNIVERSITÄT WIEN

ISABELLA R. HATAK

\title{
Kompetenz, Vertrauen und Kooperation
}




\section{Kompetenz, Vertrauen und Kooperation}

Dieses Buch beschäftigt sich mit Kompetenz als Vertrauensdeterminante. Ziel ist, die unterschiedlichen Kompetenzarten und ihren Einfluss auf den Vertrauensentstehungsprozess vor dem theoretischen Hintergrund der Neuen Institutionenökonomiezu untersuchen. Das Ergebnis ist ein experimentell geprüftes Modell, das die Kausalbeziehung zwischen unterschiedlichen Kompetenzarten und Vertrauen unter Berücksichtigung personen- und situationsbedingter Faktoren abbildet. Das Wissen um diese Kausalbeziehung erlaubt kooperationswilligen Unternehmen, Einfluss auf die Entstehung, Entwicklung und Aufrechterhaltung von Kooperationsbeziehungen zu nehmen.

Isabella R. Hatak ist Senior Researcher am Institut für KMU-Management sowie am RiCC-Forschungsinstitut für Kooperationen und Genossenschaften an der Wirtschaftsuniversität Wien (WU). Während ihrer Studien an der WU, der Karlsuniversität in Prag und der HES Amsterdam School of Business hat sie praktische Erfahrungen in verschiedenen österreichischen, deutschen und tschechischen Unternehmen gesammelt. Neben ihrer Forschungs- und Lehrtätigkeit an der WU ist sie Lektorin an mehreren Fachhochschulen. 
Kompetenz, Vertrauen und Kooperation 


\section{Forschungsergebnisse der Wirtschaftsuniversität Wien}

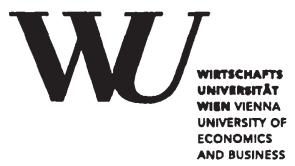

Band 50

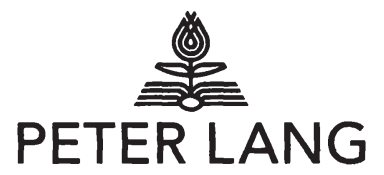

Frankfurt am Main · Berlin · Bern · Bruxelles - New York · Oxford · Wien 


\section{Kompetenz, Vertrauen und Kooperation}

Eine experimentelle Studie

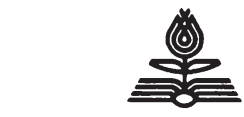

\section{PETER LANG}

Internationaler Verlag der Wissenschaften 
Bibliografische Information der Deutschen Nationalbibliothek Die Deutsche Nationalbibliothek verzeichnet diese Publikation in der Deutschen Nationalbibliografie; detaillierte bibliografische Daten sind im Internet über http://dnb.d-nb.de abrufbar.

Open Access: The online version of this publication is published on www.peterlang.com and www.econstor.eu under the international Creative Commons License CC-BY 4.0. Learn more on how you can use and share this work: http://creativecommons. org/licenses/by/4.0.

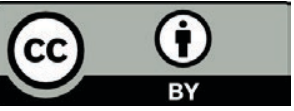

This book is available Open Access thanks to the kind support of ZBW - Leibniz-Informationszentrum Wirtschaft.

Gefördert durch die Wirtschaftsuniversität Wien.

\author{
Umschlaggestaltung: \\ Atelier Platen, nach einem Entwurf \\ von Werner Weißhappl. \\ Universitätslogo der Wirtschaftsuniversität Wien: \\ Abdruck mit freundlicher Genehmigung \\ der Wirtschaftsuniversität Wien.
} Gedruckt auf alterungsbeständigem,
säurefreiem Papier.

ISSN 1613-3056

ISBN 978-3-631-61960-5

ISBN 978-3-631-74970-8 (eBook)

(c) Peter Lang GmbH

Internationaler Verlag der Wissenschaften

Frankfurt am Main 2011

Alle Rechte vorbehalten.

Das Werk einschließlich aller seiner Teile ist urheberrechtlich geschützt. Jede Verwertung außerhalb der engen Grenzen des

Urheberrechtsgesetzes ist ohne Zustimmung des Verlages unzulässig und strafbar. Das gilt insbesondere für Vervielfältigungen, Übersetzungen, Mikroverfilmungen und die Einspeicherung und Verarbeitung in elektronischen Systemen.

www.peterlang.de 
Ich möchte mich herzlich bei all jenen bedanken, die mich bei der Erstellung dieser Arbeit unterstützt haben. Mein besonderer Dank gilt dem Hauptbetreuer meiner Dissertation Herrn Professor Dr. Dietmar Rößl, der mich für die experimentelle Forschung begeisterte und mir eine herausragende Betreuung zukommen ließ. Herrn Professor Dr. Steyrer danke ich, neben der Übernahme des Zweitgutachtens, für überaus wertvolle fachliche Anregungen und seine motivierenden Feedbacks. Bei den Mitgliedern des Doktoratskomitees Herrn Professor Dr. Mugler und Herrn Professor Dr. Zins möchte ich mich für die konstruktiven Vorschläge bedanken.

Meinen lieben Eltern gebührt besonderer Dank für ihre umfassende Unterstützung. Ihnen ist diese Arbeit gewidmet. Meine Mutter Ruth Hatak trug erheblich mit ihrer Lebensfreude und Ermutigung zu dieser Arbeit bei. Auch meinem Vater Senator KR Dr. Walter Hatak bin ich zu großem Dank verpflichtet.

Zuletzt möchte ich mich bei Alexander, Walter, Albert und Miška bedanken, die immer für mich da waren. 
Isabella Hatak - 978-3-631-74970-8 Downloaded from PubFactory at 01/11/2019 09:36:38AM via free access 
„Für den Menschen gibt es nur eine Wahrheit, das ist die, die aus ihm einen Menschen macht. “

Antoine de Saint-Exupéry 
Isabella Hatak - 978-3-631-74970-8 Downloaded from PubFactory at 01/11/2019 09:36:38AM via free access 


\section{GELEITWORT}

Die Rolle von Vertrauen wird in der Managementlehre in vielen Kontexten diskutiert. Aufgrund der Erfolgswirkung, die Vertrauen zuerkannt wird, ist die Erklärung der Vertrauensentstehung ein Forschungsfeld von hoher Bedeutung. Da darüber hinaus die Rolle von Kompetenz für die Vertrauensevolution bisher nur ansatzweise behandelt wurde, zielt die Arbeit mit der theoretisch fundierten Entwicklung und experimentellen Überprüfung eines Modells zum Einfluss von Kompetenz auf Vertrauen in zwischenbetrieblichen Kooperationen auf die SchlieBung einer zentralen Forschungslücke.

Im Rahmen der theoretischen Modellierung erarbeitet Isabella Hatak eine tragfähige konzeptive Grundlage für die weitere Arbeit - sie lotet das Vertrauensphänomen aus und bietet so eine tiefschürfende Diskussion zum Vertrauensbegriff. Auch bei der Schaffung einer tragfähigen Basis für die Operationalisierung und Messung von Kompetenz ist die Arbeit durch hohe Präzision im Umgang mit Literatur und Sprache geprägt.

Die theoretische Arbeit leistet zweifelsfrei einen wesentlichen Beitrag zur Vertrauensforschung - die besondere Herausforderung stellte aber die Entwicklung eines erfolgsversprechenden empirischen Designs dar. Neben fundierter Methodenkenntnis und ausgeprägten analytischen Fähigkeiten bedarf es der nötigen Kreativität, um die mit dem Untersuchungsgegenstand verbundene Operationalisierungsproblematik zu überwinden und das Modell einer empirischen Überprüfung zugänglich zu machen. Mit zwei großzahligen Experimenten gelingt es, diesen Anforderungen zu begegnen und so die Plausibiliät des Modells empirisch zu beurteilen.

Seit der Approbation dieser Dissertation hat Frau Dr. Isabella Hatak bereits weiterführende Forschungsarbeiten vorgelegt. Ihre Ergebnisse und ihr Forschungsdesign werden sowohl die weitere Theoriebildung als auch die empirischen Arbeiten in der Vertrauensforschung beeinflussen.

Die Auswahl des Forschungsgegenstandes, der Fragestellung und der Methode sind verdienstvoll. Die Arbeit bietet eine präzise Problemstrukturierung und löst die anspruchsvolle Zielsetzung auf heuristischer modellbildender und empirischer Ebene ein. Ich wünsche dieser Arbeit nicht zuletzt aufgrund ihrer Präzision, Klarheit und Aussagekraft und ihrer Relevanz für das Kooperationsmanagement und die empirische Vertrauensforschung eine besondere Resonanz in der Fachwelt.

Prof. Dr. Dietmar Röß। 
Isabella Hatak - 978-3-631-74970-8 Downloaded from PubFactory at 01/11/2019 09:36:38AM via free access 


\section{GELEITWORT}

In diesem Buch geht es um den Einfluss unterschiedlicher Kompetenzarten auf die Entstehung von Vertrauen in zwischenbetrieblichen Kooperationsbeziehungen. In einer Zeit, in der das wachsende Misstrauen handelnder Akteure mehr und mehr das Basisfundament eine prosperierenden Wirtschaft - nämlich Vertrauen - zu erschüttern droht, geht es also um ein Forschungsthema mit großem Aktualitätsgehalt.

Bis dato liegt $\mathrm{m}$. E. keine Arbeit vor, die die in diesem Zusammenhang relevanten Konstrukte sowohl auf theoretischer als auch auf empirischer Ebene umfassender analysiert als das vorliegende Buch.

Ganz besonders von Bedeutung sind aber die berichteten empirischen Befunde, hervorgegangen aus einem höchst originellen Laborexperiment, dass die auf individueller Ebene wahrgenommenen Kompetenzen des Vertrauensnehmers die Genese von Vertrauen in zwischenbetrieblichen Kooperationsbeziehungen positiv beeinflussen und dass dem sozial- und persönlichkeitskompetenten Akteur mehr Vertrauen als dem fach- und methodenkompetenten Akteur entgegengebracht wird. Das ist zunächst einmal für die Wissenschaft von hohem Interesse. Das Ergebnis hat aber auch eine hohe praktische Relevanz, das sich verkürzt dahingehend auf den Punkt bringen lässt: „Sozialkompetenz“ schlägt „Fachkompetenz“!

Dieses Buch bereichert zweifelsohne unseren Erkenntnisstand über die "Conditions of Trust" maßgeblich und ich wünsche ihm einen besonders großen Leserkreis.

Prof. Dr. Johannes Steyrer 
Isabella Hatak - 978-3-631-74970-8 Downloaded from PubFactory at 01/11/2019 09:36:38AM via free access 


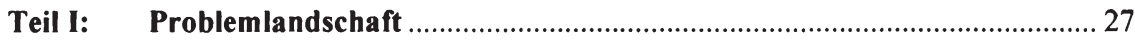

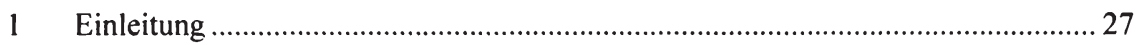

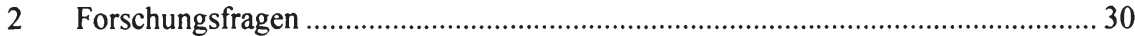

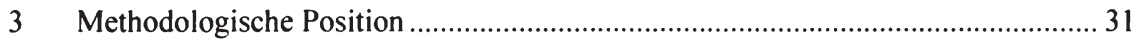

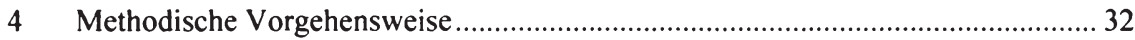

4.1 Theoriegeleitete Thesengenerierung ………….......................................... 33

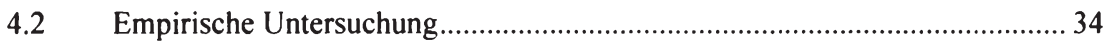

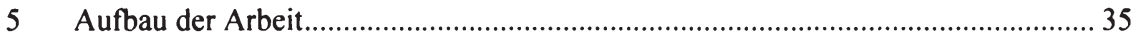

Teil II: Vertrauen als zentrales Element in zwischenbetrieblichen

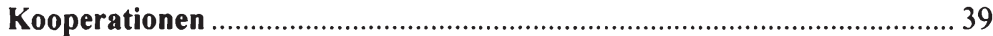

1 Die Bedeutung von Vertrauen aus verschiedenen Perspektiven ............................... 39

1.1 Vertrauen aus soziologischer Perspektive …………….................................. 40

1.1.1 Vertrauen als Mechanismus zur Reduktion sozialer Komplexität ............. 40

1.1.2 Vertrauen als einseitige Ressourcenübertragung ...................................... 47

1.2 Vertrauen aus der Perspektive der Neuen Institutionenökonomie.................... 51

1.2.1 Vertrauen aus Sicht der Transaktionskostentheorie.................................... 53

1.2.2 Vertrauen aus Sicht der Principal-Agent-Theorie ........................................ 57

1.2.3 Vertrauen aus Sicht der Property-Rights-Theorie ........................................ 58

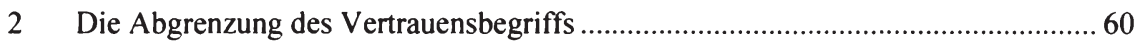

2.1 Abgrenzung gegen den Begriff, Zuversicht ${ }^{\star}$..................................................62

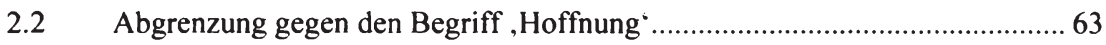

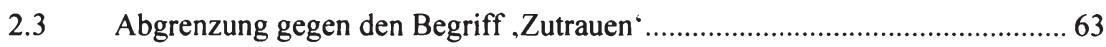

2.4 Abgrenzung gegen den Begriff .Vertrauenswürdigkeit ${ }^{\star}$................................... 65

2.5 Abgrenzung gegen den Begriff, Reputation' '.....................................................6 65

2.6 Abgrenzung gegen den Begriff, Misstrauen` ...................................................... 67

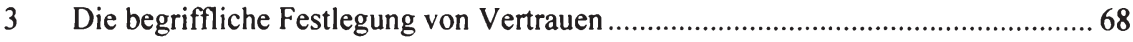

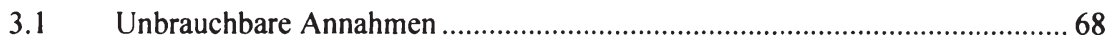

3.2 Die Annahmen der Neuen Institutionenökonomie ........................................69 
3.3 Über die Vertrauensbegründung zu einem differenzierten Vertrauensbegriff.

4 Die Principal-Agent-Theorie als Grundlage für die Analyse von Vertrauensbeziehungen

4.1 Die opportunistischen Verhaltensspielräume in Principal-Agent-

Beziehungen.

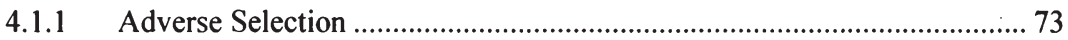

4.1.2 Hold Up ............................................................................................... 73

4.1.3 Moral Hazard ................................................................................ 75

4.2 Vertrauen als Koordinationsmechanismus in Principal-Agent-

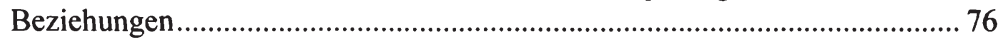

4.3 Die Vertrauensbeziehung als Principal-Agent-Beziehung ……………......... 78

4.3.1 Die Vertrauensbeziehung zwischen Vertrauensgeber und

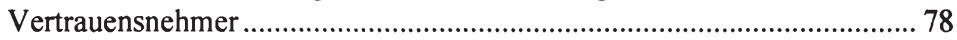

4.3.2 Die Vertrauensbeziehung zwischen Prinzipal und Agent.......................... 78

5 Vertrauensgeber und Vertrauensnehmer als Elemente der Vertrauensbeziehung ............................................................................................................. 82

5.1 Die Entscheidungssituation des Vertrauensgebers ....................................... 82

5.1.1 Die Modellierung der Vertrauensentscheidung ........................................... 84

5.1.2 Die Vertrauenserwartung als Bestandteil der Vertrauensentscheidung ..... 86

5.1.3 Die Vertrauenshandlung als Bestandteil der Vertrauensentscheidung ...... 90

5.2 Die Entscheidungssituation des Vertrauensnehmers ....................................... 93

5.2.1 Die Vertrauenswürdigkeit des Vertrauensnehmers................................... 94

5.2.2 Die intrinsischen Anreize für vertrauenswürdiges Verhalten ..................... 97

5.2.3 Die extrinsischen Anreize für vertrauenswürdiges Verhalten ................... 99

6 Die Determinanten der Entstehung von Vertrauen ................................................ 101

7 Die Vertrauensmessverfahren .......................................................................... 104

Teil III: Kompetenz als zentrales Element in zwischenbetrieblichen

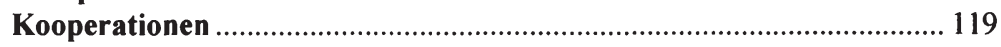

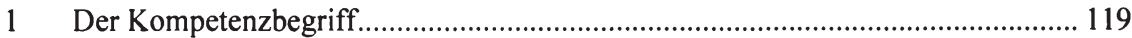

$1.1 \quad$ Begriffliche Festlegung von Kompetenz …………...................................... 119

1.2 Abgrenzung des Kompetenzbegriffs ......................................................... 120

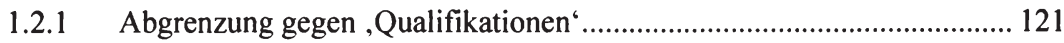


1.2.2 Abgrenzung gegen , Ressourcen'

1.2.3 Abgrenzung gegen ,Fertigkeiten ' ......................................................... 123

1.2.4 Abgrenzung gegen ,Fähigkeiten' ........................................................... 123

2 Die Kompetenzarten...................................................................................... 125

2.1 Organisationale Kompetenzen ...................................................................... 125

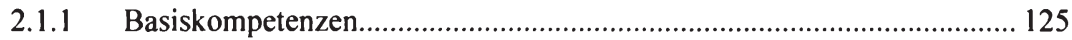

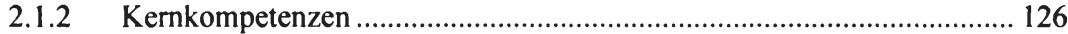

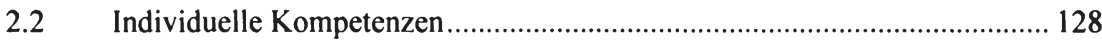

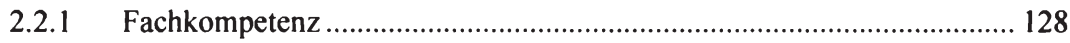

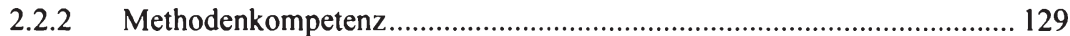

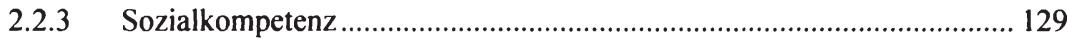

2.2.4 Persönlichkeitskompetenz................................................................ 131

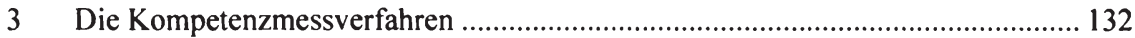

3.1 Ansätze zur Identifikation organisationaler Kompetenzen............................ 133

3.2 Ansätze zur Identifikation individueller Kompetenzen ............................... 142

4 Die Bedeutung von Kompetenz aus verschiedenen Perspektiven............................ 151

4.1 Kompetenz aus Sicht der soziologischen Handlungstheorie ........................ 152

4.2 Kompetenz aus Sicht der Principal-Agent-Theorie ...................................... 160

Teil IV: Kompetenz als Determinante der Entstehung von Vertrauen in zwischenbetrieblichen Kooperationen ................................................ 165

1 Die Forschung zur Wirkung von Kompetenz als Vertrauensdeterminante ............ 165

1.1 Der Einfluss individueller Kompetenzen auf Vertrauen.................................. 165

1.2 Der Einfluss organisationaler Kompetenzen auf Vertrauen .......................... 169

2 Die methodischen Grundlagen der Modellierung von Kompetenz als

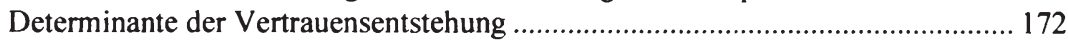

3 Die Indikatoren zur Operationalisierung des Kompetenzkonstrukts...................... 175

3.1 Die Indikatoren zur Operationalisierung individueller Kompetenzen........... 175

3.2 Die Indikatoren zur Operationalisierung organisationaler Kompetenzen ..... 180

4 Die Indikatoren zur Operationalisierung des Vertrauenskonstrukts........................ 185

5 Die Modellierung von Kompetenz als Determinante der Entstehung von Vertrauen. 
Teil V: Design zur Untersuchung der modellierten Wirkung von Kompetenz auf die Vertrauensentstehung................................................ 199

1 Die methodischen Grundlagen ........................................................................... 199

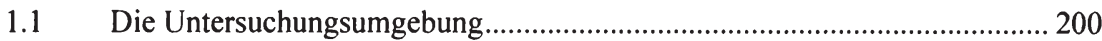

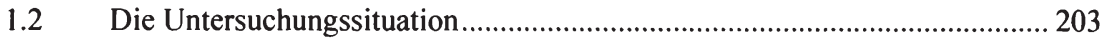

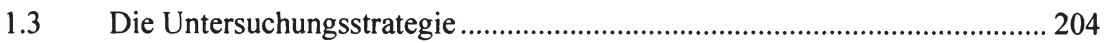

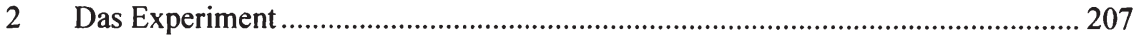

2.1 Die Integration der Vertrauenselemente in eine Fallsimulation ..................... 207

2.2 Die experimentelle Manipulation der unabhängigen Variable ...................... 214

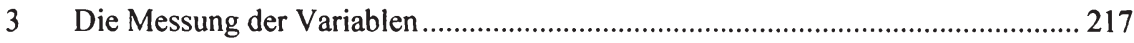

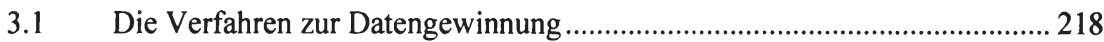

3.2 Die Messung der Dispositionen des Vertrauensgebers.................................. 219

3.3 Die Messung der Wahrnehmung des Vertrauensgebers ............................... 221

3.4 Die Messung der Handlungen des Vertrauensgebers .................................... 221

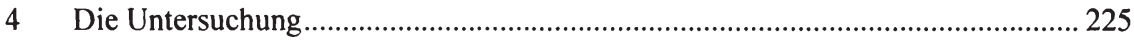

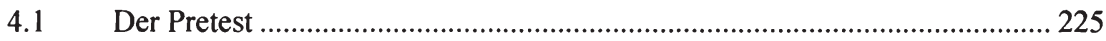

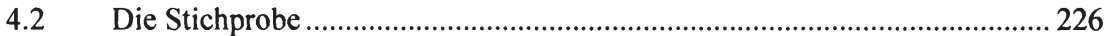

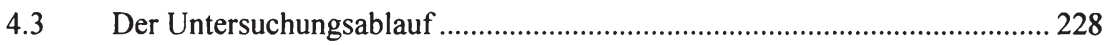

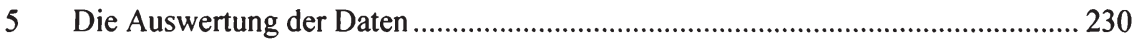

5.1 Die Gütekriterien der Messung................................................................. 230

5.2 Die statistischen Analysemethoden ............................................................. 233

5.2.1 Die statistische Modellierung................................................................ 233

5.2.2 Die Prüfung der Modellprämissen ........................................................ 236

Teil VI: Ergebnisse der Untersuchung von Kompetenz als

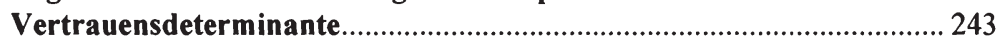

1 Die Prüfung der methodischen Effektivität des Experiments .............................. 243

1.1 Die Eignung der Fallsimulation............................................................... 243

1.2 Die Kontrolle der Manipulationseffekte der unabhängigen Variable.............246

2 Die Prüfung des Einflusses von Kompetenz auf die Entstehung von Vertrauen ... 249

2.1 Der Einfluss von Kompetenz auf Vertrauen................................................. 249

2.2 Der Einfluss von individuellen Kompetenzen auf Vertrauen ........................ 252 
2.2.1 Der Einfluss von Fach- und Methodenkompetenz auf Vertrauen............ 252

2.2.2 Der Einfluss von Sozial- und Persönlichkeitskompetenz auf Vertrauen............................................................................................. 255

2.2.3 Der Vergleich der Einflussstärke der individuellen Kompetenzen.......... 258

2.3 Der Einfluss von individuellen Kompetenzen auf Vertrauen unter Berücksichtigung von Dispositionen und situativer Wahrnehmung

2.3.1 Der Einfluss von Fach- und Methodenkompetenz auf Vertrauen unter Berücksichtigung von Dispositionen und situativer Wahrnehmung.

2.3.2 Der Einfluss von Sozial- und Persönlichkeitskompetenz auf Vertrauen unter Berücksichtigung von Dispositionen und situativer Wahrnehmung.

2.3.3 Der Vergleich der Einflussstärke der individuellen Kompetenzen unter Berücksichtigung von Dispositionen und situativer Wahrnehmung. 267

2.4 Die Analyse der Korrelationen zwischen den interessierenden Variablen .... 270

3 Die Zusammenfassung der Untersuchungsergebnisse

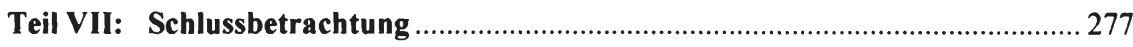

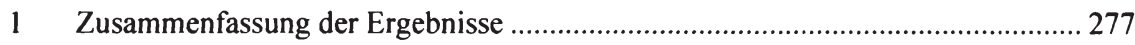

2 Erklärungsbeitrag und praktische Folgerungen ................................................. 284

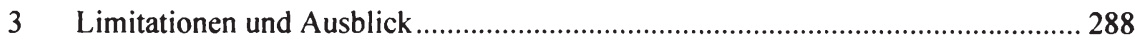

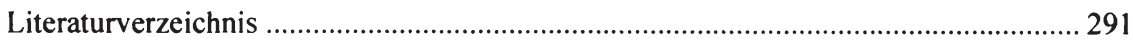

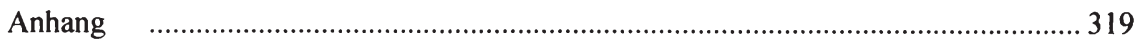


Isabella Hatak - 978-3-631-74970-8 Downloaded from PubFactory at 01/11/2019 09:36:38AM via free access 


\section{ABBILDUNGSVERZEICHNIS}

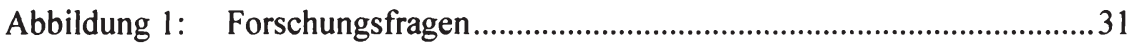

Abbildung 2: Theoriegeleitete Thesengenerierung..........................................33

Abbildung 3: Methodische Vorgehensweise ………........................................35

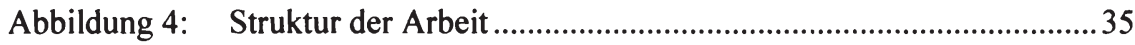

Abbildung 5: Die Vertrauensbeziehung als Principal-Agent-Beziehung ..........79

Abbildung 6: Die durch die Vertrauensbeziehung begründeten

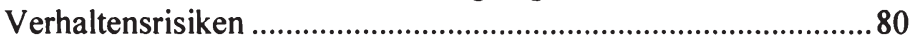

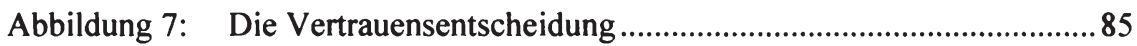

Abbildung 8: Die Informationskategorien bei der Bildung der Vertrauens-

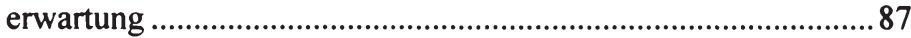

Abbildung 9: Die Determinanten der Entstehung von Vertrauen ..................... 103

Abbildung 10: Die Verfahren zur Messung von Vertrauen ................................ 105

Abbildung 11: Die Operationalisierung des Kernkompetenzkonstrukts ........... 140

Abbildung 12: Der Prozess der Modellierung .................................................. 173

Abbildung 13: Die Modellierung von Kompetenz als Determinante der Entstehung von Vertrauen .....................................................191

Abbildung 14: Ergebnismatrix zur Strategiewahl (allgemein) .........................211

Abbildung 15: Ergebnismatrix zur Strategiewahl (weiterführend)...................213

Abbildung 16: Die Prüfung der Normalverteilung der Residuen ......................238

Abbildung 17: Die Prüfung der Homoskedastizität der Residuen ......................239

Abbildung 18: Die Prüfung der Unabhängigkeit der Residuen ..........................241

Abbildung 19: Der Zusammenhang zwischen Vertrauen, FMK, RB und

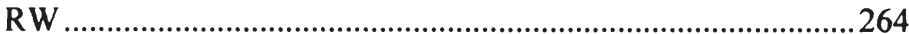

Abbildung 20: Der Zusammenhang zwischen Vertrauen, SPK, RB und RW ...266

Abbildung 21: Der Zusammenhang zwischen Vertrauen, SPK, FMK, RB

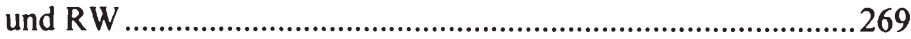

Abbildung 22: Kompetenz als Determinante der Entstehung von Vertrauen....274

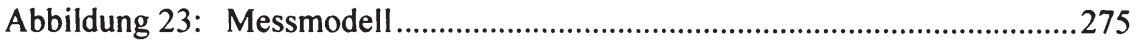


Isabella Hatak - 978-3-631-74970-8 Downloaded from PubFactory at 01/11/2019 09:36:38AM via free access 


\section{TABELLENVERZEICHNIS}

Tabelle 1: Items zur Erfassung interorganisationalen Vertrauens (OTI-

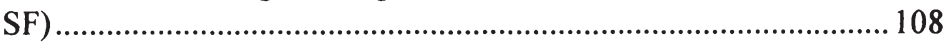

Tabelle 2: Items zur Erfassung interorganisationalen Vertrauens (BRP-

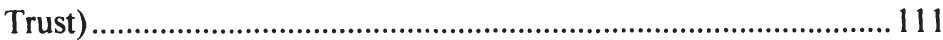

Tabelle 3: Items zur Erfassung generalisierten Vertrauens (ITS) ................... 112

Tabelle 4: Items zur Erfassung generalisierten Vertrauens (RPHNS

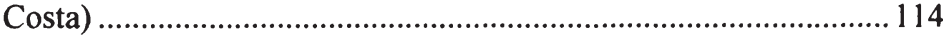

Tabelle 5: Items zur Erfassung spezifischen Vertrauens (BTI) ....................... 115

Tabelle 6: Allgemeine soziale Kompetenzen................................................ 130

Tabelle 7: Kenngrößen zur Identifikation organisationaler Kompetenzen ..... 139

Tabelle 8: Ansätze zur Identifikation organisationaler Kompetenzen ............. 141

Tabelle 9: Überblick über die Ansätze zur Identifikation individueller Kompetenzen ................................................................................ 142

Tabelle 10: Indikatoren individueller Kompetenz nach Sonntag/Schäfer-

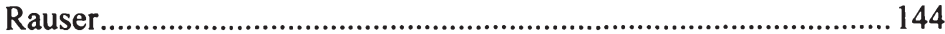

Tabelle 11: Indikatoren individueller Kompetenz nach Erpenbeck/Heyse....... 147

Tabelle 12: Indikatoren individueller Kompetenz nach Kauffeld/Grote/Freiling ............................................................. 150

Tabelle 13: Ansätze zur Identifikation individueller Kompetenz .....................151

Tabelle 14: Individuelle Kompetenzen als Vertrauensdeterminanten ............... 169

Tabelle 15: Organisationale Kompetenzen als Vertrauensdeterminanten ......... 171

Tabelle 16: Indikatoren individueller Kompetenzen......................................... 180

Tabelle 17: Indikatoren organisationaler Kompetenzen .................................... 185

Tabelle 18: Indikatoren der Vertrauenshandlung........................................187

Tabelle 19: Hypothesen zum Einfluss individueller Kompetenzen auf

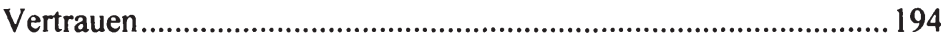

Tabelle 20: Items zur Messung der personenspezifischen Vertrauensbereitschaft ................................................................220

Tabelle 21: Items zur Messung der personenspezifischen

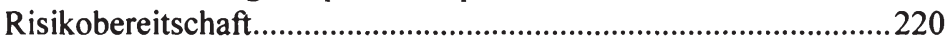

Tabelle 22: Items zur Messung der situativen Risikowahrnehmung .................221

Tabelle 23: Aufgabenstellung zur Strategiewahl ..........................................222

Tabelle 24: Aufgabenstellung zur Informationsabgabe ....................................223

Tabelle 25: Aufgabenstellung zur Kontrollintensität........................................224

Tabelle 26: Demographische Merkmale der Stichprobe...................................226

Tabelle 27: Geschlechterverteilung der Gruppenzusammensetzung .................227 
Tabelle 28: Reliabilitätskoeffizienten der Skalen

Tabelle 29: Cronbach's Alpha bei der Entfernung von Items (situative Risikowahrnehmung)

Tabelle 30: Die Prüfung auf Multikollinearität.

Tabelle 31: Deskriptive Ergebnisse zu Informations- und Kontrollhandlungen

Tabelle 32: Befund zum Zusammenhang zwischen

Kompetenzwahrnehmung und Strategiewahl .245

Tabelle 33: Ergebnisse der Prüfung auf Gruppenunterschiede bezüglich der Dispositionen

Tabelle 34: Ergebnisse der Prüfung auf Gruppenunterschiede bezüglich der Risikowahrnehmung .248

Tabelle 35: Ergebnisse der Prüfung des Einflusses von Kompetenz auf Vertrauen. 250

Tabelle 36: Ergebnisse der Prüfung des Einflusses von Kompetenz auf OI.....250

Tabelle 37: Ergebnisse der Prüfung des Einflusses von Kompetenz auf EI .....251

Tabelle 38: Ergebnisse der Prüfung des Einflusses von Kompetenz auf KI.....251

Tabelle 39: Ergebnisse der Prüfung des Einflusses von FMK auf Vertrauen...253

Tabelle 40: Ergebnisse der Prüfung des Einflusses von FMK auf $\mathrm{OI}$...............254

Tabelle 41: Ergebnisse der Prüfung des Einflusses von FMK auf EI...............254

Tabelle 42: Ergebnisse der Prüfung des Einflusses von FMK auf KI ..............255

Tabelle 43: Ergebnisse der Prüfung des Einflusses von SPK auf Vertrauen ....256

Tabelle 44: Ergebnisse der Prüfung des Einflusses von SPK auf OI................256

Tabelle 45: Ergebnisse der Prüfung des Einflusses von SPK auf EI .................257

Tabelle 46: Ergebnisse der Prüfung des Einflusses von SPK auf KI................257

Tabelle 47: Ergebnisse des Vergleichs der Einflussstärke individueller Kompetenzen auf Vertrauen

Tabelle 48: Ergebnisse des Vergleichs der Einflussstärke individueller Kompetenzen auf OI .

Tabelle 49: Ergebnisse des Vergleichs der Einflussstärke individueller Kompetenzen auf EI.

Tabelle 50: Ergebnisse des Vergleichs der Einflussstärke individueller Kompetenzen auf $\mathrm{KI}$

Tabelle 51: Ergebnisse der erweiterten Prüfung des Einflusses von FMK auf Vertrauen .263

Tabelle 52: Ergebnisse der erweiterten Prüfung des Einflusses von SPK auf Vertrauen. 
Tabelle 53: Ergebnisse des Vergleichs der Einflussstärke individueller Kompetenzen und VB, RB, RW auf Vertrauen ..............................268

Tabelle 54: Korrelationskoeffizienten zwischen FMK, VB, RB, RW und

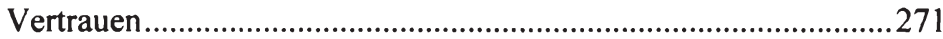

Tabelle 55: Korrelationskoeffizienten zwischen SPK, VB, RB, RW und

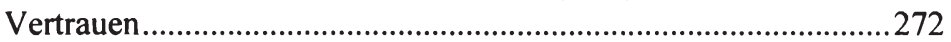

Tabelle 56: Bestätigte Hypothesen zum Einfluss individueller Kompetenzen auf Vertrauen 273 
Isabella Hatak - 978-3-631-74970-8 Downloaded from PubFactory at 01/11/2019 09:36:38AM via free access 
Abb.

Aufl.

$\beta$

bzw.

d.h.

df

EI

et al.

etc.

f.; ff.

FMK

Hrsg.

iSv

KG

KI

$\mathrm{N}$

OI

$\mathrm{p}$

PHNS

R2

RB

RW

S.

Sig.

SPK

$t$

Tab.

u.a.

VB

VG1_FMK

VG2_SPK

VIF

$\mathrm{Vgl}$.

z.B.
Abbildung

Auflage

standardisierter Regressionskoeffizient

beziehungsweise

das heißt

degrees of freedom (statistische Freiheitsgrade)

Ehrliche Informationsabgabe

et alii

et cetera

folgende; fortfolgende

Fach- und Methodenkompetenz

Herausgeber

im Sinne von

Kontrollgruppe

Kontrollintensität

Stichprobengröße

Offene Informationsabgabe

Irrtumswahrscheinlichkeit

Philosophies of Human Nature Scale

Bestimmtheitsmaß

Risikobereitschaft

Risikowahrnehmung

Seite

Signifikanz

Sozial- und Persönlichkeitskompetenz

time (Zeit)

Tabelle

unter anderem

Vertrauensbereitschaft

Versuchsgruppe 1: Fach- und Methodenkompetenz

Versuchsgruppe 2: Sozial- und Persönlichkeitskompetenz

Variance Inflation Factor

Vergleiche

zum Beispiel 
Isabella Hatak - 978-3-631-74970-8 Downloaded from PubFactory at 01/11/2019 09:36:38AM via free access 


\section{TeIl I: Problemlandschaft}

\section{EINLEITUNG}

Mit der Globalisierung kam es zu einer ,hypercompetition", die sich durch gesteigerte unternehmerische Aggressivität, schnelleren technologischen Wandel und allgemein durch höhere Unsicherheit ${ }^{2}$ und durch rasche Erschließung und Realisierung von Wettbewerbsvorteilen ${ }^{3}$ kennzeichnet. Deshalb stammt ,the only enduring advantage $[\ldots]$ from the ability to generate a continuous flow of new advantages." ${ }^{4}$ In diesem Szenario ist Flexibilität im Sinne der Fähigkeit zur schnellen Anpassung an Veränderungen von steigender Relevanz. ${ }^{5}$ Zwischenbetriebliche Kooperation $^{6}$ stellt eine mögliche Strategie zur flexiblen Anpassung dar. ${ }^{7}$

Kooperationen sind mit einer Vielzahl von Anforderungen an ein Unternehmen verbunden. $\mathrm{Zu}$ berücksichtigende Aspekte für das kooperationswillige Unternehmen sind die Definition der Kooperationsaufgabe und des korrelierenden Partnerprofils, sowie die gezielte und erfolgreiche Partnersuche, die Bestimmung der Bindungsintensität und die Regulierungen in Bezug auf die Bedingungen zur $\mathrm{Zu}$ sammenarbeit, wie beispielsweise die leistungsgerechte Aufteilung und Zuweisung von Kooperationserfolgen. ${ }^{8}$

Aufgrund des zeitlichen Auseinanderfallens der Handlungen der Kooperationsakteure ${ }^{9}$ liegt die besondere Problematik zwischenbetrieblicher Kooperationen darin, dass bestimmte Handlungen des Partners weder überwacht noch erzwungen werden können, obwohl gerade diese Handlungen einen nachhaltigen Einfluss auf die Erreichung der Kooperationsziele haben und die Kooperationspartner im Hinblick auf diese erwarteten Handlungen kooperative Vorleistungen erbringen. Somit liegt das Problem kooperativer Beziehungen in der Gefahr, im Rahmen einer Kooperation vom Partner übervorteilt zu werden. Das ist dann der Fall, wenn sich

Vgl. D’Aveni (1995); Gimeno/Woo (1996)

$\mathrm{Vgl}$. Harvey et al. (2001); Jones (2002)

Vgl. D’Aveni (1995), S. 258

Harvey et al. (2001), S. 601

Vgl. Volberda (1996)

Rößl (1994, S. 54) definiert zwischenbetriebliche Kooperationen als „Organisationsform einer Austauschbeziehung zwischen Unternehmen, die in dieser Organisation - rechtlich freiwillig - zugunsten eines längerfristigen Vorteils auf kurzfristige Vorteile verzichten und die durch Schaffung dieser Organisation selbst nicht untergehen."

7 Vgl. Ring/Van de Ven (1992), S. 483; D'Aveni (1995), S. 396 ff.: Zaheer et al. (1998), S. 141

$8 \quad$ Vgl. Sydow/Windeler (1994), S. $1 \mathrm{ff}$.

9 Kooperatives Verhalten zeigt sich darin, dass der kooperationswillige Akteur von der kurzfristigen Vorteilhaftigkeitsorientierung zugunsten einer Verhaltensweise abweicht, deren langfristige Vorteile vom unsicheren zukünftigen Verhalten des Interaktionspartners abhängen (RößI 1994, S. 52 f: Pleitner/Röß1 1995, S. 672). 
dieser mit seiner Mittelwahl ausschließlich an eigenen kurzfristigen Interessen orientiert. $^{10}$

Durch die Opportunismusspielräume des Kooperationspartners erwachsen dem kooperationswilligen Unternehmen entsprechende Risiken. Das Unternehmen hat einerseits die Möglichkeit, die Opportunismusneigung des Kooperationspartners $\mathrm{zu}$ reduzieren und die, aufgrund der unbeschränkten Opportunismusspielräume bestehen bleibenden Unsicherheiten in Bezug auf das Verhalten des Kooperationspartners und die damit zusammenhängenden Risikokosten zu akzeptieren. Andererseits kann es sich dazu entschließen, Aktionen zur Verringerung der Opportunismusspielräume und der damit korrelierenden Unsicherheiten zu setzen, und die mit diesen Maßnahmen verbundenen Kosten (z.B. Vereinbarungs- und Überwachungskosten, Kosten des Aufbaus von Sanktionspotential) zu akzeptieren. ${ }^{11}$

In diesem Zusammenhang wird die Relevanz des Vertrauenskonstrukts sichtbar, da die Akzeptanz der Unsicherheiten „nur vor dem Hintergrund einer [relativ zum Unsicherheitsausmaß und den subjektiv bewerteten Konsequenzen opportunistischen Verhaltens, Anm. d. Verf.] tragfähigen Vertrauensbeziehung ${ }^{\text {“ } 12}$ möglich ist. Um Austauschbeziehungen aufzubauen und aufrechtzuerhalten, können zwischenbetriebliche Kooperationen demnach auf der Grundlage von Vertrauen funktionieren und müssen dies sogar, wenn andere Koordinationsmechanismen nicht verfügbar oder relativ, in Bezug auf die reduzierten Risiken, teuer sind. ${ }^{13}$

Der ökonomische Wert des Vertrauens lässt sich an den möglichen Konsequenzen von fehlendem Vertrauen erkennen. Beim Aufbau von Kooperationsbeziehungen wäre ein hoher Aufwand von Nöten, um Verträge in so einer Form abzufassen, dass die Gelegenheit für opportunistisches Verhalten seitens des Vertragspartners weitgehend eingeschränkt wird. Besteht zwischen Kooperationspartnern kein Vertrauen, so müssten Art und Zweck der Beziehung detailliert festgelegt, Verhaltensregeln schriftlich niedergelegt und bei etwaigen Konflikten unabhängige Schlichtungsstellen herangezogen werden. ${ }^{14} \mathrm{Da}$ vollständige Verträge (contingent claim contracts) aber nicht formulierbar sind, können diese Beziehungen ohne jegliches Vertrauen nicht funktionieren. ${ }^{15}$ Der ökonomische Wert von Vertrauen zeigt sich nun darin, dass Vertrauen einerseits nicht vollständig geregelte $\mathrm{Be}$ ziehungen ermöglicht, und andererseits dazu beiträgt, formale Verträge zu ersetzen und die Koordination zwischen Unternehmen zu erleichtern, wodurch es zu

10 Vgl. Wurche (1994), S. $144 \mathrm{f}$.

11 Vgl. Rößl (2006), S. 39

12 Rößl (2000), S. 94

13 Vgl. Ring/Van de Ven (1992), S. 490; Rößl (1994), S. 381; Fink et al. (2010), S. 101

14 Vgl. Fukuyama (1995), S. 400

15 Vgl. Semlinger (1993), S. 341; Ripperger (1998), S. 49 
einer Senkung der mit einer Austauschbeziehung verbundenen Kosten kommt. ${ }^{16}$ Vor allem in Situationen, in denen eine spontane Kooperation als Reaktion auf neue Herausforderungen nötig ist, ist die Zeit für langfristige Verhandlungen über Kooperationsverträge knapp. ${ }^{17}$ Vertrauen kann demnach einen Beitrag zur Senkung des Koordinations- und Kontrollaufwands und zur Einsparung von Verhandlungszeit leisten. Weiters kann es dazu beitragen, einen offeneren Austausch von Informationen zu praktizieren und eventuell auf eine weitgehende schriftliche Fixierung von Verträgen und Formalisierung zwischenbetrieblicher Regelungen zu verzichten. ${ }^{18}$ Auch wird der Generierung von Vertrauen im Hinblick auf den Erfolg von zukünftigen Kooperationen Bedeutung zugesprochen, und sie schafft, wenn der Aufbau gelingt, einen schwer imitierbaren Wettbewerbsvorteil ${ }^{19}$, der besonders in Krisensituationen von Relevanz ist. ${ }^{20}$

Aufgrund des hohen Wertes von Vertrauen in zwischenbetrieblichen Kooperationen, ist es von Bedeutung, die Determinanten der Evolution von maximenbasiertem Vertrauen ${ }^{21}$ zu kennen.

Als wesentliche Determinanten der Evolution von maximenbasiertem Vertrauen in zwischenbetrieblichen Kooperationen können die Reputation eines Partners, d.h. eines Unternehmens, sowie dessen perzipierte Geschichte genannt werden. ${ }^{22}$ Determinierend auf die Vertrauensbildung wirkt weiters die Selbstsicherheit des Vertrauensgebers, die ihn befähigt, ,etwaigen Vertrauensenttäuschungen mit Fassung entgegenzusehen, ohne sie als nur vorstellbare Möglichkeit schon jetzt als

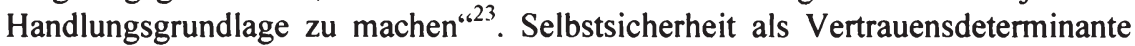
ersetzt hierbei die fehlenden Informationen, die eine Rechtfertigung der Vertrauenshandlung ermöglichen würden, womit auch die Risikobereitschaft des Vertrauensgebers eine vertrauensdeterminierende Wirkung aufweist. Die Ursache dieser Risikobereitschaft liegt in der Sicherheit des Systems, ,über genügend Ressourcen zur Gestaltung der Vertrauensbeziehung und zum Abfangen der gesteigerten Komplexität im Falle des Vertrauensbruchs zu besitzen.“24 Die

\footnotetext{
16 Vgl. Ripperger (1998), S. 270

17 Vgl. Heisig (1997), S. 151

$18 \mathrm{Vgl}$. Loose/Sydow (1994), S. 165

19 Vgl. Ripperger (1998), S. 271; Adler (2001), S. 216

$20 \quad$ Vgl. Sitkin et al. (1998), S. 394

21 Im Rahmen des maximenbasierten Vertrauens gehen die Akteure einer Vertrauensbeziehung davon aus, dass sich der Vertrauensnehmer - trotz seiner Möglichkeit opportunistisch zu handeln - kooperativ verhalten wird, und verhalten sich im Hinblick auf diese riskierte Erwartung kooperativ. Der Vertrauensgeber schenkt dem Vertrauensnehmer sein Vertrauen, weil er erwartet, dass der Vertrauensnehmer die vom Vertrauensgeber selbst herbeigeführte Verwundbarkeit nicht ausnützen wird. Vertrauen entsteht hier durch Maßnahmen, die die eigene Abhängigkeit der Parteien einer Vertrauensbeziehung erhöhen (Rößl/Fink 2006, S. 59 f.).

22 Vgl. Rößl (1994), S. 192 ff; Rößl/Fink (2006), S. 65 f.

23 Luhmann (2000), S. 102

24 Rößl (1994), S. 197
} 
folgenden ,Conditions of Trust" verfügen ebenso über eine determinierende Wirkung im Rahmen der Evolution von Vertrauen: Erreichbarkeit, Kompetenz, Konsistenz, Diskretion, Fairness, Integrität, Loyalität, Offenheit, Verlässlichkeit und Zugänglichkeit. ${ }^{25}$ Auch eine weitere empirische Untersuchung stützt die These, dass die subjektiv wahrgenommene Kompetenz, Aufrichtigkeit, Integrität, Einsatzbereitschaft, Diskretion und das Taktgefühl eines Akteurs das Vertrauen in ihn, d.h. sein Verhalten, positiv beeinflussen. ${ }^{26}$

Die Forschung zur Wirkung von Kompetenz als Vertrauensdeterminante steht am Anfang. Das gilt vor allem für Kompetenzen, die sich nicht auf Fachwissen beziehen. So nennen einige Studien Kompetenz als Vertrauensdeterminante ${ }^{27}$, unterlassen es aber zu erläutern, welche Kompetenzarten - z.B. die soziale Kompetenz im Allgemeinen oder die Kompetenz zur Signalisierung von Vertrauenswürdigkeit im Speziellen, die methodische Kompetenz zum Management einer Kooperation etc. - auf die Evolution von Vertrauen Einfluss nehmen, und wie sie auf die Vertrauensentwicklung wirken. Somit handelt es sich bei der Analyse der Rolle von Kompetenzen im Vertrauenskonstrukt und für die Vertrauensevolution um ein bestenfalls ansatzweise erschlossenes Forschungsfeld. Dabei stellt sich die Frage, ob es Kompetenzen gibt, die einen Einfluss auf die Entstehung von Vertrauen haben. Wenn die vertrauensdeterminierende Wirkung von Kompetenzen angenommen werden kann, ist in weiterer Folge zu klären, in welcher Art sie die Entstehung von Vertrauen begünstigt.

\section{FORSCHUNGSFRAGEN}

Bezugnehmend auf die Problemstellung und die in dem vorigen Kapitel identifizierten Forschungslücken wird in dieser Arbeit das Ziel verfolgt, den Beitrag der verschiedenen Kompetenzarten im Zuge der Evolution von Vertrauen zu eruieren. Es sollen folgende Forschungsfragen im Rahmen der Studie beantwortet werden:

- Wie wird Kompetenz definiert, welche Kompetenzarten gibt es und wie kann man sie operationalisieren?

- Welche Arten von Kompetenz beeinflussen die Evolution von Vertrauen?

- Wie beeinflussen die einzelnen Kompetenzarten des Vertrauensnehmers die Evolution von Vertrauen auf der Seite des Vertrauensgebers?

$25 \quad$ Vgl. Butler (1991), S. 648

26 Vgl. Moorman et al. (1993), S. 91 f.

27 Vgl. Kee/Knox (1970), S. 361; Butler (1991), S. 648; Sitkin/Roth (1993), S. 373; McKnight et al. (1998), S. 476 ff.; Adler (2001), S. 218 


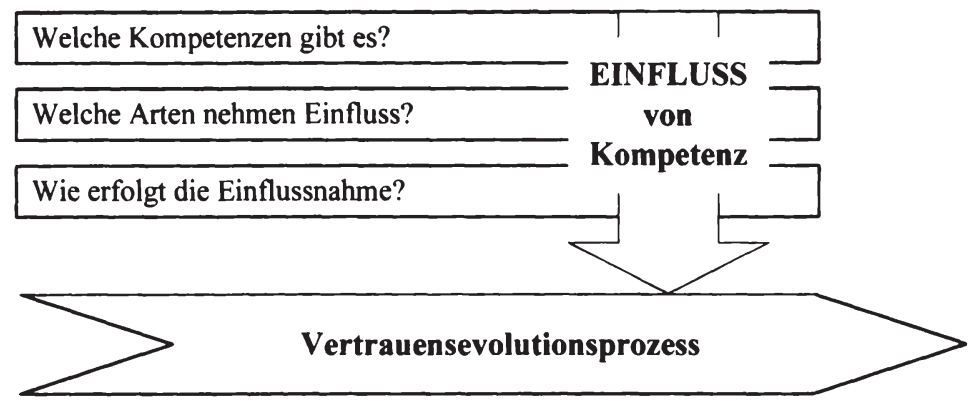

Abbildung 1: Forschungsfragen

Die Zielsetzung, die Wirkungsweise von Kompetenzen in Bezug auf die Entstehung von Vertrauen zu analysieren, umfasst verschiedene Subziele bzw. Aufgabenkomplexe.

Um eine Grundlage für weiterführende theoretische Überlegungen zu bilden, sind die Bedeutung und Einordnung von Vertrauen und Kompetenz zu klären. Dies impliziert einerseits die Herstellung von Bezügen zu anderen Theorien (z.B. Principal-Agent-Theorie, Systemtheorie, soziologische Handlungstheorie). Andererseits sind die Begriffe Vertrauen und Kompetenz von verwandten Konstrukten abzugrenzen (z.B. Zutrauen, Hoffnung, Reputation, Vertrauenswürdigkeit gegen Vertrauen, sowie Qualifikationen, Fertigkeiten und Fähigkeiten gegen Kompetenz). Die Erkenntnisse ermöglichen ein schärferes Begriffsverständnis und eine Strukturierung des Vertrauens- und des Kompetenzkonstrukts, womit die Grundlage für die Operationalisierung und Messung der Konstrukte im empirischen Teil der Arbeit geschaffen wird.

Darauf aufbauend ist es das Hauptziel, den Einfluss der Kompetenzarten (organisationale, individuelle Kompetenzen) im Vertrauensentstehungsprozess zu erforschen, sodass das Ergebnis dieser Arbeit ein Modell darstellt, das die Wirkungsweise der Kompetenzarten auf das Vertrauenskonstrukt abbildet.

\section{Methodologische Position}

Der fundamentale Zweck der im Rahmen dieser Arbeit stattfindenden Bemühungen orientiert sich an der Sichtweise Poppers, wonach das Anliegen der Wissenschaft darin besteht, ,[...] befriedigende Erklärungen zu finden für alles, was uns einer Erklärung zu bedürfen scheint. ${ }^{، 28}$ Die Forschungsaktivitäten werden von diesem Anliegen insofern beeinflusst, als das der im Zentrum des Interesses stehende Sachverhalt zunächst einer präzisen Beschreibung bedarf, um in weiterer Folge nach jenen Randbedingungen, Determinanten und Hypothesen zu suchen, 
die zu seiner Erklärung beitragen. Auf dieser Basis erfolgt dann die Entwicklung eines Modells als Abbild der komplexen Realität, welches schließlich einer empirischen Überprüfung zu unterziehen ist. ${ }^{29}$

Um Intransparenz zu vermeiden und verlässliches Wissen zu schaffen ist es erforderlich, dass der Forschungsprozess nach Regeln abläuft, deren Einhaltung überprüfbar ist. ${ }^{30} \mathrm{Da}$ sich die Anforderungen der früheren wissenschaftstheoretischen Position (im Sinne von wahren, neuen und allgemeingültigen Ergebnissen) einer strengen Überprüfbarkeit entziehen und unlösbare erkenntnistheoretische Probleme aufwerfen ${ }^{31}$, folgt die Arbeit dem heuristischen Wissenschaftsprogramm.

Dem Forschungsprojekt liegen daher folgende heuristische Prinzipien zugrunde ${ }^{32}$ :

- Nachvollziehbarkeit und intersubjektive Transparenz

- Nützlichkeit der Ergebnisse (Bestimmung jener Elemente der subjektiven Realität, die Hinweise auf relevante Wirkungsprinzipien liefern)

- Überprüfung der Aussagen unter Berücksichtigung der subjektiven Implikationen des Untersuchungsergebnisses

- Methoden- und Ideenpluralismus ${ }^{33}$

\section{METHODISCHE VoRgEHENSWEISE}

Das zur Beantwortung der Forschungsfragen gewählte Forschungsdesign umfasst eine theoretische Analyse und eine empirische Untersuchung. Im Anschluss an die theoriegestützte begriffliche Konkretisierung und Operationalisierung der beiden Forschungsobjekte ,Vertrauen' und ,Kompetenz' werden mit Hilfe von Ansätzen aus der Organisationstheorie die Grundlagen des Erkenntniszusammenhangs und des Bezugsrahmens erarbeitet. Daraus werden in einem weiteren Arbeitsschritt vorläufige Thesen zum Einfluss der Kompetenzarten auf den Entwicklungsprozess von Vertrauen generiert und in Form eines Modells dargestellt, das in weiterer Folge entsprechend der Ergebnisse der anschließenden empirischen Untersuchung adaptiert wird.

29 Vgl. Peter (1999), S. 63

30 Vgl. Leinfellner (1980), S. $161 \mathrm{ff} ;$ Mugler (2005), S. 19

31 Vgl. Feyerabend (1980), S. 191; Opp (1999a), S. 184 ff.

32 Vgl. Rößl (2005), S. 41 ff.

33 Feyerabend (1976, S. 32) schreibt dazu: „Die Welt, die wir erforschen, ist etwas weitgehend Unbekanntes. Daher müssen wir uns offenhalten, dürfen uns nicht im Voraus beschränken.“ 


\subsection{Theoriegeleitete Thesengenerierung}

Theoretische Ansätze dienen in der Dissertation an drei Stellen als Argumentationsgrundlage:

- Erstens werden die Bedeutung und die Funktionsweise des Vertrauenskonstrukts auf Basis des soziologischen Systemansatzes nach Luhmann und der Ansätze der ,Neuen Institutionenökonomie‘ erläutert. Die eigentliche Operationalisierung des Vertrauenskonstrukts erfolgt sodann auf der Grundlage der Principal-Agent-Theorie, da diese hierfür, mit Blick auf die dieser Arbeit zugrunde liegenden Fragestellungen, am zweckmäßigsten ist.

- Zweitens werden die Relevanz und die Funktionsweise des Kompetenzkonstrukts aus Sicht der soziologischen Handlungstheorie und aus der Perspektive der Principal-Agent-Theorie analysiert.

- Drittens wird bei der Erarbeitung des Wirkungsmodells (Erkenntniszusammenhang), das heißt bei der Entwicklung des Bezugsrahmens der Wirkung von Kompetenz auf die Evolution von Vertrauen, ebenso auf die PrincipalAgent-Theorie zurückgegriffen.

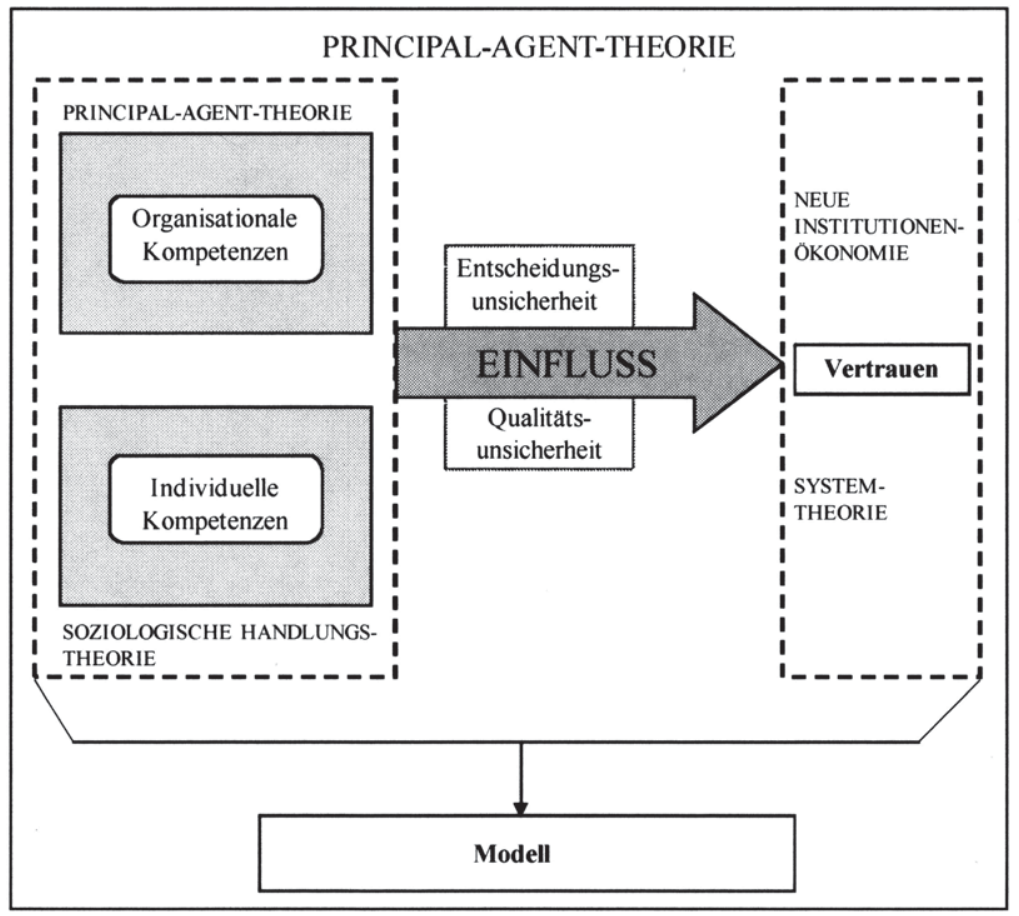

Abbildung 2: Theoriegeleitete Thesengenerierung 


\subsection{Empirische Untersuchung}

Zur Überprüfung des Modells wird eine Laboruntersuchung durchgeführt. Die Entscheidung für die Umgebung, in der das generierte Modell untersucht wird, ergibt sich aus dem Erklärungsinteresse der vorliegenden Arbeit. Da in den vergangenen Jahrzehnten in verschiedenen Fachdisziplinen bedeutende theoretische Vertrauensmodelle entwickelt wurden, die interdisziplinäre Vertrauensforschung sich aber erst im Anfangsstadium ihrer Entwicklung befindet ${ }^{34}$, verfolgt die vorliegende Arbeit das Ziel, bereits entwickelte theoretische Ansätze und empirische Forschungsergebnisse zusammenzuführen und grundlegende Zusammenhänge zu überprüfen. Der internen Validität der Untersuchung wird daher eine erhöhte Bedeutung beigemessen.

Im Hinblick auf die Untersuchungsmethoden im Labor ist grundsätzlich zwischen Beobachtung und Experiment zu differenzieren. Da die systematische Beobachtung der Entstehung von Vertrauen aber keine Variation einer oder mehrerer unabhängiger Variablen unter gleichzeitiger Kontrolle anderer Variablen zulässt, erlaubt dieses Verfahren keine intern valide Klärung der Fragen bezüglich der Determinanten der Vertrauensentstehung. Hingegen stellt das Experiment die zentrale Untersuchungsmethode betriebswirtschaftlicher Entscheidungsforschung dar, dessen einzigartige Stärke in der Überprüfung von Ursache-Wirkung-Beziehungen liegt. Durch isolierte Variation einer oder mehrerer unabhängiger Variablen, wie z.B. wahrgenommene Kompetenz/nicht wahrgenommene Kompetenz, und der Feststellung der Veränderung nachgelagerter Variablen, wie z.B. Vertrauen, wird unter kontrollierten Bedingungen eine Analyse kausaler Zusammenhänge möglich. Somit werden in der vorliegenden Untersuchung die einem Experimentaldesign zugänglichen Modellelemente auf zwischenmenschliche Beziehungen übertragen und laborexperimentell überprüft. Um eine hinreichende Adaption des Modells gewährleisten zu können, wird die laborexperimentelle Variation der unabhängigen Variable mit psychometrischen Tests zur Erfassung personen- und situationsbedingter Faktoren verbunden. An dieser Stelle sei auf Teil $\mathrm{V}$ dieser Arbeit verwiesen, wobei in Kap. 1 die Entscheidung für die Untersuchungsstrategie ausführlich begründet, in Kap. 2 die Gestaltung des Experiments detailliert beschrieben und in Kap. 3 die Messung der interessierenden Variablen erläutert wird. Nachfolgende Graphik gibt abschließend einen Überblick über die methodische Vorgehensweise. 


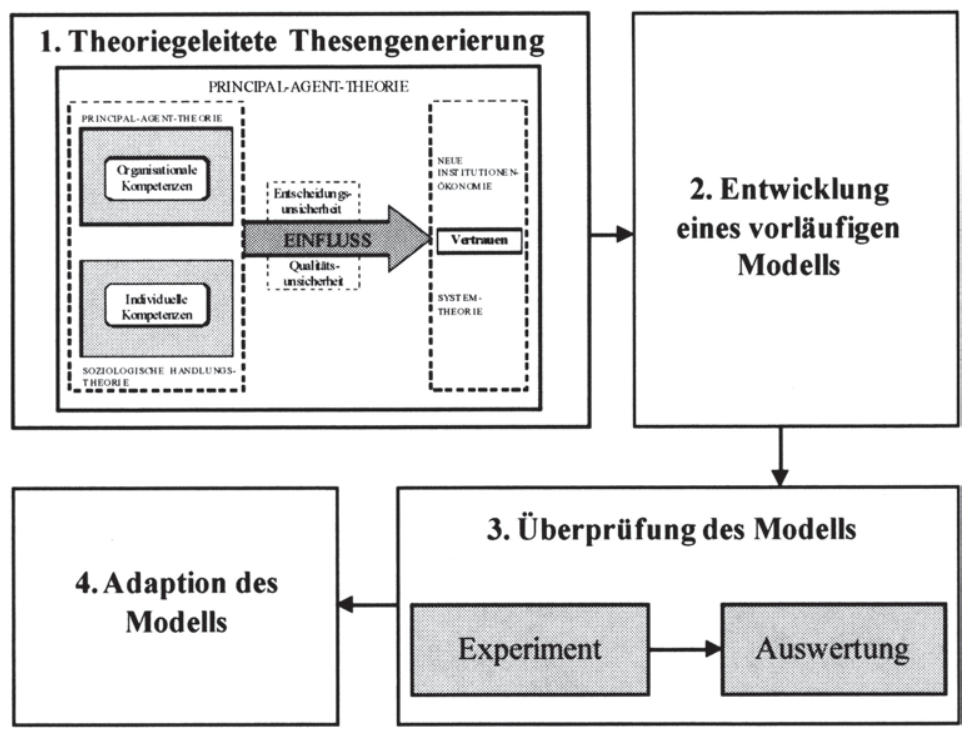

Abbildung 3: Methodische Vorgehensweise

\section{AUfBAU DER ARBEIT}

Die Arbeit besteht aus den folgenden sieben Teilbereichen:

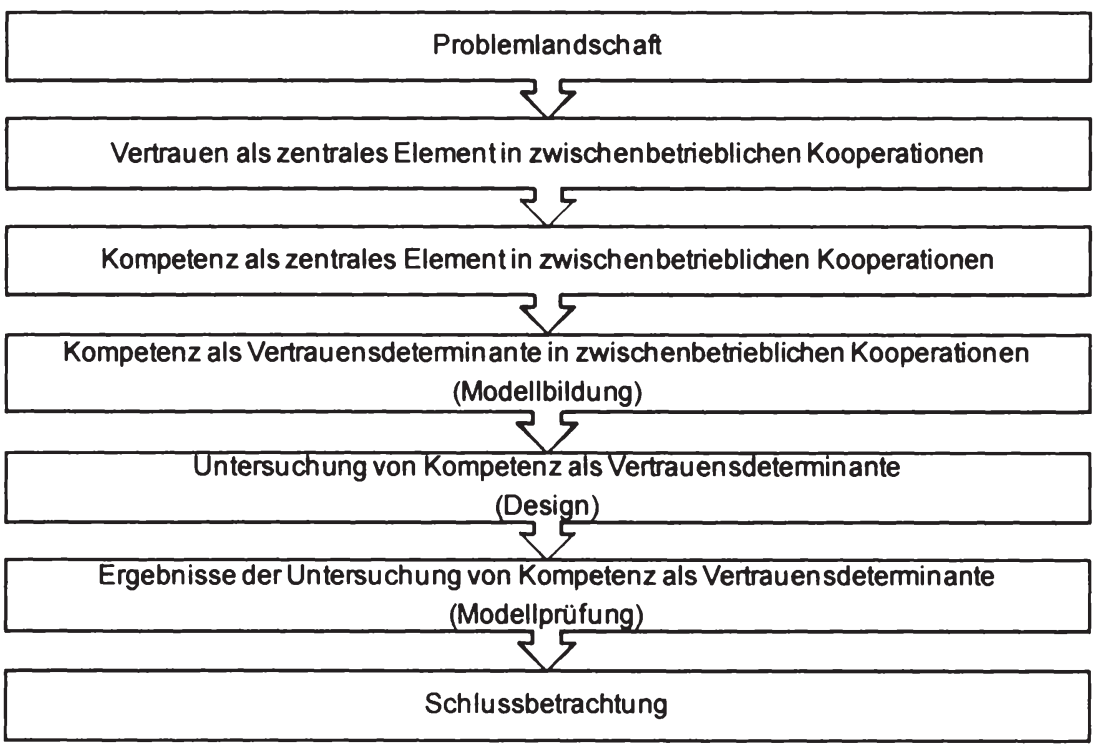

Abbildung 4: Struktur der Arbeit 
Nach der in Teil I erfolgten Einleitung mit Problemstellung und Zielsetzung, Aufbau und Methodik der Arbeit stellt Teil II den theoretischen Bezugsrahmen zur Analyse von Vertrauen in zwischenbetrieblichen Kooperationen dar. Kapitel 1 beschäftigt sich mit Vertrauen als interdisziplinäres Forschungsgebiet. So wird das Vertrauenskonstrukt aus soziologischer und ökonomischer Perspektive erläutert. Anschließend erfolgt in Kapitel 2 eine begriffliche Abgrenzung gegenüber vertrauensnahen Begriffen wie Zutrauen, Zuversicht, Hoffnung, Vertrauenswürdigkeit, Reputation und Misstrauen. In Kapitel 3 erfolgt die begriffliche Festlegung von Vertrauen. Anschließend steht in Kapitel 4 die Principal-Agent-Theorie (PAT) als Basis für die Analyse von Vertrauensbeziehungen im Mittelpunkt. Nach der Erläuterung der opportunistischen Verhaltensspielräume aus Sicht der PAT wird die Bedeutung von Vertrauen in der Agency-Theorie illustriert. Abschließend werden auf Basis der PAT die Maßnahmen zur Gestaltung der Vertrauensbeziehung mit dem Prinzipal als Vertrauensgeber und dem Agenten als Vertrauensnehmer untersucht. Im Rahmen von Kapitel 5 stehen der Vertrauensgeber und der Vertrauensnehmer als Elemente des Vertrauenskonstrukts im Vordergrund. Die Entscheidungssituation des Vertrauensgebers charakterisiert sich anhand der Vertrauenserwartung und der Vertrauenshandlung. Als zentrale Variable der Entscheidungssituation des Vertrauensnehmers wird dessen Vertrauenswürdigkeit analysiert. Nach einer Analyse der Vertrauensdeterminanten in Kapitel 6, bildet Kapitel 7 den Abschluss dieses Teils mit einem Überblick über Vertrauensmessverfahren.

Teil III stellt den theoretischen Bezugsrahmen zur Analyse von Kompetenz in zwischenbetrieblichen Kooperationen dar. So erfolgt in Kapitel 1 die begriffliche Festlegung von Kompetenz und die Abgrenzung von verwandten Begriffen wie Qualifikationen, Ressourcen, Fertigkeiten und Fähigkeiten. In Kapitel 2 werden die Kompetenzarten auf organisationaler und individueller Ebene verrastert und voneinander abgegrenzt. Kapitel 3 gibt einen Überblick über Ansätze zur Identifikation organisationaler und individueller Kompetenzen (Kompetenzmessverfahren). Abschließend beleuchtet Kapitel 4 Kompetenz aus Sicht der soziologischen Handlungstheorie und der PAT.

Nach der begrifflichen Klärung und inhaltlichen Auseinandersetzung mit den Konstrukten Vertrauen und Kompetenz erfolgt in Teil IV die Modellierung des Einflusses von Kompetenz auf die Entstehung von Vertrauen. Dass es sich bei der Analyse der Rolle von Kompetenz im Vertrauensentstehungsprozess um ein bestenfalls ansatzweise erschlossenes Forschungsfeld handelt, wird anhand des gegenwärtigen Forschungsstandes in Kapitel 1 aufgezeigt. Ausgangspunkt der Modellentwicklung stellt die Erläuterung der methodischen Grundlagen und Vorgehensweisen im Zuge der Modellgenerierung dar (Kapitel 2). Um die interdisziplinären Erkenntnisse aus der Vertrauens- und Kompetenzforschung modelltheoretisch integrieren zu können, erfolgt auf Basis der in dieser Arbeit analy- 
sierten Ansätze in Kapitel 3 die Operationalisierung von Kompetenz und in Kapitel 4 die Operationalisierung von Vertrauen. Kapitel 5 bildet den Abschluss dieses Teils mit einem Modell, das den Einfluss der Kompetenzarten auf den Vertrauensentwicklungsprozess hypothetisiert.

In Teil V erfolgt die Konzeption der Untersuchung zur Überprüfung der hypothetisierten Kausalbeziehung zwischen Kompetenz und Vertrauen. In Kapitel 1 wird das Untersuchungsziel in ein Untersuchungsdesign umgesetzt. Kapitel 2 beschreibt die von den Probanden zu bearbeitende betriebswirtschaftliche Entscheidungsaufgabe, sowie die Manipulation der unabhängigen Variable. Nach dem Überblick über die Verfahren zur Datengewinnung erfolgt in Kapitel 3 auch die Erläuterung der Messung der interessierenden Variablen. Kapitel 4 beschreibt die Ergebnisse der Pretests, sowie die zu untersuchende Stichprobe und den Untersuchungsablauf. Den Abschluss dieses Teils bildet Kapitel 5 mit einer Qualitätsanalyse der erhobenen Daten und der Beschreibung der statistischen Auswertungsmethoden zur Hypothesenprüfung.

In Teil VI werden die Ergebnisse der Untersuchung erläutert. In Kapitel 1 erfolgt die Prüfung der methodischen Effektivität des Experiments. Die Ergebnisse der Regressionsanalyse werden in Kapitel 2 beschrieben und abschließend anhand eines empirischen Modells in Kapitel 3 dargestellt.

In Teil VII erfolgt die Diskussion der Untersuchungsbefunde vor dem Hintergrund des in der Einleitung dargelegten Erklärungsinteresses der vorliegenden Arbeit. Im Anschluss an die Zusammenführung der Ergebnisse dieser Arbeit in Kapitel 1, wird in Kapitel 2 auf den Erklärungsbeitrag der Arbeit eingegangen. Abschließend werden in Kapitel 3 die inhaltlichen und methodischen Grenzen der Arbeit erläutert und Anregungen für weiterführende Forschungsaktivitäten gegeben. 
Isabella Hatak - 978-3-631-74970-8 Downloaded from PubFactory at 01/11/2019 09:36:38AM via free access 


\section{TEIL II: VERTRAUEN ALS ZENTRALES ELEMENT IN ZWISCHENBETRIEBLICHEN KOOPERATIONEN}

\section{Die BedEutung Von Vertrauen aUS VERSCHIEDENEN PERSPEKTIVEN}

Der Vertrauensbegriff wird in der Literatur auf vielfältige Art und in verschiedenen Zusammenhängen verwendet. Im allgemeinen Sprachgebrauch wird Vertrauen als Basis aller sozialen Beziehungen mit dem Glauben an die Verlässlichkeit, Ehrlichkeit und Gerechtigkeit einer Person oder einer Gruppe von Personen gleichgesetzt. Neben dieser allgemeinsprachlichen Definition finden sich hauptsächlich in den Bereichen Soziologie, Philosophie, Psychologie, Wirtschaftswissenschaft, Rechtswissenschaft und Pädagogik eine Vielzahl unterschiedlicher Begriffsverständnisse. ${ }^{35}$

Die Menge an fachspezifischen Begriffsdeutungen hat zur Folge, dass zwischen der bedeutenden Rolle, die Vertrauen in zwischenmenschlichen Beziehungen im Allgemeinen und in wirtschaftlichen Austauschbeziehungen im Besonderen einnimmt, und seiner wissenschaftlichen Erfassung und Explikation beachtliche Abweichungen bestehen. Begriffliche Unklarheiten und Unstimmigkeiten erschweren die Bildung eines gemeinsamen Verständnisses von Vertrauen ${ }^{36}$ und werden durch Forschungsdefizite im Bereich der ökonomischen Theorie verstärkt. ${ }^{37}$ Zwar betonen zahlreiche empirische Untersuchungen im Rahmen einer ökonomischen Perspektive die Bedeutung von Vertrauen für den Erfolg zwischenbetrieblicher Kooperationen und für die Zufriedenheit der Kooperationspartner, jedoch bestehen unterschiedliche Auffassungen darüber, ob Vertrauen als Voraussetzung für erfolgreiche Kooperationen oder als deren Ergebnis zu interpretieren ist. Im Rahmen der Untersuchungen zeigt sich weiters, dass Vertrauen oftmals als Explanans für kooperatives Verhalten herangezogen wird, ohne allerdings zu klären, was unter Vertrauen selbst $z u$ verstehen ist ${ }^{38}$, womit die Erfassung und Analyse des Vertrauenskonstrukts und der Vertrauensdeterminanten erschwert werden.

Für ein besseres Verständnis des Begriffs Vertrauen ist es daher erforderlich, unterschiedliche Blickwinkel einzunehmen, um die zwischen Fachdisziplinen be-

35 Vgl. Krystek/Zumbrock (1993), S. 4 f.; Plötner (1995), S. 35 f.; Krystek et al. (1997), S. 367 f.; Brockhaus (2008a)

36 Shapiro (1987, S. 625) merkt dazu an: "Its [trust, Anm. d. Verf.] conceptualization has received considerable attention in recent years, resulting in a confusing potpourri of definitions applied to a host of units and levels of analysis".

38 Vgl. Dwyer et al. (1987); Diller/Kusterer (1988); Young/Wilkinson (1989); Anderson/Narus (1990); Moorman et al. (1993); Morgan/Hunt (1994); Werner (1996); Pieper (2000) 
stehenden Barrieren zu überwinden. Da Vertrauen im Rahmen der vorliegenden Arbeit im Hinblick auf Austauschbeziehungen zwischen Unternehmen analysiert wird, ist es nicht ausreichend, es im Sinne der Psychologie ausschließlich als ein Merkmal von Individuen zu betrachten. Vielmehr ist Vertrauen in Anlehnung an die Soziologie als Merkmal sozialer Beziehungen, die sich durch die Beteiligung mindestens zweier Akteure kennzeichnen ${ }^{39}$, bei denen es sich sowohl um individuelle, als auch korporative Partner wie beispielsweise Unternehmen handeln kann, zu verstehen. In Anlehnung an diese Forderung, und unter Berücksichtigung des Anspruchs dieser Arbeit - die Determinanten der Evolution von Vertrauen im Rahmen von zwischenbetrieblichen Kooperationen zu untersuchen - wird Vertrauen im Folgenden sowohl aus soziologischer, als auch aus ökonomischer Perspektive betrachtet.

\subsection{Vertrauen aus soziologischer Perspektive}

Da sich die persönlichkeitstheoretischen Ansätze vorrangig mit der grundsätzlichen Vertrauensbereitschaft beschäftigen, dies aber nicht zu einer Erklärung der Entstehung von Vertrauensbeziehungen und deren Bedeutung beiträgt, ist eine Konzentration auf Konzepte, die sich mit der sozialen Ebene befassen, notwendig. Soziale Phänomene sind die Domäne der Soziologie. Insofern steht im folgenden Kapitel die soziologische Perspektive auf das Vertrauensphänomen, die sich stark an philosophischen, anthropologischen und ethischen Vertrauensverständnissen orientiert, im Vordergrund. Im Rahmen der soziologischen Sicht bietet sich die Option, die Bedeutung von Vertrauen innerhalb sozialer Systeme zu untersuchen $^{40}$, weshalb im Folgenden die Überlegungen der Soziologen Luhmann und Coleman, deren Perspektiven zu den bis dato wohl bedeutendsten soziologischen Arbeiten zur Vertrauensthematik zählen ${ }^{41}$, einer näheren Betrachtung unterzogen werden.

\subsubsection{Vertrauen als Mechanismus zur Reduktion sozialer Komplexität}

Nach Luhmanns systemtheoretischer Auffassung, die sich an den philosophischen Abhandlungen von Bollnow (1958) und Schottländer (1957) orientiert ${ }^{42}$, stellt Vertrauen eine grundsätzliche Voraussetzung sozialen Lebens dar: „Ohne jegliches Vertrauen könnte er [der Mensch, Anm. d. Verf.] morgens sein Bett nicht verlassen. Unbestimmte Angst, lähmendes Entsetzen befielen ihn. [...] Alles wäre

39 Vgl. Shapiro (1987), S. 626; Dasgupta (1988), S. 49 f.; Preisendörfer (1995), S. 264; Porter-Liebeskind/Lumerman-Oliver (1998), S. 119; Neubauer (1999), S. 92

$40 \quad$ Vgl. Shapiro (1987)

41 Vgl. Eberl (2003), S. 140

42 Vgl. Pieper (2000), S. 79 
möglich. Solch eine unvermittelte Konfrontierung mit der äußersten Komplexität der Welt hält kein Mensch aus. “43

Die Welt gefährdet in ihrer „unfassbaren Komplexität “44 das fortwährende Bestehen realer Systeme, einschließlich der Menschen, da sie mehr Möglichkeiten enthält, ,als die, auf die das System sich erhaltend reagieren kann “45. Um seiner Bestandsgefährdung zu entgehen und seine Handlungsfähigkeit zu sichern, muss der Mensch Verhaltensmuster konzipieren, die es ihm ermöglichen, mit dieser Komplexität zurechtzukommen bzw. diese zu verringern. ${ }^{46}$ Vertrauen stellt mit seiner „Funktion für die Erfassung und Reduktion dieser Komplexität“47 ein derartiges Handlungsmuster dar, indem es den Ausschluss bestimmter Handlungsalternativen ermöglicht ${ }^{48}$. Der Erweis von Vertrauen als Form von Sicherheit ${ }^{49}$ kann als Vergegenwärtigung der Zukunft verstanden werden. ${ }^{50}$ Indem der Mensch vertraut, schließt er ,gewisse Entwicklungsmöglichkeiten von der Berücksichtigung aus“, wodurch er ,gewisse Gefahren, die nicht ausgeräumt werden können, die aber das Handeln nicht irritieren sollen" 51 beseitigt. Vertrauen stellt somit einen bewussten Verzicht auf Informationen dar. ${ }^{52}$

Im Grunde genommen ist Vertrauen jedoch nie begründbar und kommt immer „durch Überziehen der vorhandenen Information zustande“.53 . Vertrauen ist damit eine Mischung aus Wissen und Nichtwissen, womit es als riskantes Handlungsmuster ${ }^{54}$ bzw. als „Problem der riskanten Vorleistung “55 verstanden werden kann. Vertrauen steht damit immer im Zusammenhang mit einer unsicheren Alternative und liegt nur dann vor, wenn eine Entscheidung im Endeffekt aufgrund der vertrauensvollen Erwartung getroffen wird. ${ }^{56}$ Darüber hinaus besteht der Risikoaspekt darin, dass der Nachteil eines Vertrauensbruchs größer sein kann als der Nutzen, der aus einem Vertrauenserweis resultiert. Auch ergibt sich das mit dem Vertrauensakt verbundene Risiko daraus, dass Vertrauen blind, leichtsinnig und routinemäßig erwiesen werden kann. ${ }^{57}$

\footnotetext{
43 Luhmann (2000), S. 1

44 Luhmann (2000), S. 4

45 Luhmann (2000), S. 5

46 Vgl. Luhmann (2000), S. $1 \mathrm{ff}$.

$47 \quad$ Luhmann (2000), S. 38

48 Vgl. Luhmann (2000), S. 23 f.

49 Vgl. Luhmann (2000), S. 13

50 Vgl. Pieper (2000), S. 80; Luhmann (2000, S. 9): "Wer Vertrauen erweist, nimmt Zukunft vorweg."

$51 \quad$ Luhmann (2000), S. 30

52 Vgl. Pieper (2000), S. 81; Luhmann (2000), S. 27

53 Luhmann (2000), S. 31

54 Luhmann (2000, S. 31) schreibt dazu: „Vertrauen bleibt ein Wagnis.“

$55 \quad$ Luhmann (2000), S. 27

56 Der Soziologe Simmel (1968, S. 263) schreibt dazu: „Der völlig Wissende braucht nicht zu vertrauen, der völlig Nichtwissende kann vernünftigerweise nicht einmal vertrauen."

Vgl. Schmitz (1997), S. 156; Luhmann (2000), S. 28 f
} 
Nach Luhmann kann zwischen persönlichem Vertrauen und Systemvertrauen unterschieden werden. Persönliches Vertrauen ,ist dann die generalisierte Erwartung, daß der andere seine Freiheit, das unheimliche Potential seiner Handlungsmöglichkeiten, im Sinne seiner Persönlichkeit handhaben wird - oder genauer, im Sinne der Persönlichkeit, die er als die seine dargestellt und sozial sichtbar gemacht hat. “58 Überträgt man diese Grundaussage auf Kooperationen, so lässt sich erkennen, dass Bezugspunkte des Vertrauens in Kooperationen individuelle Personen sein können. Die Erwartung, dass der Kooperationspartner sich in Zukunft nicht opportunistisch verhalten wird, kann an eine einzelne Person geknüpft sein. Individuelles Vertrauen auf persönlicher Ebene kann daher als Vertrauen innerhalb der Rollenbeziehung, in der die beruflich dargestellte und sozial sichtbar gemachte Persönlichkeit betrachtet wird, verstanden werden. ${ }^{59}$ Für die Entstehung von Vertrauen innerhalb von Kooperationsbeziehungen und damit das Erbringen einer riskanten Vorleistung, zu verstehen als supererogatorische Leistung im Sinne eines positiven Überziehens normaler Erwartungen, müssen nach Luhmann bestimmte Voraussetzungen erfüllt sein. Eine zentrale Bedeutung nimmt in diesem Zusammenhang die Vertrautheit mit dem Kooperationspartner und dem Interaktionskontext ein. Vertrautheit ist im Gegensatz zu Vertrauen unvermeidbar mit dem Leben verknüpft ${ }^{60}$ und beruht auf einem in der Vergangenheit gewonnenem Wissen. Hingegen bezieht sich Vertrauen auf die Zukunft und stellt damit ein freiwilliges Handlungsmuster dar. Eben diese Freiwilligkeit ist auch dem Kooperationspartner zu unterstellen. ${ }^{61}$ Neben der Voraussetzung eines gewissen Maßes an Vertrautheit bedarf es nach Luhmann einer Situation, die Handlungsspielräume erlaubt und nicht institutionell oder historisch bereits festgelegt ist, sodass Handlungen als persönlich motivierte Verhaltensentscheidungen zugerechnet werden können. Für die Entstehung einer Vertrauensbeziehung ist es weiters erforderlich, die Situation so zu definieren, dass der Vertrauende auf seinen Partner angewiesen ist. Nur wenn sich der Vertrauende einem potentiellen Vertrauensbruch durch Erbringung der bereits erwähnten riskanten Vorleistung aussetzt, kann gegebenenfalls eine Vertrauensbeziehung, die einen beiderseitigen Einsatz erfordert, entstehen. ${ }^{62}$ Damit stellen nach Luhmann Vertrautheit, unterstellte Handlungsspielräume und Abhängigkeit vom Partner die drei Grundvoraussetzungen für ein Überziehen der normalen Erwartungen und damit für individuelle Vertrauensbeziehungen auf persönlicher Ebene dar.

$58 \quad$ Luhmann (2000), S. 48

$59 \quad$ Vgl. Schmitz (1997), S. 161

60 Luhmann (1988), S. 95: „[...] familiarity is an unavoidable fact of life [...]."

61 Vgl. Luhmann (2000), S. $22 \mathrm{f}$.

62 Vgl. Luhmann (2000), S. $51 \mathrm{ff}$. 
Neben dem bislang behandelten Vertrauen auf persönlicher Ebene betrachtet Luhmann das Systemvertrauen als unpersönliche Vertrauensform ${ }^{63}$, die nicht unmittelbar an die Identität von Individuen anknüpft. Bei dem Vertrauen auf Systemebene wird nicht auf einzelne Individuen, sondern auf Systeme (z.B. auf eine andere Unternehmung als System, womit das Vertrauen auf der organisationalen Ebene der Kooperation entsteht ${ }^{64}$ bzw. auf ihr Funktionieren vertraut. ${ }^{65}$ Das Systemvertrauen in gesellschaftliche Funktionssysteme kann damit als Vertrauen in dessen Steuerungsmechanismen, bzw. gemäß Luhmanns Terminologie als Vertrauen in die symbolisch generalisierten Kommunikationsmedien (z.B. Geld, Macht, Wahrheit) ${ }^{66}$, verstanden werden. Das Ausmaß des Systemvertrauens in das jeweilige Kommunikationsmedium lässt sich sodann anhand der Häufigkeit seines Gebrauchs erkennen. Für Systemvertrauen ist lediglich das Zutrauen, dass die systembedingten Gefahren (z.B. Verlust des Geldwertes) in Zaum gehalten werden, erforderlich. Das bedeutet aber nicht, dass persönliches Vertrauen überflüssig wird, ganz im Gegenteil: Durch weitreichendere und intensivere Teilnahme an den Systemen entstehen nach Luhmann gleichzeitig mehr Situationen, in denen sich Vorteile erkennen und scheinbar realisieren lassen. Mit der $\mathrm{Zu}$ nahme riskanter Situationen nimmt auch der Bedarf an persönlichem Vertrauen zu. ${ }^{67}$ Während sich persönliches Vertrauen somit auf den Umgang mit Risiko bezieht, beschreibt Systemvertrauen dagegen den Umgang mit Gefahren. Beide Konzepte beruhen auf Erwartungen, die enttäuscht werden können. Der Unterschied zwischen Risiken und Gefahren besteht nach Luhmann allerdings darin, dass das Risiko, möglichen Erwartungsenttäuschungen gegenüberzustehen als vom eigenen, vorangegangenen Verhalten abhängig betrachtet wird. Während damit das Risiko durch eigenes, entsprechendes Verhalten kontrollierbar wird „you can avoid taking the risk“68 - kennzeichnen sich Gefahren dadurch, dass sie außerhalb der eigenen Kontrolle liegen. Dementsprechend zieht Systemvertrauen, anders als persönliches Vertrauen, keine Alternativen in Erwägung. Treten dann doch Erwartungsenttäuschungen ein, dann werden diese external attributiert: Die Enttäuschung wird nicht wie im Falle des persönlichen Vertrauens in Verbindung

\footnotetext{
63 Vgl. Luhmann (2000), S. 60

$64 \quad$ Vgl. Schmitz (1997), S. 163

65 Vgl. Klaus (2002), S. 89

66 Vgl. Luhmann (2000), S. 61

67 Vgl. Eberl (2003), S. 154.; Luhmann (2000), S. 60 ff., sowie Luhmann (1988), S. 102 ff., der in diesem Zusammenhang auf einen möglichen „vicious circle“ hinweist: Mangelndes Systemvertrauen erfordert zur Stabilisierung ein höheres persönliches Vertrauen, da Handeln in diesem System nun verstärkt zum Risiko wird. Andererseits hemmt das mangelnde Systemvertrauen die Entstehung von persönlichem Vertrauen. Fehlendes Systemvertrauen führt also zu mangelndem persönlichen Vertrauen, was wiederum negative Auswirkungen auf das Systemvertrauen hat: .,A lack of confidence may mean [...] a lack of trust, and a lack of trust means that behaviour which presupposes trust will be ruled out" (Luhmann 1988, S. 103). 
mit dem eigenen, vorangegangen Verhalten gebracht und damit internal attributiert, sondern anderen zugerechnet. Darüber hinaus erfordert das Systemvertrauen keine supererogatorische Leistung. Die Gefahren des täglichen Lebens und damit verbundene mögliche Erwartungsenttäuschungen werden ignoriert, um nicht in einen permanenten Zustand der Unsicherheit zu geraten, der Handlungen unmöglich macht. Dementsprechend erhöht sich durch Systemvertrauen die Anzahl der Handlungsmöglichkeiten immens. Da sich der Vertrauende beim Treffen von Entscheidungen auf fremde Informationsverarbeitung stützen kann, entfällt die allumfassende, selbstständige Informationsbeschaffung. ${ }^{69}$ Voraussetzung für den Aufbau von Systemvertrauen ist letztlich die Tatsache, dass vertrauensvolles Verhalten den Normalfall darstellt. Erst vor der dem Hintergrund, „[...] daß andere auch vertrauen und daß diese Gemeinsamkeit des Vertrauens bewußt wird" ${ }^{40}$, kann man selbst vertrauen. Das Vertrauen in Systemvertrauen bedarf allerdings keiner einzelfallbezogenen Reflektion, sondern es wird vielmehr als Verhalten (z.B. „richtiger“ Umgang mit Geld) gelernt, sodass unkontrollierbare Ängste (z.B. was geschieht, wenn alle ihr Geld abheben wollen?) schrittweise abgebaut werden, wodurch die Vertrauensbildung erleichtert wird. Beim persönlichen Vertrauen spielt die Voraussetzung, dass andere, unbeteiligte Dritte, in gleicher Weise wie man selbst Vertrauen schenken, im Vergleich zum Systemvertrauen nur eine untergeordnete Rolle. ${ }^{71}$

Damit sind beim Systemvertrauen weniger emotionale, sondern darstellungsgebundene Vertrauensgrundlagen vorherrschend. Grundsätzlich hat die Selbstdarstellung für beide Formen des Vertrauens eine hohe Relevanz. Wer Vertrauen erwerben will, muss zunächst mit der „Darstellung des eigenen Selbst als einer sozialen, sich in Interaktionen aufbauenden, mit der Umwelt korrespondierenden Identität ${ }^{\text {“72 }}$ am sozialen Leben teilnehmen. Eine soziale Distanzierung hingegen verhindert den Erwerb von Vertrauen, da sie keine Lern- und Prüfmöglichkeiten erlaubt. Um Vertrauen zu erwerben, ist es nach Luhmann weiters erforderlich, dass fremde Erwartungen in die eigene Selbstdarstellung eingebaut werden. Diese Forderung darf aber nicht mit reinem Konformismus verwechselt werden, da Rollenkonformität dazu führt, dass das Selbst überhaupt nicht sichtbar wird, womit ein Vertrauenserwerb unmöglich wird. Von Bedeutung ist vielmehr ein umformendes Eingehen auf fremde Erwartungen, was bereits als Überziehen normaler Erwartungen beschrieben wurde. ${ }^{73}$ Während allerdings beim persönlichen Vertrauen, das im Grunde emotional gebunden ist, die Selbstdarstellung bloße Voraussetzung ist, basiert Systemvertrauen geradezu darauf. Vertrauen ent-

69 Vgl. Luhmann (1988), S. 97 f., sowie S. 103; Eberl (2003), S. 155

70 Luhmann (2000), S. 92

71 Vgl. Schmitz (1997), S. 163 f; Luhmann (2000), S. 92; Eberl (2003), S. 155

72 Luhmann (2000), S. 80

73 Vgl. Luhmann (2000), S. $80 \mathrm{f}$. 
steht hier durch eine gelungene Darstellung der Funktionsweise des Systems nach außen und in der Folge durch die erfolgreiche Darstellung der Zuverlässigkeit systemimmanenter Kontrollen. Unter diesen Gegebenheiten kann davon ausgegangen werden, dass die Kontrollen unabhängig von den Motivationsstrukturen der am sozialen System Beteiligten funktionieren. Im Gegensatz zum persönlichen Vertrauen besteht im Falle des Systemvertrauens dabei kein Problem der Täuschung. Darstellungen müssen nicht durchschaut werden und es besteht kein Anlass, sich gegen Täuschungen vorzusehen und zu wappnen (z.B. dass die Bank mehr Geld ausleiht, als sie besitzt oder dass im fachlichen Rat mehr Information vermittelt wird, als logisch und empirisch stichhaltig belegt werden könnte). Es ist lediglich von Relevanz, dass die Darstellung ausreichend Systemstabilität vermittelt. Gerade weil das Systemvertrauen diffus ist, wird es widerstandsfähiger, sodass einzelne Enttäuschungen stets als Spezialfall erklärt werden können, womit Immunisierungstendenzen und damit Systemstabilität begünstigt werden. ${ }^{74}$

Nach Luhmann beruhen persönliches Vertrauen und Systemvertrauen auf unterschiedlichen Entstehungsmechanismen, doch gibt es drei strukturelle Gemeinsamkeiten, die für den Aufbau aller Vertrauensbeziehungen relevant sind: innere Sicherheit (im Sinne von Selbstvertrauen), Lernen aus vertrauensbezogenen Erfahrungen (Identitätsentwicklung) und symbolische Kontrolle. Da alle internen Prozesse mit verminderter Komplexität arbeiten und dadurch weniger Möglichkeiten und mehr Ordnung als ihre Umwelt aufweisen, ist es notwendig die Problematik von außen teilweise nach innen zu verlagern. Indem das System als Leistungsträger in Folge dieses Vorgangs äußere Sicherheit mit innerer Sicherheit substituiert, wird Komplexität reduziert und die Unsicherheitstoleranz in externen Beziehungen gesteigert. Vertrauen wird dann primär durch seine Funktionen für die systeminterne Ordnung der Informationsverarbeitung und nicht direkt durch Umweltgarantien getragen. Für diesen Vorgang der Internalisierung, das heißt, um die innere Ordnung der Erlebnisverarbeitung an die Stelle einer umweltbezogenen Begründung der , Richtigkeit' ${ }^{\star}$ der Komplexitätsreduktion zu setzen, bedarf es demnach an Selbstsicherheit. ${ }^{75}$ Ohne Selbstsicherheit, die dazu befähigt, „etwaigen Vertrauensenttäuschungen mit Fassung entgegenzusehen, ohne sie als nur vorstellbare Möglichkeit schon jetzt als Handlungsgrundlage zu machen " ${ }^{\text {"76 }}$, kann eine Vertrauensbeziehung nicht aufgebaut werden. Neben dem Lernen aus vertrauensbezogenen Erfahrungen stellt die symbolische Kontrolle eine weitere Voraussetzung für den Aufbau von Vertrauensbeziehungen dar. Hierunter ist nicht die Kontrolle des Verhaltens einer Vertrauensperson oder eines Systems, sondern die Kontrolle der eigenen Vertrauensbereitschaft zu verstehen. Nach Luhmann verlangt die Vertrauensentstehung nach einer Zügelung und Kon- 
trolle der eigenen Vertrauensbereitschaft, sodass eine Selbstvergewisserung entsteht, dass Vertrauen an Grenzen gebunden ist und nach Maßgabe bestimmter, vernünftiger Erwartungen erteilt wird. Insofern werden Rückprojektionen der Umwelt auf vertrauensvolles Verhalten zu Symbolkomplexen, deren Beobachtung und Interpretation Auskunft darüber geben, ob die Fortsetzung des Vertrauens gerechtfertigt ist oder nicht. Zur Kontrolle von Symbolen, die Vertrauenswürdigkeit suggerieren, bedarf es eines stark vereinfachten Indiziengerüsts, sowie einer ausreichend klaren Definition und Bekanntheit vertrauenskritischer Verhaltensweisen (Schwellen), außerhalb derer Vertrauen entzogen oder von vornherein nicht erwiesen wird. Die symbolische Kontrolle erfolgt im Wesentlichen undiskutiert, was zur Sicherung ihrer Funktion beiträgt. Bei einer detaillierten Artikulation von Gründen für den Vertrauenserweis besteht nämlich die Gefahr, den Aufbau von Vertrauensbeziehungen kontraproduktiv zu beeinflussen und die Entstehung von Misstrauen zu begünstigen. ${ }^{77}$ Zusammenfassend sind alle drei Komponenten für die Entstehung von Vertrauensbeziehungen von hoher Relevanz, allerdings - je nach Art des Vertrauens - mit unterschiedlicher Gewichtung. So ist insbesondere das Lernen aus vertrauensbezogenen Erfahrungen beim Systemvertrauen leichter als beim persönlichen Vertrauen. Auch ist im Rahmen des Systemvertrauens ein Verzicht auf Innengarantien im Sinne von Selbstvertrauen bzw. deren Ersatz durch funktionierende Interaktionen möglich. Hingegen wird die symbolische Kontrolle beim Systemvertrauen erschwert. Ein Grund hierfür liegt darin, dass Systemvertrauen stärker sozialisiert ist, was einen Rückgriff auf mehr, weil auch fremde, Erfahrungen erlaubt, wobei das Durchschauen und Beurteilen der Indizien für eine gelungene systemimmanente Steuerung ein hohes Maß an Fachwissen erfordert. $^{78}$

Zusammenfassend stellen die Überlegungen Luhmanns einen wertvollen Erklärungsansatz für die Bedeutung von Vertrauen in modernen Gesellschaftssystemen dar. Indem Luhmann Vertrauen grundsätzlich als Beziehungsphänomen ansieht, das etwas über den sozialen Zusammenhalt der an dieser Beziehung Beteiligten aussagt, geht sein Ansatz über die psychologischen Erkenntnisse hinaus, ohne sie allerdings aufzugeben. Darüber hinaus macht er deutlich, dass es sich bei Vertrauen keineswegs um eine kalkulatorische Vorgehensweise handelt. Hervorgehoben werden kann auch, dass Luhmann, auf der Ebene einer sich anbahnenden Interaktion, eine Differenzierung der Begriffe Vertrauen und Vertrautheit vornimmt. Weiters unterscheidet Luhmann zwischen persönlichem Vertrauen und Systemvertrauen, wobei er letzteres mit Zutrauen gleichsetzt. Während beim persönlichen Vertrauen in Personen vertraut wird, geht es beim Systemvertrauen um das Vertrauen in Systeme bzw. in deren Funktionieren. Luhmann gründet die Differenzierung der beiden Vertrauensarten auch darauf, dass er persönliches Ver-

Vgl. Luhmann (2000), S. 75 ff.; Eberl (2003), S. 157 
trauen an die Existenz von Risiko koppelt, wohingegen er Systemvertrauen in Verbindung mit Gefahren setzt und in Zusammenhang mit überzeugender Selbstpräsentation des Systems bringt. Angesichts dieser grundlegenden Unterschiede wirken die von Luhmann erfassten strukturellen Gemeinsamkeiten von persönlichem Vertrauen und Systemvertrauen, die den Aufbau aller Vertrauensbeziehungen begünstigen, allerdings relativ allgemein und unspezifisch. Zusammenfassend bietet der Ansatz von Luhmann jedoch Anregungen für ein fundiertes Verständnis des Vertrauenskonstrukts und seiner Bedeutung. Für diese Arbeit sind allerdings entsprechende Präzisierungsarbeiten, vor allem im Hinblick auf den Entstehungsprozess von Vertrauen, notwendig, weshalb im Folgenden auf den Ansatz von Coleman eingegangen wird.

\subsubsection{Vertrauen als einseitige Ressourcenübertragung}

Die Überlegungen Colemans stützen sich weitgehend auf das Menschenbild des rational und zielgerichtet handelnden Akteurs. Im Rahmen dieser Auffassung wird den individuellen Akteuren die Möglichkeit zugestanden, nach ihrem eigenen Willen zu handeln und dieses Handeln als rational verstanden. ${ }^{79}$

Kernelemente dieser individuellen Handlungsebene sind: Interessen, Kontrolle, Ressourcen (bzw. Ereignisse). Individuen verfolgen spezifische Interessen, die wiederum nur mit entsprechenden Ressourcen oder Ereignissen verwirklicht werden können. Typischerweise handelt es sich bei Ressourcen um private Güter, allerdings können auch individuelle Handlungen darunter verstanden werden. Die Erweiterung um die Kategorie Ereignisse weist daraufhin, dass auch Handlungsergebnisse Gegenstand der Interessen sein können. Für die Verwirklichung der Interessen bedarf es der Kontrolle über Ressourcen oder Ereignisse. Ist es nicht möglich, die Ressourcen, die zur Verwirklichung der eigenen Interessen dienen, selbst zu kontrollieren, sind durch das Auseinanderfallen von Interessen und Kontrolle die Grundvoraussetzungen für die Bildung eines sozialen Systems gegeben. Denn nur in den Situationen, in denen eigene Interessen durch Ressourcen, die ein anderer Akteur kontrolliert, verwirklicht werden können, ist die Motivation zu Interaktionen gegeben. Rational und zielgerichtet handelnde Akteure sind also bestrebt, die ihrer Kontrolle unterliegenden Ressourcen, an denen sie wenig bis gar nicht interessiert sind, einzusetzen, um jene Ressourcen zu gewinnen, die der Verwirklichung ihrer Interessen dienen, aber der Kontrolle anderer Akteure unterliegen. ${ }^{80}$

Vor diesem handlungstheoretischen Hintergrund versteht Coleman Vertrauen als einseitigen, willentlichen Ressourcentransfer seitens des Vertrauensgebers, aus

Vgl. Coleman (1991), S. 121; Lane/Bachmann (1996), S. 369; Pieper (2000), S. 83; Klaus (2002), S. 90

Vgl. Coleman (1991), S. 34 ff.; Eberl (2003), S. 62 
dem dem Vertrauensnehmer keine Verpflichtung zur Gegenleistung erwächst ${ }^{81}$, wodurch der Vertrauensgeber eine risikoreiche Vorleistung, ähnlich wie bei den Überlegungen Luhmanns, erbringt. Situationen, in denen Vertrauen eine Rolle spielt, stellen damit eine Untergruppe der Situationen, die mit einem gewissen Risiko, dessen Höhe in Abhängigkeit von der Leistung des Vertrauensnehmers besteht, behaftet sind, dar. ${ }^{82}$ Vertrauensgeber nehmen dieses Risiko auf sich, wenn „[...] das Verhältnis der Gewinnchance zur Verlustchance größer ist als das Verhältnis des Ausmaßes des möglichen Verlustes zum Ausmaß des möglichen Gewinnes $^{\text {“83 }}$. Vertrauen kann somit als „erfolgsorientiertes Kalkül ${ }^{184}$ verstanden werden. Die Variablen, die die Entscheidung für eine Vertrauensvergabe beeinflussen sind demnach: Erstens die Wahrscheinlichkeit, dass sich der Vertrauensnehmer als vertrauenswürdig erweist (p), zweitens der Wert, der verloren werden könnte (L), und drittens der Wert, der gewonnen werden könnte (G). Der potentielle Vertrauensgeber wird sich für einen Vertrauenserweis entscheiden, wenn $\mathrm{p} /(1-\mathrm{p})>\mathrm{L} / \mathrm{G}$. Für den Fall, dass $\mathrm{p} /(1-\mathrm{p})=\mathrm{L} / \mathrm{G}$ wird sich der Vertrauensgeber indifferent verhalten, und in einer Situation, die durch $\mathrm{p} /(1-\mathrm{p})<\mathrm{L} / \mathrm{G}$ gekennzeichnet ist, wird er dem potentiellen Vertrauensnehmer kein Vertrauen entgegenbringen. ${ }^{85}$ Damit lässt sich Vertrauen nach Coleman vollkommen formallogisch erfassen.

Des Weiteren stellt nach Coleman nicht nur der Vertrauensgeber erfolgsorientierte Vertrauenskalküle auf, sondern auch der Vertrauensnehmer nimmt Kalkulationen vor. Und zwar kann er das in ihn gesetzte Vertrauen rechtfertigen oder enttäuschen. Grundsätzlich erlaubt der im Voraus geleistete Vertrauensakt des Vertrauensgebers dem Vertrauensnehmer Handlungen, die er sonst nicht realisieren könnte. Der vertrauensbedingte Gewinn des Vertrauensgebers ist im Regelfall nicht bloß von den Fähigkeiten des Vertrauensnehmers, sondern auch von dessen Bemühungen abhängig. Demnach ist es für den Vertrauensnehmer oftmals ein Gewinn, dass ihm auch in Zukunft vertraut wird, sei es von demselben oder potentiell anderen Vertrauensgebern. Coleman konzentriert sich hier also vorrangig auf die Reputation des Vertrauensnehmers, woraus sich zwei Konsequenzen ergeben: 1. Je länger der Vertrauensnehmer die Dauer der zukünftigen Beziehung einschätzt und je höher die daraus zu erwartenden Gewinne sind, desto eher wird er sich als vertrauenswürdig erweisen. 2. Je intensiver der Vertrauensgeber mit anderen potentiellen, zukünftig bedeutenden Vertrauensgebern in Kontakt steht, desto eher wird der Vertrauensnehmer ebenfalls versuchen, das in ihn gesetzte Vertrauen zu rechtfertigen. Dementsprechend kann unter der Kalku-

81 Vgl. Coleman (1991), S. 124

82 Vgl. Coleman (1991), S. 115

83 Coleman (1991), S. 126

84 Pieper (2000), S. 83

85 Vgl. Coleman (1991), S. 126 
lation des Vertrauensnehmers das Abwägen von kurzfristigen Gewinnen aus einem gegenwärtigen Vertrauensbruch gegen die entgangenen Gewinne aus zukünftigen vertrauensbasierten Vereinbarungen verstanden werden. Der Vertrauensgeber seinerseits hat allerdings die Möglichkeit, durch entsprechende Gestaltung der Rahmenbedingungen im Sinne einer Schaffung sozialer Strukturen, das Kalkül des Vertrauensnehmers in Richtung Vertrauenswürdigkeit zu lenken. ${ }^{86}$

Generell differenziert Coleman zwischen drei Arten von Vertrauenssystemen, die über das skizzierte einfache Vertrauensnehmer-Vertrauensgeber-Modell hinausgehen: 1. Gegenseitiges Vertrauen, bei dem der Vertrauensgeber dem Vertrauensnehmer vertraut und ersterer gleichzeitig der Vertrauensnehmer des Vertrauens ist, das der ursprüngliche Vertrauensnehmer und nun auch Vertrauensgeber in ihn setzt. 2. Vertrauenssysteme mit intermediären Akteuren, bei denen ein- und derselbe Akteur als Vertrauensnehmer für einen Akteur und als Vertrauensgeber für einen anderen Akteur fungiert. 3. Vertrauenssysteme, bei denen Drittparteien-Vertrauen eine Rolle spielt, indem ein Akteur dem Versprechen eines zweiten Akteurs nicht vertraut, sondern die Interaktion nur durch das Vertrauen in eine andere, an der Transaktion unbeteiligte Drittpartei zustande kommt. ${ }^{87}$ Das System gegenseitigen Vertrauens kennzeichnet sich durch eine positive Rückkoppelungsschleife, die in einem gesteigerten $M a ß$ an Vertrauensvergabe und Vertrauenswürdigkeit resultiert. Das Kalkül in einer symmetrischen Vertrauensbeziehung verändert sich nämlich dahingehend, dass einerseits der Anreiz, das Vertrauen zu enttäuschen, für den Vertrauensnehmer geringer wird, da er davon ausgehen muss, dass bei einem Rollentausch sein Vertrauen im Rahmen eines Racheakts ebenso enttäuscht wird (der entgangene Gewinn wird größer). Andererseits wächst für den Vertrauensgeber der Anreiz, Vertrauen zu schenken, da sowohl die Beurteilung der Vertrauenswürdigkeit des Vertrauensnehmers aufgrund eigener Sanktionspotentiale erleichtert wird, und auch der mögliche Gewinn aufgrund eines wahrscheinlicheren zukünftigen Rollentauschs größer wird. Damit bestehen sowohl für den Vertrauensgeber, als auch für den Vertrauensnehmer in einer asymmetrischen Beziehung Anreize, diese in eine symmetrische Beziehung gegenseitigen Vertrauens zu transformieren. Für diese Transformation gibt es zwei Möglichkeiten: Die erste Möglichkeit, mit der der Vertrauensgeber den Vertrauensnehmer dazu bringen kann, Vertrauen in ihn zu setzen, besteht darin, Situationen zu schaffen, in denen der Vertrauensnehmer zum Vertrauensgeber wird (z.B. wenn der ursprüngliche Vertrauensnehmer dem Vertrauensgeber einen Gefallen erweisen kann), sofern derartige Situationen nicht von selbst entstehen. Eine andere Möglichkeit ist, dass der Vertrauensgeber den Nutzen des Aufrechterhaltens der Beziehung für den Vertrauensnehmer erhöht, indem er hohe Gewinne in Aussicht stellt. Dadurch entstehen auf eine Fortführung der Beziehung 
gerichtete Erwartungen beim Vertrauensnehmer, die diesen den Wert der Beziehung höher einschätzen lassen. Gewöhnt sich der Vertrauensnehmer an die hohen Gewinne, wird er alles tun, um die Beziehung aufrechtzuerhalten - und damit auch Vertrauen schenken. Auch für den Vertrauensnehmer bieten sich Strategien, um asymmetrische Beziehungen in symmetrische Relationen zu transformieren. So kann er sich beispielsweise dazu entscheiden, die Erwartungen des Vertrauensgebers zu übertreffen, womit er das in ihn gesetzte Vertrauen nicht bloß rechtfertigt, sondern gleichzeitig eine Verpflichtung seitens des Vertrauensgebers schafft. Eine derartige Handlung würde zwar für den Vertrauensnehmer anfänglich zu höheren Kosten führen, allerdings könnten diese durch mögliche zukünftige Gewinne ausgeglichen werden. Beim zweiten Vertrauenssystem differenziert Coleman zwischen drei Typen von Intermediären: Berater, Bürge, Unternehmer. In den Situationen, wo der Vertrauensgeber dem letztendlichen Vertrauensnehmer nicht auf direktem Wege vertraut, sind diese Intermediäre notwendig. Wird ein Berater als Intermediär eingesetzt, dann wird auf dessen Urteilsfähigkeit vertraut. Sollte sich der Vertrauensnehmer nicht als vertrauenswürdig erweisen, verliert der Berater lediglich das Vertrauen in seine Urteilskraft, der Vertrauensgeber jedoch Ressourcen. Im Gegensatz dazu erleidet der Bürge anstelle des Vertrauensgebers einen Ressourcenverlust, wenn der letztendliche Vertrauensnehmer das Vertrauen enttäuscht, wogegen seine Vertrauenswürdigkeit in den Augen des Vertrauensgebers nicht verringert wird. Dasselbe gilt, wenn es sich bei dem Intermediär um ein Unternehmen handelt, dass das Vertrauen verschiedener Vertrauensgeber zusammenfasst und in Form von Ressourcen auf verschiedene Vertrauensnehmer aufteilt. Rechtfertigen die Vertrauensnehmer das in sie gesetzte Vertrauen nicht, so erleidet das Unternehmen als Intermediär einen Ressourcenverlust, nicht die Vertrauensgeber. Das Kalkül der Vertrauensvergabe des Vertrauensgebers stützt sich bei allen drei Typen wieder auf wahrscheinliche Gewinne und Verluste. Das Drittparteien-Vertrauen als drittes Vertrauenssystem ähnelt der Beziehung, in der ein Bürge als Intermediär in einer Transaktion auftritt, allerdings mit dem Unterschied, dass die Drittpartei (z.B. Banken, Kreditunternehmen, Staatsregierungen) eine passive Rolle innehat. Damit eine Transaktion zustande kommt, wird im Rahmen eines derartigen Systems die Notwendigkeit für den Vertrauensgeber, Vertrauen in das Versprechen des Vertrauensnehmers zu setzen, durch Vertrauen in eine Drittpartei (und als Konsequenz davon, das Vertrauen in z.B. die Gültigkeit der Währung) ersetzt, obwohl diese an der Transaktion nicht direkt beteiligt ist. ${ }^{88}$

Zusammenfassend werden Colemans Überlegungen weitgehend vom Bild des rational und zielgerichtet handelnden Akteurs gestützt. ${ }^{89}$ Der potentielle Ver- 
trauensgeber legt durch eine rationale Entscheidung fest, ob er vertraut und demnach Ressourcen an den potentiellen Vertrauensnehmer überträgt oder ob er, infolge von Misstrauen, Vertrauen und Ressourcen zurückhält. Der potentielle Vertrauensnehmer wiederum entscheidet nach rationalem Kalkül, ob er das in ihn gesetzte Vertrauen rechtfertigt oder enttäuscht. Die Entscheidungen im Rahmen des Vertrauensprozesses beruhen demnach auf dem Kalkül der „Nutzenmaximierung unter Risiko" “90. Die von Coleman beschriebene Kalküldimension des Vertrauens, nach der eine Person dann vertraut, wenn das Produkt aus potentiellem Gewinn und Gewinnwahrscheinlichkeit größer ist als das Produkt aus potentiellem Verlust und Verlustwahrscheinlichkeit, kann in operationalisierter Form zur Erklärung des Vertrauens in zwischenbetrieblichen Kooperationen herangezogen werden.

\subsection{Vertrauen aus der Perspektive der Neuen Institutionen- ökonomie}

Um die ökonomische Sinnhaftigkeit von Vertrauen zu eruieren ist eine Gegenüberstellung der positiven Konsequenzen der Vertrauenshandlung, einer Nutzensteigerung und einer Kostensenkung, einerseits und der negativen Konsequenzen der Vertrauenshandlung, den Risikokosten, den Kosten der Risikobegrenzung und den Kosten des Vertrauensaufbaus, andererseits erforderlich. ${ }^{91}$ Für diese Untersuchung bieten sich die Ansätze der ,Neuen Institutionenökonomie‘ an, weshalb im Anschluss an einen Überblick die Transaktionskostentheorie, sowie die Principal-Agent-Theorie (die eigentliche Operationalisierung des Vertrauenskonstrukts erfolgt auf der Grundlage der Principal-Agent-Theorie, vgl. Teil II Kap. 4 und 5, sowie Teil IV Kap. 4 und 5) und die Property-Rights-Theorie im Zusammenhang mit dem Vertrauensbegriff näher erläutert werden.

Die Neue Institutionenökonomie geht grundsätzlich von einer begrenzten Rationalität der Akteure aus, womit sie Raum für subjektiv und objektiv unsichere Erwartungen, sowohl im Hinblick auf Umweltzustände, als auch in Bezug auf das Verhalten Dritter lässt. Letzteren eröffnen sich dadurch Opportunismusspielräume. ${ }^{92}$

Generell werden unter dem Oberbegriff, Neue Institutionenökonomie‘ Ansätze der Forschungsrichtungen ,Institutional Environment' und ,Institutional Arrangement' zusammengefasst. Der Richtung des ,Institutional Environment' werden

der gesellschaftlichen Struktur und deren sozialer Normen, sowie des emotionalen Zustands und der Erfahrungen der beteiligten Akteure für die Entstehung von Vertrauen vernachlässigt (Antfang/ Urban (1994), S. 17; Loose/Sydow (1994), S. 168; Preisendörfer (1995), S. 269; Lane/Bachmann (1996), S. 369; Klaus (2002), S. 95).

$90 \quad$ Coleman (1991), S. 125

91 Vgl. Pieper (2000), S. 130

92 Vgl. Ripperger (1998), S. 22 
die ökonomische Theorie der Politik (Public-Choice-Theorie) und die Theorie der Verfügungsrechte (Property-Rights-Theorie) zugerechnet. ${ }^{93}$ Im Zentrum dieser beiden Ansätze stehen Institutionen, die das Zusammenleben in der Gesellschaft bestimmen. ${ }^{94}$ Während sich die Public-Choice-Theorie vorrangig mit der Untersuchung öffentlich-rechtlicher und privatrechtlicher Institutionen und der Interdependenzen von Wirtschaft und Politik beschäftigt, befasst sich die PropertyRights-Theorie mit der Untersuchung der Allokationsstruktur der Verfügungsrechte, über die die Wirtschaftssubjekte verfügen. ${ }^{95}$ Die Ansätze der Forschungsrichtung des ,Institutional Arrangement', wozu die Transaktionskostentheorie (Transaction Cost Economics) und die Principal-Agent-Theorie zählen, beschäftigen sich dagegen mit konkreten Organisationsbelangen, wie der Analyse von Mechanismen zur effizienten Gestaltung von Austauschbeziehungen, weshalb sie im Rahmen dieser Arbeit von hoher Relevanz für die Erklärung der Funktionsweise und der Bedeutung des Vertrauenskonstrukts sind.

Abschließend wird auf den Begriff der Institution eingegangen, den die Institutionenökonomie wesentlich zugrunde legt. Neben den vielfältigen Einsatzmöglichkeiten im alltäglichen Sprachgebrauch, dient der Institutionenbegriff in der Ökonomik sowohl der Bezeichnung korporativer Gebilde, als auch der Beschreibung normativer Regeln und Verhaltensmuster. ${ }^{96}$ Wenngleich ein allgemein akzeptierter Institutionenbegriff in der Neuen Institutionenökonomie nicht existiert, wird im Folgenden ein Institutionenverständnis zugrunde gelegt, das diese als „humanly devised constraints imposed on human interaction [...]", bestehend aus ,[...] formal rules, informal constraints (norms of behavior, conventions, and selfimposed codes of conduct), and their enforcement characteristics [...] ${ }^{\text {(67 }}$, interpretiert. Institutionen stellen demnach Spielregeln dar, die menschliche Interaktionen durch Verringerung sozialer Komplexität erleichtern. Diese können nach North sowohl formeller (z.B. Verträge), als auch informeller Natur (z.B. Sitten, Bräuche) sein. Im Rahmen der Neuen Institutionenökonomie steht vor allem die formelle Seite und auf dieser besonders die Institution des Vertrags im Zentrum der Betrachtungen. Verträge werden vereinbart, um Transaktionen zwischen individuellen oder korporativen Akteuren möglichst effizient zu gestalten. Darauf aufbauend können die verschiedenen ökonomisch relevanten Vertragsformen wiederum entsprechenden Institutionen als Beherrschungssysteme dieser Vertragsbeziehungen zugeordnet werden. So fasst beispielsweise Williamson im Rahmen der

\footnotetext{
93 Vgl. Bayon (1997), S. 30; Pieper (2000), S. 131

94 Vgl. Williamson (1990a), S. 64

95 Vgl. zur Property-Rights-Theorie z.B. Picot (1981); Picot (1991), S. 145 ff.; Dietl (1993); Picot (1995), S. 2106 f.; Ripperger (1998), S. 25

96 Vgl. Albach/Albach (1989); Dietl (1993); Ripperger (1998), S. 23 f.

97 North (1991), S. 4
} 
Transaktionskostentheorie ,Markt ${ }^{\star}$ und ,Hierarchie ${ }^{6}$ als Institutionen auf, die alternative Beherrschungs- und Überwachungssysteme darstellen. ${ }^{98}$

\subsubsection{Vertrauen aus Sicht der Transaktionskostentheorie}

Die Transaktionskostentheorie, deren Wurzeln auf den späteren Nobelpreisträger Coase (1937) zurückgehen, behandelt die Kosten, die durch die Koordination wirtschaftlicher Aktivitäten ${ }^{99}$ bzw. durch den Transfer der Verfügungsgewalt über Güter ausgelöst werden. ${ }^{100}$ Indem sie durch ein Denken in Transaktionen ${ }^{101}$ geprägt ist, stellt sie einen Bezugsrahmen dar, dessen Anwendung die Untersuchung und Strukturierung von Austauschbeziehungen und deren Kosten erleichtert ${ }^{102}$, und dementsprechend das Verständnis institutioneller Entwicklungen und den Vergleich institutioneller Koordinationsformen ermöglicht. ${ }^{103}$ Williamson, der Coases Gedanken vertiefte und dessen Werk die Transaktionskostentheorie maßgeblich prägte, beschäftigte sich eingehend mit der Frage, welche Mechanismen für eine effiziente Koordination von Transaktionsbeziehungen geeignet sind. Nach Williamson muss die Wahl zwischen den Koordinationsmechanismen ,Markt' und ,Hierarchie' davon geleitet werden, dass die mit der Ausführung der Transaktionen verbundenen Kosten minimal sind. ${ }^{104}$ Williamson versteht unter den Transaktionskosten nicht bloß die monetär erfassbaren Kosten, sondern auch alle mit einer Transaktion verbundenen Opfer und Anstrengungen. ${ }^{105}$ Allerdings gestaltet sich die Erhebung dieser nicht monetären Kosten in der Praxis als schwierig. ${ }^{106}$

Bezugnehmend auf die vorliegende Problemstellung lassen sich die Transaktionskosten in Abhängigkeit der Entwicklung einer zwischenbetrieblichen Kooperation folgendermaßen untergliedern: die ex ante (vor Übertragung der Property Rights) anfallenden Suchkosten (Informationen, Kooperationspartner) und Vereinbarungskosten (Anbahnung und Abschluss von Transaktionen), sowie die ex post (nach Übertragung der Property Rights) anfallenden Vertragsdurchsetzungskosten (Kontrolle, Qualitätsprüfung) und Anpassungskosten (Vertragsaufhebung, Beendigung). ${ }^{107}$ Für die Entstehung von Transaktionskosten sind nach Williamson zwei Gruppen von Faktoren verantwortlich: Humanfaktoren und Um-

\footnotetext{
98 Vgl. Williamson (1990a), S. 14; siehe hierzu auch Ripperger (1998), S. 24; Eberl (2003), S. 76 f.

99 Vgl. Schanz (2000), S. 135

$100 \quad$ Vgl. Mugler (1995), S. 498

101 Picot et. al (1997, S. 108) definieren Transaktion als „transfer of property rights between two parties."

102 Vgl. Albach (1989), S. 27 ff.

$103 \mathrm{Vgl}$. Picot/Dietl (1990), S. $182 \mathrm{f}$.

104 Vgl. Williamson (1975); Williamson (1985)

105 Vgl. Picot (1985), S. 224

106 Vgl. Albach (1989), S. 38

107 Vgl. Picot et al. (1997), S. 111; Ripperger (1998), S. 26; Pieper (2000), S. 135
} 
weltfaktoren. ${ }^{108} \mathrm{Zu}$ den Humanfaktoren sind die beschränkte Rationalität des Menschen, sowie das mögliche opportunistische Verhalten der an der Transaktion beteiligten Akteure, das zu einem Risiko im Hinblick auf die Zielerreichung führt, zu zählen. ${ }^{109}$ Als Umweltfaktoren sind die Unsicherheit, der die Transaktion unterliegt, die Wiederholungshäufigkeit der Transaktion, sowie die Besonderheiten der zur Abwicklung einer Transaktion erforderlichen Investitionen zu nennen. ${ }^{110}$

Im Hinblick auf die für Transaktionskosten verantwortlichen Umweltfaktoren zeigt sich bei auf langfristige Vorteile ausgerichteten Transaktionen, die hochgradig unsicher und komplex sind, ein Auseinanderfallen von Leistungen und Gegenleistungen. Die damit verbundenen Unsicherheiten und Mehrdeutigkeiten führen zu gestiegenen Transaktionsrisiken (Opportunismusspielraum des Kooperationspartners), da der langfristige Vorteil vom zukünftigen Verhalten des Kooperationspartners abhängt. Während kurzfristige, triviale Transaktionen über das kurzfristige Vorteilhaftigkeitskalkül des Marktes flexibel und verlässlich gesteuert werden können, würde eine marktbasierte Steuerung von langfristigen Tauschbeziehungen dazu führen, dass die sich im Rahmen der Transaktion ergebenden Opportunismusspielräume regelmäßig wahrgenommen werden. Dementsprechend müssen langfristige Tauschbeziehungen auf Koordinationsmechanismen beruhen, die nicht auf einem kurzfristigen Vorteilhaftigkeitskalkül basieren. Das heißt, man entschließt sich zu einer Verhaltensweise, deren Vorteile sich erst in der Zukunft und in Abhängigkeit des unsicheren Verhaltens des Interaktionspartners ergeben, und die bei Betrachtung nur einer Entscheidungssequenz nicht über den höchsten Zielerreichungsgrad unter den Alternativen verfügt. ${ }^{11}$ Mit zunehmender Komplexität der Transaktion, die entsprechend hohe Opportunismusspielräume impliziert, welche aufgrund der Relevanz der Transaktion nicht einfach riskiert oder akzeptiert werden können, ist eine Sicherstellung des Verhaltens des Austauschpartners erforderlich. Nach der Transaktionskostentheorie erfordern durch hohe Opportunismusspielräume gekennzeichnete Transaktionen daher eine hierarchische Steuerung zur Reduktion der Opportunismusspielräume und der Transaktionskosten. ${ }^{112}$ Aber auch die hierarchische Koordination kann das Verhalten nicht uneingeschränkt steuern. Glaubhafte Sanktionen verlangen nach einem ausreichenden Sanktionspotential ${ }^{113}$, sowie nach der im Voraus bestehenden Kenntnis des gewünschten ${ }^{114}$ und der im Nachhinein be-

\footnotetext{
108 Vgl. Williamson (1975), S. 40

109 Vgl. Rößl (2006), S. 25

110 Vgl. Williamson (1975), S. 40

111 Vgl. Kaas (1992), S. 884 f; Rößl (1996), S. 313 f.; Rößl/Fink (2007)

112 Vgl. Williamson (1991), S. 22 f;; Rößl (1996), S. 314

113 Vgl. Backhaus (1992), S. 787; Kaas (1992), S. $886 \mathrm{ff}$.

114 Vgl. Eberl (2004), S. 267
} 
stehenden Feststellbarkeit des realisierten Verhaltens ${ }^{115}$ der Kooperationspartner. Dies ist jedoch vor allem bei zwischenbetrieblichen Kooperationen oftmals nicht möglich bzw. nicht wünschenswert (z.B. R\&D-Kooperationen: unbekanntes Ziel, Flexibilitätsverlust, Begrenzung des Selbstbestimmungspotentials). ${ }^{116}$ Es zeigt sich somit, dass die hierarchische Koordinationsform vor allem im Rahmen zwischenbetrieblicher Kooperationen dann ihre Grenzen im Sinne eines Organisationsversagens erreicht, wenn die Ziele nicht oder nur zu prohibitiv hohen Kosten programmiert werden können. ${ }^{117}$ Daher sind in Fällen langfristiger Tauschbeziehungen, die hochgradig unsicher und komplex sind, sodass ein hierarchisches Festlegen des Verhaltens der Unsicherheit nicht angepasst wäre, weder der Markt noch die Hierarchie geeignete Koordinationsmechanismen. Denn „einerseits steht die Langfristigkeit einer verlässlichen Koordination durch den Marktmechanismus entgegen, umgekehrt erlaubt die mangelnde Programmierbarkeit der Beziehungen keine hierarchische Koordination."118 Der Versuch, diese Transaktionen - trotz ihrer Spezifität - über den Markt im Sinne einer Selbstabstimmung auf Basis kurzfristiger Vorteilhaftigkeitskalküle zu steuern, resultiert aufgrund der hohen Opportunismusspielräume in Chaos. Der Versuch, diese Transaktionen hierarchisch im Sinne einer Fremdabstimmung auf Basis langfristiger Kalküle zu steuern, resultiert in Systemrigidität, die vor allem vor dem Hintergrund hoher Komplexität ein großes Problem darstellt. ${ }^{119}$

Aufgrund der Schwächen der Koordinationsmechanismen Markt und Hierarchie wird ein alternativer Koordinationsmechanismus für Steuerungsaufgaben, bei denen sowohl die marktbasierte als auch die hierarchische Koordination versagen, diskutiert. ${ }^{120}$ Dieser Koordinationsmechanismus wird als ,trust ${ }^{121}$, ,relational contracting $^{6122}$, ,self-commitment ${ }^{123}$ oder ,Selbstverpflichtung ${ }^{6124}$ bezeichnet. Seine Funktionsweise und damit die Bedeutung von Vertrauen im Rahmen der Transaktionskostentheorie für Transaktionsbeziehungen erklärt sich folgendermaßen: In Transaktionsbeziehungen, die auf der Basis von Vertrauen, das seine koordinierende Wirkung aus der Normierung vereinbarungskonformen Verhaltens durch Selbstverpflichtung der Akteure bezieht, gesteuert werden, begrenzen keine Kontrollen und Sanktionen die Handlungsfreiheit der Akteure. ${ }^{125}$ Ihnen stehen jederzeit die Wahrnehmung eigener, kurzfristiger Vorteile und damit die Aus-

115 Vgl. Dwyer et al. (1987), S. 13

116 Vgl. dazu ausführlich Rößl (1994), S. 6 ff.; Shane (1994); Rößl (1996), S. 315 ff.; Rößl/Fink (2007)

117 Vgl. Ring/Van de Ven (1992); Ring/Van de Ven (1994)

118 Rößl (1996), S. 320

119 Vgl. Ring/Van de Ven (1992); Rößl (1994), S. 11

120 Vgl. Rößl (1996); Adler (2001); Eberl (2004); Rößl/Fink (2007)

121 Vgl. Adler (2001); Eberl (2004)

122 Vgl. Carson et al. (2006)

123 Vgl. Frey/Osterloh (2002)

124 Vgl. Rößl (1996); Sydow/Windeler (2000); Rößl/Fink (2007)

$125 \mathrm{Vgl}$. Roßl/Fink (2007) 
übung eines defektiven Verhaltens, das die Kooperation und die Kooperationspartner schädigt, frei. ${ }^{126}$ Indem sich die einzelnen Akteure aber auf ein kooperatives Verhalten festlegen, begrenzen sie ihre Opportunismusneigung (Wahrscheinlichkeit der Ausnutzung eines vorhandenen Opportunismusspielraums) freiwillig, womit ein defektives Verhalten weniger wahrscheinlich wird. ${ }^{127}$ Legen sich alle Kooperationspartner auf ein kooperatives Verhalten fest und verpflichten sich damit dem langfristigen Vorteil selbst, entfaltet Vertrauen seine Koordinationswirkung und ermöglicht Beziehungen, die sonst nicht stattfänden. ${ }^{128}$ Es ist jedoch darauf hinzuweisen, dass sich im Rahmen der Koordination durch Vertrauen der Opportunismusspielraum der Akteure nicht verringert, womit die Möglichkeit für defektives Verhalten bestehen bleibt. Hinzu kommt, dass mit der zunehmenden Erfahrung, dass der Transaktionspartner den Opportunismusspielraum bisher noch nicht ausgenützt hat, das wahrgenommene Risiko sinkt. Damit stellen die nicht reduzierten Opportunismusspielräume in Verbindung mit der gesunkenen Risikowahrnehmung ein permanentes Gefährdungspotential für den Vertrauensgeber dar. ${ }^{129}$

Zusammenfassend zeigt sich, dass Vertrauen im Rahmen der Transaktionskostentheorie ein effizienter Mechanismus für die Koordination von Austauschbeziehungen ist - vor allem dann, wenn komplexe Güter betroffen sind. Durch Nutzung des Transaktionskoordinationsmechanismus Vertrauen können Wettbewerbsvorteile erlangt werden: Wurde Vertrauen in die Selbstverpflichtung des Kooperationspartners gewonnen, dann ersetzt dieses Vertrauen Produktionsund Transaktionskosten. Indem in die Leistungsfähigkeit und den Leistungswillen vertraut werden, verlieren input- und outputbezogene Selbstbindungen an Bedeutung, womit Kosten für Sicherheitsäquivalente eingespart werden. Hinzu kommt, dass wechselseitige Selbstverpflichtungen stabile und flexible Austauschbeziehungen begünstigen, und zwar auch im Hinblick auf jene Beziehungen, die mit hierarchisch mechanistischen Mechanismen gar nicht oder nur zu prohibitiv hohen Kosten koordinierbar wären. ${ }^{130}$ Der zentrale Vorteil von Vertrauen als Koordinationsmechanismus zeigt sich im Rahmen dieser Theorie somit in seiner Fähigkeit, hochkomplexe Transaktionen mit vergleichsweise geringeren Transaktionskosten zu ermöglichen und aufrechtzuerhalten. ${ }^{131}$

\footnotetext{
126 Vgl. Wurche (1994), S. 149

127 Vgl. Nooteboom et al. (1997), S. 310

128 Vgl. Osterloh/Weibel (2000); Rößl/Fink (2007)

129 Vgl. Siegrist (2001); S. 23 f.; Rößl/Fink (2007)

130 Vgl. Rößl (1996), S. 331

131 Vgl. Vogt (1997); S. 206; Adler (2001), S. 219
} 
Allerdings beschränkt die mangelnde Messbarkeit der Transaktionskosten und die Unmöglichkeit der empirischen Trennung von Produktions- und Transaktionskosten die Anwendung des Transaktionskostenmodells in empirischen Studien. ${ }^{132}$

\subsubsection{Vertrauen aus Sicht der Principal-Agent-Theorie}

Gegenstand der Principal-Agent-Theorie, die auf den gleichen Annahmen wie die Transaktionskostentheorie beruht, sind im weitesten Sinne Auftragsbeziehungen. ${ }^{133}$ Die Grundlage dieser Delegationsbeziehungen bilden die Informationsdefizite des Prinzipals als Auftraggeber gegenüber dem Agenten als Auftragnehmer, sowie die Umweltunsicherheit. Letztgenannte Größe beeinflusst, neben der Qualifikation und dem Arbeitseinsatz des Agenten, das Ergebnis seiner Arbeit. Nach Erbringung des Arbeitseinsatzes durch den Agenten ist es dem Prinzipal daher weder vollständig, noch kostenlos möglich festzustellen, welcher Teil des Ergebnisses auf die Qualifikation, welcher auf das Verhalten des Agenten und welcher Teil auf die Umweltunsicherheit zurückzuführen ist. Aus dieser Tatsache kann ein Informationsvorsprung für den Agenten abgeleitet werden, der ihm Raum für opportunistisches Handeln und damit die Verfolgung persönlicher Ziele lässt. ${ }^{134}$ Aus dieser Informationsasymmetrie zwischen Prinzipal und Agenten erwachsen dem Prinzipal spezifische Risiken. Um sie abzuwenden, hat der Prinzipal einerseits die Möglichkeit, die Unsicherheiten in Bezug auf das Verhalten des Agenten und die damit zusammenhängenden Risikokosten hinzunehmen. Andererseits kann er sich dazu entschließen, Aktionen zur Verringerung der Unsicherheiten zu setzen und die damit korrelierenden Kosten (Agency-Costs ${ }^{135}$ ) zu akzeptieren. ${ }^{136}$

In diesem Zusammenhang wird die Relevanz des Vertrauenskonstrukts sichtbar, da die Akzeptanz der Unsicherheiten ,nur vor dem Hintergrund einer [relativ zum Unsicherheitsausmaß und den subjektiv bewerteten Konsequenzen opportunistischen Verhaltens, Anm. d. Verf.] tragfähigen Vertrauensbeziehung“137 möglich ist. Aus principal-agent-theoretischer Sicht fungiert Vertrauen als Explanans für nicht durch explizite Steuerungsmaßnahmen initiiertes kooperatives Verhalten des Agenten bzw. den Verzicht auf diese seitens des Prinzipals. Indem der Prinzipal

132 Vgl. Albach (1989), S. 38; Weber (1993), S. 53 ff.

133 Vgl. Rasmusen (1989), S. 133 ff.; Picot (1991), S. 150 ff.; Milgrom/Roberts (1992), S. $214 \mathrm{ff}$; Bayon (1997), S. 40; Schanz (2000), S. 139

$134 \mathrm{Vgl}$. Spremann (1989), S. $3 \mathrm{ff}$.

135 Ripperger (1998, S. 65) versteht unter Agency-Costs „das prinicpal-agent-theoretische Äquivalent zu denjenigen Transaktionskosten, die im Rahmen von Schutzmaßnahmen gegen opportunistisches Verhalten anfallen." Sie setzen sich aus folgenden Komponenten zusammen (Schanz 2000, S. 140): Kontrollkosten des Prinzipals (z.B. Überwachungssysteme), Garantiekosten des Agenten (z.B. Schadenersatzleistungen) und Wohlfahrtsverluste (z.B. Agent verzichtet auf die Ausführung bestimmter Handlungen, die einen positiven Beitrag zur Zielerreichung leisten würden).

136 Vgl. Rößl (2006), S. 39

137 Rößl (2000), S. 94 
vertraut, geht er davon aus, dass der Agent weder bestehende Informationsasymmetrien noch spezifische Investitionen zum Schaden des Prinzipals ausnützt. ${ }^{138}$ Vertrauen ,absorbiert' demnach die in einer Vertragsbeziehung präsenten Verhaltensrisiken $^{139}$, womit es einen wesentlichen Beitrag zur Lösung von AgencyProblemen (vgl. dazu Kap. 4) leisten kann. ${ }^{140}$ Durch Investitionen in Vertrauenskapital können Agency-Kosten gesenkt werden ${ }^{141}$, ohne die wirtschaftlichen Erträge der Akteure zu schmälern. ${ }^{142}$ Ähnlich wie bei den Transaktionskosten reduziert Vertrauen die in den Agency-Kosten verankerten Reibungsverluste. ${ }^{143}$

Zusammenfassend zeigt sich Vertrauen im Rahmen der Principal-Agent-Theorie in einem weitgehenden Verzicht auf explizite Kontroll- und Sicherungsinstrumente zum Schutz gegen opportunistisches Verhalten. Als Element innerhalb einer Principal-Agent-Beziehung kann Vertrauen die Agency-Kosten reduzieren und auch dann zu einer Überwindung der Agency-Probleme beitragen, wenn andere Koordinationsmechanismen nicht verfügbar oder relativ, in Bezug auf die reduzierten Risiken, teuer sind. ${ }^{144}$ Indem die Principal-Agent-Theorie zwischen unterschiedlichen Szenarien von Verhaltensrisiken unterscheidet und die Risikobereitschaft der Akteure berücksichtigt ${ }^{145}$, bietet sie eine Erweiterung gegenüber der Transaktionskostentheorie und ist damit anwendungsnäher, während der Transaktionskostentheorie Modellplatonismus vorgeworfen werden kann. Daher wird die Principal-Agent-Theorie im Zuge dieser Arbeit als theoretischer Rahmen für die Untersuchung von Kompetenz als Determinante der Entstehung von Vertrauen in zwischenbetrieblichen Kooperationen herangezogen.

\subsubsection{Vertrauen aus Sicht der Property-Rights-Theorie}

Unter Property-Rights (Verfügungsrechte) sind die mit einer Ressource verbundenen Rechte zu verstehen, nämlich das Recht (1) die Ressource zu nutzen (usus), (2) sich ihren Ertrag anzueignen (usus fructus), (3) ihre Form und Substanz zu verändern (abusus) und (4) sie Anderen ganz oder teilweise zu überlassen bzw.

138 Vgl. Bradach/ Eccles (1989), S. 104; Currall/Judge (1995), S. 153; Madhok (1995), S. 120; Cummings/Bromiley (1996), S. 303; Child (1998), S. 244

139 Ripperger (1998, S. 72) schreibt hierzu: „Die beschriebenen Risiken lösen sich nicht einfach auf, sondern müssen erst auf eine Art und Weise transformiert werden, welche es einem Akteur überhaupt erst ermöglicht, auf eine explizite vertragliche Absicherung gegen diese zu verzichten." Ähnlich auch Meijboom et al. (2006, S. 428): „As long as trust exists, the lack of control is often not experienced, or at least not considered as an unpleasant vulnerability.", sowie Lane/Bachmann (1996, S. 367): "Trust absorbs complexity in so far as someone who trusts acts as if alter ego's actions are, at least to some extent, predictable."

140 Vgl. Irlenbusch/Schade (1999), S. 748

141 Vgl. Kaas (1992); Adler (2001), S. 219; Gilbert (2007), S. 66

142 Vgl. Koslowski (2000), S. 430

143 Vgl. Ripperger (1998), S. 68

144 Vgl. Rößl (1994), S. 381

$145 \mathrm{Vgl}$. Roeder (2000), S. $118 \mathrm{f}$. 
zu veräußern. ${ }^{146}$ Aus Sicht der Property-Rights-Theorie sind daher nicht materielle Güter der eigentliche Gegenstand ökonomischer Transaktionen, sondern spezifisch zusammengesetzte Bündel von Rechten: „It is not the resource itself that is owned; it is a bundle, or a proportion of rights to use a resource that is owned." 147 Property-Rights können sowohl auf Sitten, Bräuchen und Konventionen beruhen, als auch gesetzlich begründet sein. Bei gesetzlich begründeten Verfügungsrechten ist zwischen absoluten, die gegenüber jedermann gelten, und relativen Verfügungsrechten, die Ansprüche zwischen bestimmten Akteuren begründen und im Regelfall aus vertraglichen Schuldverhältnissen oder Haftungsregeln resultieren, zu differenzieren. ${ }^{148}$

Die Grundzüge der Property-Rights-Theorie lassen sich anhand der drei folgenden Fragestellungen konkretisieren:

1. Welche Verfügungsrechtsstruktur hat eine bestimmte Gesellschaft (z.B. Privateigentum, Staatseigentum, ,open access', Gemeineigentum ${ }^{149}$ )?

2. Welche Auswirkungen hat diese Verfügungsrechtsstruktur auf den Wirtschaftsablauf?

3. Welche Faktoren bedingen die Entstehung und Veränderung von Verfügungsrechten? ${ }^{150}$

Die zentrale Hypothese, die der Property-Rights-Theorie und damit den aufgezählten Fragestellungen zugrunde liegt, ist, dass eine adäquate Allokation von Verfügungsrechten an Ressourcen deren effiziente Nutzung und damit einhergehend eine hohe Produktivität garantiert. Die Voraussetzung für eine effiziente Allokation ist, dass der Einnahmenrückfluss, der aus der produktiven Nutzung der Ressourcen, abzüglich des zu Marktpreisen bewerteten Faktoreneinsatzes, resultiert, kontrolliert wird. Um diese Kontrolle zu gewährleisten, ist es notwendig, dass der Besitzer von Ressourcen - als ,residual claimant on economic returns "“151 - nach Gewinnmaximierung strebt, deren Realisierung wiederum einen effizienten Ressourceneinsatz erfordert. Indem die effiziente Nutzung von Ressourcen zu ökonomischem Wohlstand führt, sind die Verfügungsrechte an Ressourcen gemäß der Property-Rights-Theorie daher jenen Akteuren zu überantworten, die sie am effizientesten nutzen. ${ }^{152}$

Aufgefasst als „motivation force that determines economic productivity“" 153 besitzen Property-Rights eine hohe Relevanz für das Funktionieren von Märkten.

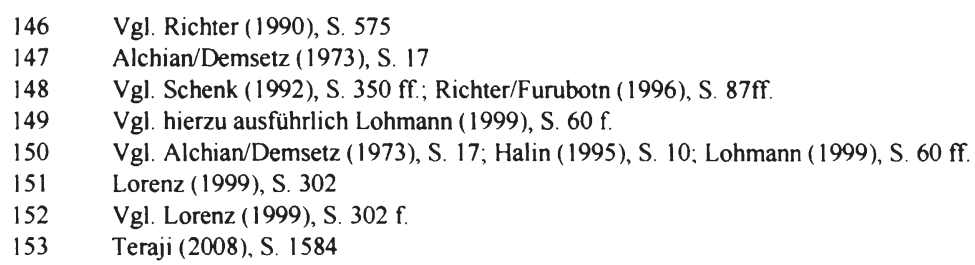

153 Teraji (2008), S. 1584 
Sofern die Verfügungsrechte an wirtschaftlichen Ressourcen unsicher sind, fallen Transaktionskosten an, die sich dadurch ergeben, dass besondere und kostenintensive Schutzmaßnahmen seitens der Ressourcenbesitzer erforderlich sind. Zur Reduktion von Transaktionskosten und zur Sicherstellung von Produktivität und Effizienz stellt sich daher die Frage nach einem alternativen Mechanismus, der hinter sicheren Property-Rights steht. Nach Teraji handelt es sich dabei um das Konstrukt ,Commitment', das sich auf die Intention eines Akteurs, eine Beziehung mit einer anderen Entität aufrechtzuerhalten, bezieht. Voraussetzung für die Entstehung von Commitment ist die Existenz von Vertrauen. Ein Akteur wird sich stärker zum Schutz von Property-Rights verpflichten, d.h. ein Commitment eingehen und aufrechterhalten, je mehr er erwartet, dass der andere Akteur seine selbstherbeigeführte Verwundbarkeit nicht ausnützt, oder in anderen Worten, je mehr er das Commitment des anderen Akteurs erwartet. Die Entstehung sicherer Property-Rights verlangt daher von jedem Akteur, darauf zu vertrauen, dass der andere Akteur seine Rechtsoptionen nicht opportunistisch ausnützen wird. Zusammenfassend kann Vertrauen im Rahmen dieser Theorie nicht bloß als Kalkulationsproblem, sondern als Voraussetzung für die Entstehung sicherer PropertyRights, die Reduktion von Unsicherheiten und Transaktionskosten und allgemein für Effizienzsteigerungen im Hinblick auf ökonomische Aktivitäten aufgefasst werden. ${ }^{154}$

\section{DiE ABGRENZUNG DES VERTRAUENSBEGRIFFS}

Die vorangegangene Diskussion hat gezeigt, dass Vertrauen als interdisziplinäres Forschungsgebiet höchst unterschiedlich konzipiert wird. Ein eindeutiges Verständnis von Vertrauen verlangt daher nach einer begrifflichen Abgrenzung von verwandten Mechanismen. Um dieser Forderung gerecht zu werden, ist es notwendig, zunächst die beiden Kategorien ,Unsicherheit' und ,Risiko', die eine Differenzierung zwischen Vertrauen - als Mechanismus zur Stützung unsicherer Erwartungen - und vertrauensnahen Begriffen ermöglichen, voneinander abzugrenzen.

Eine einheitliche Definition von Risiko und Unsicherheit existiert nicht, vielmehr wird unter diesen Begriffen eine Vielzahl verschiedener Bedeutungen zusammengefasst. ${ }^{155}$ Im Bereich der ,Unsicherheit' kann generell zwischen objektiver und subjektiver, sowie zwischen endogener und exogener Unsicherheit unterschieden werden. ${ }^{156}$ Nach Ripperger ist die Ursache objektiver Unsicherheit - als Dimension der Welt - in der Zufälligkeit zukünftiger Ereignisse begründet. Hingegen resultiert die subjektive Unsicherheit - als Dimension des Menschen - aus

154 Vgl. Morgan/Hunt (1994), S. 24; Teraji (2008), S. 1586 ff.; ähnlich hierzu: Lohmann (1999), S. 62 f.; James/Sykuta (2005), S. 547 ff.; Berggren/Jordahl (2006), S. 161

155 Vgl. Williamson (1990b); Schrader et al. (1993); Bamberg/Coenenberg (1994)

156 Vgl. Hoyos (1987), S. 50; Kaufmann (1987), S. 38 
einem zusätzlichen Informationsmangel bzw. aus der mangelnden Fähigkeit, Informationen richtig zu verarbeiten. Dementsprechend liegt die Ursache für objektive Unsicherheit im Zufall, während subjektive Unsicherheit durch die Möglichkeit des Irrtums - als menschliche Schwäche - entsteht. ${ }^{157}$ Unter der Annahme begrenzter Rationalität kann davon ausgegangen werden, dass Akteure in Entscheidungssituationen sowohl mit objektiver, als auch mit subjektiver Unsicherheit konfrontiert sind.

Während die Ursache einer unsicheren Erwartung das Unterscheidungskriterium zwischen objektiver und subjektiver Unsicherheit darstellt, ist deren Gegenstand das Kriterium bei der Differenzierung zwischen exogener und endogener Unsicherheit. Unter exogener Unsicherheit (Umweltunsicherheit) sind jene Ereignisse zu verstehen, die durch Faktoren ausgelöst werden, die außerhalb des direkten Einflussbereichs von Akteuren liegen (z.B. Handlungen der Natur und Variablen wie Technologien und Preisentwicklungen). Demzufolge können ihre Eintrittswahrscheinlichkeiten auch nicht durch Vertragsabschlüsse mit den sie auslösenden Akteuren beeinflusst werden. Hingegen umfasst die endogene Unsicherheit eines Akteurs jene Ereignisse, die aus den - seinen Nutzen direkt oder indirekt beeinflussenden - Entscheidungen bzw. Handlungen seiner Interaktionspartner resultieren. Auf die Eintrittswahrscheinlichkeiten endogen unsicherer Ereignisse kann durch Vertragsabschlüsse mit den sie auslösenden Akteuren Einfluss genommen werden. In diesem Zusammenhang dienen Verträge der Koordination von Austauschbeziehungen und der Begrenzung von Handlungsrisiken. ${ }^{158}$

Vor dem Hintergrund einer von Unsicherheit gekennzeichneten Welt weisen Entscheidungen mehr als eine Konsequenz auf, womit der Nutzen einer Handlung unsicher ist. Grundsätzlich stellt Unsicherheit erst dann ein Problem für einen Akteur dar, wenn mehrere Kontingenzen verschiedene ökonomische Konsequenzen haben. Für den Fall, dass Handlungsalternativen zu unsicheren Ereignissen mit unterschiedlichen Auswirkungen auf den Nutzen des Akteurs führen, befindet er sich in einer durch Risiko gekennzeichneten Situation. ,Risiko' stellt demnach die Möglichkeit eines Schadens (Nutzenverlust, der auch in einem entgangenen Nutzengewinn durch die Nichtwahrnehmung einer alternativen Entscheidung (Opportunitätskosten) bestehen kann) dar, die sich in Folge der Entscheidung für eine konkrete Handlungsalternative ergeben kann. Die Höhe des Handlungsrisikos bestimmt sich nach den Eintrittswahrscheinlichkeiten der mit der Handlung verbundenen Kontingenzen und der dadurch bedingten ökonomischen Folgen. Während sich Unsicherheit also durch Wertneutralität kennzeichnet, umfasst der

157 Vgl. Ripperger (1998), S. 16

158 Vgl. Lorenz (1988), S. 197; Ripperger (1998), S. 17 
Begriff des Risikos die bewertbaren negativen Konsequenzen eines unsicheren Ereignisses. $^{159}$

\subsection{Abgrenzung gegen den Begriff, Zuversicht ${ }^{6}$}

Die begriffliche Abgrenzung von Vertrauen gegen Zuversicht basiert auf der Unterscheidung zwischen Unsicherheit und Risiko. Akteure können nicht existieren, ohne Erwartungen über zukünftige Ereignisse zu bilden, und zwar unter Vernachlässigung der Möglichkeit, dass diese Erwartungen enttäuscht werden können. Der Grund für diese Vernachlässigung liegt zum einen darin, dass die Wahrscheinlichkeit einer Enttäuschung gering ist und zum anderen, dass dauerhafte Angst und Verzweiflung keine sinnvollen Alternativen darstellen. So erwartet man beispielsweise bei der Inanspruchnahme eines öffentlichen Verkehrsmittels, nicht im Rahmen eines Unfalls verletzt zu werden oder beim Kauf von Nahrungsmitteln, dass diese nicht vergiftet sind. Die einzige Alternative zu Erwartungen dieser Art wäre, seine Erwartungen komplett zurückzuziehen, ohne über etwas zu verfügen, mit dem man sie substituieren könnte, das heißt also, in einem Zustand dauerhafter Angst und Unsicherheit zu leben. Zuversicht stellt damit eine generelle Reaktion auf die immer vorhandenen objektiven Unsicherheiten des Alltags dar. Vertrauen hingegen ist die potentielle Reaktion auf spezifische Risikosituationen, womit es sowohl die objektive Möglichkeit, als auch das subjektive Bewusstsein eines potentiellen Schadens voraussetzt. Dementsprechend beruhen Risiken auf dem Bewusstsein, dass das eigene Verhalten zukünftige Ereignisse beeinflussen kann, womit sie erst als Bestandteile konkreten Handelns und Entscheidens entstehen. Die Voraussetzungen für Risiken sind somit die Möglichkeit ihrer Vermeidung, sowie das damit einhergehende Vorliegen von Handlungsalternativen. ${ }^{160}$

Der Unterschied zwischen Zuversicht und Vertrauen liegt also in der subjektiven Wahrnehmung einer Situation. Um eine Vertrauenssituation handelt es sich, wenn sich der Vertrauensgeber der Möglichkeit eines Schadens bewusst ist und er eine Vermeidung dieser Situation durch Entscheidung zwischen mehreren Handlungsalternativen in Erwägung zieht. Hingegen ist von einer Zuversichtssituation zu sprechen, wenn der Vertrauensgeber keine Handlungsalternativen im Umgang mit Unsicherheitssituationen erwägt. Für den Fall, dass eine Zuversichtssituation als riskant empfunden wird, wodurch ihre Vermeidung in Erwägung gezogen wird, kann sie sich in eine Situation des Vertrauens umwandeln. Demgegenüber kommt es zu einer Umwandlung einer Vertrauenssituation in eine Zuversichts-

159 Vgl. Ripperger (1998), S. 19 f.

160 Vgl. Deutsch (1962), S. 302; Luhmann (1988), S. 97 ff.; Giddens (1995), S. 45 f.; Ripperger (1998), S. 36 f.; Klaus (2002), S. 113 f.; Fink (2004), S. 36 f.; Röß1/Fink (2006), S. 59 
situation, wenn die Erwartung durch Sicherheit gekennzeichnet ist und es zu einer Vernachlässigung der Möglichkeit ihrer Enttäuschung kommt. ${ }^{161}$

\subsection{Abgrenzung gegen den Begriff, Hoffnung}

Nach der Abgrenzung von Vertrauen gegen Zuversicht, sowie der Begründung der Notwendigkeit von Vertrauen in Risikosituationen, stellt sich die Frage, ob Vertrauen bei allen Risikoarten gleichermaßen relevant ist. Vertraut beispielsweise ein Akteur, der einen Einsatz im Rahmen eines Glücksspiels (z.B. Lotterie, Roulette) riskiert? Die Frage kann verneint werden, da ein Vertrauen in den Zufall nicht möglich ist. Dadurch, dass in einer derartigen Situation allenfalls die Hoffnung auf ein günstiges Ergebnis besteht, ist Vertrauen vor diesem Hintergrund nicht relevant. Im Gegensatz zur Hoffnung stellt sich beim Vertrauen zusätzlich die Frage, wem vertraut wird und nicht bloß, worauf man vertraut. Vertrauen bezieht sich damit auf endogene Unsicherheit, die dann vorliegt, wenn das eingegangene Risiko vom Verhalten des potentiellen Vertrauensnehmers abhängig ist. Um die Unsicherheit im Hinblick auf die Entscheidungen der Mitakteure und ihre Auswirkungen zu akzeptieren, kann der Akteur auf erwartungskonforme Handlungen vertrauen. ${ }^{162}$ Vertrauen reflektiert damit die prinzipielle Offenheit menschlicher Einstellungen und Handlungen. ${ }^{163}$ Hoffnung hingegen bezieht sich grundsätzlich auf exogene Unsicherheit, die vorliegt, wenn keiner der Akteure die Eintrittswahrscheinlichkeit der Ereignisse beeinflussen kann. ${ }^{164}$ Auf diese Art von Unsicherheit kann der Akteur nur mit Hoffnung reagieren. Hoffnung eliminiert demnach Kontingenz. ${ }^{165}$

\subsection{Abgrenzung gegen den Begriff ,Zutrauen`}

Vertrauen stellt ein Instrument dar, das es ermöglicht, endogene Unsicherheit in Bezug auf das Verhalten eines Akteurs zu akzeptieren. In unsicheren Interaktionssituationen ist der Vertrauensgeber gezwungen, Erwartungen bezüglich der Handlungen eines potentiellen Vertrauensnehmers anzustellen. Stimmen diese Erwartungen mit den von diesem möglichen Vertrauensnehmer ausdrücklich oder implizit angekündigten Handlungen überein, so handelt es sich um Vertrauen. ${ }^{166}$ Vertrauen bezieht sich also im Endeffekt immer auf die Erwartung kooperativen

\footnotetext{
161 Vgl. Ripperger (1998), S. 37

162 Vgl. Lorenz (1988), S. 197 f.; Ripperger (1998), S. 37 f.: Luhmann (2000), S. 35: Roeder (2000), S 24; Fink (2004), S. 37; Röß1/Fink (2006), S. 59

163 Vgl. Luhmann (2000), S. $28 \mathrm{f}$.

164 Vgl. Antfang/Urban (1994), S. 6; Ripperger (1998), S. 38; Rößl/Fink (2007)

165 Vgl. Luhmann (2000), S. 29

166 Vgl. Robinson (1996), S. 578; Becerra/Gupta (2003), S. 33: Meijboom et al. (2006), S. 428: Rößl/Fink (2006), S. 58
} 
Verhaltens, sei es auch nur die Vermeidung gegenseitiger Schädigung als Minimalbedingung wechselseitiger Kooperation. ${ }^{167}$

Die Erfüllung der Erwartungen des Vertrauensgebers ist von der Existenz zweier Größen abhängig: der technischen Kompeten $z^{168}$ und dem Willen des potentiellen Vertrauensnehmers. Die Bewertung dieser beiden Einflussfaktoren ist durch Unsicherheit gekennzeichnet. ${ }^{169}$ Ist der Vertrauensgeber der Meinung, dass der potentielle Vertrauensnehmer über die technische Kompetenz verfügt, die in ihn gesetzte Erwartung zu erfüllen, so spricht man von Zutrauen. Zutrauen bezieht sich demnach rein auf jene Erwartungen, die in Zusammenhang mit der technischen Kompetenz des potentiellen Vertrauensnehmers in Bezug auf ein bestimmtes Verhalten stehen. Während im Fokus von Zutrauen technische Kompetenzunsicherheit steht, bezieht sich Vertrauen auf die Unsicherheit, ob der potentielle Vertrauensnehmer seine Fähigkeiten korrekt darstellt und sie entsprechend der Erwartungen des Vertrauensgebers einsetzt, und sich demnach erwartungskonform verhält. Vertrauen betrifft daher ausschließlich motivationale Aspekte in Kooperationsbeziehungen und damit Verhaltensrisiken. ${ }^{170}$ Diese ergeben sich dadurch, dass die Vertrauensentscheidung des Vertrauensgebers besonders durch opportunistisches Verhalten seitens des Vertrauensnehmers enttäuscht werden kann. Von zentraler Bedeutung für die Entstehung von Vertrauen ist daher die generelle Vertrauensbereitschaft des Vertrauensgebers: Je stärker die subjektive Neigung des Vertrauensgebers ist, dem potentiellen Vertrauensnehmer vertrauenswürdiges Verhalten zu unterstellen, desto eher kann sie durch das Verhalten des Vertrauensnehmers begründete Risiken absorbieren, womit sie sich positiv auf die Höhe der Vertrauenserwartung auswirkt. ${ }^{171}$ Hingegen ist das Vorliegen einer Zutrauensbereitschaft für die Entstehung von Zutrauen nicht erforderlich, da sich Zutrauen auf technische Aspekte in Kooperationsbeziehungen und Gefahren anstelle von Risiken bezieht: Da Gefahren im Gegensatz zu Risiken nämlich außerhalb der Kontrolle des Vertrauensgebers liegen, muss der Vertrauensgeber nicht zutrauensbereit sein, um eine Zutrauenserwartung bezüglich der technischen Kompetenz des Vertrauensnehmers bilden zu können - er ist der Gefahr einer Zutrauenserwartungsenttäuschung ohnehin ausgeliefert. ${ }^{172}$

167 Vgl. Ripperger (1998), S. 38; Sako/Helper (1998), S. 388

168 Darunter sind sowohl die Basis- und Kernkompetenzen auf organisationaler Ebene, als auch die Fach- und Methodenkompetenzen auf individueller Ebene des Vertrauensnehmers zu verstehen (vgl. hierzu die Ausführungen in Teil III dieser Arbeit).

169 Vgl. Fink (2004), S. 37; Rößl/Fink (2006), S. 59

170 Vgl. Dasgupta (1988), S. 52; Ripperger (1998), S. 39 f.

171 Vgl. hierzu die Ausführungen in Teil II Kap. 5.1 und Teil IV Kap. 5, sowie die dort zit. Literatur.

172 Vgl. Luhmann (1988), S. 98, sowie vor allem die Ausführungen in Teil IV Kap. 5 und die dort zit. Literatur. 


\subsection{Abgrenzung gegen den Begriff, Vertrauenswürdigkeit ${ }^{\star}$}

Vertrauen beruht auf der Erwartungshaltung des Vertrauensgebers, dass sich der Vertrauensnehmer als vertrauenswürdig erweist und freiwillig auf opportunistisches Verhalten verzichtet. ${ }^{173}$ Vertrauenswürdigkeit ist hierbei als ,[...] the motivation (or lack thereof) to lie ${ }^{\text {“ } 174}$ zu verstehen. Diese Definition ist in dem Sinne zu interpretieren, als dass der Vertrauensnehmer dann als vertrauenswürdig eingestuft wird, wenn er sich freiwillig gegen opportunistisches Verhalten entscheidet. Auch für Luhmann ist ein Akteur vertrauenswürdig, wenn er ,[...] bei dem bleibt, was er bewußt oder unbewußt über sich selbst mitgeteilt hat." ${ }^{175}$ Die Neigung des potentiellen Vertrauensnehmers, sich entsprechend seiner expliziten oder impliziten Mitteilungen zu verhalten, steigt in Abhängigkeit von der Dauer der Vertrauensbeziehung, sowie der Kommunikationshäufigkeit zwischen den beteiligten Akteuren. Auch wird sich der Vertrauensnehmer als umso vertrauenswürdiger erweisen, je größer der Gewinn ist, den er sich von der Vertrauensbeziehung erhofft. ${ }^{176}$

Die Vertrauenswürdigkeit des Vertrauensnehmers ist insofern relevant, als dass sie die Vertrauensentscheidung des Vertrauensgebers beeinflusst. Über das Aus$\mathrm{maß}$ der Vertrauenswürdigkeit urteilt der Vertrauensgeber anhand der subjektiven Einschätzung von Merkmalen des potentiellen Vertrauensnehmers. ${ }^{177}$ Einerseits resultiert die Entscheidung zu vertrauen aus einer rationalen Bewertung der Vertrauenswürdigkeit des potentiellen Vertrauensnehmers, basierend auf dem Wissen über ihn (abgeleitet aus z.B. Reputation, etablierten Normen und Standards, eigenen Erfahrungen). Andererseits hat die Vertrauensentscheidung auch psychologische Ursachen, die Einfluss auf die Erwartung und Zuschreibung von Vertrauenswürdigkeit nehmen. ${ }^{178}$

\subsection{Abgrenzung gegen den Begriff, Reputation“}

Um eine Vertrauensbeziehung zu etablieren, benötigen Akteure Ressourcen, die oder deren Wertentwicklung sie anderen Akteuren zur Erhöhung ihrer eigenen Abhängigkeit übertragen können. Die wohl am häufigsten überantwortete Ressource bzw. das gebräuchlichste Pfand ist die Reputation eines Akteurs in seiner

$173 \mathrm{Vgl}$. dazu Bradach/Eccles (1989, S. 104): „Trust is a type of expectation that alleviates the fear that one's exchange partner will act opportunistically." Ähnlich auch Madhok (1995, S. 120): "[...] trust is the perceived likelihood of the other not behaving in a self-interested manner."

174 Mayer et al. (1995), S. 716

175 Luhmann (2000), S. 48

176 Vgl. Coleman (1991), S. 138

177 Vgl. Mayer et al. (1995), S. 717, und die Ausführungen in Teil II Kap. 6, sowie die dort zit Literatur

178 Vgl. Nooteboom (2002), S. 11 ff.; ähnlich auch Kramer (1999, S. 571): „[...] most trust theorists agree that trust $[\ldots]$ is fundamentally a psychological state." 
Umwelt, aber auch sich selbst gegenüber ${ }^{179}:$,Wer moralisch kommuniziert und damit bekannt gibt, unter welchen Bedingungen er andere und sich selbst achten bzw. missachten wird, setzt seine Selbstachtung ein - und damit aufs Spiel.“180 Die Reputation, über die ein Akteur verfügt, ist damit ein Ausgangspunkt, um Vertrauensbeziehungen zu etablieren - und das ist auch der Punkt, an dem sich Vertrauen und Reputation treffen: der Vertrauensgeber vertraut einem Vertrauensnehmer, nicht weil dieser eine Reputation als vertrauenswürdiger Akteur hat, sondern der Vertrauensnehmer hat eine Reputation als vertrauenswürdiger Akteur, weil er seine Reputation in der Vertrauensbeziehung immer neu aufs Spiel zu setzen bereit ist. ${ }^{181}$

Im allgemeinen Sprachgebrauch ist Reputation, abgeleitet von ,reputatio“ (lat.), als der Ruf oder das Ansehen zu interpretieren. ${ }^{182}$ Genauer gesagt wird sie als „impression of public esteem or high regard judged by others“ ${ }^{\text {“183 }}$ definiert. Reputation spiegelt sich in den Annahmen anderer über die Strategie, die ein Akteur verfolgt, wider. ${ }^{184}$ Sie umfasst sowohl Annahmen über das allgemeine Geschäftsgebaren eines Akteurs, als auch Annahmen über die Bereitschaft und Fähigkeit des Akteurs, Vertrauensbeziehungen aufzubauen. Sie kann damit als Ergebnis der Beobachtungen des Verhaltens eines Akteurs interpretiert werden, was dazu führt, dass sich jede Handlung des Partners auf dessen Reputation auswirkt. ${ }^{185}$

Reputation entsteht durch Addition der Einzelerwartungen in Bezug auf Vertrauenswürdigkeit und technische Kompetenz: ,[...] Reputation ist gewissermaßen die öffentliche Information über die bisherige Vertrauenswürdigkeit eines Akteurs. Sie spielt z.B. bei Kooperationen eine große Rolle, wenn keine nachprüfbaren Informationen über die Leistungsfähigkeit potentieller Partner [...] $]^{\star 186}$ vorhanden sind. Wird einem Akteur ein hohes Ausmaß an Vertrauenswürdigkeit und technischer Kompetenz zugeschrieben, so hat er eine gute Reputation. Um eine schlechte Reputation handelt es sich, wenn dem Akteur ein geringes Ausmaß an Vertrauenswürdigkeit und Kompetenz zugeschrieben wird. ${ }^{187}$

Reputation kann als Grad des dem Akteur in der Vergangenheit in Summe entgegengebrachten Vertrauens verstanden werden. Sie fungiert damit als Voraussetzung der zukünftigen Einnahmenerzielung des Akteurs, und kann als multi-

\footnotetext{
179 Vgl. Rößl/Fink (2004); Rößl/Fink (2006), S. 65

180 Luhmann (1991), S. 26

181 Vgl. Rößl/Fink (2006), S. 65 f.

182 Vgl. Brockhaus (2008b)

183 Merriam Webster's Collegiate Dictionary (1996), S. 1001

184 Vgl. Axelrod (1991), S. 135

185 Vgl. Axelrod (1991), S. 135; Pieper (2000), S. 141 f.; Perry (2001), S. 71 f.

186 Picot et al. (2001), S. 126 f.; ähnlich auch Ripperger (1998, S. 183): "Die Reputation eines Akteurs reflektiert den Informationsstand Dritter darüber, wie vertrauenswürdig er sich in der Vergangenheit anderen gegenüber verhalten hat." 
attributives Konstrukt zur Erfassung des von Anderen bereits erhaltenen Vertrauenskapitals aufgefasst ${ }^{188}$ und damit als Indikator zur Messung seiner Vertrauenswürdigkeit herangezogen werden. ${ }^{189}$

\subsection{Abgrenzung gegen den Begriff, Misstrauen“}

Misstrauen stellt eine Alternative zu Vertrauen dar, die genauso wenig wie Vertrauen als „universelle Einstellung durchführbar“"190 ist. Während ganzheitliches Vertrauen zu riskant ist, ist generelles Misstrauen zu belastend. ${ }^{191}$

Vor dem Hintergrund der Funktion von Vertrauen wird die qualitative Unterschiedlichkeit und funktionale Äquivalenz von Vertrauen und Misstrauen deutlich: Vertrauen reduziert soziale Komplexität, sichert also durch Übernahme eines Risikos die Handlungsfähigkeit. Trifft ein Akteur hingegen bloß die Entscheidung nicht zu vertrauen, so stellt er die ursprüngliche Komplexität der Geschehensmöglichkeiten wieder her. Da ihn dieses Übermaß an Komplexität überfordern und handlungsunfähig machen würde, muss er - wenn er nicht vertrauen will - auf eine funktional äquivalente Strategie der Komplexitätsreduktion zurückgreifen. Er muss seine Erwartungen in eine negative Richtung lenken, muss also in bestimmten Hinblicken misstrauisch werden. Dementsprechend trägt auch Misstrauen zu einer Vereinfachung im Umgang mit der sozialen Komplexität bei. ${ }^{192}$

Misstraut also ein Akteur einem anderen Akteur, so verzichtet er bewusst auf Kontrollinformationen obwohl er mit hoher Sicherheit ein negatives Ereignis erwartet. Verfügt er weder über die Bereitschaft, sich Kontrollinformationen zu beschaffen, noch über die Bereitschaft, Risiko zu übernehmen, bleibt ihm nur noch die Alternative, seine Erwartungen ins Negative zu verschieben, um die Umweltkomplexität zu reduzieren. Diese Haltung wird als Misstrauen bezeichnet. Der Unterschied zwischen Misstrauen und Vertrauen liegt demnach in einer mit einer negativen statt einer positiven Wertschätzung verbundenen Erwartung. ${ }^{193}$ So wird sich ein Akteur entscheiden, zu vertrauen, wenn er davon ausgeht, dass sich das mögliche Verhalten anderer Akteure positiv in seinen eigenen Lebensführungsplan einfügen wird. Hingegen wird er misstrauen, wenn er davon ausgeht, dass dies nicht der Fall sein wird. Weiters unterscheidet sich Misstrauen im Hinblick

\footnotetext{
$188 \quad$ Vgl. Weigelt/Camerer (1988), S. 443

189 Vgl. Perry (2001), S. 71 f.

190 Luhmann (2000), S. 94

191 Vgl. Luhmann (2000), S. 95

192 Vgl. Luhmann (2000), S. 93

193 Vgl. Platzköster (1990), S. 48; Pieper (2000), S. 68 f.; ahnlich auch Sitkin/Roth (1993, S. 367) „[...] distrust is engendered when expectations about the compatibility of an employee's belief and values with the organization's cultural values are called into question."
} 
auf seine Erscheinungsformen von Vertrauen. Es ist beispielsweise mit Stress, Angst und Hoffnungslosigkeit verbunden. ${ }^{194}$

\section{DiE BEGRIFFLICHE FESTLEGUNG VON VERTRAUEN}

Die Verschiedenartigkeit der Vertrauenskonzeptionen von Wissenschaftlern und der nahe Bezug zu verwandten Begriffen ist ein deutliches Zeichen für die Vielschichtigkeit des Vertrauensphänomens. Je nach Perspektive und Forschungsintention stehen unterschiedliche Aspekte im Vordergrund, weshalb keine dominierende Vertrauensdefinition existiert.

\subsection{Unbrauchbare Annahmen}

Vor allem die Perspektive der klassischen Theorie der Ökonomik, die von einem vollkommen rationalen Homo Oeconomicus ausgeht, hat wesentlich dazu beigetragen, dass der Vertrauensbegriff als weitgehend unbestimmte ,Black Box' verwendet wurde: Vor dem Hintergrund vollkommener Rationalität und vollständiger Informationen ist es dem Homo Oeconomicus möglich, sämtliche Handlungsalternativen aller Akteure des Wirtschaftslebens - seine inbegriffen - zu kennen und zu erfassen. Während ihm die Annahme vollständiger Information unbeschränkte Kenntnis über die Handlungsmöglichkeiten aller Akteure unterstellt, weist ihm die Annahme unbegrenzter Rationalität die kognitive Kapazität zu, diese Informationen fehlerfrei zu verarbeiten. Hinzu kommt, dass dem Homo Oeconomicus nicht nur alle Handlungsmöglichkeiten, sondern auch die Handlungsfolgen bekannt sind. Ausgehend vom unbeschränkten Opportunismus aller Akteure, kommt es zu einer Situation, in der alle alles wissen. Unsicherheit ist damit denkunmöglich, denn jeder Akteur wählt aus seinen Handlungsmöglichkeiten jene aus, deren Folgen für ihn am günstigsten sind. Bei Vorliegen dieser Annahmen kann das Verhalten aller Akteure vorhergesagt werden. Aufgrunddessen, dass Sicherheit im Hinblick auf das Verhalten aller Akteure besteht, ist Vertrauen für den Homo Oeconomicus nicht nur überflüssig, es ist ihm gar nicht möglich zu vertrauen. ${ }^{195}$

194 Vgl. Luhmann dazu (2000, S. 93): "Diese negativen Strategien geben dem Misstrauen jenes emotional gespannte, oft krampfhafte Naturell, das es vom Vertrauen unterscheidet."

195 Vgl. Simmel (1968), S. 263; Rößl/Fink (2006), S. 58; ännlich dazu auch Albach (1980, S. 3): „In der Theorie des vollkommenen Marktes bei Sicherheit braucht man kein Vertrauen. Für den homo oeconomicus sind alle Partner gleich - und gleich gute Optimierungsmaschinen." 


\subsection{Die Annahmen der Neuen Institutionenökonomie}

Vertrauen kann demnach nur in einem Denkrahmen analysiert werden, in dem das Verhalten der Akteure und damit die Handlungsfolgen unsicher sind. ${ }^{196}$ Einen solchen Denkrahmen stellt die Neue Institutionenökonomie dar, indem sie von begrenzter Rationalität, unvollständiger Information und von einem durch langfristige Perspektiven begrenzten kurzfristigen Opportunismus ausgeht. Nachdem im vorangegangen Kapitel Vertrauen als eine mögliche Reaktion auf Verhaltensrisiken eingegrenzt wurde, soll nun überprüft werden, ob sich die realitätsnäheren Verhaltensannahmen der Neuen Institutionenökonomie tatsächlich zur Erfassung der Vertrauensproblematik eignen: Vertrauen wird erst durch die Verhaltensunsicherheit, das heißt durch Unsicherheit über die Intentionen der am Austausch beteiligten Akteure als Problem begründet. Es basiert auf einer mit einer positiven Wertschätzung verbundenen Erwartung, schließt die Möglichkeit einer Enttäuschung aber nicht aus. Die Motivation eines Akteurs resultiert ökonomisch aus dessen Präferenzen und Handlungsbeschränkungen. Verhaltensunsicherheit resultiert vorrangig aus subjektiver Unsicherheit, das heißt aus mangelnden Informationen über die tatsächlichen Präferenzen eines anderen Akteurs, umfasst allerdings auch objektive Unsicherheit über mögliche Handlungsrestriktionen. Ein Akteur verfügt über private Informationen in Bezug auf seine Präferenzen, die er gegenüber dem Vertrauensgeber verbergen kann. Das Vorliegen von Informationsasymmetrien, gemeinsam mit der Möglichkeit eines Irrtums stellt somit eine wesentliche Voraussetzung für die Erfassung der Vertrauensproblematik dar. Allein deshalb wird der Homo Oeconomicus der klassischen Theorie der Ökonomik nie der Vertrauensproblematik gegenüberstehen. Erst durch die Annahme begrenzter Rationalität können subjektive Unsicherheit und damit die Möglichkeit eines Irrtums als eine notwendige Bedingung für Vertrauen entstehen. Das Vertrauensrisiko, das ein weiteres grundlegendes Element jeder Vertrauenssituation ist, wird durch die Möglichkeit der Vertrauensenttäuschung und des sich daraus ergebenden Schadens begründet. Das Vorliegen eines derartigen Risikos verlangt nach der potentiellen Bereitschaft des Vertrauensnehmers, Handlungsspielräume auch zum Schaden des Vertrauensgebers auszunutzen. Die in der klassischen Theorie der Ökonomik unterstellte Annahme, dass der Homo Oeconomicus bloß seinen Eigeninteressen verpflichtet ist, kann diese Möglichkeit eines Schadens nicht erklären und deshalb die Vertrauensproblematik nicht erfassen. Dementsprechend schafft erst begrenzte Rationalität Raum für Opportunismus und das Problem des Vertrauens wird allein durch die Gefahr opportunistischen Verhaltens bedingt. Damit stehen die Verhaltensannahmen der Neuen Institutionenökonomie in keinem Widerspruch zur Vertrauens- 
problematik, sondern sind sogar eine notwendige Voraussetzung für ihre Erfassung im Rahmen der vorliegenden Arbeit. ${ }^{197}$

\section{3 Über die Vertrauensbegründung zu einem differenzierten Vertrauensbegriff}

Nachdem Vertrauen als eine mögliche Reaktion auf Verhaltensrisiken, die durch die motivationale Disposition eines anderen Akteurs begründet werden, weiter eingegrenzt wurde, stellt sich nun die Frage nach einer konkreten Definition des Vertrauensbegriffs. Zu diesem Zweck ist es sinnvoll, sich an der Begründung bzw. Rechtfertigung des Vertrauensschenkens zu orientieren. Ebenso wie Osterloh und Weibel zwischen instrumentellem und maximenbasiertem Vertrauen unterscheiden ${ }^{198}$, differenziert Luhmann nach der Begründbarkeit des Vertrauensschenkens zwischen gerechtfertigtem und ungerechtfertigtem Vertrauen. Gerechtfertigtes Vertrauen bzw. instrumentelles Vertrauen stellt ein Verhalten dar, das auf einem rationalen Kalkül basiert - es wandelt das Vertrauensproblem in ein Erkenntnisproblem ab. Ein Akteur kann einem anderen Vertrauen schenken, weil die anderen Verhaltensmöglichkeiten für diesen wenig attraktiv sind. „Man könnte dieser „Lösung“ daher auch die Fassung geben: Vertraue dort, wo es nicht nötig ist. Das eigentlich Problem ist aber das ungerechtfertigte Vertrauen, das sich selbst rechtfertigt und dadurch schöpferisch wird." "199 Im Rahmen des ungerechtfertigten bzw. maximenbasierten Vertrauen legen sich die Akteure darauf fest, dass der andere sich - trotz Bestehens seiner Opportunismusspielräume - kooperativ verhalten wird, und verhalten sich im Hinblick auf diese riskierte Erwartung kooperativ. Aus der übereinstimmenden kooperativen Antwort entsteht maximenbasiertes Vertrauen - ein Vertrauen, das sich selbst rechtfertigt: Der Vertrauensgeber schenkt dem Vertrauensnehmer sein Vertrauen, weil er erwartet, dass der Vertrauensnehmer die von ihm selbst herbeigeführte Verwundbarkeit nicht ausnützen wird. Vertrauen entsteht hier durch Maßnahmen, die die eigene Abhängigkeit der Parteien einer Vertrauensbeziehung erhöhen. ${ }^{200}$

Für die Festlegung der dieser Arbeit zugrunde liegende Vertrauensdefinition ist es erforderlich, zwischen instrumentellem und maximenbasiertem Vertrauen zu differenzieren, da es einen gravierenden Unterschied macht, ob auf ein bestimmtes Verhalten eines Akteurs vertraut werden kann, weil sein Verhaltensspielraum so eingeschränkt ist oder eingeschränkt wurde, dass ein bestimmtes Verhalten erwartet werden kann (instrumentelles Vertrauen), oder weil dieses Verhalten durch Etablierung maximenbasierten Vertrauens moralisch normiert

197 Vgl. Ripperger (1998), S. 41f.; ähnlich Bradach/Eccles (1989), S. 104

198 Vgl. Osterloh/Weibel (2000), S. 103

199 Vgl. Luhmann (2000), S. 102

200 Vgl. Rößl/Fink (2006), S. 59 f. 
wurde $^{201}$ : Einerseits können bestimmte Austauschbeziehungen nur über maximenbasiertes Vertrauen gesteuert werden. Hierbei handelt es sich um jene Fälle, bei denen Möglichkeiten zur effizienten Begrenzung von Opportunismusspielräumen begrenzt sind. In diesen Situationen ist die Vertrauenswürdigkeit der Kooperationspartner aufgrund des Fehlens von Kontrollmechanismen und der sich daraus ergebenden Unmöglichkeit von Sanktionen von besonderer Relevanz. Andererseits haben die Entstehung instrumentellen bzw. maximenbasierten Vertrauens verschiedene Ausgangspunkte: Bei erstem versucht ein Kooperationspartner die Opportunismusspielräume des anderen zu begrenzen und damit den anderen zu beherrschen, bei zweitem liefert sich der Kooperationspartner freiwillig dem anderen aus und reduziert damit seine eigenen Handlungsspielräume. Während instrumentelles Vertrauen also ein Mittel darstellt, um einen anderen Zweck als die Schaffung einer Vertrauenssituation zu erfüllen, bezieht maximenbasiertes Vertrauen, das auf eine Reduzierung von Opportunismusneigungen abzielt, seine Rechtfertigung aus sich selbst. Sein Nutzen liegt in der Verhaltenskoordination, deren Verlässlichkeit sich aus der Normierung vereinbarungskonformen Verhaltens durch Selbstverpflichtung der Akteure ergibt. ${ }^{202}$

Instrumentelles Vertrauen resultiert aus hierarchischem Steuerungshandeln und zielt mit Anordnungen, Kontrollen und Sanktionen auf eine Reduktion der Opportunismusspielräume ab. Da es im Rahmen der vorliegenden Arbeit aber gerade um Kooperationsbeziehungen geht, konzentrieren sich die folgenden Ausführungen auf maximenbasiertes und damit intrinsisch motiviertes Vertrauen. Aus den vorangegangen Überlegungen ergibt sich damit folgende Vertrauensdefinition:

Vertrauen ist die freiwillige Erbringung einer riskanten Vorleistung als Reaktion auf die subjektive Unsicherheit über das Verhalten des Kooperationspartners, und zwar unter Verzicht auf explizite vertragliche Sicherungs- und Kontrollmaßnahmen zur Begrenzung von Opportunismusspielrăumen, und in der Erwartung, dass sich der Kooperationspartner, trotz dem Fehlen dieser Schutzmaßnahmen, kooperativ verhalten wird.

\section{Die Principal-Agent-Theorie als Grundlage FÜr DIE ANALYSE VON VERTRAUENSBEZIEHUNGEN}

Um von Vertrauen sprechen zu können, ist grundsätzlich das Vorliegen einer Beziehung zwischen mindestens zwei Akteuren, die im Folgenden als Vertrauensbeziehung bezeichnet wird, erforderlich. Das Zustandekommen einer Vertrauens-

201 Vgl. Rößl (1996); Osterloh/Weibel (2000)

202 Vgl. Rößl/Fink (2006), S. 60; ähnlich auch McAllister (1995); Rousseau et al. (1998) 
beziehung setzt sowohl die Schenkung, als auch die Annahme von Vertrauen voraus. Für eine weitere Genese von Vertrauen ist zudem dessen Honorierung erforderlich. Die Entscheidung, Vertrauen zu schenken, unterscheidet sich in ihrem zugrunde liegenden Kosten-Nutzen-Kalkül, sowie den mit ihr einhergehenden Anreizen, Handlungsalternativen und Risiken grundlegend von der Entscheidung, Vertrauen anzunehmen bzw. zu honorieren. Aufgrunddessen verlangt die Beschreibung und Erklärung des Vertrauenskonstrukts nach einer analytischen Trennung der Rolle des Vertrauensgebers auf der einen Seite, und der Rolle des Vertrauensnehmers auf der anderen Seite. ${ }^{203}$

Zentrum der Vertrauensproblematik ist ein Motivationsproblem, das durch Anreize und Sanktionen bewältigt werden muss. Hierfür stellt die Principal-AgentTheorie ein geeignetes theoretisches Instrumentarium dar, das ermöglicht, motivationsbedingte Risiken in der Vertrauensbeziehung zu erkennen und zu klassifizieren, sowie Empfehlungen zur Eingrenzung dieser Risiken abzuleiten (vgl. Kap. 4.1). Bisher wird Vertrauen überwiegend als Koordinationsmechanismus innerhalb von Principal-Agent-Beziehungen diskutiert (vgl. Kap. 4.2). Um den Vertrauensmechanismus und die ihm zugrunde liegenden Anreizmechanismen allerdings erfassen und erklären zu können, ist eine analytische Betrachtung auf einer Metaebene notwendig: Eine Modellierung der Vertrauensbeziehung als PrincipalAgent-Beziehung kann erfolgen, indem die Vertrauensbeziehung als implizite Vertragsbeziehung zwischen Vertrauensgeber und Vertrauensnehmer interpretiert wird (vgl. Kap. 4.3).

\subsection{Die opportunistischen Verhaltensspielräume in Principal- Agent-Beziehungen}

Nachdem der Gegenstand der Principal-Agent-Theorie bereits in Kap. 1.2.2 erläutert wurde, wird im Folgenden detaillierter auf die opportunistischen Verhaltensspielräume des Agenten, die sich aus seinem Informationsvorsprung gegenüber dem Prinzipal ergeben, eingegangen. Hierbei kann zwischen drei Möglichkeiten differenziert werden: Erstens hat der Agent die Möglichkeit, dem Prinzipal seine Eigenschaften (z.B. Qualifikationen) zu verheimlichen (,hidden characteristics'). Zweitens ist es dem Agenten möglich, seine Absichten vor dem Prinzipal zu verbergen (,hidden intentions'). Drittens kann der Agent Handlungen ausführen, die vom Prinzipal nicht beobachtet werden können (,hidden actions'). ${ }^{204}$

203 Vgl. Ripperger (1998), S. 63

204 Vgl. Schanz (2000), S. 139 


\subsubsection{Adverse Selection}

Das Problem der Adverse Selection liegt in dem Risiko der nicht optimalen Auswahl des Vertragspartners und beruht auf Informationsasymmetrien, die ex ante bzw. vor Vertragsabschluss zwischen Prinzipal und Agenten auftreten. ${ }^{205}$ Dem Prinzipal bleiben wesentliche Qualitätseigenschaften des Vertragspartners bzw. der von ihm angebotenen Dienstleistungen und Produkte (,hidden characteristics') vor Eingehen der Vertragsbeziehung verborgen. Durch die Möglichkeit, seine Eigenschaften geheim zu halten und damit durch den ihm zur Verfügung stehenden Informationsvorsprung, eröffnen sich dem Agenten Chancen der opportunistischen Ausnutzung dieser Asymmetrien, indem er eine für ihn vorteilhafte, aber für den Prinzipal unvorteilhafte Vertragsbeziehung eingeht. Unter Umständen können Adverse Selection-Probleme das Zustandekommen von Vertragsbeziehungen hemmen oder im Extremfall zum Zusammenbruch eines gesamten Marktes führen. ${ }^{206}$

Das Adverse Selection-Risiko, d.h. die Qualitätsunsicherheit, kann durch zwei Mechanismen begrenzt werden: Signaling und Screening. Das Ziel dieser Maßnahmen liegt im Abbau der zwischen Prinzipal und Agent bestehenden vorvertraglichen Informationsdifferenzen. Im Rahmen des Signaling gehen die Aktionen zum Abbau der ex ante existierenden Informationsasymmetrien vom Agenten als besser informierte Partei aus. Dementsprechend kann Signaling als aktiver Versuch, entscheidungsrelevante Informationen (z.B. in Form von Gütesiegeln, Garantien oder Zeugnissen über die Leistungsfähigkeit des Agenten) zu übermitteln, interpretiert werden. Voraussetzung für die Glaubwürdigkeit und somit Wirksamkeit von Signaling-Aktivitäten ist die Erfüllung der Self-Selection-Bedingung. Hierfür ist es erforderlich, dass das Signal nur von einem Agenten angeboten wird, der tatsächlich über die signalisierte Eigenschaft verfügt, und dass das Fehlen eines solchen Signals für ein Fehlen der entsprechenden Eigenschaft steht. Beim Screening gehen die Aktivitäten zur Reduzierung der vorvertraglichen Informationsasymmetrien vom Prinzipal als schlechter informierte Partei aus. Ziel ist die Differenzierung von Agenten anhand verschiedener Merkmale, die im Rahmen von Prüfungsaktivitäten oder unter Vorgabe von Selbstselektionsschemata erfolgen kann. ${ }^{207}$

\subsubsection{Hold Up}

Das Problem des Hold Up als Gefahr opportunistischen Verhaltens nach Vertragsabschluss entsteht durch Vorleistungen bzw. spezifische Investitionen des Prin-

205 Vgl. Arrow (1985), S. 38 ff.; Vogt (1997), S. 191; Ebers/Gotsch (1999), S. 213

206 Vgl. Stiglitz (1989); Picot (1991), S. 152; Vogt (1997), S. 28; Ripperger (1998), S. 65; Ebers/ Gotsch (1999), S. 213

207 Vgl. Stiglitz (1974); Spremann (1990); Vogt (1997), S. 110; Ripperger (1998), S. 65 
zipals. Diese führen zu einer Einschränkung bei der Auswahl zwischen verschiedenen Beziehungspartnern, wodurch eine einseitige Abhängigkeit des Prinzipals vom Agenten entsteht, die dieser nach Vertragsabschluss zum Schaden des Prinzipals ausnutzen kann. ${ }^{208}$ Je spezifischer die vorvertraglichen Investitionen des Prinzipals sind, desto höher ist seine Abhängigkeit vom Agenten. Als Beispiel für die Ausnutzung einer Hold Up-Situation kann das opportunistische Verhalten eines Automobilherstellers (Agent), der einem Zulieferunternehmen (Prinzipal) eine intensivierte Zusammenarbeit in Aussicht stellt und diesen dadurch zum Bau einer Produktionsanlage in seiner unmittelbaren Nähe veranlasst, genannt werden. Nach Vornahme der Investitionen durch das Zulieferunternehmen nutzt der Automobilhersteller seine Machtposition aus, indem er androht, sich aus dem bestehenden Vertrag zurückzuziehen. Aufgrunddessen, dass die spezifischen Investitionen für das Zulieferunternehmen bei Auflösung des Vertrages wertlos werden würden, ergibt sich für den Automobilhersteller die Möglichkeit, nach Vertragsabschluss Preiszugeständnisse zu erwirken, und damit seinen Opportunismusspielraum auszunützen. Das Hold Up-Problem zeigt sich also in der subjektiven Unsicherheit über die wahren Absichten des Agenten (,hidden intention'). Im Gegensatz zu ,hidden actions', die nach Vertragsabschluss weitgehend unentdeckt bleiben, kann der Prinzipal die zunächst verborgenen Absichten im Laufe der Vertragsbeziehung als grundsätzliche Handlungen des Agenten beobachten. Hidden intention ist vor allem im Zusammenhang mit spezifischen Investitionen des Prinzipals problematisch - diese stellen, anstelle von Informationsasymmetrien, den Kern des Hold Up-Problems dar. Die Angst vor der opportunistischen Ausnutzung einer Hold Up-Situation kann eine für beide Beziehungspartner vorteilhafte Kooperation verhindern. ${ }^{209}$

Das Hold Up-Risiko kann durch den Erwerb von Eigentum an spezifischen Investitionen reduziert werden (z.B. in Form einer vertikalen Integration von Leistungsaktivitäten). Eine andere Möglichkeit, um spezifische Investitionen zu schützen, stellt die ex ante zu erlangende Verfügungsgewalt über ein Pfand, das der Vertragspartner als Folge opportunistischen Verhaltens verlieren würde, dar. Das Ziel dieser Absicherung liegt im künstlichen Aufbau eines Gleichgewichts der Verhandlungspositionen innerhalb der Vertragsbeziehung. Indem die investierende Partei die Möglichkeit einer Gegenandrohung erhält, wird das einseitige Abhängigkeitsverhältnis in ein beiderseitiges umgewandelt. ${ }^{210}$

208 Vgl. Alchian/Woodward (1988), S. 67 f.; Vogt (1997), S. 30

209 Vgl. Vogt (1997), S. 30; Ripperger (1998), S. 67; Klaus (2002), S. 135

210 Vgl. Hart (1995); Vogt (1997), S. 230 


\subsubsection{Moral Hazard}

Das Moral Hazard-Problem entsteht während der Leistungsbeziehung und beruht auf Informationsasymmetrien, die ex post, d.h. nach Vertragsabschluss, zwischen Prinzipal und Agent auftreten. Nach Aufnahme der Vertragsbeziehung kann der Prinzipal bestimmte Anstrengungen des Agenten nicht beobachten bzw. nicht beurteilen (,hidden action'). Dem Agenten eröffnen sich durch seinen ihm zur Verfügung stehenden Informationsvorsprung Möglichkeiten der opportunistischen Ausnutzung dieser Asymmetrien, für den Fall, dass seine Leistung nicht beurteilt werden kann bzw. sehr hohe Kosten dafür anfallen würden. ${ }^{211}$

Die Anstrengungen des Agenten und die Wahrscheinlichkeit von günstigen Handlungsergebnissen für den Prinzipal verhalten sich prinzipiell positiv zueinander. Vorteilhafte Handlungsergebnisse sind allerdings nicht bloß vom Anstrengungsniveau des Agenten, sondern auch von vertragsexogenen Faktoren in Form von Umwelteinflüssen abhängig. Obwohl der Prinzipal das Handlungsergebnis des Agenten ex post feststellen kann, ist es ihm nicht möglich, dessen Handlungen an sich zu beobachten. Somit kann er nicht beurteilen, welchen Anteil die Anstrengungen des Agenten und welchen Anteil Umwelteinflüsse an dem zustandegekommenen Ergebnis nehmen. ${ }^{212}$ So können beispielsweise die Eigenkapitalgeber eines Unternehmens nicht unmittelbar erkennen, ob ein positives Geschäftsergebnis auf die Leistungen des Managements oder auf günstige exogene Einflüsse zurückzuführen ist. ${ }^{213}$

Das Problem des Moral Hazard kann durch den Einsatz von Monitoring-Aktivitäten begrenzt werden. Monitoring umfasst all jene Aktivitäten, die der Feststellung, ob der Agent seinen vertraglichen Pflichten nachkommt bzw. diese erfüllt hat, dienen. Für den Fall, dass Monitoring-Aktivitäten aufgrund situativer Gegebenheiten (z.B. technische Unmöglichkeit, hohe Kosten) kein geeignetes Kontrollinstrument darstellen, können Moral Hazard-Risiken durch ausdrückliche vertragliche Anreize teilweise reduziert werden. Das Ziel dieser Anreize ist es, die Entlohnung des Agenten derart an sein Handlungsergebnis zu knüpfen, dass eine möglichst weitreichende Übereinstimmung im Hinblick auf die Ziele von Prinzipal und Agent erreicht wird. Neben expliziten vertraglichen Regelungen kann der Besitz eines Pfandes (z.B. Reputation), das der Agent im Falle eines aufgedeckten opportunistischen Verhaltens verlieren würde, als Kontrollinstrument herangezogen werden. ${ }^{214}$

211 Vgl. Arrow (1985), S. 43 ff.; Plötner (1995), S. 47; Ripperger (1998), S. 66; Ebers/Gotsch (1999), S. 213

212 Vgl. Vogt (1997), S. 31; Ebers/Gotsch (1999), S. 213

213 Vgl. Ebers/Gotsch (1999), S. 213; Klaus (2002), S. 133

214 Vgl. Arrow (1985), S. 45 f;; Vogt (1997), S. 31 f.; Ripperger (1998), S. 66 f.; Ebers/Gotsch (1999), S. 214 


\subsection{Vertrauen als Koordinationsmechanismus in Principal- Agent-Beziehungen}

Vertrauen wird bisher vorrangig als alternativer Steuerungsmechanismus innerhalb der Beziehung zwischen Prinzipal und Agent diskutiert. Es dient in diesem Kontext als Erklärungsvariable für nicht durch explizite Kontroll- und Sicherungsinstrumente initiiertes kooperatives Verhalten des Agenten bzw. den Verzicht auf diese seitens des Prinzipals. ${ }^{215}$

Da explizite Kontroll- und Sicherungsinstrumente vielfach nicht oder nur zu unverhältnismäßig hohen Kosten durchsetzbar sind, erlangt Vertrauen bei der Überwindung der in Kapitel 4.1 beschriebenen opportunistischen Verhaltensspielräume eine entscheidende Bedeutung. So zeigt sich die Relevanz von Vertrauen bei der Überwindung von Adverse Selection-Problemen darin, dass es einerseits sowohl die Kosten des Signaling auf der Seite des Agenten, als auch die Kosten des Screening auf der Seite des Prinzipals reduziert. Andererseits können Signalingund Screening-Aktivitäten auch selbst als vertrauensfördernde Maßnahmen fungieren. ${ }^{216}$ Beispielsweise wird ein Zulieferunternehmen (Prinzipal), das einem Automobilhersteller (Agent) bereits ein gewisses Maß an Vertrauen entgegenbringt, weniger Zeit und Mühe für Nachforschungen über dessen Geschäftspraktiken aufwenden, als ein misstrauisches Unternehmen. Auf der anderen Seite können freiwillige Qualitätsgarantien des Automobilherstellers, d.h. des potentiellen Kooperationspartners, das Vertrauen des Zulieferunternehmens in ihn begründen bzw. erhöhen.

Sofern vertragliche Sicherungsmaßnahmen nicht oder nur zu sehr hohen Kosten implementierbar sind, können Hold Up-Probleme allein durch Vertrauen bewältigt werden. In diesem Zusammenhang ist darauf hinzuweisen, dass sich Vertrauen und explizite vertragliche Sicherungsmaßnahmen bei einem komplementären Einsatz innerhalb einer spezifischen Principal-Agent-Beziehung nicht beliebig substituieren lassen. So wird eine Misstrauensspirale in Gang gesetzt, wenn der Prinzipal in einer Hold Up-Situation bisher auf ein vertrauenswürdiges Verhalten des Agenten vertraut hat und dann plötzlich von letzterem Sicherungsgüter verlangt. Dieses Verlangen kann unter Umständen dazu führen, dass die Bereitschaft des Agenten, sich in zukünftigen Hold Up-Situationen - ohne extrinsische Anreizwirkungen, begründet durch entsprechende Sicherungsgüter - vertrauenswürdig zu verhalten, sinkt. ${ }^{217}$

Die Bedeutung von Vertrauen bei der Überwindung von Moral Hazard-Problemen kommt darin zum Ausdruck, dass die mit dem Vertrauenskonstrukt ver-

Vgl. Porter-Liebeskind/Lumerman-Oliver (1998), S. 119; Adler (2001), S. 219; Reed (2001), S. 201

Vgl. Luhmann (2000), S. 48 
bundenen sozialen und psychologischen Anreize und Sanktionen als Substitut für monetäre Anreizsysteme herangezogen werden können, was zu einer Kostensenkung führt. Es ist jedoch darauf hinzuweisen, dass diese bei einem komplementären Einsatz innerhalb einer spezifischen Principal-Agent-Beziehung nicht beliebig substituierbar sind. So kann die Intensivierung extrinsischer Motivationsmaßnahmen seitens des Prinzipals negative Auswirkungen auf die intrinsische Motivation des Agenten haben, womit eine Misstrauensspirale, ähnlich jener in der zuvor beschriebenen Hold Up-Situation, ausgelöst wird. Dieses Risiko ist besonders dann gegeben, wenn zwischen Prinzipal und Agent bereits Vertrauen besteht und der Agent das entgegengebrachte Vertrauen des Prinzipals selbst als Gegenstand eines impliziten Vertrags interpretiert. Unter diesen Umständen wird der Agent die Intensivierung extrinsischer Motivationsmaßnahmen als ein Zeichen von Misstrauen auffassen, und dementsprechend von einem einseitigen Bruch des impliziten Vertrags durch den Prinzipal ausgehen. Als Folge dieser Auffassung wird sich der Agent ebenfalls weniger stark an den impliziten Vertrag gebunden fühlen und aufgrund des Entfalls der psychologischen Kosten eines schlechten Gewissens, der sich durch den Zusammenbruch der Vertrauensbeziehung ergibt, sein Anstrengungsniveau deutlich reduzieren, was mit einer Verschlechterung des für den Prinzipal beobachtbaren Handlungsergebnisses einhergeht. Das verschlechterte Handlungsergebnis wird wiederum den Prinzipal zu Misstrauen veranlassen, weshalb er versuchen wird, das Anstrengungsniveau des Agenten durch intensivierte Monitoring-Aktivitäten bzw. weitere Maßnahmen zur Steigerung der extrinsischen Motivation wieder zu erhöhen. Es kommt damit zu einer Erosion von Vertrauen, die im Extremfall in einen völligen Zusammenbruch der Vertrauensbeziehung münden kann. ${ }^{218}$ Misstrauen gerät in diesem Szenario also zur selbsterfüllenden Prophezeiung, da „a decline in trust often leads to further decline in trust. “219

Zusammenfassend zeigt sich Vertrauen innerhalb von Principal-Agent-Beziehungen in einem weitgehenden Verzicht auf explizite Kontroll- und Sicherungsmaßnahmen zum Schutz gegen opportunistisches Verhalten. Indem der Prinzipal vertraut, geht er davon aus, dass der Agent weder bestehende Informationsasymmetrien noch spezifische Investitionen zu seinem Schaden ausnützt. Vertrauen stellt damit einen Koordinationsmechanismus dar, der durch die ,Absorption" von in Principal-Agent-Beziehungen bestehenden Verhaltensrisiken einen wesentlichen Beitrag zur Senkung von Agency-Kosten leisten kann.

218 Vgl. Arrow (1985), S. 50; Sitkin/Roth (1993); Robinson/Rousseau (1994); Robinson (1996): Ripperger (1998), S. 69 f.

219 Robinson/Rousseau (1994), S. 255 


\subsection{Die Vertrauensbeziehung als Principal-Agent-Beziehung}

Ebenso wie explizite Steuerungsinstrumente setzt der Vertrauensmechanismus für sein Funktionieren und damit für die Ermöglichung des Prozesses der Risikoabsorption entsprechende Anreizmechanismen voraus. Um die der Vertrauensbeziehung immanenten Anreizwirkungen zu identifizieren, einhergehend mit einer Erklärung und Erfassung des Vertrauensmechanismus, ist eine analytische Betrachtung der Vertrauensbeziehung auf einer Metaebene erforderlich. In diesem Sinne ist Vertrauen nicht länger bloß als mögliches Explanans für kooperatives Verhalten in Principal-Agent-Beziehung zu betrachten, sondern es wird selbst zum Gegenstand der Principal-Agent-Problematik. Dementsprechend wird die Vertrauensbeziehung als Principal-Agent-Beziehung in Form einer impliziten Vertragsbeziehung zwischen Prinzipal und Agent modelliert.

\subsubsection{Die Vertrauensbeziehung zwischen Vertrauensgeber und Vertrauens- nehmer}

Eine Vertrauensbeziehung setzt die Existenz von mindestens zwei Parteien voraus, d.h. von Vertrauensgeber und Vertrauensnehmer. Generell kann die Vertrauensbeziehung auch wechselseitig sein, sodass jeder Akteur gleichzeitig die Rolle von Vertrauensgeber und Vertrauensnehmer einnimmt. Aufgrunddessen, dass aber jede Partei über verschiedene Informationen und Handlungsalternativen verfügt, andere Interessen verfolgt und unterschiedlichen Anreiz- und Sanktionsmöglichkeiten ausgesetzt ist, ist die vorgenommene Rollentrennung aus analytischen Gründen unverzichtbar. Grundsätzlich können jeweils mehrere Akteure die Rolle des Vertrauensgebers bzw. des Vertrauensnehmers einnehmen (z.B. Vertrauen zwischen kooperierenden Unternehmen). Da hier die Auffassung vertreten wird, dass Vertrauen zwischen Unternehmen letztlich immer durch Individuen, die diesen Unternehmen angehören, begründet und zerstört wird ${ }^{220}$, wird die Vertrauensbeziehung im Folgenden, sowie im empirischen Teil dieser Arbeit als eine Beziehung zwischen Individuen analysiert.

\subsubsection{Die Vertrauensbeziehung zwischen Prinzipal und Agent}

In der Erbringung einer riskanten Vorleistung zeigt sich das Vertrauen des Vertrauensgebers. Indem der Vertrauensnehmer diese riskante Vorleistung annimmt, wird ein impliziter Vertrag zwischen ihm und dem Vertrauensgeber und damit die Entstehung einer Vertrauensbeziehung begründet. Diese charakterisiert sich durch Informationsasymmetrien und externe Effekte: Der Vertrauensnehmer verfügt über private Informationen im Hinblick auf seine wahren Präferenzen und Hand- 
lungsintentionen und hat die Macht, dem Vertrauensgeber durch sein Handeln einen Schaden zuzufügen oder einen Nutzen zu stiften. Der Kern der Vertrauensproblematik besteht daher in einem Principal-Agent- bzw. Motivationsproblem, das sich darin zeigt, dass der Vertrauensgeber nur vertraut, wenn er davon ausgeht, dass der Vertrauensnehmer motiviert ist, sich ihm gegenüber vertrauenswürdig zu verhalten. ${ }^{221}$ Damit kann die Vertrauensbeziehung als Principal-AgentBeziehung modelliert werden, in der der Vertrauensgeber die Rolle des Prinzipals und der Vertrauensnehmer die Rolle des Agenten einnimmt.

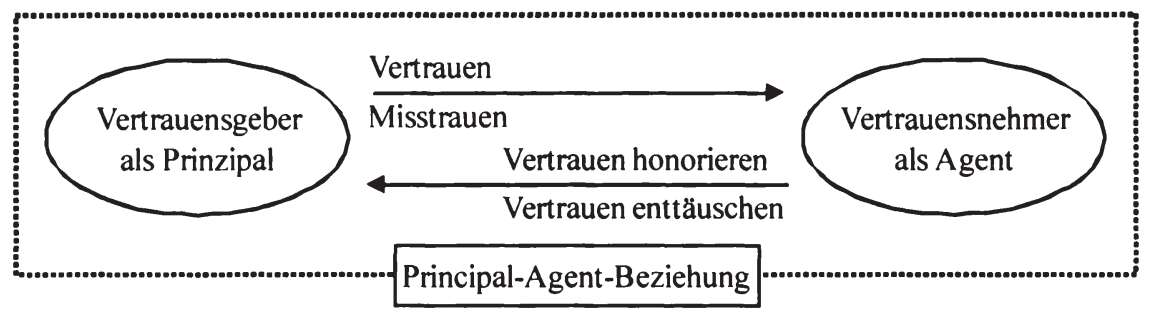

Abbildung 5: Die Vertrauensbeziehung als Principal-Agent-Beziehung

Quelle: In Anlehnung an Ripperger (1998), S. 74

\subsubsection{Der Vertrauensgeber als Prinzipal}

Vor dem Eintritt in eine Vertrauensbeziehung sieht sich der Vertrauensgeber grundsätzlich mit einem Adverse Selection-Problem konfrontiert, da er einen vertrauenswürdigenVertrauensnehmer auswählen bzw. entscheiden muss, ob der potentielle Vertrauensnehmer vertrauenswürdig ist. Zwar ist die Vertrauenswürdigkeit des Agenten in jeder Adverse Selection-Situation relevant, da sie die Glaubwürdigkeit seiner Aussagen im Hinblick auf die Qualität der von ihm angebotenen Leistungen erhöht. Allerdings stellen derartige qualitative Eigenschaften, wie zum Beispiel ISO-Zertifizierungen, zugleich das zentrale Kriterium für den Selektionsprozess dar. Wird jedoch die Vertrauensbeziehung als Principal-AgentBeziehung interpretiert, so stellt die Vertrauenswürdigkeit des Agenten selbst das zentrale Kriterium bzw. die zentrale Eigenschaft dar, anhand dessen bzw. der der Prinzipal verschiedene Agententypen unterscheidet. Während das Ziel der Screening-Aktivitäten des Prinzipals damit in der Identifizierung von Agenten, die über diese Eigenschaften verfügen, besteht, sind die Agenten bestrebt, diese Eigenschaften zu signalisieren, um vorvertragliche Informationsasymmetrien abzubauen. ${ }^{222}$

221 Vgl. Mayer et al. (1995); Ripperger (1998), S. 73; Zaheer et al. (1998); Roeder (2000), S. 124: Slangen (2003), S. 1096

222 Vgl. Ripperger (1998), S. 74; Roeder (2000), S. 125; Coyle-Shapiro/Morrow (2001), S. 325; Klaus (2002), S. 145 
Vertrauensvolles Verhalten zeigt sich in der Erbringung einer riskanten Vorleistung durch den Vertrauensgeber, womit Vertrauen grundsätzlich ein Hold UpRisiko begründet: Indem es sich bei der riskanten Vorleistung um eine irreversible Investition handelt, führt ihre Erbringung den Vertrauensgeber in ein einseitiges Abhängigkeitsverhältnis vom Vertrauensnehmer. So macht sich ein $\mathrm{Zu}$ lieferunternehmen (Prinzipal) von einem Automobilhersteller (Agent) abhängig, wenn es eine Produktionsanlage in dessen unmittelbarer Nähe errichtet, weil es dem Automobilhersteller, der ihm eine intensivierte Zusammenarbeit in Aussicht gestellt hat, vertraut. Grundsätzlich steigt die Höhe des Hold Up-Risikos, dem sich der Vertrauensgeber als Prinzipal aussetzt, mit der Spezifität und dem Wert der riskanten Vorleistung. ${ }^{223}$

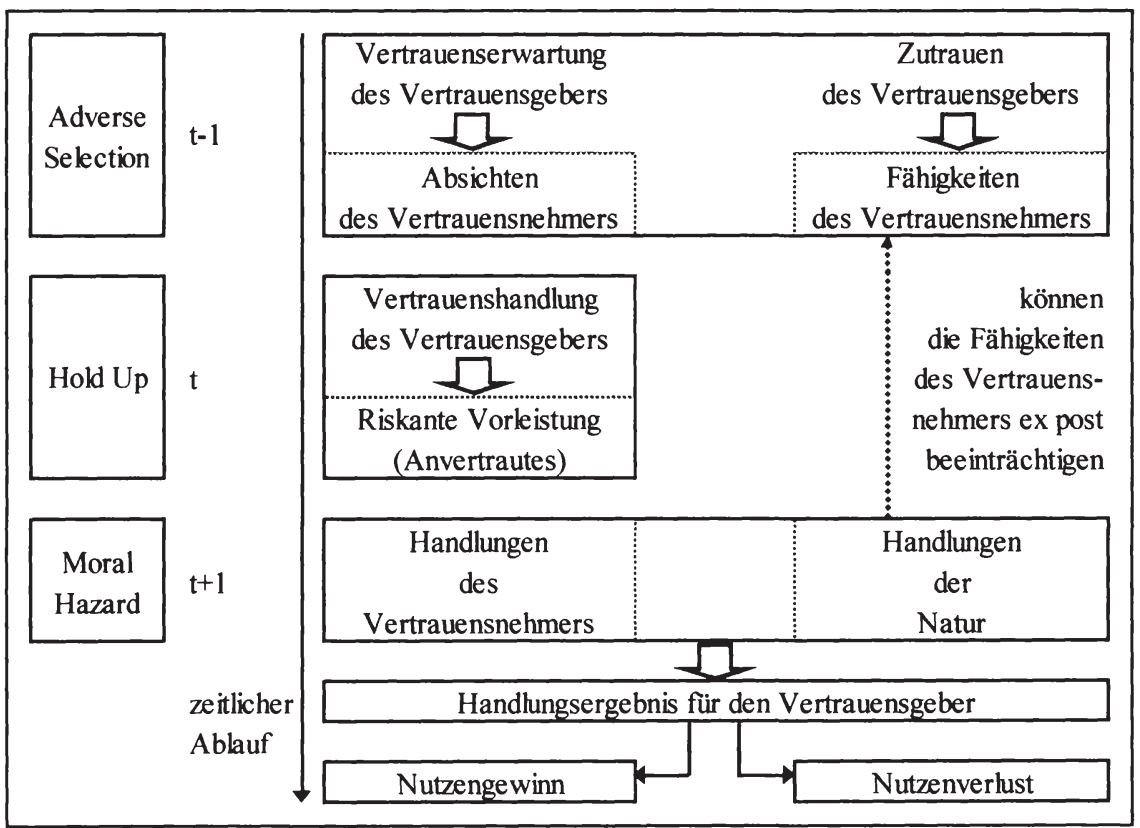

Abbildung 6: Die durch die Vertrauensbeziehung begründeten Verhaltensrisiken

Quelle: In Anlehnung an Ripperger (1998), S. 76

Sind Vertrauensgeber und Vertrauensnehmer eine Vertrauensbeziehung eingegangen, kann der Vertrauensnehmer zwischen mehreren Handlungsalternativen wählen. Diese liegen auf einem Kontinuum, das an seinen Extrempunkten mit ,Vertrauen honorieren' bzw. ,Vertrauen enttäuschen' begrenzt wird. Die Beurteilung des Handlungsergebnisses ist für den Vertrauensgeber unter Umständen erschwert, da er das Anstrengungsniveau des Vertrauensnehmers oft nur mangel- 
haft von exogenen Umwelteinflüssen abgrenzen kann, was einer Moral HazardSituation gleichkommt. Er ist dann nicht in der Lage, korrekt zu beurteilen, inwieweit der Eintritt eines schädigenden oder nutzenstiftenden Ereignisses auf die Handlung des Vertrauensnehmers bzw. auf nicht kontrollierbare exogene Einflüsse zurückzuführen ist. ${ }^{224}$ Beispielsweise kann ein KMU (Prinzipal), das sich im Rahmen einer Internationalisierungsstrategie mit einem ausländischen KMU (Agent) zur gemeinsamen Marktbearbeitung zusammengeschlossen hat, nicht eindeutig beurteilen, inwieweit die schlechte Position seiner Produkte am ausländischen Markt auf einen Vertrauensmissbrauch durch das ausländische KMU zurückzuführen ist, oder ob letzteres nur durch Ereignisse außerhalb seiner Kontrolle an der Honorierung der Vertrauensentscheidung gehindert wurde.

Aufgrunddessen, dass der Vertrauensgeber in der Vertrauensbeziehung auf den Einsatz expliziter vertraglicher Steuerungsmechanismen verzichtet, muss er sich beim Treffen seiner Vertrauensentscheidung an den bereits vorhandenen sozialen und psychologischen Steuerungsmechanismen orientieren. Von besonderer Bedeutung in diesem Szenario sind daher die Präferenzen des Vertrauensnehmers, sowie seine, durch sein soziales Umfeld determinierten Handlungsrestriktionen in Bezug auf kritische Ereignisse. Vor Eintritt in eine Vertrauensbeziehung wird der Vertrauensgeber also bestrebt sein, die Handlungsintentionen des Vertrauensnehmers, sowie die sich daraus ergebenden verhaltensbedingten Risiken zu antizipieren. ${ }^{25}$ Das der Vertrauensentscheidung zugrunde liegende Entscheidungskalkül des Vertrauensgebers in seiner Rolle als Prinzipal wird in Kapitel 5.1 erläutert.

\subsubsection{Der Vertrauensnehmer als Agent}

Analog zum Vertrauensgeber muss sich der Vertrauensnehmer für oder gegen den Eintritt in eine Vertrauensbeziehung mit dem Vertrauensgeber entscheiden. Entscheidet er sich für die Annahme der riskanten Vorleistung des Vertrauensgebers, so begründet er einen impliziten Vertrag zwischen sich und dem Vertrauensgeber. In seiner Rolle als Agent hat er sodann die Möglichkeit, das in ihn gesetzte Vertrauen zu honorieren oder zu enttäuschen und damit das Nutzenniveau des Vertrauensgebers $\mathrm{zu}$ beeinflussen. Um ein für den Vertrauensgeber nutzenstiftendes Ereignis zu erzielen, muss der Vertrauensnehmer Ressourcen einsetzen. Er wird sich für diesen Ressourceneinsatz entscheiden, d.h. sich vertrauenswürdig verhalten, wenn die Kosten dieses Verhaltens niedriger sind als der dadurch erzielbare Nutzen. Da die Vertrauensbeziehung bloß einen impliziten Vertrag begründet, ist es erforderlich, dass die Erfüllung der Vertrauenserwartung selbstdurchsetzend ist, womit intrinsische, sowie durch die Vertrauensbeziehung selbst be-

224 Vgl. Ripperger (1998), S. 75

225 Vgl. Ripperger (1998), S. 76; Roeder (2000), S. 125 f.; Klaus (2002), S. 146 f. 
gründete extrinsische Anreize für vertrauenswürdiges Verhalten besondere Bedeutung erlangen (vgl. Kap. 5.2). ${ }^{226}$

\subsubsection{Die Vertrauensbeziehung zwischen Prinzipalen und Agenten}

Wird die Vertrauensbeziehung zwischen Vertrauensgeber in seiner Rolle als Prinzipal und Vertrauensnehmer in seiner Rolle als Agent in den dynamischen Kontext ihres sozialen Umfelds verlagert, so ergeben sich zusätzliche Anreizwirkungen für vertrauenswürdiges Verhalten. Diese sind unter anderem auf die Wechselseitigkeit der Vertrauensbeziehung zwischen einer Vielzahl von Akteuren zurückzuführen, von denen jeder abwechselnd die Rolle des Vertrauensgebers, des Vertrauensnehmers oder einer Drittpartei einnehmen kann. Wechselseitige Vertrauensbeziehungen innerhalb eines sozialen Systems verschaffen dem Vertrauensgeber ein zusätzliches Drohpotential zur Sicherstellung von vertrauenswürdigem Verhalten seitens des Vertrauensnehmers: Durch die soziale Einbettung der Vertrauensbeziehung kann der Vertrauensgeber auf einen Vertrauensbruch mit einem Vertrauensbruch seinerseits antworten, oder über Reputationsmechanismen das zukünftige Verhalten von Drittparteien gegenüber dem Vertrauensnehmer negativ beeinflussen. ${ }^{227}$ Gehen beispielsweise ein Automobilhersteller und ein $\mathrm{Zu}$ lieferunternehmen, die beide einem Automobilcluster angehören, eine Vertrauensbeziehung ein und enttäuscht der Automobilhersteller das Vertrauen des Zulieferunternehmens (z.B. indem er dessen Produkte nicht zum vereinbarten Preis abnimmt), so hat letzteres die Möglichkeit, das zukünftige Verhalten anderer $\mathrm{Zu}$ lieferunternehmen im Cluster (oder z.B. auch von Banken) gegenüber dem Automobilhersteller negativ zu beeinflussen.

\section{VERTRAUENSGEBER UND VERTRAUENSNEHMER ALS ELEMENTE DER VERTRAUENSBEZIEHUNG}

\subsection{Die Entscheidungssituation des Vertrauensgebers}

Einleitend wird auf die grundlegenden Bedingungen für das Vorliegen einer vertrauensbezogenen Entscheidungssituation eingegangen. Bezugnehmend auf das dieser Arbeit zugrunde liegende Vertrauensverständnis stellt die Vertrauensentscheidung eine riskante Vorleistung und zugleich irreversible Investition dar: Der Vertrauensgeber überantwortet dem Vertrauensnehmer Ressourcen in Form des Anvertrauten, die dieser zum Nutzen oder Schaden des Vertrauensgebers einsetzen kann, womit er sich gegenüber dem Vertrauensnehmer in eine Position der Verwundbarkeit begibt. Dieser ,Einsatz', der ein konstitutives Merkmal der Ver- 
trauenssituation darstellt, begründet eine einseitige Abhängigkeit des Vertrauensgebers vom Vertrauensnehmer, die dieser zum Schaden des ersteren ausnutzen kann. Der Bruch von Vertrauen geht grundsätzlich mit einer Schädigung, wenn nicht sogar dem völligen Verlust des Anvertrauten einher und damit ist die Vertrauensinvestition des Vertrauensgebers irreversibel. Die riskante Vorleistung stellt also eine spezifische Investition seitens des Vertrauensgebers dar und damit begründet die Vertrauensentscheidung für den Vertrauensgeber grundsätzlich ein Hold Up-Risiko. ${ }^{228}$ Eine weitere Bedingung, neben dem Moment des Einsatzes, die vorliegen muss, um von einer Vertrauenssituation sprechen zu können, ist die Freiheit auf beiden Seiten der Vertrauensbeziehung: In Bezug auf den Vertrauensnehmer setzt Vertrauen voraus, dass dieser Wahlfreiheit zwischen der Honorierung und der Enttäuschung des in ihn gesetzten Vertrauens hat. Erst dieser Handlungsspielraum bezüglich der ihm anvertrauten Ressourcen begründet die Möglichkeit eines Schadens durch den Vertrauensnehmer und dadurch das Vertrauensrisiko. ${ }^{229}$ Indem der Vertrauensgeber vertraut, handelt er so, als ob der Vertrauensnehmer seine Wahlfreiheit im Sinne des Vertrauensgebers nutzen würde, ohne ihn jedoch in irgendeiner Form in dieser Freiheit einzuschränken. Je stärker nämlich der Handlungsspielraum des Vertrauensnehmers ex ante, z.B. durch explizite vertragliche Sicherungsmechanismen, eingeschränkt wird, desto weniger Raum bleibt für Vertrauen. ${ }^{230}$ Andererseits setzt Vertrauen auch in Bezug auf den Vertrauensgeber Wahlfreiheit voraus. Die Platzierung von Vertrauen ist mit dem Eingehen eines Risikos verbunden. Die Freiheit des Vertrauensgebers besteht nun darin, das Eingehen dieses Risiko vermeiden oder zumindest verringern zu können, indem er sich gegen eine potentielle Kooperationsbeziehung entscheidet, oder aber die damit verbundenen Risiken durch explizite Sicherungsmaßnahmen reduziert. Zusammenfassend setzt eine Vertrauenssituation damit, neben dem Merkmal der riskanten Vorleistung, die Wahlfreiheit des Vertrauensgebers zwischen Vertrauen und Misstrauen - in anderen Worten die Möglichkeit der Vertrauensplatzierung - voraus, ansonsten würde es sich maximal um eine Situation der Zuversicht handeln. ${ }^{231}$

Nachdem die riskante Vorleistung, sowie die Wahlfreiheit von Vertrauensgeber und Vertrauensnehmer als Voraussetzungen für das Vorliegen einer Vertrauenssituation identifiziert worden sind, stellt sich die Frage, welches Kalkül der Vertrauensentscheidung des Vertrauensgebers zugrunde liegt. Bisherige Modelle der Vertrauensentscheidung weisen folgende Schwächen auf: Sie basieren vorrangig auf einem reinen Eigennutzkriterium und berücksichtigen nicht ausreichend die Relevanz des mit der Vertrauensentscheidung verbundenen Risikos für den Ver-

228 Vgl. Ripperger (1998), S. 85 f.; Luhmann (2000), S. 27

229 Vgl. Gambetta (1998), S. 219

230 Vgl. Rößl (1994); Rößl (2000)

231 Vgl. Ripperger (1998), S. 86 f. 
trauensgeber. Darüber hinaus unterscheiden sie nicht analytisch zwischen Vertrauen als subjektiver Erwartungshaltung und Vertrauen als sichtbares kooperatives Verhalten: Während Vertrauen beispielsweise in der Psychologie häufig als subjektive Erwartungshaltung verstanden wird, wird es vor allem in der Spieltheorie als sichtbares kooperatives Verhalten in Gefangenen-Dilemma-Situationen analysiert. ${ }^{232} \mathrm{Um}$ aber die Vertrauensentscheidung als Prozessergebnis in Form eines integrativen theoretischen Modells erfassen und erklären zu können, d.h. eine Identifikation jener Variablen, die das Entscheidungskalkül des Vertrauensgebers beeinflussen, zu ermöglichen, ist eine Differenzierung zwischen subjektivem Entscheidungskalkül und objektiv beobachtbarem Verhalten unerlässlich (Kap. 5.1.1). Die Vertrauenserwartung, die auf der Extrapolation von verfügbaren Informationen (Kap. 5.1.2) basiert, steht am Anfang des Entscheidungsprozesses und kann den Vertrauensgeber zu einer Vertrauenshandlung (Kap. 5.1.3) motivieren, deren Vornahme das Vorliegen einer Vertrauensentscheidung impliziert.

\subsubsection{Die Modellierung der Vertrauensentscheidung}

Für die modelltheoretische Erfassung und Erklärung der Vertrauensplatzierung als Entscheidungsprozessergebnis muss zunächst zwischen subjektivem Entscheidungskalkül und objektiv beobachtbarem Verhalten differenziert werden. Darauf aufbauend ist ein Klassifikationsschema zu entwickeln, das es erlaubt, aus der Vielzahl der Kombinationen von Verhaltensweisen und zugrunde liegenden Kalkülen jene zu erfassen, die tatsächlich eine Vertrauensentscheidung markieren.

Generell ist zwischen Handlungen und den ihnen zugrunde liegenden Erwartungen zu differenzieren: Während identischen Handlungen unterschiedliche Erwartungen zugrunde liegen können, lösen dieselben Erwartungen unter Umständen unterschiedliche Handlungen aus. ${ }^{233}$ Die Vertrauenshandlung zeigt sich im Erbringen der riskanten Vorleistung. Durch seinen Einsatz in Form des Anvertrauten begibt sich der Vertrauensgeber in eine Position, die seine Verwundbarkeit gegenüber dem Verhalten des Vertrauensnehmers und dadurch ein Risiko für ihn begründet: „Für den Vertrauenden ist seine Verwundbarkeit das Instrument, mit dem er eine Vertrauensbeziehung in Gang bringt. ${ }^{‘ 234}$ Die Vertrauenserwartung des Vertrauensgebers besteht in der subjektiven Einschätzung, dass der Vertrauensnehmer freiwillig auf opportunistisches Verhalten, also auf die Veruntreuung des Einsatzes, verzichtet. Dementsprechend liegt eine Vertrauensentscheidung (Feld I in Abbildung 7) im Sinne eines Schenkens von Vertrauen durch den Vertrauensgeber nur dann vor, wenn eine Vertrauenshandlung vorgenommen,

\footnotetext{
232 Vgl. Deutsch (1958, 1962); Rotter (1980); Coleman (1991), S. 125; Ripperger (1998), S. 87 ff.

233 Vgl. Ajzen/Fishbein (1980); Heckhausen (1980), S. 8 ff.; Nieschlag/Dichtl/Hörschgen (1997), S. 169

234 Luhmann (2000), S. 55
} 
und diese Handlung durch eine Vertrauenserwartung motiviert wurde. Allerdings muss nicht jedes kooperative Verhalten auf einer vertrauensvollen Erwartung basieren und nicht immer resultiert eine Vertrauenserwartung in einem vertrauensvollen Verhalten. Damit ist Kooperation nicht notwendigerweise ein Zeichen für Vertrauen und Vertrauen alleine nicht immer eine ausreichende Bedingung für Kooperation. ${ }^{235}$

\begin{tabular}{|c|c|c|c|}
\hline & \multicolumn{2}{|c|}{ Vertrauenshandlung } \\
\hline & & ja & nein \\
\hline \multirow{8}{*}{ 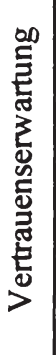 } & & I & II \\
\hline & . & Kooperation & Vertrauen \\
\hline & & aufgrund von & ohne \\
\hline & & Vertrauen & Kooperation \\
\hline & & III & IV \\
\hline & .등 & Kooperation & weder Vertrauen \\
\hline & & ohne & noch Kooperation \\
\hline & & vertrauen & \\
\hline
\end{tabular}

Abbildung 7: Die Vertrauensentscheidung

Quelle: In Anlehnung an Ripperger (1998), S. 93

Die Lücke zwischen Vertrauenserwartung und Vertrauenshandlung (Feld II und III) kann durch Einbeziehung des Faktors Risiko in den Entscheidungsprozess theoretisch geschlossen werden. So kann der Vertrauensgeber den Vertrauensnehmer in Situationen mit hohem Risiko ${ }^{236}$ zwar als vertrauenswürdig einschätzen, sich aber dennoch nicht zur Vertrauenshandlung entschließen (Feld II). Dagegen erfordert eine Situation mit niedrigem Risiko eine niedrigere Vertrauenserwartung bzw. kann ein scheinbar vertrauensvolles kooperatives Verhalten auch durch Nichtwissen um die damit verbundenen Risiken ausgelöst werden (Feld III). Da Vertrauen jedoch das Wissen um einen möglichen Schaden voraussetzt, liegt bei fehlendem Bewusstsein subjektiv betrachtet kein Verhaltensrisiko und damit auch kein Bedarf für Vertrauen vor. Definiert man als Handlungsschwellenwert die Höhe der Vertrauenserwartung, ab der sich eine solche Erwartung auch in einer Vertrauenshandlung zeigt, dann schwankt dieser Schwellenwert somit

235 Vgl. Kee/Knox (1970), S. 359; Lewis/Weigert (1985); Clark/Payne (1995), S. 17; Ripperger (1998), S. 93

236 Die Höhe des Risikos einer Vertrauensentscheidung kann als Funktion der absoluten Größe des potentiellen Schadens im Falle eines Vertrauensbruchs bzw. des potentiellen Nutzens im Falle einer Honorierung des geschenkten Vertrauens sowie der Eintrittswahrscheinlichkeit des nutzenstiftenden Ereignisses bzw. des schädigenden Ereignisses dargestellt werden. Die Eintrittswahrscheinlichkeit des nutzenstiftenden Ereignisses ergibt sich als Produkt aus der Wahrscheinlichkeit, dass der Vertrauensnehmer die Intention hat, sich vertrauenswürdig zu verhalten und der Wahrscheinlichkeit, dass seine Făhigkeiten, diese Intention zu realisieren, nicht durch Ereignisse außerhalb seiner Kontrolle behindert werden (Ripperger 1998, S. 131). 
situativ in Abhängigkeit des wahrgenommenen Risikos und individuell mit der Risikobereitschaft des Vertrauensgebers. ${ }^{237}$ Zusammenfassend ist eine Vertrauensentscheidung dann gegeben, wenn die Vertrauenshandlung tatsächlich erfolgt und durch die ihr zugrunde liegende Vertrauenserwartung motiviert wurde.

\subsubsection{Die Vertrauenserwartung als Bestandteil der Vertrauensentscheidung}

Bei der Bildung der Vertrauenserwartung sieht sich der Vertrauensgeber mit einem Adverse Selection-Problem konfrontiert, da er in seiner Rolle als Prinzipal einen vertrauenswürdigen Agenten auswählen bzw. entscheiden muss, ob der potentielle Vertrauensnehmer vertrauenswürdig ist, ihm jedoch diese Eigenschaft vor Eingehen der Vertrauensbeziehung weitgehend verborgen bleibt. Die Vertrauenserwartung basiert damit auf der zugeschriebenen Motivation des Handelns des Vertrauensnehmers, genauer auf der Einschätzung der Intensität und Stabilität der Motivation des Vertrauensnehmers und damit der Antizipation von dessen Handlungsabsicht seitens des Vertrauensgebers. Ein Agent ist grundsätzlich motiviert, jene Handlungsalternative zu wählen, die seinen Nutzen auf Basis seiner Präferenzen unter den jeweiligen Handlungsrestriktionen maximiert. Die Zuverlässigkeit einer vertrauenswürdigen Absicht im Sinne eines Fortbestehens über einen gewissen Zeitraum und sich ändernde Umstände, ist eine Funktion ihrer Intensität und Stabilität als Ausprägungen der ihr zugrunde liegenden Motivation. Die Intensität einer vertrauenswürdigen Handlungsabsicht kann als die Höhe des Nutzens, den der Vertrauensnehmer unter einem gegebenen Umweltzustand aus vertrauenswürdigem Verhalten zieht bzw. als die Höhe der Kosten, die er bereit ist, für die Honorierung der in ihn gesetzten Vertrauenserwartung unter einem gegebenen Umweltzustand zu akzeptieren, operationalisiert werden. Die Stabilität einer vertrauenswürdigen Handlungsabsicht hängt davon ab, inwieweit der Nutzen und somit die Motivation des Vertrauensnehmers, sich vertrauenswürdig zu verhalten, in Abhängigkeit von möglichen Umweltzuständen variieren. Eine Absicht wird umso stabiler sein, je intensiver die ihr zugrunde liegenden Motive auf den Vertrauensgeber selbst bzw. auf Ereignisse und Ressourcen innerhalb seiner Kontrolle ausgerichtet sind. Generell kann davon ausgegangen werden, dass je größer der Zeitraum zwischen der Entscheidung des Vertrauensgebers, Vertrauen zu schenken und seiner möglichen Honorierung durch den Vertrauensnehmer ist, und je höher die Unsicherheit in Bezug auf die jeweiligen Handlungsrestriktionen innerhalb dieses Zeitraums ist, desto stärker ist die Gewichtung der wahrgenommenen Zuverlässigkeit der vertrauenswürdigen Handlungsabsicht des Vertrauensnehmers in der Vertrauensentscheidung des Vertrauensgebers. ${ }^{238}$

237 Vgl. Ripperger (1998), S. 94

238 Vgl. Ripperger (1998), S. 94 ff.; Klaus (2002), S. 153 
Bei der Einschätzung der Handlungsabsicht des Vertrauensnehmers sieht sich der Vertrauensgeber mit subjektiver Unsicherheit im Hinblick auf dessen wahre Präferenzen, sowie mit objektiver Unsicherheit in Bezug auf das Eintreten möglicher Umweltzustände und der sich daraus ergebenden Handlungsrestriktionen konfrontiert. Im Rahmen der Bildung der Vertrauenserwartung, die auf der subjektiven Einschätzung der Vertrauenswürdigkeit des Vertrauensnehmers beruht, wird der Vertrauensgeber daher bestrebt sein, seine subjektive Unsicherheit durch zusätzliche Informationen zu reduzieren, wobei das Ausmaß seiner Informationsbeschaffungsaktivitäten dem Trade-off von zusätzlichem Nutzen und zusätzlichen Kosten der Informationen unterliegt. Da Informationslücken aber nicht vollständig ausgefüllt werden können, erfordert Vertrauen grundsätzlich die Extrapolation von Informationen: Aufgrunddessen, dass der Vertrauensgeber nicht über alle Informationen verfügt, die er bräuchte, um erfolgssicher handeln zu können, extrapoliert er seine vorhandenen Informationen aus der Vergangenheit in die Zukunft. ${ }^{239}$ Hierbei greift er auf interdependente Informationssphären zurück, die sich anhand der Kategorien ,Bezugsobjekt der Information' und ,Quelle der Information' in spezifisches Vertrauen, generalisiertes Vertrauen, Reputation und Vertrauensatmosphäre klassifizieren lassen.

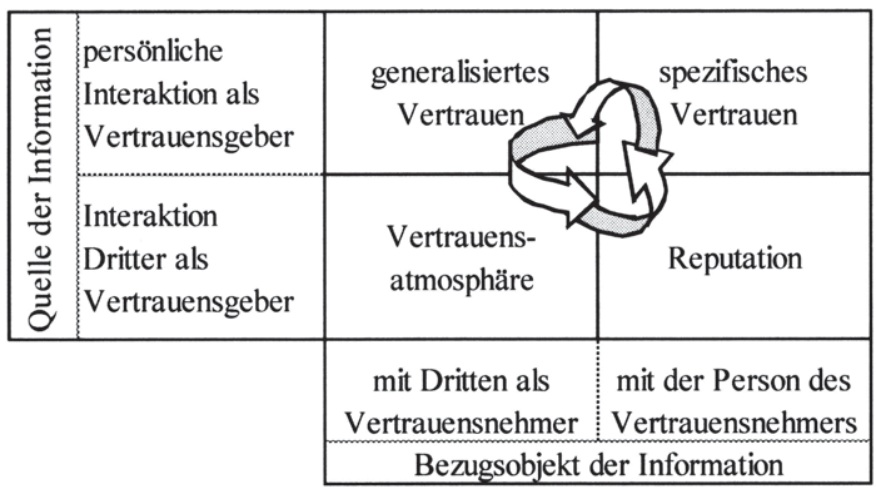

Abbildung 8: Die Informationskategorien bei der Bildung der Vertrauenserwartung Quelle: In Anlehnung an Ripperger (1998), S. 99

Bei der Bildung der Vertrauenserwartung bedient sich der Vertrauensgeber nicht nur eigener Erfahrungen, sondern häufig auch der Erfahrungen Dritter. Sofern sich diese nicht direkt auf den Vertrauensnehmer, sondern auf die Interaktion mit Dritten innerhalb eines gemeinsamen Kontextes beziehen, werden sie im Folgenden als konstitutive Elemente der Vertrauensatmosphäre, die sich grundsätzlich auf ein soziales System bezieht (z.B. wirtschaftliche Organisation, Gruppe von Akteuren), verstanden. Je höher der Anteil an positiven Erfahrungen mit Ver- 
trauensbeziehungen innerhalb eines sozialen Systems ist, desto besser ist auch die Qualität der Vertrauensatmosphäre. ${ }^{240}$

Die Erfahrungen Dritter mit dem Vertrauensnehmer selbst äußern sich dagegen in dessen Reputation, womit sie im hier verwendeten Kontext als öffentliche Information über die Vertrauenswürdigkeit eines Akteurs zu verstehen ist. Zwischen der Reputation und der Vertrauensatmosphäre bestehen insofern Interdependenzen, als dass sowohl das Vorhandensein effizienter Reputationsmechanismen, als auch die Art der Reputationen innerhalb eines sozialen Systems Einfluss auf die subjektive Wahrnehmung und die Qualität der Vertrauensatmosphäre innerhalb dieses Systems nehmen. Trotz ihres maßgeblichen Einflusses auf die Vertrauenserwartung des Vertrauensgebers, stellt die Reputation eines Akteurs kein ausreichendes Substitut für eigene Erfahrungen dar. ${ }^{241}$ Zusammenfassend stellen die Vertrauensatmosphäre und die Reputation Informationskategorien dar, die sich aus den Erfahrungen Dritter und dem Zusammenwirken einer Vielzahl von Akteuren ergeben.

Bei der Bildung der Vertrauenserwartung kann sich der Vertrauensgeber auch eigener Erfahrungen bedienen. Seine persönlichen Erfahrungen in Bezug auf die generelle Vertrauenswürdigkeit von Dritten (z.B. Person oder Gruppe) bilden die Grundlage des generalisierten Vertrauens, das als die grundsätzliche Vertrauensbereitschaft eines Akteurs interpretiert werden kann. ${ }^{242}$ Als relativ stabiles Merkmal der Persönlichkeit des Vertrauensgebers ist der Grad an generalisiertem Vertrauen unabhängig von den Spezifika einer bestimmten Situation. Hingegen wird die Vertrauenserwartung in einer spezifischen Situation von dem Grad an generalisiertem Vertrauen maßgeblich beeinflusst: Bei der Bildung der Vertrauenserwartung in einer spezifischen Situation ist der Vertrauensgeber mit einem Informationsmangel konfrontiert. Um dieses Problem zu bewältigen, kann er auf Basis vergangener Erfahrungen mit Dritten generalisierte Erwartungen entwickeln. Diese beeinflussen sodann als Informationssubstitute in Form von generalisiertem Vertrauen die Vertrauenserwartung in einer spezifischen Situation. Da der Grad an generalisiertem Vertrauen den Erfolg der Strategie ,Vertrauen schenken' in seiner Vergangenheit reflektiert, wird die Erwartung des Vertrauensgebers, dass sich der Vertrauensnehmer vertrauenswürdig verhalten wird, in einer spezifischen Situation umso höher sein, je vertrauenswürdiger sich Dritte ihm gegenüber in einer ähnlichen Situation in der Vergangenheit verhalten haben. ${ }^{243}$

Dagegen beruht das spezifische Vertrauen des Vertrauensgebers auf seiner subjektiven Einschätzung der Vertrauenswürdigkeit eines bestimmten Vertrauens-

240 Vgl. Ripperger (1998), S. 100

241 Vgl. Perry (2001), S. 71 f.; Picot et al. (2001), S. 126 f

242 Vgl. Petermann (1996), S. 20

243 Vgl. Rotter (1967), S. 651; Rotter (1980), S. 2; Currall/Judge (1995); Mayer et al. (1995), S. 716 
nehmers in einer spezifischen Situation, was in dieser Hinsicht eine Gegenwartsorientierung impliziert. Spezifisches Vertrauen basiert auf zwei Komponenten, und zwar einerseits auf dem situationsspezifischen Vertrauen, das auf konkreten Merkmalen der Verhaltenssituation beruht, und andererseits auf dem personenspezifischen Vertrauen, das auf den Charakteristika des Vertrauensnehmers beruht. In erster Linie wird es jedoch von letzterem, also der Persönlichkeit des Vertrauensnehmers, beeinflusst. In diesem Zusammenhang nehmen folgende Faktoren als persönliche Merkmale des Vertrauensnehmers einen positiven Einfluss auf die subjektive Einschätzung seiner Vertrauenswürdigkeit: Erreichbarkeit, Kompetenz, Konsistenz, Diskretion, Fairness, Integrität, Loyalität, Offenheit, Verlässlichkeit und Zugänglichkeit. ${ }^{244}$ Auch eine weitere empirische Untersuchung bestätigt die Annahme, dass die subjektiv wahrgenommene Kompetenz, Aufrichtigkeit, Integrität, Einsatzbereitschaft, Diskretion und das Taktgefühl eines Akteurs das Vertrauen in ihn, d.h. sein Verhalten, positiv beeinflussen. ${ }^{245}$ Damit setzt spezifisches Vertrauen Kenntnisse des Vertrauensgebers über die Persönlichkeit des Vertrauensnehmers und daher auch einen geringen Grad an persönlicher Interaktion mit diesem voraus. In diesem Sinne beruht es auch auf vergangenen Erfahrungen aus der direkten Interaktion mit dem betreffenden Akteur, wird jedoch als spezifische Erwartungshaltung in jeder gegenwärtigen Situation neu gebildet, womit sich spezifisches Vertrauen im Wesentlichen auf die dem Vertrauensnehmer zugeschriebene Motivation, sich im Rahmen der Vertrauensbeziehung vertrauenswürdig zu verhalten, bezieht. Voraussetzung für die Entwicklung von spezifischem Vertrauen ist die Verfügbarkeit spezifischer Informationen über die Person des potentiellen Vertrauensnehmers, sowie über die besonderen situativen Gegebenheiten. In dem Grad, in dem diese Informationen dem Vertrauensgeber nicht zur Verfügung stehen, muss er seine Vertrauenserwartung stärker auf Informationssubstitute in Form von generalisiertem Vertrauen, Reputation und Vertrauensatmosphäre stützen, d.h. auf weniger spezifische Informationen zurückgreifen. ${ }^{246}$ Zwischen spezifischem Vertrauen und den anderen genannten Informationssphären bestehen jedoch auch Interdependenzen. So wird der Vertrauensgeber einem Akteur mit guter Reputation eher spezifisches Vertrauen entgegenbringen als einem Akteur, der über eine schlechte Reputation verfügt. Auch wird das Ausmaß an generalisiertem Vertrauen, das seinerseits von der Vertrauensatmosphäre in einem sozialen System determiniert werden kann, in der Regel das Vertrauen des Vertrauensgebers in einer spezifischen Situation positiv beeinflussen. ${ }^{247}$

244 Vgl. Butler (1991), S. 648

245 Vgl. Moorman et al. (1993), S. $91 \mathrm{f}$

246 Vgl. Ripperger (1998), S. $105 \mathrm{f}$.

247 Vgl. Ripperger (1998), S. 100 
Grundsätzlich hängt die Gewichtung der einzelnen Informationssphären in einer Entscheidungssituation von der Verfügbarkeit vergangener Erfahrungen in ähnlichen Situationen, d.h. der Vertrautheit einer Situation, sowie von den Beschaffungs- und Verarbeitungskosten situationsspezifischer Informationen ab. Sind letztere nur zu sehr hohen Kosten erhältlich und ist die Situation durch ein hohes $\mathrm{Ma} ß$ an Vertrautheit gekennzeichnet, so wird die Vertrauenserwartung des Vertrauensgebers überwiegend von generalisiertem Vertrauen, Informationen über die allgemeine Reputation des potentiellen Vertrauensnehmers und der Vertrauensatmosphäre des sozialen Umfelds beeinflusst. Mit sinkenden Kosten situationsspezifischer Informationen und fallendem Grad an Vertrautheit einer Situation wird die Vertrauenserwartung des Vertrauensgebers weniger auf generalisiertem Vertrauen und mehr auf situations- und personenspezifischen Zusatzinformationen gründen. ${ }^{248}$ Zusammenfassend steigt der Einfluss des generalisierten Vertrauens mit der wachsenden Vertrautheit einer Situation. Vertrautheit ermöglicht nämlich eine Generalisierung vergangener Erfahrungen im Sinne ihrer Fortschreibung auf zukünftige Erwartungen. Obwohl die Anwendung einer in der Vergangenheit erfolgreichen Vertrauensstrategie auf neue, aber vertraute Situationen nicht immer Effektivität im Sinne einer richtigen Einschätzung der vertrauensvollen Absicht des Vertrauensnehmers gewährleistet, ist sie grundsätzlich effizient: Die Beschaffung und Verarbeitung situationsspezifischer Informationen verursacht dem begrenzt rationalen Vertrauensgeber Kosten und im Rückgriff auf generalisiertes Vertrauen zeigt sich der wirtschaftliche Umgang mit seiner begrenzten Rationalität. ${ }^{249}$

\subsubsection{Die Vertrauenshandlung als Bestandteil der Vertrauensentscheidung}

Die Bildung der Vertrauenserwartung steht am Anfang des Entscheidungsprozesses, an dessen Ende vertrauensvolles Verhalten im Sinne einer Vertrauenshandlung stehen kann, aber nicht stehen muss. Die Vertrauenshandlung des Vertrauensgebers zeigt sich erst in der freiwilligen Erbringung einer riskanten Vorleistung unter Verzicht auf explizite Sicherungsmaßnahmen gegen opportunistisches Verhalten und ergibt sich aus der Entscheidung des Vertrauensgebers, sich dem Hold Up-Risiko ungeschützt auszusetzen. Dieser Entschluss ist neben der Höhe seiner Vertrauenserwartung zusätzlich abhängig von der Höhe des wahrgenommenen situationsspezifischen Risikos, sowie der subjektiven Risikobereitschaft des Vertrauensgebers.

Grundsätzlich ist davon auszugehen, dass der Vertrauensgeber dann Vertrauen schenken wird, wenn der erwartete Nutzengewinn größer ist als der erwartete Nutzenverlust: $p^{*} G>|(1-p) * L|$ wobei $G$ (Höhe des Nutzengewinns für den Ver- 
trauensgeber bei Eintreten des gewünschten Ereignisses) $>0$ und L (Höhe des Nutzenverlustes bei Eintreten des unerwünschten Ereignisses) $<0 .{ }^{250}$ Der Wahrscheinlichkeitswert $p$ als Eintrittswahrscheinlichkeit des Nutzengewinns $G$ ist nicht gleichzusetzen mit der Wahrscheinlichkeit, dass sich der Vertrauensnehmer vertrauenswürdig verhalten wird (bzw. ist ( 1 -p) als Eintrittswahrscheinlichkeit des Nutzenverlustes L nicht unbedingt identisch mit der Wahrscheinlichkeit des Vertrauensbruchs). Während sich die Vertrauenserwartung nämlich ausschließlich auf die Absicht des Vertrauensnehmers, seine Fähigkeiten im Sinne des Vertrauensgebers einzusetzen, bezieht, kann hingegen die Eintrittswahrscheinlichkeit des möglichen Nutzengewinns $G$ bzw. des potentiellen Nutzenverlustes $L$ während der Vertrauensbeziehung auch durch exogene Einflüsse außerhalb der Kontrolle des Vertrauensnehmers verändert werden. In diesem Fall sieht sich der Vertrauensgeber in der Vertrauensbeziehung mit einem Moral Hazard-Problem konfrontiert: In seiner Rolle als Prinzipal kann er zwar das Handlungsergebnis in Gestalt seiner eigenen Nutzenveränderung feststellen, jedoch ist es ihm nicht möglich, zu beurteilen, inwieweit dieses Ergebnis auf die Anstrengungen des Vertrauensnehmers, also auf dessen vertrauenswürdiges Verhalten, oder aber auf besonders günstige bzw. ungünstige exogene Umstände zurückzuführen ist. Da exogen unsichere Ereignisse ex post die Fähigkeiten des Vertrauensnehmers, in der vom Vertrauensgeber erwarteten Art und Weise zu handeln, beeinflussen können, ist es dem Vertrauensgeber nicht immer möglich festzustellen, ob die Bewahrung des Anvertrauten tatsächlich auf vertrauenswürdigem Verhalten bzw. dessen Schädigung tatsächlich auf Vertrauensbruch im Sinne einer opportunistischen Ausnutzung der Hold Up-Situation beruht, oder aber aus exogenen Ereignissen außerhalb der Kontrolle des Vertrauensnehmers resultiert. ${ }^{251}$ Dementsprechend können die Fähigkeiten des Vertrauensnehmers - trotz seiner hohen Motivation - durch exogene Ereignisse außerhalb seiner Einflusssphäre so eingeschränkt werden, dass er seiner wohlwollenden Absicht nicht mehr nachkommen kann und gegen seinen Willen ein schädigendes Ereignis herbeiführt. Um Vertrauensbruch handelt es sich jedoch nur dann, wenn der Vertrauensnehmer opportunistisch handelt, also den Schaden mit der Absicht, seinen eigenen Nutzen auf Kosten des Vertrauensgebers zu vergrößern, herbeiführt. ${ }^{252}$

Im Rahmen seiner Vertrauensentscheidung muss der Vertrauensgeber daher, neben der Absicht des Vertrauensnehmers, zugleich mögliche exogene Einflüsse, die dessen Fähigkeiten ex post beeinträchtigen können, antizipieren. Da die Vertrauenserwartung aber lediglich Verhaltensrisiken, nicht aber Umweltrisiken absorbieren kann, beinhaltet die Erwartung des Vertrauensgebers auch ein Element

$250 \quad$ Vgl. Coleman (1991), S. 126

251 Vgl. Arrow (1985), S. 43 ff; Plötner (1995), S. 47: Ripperger (1998), S. 119: Ebers/Gotsch (1999), S. 213

252 Vgl. Bhide/Stevenson (1990), S. 125 
der Hoffnung. Die Vertrauenserwartung setzt sich damit einerseits aus der vertrauensvollen Erwartung im Sinne der subjektiven Einschätzung der Handlungsabsicht des Vertrauensnehmers und andererseits aus der hoffnungsvollen Erwartung, dass das schädigende Ereignis nicht durch exogene Einflüsse herbeigeführt wird, zusammen, womit die geschätzte Eintrittswahrscheinlichkeit $p$ des nutzenstiftenden Ereignisses $G$ als Funktion der vertrauensvollen und der hoffnungsvollen Erwartung aufzufassen ist. ${ }^{253}$

Das Ausgangskriterium, wonach sich der Vertrauensgeber als rational handelnder Akteur für eine Vertrauenshandlung entscheidet, wenn der erwartete Nutzen positiv ist, ist jedoch nicht ausreichend, da die Vertrauenshandlung nicht nur von der Höhe der Vertrauenserwartung, aber auch von der Höhe des wahrgenommenen situationsspezifischen Risikos beeinflusst wird: In der Wahrnehmung des Vertrauensgebers beinhaltet die Entscheidungssituation ein Risiko, da die Entscheidung für oder gegen die Vornahme einer Vertrauenshandlung zu unsicheren Ereignissen mit unterschiedlichen Konsequenzen auf seinen Nutzen führen kann. ${ }^{254}$ Das wahrgenommene Risiko hängt seinerseits allerdings nicht bloß vom Verhältnis zwischen möglichen Gewinnen und Verlusten ab, die der Entscheidungssituation inhärent sind, sondern steigt auch mit deren Höhe. Die Situationsspezifität der Risikowahrnehmung ergibt sich daraus, dass die Art und die Höhe der Konsequenzen der Vertrauenshandlung als gewählte Alternative vorwiegend situationsbedingt und kaum vom Vertrauensnehmer beeinflussbar sind. ${ }^{255}$ Aber nicht nur die Höhe des situationsspezifischen Risikos, sondern auch die Höhe der personenspezifischen Risikobereitschaft des Vertrauensgebers als Bereitschaft, ein gegebenes Risiko zu akzeptieren, beeinflusst die Vertrauenshandlung maßgeblich. In diesem Zusammenhang ist Risikobereitschaft von Vertrauensbereitschaft abzugrenzen. Vertrauensbereitschaft betrifft ausschließlich die subjektive Neigung des Vertrauensgebers, dem potentiellen Vertrauensnehmer vertrauenswürdiges Verhalten zu unterstellen, sodass sie sich positiv auf die Höhe der Vertrauenserwartung auswirkt. Risikobereitschaft dagegen zeigt sich erst als Komponente des Entscheidens und Handelns und damit in der konkreten Vertrauenshandlung des Vertrauensgebers. Die Risikobereitschaft des Vertrauensgebers korreliert mit der Höhe des Risikos, das er durch die Erbringung der riskanten Vorleistung einzugehen bereit ist. Risikobereitschaft umfasst auch jene Risiken, die nicht ausschließlich durch die Motivation des Vertrauensnehmers, sondern auch durch unsichere exogene Ereignisse begründet werden. Während

\footnotetext{
253 Vgl. Ripperger (1998), S. 120

254 Vgl. Meifert (2003), S. 33

255 Vgl. Mayer et al. (1995, S. 726): „[...] the perception of risk involves the trustor's beliefs about likelihoods of gains and losses outside of considerations that involve the relationship with the particular trustee." Ähnlich dazu auch Köszegi (2001, S. 69): “[ ...] Konsequenzen von vertrauensvollem Verhalten sind in der Regel situationsbedingt und kaum durch das Vertrauensobjekt beeinflussbar."
} 
Risikobereitschaft also sowohl Verhaltensrisiken, als auch Umweltrisiken umfasst, bezieht sich Vertrauensbereitschaft ausschließlich auf die Bereitschaft, durch das Verhalten des Vertrauensnehmers begründete Risiken in der Vertrauenserwartung $\mathrm{zu}$ absorbieren. Vertrauensbereitschaft und Risikobereitschaft hängen jedoch insofern zusammen, als dass der Vertrauensgeber durch eine hohe Vertrauensbereitschaft einen großen Anteil der Verhaltensrisiken ex ante durch sein Vertrauen absorbieren kann, sodass die für die Vertrauenshandlung notwendige Risikobereitschaft niedriger sein kann, als wenn er nicht vertrauen würde. Zusammenfassend wird sich der Vertrauensgeber also für eine Vertrauenshandlung entscheiden, wenn (1) der erwartete Nutzen einer solchen Handlung positiv ist und (2) seine Risikoprämie nicht seinen erwarteten Nutzen übersteigt, d.h. sein Sicherheitsäquivalent positiv ist. ${ }^{256}$

Insgesamt ist die Vertrauensentscheidung des Vertrauensgebers als Ergebnis eines Prozesses zu verstehen, der mit der Bildung der Vertrauenserwartung beginnt und zu einer Vertrauenshandlung im Sinne einer riskanten Vorleistung führen kann, aber nicht zwingend muss. Für den Fall, dass das mit der Vertrauenshandlung verbundene Risiko die persönliche Risikobereitschaft des Vertrauensgebers übersteigt, wird er versuchen, dieses durch den komplementären Einsatz expliziter vertraglicher Sicherungs- und Kontrollmechanismen zu verringern, sofern die damit einhergehenden Transaktionskosten seine Risikoprämie und den mit der Austauschbeziehung assoziierten Erwartungsnutzen nicht übersteigen. ${ }^{257}$

\subsection{Die Entscheidungssituation des Vertrauensnehmers}

Mit der Annahme der riskanten Vorleistung begründet der Vertrauensnehmer einen impliziten Vertrag zwischen sich und dem Vertrauensgeber, dessen Gegenstand die Erfüllung der Vertrauenserwartung ist. Das persönliche Kalkül des Vertrauensnehmers wird darüber entscheiden, ob er der Vertrauenserwartung gerecht wird. Ziel dieses Kapitels ist es daher, die intrinsischen und extrinsischen Anreize für vertrauenswürdiges Verhalten zu erfassen und zu erklären. Das Entscheidungskalkül des Vertrauensnehmers bzw. die intrinsischen und extrinsischen Anreize, die in dieses Kalkül eingehen, sind für die Erfassung des Vertrauenskonstrukts aus folgenden Gründen relevant: Einerseits determiniert es das Verhalten des Vertrauensnehmers und damit die Wahrscheinlichkeit der Entsprechung der Vertrauenserwartung. Andererseits beeinflusst es die Vertrauensentscheidung des Vertrauensgebers und damit die Platzierung von Vertrauen, indem der Vertrauens-

256 Vgl. Ripperger (1998), S. 122 f.; Anm. d. Verf.: Unter der Risikoprämie ist der Preis, den der Vertrauensgeber zu zahlen bereit wäre, um von einem unsicheren zu einem sicheren Nutzenzuwachs zu gelangen, zu verstehen - subtrahiert man diesen subjektiven Risikopreis vom unsicheren Erwartungsnutzen, so verbleibt als Betrag das Sicherheitsäquivalent, d.h. der sichere Nutzen, den der Vertrauensgeber als äquivalent zum unsicheren Nutzen einstufen würde. 
geber seine Einschätzung des Entscheidungskalküls des Vertrauensnehmers und die Antizipation der daraus resultierenden Handlungsabsicht zur Grundlage seiner Vertrauenserwartung macht. ${ }^{258}$

\subsubsection{Die Vertrauenswürdigkeit des Vertrauensnehmers}

Vertrauenswürdiges Verhalten zeigt sich in der freiwilligen Nutzenstiftung für den Vertrauensgeber und setzt den Verzicht auf die Ausnutzung opportunistischer Verhaltensspielräume voraus. ${ }^{259}$ Bei opportunistischem Verhalten geht die Absicht zu täuschen der konkreten Realisierung des eigenen Vorteils unter Schädigung des Vertrauensgebers voraus. ${ }^{260}$ Damit ist derjenige nicht vertrauenswürdig, der unter Vortäuschung wohlwollender Absichten Vertrauen erweckt und anschließend das für den Vertrauensgeber schädigende Ereignis $\mathrm{zu}$ seinem eigenen Vorteil bewusst herbeiführt. Hingegen ist derjenige vertrauenswürdig, der die Intention realisiert, die er dem Vertrauensgeber in Worten oder durch konkludentes Verhalten, wie z.B. die stillschweigende Annahme der vom Vertrauensgeber erbrachten riskanten Vorleistung, bewusst oder unbewusst kommuniziert hat. ${ }^{261}$

Grundsätzlich wird sich der Vertrauensnehmer als rational handelnder Akteur vertrauenswürdig verhalten, wenn sein Nutzen höher ist als die Kosten der Honorierung des in ihn gesetzten Vertrauens. Sein Entscheidungskalkül ergibt sich hierbei aus seinen Präferenzen und den jeweiligen Handlungsrestriktionen. Die Art seiner Präferenzen bestimmt seine Vertrauenswürdigkeit als dispositionale Variable seiner Persönlichkeit und damit seine intrinsische Motivation, sich einem spezifischen Vertrauensgeber gegenüber vertrauensvoll zu verhalten. Aus der Art der Handlungsrestriktionen in einer gegebenen Vertrauensbeziehung ergeben sich entsprechende extrinsische Anreize für vertrauenswürdiges Verhalten. ${ }^{262}$

Um Vertrauenswürdigkeit als dispositionale Variable der Persönlichkeit des Vertrauensgebers ökonomisch erfassen zu können, bedarf es eines ökonomisch operationalisierbaren Kriteriums für eine vertrauenswürdige Disposition, das zugleich als Indikator für die Wahrscheinlichkeit vertrauenswürdigen Verhaltens fungiert. Von besonderer Bedeutung in diesem Zusammenhang sind die moralischen Qualitäten des Vertrauensnehmers, denn sowohl moralisches, als auch vertrauenswürdiges Handeln charakterisiert sich anhand des bewussten Verzichts auf

\footnotetext{
258 Vgl. Mayer et al. (1995), S. 717; Ripperger (1998), S. 137

259 Vgl. Bradach/Eccles (1989), S. 104

260 Nach Williamson (1990b, S. 54) bezieht sich Opportunismus ,insbesondere auf vorsätzliche Versuche irrezuführen, zu verzerren, verbergen, verschleiern oder sonstwie zu verwirren.“

261 Vgl. Luhmann (2000), S. 48

262 Vgl. Ripperger (1998), S. 137
} 
den eigenen Vorteil zugunsten des Vorteils eines anderen. Der vertrauenswürdige Vertrauensnehmer verzichtet freiwillig auf den Konsum bestimmter Ressourcen und setzt sie stattdessen zur Herbeiführung des für den Vertrauensgeber nutzenstiftenden Ereignisses ein. Die Vertrauenswürdigkeit eines Akteurs ist also nicht zuletzt eine Frage seiner moralischen Qualitäten und das Handeln innerhalb einer Vertrauensbeziehung kann moralisch nicht wertfrei sein. Die moralischen Qualitäten eines Akteurs sind allerdings schwer zu erfassen und noch problematischer ist es, sie ökonomisch zu quantifizieren, sodass sie nicht als Indikator für die Wahrscheinlichkeit vertrauenswürdigen Verhaltens des Vertrauensnehmers fungieren können. Erforderlich ist daher ein Kriterium vertrauenswürdigen bzw. moralischen Handelns, das eine bessere Operationalisierung im ökonomischen Sinne ermöglicht. $^{263}$

Intrinsisch motiviertes moralisches Handeln erfordert in ökonomischer Hinsicht eine positive Korrelation zwischen dem Nutzen des Vertrauensnehmers und dem Nutzen des von seinem Verhalten betroffenen Vertrauensgebers und setzt in diesem Sinne altruistische Präferenzen, in denen sich Altruismus als dispositionale Variable manifestiert, voraus. Altruistisches Verhalten und damit auch moralisches Handeln kann damit, unabhängig von äußeren Handlungsrestriktionen, durch altruistische Präferenzen intrinsisch motiviert sein. Allerdings kann moralisches Handeln auch extrinsisch, d.h. durch institutionelle Anreize und Sanktionen in der jeweiligen Situation, motiviert sein. Die Differenzierung zwischen altruistischem Verhalten als manifeste Handlung einerseits und altruistischen Präferenzen als intrapersonale, dispositionale Variable der Persönlichkeit eines Akteurs andererseits ist hier maßgeblich: Ein Akteur A verhält sich altruistisch, wenn er einen unkompensierten Nutzentransfer an Akteur B tätigt, unabhängig davon, welche Motivation dieser Handlung zugrunde liegt. Ein Akteur A ist altruistisch, d.h. besitzt altruistische Präferenzen dem Akteur B gegenüber, zu dem Grad, in dem sein eigener Nutzen positiv funktional abhängig ist vom Nutzen des Akteurs B und daher mit dessen Nutzen positiv korreliert. ${ }^{264}$

Nachdem altruistisches Verhalten als (zunächst) unkompensierter Nutzentransfer zur Operationalisierung moralischen Handelns und altruistische Präferenzen im Sinne einer positiven Nutzenkorrelation zur Operationalisierung einer moralischen Disposition herangezogen worden sind, bedarf es der Prüfung, inwieweit diese Kriterien auch als Indikator für vertrauenswürdiges Verhalten bzw. für Vertrauenswürdigkeit als intrapersonale Disposition eingesetzt werden können. Von besonderer Bedeutung bei der Betrachtung des Vertrauenskonstrukts anhand dieser Kriterien sind hierbei folgende Unterscheidungen: Einerseits ist es

263 Vgl. Ripperger (1998), S. $140 \mathrm{f}$.

264 Vgl. Ripperger (1998), S. 144 f.; ähnlich dazu Vanberg (1994), sowie Palfrey/Rosenthal (1988, S. 310 ), die unter Altruismus ,a component of utility that depends solely on how much the individual contributes" verstehen 
erforderlich zwischen Vertrauen seitens des Vertrauensgebers und Vertrauenswürdigkeit seitens des Vertrauensnehmers zu differenzieren. Andererseits ist eine Trennung zwischen zugrunde liegender Disposition und manifestem Verhalten notwendig.

Die Unterscheidung zwischen Vertrauen seitens des Vertrauensgebers und Vertrauenswürdigkeit seitens des Vertrauensnehmers ist insofern relevant, als dass die Vertrauensentscheidung des Vertrauensgebers ein altruistischer Akt sein kann, aber nicht sein muss, da die riskante Vorleistung nicht notwendigerweise einen positiven Nutzentransfer für den Vertrauensnehmer darstellt. Hingegen handelt es sich bei der Entscheidung des Vertrauensnehmers für oder gegen vertrauenswürdiges Verhalten grundsätzlich auch um eine Entscheidung für oder gegen altruistisches bzw. moralisch korrektes Verhalten dem Vertrauensgeber gegenüber. Dem Vertrauensnehmer verursacht vertrauenswürdiges Verhalten grundsätzlich Kosten, seien es auch nur die Opportunitätskosten des entgangenen Nutzengewinns im Falle der Nichtausnutzung der Opportunismusspielräume. Darüber hinaus geht die Erfüllung der Vertrauenserwartung in der Regel mit dem Einsatz eigener Ressourcen einher. Indem der vertrauenswürdige Agent freiwillig auf den eigenen Konsum von Ressourcen verzichtet und sie einsetzt, um das Nutzenniveau des Prinzipals zu heben, nimmt er einen (zunächst) unkompensierten Nutzentransfer vor und verhält sich in diesem Sinne altruistisch. Zusammenfassend nimmt also das Moment altruistischen Verhaltens eine unterschiedliche Bedeutung im Handeln von Vertrauensgeber und Vertrauensnehmer ein: Vertrauensvolles Verhalten seitens des Vertrauensgebers im Sinne eines Anvertrauens der Kontrolle über Ressourcen oder Ereignisse umfasst nicht notwendigerweise ein altruistisches Moment, sondern kann vielmehr als Aufforderung zu altruistischem bzw. moralisch korrektem Verhalten seitens des Vertrauensnehmers verstanden werden. Der Vertrauensnehmer kommt dieser Aufforderung nach, indem er darauf verzichtet, das ihm Anvertraute zum Schaden des Vertrauensgebers und zu seinem eigenen Vorteil einzusetzen und darüber hinaus, indem er eigene Ressourcen zur Erfüllung der in ihn gesetzten Vertrauenserwartung aufwendet. Damit erfordert vertrauenswürdiges Handeln einen (zunächst) unkompensierten Nutzentransfer bzw. altruistisches Verhalten gegenüber dem Vertrauensgeber. ${ }^{265}$

Die Bedeutung der Unterscheidung zwischen manifestem Verhalten und den einem solchen Verhalten zugrunde liegenden Dispositionen zeigt sich hingegen darin, dass vertrauenswürdiges Handeln nicht unbedingt zugleich eine vertrauenswürdige Disposition, d.h. eine in der Persönlichkeitsstruktur verankerte Moralität in Form altruistischer Präferenzen, erfordert. Der Entschluss, die Vertrauenserwartung zu erfüllen, kann auf sehr unterschiedlichen 
Motiven basieren. Allerdings wird der Grad, zu dem der Vertrauensnehmer tatsächlich altruistische Präferenzen besitzt, seine intrinsische Motivation in Bezug auf vertrauenswürdiges Verhalten und damit seine Vertrauenswürdigkeit als dispositionale Variable seiner Persönlichkeit selbst beeinflussen. Unter diesen Gegebenheiten wird ein vertrauenswürdiger Agent auch dann sein Anstrengungsniveau nicht verringern bzw. zum Schaden des Prinzipals handeln, wenn letzterer das Verhalten von ersterem aufgrund von Moral Hazard-Problemen entweder nicht beobachten oder aufgrund einer Hold Up-Situation nicht sanktionieren kann. In anderen Worten wird der vertrauenswürdige Agent auch dann weiterhin eigene Ressourcen zur Herbeiführung des für den Vertrauensgeber nutzenstiftenden Ereignisses einsetzen, wenn keine entsprechenden extrinsischen Anreizmechanismen vorhanden sind. Zusammenfassend wird die Stärke und Stabilität der vertrauenswürdigen Absicht des Agenten über Zeit und sich ändernde Umstände hinweg generell größer sein, als die eines Akteurs, in dessen Persönlichkeit keine vertrauenswürdige Disposition verankert ist. ${ }^{266}$ Im Folgenden wird zunächst intrinsisch motiviertes vertrauenswürdiges Verhalten erläutert, um dann anschließend auf extrinsische Anreize für die Erfüllung der Vertrauenserwartung einzugehen.

\subsubsection{Die intrinsischen Anreize für vertrauenswürdiges Verhalten}

Die Stärke einer vertrauenswürdigen Absicht ist eine Funktion der Kosten, inklusive der Opportunitätskosten des entgangenen Nutzengewinns durch einen potentiellen Vertrauensbruch, die der Vertrauensnehmer bereit ist, für die Erfüllung der Vertrauenserwartung des Vertrauensgebers zu tragen. Der Vertrauensnehmer wird das in ihn gesetzte Vertrauen rechtfertigen, wenn die Kosten vertrauenswürdigen Verhaltens für ihn niedriger sind als sein Nutzen. Ob dies der Fall ist, hängt neben extrinsischen Anreizen und Sanktionen vor allem von der intrinsischen Motivation des Vertrauensnehmers ab. Die intrinsische Motivation des Vertrauensnehmers bezüglich altruistischen Verhaltens, das sich durch eine positive Korrelation seines Nutzens mit der von ihm verursachten Nutzenveränderung bei dem Vertrauensgeber kennzeichnet, wird von seinem Grad an Altruismus determiniert. Als dispositionale Variable, die sich ökonomisch in altruistischen Präferenzen manifestiert, kann Altruismus daher als Indikator für die Vertrauenswürdigkeit des Vertrauensnehmers fungieren. ${ }^{267}$

Motivationale Grundorientierungen des Vertrauensnehmers lassen sich annähernd durch eine Variation der funktionalen Abhängigkeit zwischen dem Nutzen des Vertrauensgebers und jenem des Vertrauensnehmers ökonomisch operationalisieren: Ein Vertrauensnehmer, dessen Nutzenfunktion negativ mit der 
des Vertrauensgebers korreliert (opportunistische Präferenzen), verfügt über eine kompetitive Motivation und ist grundsätzlich geneigt, opportunistisch zu handeln. Bei einem individualistisch orientierten Vertrauensnehmer (egoistische Präferenzen) existiert keine Korrelation mit dem Nutzen des Vertrauensgebers und somit müssen die durch sein Handeln verursachten positiven und negativen Externalitäten auf das Nutzenniveau des Vertrauensgebers allein über extrinsische Anreize und Sanktionen, also über die institutionellen Einwirkungen auf seine Handlungsrestriktionen, internalisiert werden. Hingegen ist ein kooperativ orientierter Vertrauensnehmer (altruistische Präferenzen) aufgrund der positiven Korrelation seines Nutzens mit dem Nutzen des Vertrauensgebers zu einem gewissen Grad intrinsisch motiviert, sich diesem gegenüber vertrauenswürdig zu verhalten. ${ }^{268}$

Je höher der Grad der Ausprägung altruistischer Präferenzen des Vertrauensnehmers in Bezug auf den Vertrauensgeber ist, desto geringer wird er seinen eigenen Nutzen im Verhältnis zum Nutzen des Vertrauensgebers gewichten, und umso größer wird seine intrinsische Motivation zu vertrauenswürdigem Verhalten sein. In dem Maße, in dem der Vertrauensgeber über die Kosten der Ressourcen, die der Vertrauensnehmer für die Erfüllung der Vertrauenserwartung einsetzt, und über extrinsisch wirkende Anreize auf den Vertrauensnehmer informiert ist, kann er, in Verbindung mit seiner durch das Verhalten des Vertrauensnehmers bedingten Nutzenveränderung, Rückschlüsse auf dessen Grad altruistischer Präferenzen ziehen und dadurch die Stärke und Stabilität von dessen vertrauenswürdiger Absicht in zukünftigen Vertrauenssituationen antizipieren und seine Vertrauensentscheidung daran ausrichten. Je stärker vertrauenswürdiges Verhalten aus altruistischen Präferenzen gegenüber dem Vertrauensgeber und damit aus intrinsischen Motiven heraus resultiert, umso stabiler wird die vertrauenswürdige Absicht des Vertrauensnehmers auch in Zukunft sein. ${ }^{269}$

Zusammenfassend kann die Befriedigung altruistischer Präferenzen durch die Herbeiführung des für den Vertrauensgeber nutzenstiftenden Ereignisses als intrinsischer Anreiz für vertrauenswürdiges Verhalten aufgefasst werden. Unter der Vernachlässigung der Existenz extrinsischer Anreize und Sanktionen wird sich der Vertrauensnehmer dem Vertrauensgeber gegenüber so lange vertrauenswürdig verhalten, wie der Nutzentransfer, den er im Rahmen vertrauenswürdigen Verhaltens an den Vertrauensgeber leistet, durch den Nutzen, den er aus seinen moralischen Qualitäten zieht, kompensiert wird. Der Grad der Ausprägung altruistischer Präferenzen, d.h. das Ausmaß der positiven Nutzenkorrelation zwischen Vertrauensnehmer und Vertrauensgeber, stellt damit einen geeigneten Indikator

268 Vgl. Landes/Posner (1978), S. 418

269 Vgl. Ripperger (1998), S. $151 \mathrm{f}$. 
für die Vertrauenswürdigkeit eines Akteurs im Sinne seiner motivationalen Grunddisposition dar. ${ }^{270}$

\subsubsection{Die extrinsischen Anreize für vertrauenswürdiges Verhalten}

Der implizite Vertrag zwischen Vertrauensgeber und Vertrauensnehmer begründet Rechte und Pflichten auf beiden Seiten der Vertrauensbeziehung. Durch den Einsatz von Ressourcen zur Erfüllung der Vertrauenserwartung und damit des impliziten Vertrags, begründet der Vertrauensnehmer einen moralischen Anspruch gegenüber dem Vertrauensgeber, ihn zu einem späteren Zeitpunkt für diesen Nutzentransfer zu kompensieren. Die Bereitschaft des Vertrauensgebers, die empfangene Leistung zu einem späteren Zeitpunkt zu erwidern, ist sowohl von seinem eigenen Nutzengewinn, als auch von seiner Einschätzung der Kosten, die der Vertrauensnehmer für sein vertrauenswürdiges Verhalten zu tragen bereit war, abhängig. Damit ergibt sich die Höhe des durch die Erfüllung der Vertrauenserwartung begründeten moralischen Anspruchs aus dem Kosten/Nutzen-Verhältnis vertrauenswürdigen Verhaltens. ${ }^{271}$

Die Begründung eines moralischen Anspruchs gegenüber dem Vertrauensgeber, als Folge des Ressourceneinsatzes zur Erfüllung der Vertrauenserwartung, kann als extrinsischer Anreiz für vertrauenswürdiges Verhalten fungieren. Die Bedeutung dieses Anreizes ist davon abhängig, inwieweit der Vertrauensgeber als Nutznießer vertrauenswürdigen Verhaltens seitens des Vertrauensnehmers dieses Verhalten zu einem späteren Zeitpunkt reziproziert. Reziprok altruistisches Verhalten wird reguliert über die universell gültige Reziprozitätsnorm und kann - als ein der Vertrauensbeziehung immanenter Anreiz für vertrauenswürdiges Verhalten - einen entscheidenden Beitrag dazu leisten, dass der implizite Vertrag zwischen Vertrauensgeber und Vertrauensnehmer selbstdurchsetzend ist. ${ }^{272}$

Die Minimalansprüche der Norm reziproken Verhaltens können als strategische Verhaltensregeln folgendermaßen formuliert werden: (1) Erwidere altruistisches Verhalten nicht mit opportunistischem Verhalten und (2) gleiche die durch altruistisches Verhalten Dritter erzielten Nutzengewinne durch unkompensierte Nutzentransfers an diese langfristig aus. Damit begründet die Reziprozitätsnorm ein wechselseitiges System sozialer Verpflichtungen und erzeugt dadurch Gläubiger-Schuldner-Beziehungen sozialer Art. Die Höhe einer Verpflichtung ist im Wesentlichen vom wahrgenommenen Wert der erhaltenen Leistung abhängig und variiert mit dem Status der an einer Transaktion teilnehmenden Akteure: Sie verhält sich proportional zu der Intensität der Bedürftigkeit des Schuldners zum Zeitpunkt des Erhalts der Leistung, zu den Ressourcen, die dem

270 Vgl. Ripperger (1998), S. 152

271 Vgl. Trivers (1971), S. 49; Bierhoff (2000), S. $80 \mathrm{ff}$.

272 Vgl. Ripperger (1998), S. 153 
Gläubiger zu diesem Zeitpunkt zur Verfügung standen, sowie zu den Motiven, die der Handlung des Gläubigers zugrunde lagen, und zu den wahrgenommenen Handlungsrestriktionen des Gläubigers. Die Aufgabe der Reziprozitätsnorm besteht in der Verhinderung von ungleichen Austauschbeziehungen und damit in der Aufrechterhaltung eines leistungsbezogenen Gleichgewichts zwischen Vertrauensgeber und Vertrauensnehmer. Die durch ausstehende Verpflichtungen geschaffenen Gläubiger-Schuldner-Beziehungen leisten einen wesentlichen Beitrag zur Stabilisierung sozialer Systeme, da ein Abbruch der Beziehung im Falle unausgeglichener ,sozialer Konten' für beide Parteien unvorteilhaft ist: Der Gläubiger würde durch den Abbruch der Beziehung seine ihm zustehenden Ansprüche gegenüber dem Schuldner aufgeben. Der Schuldner könnte sich durch den Abbruch der Beziehung zwar seinen Leistungsverpflichtungen entziehen, würde dabei aber seine Reputation als Schuldner verschlechtern und sich dadurch dem Risiko aussetzen, dass weder der Gläubiger noch Dritte ihm eine erneute ,soziale Verschuldung' gewähren. Hinzu kommt, dass der Abbruch von Beziehungen oder die Schädigung derer, mit denen der Schuldner in einem Schuldverhältnis steht, für ihn mit psychischen Kosten verbunden ist, und zwar in dem Ausmaß, in dem die Reziprozitätsnorm tatsächlich internalisiert ist. Die zentrale Bedeutung der Reziprozitätsnorm als universell gültige Verhaltensregel besteht darin, dass sie altruistisches Verhalten auch für einen Egoisten unter rationalen Gesichtspunkten lohnend erscheinen lässt. ${ }^{273}$

Die Vorteile reziprok altruistischen Verhaltens resultieren aus dessen Reziprozität und dem günstigen Verhältnis zwischen Kosten und Nutzen eines altruistischen Akts. ${ }^{274}$ Wenn der Nutzen einer altruistischen Handlung für den Begünstigten größer ist als deren Kosten für den Handelnden, und wenn letzterer davon ausgehen kann, dass ersterer den altruistischen Akt zu einem späteren Zeitpunkt erwidert - womit reziproker Altruismus dann analog einer Versicherung auf Gegenseitigkeit funktioniert - dann führt altruistisches Verhalten für beide Seiten langfristig zu einem Nutzenzuwachs. Reziprok altruistisches Verhalten wird vor allem stark ausgeprägt sein, wenn (1) ein Akteur häufig mit altruistischen Situationen konfrontiert ist, (2) wenn er wiederholt mit der gleichen Gruppe von Individuen (z.B. Unternehmen) interagiert und (3) wenn Paare altruistischer Akteure symmetrisch altruistischen Situationen ausgesetzt sind, d.h. die Möglichkeit haben, Kosten und Nutzen gegenseitig so auszugleichen, dass diese gleichwertig sind. Eine langfristige Ausrichtung der Beziehung, ein hoher Grad an gegenseitiger Abhängigkeit, die Einbindung in ein relativ geschlossenes System und die Abwesenheit von stark hierarchischen Strukturen stellen weitere Parameter dar,

273 Vgl. Brennan/Buchanan (1993), S. 87; Ripperger (1998), S. 153 ff.; Klaus (2002), S. 187 f.

274 Vgl. Trivers (1971); Palfrey/Rosenthal (1988) 
die die Konfrontation mit altruistischen Situationen und damit die Möglichkeit zu altruistischem Verhalten positiv beeinflussen. ${ }^{275}$

Zusammenfassend begründet der explizite Vertrag zwischen Vertrauensgeber und Vertrauensnehmer Rechte und Pflichte auf beiden Seiten der Vertrauensbeziehung: Wer sich einem anderen gegenüber in einer für diesen bedeutenden Situation als vertrauenswürdig gezeigt hat, der erwirbt einen moralischen Anspruch darauf, dass sich der andere ebenfalls vertrauenswürdig verhalten wird, wenn er sich selbst in einer für ihn bedeutenden Situation befindet. In diesem Sinne kann der Mechanismus des reziproken Altruismus - als ein durch die Vertrauensbeziehung selbst begründeter, extrinsischer Anreiz für vertrauenswürdiges Verhalten - einen entscheidenden Beitrag dazu leisten, dass der Vertrauensnehmer die Vertrauenserwartung des Vertrauensgebers erfüllt.

\section{Die DETERMINANTEN DER ENTSTEHUNG VON VERTRAUEN}

Die Vertrauensentscheidung durch den Vertrauensgeber zeigt sich in der Vornahme der Vertrauenshandlung, d.h. in der freiwilligen Erbringung einer riskanten Vorleistung, die durch die Vertrauenserwartung, d.h. durch die subjektive Einschätzung, dass der Vertrauensnehmer freiwillig auf opportunistisches Verhalten verzichtet, motiviert wird. Nachdem sowohl die Höhe der Vertrauenserwartung und der situationsspezifischen Risikowahrnehmung, sowie die Risikobereitschaft des Vertrauensgebers bereits im Zusammenhang mit der Vertrauenshandlung, als auch die Vertrauensatmosphäre, die Reputation des Vertrauensnehmers und die grundsätzliche Vertrauensbereitschaft des Vertrauensgebers bereits im Zusammenhang mit der Vertrauenserwartung als grundlegende Vertrauensdeterminanten identifiziert wurden (vgl. Kap. 5), werden im Folgenden jene Faktoren analysiert, die einen positiven Einfluss auf die Entstehung von Vertrauen seitens des Vertrauensgebers gegenüber einem bestimmten Vertrauensnehmer in einer spezifischen Situation nehmen.

Das spezifische Vertrauen des Vertrauensgebers, das auf seiner subjektiven Einschätzung der Vertrauenswürdigkeit eines bestimmten Vertrauensnehmers in einer spezifischen Situation beruht ${ }^{276}$, wird vorrangig von den persönlichen Merkmalen des Vertrauensnehmers beeinflusst. In diesem Zusammenhang identifiziert Butler zehn Faktoren als persönliche Merkmale des Vertrauensnehmers, die einen positiven Einfluss auf die Einschätzung seiner Vertrauenswürdigkeit nehmen: Erreichbarkeit, Kompetenz, Konsistenz, Diskretion, Fairness, Integrität, Loyalität,

275 Vgl. Trivers (1971); Harbach (1992), S. $161 \mathrm{f}$

276 Vgl. dazu Möllering (2006, S. 13): ,[T]he more trustworthy others appear to us, the more likely we are to trust them at all, the stronger our trust will be and the more we will be prepared to enact this trust." 
Offenheit, Verlässlichkeit und Zugänglichkeit. ${ }^{277}$ Mayer et al. gehen davon aus, dass Können (ability), Wohlwollen (benevolence) und Integrität (integrity) eines Vertrauensnehmers die Entstehung von spezifischem Vertrauen determinieren. ${ }^{278}$ Aufgrund der inhaltlichen Überschneidungen zwischen dem ,Conditions of Trust Inventory' von Butler und den ,Factors of Trustworthiness' von Mayer et al. wird auf eine separate Erläuterung der Vertrauensdeterminanten nach Butler verzichtet. Im Rahmen der folgenden Analyse der drei Faktoren von Mayer et al. wird jedoch auf sie Bezug genommen.

Der erste Faktor nach Mayer et al. umfasst das aufgaben- und situationsspezifische Können eines Vertrauensnehmers. Dieses wird von seinen Fertigkeiten, Fähigkeiten und Qualifikationen beeinflusst. ${ }^{279}$ In diesem Kontext soll daher ,Können' nach Mayer et al. mit ,Kompetenz' nach Butler, im Rahmen dieser Arbeit verstanden als Übereinstimmung der Anforderungen einer Aufgabe mit den Fähigkeiten des Aufgabenerfüllers ${ }^{280}$, gleichgesetzt werden. Während eine Vielzahl von Studien die determinierende Wirkung von Kompetenz auf die Vertrauensentstehung nennt ${ }^{281}$, wurde gezeigt, dass auf individueller Ebene vor allem die fachliche und die soziale Kompetenz des Vertrauensnehmers von herausragender Bedeutung für die Entstehung von Vertrauen sind. ${ }^{282}$ Generell ist der Vertrauensgeber bei dem Treffen seiner Vertrauensentscheidung mit zwei Formen von Unsicherheit konfrontiert: So besteht einerseits Unsicherheit darüber, ob der potentielle Vertrauensnehmer die Vertrauensentscheidung honorieren kann und andererseits ist der Vertrauensgeber unsicher, ob sich der potentielle Vertrauensnehmer vertrauenswürdig verhalten will. Die wahrgenommene Fachkompetenz des Vertrauensnehmers kann nun die Entstehung von Vertrauen seitens des Vertrauensgebers insofern begünstigen, als dass sie seine Unsicherheit bezüglich des Könnens des Vertrauensnehmers reduziert. Verfügt der Vertrauensgeber also über die Einschätzung, dass der potentielle Vertrauensnehmer die fachliche Kompetenz zur Honorierung der Vertrauensentscheidung besitzt, dann kann er aufgrund seiner Zutrauenserwartung eher geneigt sein, eine Vertrauenshandlung zu setzen. Die wahrgenommene soziale Kompetenz des Vertrauensnehmers kann die Entstehung von Vertrauen begünstigen, indem sie die Unsicherheit des Vertrauensgebers bezüglich des Willens des Vertrauensnehmers, sich vertrauenswürdig zu verhalten, reduzieren kann. Die detaillierte Analyse des Einflusses von Kompetenz auf die Entstehung von Vertrauen seitens des Vertrauensgebers

277

278

279

280

281

282

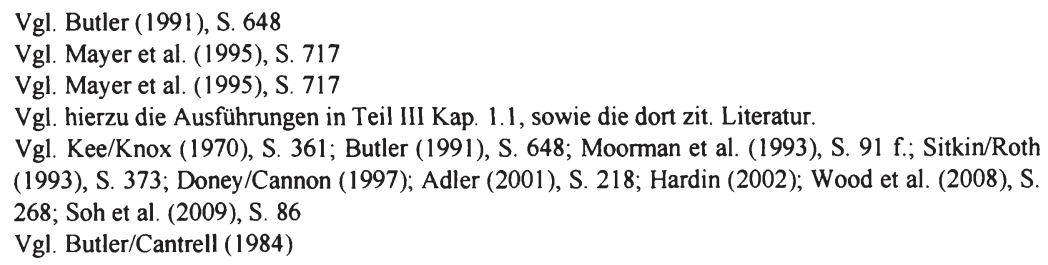


gegenüber einem bestimmten Vertrauensnehmer in einer spezifischen Situation ist Gegenstand des Teils IV, sowie des empirischen Teils dieser Arbeit.

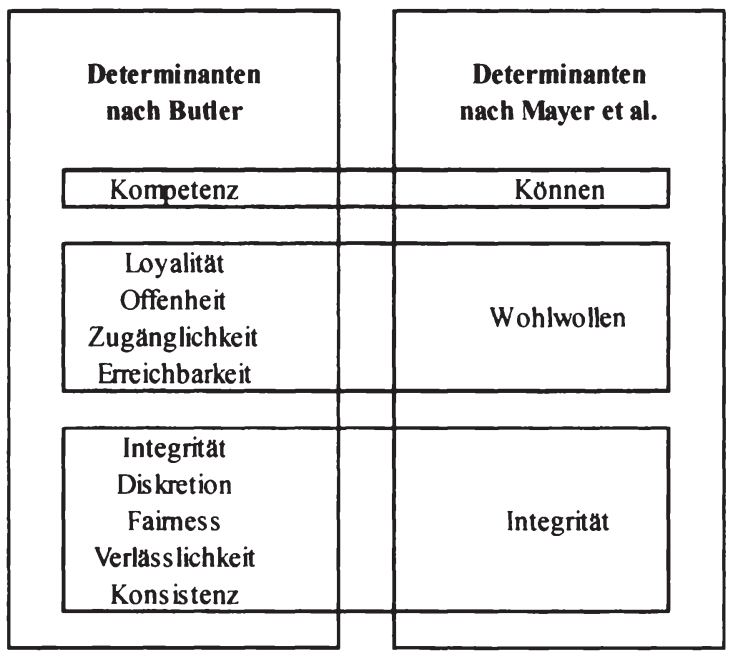

Abbildung 9: Die Determinanten der Entstehung von Vertrauen

Wohlwollen als zweite Determinante der Entstehung von Vertrauen bezeichnet die Wahrnehmung einer positiven Orientierung des Vertrauensnehmers gegenüber dem Vertrauensgeber. ${ }^{283}$ Mayer et al. subsumieren unter Wohlwollen die Faktoren Loyalität, Offenheit, Zugänglichkeit und Erreichbarkeit eines Vertrauensnehmers nach Butler. ${ }^{284}$ Während die Ergebnisse einer experimentellen Untersuchung von Butler und Cantrell eine geringe Bedeutung der Faktoren Loyalität und Offenheit für die Entstehung von Vertrauen implizieren ${ }^{285}$, bestätigen die Resultate der Erhebung von Davis et al. ihre Annahme, dass die Entstehung von Vertrauen von dem wahrgenommenen Wohlwollen des Vertrauensnehmers determiniert wird. ${ }^{286}$ $\mathrm{Da}$,Wohlwollen' impliziert, dass der Vertrauensnehmer dem Vertrauensgeber keinen Schaden zufügt, auch wenn der Anreiz dazu besteht ${ }^{287}$, besitzt dieser Faktor insbesondere in interdependenten Entscheidungssituationen eine hohe Relevanz: So ist der Vertrauensgeber eher bereit, sich gegenüber einem wohlwollenden Interaktionspartner verwundbar $\mathrm{zu}$ machen, als gegenüber einem Interaktionspartner, dessen Wohlwollen ihm fraglich erscheint. ${ }^{288}$ Dies führt zu dem

283 Vgl. Mayer et al. (1995), S. 719

284 Vgl. Butler (1991), S. 648; Mayer et al. (1995), S. 723

$285 \mathrm{Vgl}$. Butler/Cantrell (1984)

286 Vgl. Davis et al. (2000)

287 Vgl. Gambetta (1988), S. 217

$288 \quad$ Vgl. Kee/Knox (1970), S. 361 
Schluss, dass das wahrgenommene Wohlwollen des Vertrauensnehmers die Entstehung von Vertrauen positiv beeinflusst. ${ }^{289}$

Die Wahrnehmung, dass der Vertrauensnehmer Prinzipien treu ist, die der Vertrauensgeber akzeptiert, definiert die Integrität des Vertrauensnehmers. ${ }^{290}$ Integrität als dritte Vertrauensdeterminante nach Mayer et al. wird vorrangig mit Diskretion, Fairness, Verlässlichkeit und konsistentem Verhalten des Vertrauensnehmers in Verbindung gebracht. ${ }^{291}$ Charakterisiert sich der Vertrauensnehmer dadurch, dass er seine Versprechen einhält, geltende Normen beachtet, über einen ausgeprägten Gerechtigkeitssinn verfügt, sowie, dass seine Handlungen in der Vergangenheit konsistent waren, so wird ihm ein hoher Grad an Integrität zugesprochen. Im Gegensatz zum Faktor, Wohlwollen' kann der Vertrauensgeber die Informationen zur Einschätzung der Integrität eines potentiellen Vertrauensnehmers nicht bloß aus vergangenen Interaktionen mit diesem beziehen, sondern er kann seine Wahrnehmung auch auf glaubwürdige Informationen von Drittparteien über den Vertrauensnehmer stützen. ${ }^{292}$ In den Studien von Butler und Cantrell wurde gezeigt, dass die Integrität des Vertrauensnehmers, direkt nach der Kompetenz, die bedeutendste Determinante der Entstehung von Vertrauen seitens des Vertrauensgebers darstellt. ${ }^{293}$

Zusammenfassend stellen, neben der situationsspezifischen Risikowahrnehmung, der vertrauensgeberspezifischen Risikobereitschaft und der grundsätzlichen Vertrauensbereitschaft des Vertrauensgebers, sowie der Reputation des Vertrauensnehmers, und der Vertrauensatmosphäre als grundlegende Einflussfaktoren auf die Vertrauensentstehung, die Faktoren Kompetenz, Wohlwollen und Integrität bedeutende Determinanten der Entstehung von Vertrauen seitens des Vertrauensgebers gegenüber einem Vertrauensnehmer in einer spezifischen Situation dar.

\section{DiE VERTRAUENSMESSVERFAHREN}

Im folgenden Kapitel werden für diese Arbeit relevante Verfahren zur Erfassung von Vertrauen beleuchtet. Auf Basis der erläuterten Vertrauensmessansätze erfolgt in Teil IV die Ableitung konsensfähiger Indikatoren, die als empirische Äquivalente von Vertrauen fungieren.

Vertrauensmessverfahren werden hier als Ergebnis der Grundauffassung von Vertrauen verstanden. Aus dieser Auffassung resultiert der Blickwinkel auf die verschiedenen Vertrauensebenen und Vertrauensarten. Er wiederum determiniert die

\footnotetext{
289 Vgl. Späth (2008), S. 195

290 Vgl. Mayer et al. (1995), S. 719

291 Vgl. Butler (1991), S. 648; Mayer et al. (1995), S. 723

292 Vgl. Mayer et al. (1995), S. 719

$293 \mathrm{Vgl}$. Butler/Cantrell (1984)
} 
Sicht auf die Vertrauensentstehung, die Neigung zu einer eher objektiven oder subjektiven Vertrauenserfassung und die Präferenz eher quantitativer oder qualitativer Methoden der Vertrauensforschung.

Im Zuge der wissenschaftlichen Auseinandersetzung mit Vertrauen wurde eine Vielzahl von Verfahren entwickelt, um dieses latente Konstrukt zu erfassen. ${ }^{294}$ Grundsätzlich ist zwischen objektiven und subjektiven Verfahren zu differenzieren:

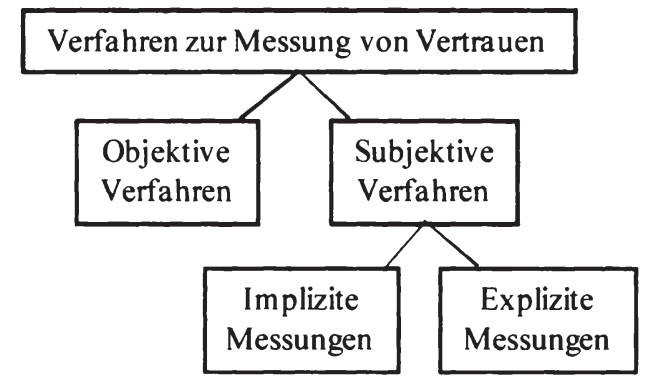

Abbildung 10: Die Verfahren zur Messung von Vertrauen Quelle: In Anlehnung an Plötner (1995), S. 167

Die sogenannten objektiven Vertrauensmessverfahren kennzeichnen sich dadurch, dass ökonomische Größen, wie beispielsweise Produktivität, Rentabilität, Liquidität, als Vertrauensindikatoren fungieren. Diese Vorgehensweise ist sowohl mit Vor- als auch Nachteilen verbunden: Einerseits ermöglicht das Heranziehen objektiver Indikatoren, die sich durch eine hohe Reliabilität kennzeichnen, eine Erfassung des Konstrukts mit geringem Aufwand, da die relevanten Informationen leicht zugänglich sind. ${ }^{295}$ Andererseits berücksichtigen diese Verfahren nicht die in dieser Arbeit erläuterten Charakteristika des maximenbasierten Vertrauenskonstrukts, wozu die freiwillige Erbringung einer riskanten Vorleistung als Reaktion auf die subjektive Unsicherheit über das Verhalten eines anderen Akteurs, der Verzicht auf explizite vertragliche Sicherungs- und Kontrollmaßnahmen zur Begrenzung von Opportunismusspielräumen, und die Erwartung, dass sich der andere, trotz dem Fehlen dieser Schutzmaßnahmen, kooperativ verhalten wird, zählen. Der Schluss von hohen Produktivitätskennzahlen oder einer hohen Anzahl von Kooperationsbeziehungen auf ein hohes Vertrauensniveau mag daher pragmatisch wünschenswert sein, allerdings stehen ihm erhebliche Zweifel an der Validität der Indikatoren gegenüber. Bei den sogenannten subjektiven Verfahren, im Rahmen derer psychische Variablen bzw. die damit unmittelbar zusammenhängenden Verhaltensweisen - anstelle quantitativ eindeutig messbarer ökono-

294 Vgl. dazu den Überblick über Verfahren der Vertrauenserfassung von Raimondo (2000); Kenning (2002), S. $50 \mathrm{f}$

295 Vgl. Plötner (1995), S. 167; Kenning (2002), S. 46 
mischer Größen - zur Erfassung von Vertrauen herangezogen werden, ist zwischen expliziten und impliziten Verfahren zu differenzieren: Explizite Verfahren zielen auf die direkte Erfassung von Vertrauen ab. Durch die Verwendung von 1-Item-Skalen, die explizit nach dem Vertrauen in einen Kooperationspartner fragen, wird im Zuge dieser Verfahren das Problem der Indikatorvalidität umgangen. Zu den Nachteilen dieser Art von Befragung zählen allerdings die mit ihr verbundenen ergebnisverzerrenden Störfaktoren, sowie die durch die unterschiedliche Auffassung des Vertrauensbegriffs der Befragten bedingte mangelnde Aussagekraft der Ergebnisse. ${ }^{296}$ Aufgrunddessen, dass es sich bei Vertrauen um ein Phänomen handelt, das sich in empirischen Untersuchungen nicht unmittelbar erfassen lässt, sind explizite Verfahren zu vernachlässigen. ${ }^{297}$ Hingegen stellen implizite Verfahren einen geeigneten Ansatzpunkt zur Erfassung latenter Konstrukte wie Vertrauen dar. Im Rahmen dieser Ansätze wird von beobachteten Verhaltensweisen oder erfragten Einstellungen und deren Verhaltenswirksamkeit, die als Indikatoren oder konstitutive Bestandteile des Vertrauenskonstrukts aufgefasst werden, auf Vertrauen geschlossen. ${ }^{298}$ Die impliziten Verfahren zur Erfassung von Vertrauen können nach ihrem Untersuchungsgegenstand in Ansätze zur Messung interorganisationalen Vertrauens, generalisierten Vertrauens und Instrumente zur Erhebung spezifischen Vertrauens differenziert werden.

Während die nachfolgend erläuterten Ansätze von Cummings/Bromiley (1996) und Currall/Judge (1995) das Konstrukt des interorganisationalen Vertrauens fokussieren, stehen bei dem im Anschluss diskutierten Verfahren von Rotter (1967), und dem in diesem Zusammenhang dargestellten Instrument von Costa (2000), das generalisierte Vertrauen, und bei dem Ansatz von Gillespie (2003) das spezifische Vertrauen im Zentrum der Betrachtung. Die Auswahl dieser Ansätze aus der Fülle von impliziten Vertrauensmessverfahren beruht auf ihrer Eignung für die in Teil IV dieser Arbeit verfolgte Operationalisierung des Vertrauenskonstrukts, sowie auf der Anwendbarkeit ihrer Instrumente im Rahmen der empirischen Untersuchung des Einflusses von Kompetenz auf die Entstehung von Vertrauen. Die Zielsetzungen, theoretischen Grundlagen, methodologischen Einordnungen und verfahrensbezogenen Beurteilungen der Gütekriterien der ausgewählten Verfahren lassen darauf schließen, dass ihre Anwendung zur Erhebung der Vertrauensbereitschaft, sowie zur Erfassung der Entstehung spezifischen Vertrauens seitens des Vertrauensgebers im Rahmen dieser Arbeit geeignet ist. So teilen auch Cummings/Bromiley und Currall/Judge die in dieser Arbeit vertretene Auffassung, dass Vertrauen zwischen Organisationen letztlich immer durch In-

296 Vgl. Plötner (1995), S. 168; Kenning (2002), S. 47; Schweer/Thies (2003), S. 38

297 Vgl. Putnam (1995); Glaeser et al. (2000), S. 812; Schweer/Thies (2003), S. 37; Pirson (2007), S. 65; Soh et al. (2009), S. 85

298 Vgl. Plötner (1995), S. 168 
dividuen, die diesen Organisationen angehören, begründet und zerstört wird ${ }^{299}$, was sich darin zeigt, dass ihre Verfahren zur Erfassung interorganisationalen Vertrauens auf personaler Ebene ansetzen.

\section{Der Ansatz von Cummings/Bromiley}

Im Rahmen dieses Ansatzes von Cummings und Bromiley wird Vertrauen als ,individual's belief or a common belief among a group of individuals that another individual or group (a) makes good-faith efforts to behave in accordance with any commitments both explicit or implicit, (b) is honest in whatever negotiations preceded such commitments, and (c) does not take excessive advantage of another even when the opportunity is available ${ }^{\text {‘300 }}$ aufgefasst. Vertrauenswürdiges Verhalten liegt demnach vor, wenn sich ein Individuum entsprechend (a), (b) und (c) verhält.

Basierend auf diesem Verständnis zielt die Arbeit von Cummings und Bromiley darauf ab, ein validiertes Instrument zur Erfassung von interorganisationalem Vertrauen zu entwickeln, wobei sich interorganisationales Vertrauen auf das Ausmaß von Vertrauen zwischen Organisationen oder zwischen Abteilungen einer Organisation bezieht. ${ }^{301} \mathrm{Im}$ Zuge dieses Entwicklungsprozesses identifizieren die Autoren auf Basis ihrer Vertrauensdefinition drei Indikatoren (Integrität, Ehrlichkeit in Verhandlungen und Verzicht auf die Ausnützung von Opportunismusspielräumen), die als empirische Äquivalente des dreidimensionalen Vertrauenskonstrukts $^{302}$ fungieren. Das in Folge generierte Set von Items zur Messung von Vertrauen in Organisationen oder zwischen Organisationen bildet die Indikatoren des Vertrauenskonstrukts reliabel ab. Das Ergebnis umfasst zwei Versionen des Instruments zur Erfassung interorganisationalen Vertrauen (,The Organisational Trust Inventory - OTI'): Während das OTI-Long Form aus 62 Items besteht, setzt sich das OTI-Short Form aus 12 Items zusammen, deren modelltheoretische $\mathrm{Zu}$ ordnung zu den identifizierten Indikatoren durch eine konfirmatorische Faktorenanalyse bestätigt wird. Nachfolgende Tabelle gibt einen Überblick über das von Cummings und Bromiley generierte validierte Instrument $\left(\mathrm{OTI}^{-\mathrm{SF}^{303}}\right)$ zur Erfassung interorganisationalen Vertrauens.

\footnotetext{
299 Vgl. hierzu die Ausführungen in Teil II Kap. 4.3.1, sowie die dort zit. Literatur.

300 Cummings/Bromiley (1996), S. 303

301 Die Relevanz der Messung interorganisationalen Vertrauens ergibt sich dadurch, dass Vertrauen in und zwischen Organisationen Transaktionskosten reduziert (Cummings/Bromiley 1996, S. 303).

302 Cummings/Bromiley (1996, S. 305) schreiben hierzu: ,[...] as a belief, trust should be assessed across three components. These are trust as an affective state, as a cognition, and as an intended behavior."

303 Die Items zur Erfassung der Dimension „intendiertes Verhalten“ wurden im OTI-SF eliminiert, da sie einzeln und im Durchschnitt gesehen, niedrigere item-to-factor Korrelationen aufweisen, als die Items zur Erfassung der affektiven und kognitiven Dimension. Von diesen wurden aus dem OTI-LF jene für das OTI-SF ausgewählt, die die höchsten item-to-factor Korrelationen aufweisen (Cummings/Bromiley 1996, S. 317).
} 


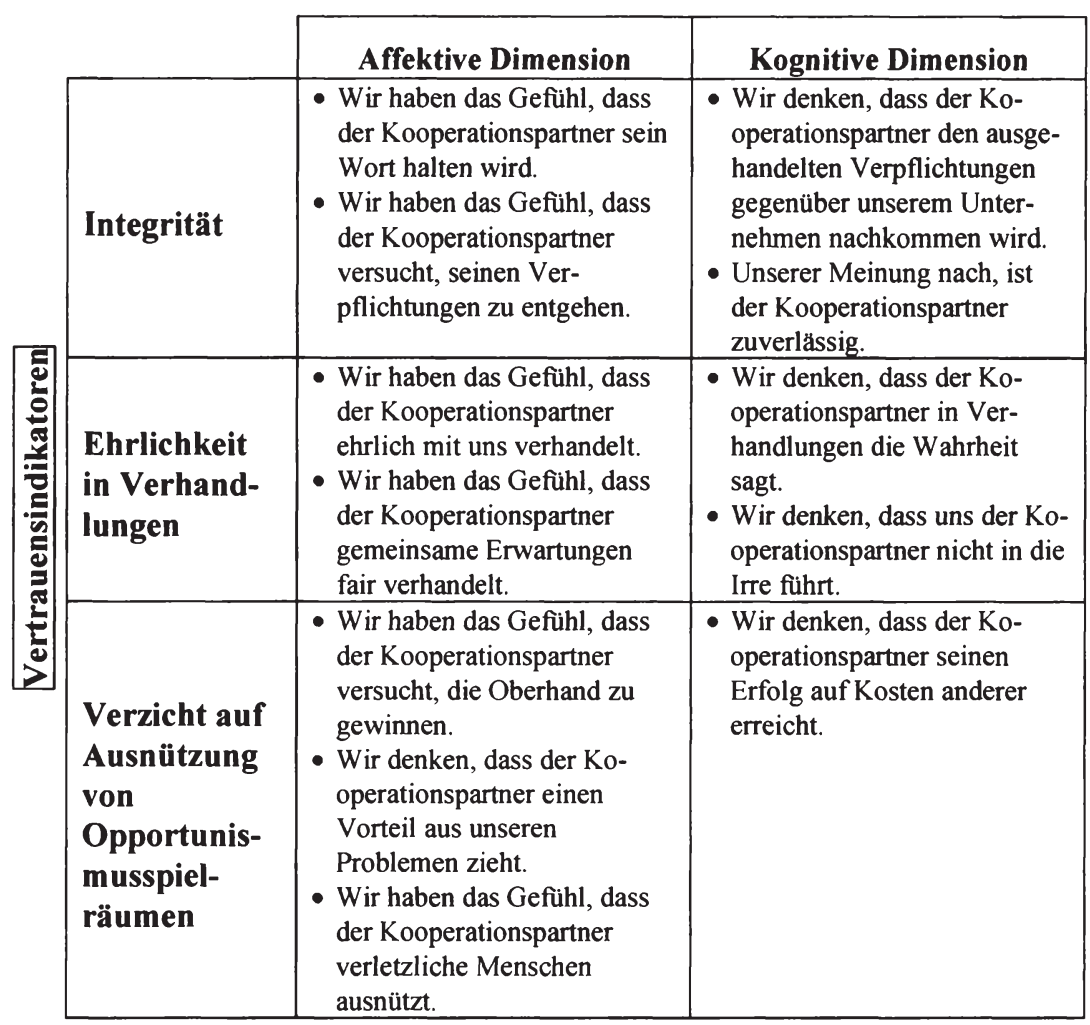

Tabelle 1: Items zur Erfassung interorganisationalen Vertrauens (OTI-SF)

Quelle: In Anlehnung an Cummings/Bromiley (1996, S. 328 f.)

Die Eignung des OTIs für den vorliegenden Untersuchungskontext zeigt sich darin, dass sich die ihm zugrunde liegende Vertrauensdefinition mit dem Vertrauensverständnis dieser Arbeit deckt. Ein weiterer Vorteil liegt in der Operationalisierung des Vertrauenskonstrukts: Es wird eine Vielzahl von Items entwickelt und den Vertrauensindikatoren und -dimensionen zugewiesen. Für eine Berücksichtigung des OTIs im Rahmen der in Teil IV dieser Arbeit verfolgten Operationalisierung des Vertrauenskonstrukts spricht auch, dass die von Cummings und Bromiley identifizierten Indikatoren empirisch bestätigt wurden. Auf die Frage, ob es sich bei gewissen Indikatoren nicht eher um Determinanten der Vertrauenserwartung und damit Indikatoren der Vertrauenswürdigkeit anstelle von empirischen Äquivalenten von Vertrauen handelt, wird im Zuge der Operationalisierung von Vertrauen eingegangen. 


\section{Der Ansatz von Currall/Judge}

Im Rahmen dieser Arbeit von Currall und Judge wird Vertrauen als ,an individual's behavioral reliance on another person under a condition of risk “304 verstanden. Dementsprechend manifestiert sich vertrauendes Verhalten seitens des Vertrauensgebers darin, dass er sein Schicksal in einer Situation, die sich durch Risiko kennzeichnet, in die Hände des Vertrauensnehmers legt.

Basierend auf diesem Verständnis zielt der Ansatz von Currall und Judge auf die Entwicklung eines Instruments zur Erfassung von interorganisationalem Vertrauen, d.h. von Vertrauen zwischen Boundary Role Persons (BRPs) ${ }^{305}$, wozu beispielsweise die für eine zwischenbetriebliche Kooperation zuständigen Akteure kooperierender Betriebe zählen, ab. Die Relevanz dieses Untersuchungsgegenstandes ergibt sich aus der Annahme, dass Vertrauen zwischen BRPs zu einer Verbesserung der Kommunikation zwischen den kooperierenden Betrieben, sowie zu einem Verzicht auf kostenintensive Sicherungs- und Kontrollinstrumente führt. ${ }^{306}$ Im Rahmen des Prozesses der Entwicklung eines validierten Instruments zur Erfassung des Vertrauens zwischen BRPs identifizieren die Autoren auf Basis einer Literaturrecherche vier Vertrauensindikatoren:

- Offene und ehrliche Kommunikation mit dem Kooperationspartner: Vertrauen zeigt sich darin, dass der Vertrauensgeber dem Kooperationspartner Informationen offenlegt, die zu seinem Nachteil genutzt werden können, sowie in der Korrektheit der Kommunikation, d.h. in dem Verzicht auf Informationsfilterung und-verzerrung.

- Eingehen informeller Vereinbarungen mit dem Kooperationspartner: Vertrauen zeigt sich in dem Verzicht auf den Einsatz schriftlicher Sanktionsvereinbarungen, die für den Kooperationspartner im Falle der Nichterfüllung seiner Pflichten Wirksamkeit entfalten würden. Dadurch, dass sich der Vertrauensgeber durch diesen Verzicht verwundbar macht, ist die Vertrauensentscheidung für ihn mit dem Risiko vertrauensunwürdigen Verhaltens seitens des Vertrauensnehmers verbunden.

- Überwachung des Kooperationspartners: Vertrauen zeigt sich in dem Verzicht auf den Einsatz expliziter Überwachungsinstrumente zur Kontrolle des Verhaltens des Kooperationspartners.

- Aufgabenkoordination mit dem Kooperationspartner: Vertrauen zeigt sich in der Koordination von komplementären Ressourcen, Informationen und Fähig-

304 Currall/Judge (1995), S. 153

305 Hierunter verstehen Currall/Judge (1995, S. 151) "the individuals who provide the linking mechanism across organizational boundaries".

$306 \mathrm{Vgl}$. Currall/Judge (1995), S. 152 
keiten zur Bewältigung von Aufgaben, die der Vertrauensgeber ohne den Kooperationspartner nicht lösen könnte. ${ }^{307}$

Zur Erfassung dieser Vertrauensindikatoren entwickeln Currall und Judge 25 Items, deren modelltheoretische Zuordnung zu den vier Beurteilungsdimensionen durch eine konfirmatorische Faktorenanalyse bestätigt wird. Aufgrund ihrer Relevanz für den vorliegenden Untersuchungskontext gibt nachfolgende Tabelle einen Überblick über das von Currall und Judge entwickelte Instrument zur Erfassung interorganisationalen Vertrauens. ${ }^{308}$

\begin{tabular}{|l|l|}
\hline Indikator & Item (* steht für inverse Aussagen) \\
\hline & $\begin{array}{l}\text { - Ich denke genau nach, bevor ich dem Kooperationspartner meine Meinung } \\
\text { sage.* }\end{array}$ \\
- Ich gebe dem Kooperationspartner alle mir bekannten und relevanten Infor- \\
mationen über wichtige Angelegenheiten weiter, auch wenn die Möglichkeit \\
besteht, dass diese Vorgehensweise meine eigene Organisation gefährdet. \\
- Ich gebe dem Kooperationspartner alle mir bekannten und relevanten Infor- \\
mationen über wichtige Angelegenheiten weiter, auch wenn die Möglichkeit \\
besteht, dass diese Vorgehensweise meinen Arbeitsplatz gefährdet. \\
- Ich begrenzte die Informationen, die ich an den Kooperationspartner weiter- \\
gebe, auf ein Minimum.* \\
- Ich halte absichtlich Informationen zurück, wenn ich mit dem Kooperations- \\
partner kommuniziere.*
\end{tabular}

307 Vgl. Currall/Judge (1995), S. 153 f.

308 Aufgrund ihrer zu vernachlässigenden Bedeutung für den vorliegenden Untersuchungskontext werden die dem Indikator, Aufgabenkoordination' zugeordneten Items nicht aufgelistet. Des weiteren wurde die Formulierung , the counterpart BRP‘ durch , der Kooperationspartner` ersetzt. 


\begin{tabular}{|l|l|}
\hline & $\begin{array}{l}\text { - Ich beobachte den Kooperationspartner genau, um sicherzugehen, dass er } \\
\text { kein Verhalten setzt, das meinem Unternehmen schadet.* } \\
\text { - Ich überwache den Kooperationspartner (z.B. ihm über die Schulter schauen) } \\
\text { nachdem ich ihn gebeten habe, etwas zu tun.* } \\
\text { - Ich fühle mich sicher, nachdem ich den Kooperationspartner gebeten habe, } \\
\text { etwas zu tun. } \\
\text { - Ich setze andere ein, um das Verhalten des Kooperationspartners zu über- } \\
\text { prüfen, sodass ich sicher sein kann, dass er nicht versucht, mit etwas ,davon- } \\
\text { zukommen“** } \\
\text { - In Situation ohne schriftlich fixierte Vereinbarungen überprüfe ich Auf- } \\
\text { zeichnungen, um die Angaben, die der Kooperationspartner gegeben hat, zu } \\
\text { verifizieren.* }\end{array}$ \\
\hline
\end{tabular}

Tabelle 2: Items zur Erfassung interorganisationalen Vertrauens (BRP-Trust)

Quelle: In Anlehnung an Currall/Judge (1995, S. 168)

Zusammenfassend liefert der Ansatz von Currall und Judge mit seiner Entwicklung eines Instruments zur Messung interorganisationalen Vertrauens wichtige Ansatzpunkte für die in Teil IV dieser Arbeit verfolgte Operationalisierung des Vertrauenskonstrukts.

\section{Der Ansatz von Rotter}

Rotter versteht unter Vertrauen,,an expectancy held by an individual or a group that the word, promise, verbal or written statement of another individual or group can be relied upon. “309 Dieses Verständnis beruht auf der Annahme, dass sich Individuen auf Erfahrungen berufen, um neue und unsichere Situationen zu bewältigen. Auf Basis dieser Erfahrungen werden sodann Erwartungen entwickelt, die relativ stabile Persönlichkeitsmerkmale darstellen, zu denen auch Vertrauen zählt. Rotters Auffassung, dass es sich bei Vertrauen um eine personenspezifische Einstellung handelt, zeigt sich auch in seiner Differenzierung zwischen ,High Trusters $^{\text {‘310 }}$, die in höherem Ausmaß bereit sind, Vertrauensbeziehungen einzugehen, und ,Low Trusters', die generell weniger vertrauensbereit sind. ${ }^{311}$

Basierend auf diesem Verständnis zielt die Arbeit von Rotter auf die Entwicklung eines Instruments zur Erfassung von generalisiertem Vertrauen ab. Die im Zuge dieses Entwicklungsprozesses generierten Items zur Messung des eindimensionalen Vertrauenskonstrukts beziehen sich auf individuelle generelle Erwartungen in verschiedenen Lebenssituationen. Im Ergebnis umfasst die validierte „Interpersonal Trust Scale“ (ITS) 40 Items $^{312}$, wobei es sich bei 15 davon um

\footnotetext{
309 Rotter (1967), S. 651

310 Verglichen mit ,Low Trusters` kennzeichnen sich ,High Trusters` durch eine höhere Konsensorientierung, einen offeneren Zugang zu hoffnungsvollen Kooperationen und eine geringere Bereitschaft zu lügen (Rotter 1980, S. 2 f.).

311 Vgl. Rotter (1980), S. $1 \mathrm{ff}$.

312 Vgl. Rotter (1967), S. 653 ff.; Rotter (1971), S. 446
} 
- Die Heuchelei nimmt in unserer Gesellschaft zu.

- Dieses Land hat eine dunkle Zukunft, wenn wir nicht bessere Leute in die Politik bringen können.

- Ohne die Kontrolle der Lehrer wăhrend der Prüfungen würde wahrscheinlich das Schummeln zunehmen.

- Die Vereinten Nationen (UNO) werden niemals eine wirksame Kraft bei der Erhaltung des Weltfriedens sein.

- Die meisten Leute wären entsetzt, wenn sie wüssten, wie viele Nachrichten, die man sieht oder hört, verfalscht sind.

- Trotz vieler Berichte in Zeitungen, Radio und Fernsehen ist es schwer, eine objektive Darstellung von öffentlichen Ereignissen zu erhalten.

- Wenn wir wirklich wüssten, was in der internationalen Politik vor sich geht, dann hätten wir viel mehr Grund zur Sorge, als dies heute der Fall ist.

- Viele bedeutende nationale Sportwettkämpfe sind in der einen oder anderen Weise manipuliert.

- Bei den meisten Leuten kann man sich darauf verlassen, dass sie das tun werden, was sie ankündigen.

- Im Umgang mit Fremden ist man besser solange vorsichtig, bis diese bewiesen haben, dass sie vertrauenswürdig sind.

- Die Leute werden eher durch Furcht vor sozialer Missbilligung oder Bestrafung, als von ihrem Gewissen davon abgehalten, Gesetze zu übertreten.

- Bei Eltern kann man sich normalerweise darauf verlassen, dass sie ihre Versprechen halten.

- Das Gericht ist der Ort, wo wir alle eine unvoreingenommene Behandlung erfahren können.

- Man kann, trotz anders lautender Aussagen, davon ausgehen, dass die meisten Leute hauptsächlich an ihrem eigenen Wohlergehen interessiert sind.

- Die Zukunft scheint vielversprechend zu sein.

- Die meisten gewählten Volksvertreter meinen es bei ihren Wahlversprechen wirklich ehrlich.

- Bei den meisten Fachleuten kann man sicher sein, dass wie wahrheitsgemäß die Grenzen ihres Wissens zugeben.

- Bei den meisten Eltern kann man sich darauf verlassen, dass sie angedrohte Strafen auch ausführen.

- In der heutigen, vom Konkurrenzdenken geprägten Zeit muss man auf der Hut sein, wenn man nicht ausgenutzt werden will.

- Die meisten Idealisten sind aufrichtig und tun gewöhnlich auch selber das, was sie anderen predigen.

- Die meisten Kaufleute sind bei der Beschreibung ihrer Waren ehrlich.

- Die meisten Schüler würden in der Schule nicht schummeln, selbst wenn sie sicher wären, selbst wenn sie sicher wären, damit durchzukommen.

- Die meisten Kundendienstmechaniker berechnen nicht zuviel, selbst wenn sie glauben, dass man sich in ihrem Spezialgebiet nicht auskennt.

- Ein großer Teil von Schadenersatzansprüchen, die gegen Versicherungsgesellschaften erhoben werden, beruht auf Schwindel.

- Die meisten Leute beantworten Meinungsumfragen ehrlich.

Tabelle 3: Items zur Erfassung generalisierten Vertrauens (ITS)

Quelle: In Anlehnung an Rotter (1991, S. 395 f.) 
Zusammenfassend liefert die Skala von Rotter wichtige Anhaltspunkte für die Erfassung generalisierten Vertrauens. Allerdings wurde im Zuge einer Faktorenanalyse gezeigt, dass die als Instrument zur Erfassung des eindimensionalen Vertrauenskonstrukts entwickelte ITS drei Dimensionen (Vertrauen in Institutionen, wahrgenommene Aufrichtigkeit anderer Individuen, die Notwendigkeit der Vorsicht vor anderen Individuen) erhebt, womit die von Rotter generierten Items nicht mit der theoretischen Fundierung übereinstimmen, in der ausschließlich das interpersonale Vertrauen den Untersuchungsgegenstand darstellt. ${ }^{313}$ Aufgrunddessen, dass auch weitere Arbeiten die Validität der ITS in Frage stellen ${ }^{314}$, wird auf den Einsatz der von Rotter entwickelten Skala im Zuge der in dieser Arbeit verfolgten Erfassung des Einflusses von Kompetenz auf die Entstehung von Vertrauen verzichtet.

Hingegen stellt die von Costa (2000) entwickelte und validierte Skala ein geeignetes Instrument zur Erfassung des generalisierten Vertrauens im Rahmen des empirischen Teils dieser Arbeit dar. Sie wurde, ähnlich der Instrumente von Cummings/Bromiley und Currall/Judge, auf Grundlage explorativer Interviews, Literaturrecherchen und Expertenurteilen entwickelt. Ihre Items basieren auf der Subskala ,Trust' der ,Revised Philosophies of Human Nature Scale (RPHNS) ${ }^{315}$, bei der es sich um ein theoretisch fundiertes und empirisch geprüftes Instrument zur Erfassung der „expectancies that people have about the ways in which other people generally behave ${ }^{\text {(3) }} 16$ handelt. Obwohl die RPHNS ebenso wie die ITS von Rotter generelle Erwartungshaltungen von Individuen misst, ist davon auszugehen, dass die Items der RPHNS eher interpersonale Beziehungen fokussieren als die Items der ITS. ${ }^{317}$ Im Rahmen dieser Arbeit wird daher die von Costa entwickelte Skala zur Erfassung generalisierten Vertrauens dem Instrument von Rotter vorgezogen, da sie ihren Fokus auf interpersonale Beziehungen richtet und sich die ihr zugrunde liegende Vertrauensdefinition ${ }^{318}$ mit dem Vertrauensverständnis dieser Arbeit deckt.

\footnotetext{
313 Vgl. Kaplan (1973), S. 13 f.; ähnlich auch Corazzini (1977), S 75 ff.

314 Vgl. Amelang et al. (1984); Plőtner (1995), S. 168 f.; Pieper (2000), S. 89 f.; Klaus (2002), S. 84

315 Die ursprüngliche Version dieses Instruments, die PHNS, umfasst 84 Items und die folgenden Subskalen: Vertrauenswürdigkeit, Altruismus, Unabhängigkeit, Willensstärke, Komplexität des menschlichen Wesens und Veränderlichkeit des menschlichen Wesens (Wrightsman 1992, S. 305). Die revidierte Version der PHNS, die RPHNS, setzt sich hingegen aus 20 Items mit den zwei Subskalen Vertrauen und Zynismus zusammen (Wrightsman 1991, S. 392 f.).

316 Wrightsman (1991), S. 385

317 Vgl. Costa (2000), S. 101

318 Nach Costa et al. (2001, S. 228) manifestiert sich Vertrauen, ,in the behaviours towards others, is based on the expectations made upon behaviours of these others, and on the perceived motives and intentions in situations entailing risk for the relationship."
} 
Nachfolgende Tabelle gibt einen Überblick über das von Costa entwickelte und validierte, sieben Items umfassende reliable Instrument ${ }^{319}$ zur Messung der Vertrauensbereitschaft.

- Die meisten Menschen helfen jemandem in Not ohne zu zögern.

- Die meisten Menschen folgen dem Motto,Verhalte dich anderen gegenüber so, wie du willst, dass sie sich dir gegenüber verhalten".

- Die meisten Menschen sagen was sie denken.

- Die meisten Menschen sagen im Grunde die Wahrheit; sogar dann, wenn sie wissen, dass sie besser dran wären, wenn sie lügen würden.

- Menschen sind normalerweise aufrichtig um die Probleme anderer besorgt.

- Die meisten Menschen stehen zu ihrer Überzeugung.

- Menschen bleiben normalerweise bei ihrer Meinung, wenn sie denken, dass sie Recht haben, auch wenn die anderen nicht ihrer Meinung sind.

Tabelle 4: Items zur Erfassung generalisierten Vertrauens (RPHNS Costa)

Quelle: In Anlehnung an Costa (2000, S. 182)

\section{Der Ansatz von Gillespie}

Gillespie versteht Vertrauen als "willingness of a party to be vulnerable to the actions of another party, as evidenced by the trustor's willingness to engage in behavior which would objectively open them to vulnerability if the trust was violated." ${ }^{320}$ Demnach manifestiert sich Vertrauen in interpersonalen Relationen in der Bereitschaft zu vertrauendem Verhalten. ${ }^{321}$

Basierend auf diesem Verständnis zielt der Ansatz von Gillespie auf die Entwicklung eines Instruments zur Erfassung spezifischen Vertrauens, und damit auf die Messung der Bereitschaft eines Individuums, sich in einer Beziehung mit einer spezifischen Person über verschiedene Formen vertrauenden Verhaltens verwundbar zu machen, ab. ${ }^{322}$ Die Relevanz dieses Untersuchungsgegenstands ergibt sich aus der Annahme, dass interpersonales Vertrauen in Arbeitsbeziehungen positiv mit einer Vielzahl produktivitätsbezogener Verhalten und Ergebnisse ${ }^{323}$ korreliert. Im Rahmen des Prozesses der Entwicklung eines validierten Instruments zur Erfassung des spezifischen Vertrauens identifiziert Gillespie auf Basis von Literaturrecherche und Experteninterviews zwei Indikatoren vertrauenden Verhaltens:

- Reliance: Vertrauen zeigt sich darin, dass sich der Vertrauensgeber auf die wahrgenommenen Fertigkeiten, das Wissen, die Ansichten oder Handlungen,

319 Vgl. Costa (2003), S. 611; zur Reliabilität der Skala vgl. Späth (2008)

$320 \quad$ Gillespie (2003), S. 4

321 Vgl. Gillespie (2003), S. 7

322 Vgl. Gillespie (2003), S. 8

323 Nach Gillespie (2003, S. 2) hat interpersonales Vertrauen einen positiven Einfluss auf „organizational citizenship behaviors, individual job performance, and group level performance, as well as work-place attitudes and cognitions, such as job satisfaction, commitment and acceptance of decisions and goals.“ Ähnlich dazu äußern sich auch Dirks/Ferrin (2001). 
einschließlich Delegationen und Autonomiezuerkennung, des Vertrauensnehmers verlässt.

- Disclosure: Vertrauen zeigt sich darin, dass der Vertrauensgeber dem Vertrauensnehmer arbeitsbezogene oder persönliche Informationen sensibler Art offenlegt, die zu seinem Nachteil genutzt werden können. ${ }^{324}$

Zur Erfassung dieser Vertrauensindikatoren entwickelte Gillespie 10 Items, deren modelltheoretische Zuordnung zu den zwei Beurteilungsdimensionen durch eine konfirmatorische Faktorenanalyse bestätigt wird. Aufgrund ihrer Relevanz für den vorliegenden Untersuchungskontext gibt nachfolgende Tabelle einen Überblick über das valide und reliable multidimensionale Instrument (,The Behavioral Trust Inventory - BTI') zur Erfassung spezifischen Vertrauens. ${ }^{325}$

\begin{tabular}{|c|c|c|c|}
\hline \multicolumn{3}{|c|}{ Indikator } & \multirow[b]{2}{*}{$\begin{array}{l}\text { Item } \\
\text { - Ich bin gewillt, mich auf die aufgabenbezogenen Fertigkeiten und } \\
\text { Fahigkeiten des Kooperationspartners zu verlassen. } \\
\text { - Ich bin gewillt, von dem Kooperationspartner abhängig zu sein, wenn er } \\
\text { eine wichtige Kooperationsaufgabe für mein Unternehmen wahmimmt. } \\
\text { - Ich bin gewillt, mich darauf zu verlassen, dass der Kooperationspartner } \\
\text { mein Unternehmen anderen akkurat darstellt. } \\
\text { - Ich bin gewillt, von dem Kooperationspartner abhăngig zu sein, wenn er } \\
\text { mich in einer schwierigen Situation unterstützt. } \\
\text { - Ich bin gewillt, mich auf die kooperationsbezogenen Urteile des Koopera- } \\
\text { tionspartners zu verlassen. }\end{array}$} \\
\hline & 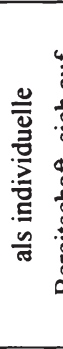 & & \\
\hline $\begin{array}{l}\text { 늘 } \\
\frac{0}{0} \\
\frac{0}{0}\end{array}$ & 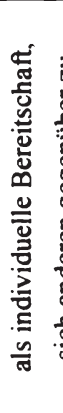 & 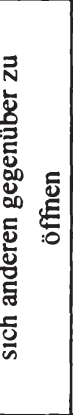 & $\begin{array}{l}\text { - Ich bin gewillt, meine persönlichen Gefühle mit dem Kooperationspartner } \\
\text { zu teilen. } \\
\text { - Ich bin gewillt, unternehmensbezogene Probleme oder Schwierigkeiten mit } \\
\text { dem Kooperationspartner zu diskutieren, die potentiell dazu benutzt } \\
\text { werden könnten, meinem Unternehmen zu schaden. } \\
\text { - Ich bin gewillt, dem Kooperationspartner persönliche Informationen } \\
\text { anzuvertrauen, die mein Unternehmen betreffen. } \\
\text { - Ich bin gewillt, meine ehrlichen Gefuhle, auch negative Emotionen und } \\
\text { Frustration, in Bezug auf mein Unternehmen mit dem Kooperationspartner } \\
\text { zu diskutieren. } \\
\text { - Ich bin gewillt, meine persönlichen Überzeugungen mit dem Koopera- } \\
\text { tionspartner zu teilen. }\end{array}$ \\
\hline
\end{tabular}

Tabelle 5: Items zur Erfassung spezifischen Vertrauens (BTI)

Quelle: In Anlehnung an Gillespie (2003, S. 41)

324 Vgl. Gillespie (2003), S. 10. Der Zusammenhang zwischen den von Gillespie identifizierten Indikatoren besteht darin, dass ,people are typically more willing to disclose to others whom they feel they can also rely, and vice versa." (Gillespie 2003, S. 35).

325 Vgl. Gillespie (2003), S. 13 ff.; Das Instrument wurde an den vorliegenden Untersuchungskontext angepasst, was nach den Ergebnissen der Cross-Validation-Study von Gillespie (2003, S. 38) zulässig ist. Zur Reliabilităt der Skala vgl. auch Spăth (2008). 
Die Eignung des BTIs für den vorliegenden Untersuchungskontext zeigt sich darin, dass sich die ihm zugrunde liegende Vertrauensdefinition mit dem Vertrauensverständnis dieser Arbeit deckt: So impliziert einerseits die von Gillespie identifizierte Vertrauensdimension ,Reliance' einen Verzicht auf explizite vertragliche Sicherungs- und Kontrollinstrumente, indem die Entscheidung getroffen wird, sich auf den Vertrauensnehmer zu verlassen. Andererseits impliziert Gillespies Dimension ,Disclosure' die freiwillige Erbringung einer riskanten Vorleistung, indem der Vertrauensgeber mit dem Vertrauensnehmer Informationen teilt, die zu seinem Nachteil genutzt werden können. Für eine Berücksichtigung des BTIs im Rahmen der in Teil IV dieser Arbeit verfolgten Operationalisierung des Vertrauenskonstrukts spricht auch, dass die von Gillespie identifizierten Indikatoren durch qualitative und quantitative Daten aus Querschnittuntersuchungen und Längsschnitterhebungen bestätigt wurden. ${ }^{326}$

Nachdem für diese Arbeit relevante Instrumente zur Erfassung des Vertrauenskonstrukts erläutert wurden, wird abschließend auf problematische Vorgehensweisen bei der Erhebung von Vertrauen eingegangen, die sich im Zuge der diesem Kapitel vorangegangen Literaturrecherche gezeigt haben:

- Der alleinige Schluss aus Beobachtungen von Handlungen und Entscheidungen, die auf Vertrauen beruhen, jedoch auch durch andere Faktoren bedingt sein können, auf Vertrauen.

- Die mangelnde Differenzierung zwischen generalisiertem Vertrauen und spezifischem Vertrauen. So erfassen die Skalen von Rotter (ITS) und Wrightsman (RPHNS) nicht das Vertrauen in einen spezifischen Kooperationspartner, sondern generalisiertes Vertrauen im Sinne der generellen Vertrauensbereitschaft eines Individuums.

- Der Schluss aus der Wahrnehmung der Vertrauenswürdigkeit eines Individuums auf das Vertrauen in einer spezifischen Situation. Bildet sich der Vertrauensgeber ein Urteil über seinen potentiellen Kooperationspartner, impliziert das weder ein Risiko, noch den Verzicht auf Kontroll- und Sicherungsmaßnahmen zur Begrenzung von Opportunismusspielräumen. Dies sind jedoch zentrale Elemente des Vertrauenskonstrukts.

- Die Verwendung von 1-Item-Skalen zur expliziten Erfassung von Vertrauen. Ihre Bedeutung liegt im Einsatz zur Konstruktvalidierung. ${ }^{327}$

326 Gillespie (2003, S. 9 ff.) führte eine Interview-Studie mit 96 Probanden, eine Pilot-Studie mit 39 Teilnehmern, eine Studie zur Validierung mit 77 Projektleitern und 234 Teammitgliedern aus dem Bereich F\&E, sowie eine Vergleichsprüfung mit 67 Managern und 214 direkt unterstellten Mitarbeitern durch.

327 Ähnlich hierzu äußern sich auch Gillespie (2003), S. 5 f.; Schweer/Thies (2003), S. 38 f.; Späth (2008), S. $132 \mathrm{f}$. 
Nach Gillespie gründen sich diese problematischen Vorgehensweisen bei der Erfassung des Vertrauenskonstrukts in der fehlenden Kongruenz zwischen den Bemühungen zur Definition und jenen zur Operationalisierung von Vertrauen: „In summary, the organizational trust literature is characterized by a mismatch between the defining features of trust and its measurement. A contributing factor to this problem is a lack of well-validated trust measures that are congruent with its defining features. “328 Um der Forderung nach einer engen Übereinstimmung von Definition und Messinstrument ${ }^{329}$ nachkommen und damit den genannten Defiziten begegnen zu können, wird in Kapitel 4 des Teils IV auf Basis der dieser Arbeit zugrunde liegenden Vertrauensdefinition festgelegt, welche Operationen im Sinne von Handlungen, Reaktionen, Zuständen unter Bezugnahme auf den vorliegenden Untersuchungskontext als indikativ für das Vertrauenskonstrukt anzusehen sind. Die Absicherung dieser Vertrauensindikatoren erfolgt anhand der validierten Ergebnisse dieses Kapitels, sowie durch die Ergebnisse einer weiterführenden Literaturrecherche, da eine ausführliche Bedeutungsanalyse des im Fokus stehenden Konstrukts die Voraussetzung für eine erfolgreiche Operationalisierung darstellt. ${ }^{330}$ Auf Basis dieser Operationalisierung wird sodann in Kapitel 5 des Teils IV ein Messmodell generiert, um den Einfluss von Kompetenz auf die Entstehung von Vertrauen zu hypothetisieren. Die besondere Herausforderung hypothesentestender Forschung liegt in der Übersetzung des latenten Vertrauenskonstrukts in quantitative Daten. Besondere Sorgfalt bei der Auswahl der Messinstrumente ist daher unerlässlich, da diese die Aussagekraft der gesamten Untersuchung determinieren. ${ }^{331}$ Aufgrund des Vertrauensverständnisses und des Ziels dieser Arbeit, die Wirkung von Kompetenz als Determinante der Entstehung von Vertrauen zu analysieren, ist eine Befragung der Probanden nicht ausreichend. Vielmehr soll ein Experiment zum Einsatz kommen, um durch die Beobachtung der Verhaltensweisen der Probanden, einen möglichen Kausalzusammenhang zwischen Kompetenz und Vertrauen aufdecken zu können. Beispiele dieser Art impliziter Verfahren finden sich in der Vertrauensforschung vor allem bei den Untersuchungen zum Gefangenen-Dilemma, im Rahmen derer die Entscheidung für kooperative Verhaltensweisen als Indikator für Vertrauen fungiert. Der Grund für diese Annahme liegt darin, dass die Wahl der kooperativen Strategie mit dem Risiko verbunden ist, dass sich der Gegenspieler A nicht kooperativ verhält, weshalb die freiwillige Entscheidung für eine kooperative Verhaltensweise seitens des Spielers B die Erbringung einer riskanten Vorleistung, durch die er sich verwundbar macht, impliziert. Mit dieser Annahme wird an die in dieser Arbeit formulierten theoretischen Überlegungen zum Vertrauenskonstrukt angeknüpft, wo

$328 \quad$ Gillespie (2003), S. 7

329 Vgl. Lewicki et al. (2006), S. 1014

$330 \quad$ Vgl. Opp (1999b), S. 58; Spăth (2008), S. 132

331 Vgl. Späth (2008), S. 112 
dargelegt wurde, dass die Vertrauenshandlung mit der Wahrnehmung von Risiko verbunden ist. Den Untersuchungen zum Gefangen-Dilemma folgend, kann davon ausgegangen werden, dass das Vertrauen des Spielers B umso höher ist, je höher die im Experiment eingesetzte Summe ist, die er durch die Kooperation mit dem Partner A aufs Spiel setzt. Daneben zeigt sich das Vertrauen des Spielers B in den Partner A auch darin, inwieweit er durch den Einsatz von Kontroll- und Sicherungsmaßnahmen bestrebt ist, seine subjektive Unsicherheit zu reduzieren. Den Untersuchungen zum Gefangenen-Dilemma folgend, kann davon ausgegangen werden, dass das Vertrauen umso geringer ist, je höher der Einsatz von Kontroll- und Sicherungsinstrumenten (z.B. schriftliche fixierte Vereinbarungen) zur Begrenzung der Opportunismusspielräume des Partners A ist. ${ }^{332}$ Um einen Schluss auf die Kompetenz-Vertrauen-Beziehung ziehen zu können, ist die Anwendung eines Experimentaldesigns geeignet, da es erlaubt, die Kooperationsbeziehung zwischen Spieler B und A als Vertrauensbeziehung zwischen Vertrauensgeber und Vertrauensnehmer zu beobachten, und zwar unter den konstitutiven Situationsbedingungen der subjektiven Unsicherheit und des Risikos der Vertrauensenttäuschung - nämlich, indem eine Beobachtung des Vertrauensgebers bei der Entscheidung für Verhaltensoptionen erfolgt, die eine Vertrauensentscheidung reflektieren. Um diesen vermuteten Kausalzusammenhang zwischen Kompetenz und Vertrauen hinreichend zu erforschen und abzusichern, ist es allerdings von hoher Bedeutung, vorab die generelle Vertrauensbereitschaft und die personenspezifische Risikobereitschaft, sowie im Zuge des Experiments die situationsspezifische Risikowahrnehmung der Probanden in ihrer Rolle als Vertrauensgeber in Form von Befragungen abzutesten. ${ }^{333}$ Demnach liegt die Relevanz der in diesem Kapitel erfolgten Analyse der validierten Messinstrumente, neben ihrer Bedeutung für die Ableitung von Indikatoren in Teil IV Kap. 4, die als empirische Äquivalente der Merkmalsdimensionen des Vertrauenskonstrukts fungieren, in ihrem Ergebnis der Anwendbarkeit der Skalen im Rahmen des empirischen Teils dieser Arbeit.

332 Vgl. dazu auch Plötner (1995), S. $170 \mathrm{f}$.

333 Glaeser et al. (2000, S. 812) schreiben hierzu: „[...] experiments can be integrated with surveys to measure individual-level variation in traditionally hard-to-measure characteristics such as trust and trustworthiness. “ Der Grund hierfür liegt darin, dass „Experiments measure preferences, behavioral propensities, and other individual attributes much more convincingly than surveys, since experiments provide direct observations of behavior. By connecting experiments and surveys, we can determine the socioeconomic correlates of hard-to-measure individual attributes, and test the validity of survey measures of these attributes." (Glaeser et al. 2000, S. 841). 
TEIL III: KOMPETENZ ALS ZENTRALES ELEMENT IN ZWISCHENBETRIEBLICHEN KOOPERATIONEN

\section{DER KOMPETENZBEGRIFF}

\subsection{Begriffliche Festlegung von Kompetenz}

Der Kompetenzbegriff wird, wie auch der Vertrauensbegriff, auf vielfältige Art und in verschiedenen Zusammenhängen verwendet. Im allgemeinen Sprachgebrauch wird Kompetenz mit Sachverstand, Zuständigkeit und Fähigkeit gleichgesetzt. Sie kann sich auf verschiedene Aufgabenstellungen, wie beispielsweise auf die Kommunikationskompetenz, und Bereiche, wie zum Beispiel auf den zwischenmenschlichen Bereich (Sozialkompetenz) oder bestimmte Wissens- und Arbeitsgebiete (Fachkompetenz), beziehen. Neben dieser allgemeinsprachlichen Definition finden sich in den Bereichen der Wirtschafts-, Sprach- und Rechtswissenschaften höchst unterschiedliche Begriffserklärungen ${ }^{334}$, was die Erforschung von Kompetenz zusätzlich erschwert. Bruch verweist deutlich auf die Forschungslücke: „Besondere Schwierigkeiten gibt es bisher unabhängig von der jeweiligen Kompetenzebene bei der Identifizierung von Kompetenzen. Sowohl bezüglich der Konstrukte selbst - ihre Definition, Operationalisierung und Skalierung - als auch bezogen auf Verfahren zur Diagnose und Evaluation von Wissen und Kompetenzen sind noch erhebliche Anstrengungen in Theorie und Praxis erforderlich. ${ }^{\text {3335 }}$

Im organisationalen Kontext kann Kompetenz als rational bestimmte und abgegrenzte Zuständigkeit verstanden werden, die sich anhand folgender Merkmale charakterisiert:

- Ein bedingt durch Leistungsverteilung sachlich abgegrenzter Bereich von Leistungspflichten.

- Die Zuordnung der dafür notwendigen Befehlsgewalten, sowie

- die klare Abgrenzung der eventuell zulässigen Zwangsmittel und ihrer Anwendungsvoraussetzungen. ${ }^{336}$

Da diese Arbeit jedoch den Aspekt der Fähigkeit und nicht jenen der Berechtigung fokussiert, wird im Folgenden auf einige, für diese Arbeit relevante Merkmale der Kompetenz eingegangen. Grundsätzlich stellt Kompetenz ein mehrdimensionales Konstrukt dar, das schwer imitierbar, transferierbar und handelbar

335 Bruch (1999), S. 136

336 Vgl. Weber(1972), S. 125 
ist, und kann als komplexes, auf Lernprozessen basierendes Interaktionsmuster verstanden werden. ${ }^{337}$

Als dynamisches Konzept lässt sich Kompetenz anhand der Dimensionen ,Aufgabe' und ,Fähigkeit' eingrenzen. Kompetenzen „,do not exist in the way a car does; they exist only when the knowledge (and the skill) meets the task." ${ }^{338}$ Eine weitere bedeutende Komponente des Kompetenzkonstrukts, neben jener der Fähigkeit und der der Aufgabe, ist daher die Aufgabenerfüllung. So kann Kompetenz als Vermögen, die Lücke zwischen Aufgabe und deren Erfüllung zu schließen, verstanden werden: „To be capable of some thing is to have a generally reliable capacity to bring that thing about as a result of intended action. Capabilities fill the gap between intention and outcome, and they fill it in such a way that the outcome bears a definite resemblance to what was intended. ${ }^{\prime 339}$ Auch für Sanchez et al. besteht ein Zusammenhang zwischen Kompetenz und Aufgabe, Fähigkeit, sowie der erfolgreichen Erfüllung einer geplanten Aufgabe: „Since competence implies an intention to achieve some desired result (goal) through action, and since action-taking requires some notions of cause and effect, knowledge and the application of knowledge through action are at the foundation of the concepts of skills, capabilities and (ultimately) competence““. ${ }^{340}$ Zusammenfassend lässt sich daher eine dreidimensionale Struktur des Kompetenzkonstrukts beobachten, die sich aus Aufgabe, Fähigkeiten und Aufgabenerfüllung zusammensetzt. Kompetenz kann demnach als Übereinstimmung der Anforderungen der Aufgabe an den Aufgabenerfüller mit den Fähigkeiten des Aufgabenerfüllers definiert werden. Damit lässt sich das Kompetenzausmaß am Grad der Aufgabenerfüllung erkennen.

\subsection{Abgrenzung des Kompetenzbegriffs}

Ein eindeutiges Verständnis des Kompetenzkonstrukts verlangt nach einer begrifflichen Abgrenzung von verwandten Konstrukten. Im folgenden Kapitel erfolgt daher eine Analyse der kompetenznahen Begriffe Qualifikationen, Ressourcen, Fertigkeiten und Fähigkeiten, um die Verrasterung der Kompetenzarten auf organisationaler und individueller Ebene, sowie deren Abgrenzung voneinander (Kap.

2) zu ermöglichen.

337 Vgl. Rasche (1994), S. 141 ff.; Doz (1996), S. 156 f.; Heines (1998), S. 61; Fearns (2004), S. 45

338 Krogh/Roos (1996), S. 425. Die Verbindung von Fähigkeit und Aufgabe zur Spezifikation von Kompetenz leiten die Autoren aus dem ursprünglichen Kompetenzverständnis ab: „The term competence is derived from "competentia" (lat.) which literally means "agreement". Only where there exists an agreement or fit between "knowledge" (or subject) and "task", we speak of competence" (Krogh/Roos 1992, S. 11).

339 Dosi et al. (2000), S. 2

340 Sanchez (1996), S. 9 


\subsubsection{Abgrenzung gegen, Qualifikationen“}

Unter Qualifikation ist das individuelle Arbeitsvermögen zu verstehen, d.h. die Gesamtheit der subjektiv-individuellen Fertigkeiten, Kenntnisse und Verhaltensmuster, die es einer Person ermöglichen, die Anforderungen in bestimmten Arbeitsfunktionen auf Dauer zu erfüllen. ${ }^{341}$ Der Qualifikationsbegriff umfasst jedoch nicht nur den Aspekt der Befähigung, und dementsprechend jenen der Eignung, sondern auch den Berechtigungsaspekt. ${ }^{342}$

Kompetenz lässt sich von Qualifikation dahingehend abgrenzen, dass sie den Aspekt der Befähigung stärker fokussiert als jenen der Berechtigung einer Person. ${ }^{343}$ Weiters unterscheiden sich Kompetenzen von Qualifikationen dadurch, dass sie sowohl auf individueller, als auch auf organisationaler Ebene vorliegen können. Während individuelle Kompetenzen, die sich anhand der Dimensionen Wissen, Erfahrung, Einstellung und Persönlichkeitsmerkmale charakterisieren las$\operatorname{sen}^{344}$, rein personengebunden sind und dementsprechend dem Betrieb mit dem Ausscheiden eines kompetenten Mitarbeiters verloren gehen, verbleiben organisationale Kompetenzen aufgrund ihrer fehlenden Personenbindung tendenziell im Unternehmen und können nicht auf einzelne Personen zurückgeführt werden. Unter Zuhilfenahme des Kriteriums der Personenbindung wird nun auch der Unterschied zwischen Kompetenzen und Qualifikationen deutlich: Qualifikationen beschreiben rein individuelle Fertigkeiten - eine Verankerung auf organisationaler Ebene ist nicht gegeben. ${ }^{345}$ Auf individueller Ebene lassen sie sich von Kompetenzen insofern abgrenzen, als dass sie Fertigkeiten bezeichnen, die notwendig sind, um spezifische Aufgaben zu erfüllen, während sich individuelle Kompetenzen auf eher unbestimmte Aufgaben beziehen, die selbstorganisierte Handlungen erfordern. ${ }^{346}$ Kompetenz ist demnach als breiteres Konzept zu interpretieren, welches das Qualifikationskonstrukt einschließt.

\subsubsection{Abgrenzung gegen, Ressourcen'}

Ressourcen können als Stärken und Schwächen eines Unternehmens verstanden werden, die zu einem gewissen Grad langfristig mit dem Unternehmen verbunden sind. Generell kann zwischen tangiblen und intangiblen Ressourcen differenziert werden. Während beispielsweise Wissen, Personal und Verträge zu den intangiblen Ressourcen zählen, handelt es sich bei Finanzierungsmitteln und Maschinen um tangible Ressourcen. ${ }^{347}$ Entsprechend ihrer Form kann auch zwischen

\footnotetext{
341 Vgl. Gabler-Wirtschaftslexikon (1997)

342 Vgl. Brockhaus (2009b)

343 Vgl. Becker/Rother (1998), S. 1

344 Vgl. Krogh/Roos (1995), S. 66

345 Vgl. Strasmann/Schüler (1996), S. 12; Bouncken (2003), S. 62

$346 \mathrm{Vgl}$. Erpenbeck/Rosenstiel (2007)

347 Vgl. Wernerfelt (1984), S. 172
} 
physischen, humankapitalbezogenen (z.B. Wissen, Fertigkeiten, Erfahrungen, Beziehungen und Einstellungen der Organisationsmitglieder ${ }^{348}$ ) und organisationalen Ressourcen (z.B. Informationssysteme, Planungs- und Kontrollsysteme, Organisationsstruktur, formelle Beziehungen) unterschieden werden. ${ }^{349}$ Auch die Differenzierung zwischen „facilities and equipement", „personal skills“, „organizational capabilities" und „management capabilites" lässt darauf schließen, dass unter den Ressourcenbegriff Fertigkeiten (skills) und Fähigkeiten (capabilities) subsumiert werden können. ${ }^{350}$ Auch anhand ihres Potentials langfristige Wettbewerbsvorteile generieren zu können, lassen sich Ressourcen klassifizieren: Bei Ressourcen erster Klasse handelt es sich um Managementaktivitäten, die grundlegende Geschäftsprozesse unterstützen. Zur zweiten Klasse zählen Ressourcen, die der Integration verschiedener Geschäftsbereiche dienen (z.B. administrative Fähigkeiten wie die Infrastruktur eines Betriebs, Managementfähigkeiten und systeme). Ressourcen dritter Klasse, wie beispielsweise die Lernfähigkeit der Organisation, erlauben eine bessere Ressourcennutzung und fördern Innovationen. Die Ressourcen vierter Klasse verbessern die Entwicklung und Durchsetzung der Unternehmungsvision. ${ }^{351}$

Kompetenzen lassen sich von Ressourcen nun dahingehend abgrenzen, dass bei ersteren das intangible und wissensorientierte Element dominiert, während der Ressourcenbegriff häufig mit Assoziationen verbunden ist, die tangible Ressourcen (z.B. Maschinen) betreffen. ${ }^{352}$ In diesem Zusammenhang können Kompetenzen als Kombination unternehmensspezifischer Faktoren verstanden werden, die es ermöglichen, gegebene Ziele zu erreichen. Sie stellen die Fähigkeit einer Organisation, Ressourcen zu integrieren, dar. ${ }^{353}$ Diese Integrationsleistung bezieht sich unter anderem auf die Koordination von Personen und deren Fertigkeiten. ${ }^{354}$

Charakteristisch für Kompetenzen ist somit die Bündelung verschiedener Ressourcen, Fertigkeiten und Fähigkeiten, sowie ihr Potentialcharakter: „Competence refers to an ability to sustain the coordinated deployment of assets (both tangible and intangible) in ways that help a firm achieve its goals" ${ }^{355}$ Mit ihrem Potentialcharakter bzw. ihrer Komponente der Aufgabenerfüllung ist auch die dynamische Wirkung von Kompetenzen verbunden: Sie charakterisieren sich in stärkerem Ausmaß durch dynamische Potentiale, die zukünftige Optionen ermöglichen, denn durch Bestände. ${ }^{356}$

\footnotetext{
348 Vgl. Grant (1995), S. $91 \mathrm{ff}$.

349 Vgl. Barney (1991), S. 101

350 Vgl. Ansoff (1965), S. $85 \mathrm{ff}$.

351 Vgl. Brumagim (1994), S. 85; Bouncken (2003), S. 57

352 Vgl. Hall (1993), S. 611

353 Vgl. Amit/Schoemaker (1993), S. 35; McGrath et al. (1995), S. 254

354 Vgl. Grant (1991), S. 122; Bouncken (2003), S. 62

355 Sanchez/Thomas (1996), S. 64

356 Vgl. Bouncken (2003), S. 63
} 


\subsubsection{Abgrenzung gegen, Fertigkeiten'}

Fertigkeiten (Skills) werden als angelegte, nicht aggregierte Eigenschaften verstanden, die stark mit ihrer Anwendung in einem spezifischen Sachverhalt verknüpft $\operatorname{sind}^{357}$, wodurch sie sich von Kompetenzen abgrenzen. ${ }^{358}$ Ein weiteres Kennzeichen ist, dass sie sowohl dem kognitiven, als auch dem psychomotorischen Bereich unterliegen. Mit steigender Fertigkeit und damit ihrer Automatisierung wird die Ausführung von Tätigkeiten immer weniger bewusst. ${ }^{359}$

Allerdings wird auch die Auffassung vertreten, Skills nicht bloß in Abhängigkeit von Personen zu verstehen. So interpretieren Edge et al. Skills als systematische Organisationseigenschaften, die aus menschlichen und organisatorischen Faktoren bestehen. ${ }^{360}$ Im Rahmen dieser Arbeit werden Fertigkeiten hingegen als individuell personengebunden verstanden, um eine Abgrenzung gegen Fähigkeiten und Kompetenzen zu gewährleisten. Die fehlende organisationale Betrachtung ermöglicht vor allem eine schärfere Abgrenzung des Begriffs gegenüber Fähigkeiten.

Zusammenfassend lässt sich sagen, dass Fertigkeiten auf die spezifische Erfüllung bestimmter Aufgaben bzw. auf das Erreichen bestimmter Ergebnisse ausgerichtet sind, wodurch sie sich besonders von den ebenfalls personengebundenen Individualkompetenzen abgrenzen. Neben dieser ergebnisorientierten Ausrichtung, bieten Fertigkeiten zugleich auch das Potential, eine bestimmte Aufgabe zu erfüllen. $^{361}$ Eine Fertigkeit impliziert, etwas besonders gut zu können, da eine Fertigkeit oft erst dann als solche begriffen wird, wenn sie über ein bestimmtes Anspruchs- bzw. Zufriedenheitsniveau hinausgeht.

\subsubsection{Abgrenzung gegen, Fähigkeiten`}

Die Abgrenzung von Kompetenz gegen Fähigkeit gestaltet sich als schwierig, da die Termini eine enge Bindung aufweisen und vielfach als synonym angesehen werden. ${ }^{362}$ In der angloamerikanischen Literatur beispielsweise wird der Begriff ,capability" oft gleichbedeutend mit ,competence' verwendet.

Fähigkeiten können sowohl auf organisationaler, als auch auf individueller Ebene vorliegen. Auf organisationaler Ebene stellen Fähigkeiten Bündel betrieblicher

$357 \quad$ Vgl. Edge et al. (1995), S. 191

358 Vgl. Rasche (1994), S. 93 f.; Erpenbeck/Rosenstiel (2007)

359 Vgl. Edelmann (1993), S. 229

360 Vgl. Edge et al. (1995), S. $188 \mathrm{ff}$.

361 Vgl. Nelson/Winter (1982), S. 73 ff.; Edge et al. (1995), S. 191

362 Zum Beispiel zeigt sich bei Stahl (2000, S. 414 f.) im Rahmen der Differenzierung zwischen individualen Kompetenzarten eine gleichbedeutende Verwendung der Begriffe ,Kompetenz' und ,Fähigkeit 
Faktoren dar, die der Koordination von Ressourcen im Unternehmen dienen. ${ }^{363}$ Dazu zählen beispielsweise: „External integration, leading to quality; Internal integration, leading to speed and efficiency; Flexibility, leading to responsiveness and variety; The capacity to experiment, leading to continuous improvement; The capacity to cannibalize, leading to radical innovation. ${ }^{6364}$ Mit ihrem koordinativen Potential bilden sie die Grundlage für Wettbewerbsvorteile und lassen sich von Ressourcen folgendermaßen abgrenzen: „While resources are the source of a firm's capabilities, capabilities are the main source of its competitive advantage“ ${ }^{365}$ Auf individueller Ebene können Fähigkeiten als „capability for a smooth sequence of coordinated behavior “366 definiert werden. Indem sich ihre Anwendung als Verhaltensstereotyp vollzieht, ist eine gezielte Bewusstseinssteuerung nur in Ausnahmefällen möglich. Dementsprechend beruhen individuelle Fähigkeiten auf implizitem Wissen und kennzeichnen sich durch sinkende Transparenz und Kodifizierbarkeit bei zunehmender Komplexität. Neben dem Merkmal der unterbewussten Steuerung, charakterisiert sich der Einsatz individueller Fähigkeiten durch die Wahl zwischen verschiedenen Entscheidungsalternativen, welche „routiniert und dezentral getroffen werden.“367

Fähigkeiten lassen sich von Kompetenzen nun dahingehend abgrenzen, als dass sie als Vorstufe zu letzteren begriffen werden können: „[...] knowledge and the application of knowledge through action are the foundation oft the concept of skills, capabilities and (ultimately) competence.“6368 Sie können als „complex patterns of coordination between people and between people and other resources" verstanden und mittels "learning through repetition" ${ }^{\text {“369 }}$ perfektioniert werden. Anhand dieser Charakterisierung wird eine Verbindung zwischen Capabilities, Lernen und Erfahrung ersichtlich. Das Lernen durch Wiederholungen impliziert eine Nahebeziehung der Fähigkeiten zu Routinen.

Fähigkeiten können somit als wiederholbare, wissensbasierte Handlungsmuster verstanden werden, die eine Ausübung von Handlungen gestatten. Aufgrunddessen, dass sie über eine relativ hohe Spezifität zur Erreichung eines bestimmten Ergebnisses verfügen, kann ihnen eine gewisse Zielorientierung unterstellt werden. Bezugnehmend auf die Ziel- bzw. Aufgabenorientierung sind sie im Vergleich zu Kompetenzen spezifischer und enger zu interpretieren. ${ }^{370}$ Kompetenzen

\footnotetext{
363 Vgl. Amit/Schoemaker (1993); Fearns (2004), S. 90

364 Baldwin/Clark (1994), S. 89

365 Grant (1991), S. 119

366 Nelson/Winter (1982), S. 73

367 Rasche (1994), S. 97

368 Sanchez (1996), S. 9

$369 \quad$ Vgl. Grant (1991), S. 122

370 Vgl. Bouncken (2003), S. 61; Morgeson et al. (2004)
} 
weisen demgegenüber einen allgemeineren Potentialcharakter und damit eine stärkere Bedeutung auf.

Diese Arbeit differenziert zwischen den Termini Fähigkeiten und Kompetenzen, wobei unter Kompetenz die Übereinstimmung von Aufgabe und Fähigkeiten, welche zur Schließung der Lücke zwischen Aufgabe und deren Erfüllung eingesetzt werden, verstanden wird.

\section{DIE KOMPETENZARTEN}

Nachdem Kompetenz definiert und von verwandten Konstrukten abgegrenzt wurde, kann im Folgenden zwischen individuellen und organisationalen Kompetenzen differenziert werden. An der Entstehung organisationaler Kompetenzen sind die individuellen Kompetenzen maßgeblich beteiligt. ${ }^{371}$ Doz schreibt hierzu: „[...] most organizational competences start with the individual."

\subsection{Organisationale Kompetenzen}

Unter organisationalen Kompetenzen ist die aufgabenbezogene Bündelung organisationaler Fähigkeiten, die in Bezug auf ihren Beitrag zur Erfüllung von Aufgaben bewertet werden, zu verstehen. Hierunter fallen sowohl Basis- als auch Kernkompetenzen, deren Unterschied in ihrer qualitativen Ausprägung und strategischen Relevanz liegt. ${ }^{373}$

\subsubsection{Basiskompetenzen}

Basiskompetenzen können als „necessary competences“, über die das Unternehmen verfügen muss, um am Markt agieren zu können, verstanden werden. Sie setzen sich aus organisationalen Routinen zusammen und stellen die Wettbewerbsfähigkeit eines Unternehmens sicher, indem sie auf zufriedenstellendem Niveau die Erfüllung einer Vielzahl notwendiger betrieblicher Abläufe (z.B. Marketing, Rechnungslegung, Personalbeschaffung) gewährleisten. ${ }^{374}$ So können die von Lado et al. beschriebenen funktionalen Kompetenzen, die auf der Ebene einzelner Abteilungen ihren Beitrag zum Unternehmenserfolg leisten, zu den Basiskompetenzen gezählt werden. ${ }^{375}$ Auch die von Krüger und Homp beschriebenen Kompetenzen erster Ordnung, die dazu dienen, die branchendurchschnittliche Wettbewerbsfähigkeit eines Unternehmens sicherzustellen ${ }^{376}$, sowie

371 Vgl. Rasche (1994); Bruch (1999); Probst et al. (2000)

372 Doz (1996), S. 157

373 Vgl. Fearns (2004), S. $102 \mathrm{ff}$

374 Vgl. Fearns (2004), S. $103 \mathrm{f}$.

375 Vgl. Lado et al. (1992), S. $82 \mathrm{ff}$.

376 Vgl. Krüger/Homp (1997), S. 26 
die von Turner und Crawford beschriebenen Managementkompetenzen, die die Beherrschung der Planungs-, Organisations-, Führungs-, Kontroll- und Koordinationsprozesse ermöglichen ${ }^{377}$, sind als Basiskompetenzen zu verstehen.

\title{
2.1.2 Kernkompetenzen
}

Die Kernkompetenz als Sonderform unternehmensspezifischer Ressourcen hat ihren Ursprung innerhalb des Gedankenguts des Resource-Based-View ${ }^{378}$, der die unternehmenseigenen Ressourcen als Voraussetzung für die Einzigartigkeit bzw. die Wettbewerbsvorteile der Unternehmung erachtet. ${ }^{379}$ Ihr Begriff (,Core Competence') wurde von Prahalad und Hamel geprägt ${ }^{380}$, die darunter ein Bündel verschiedener Technologien und Fähigkeiten verstehen, das einen besonderen Kundennutzen generiert und den Zugang zu einer Vielzahl von Märkten eröffnet. ${ }^{381}$

Kernkompetenzen tragen maßgeblich zur Durchsetzung eines langfristigen Wettbewerbsvorteils bei: "The real sources of advantage are to be found in management's ability to consolidate corporatewide technologies and production skills into competencies that empower individual businesses to adapt quickly to changing opportunities [...] Core competencies are the collective learning in the organization, especially how to coordinate diverse production skills and integrate multiple streams of technologies. ${ }^{382}$ Um dauerhafte Wettbewerbsvorteile für ein Unternehmen zu generieren, müssen Kernkompetenzen verschiedene Merkmale wie Nicht-Imitierbarkeit ${ }^{383}$, Transferierbarkeit ${ }^{384}$ und Stiftung von Kundennutzen $^{385}$ aufweisen. Dementsprechend verstehen Krüger und Homp unter einer

377

378

379

380

381

382

383

384

\begin{abstract}
Vgl. Turner/Crawford (1994), S. 243
Vgl. Rasche (1994), S. $91 \mathrm{ff}$.

Vgl. Fearns (2004), S. 35

Vgl. Prahalad/Hamel (1990), S. $80 \mathrm{ff}$.

Vgl. Hamel/Prahalad (1995), S. 302

Prahalad/Hamel (1990), S. 81 f.

Um dauerhafte Wettbewerbsvorteile zu generieren, ist die Nicht-Imitierbarkeit der Kernkompetenz durch die Konkurrenz von zentraler Bedeutung: „To qualify as a core competence, a capability must also be competitively unique" (Hamel/Prahalad 1994, S. 205). Einzigartigkeit bedeutet nicht, dass eine Kernkompetenz nur in einem einzigen Unternehmen vorliegen darf, vielmehr muss der Grad ihrer Beherrschung im Vergleich zur Konkurrenz überdurchschnittlich hoch sein, um die dauerhafte Nicht-Imitierbarkeit der Kernkompetenz zu sichern.

Um den Unternehmenserfolg dauerhaft sicherzustellen, müssen Kernkompetenzen transferierbar sein, d.h. sie bedürfen eines hohen Abstraktionsgrades, um auf verschiedenen Absatzmärkten eingesetzt werden zu können: Wenn beispielsweise einzelne Produkte im Zeitablauf ihren Wert auf einem stagnierenden Absatzmarkt verlieren, kann durch den Transfer der Kernkompetenzen in andere Produkte, deren Markt sich in der Entstehungs- oder Wachstumsphase befindet, langfristig der Unternehmenserfolg gesichert werden (Prahalad/Hamel 1990; Grant 1991; S. 116 f.; Hamel/ Prahalad 1994; Krüger/Homp 1997, S. 95; Fearns 2004, S. 38).

Kernkompetenzen müssen einen erheblichen Beitrag zu den von Kunden wahrgenommen Vorzügen des Endprodukts leisten (Hamel 1994, S. 13). Erst mit dem Fokus des Kundennutzens wird sicher-
\end{abstract}


Kernkompetenz „die dauerhafte und transferierbare Ursache für den Wettbewerbsvorteil einer Unternehmung, die auf Ressourcen und Fähigkeiten basiert. ${ }^{\text {“386 }}$

Unter Zuhilfenahme von Porters Wertkettenkonzept und mittels Prozessmodellen können Kernkompetenzen kategorisiert werden. So differenziert Hamel zwischen

- marktzugangsbezogenen Kernkompetenzen (z.B. Marktentwicklung, Marketing- und Vertriebsfähigkeiten, Servicefähigkeiten, Händlermanagement) zur Forcierung von Kundennähe

- integritätsbezogenen Kernkompetenzen (z.B. Qualitäts-, Just-in-Time-Management, Simultaneous Engineering) zur Unterstützung eines schnelleren, flexibleren und verlässlicheren Operierens im Vergleich zu Konkurrenzunternehmen

- funktionalitätsbezogenen Kernkompetenzen (z.B. bedarfsgerechte Fertigung, individuelle Problemlösungen) ${ }^{387}$ zur Generierung von Produkten mit hohem Kundennutzen

Eine weitere Kategorie stellen die technologiebezogenen Kernkompetenzen dar, die Produkt- und Prozessinnovationen ermöglichen, sowie die Beherrschung von Produktions-, Logistik-, Service- und technologieorientierten Kooperationsprozessen gewährleisten. Aufgrund ihres hohen Komplexitäts- und Intransparenzgrades sind sie für Konkurrenten schwer zu imitieren. ${ }^{388} \mathrm{Zu}$ ihren Bestandteilen zählen physisch-technische Systeme, Managementsysteme, Fertigkeiten und Wissen, sowie bestimmten Ausbildungs- und Belohnungssysteme, womit sie auch personengebundene Facetten umfassen. Physisch-technische Systeme beinhalten eine Vielzahl unterschiedlicher Elemente wie beispielsweise Produktionsanlagen, EDV und Systeme, die das Wissen der Individuen speichern. Managementsysteme als organisierte Verhaltensweisen zur Ressourcenbildung und-steuerung unterstützen die Entwicklung von Wissen, indem sie bestimmte Vorgehensweisen in Organisationen vorgeben und eine Erziehungsfunktion aufweisen. ${ }^{389}$

Zusammenfassend handelt es sich bei Kernkompetenzen um eine besondere Form organisationaler Kompetenzen, die sich von Basiskompetenzen anhand der wettbewerbsbezogenen Merkmale der Nicht-Imitierbarkeit, Stiftung von Kundennutzen und Transferierbarkeit abgrenzen lassen. Während Basiskompetenzen auf zufriedenstellendem Level übliche Unternehmensaufgaben erfüllen, stellen Kern-

gestellt, dass das Unternehmen seine Strategie an den Kunden orientiert, welche in Folge den wahrgenommenen Zusatznutzen mit einer positiven Kaufentscheidung belohnen (Rasche 1994, S. 89).

386 Krüger/Homp (1997), S. 27

387 Vgl. Hamel (1994), S. 16. Hamels Kategorisierung liefert erste Ansatzpunkte für die in Teil IV dieser Arbeit verfolgte Operationalisierung der organisationalen Kompetenz.

388 Vgl. Rasche (1994), S. 114; Teece et al. (1994), S. 19; Turner/Crawford (1994), S. 243

389 Vgl. Leonard (1995), S. 18 ff; Bouncken (2003), S. 69 
kompetenzen einzigartige, spezifische organisationale Fähigkeiten dar, die dem Unternehmen einen Wettbewerbsvorteil verschaffen, indem sie strategische Aufgaben auf überdurchschnittlichem Level erfüllen. Ein zentraler Unterschied zwischen Basis- und Kernkompetenzen liegt damit in der geringeren Außenwirkung und-wahrnehmung der Basiskompetenzen (z.B. spielt der Kundennutzen im Rahmen der Diskussion um Basiskompetenzen keine bedeutende Rolle). Während Kernkompetenzen vorrangig an ihrer Wettbewerbswirkung festgemacht werden, verbleibt die Auseinandersetzung mit der Qualität von Basiskompetenzen innerhalb der Organisationsgrenzen. ${ }^{390}$

\subsection{Individuelle Kompetenzen}

Individuelle Kompetenzen lassen sich von organisationalen Kompetenzen durch das Kriterium der Personenbindung abgrenzen. So kann unter einer individuellen Kompetenz die Summe ,[...] of a person's knowledge, experience, skills and attitude ${ }^{\text {“391 }}$ verstanden werden.

Anhand der vier Dimensionen Wissen, Erfahrungen, Einstellungen und Persönlichkeitsmerkmale kann zwischen verschiedenen Arten individueller Kompetenz differenziert werden. ${ }^{392}$ Unter Berücksichtigung des Anspruchs und Forschungsinteresses dieser Arbeit, wird im Folgenden zwischen fachlicher, methodischer, sozialer und personaler Kompetenz unterschieden. Diese Form der Differenzierung ist in der fachspezifischen Literatur stark vertreten. ${ }^{393}$

\subsubsection{Fachkompetenz}

Unter der fachlichen oder epistemischen Kompetenz ist die Übereinstimmung der technischen Anforderungen einer Aufgabe mit den fachlichen Fertigkeiten und Fähigkeiten des Aufgabenerfüllers zu verstehen. Dazu zählt beispielsweise die manuelle Geschicklichkeit von Handwerkern, aber auch das fachlich breite und tiefe Wissen von Ingenieuren und Kaufleuten. Ein Teil dieses Fachwissens resultiert aus den curricularen Stationen eines Individuums in Schule und Lehre oder Universität. Im Rahmen späterer Berufserfahrung und durch Weiterbildungsmaßnahmen wird dieses Fachwissen systematisch gefestigt und vertieft. Demnach beruht die epistemische Kompetenz eines Individuums auf dem Fachwissen, das

\footnotetext{
$390 \quad$ Vgl. Fearns (2004), S. 104

391 Schreinemakers (1999), S. 136

392 Vgl. Krogh/Roos (1995), S. 66

393 Vgl. Jäger (2001); Schaeper/Briedis (2004). Das hier vorgestellte Konstruktverständnis soll dennoch nicht über die in der Literatur kritisierte Heterogenităt der definitorischen Erfassung spezifischer Kompetenzausprägungen hinwegtäuschen. Vgl. zur diesbezüglichen Kritik Schuler/ Barthelme (1995, S. 115) und Stahl (1996, S. 229).
} 
es sich im Rahmen seiner Aus- und Weiterbildung angeeignet hat, sowie auf den Erfahrungen, die es im Umgang mit gleichartigen Situationen gemacht hat. ${ }^{394}$

\subsubsection{Methodenkompetenz}

Die methodische oder heuristische Kompetenz stellt die Summe der persönlichen Problemlösungsverfahren, die die Bewältigung neuartiger Situationen, für die das Fachwissen nicht mehr ausreicht, ermöglicht, dar. Hierzu zählt beispielsweise die kognitive Methodenkompetenz, die analytische und konzeptionelle Fähigkeiten umfasst, welche es dem Individuum ermöglichen, Verantwortung für die Planung und Kontrolle der Aufgabenerfüllung zu übernehmen. Weiters erleichtert sie seinen Umgang mit unvorhersehbaren Ereignissen. Daneben kann zwischen der berufsspezifischen Methodenkompetenz, die sich durch den problem-, aufgaben-, situationsspezifischen Einsatz des Fachwissens charakterisiert, und der anspruchsvolleren Sprachkompetenz, wozu die rhetorische Eloquenz, die Artikulation der Aussprache und die Fremdsprachenkenntnisse zählen, differenziert werden. Zusammenfassend zählen zur methodischen Kompetenz fachspezifische Methoden, allgemeine Arbeitstechniken, persönliche Planungs- und Arbeitsmethoden und Problemlösungsmethoden in Arbeitsteams, die die Bewältigung neuartiger Situationen unterstützen. ${ }^{395}$

Die methodische Kompetenz umfasst daher auch den Aspekt ,Lernen lernen“. Individuen müssen Lernziele oder Aufgaben selbst erkennen können und mit den richtigen Verfahren angehen. Ihnen obliegt die Beschaffung, Filterung und Anwendung der global verfügbaren Informationen. ${ }^{396}$ Somit kann die heuristische Kompetenz als Wissen darüber, wie man sich Wissen verschaffen kann, definiert werden.

Zusammenfassend ergänzt die Methodenkompetenz die Fachkompetenz. Im Individuum selbst ruft sie Kriterien ab, die neben analytische und logische Denkprozesse auch strukturierende und kontextuelle Denkprozesse setzen. ${ }^{397}$

\subsubsection{Sozialkompetenz}

Bei der Sozialkompetenz handelt es sich um die Kompetenz, soziale Austauschbeziehungen mit Hilfe von kommunikativen Mitteln zu initiieren, zu erhalten und zu beenden. ${ }^{398}$ Als „Fähigkeit zum konstruktiven Umgang mit sich und anderen“399

394 Vgl. Winnes (1993), S. 92; Backhaus (1995), S. 410; Hinterhuber/Stahl (1996), S. 103; Stahl (1996), S. 224; Möhringer (1998), S. 27; Stahl (2000), S. 414 f.; Bühner (2004), S. 29: Erpenbeck/ Rosenstiel (2007), S. XXIV

395 Vgl. Docherty/Marking (1997), S. 27; Bühner (2004), S. 29; Stahl (2008), S. 30

396 Vgl. Winnes (1993), S. 93

397 Vgl. Faix/Laier (1991), S. 29

398 Für einen Definitionsüberblick vgl. z.B. Friede (1994) und Edinsel (1998). 
ist sie vor allem in Bezug auf den Erhalt zwischenmenschlicher Interaktionen relevant, da sie die Entstehung enger persönlicher Beziehungen, sowie einen vertrauensvollen Wissensaustausch über hierarchische und funktionale Grenzen hinweg forciert. Damit stellt sie auch eine wesentliche Voraussetzung für die „Nutzbarmachung der Kernkompetenzen“"400 dar.

Das Ausmaß der sozialen Kompetenz lässt sich einerseits anhand der Ausprägungen ihrer beobachtbaren Komponenten, die einen konkreten Verhaltensbezug aufweisen, und andererseits anhand ihrer nicht-beobachtbaren Komponenten, die die Entstehung dieses beobachtbaren Verhaltens verantworten, bestimmen. $\mathrm{Zu}$ den beobachtbaren Komponenten zählen die Kommunikationsfähigkeit, die Kooperations- und Koordinationsfähigkeit bzw. Teamfähigkeit, sowie die Konfliktfähigkeit. ${ }^{401}$ Die Konstrukte Empathie (Fähigkeit, sich in andere Personen hineinzuversetzen), Ambiguitätstoleranz (Fähigkeit, den Interaktionspartnern die Chance zur Entfaltung ihrer eigenen Ich-Identität zu ermöglichen) und Selbstöffnung (Bereitschaft, den Interaktionspartnern Einblick in sein ,Selbst' ${ }^{*} \mathrm{zu}$ geben ${ }^{402}$ stellen die Komponenten der sozialen Kompetenz dar, die nicht beobachtbar sind, weshalb auf sie nur anhand des beobachtbaren Verhaltens Rückschlüsse gezogen werden können. ${ }^{403}$ Entsprechend der Auffassung, dass es sich bei der sozialen Kompetenz nicht um eine einzelne Eigenschaft, sondern um einen Überbegriff für eine Vielzahl von allgemeinen sozialen Kompetenzen handelt, können die allgemeinen sozialen Kompetenzen nach folgenden drei Kategorien systematisiert werden ${ }^{404}$ :

\begin{tabular}{|c|c|c|}
\hline $\begin{array}{c}\text { perzeptiv-kognitiver } \\
\text { Bereich }\end{array}$ & $\begin{array}{c}\text { motivational- } \\
\text { emotionaler Bereich }\end{array}$ & behavioraler Bereich \\
\hline 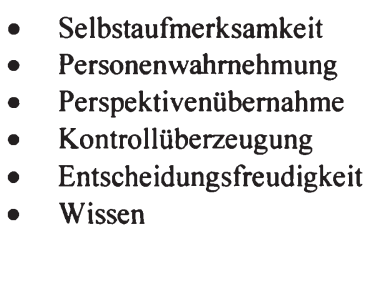 & $\begin{array}{ll}\text { - } & \text { emotionale Stabilität } \\
\text { - } & \text { Prosozialität } \\
\text { - } & \text { Wertepluralismus }\end{array}$ & $\begin{array}{ll}\text { - } & \text { Extraversion } \\
\text { - } & \text { Durchsetzungsfähigkeit } \\
\text { - } & \text { Handlungsflexibilität } \\
\text { - } & \text { Kooperationsfähigkeit } \\
\text { - Kommunikations- } \\
\text { - } \\
\text { - Kertigkeiten } \\
\text { - Selbststeuerung }\end{array}$ \\
\hline
\end{tabular}

Tabelle 6: Allgemeine soziale Kompetenzen

Quelle: In Anlehnung an Kanning (2005, S. 9)

399 Zürn (1993), S. 191

400 Bühner (2004), S. 30; ähnlich dazu auch Hafeez/Essmail (2007)

401 Vgl. Docherty/Marking (1997), S. 27; Erpenbeck/Sauer (2001); Konradt et al. (2003); GreimelFuhrmann/Pachlinger (2008), S. $68 \mathrm{ff}$.

$402 \quad$ Vgl. Hargie et al. (1994)

403 Vgl. Schuler/Barthelme (1995), S. 82; Stahl (2000), S. 414 f;; Stahl (2005)

404 Vgl. Kanning (2005), S. 6; ähnliche Kataloge allgemeiner sozialer Kompetenzen auch bei Argyle (1969), Faix/Laier (1989, 1991) und Schuler/Barthelme (1995) 
Zusammenfassend zeigt sich die soziale oder relationale Kompetenz in dem Verständnis des Verhaltens anderer Personen, sowie in den kommunikativen und kooperativen Verhaltensweisen, die bezüglich einer erfolgreichen Zielerreichung zu einer Optimierung sozialer Interaktionsprozesse im Umgang mit anderen Personen beitragen. Notwendige Voraussetzung hierfür ist das Vorliegen eines breiten Spektrums sozialer Fähigkeiten, wie Empathie, Ambiguitätstoleranz und Selbstöffnung.

\subsubsection{Persönlichkeitskompetenz}

Die persönliche oder personale Kompetenz bezieht sich auf die Merkmale und Eigenschaften, die von anderen an der eigenen Person wahrgenommen werden. ${ }^{405}$ Dementsprechend ist unter personaler Kompetenz die Übereinstimmung motivationaler Anforderungen einer Aufgabe mit den von anderen an der Person des Aufgabenerfüllers wahrgenommenen personalen Fähigkeiten zu verstehen.

In der charakteristischen Motivstruktur eines Individuums liegt die Basis zur persönlichen Bewertung von Handlungen, deren Realisierbarkeit und deren Folgen, sowie darauf aufbauend zur Anregung neuer Handlungen. Sie erlaubt ihm den erfolgreichen Einsatz seiner Fertigkeiten und Fähigkeiten. Erst die Fähigkeit, seine Persönlichkeit realitätsnah einzuschätzen, ermöglicht ihm, wirksam in seine Umwelt einzugreifen, Verantwortung für sich und seine Handlungen zu übernehmen und Erfolg von Misserfolg zu unterscheiden, d.h. ,Person' zu sein. Das In-Frage-Stellen der eigenen Person, die Fähigkeit zur Selbstkritik und zur Annahme der Kritik Dritter, ist eine notwendige Voraussetzung zur persönlichen Weiterentwicklung. ${ }^{406}$ Als Element der personalen Kompetenz ist daher das Selbstkonzept mit Selbstvertrauen und Selbstwertgefühl aufzufassen, das einhergeht mit den Fähigkeiten zur Selbstwahrnehmung und Selbstreflexion ${ }^{407}$. Erst wenn Misserfolge als Herausforderungen aufgefasst werden und persönliche Kritik emotional aufgefangen und als Möglichkeit zur Überprüfung persönlicher Überzeugungen und Einstellungen begriffen wird, werden Eigenmotivation und ein Abgleich mit den Zielen und Wertvorstellungen Dritter möglich. Die Fähigkeit, sich und sein Tun kritisch zu reflektieren, setzt Ambiguitätstoleranz, sowie eine gewisse Distanz zu sich selbst voraus (kritische und objektive Selbstwahrnehmung, konstruktive Selbstkritik, Kenntnis der persönlichen Stärken und Schwächen, Pflicht- und Wertbewusstsein). Weitere Komponenten der personalen Kompetenz stellen daher realitätsnahe Einstellungen, Motive, Werthaltungen und

\footnotetext{
405 Vgl. Bühner (2004), S. 30

406 Vgl. Erpenbeck/Heyse (2007), S. 98

407 Darunter ist die Fähigkeit, vom Alltagsgeschehen Abstand zu gewinnen und dieses zu analysieren und zu begreifen, zu verstehen. Durch diese Reflexion kann der Mensch Rückschlüsse aus der Vergangenheit ziehen und dadurch Handlungsempfehlungen für die Zukunft generieren (Stahl 2000, S 414 f.: Stahl 2005).
} 
persönliche Begabungen dar. Auch die energetischen Persönlichkeitsmerkmale Tatkraft, Zielstrebigkeit, Ausdauer, Experimentierfreudigkeit, Neugier und Belastbarkeit sind Aspekte der personalen Kompetenz. ${ }^{408}$

Mit Blick auf die im vorangegangenen Kapitel beschriebenen Komponenten der sozialen Kompetenz zeigt sich, dass dieses Konstrukt eng mit jenem der personalen Kompetenz verwoben ist. Das kann darauf zurückgeführt werden, dass sich die beiden Konstrukte bedingen und voneinander abhängen. Die soziale Kompetenz stellt die Summe aller Verfahren dar, die es dem Individuum ermöglichen, persönliche und übergeordnete Ziele mit anderen Personen in partnerschaftlichen Relationen bzw. Gruppenprozessen erfolgreich und kooperativ zu verfolgen. Hierfür sind Komponenten der personalen Kompetenz wie Aufrichtigkeit, Kritik- und Konfliktfähigkeit, Selbstorganisation und Rollendistanz im Umgang mit sich selbst notwendig. ${ }^{409}$

Zusammenfassend bezieht sich die personale Kompetenz auf die Behandlung, Wahrnehmung und Entwicklung der eigenen Person. Sie ist maßgeblich von den individuellen Bedürfnissen, Motiven, Wertehaltungen, Einstellungen und Erwartungen geprägt und dient dazu, Entwicklungschancen, Anforderungen und Einschränkungen im sozialen Leben zu klären, zu analysieren und $\mathrm{zu}$ beurteilen, sowie kreative Entwicklungs- und Lernprozesse zu initiieren.

\section{DIE KOMPETENZMESSVERFAHREN}

Nachdem die Kompetenzarten auf organisationaler und individueller Ebene verrastert und voneinander abgegrenzt wurden, wird im Folgenden auf für diese Arbeit relevante Verfahren zur Messung organisationaler und individueller Kompetenzen eingegangen. Auf Basis der erläuterten Kompetenzmessansätze erfolgt in Teil IV der Arbeit die Ableitung konsensfähiger und gültiger Indikatoren, die als empirische Äquivalente der einzelnen Kompetenzarten fungieren (Operationalisierung der Kompetenzarten).

Kompetenzmessverfahren werden hier als Ergebnis der Grundauffassung von Kompetenz verstanden. Aus dieser Auffassung resultiert der Blickwinkel auf die verschiedenen Kompetenzebenen und Kompetenzarten. Er wiederum determiniert die Sicht auf die Kompetenzentwicklung, die Neigung zu einer eher objektiven oder subjektiven Kompetenzbeobachtung und die Präferenz eher quantitativer oder qualitativer Methoden der Kompetenzforschung. ${ }^{410}$

410 Vgl. Erpenbeck/Rosenstiel (2007), S. XXX 


\subsection{Ansätze zur Identifikation organisationaler Kompetenzen}

Die Ansätze zur Identifikation organisationaler Kompetenz können nach ihrem Untersuchungsgegenstand im Sinne von aktueller bzw. wahrgenommener Kompetenz unterschieden werden. Unter aktueller Kompetenz ist die Einschätzung der Übereinstimmung von Aufgabe und Fähigkeiten durch den Kompetenzinhaber, beispielsweise das kooperierende Unternehmen A, zu verstehen. Sie ist damit situations- und selbstbezogen. ${ }^{411}$ Die wahrgenommene Kompetenz stellt die Einschätzung der Übereinstimmung von Aufgabe und Fähigkeiten des kooperierenden Unternehmens A durch den Kooperationspartner B dar. Sie ist damit bewertungs- und außenbezogen. ${ }^{412}$ Ihre Relevanz steigt in Principal-AgentSituationen, die sich dadurch kennzeichnen, dass zwischen Agent A und Prinzipal B Informationsasymmetrien bestehen. Vor dem Hintergrund, dass der Prinzipal B bestrebt ist, die damit einhergehende Unsicherheit bezüglich der Eigenschaften und Handlungsabsichten des Agenten A zu reduzieren, kann das Konstrukt der wahrgenommenen Kompetenz als Ergebnis eines auf den Agenten A ausgerichteten Wahrnehmungsprozesses von Zusatzinformationen aufgefasst werden. Im Hinblick auf den Entstehungsprozess von Vertrauen, dessen Erforschung Ziel dieser Arbeit ist, besitzt das Konstrukt der wahrgenommenen Kompetenz gegenüber jenem der aktuellen Kompetenz eine höhere Relevanz, da die Entscheidung des kooperierenden Unternehmens B, einem Kooperationspartner A zu vertrauen weniger auf dem objektiven Vorliegen aktueller Kompetenzen von A, sondern stärker auf der subjektiven Wahrnehmung, positiven Beurteilung und Attribution dieser Kompetenzen durch das kooperierende Unternehmen B beruht. ${ }^{413}$ Während die nachfolgend erläuterten Ansätze von Weiss (1992) und Möhringer (1998) das Konstrukt der wahrgenommenen organisationalen Kompetenz fokussieren, steht bei den im Anschluss diskutierten Ansätzen von Bouncken (2003), Faix/Krupp (2002), Heyse/Erpenbeck (1997) und Schiller (2000) die aktuelle (Kern-) Kompetenz im Zentrum der Betrachtung.

\section{Der Ansatz von Weiss zur Kompetenzwahrnehmung}

Die Arbeit von Weiss zielt darauf ab, einen Erklärungsansatz für die Relevanz des Kompetenzkonstrukts abzuleiten und darauf aufbauend einen Operationalisierungsvorschlag für die Messung von wahrgenommener Kompetenz zu generieren. $^{414}$

Bezüglich der Relevanz des Kompetenzkonstrukts wird zunächst geklärt, in welchen Situationen ein Akteur B seine Wahrnehmung der Kompetenzen eines anderen Akteurs A als Zusatzinformation heranzieht. Unter Bezugnahme auf in-

411 Vgl. Weiss (1992), S. 61; Stahl (1996), S. 221; Möhringer (1998), S. 28

412 Vgl. Stahl (1996), S. 221 f.; Weiss (1996), S. 63

413 Vgl. hierzu die Ausführungen in Teil III Kap. 4.2 und Teil IV Kap. 1.2, sowie die dort zit. Literatur.

414 Vgl. Weiss (1992), S. 185 
formationsökonomische Erkenntnisse wird der Relevanzbereich auf solche Transaktionssituationen begrenzt, in denen der Beurteilungsprozess des Akteurs B durch einen Mangel an Kontrollinformationen gekennzeichnet ist und damit mit spezifischen Risiken einhergeht. In diesen Situationen ist zu erwarten, dass B bestrebt sein wird, seine Unsicherheit bezüglich der Handlungsabsichten von A durch eine Informationsselektion und durch einen Rückgriff auf Surrogatinformationen zu reduzieren. Der bewusste Verzicht auf eine ausgiebige Suche nach Kontrollinformationen und damit die Bildung einer vertrauensvollen Erwartung bezüglich der Handlungsabsichten von A kann allerdings nur antizipiert werden, wenn Informationen wahrgenommen werden, die B dazu veranlassen, auf eine höhere Eintrittswahrscheinlichkeit seiner Erwartung zu schließen. Die Kompetenz von A wird in diesem Kontext als eine solche Information zur Unsicherheitsreduktion aufgefasst. Die Beurteilung der Kompetenz von A durch B sieht Weiss damit als Ergebnis eines extensiven Wahrnehmungsprozesses von Surrogatinformationen, in dem eine Bewertung der einzelnen für die Kompetenz relevanten Eigenschaften von A erfolgt. ${ }^{415}$ Entsprechend dieser Annahme wählt Weiss zur Identifikation der kompetenzrelevanten Beurteilungsmerkmale ein dekompositionelles Verfahren auf Basis der Conjoint-Analyse. Im Ergebnis umfasst der Operationalisierungsvorschlag zur Messung der wahrgenommenen Kompetenz des Akteurs A 44 Beurteilungsmerkmale ${ }^{416}$, deren modelltheoretische Zuordnung $\mathrm{zu}$ den folgenden 6 Beurteilungsdimensionen durch eine konfirmatorische Faktorenanalyse bestätigt wird: gegenwärtige Marktstellung, zukünftige Marktpräsenz, Anpassungsfähigkeit, Struktur, Dienstleistungsangebot und Nachweis der Leistungsfähigkeit. ${ }^{417}$ Diese Dimensionen, einschließlich ihrer Beurteilungsmerkmale, liefern wichtige inhaltliche Anhaltspunkte für die in Teil IV dieser Arbeit verfolgte Operationalisierung der organisationalen Kompetenzen.

\section{Der Ansatz von Möhringer zur Kompetenzkommunikation}

Die Arbeit von Möhringer hat zum Ziel, einen Erklärungsansatz für die Kompetenzkommunikation zu generieren. Die zu kommunizierenden Kompetenzen werden in Anlehnung an Weiss als Ersatzinformationen eines Akteurs B, die er

415 Vgl. Weiss (1992), S. $56 \mathrm{ff}$.

416 Auf eine Auflistung der 44 Kompetenzmerkmale wird an dieser Stelle verzichtet. Die im Rahmen der Modellentwicklung berücksichtigten Kompetenzmerkmale finden sich als Indikatornachweise in Teil IV Kapitel 3.

417 Vgl. Weiss (1992), S. 109 ff. Die Überprüfung des Modells zur Kompetenzwahrnehmung basiert auf einer Stichprobe von $\mathrm{N}=93$, deren Umfang im Hinblick auf den Forschungsbereich seitens des Autors als relativ klein beurteilt wird (Weiss 1992, S. 122). Unter Berücksichtigung der fehlenden Repräsentativität liefern die Ergebnisse der konfirmatorischen Faktorenanalyse dennoch relevante Tendenzaussagen zur Wahrnehmungsstruktur organisationaler Kompetenzen. 
bei der Einschätzung eines Akteurs A heranzieht, begriffen, womit auch Möhringers Ansatz das Konstrukt der wahrgenommenen Kompetenz fokussiert. ${ }^{418}$

Im Zentrum der Untersuchung stehen die transaktionsspezifische Relevanz der Kompetenzkommunikation, die Dimensionen der Kompetenzbotschaften, die Medien zur Kompetenzkommunikation, sowie deren phasengerechte Anwendung. Im Hinblick auf die Relevanz der Kompetenzkommunikation im Rahmen von Transaktionen wird empirisch nachgewiesen, dass der Akteur B spezifischen Informationen bezüglich der Kompetenz des Akteurs A beim Erstkontakt eine höhere Bedeutung beimisst als bei nachfolgenden Kontakten. ${ }^{419}$ Im Zuge der Identifikation phasengerechter Kompetenzbotschaften werden die von Weiss ermittelten Beurteilungsdimensionen zur Wahrnehmung der Kompetenz des Akteurs A empirisch geprüft und bestätigt, wobei an die Stelle von Weiss' Dimension ,Struktur' die Dimension ,Eigenschaften des Konzepts' gesetzt wird. Im Zuge einer Diskrepanz-Analyse zeigt sich sodann, dass je nach Kontaktphase unterschiedliche Kompetenzdimensionen und dementsprechend unterschiedliche Kompetenzbotschaften von Relevanz sind, um zukünftige Kontakte zu forcieren. Durch diese Analyse, die der Erfassung der Differenz zwischen der von Akteur A angenommenen Kompetenzwahrnehmung durch den Akteur B und der tatsächlichen Kompetenzwahrnehmung des Akteurs B dient, werden Stärken und Schwächen der Kompetenzkommunikation aufgedeckt. ${ }^{420}$ Bezüglich der Identifikation der Medien zur Kompetenzkommunikation zeigt sich, dass der Akteur B seine Informationen über die Kompetenz des Akteurs A vorrangig über das Medium der persönlichen Kommunikation mit dem Transaktionspartner A bezieht. ${ }^{421}$ Zusammenfassend liefert der Ansatz von Möhringer mit seiner Ermittlung der phasenbezogenen Relevanz einzelner Kompetenzdimensionen, sowie der Berücksichtigung der Perspektiven beider Transaktionspartner wichtige Anhaltspunkte für die im Rahmen dieser Arbeit verfolge Operationalisierung des Kompetenzkonstrukts.

Während die Ansätze von Weiss und Möhringer das Konstrukt der wahrgenommenen Kompetenz fokussieren, steht bei den nachfolgenden Ansätzen die aktuelle (Kern-)Kompetenz im Zentrum der Betrachtung. Hierbei ist zwischen methodisch orientierten und praxeologisch orientierten Ansätze zur Identifikation von (Kern-)Kompetenzen unterscheiden. Während die methodisch orientierten

\footnotetext{
418 Vgl. Möhringer (1998), S. 29. Hinsichtlich des empirischen Untersuchungsteils ist festzuhalten, dass die zugrunde liegende Stichprobe $\mathrm{N}=62$ betrug. Da die Grundgesamtheit als relativ klein angenommen werden kann, lässt die Stichprobe repräsentative Schlüsse zu (Möhringer 1998, S. 123 f.)

419 Vgl. Möhringer (1998), S. 147

420 Vgl. Möhringer (1998), S. 94, sowie S. $142 \mathrm{f}$

421 Vgl. Möhringer (1998), S. 50, sowie S. $133 \mathrm{f}$.
} 
Ansätze ${ }^{422}$ darauf abzielen, eine allgemeingültige Konzeption zur Messung von (Kern-)Kompetenzen zu entwickeln, liegt der Fokus der praxeologisch orientierten Ansätze ${ }^{423}$ auf der Identifikation branchenbezogener (Kern-) Kompetenzen.

Grundsätzlich ist die systematische Erfassung von Kernkompetenzen mit einer Vielzahl von Schwierigkeiten verbunden. Aufgrund der Intransparenz, Heterogenität und Vielschichtigkeit der Entstehungsgründe und -orte dieser Art von Kompetenz ist die Identifikation von direkt wahrnehmbaren und empirisch erfassbaren Korrelaten, die Rückschlüsse auf das theoretische Konstrukt der Kernkompetenz erlauben bzw. selbiges einer Operationalisierung zugänglich machen, erschwert. Dies zeigt sich auch darin, dass kaum Arbeiten vorliegen, die sich in diesem Zusammenhang mit der Operationalisierung des Konstrukts befassen bzw. darin, dass die wenigen Arbeiten, die eine Operationalisierung des Konstrukts vornehmen, nicht über eine Methodendiskussion zur Kernkompetenzidentifikation hinausgehen. ${ }^{424}$ Der nachfolgenden Relevanzeinschätzung der Ansätze zur Identifikation von (Kern-)Kompetenzen sei deshalb vorangestellt, dass ihre Ergebnisse durchwegs unter dem Vorbehalt der ausstehenden empirischen Überprüfung zu beurteilen sind.

\section{Der Ansatz von Bouncken}

Der Ansatz von Bouncken zielt darauf ab, eine Theorie organisationaler Kompetenzen unter besonderer Berücksichtigung organisationaler Metakompetenzen $^{425}$ zu entwickeln. Dabei wird den Fragen nach der Entstehung und Entwicklung organisationaler Kompetenzen nachgegangen. Bouncken entwickelt in diesem Zusammenhang einen Operationalisierungsvorschlag zur Identifikation organisationaler Kompetenzen, wobei zwischen folgenden Kategorien differenziert wird: Routinisierungskompetenzen, Kreationskompetenzen, Kooperationskompetenzen, externe Kommunikationskompetenzen, Marktkommunikationskompetenzen, Um- und Restrukturierungskompetenzen, sowie Verlern- und Ausstiegskompetenzen. Diese Kategorien charakterisieren sich dadurch, dass sie eine Vermischung der unterschiedlichen Arten von Metakompetenzen und ihrer Ausprägungen darstellen. Dadurch und aufgrund ihrer geringeren Abstraktheit verfügen sie, verglichen mit reinen Metakompetenzen, über einen höheren Anwendungsbezug im Unternehmen. Diese stärkere Anwendungsnähe verbessert die Identifikation von organisationalen Kompetenzen. Werden die Kategorien organi-

422 Vgl. dazu beispielsweise Tampoe (1994), S. 68; Bullinger et al. (1995), S. 194 ff.; Edge et al. (1995), S. 191; Steinle et al. (1997), S. 13 ff.

423 Vgl. dazu beispielsweise Bakker et al. (1994); Friedrich (1995); Hamel/Prahalad (1995); Krüger/ Homp (1997); Hinterhuber et al. (2003)

424 Vgl. Faix/Krupp (1999), S. 11; Faix/Krupp (2002), S. 60; Bouncken (2003), S. 328 ff.

425 Darunter sind grundlegende Kompetenzen zu verstehen, die auf hohem Abstraktionsniveau der Vernetzung, Weitergabe und Entwicklung von Ressourcen im Unternehmen dienen (Bouncken 2003, S. 2). 
sationaler Kompetenzen ex post bewertet und wird dabei das Kriterium der Stiftung von Kundennutzen einbezogen, so können sie deckungsgleich mit Kernkompetenzen sein. ${ }^{426}$ Für die Identifikation von Kernkompetenzen sind sowohl externe, als auch interne Informationsquellen heranzuziehen. Externe Informationen von Kunden und Lieferanten ermöglichen Rückschlüsse auf die Außenwirkung und Reputation des Unternehmens und damit, in Verbindung mit internen Informationen, die auf die Beurteilung der Organisationsstruktur und kultur und der Technologien gerichtet sind, die Identifikation von Kernkompetenzen. ${ }^{427}$ Bezugnehmend auf die vorliegende Arbeit liefern die von Bouncken beschriebenen Kategorien organisationaler Kompetenzen und deren verbale Ausdifferenzierung, sowie die kernkompetenzspezifischen Informationsquellen, vorbehaltlich ihrer ausstehenden empirischen Überprüfung, Ansatzpunkte für die in Teil IV verfolgte Operationalisierung organisationaler Kompetenzen.

\section{Der Ansatz von Schiller}

Die Arbeit von Schiller zielt darauf ab, einen Kompetenz-Management-Ansatz zu entwickeln. Im Zentrum der Analyse steht die Evaluation von organisationalen Kompetenzprofilen und damit einhergehend die Erfassung von wettbewerbsrelevanten Kompetenzen und erfolgskritischen Kernkompetenzen. ${ }^{428}$ Dabei wird ein Operationalisierungsvorschlag zur Identifikation von (Kern-)Kompetenzen entwickelt, der neun primäre und vier sekundäre Kompetenzfelder umfasst. Während sich die primären Kompetenzfelder Projektmanagement, Technologiemanagement, kunden- und technologieorientierte Angebotskonzipierung, Know-how-Transfer, Kooperationsmanagement, Projektdevelopment, Financial Engineering, Pre-Sale- sowie After-Sale-Dienstleistungen auf Prozessphasen beziehen, begleiten die sekundären Kompetenzfelder Kostenmanagement, Beziehungsmanagement, Informations- und Kommunikationsmanagement, sowie Interkulturelles Management diese übergreifend. ${ }^{429}$ Die Eignung dieses Operationalisierungsvorschlags wurde mithilfe einer explorativen Studie ${ }^{430}$ überprüft und bestätigt. Vorbehaltlich ihrer ausstehenden testkritischen Prüfung liefern die identifizierten Kompetenzfelder, sowie deren verbale Ausdifferenzierung inhaltliche

\footnotetext{
426 Vgl. Bouncken (2003), S. $309 \mathrm{ff}$.

427 Vgl. Bouncken (2000), S. 870; Bouncken (2003), S. $328 \mathrm{f}$.

428 Vgl. Schiller (2000), S. 2 und 121. Der Unterschied zwischen wettbewerbsrelevanten Kompetenzen, im Rahmen dieser Arbeit verstanden als Basiskompetenzen, und erfolgskritischen Kernkompetenzen liegt darin, dass letztere, verglichen mit der Konkurrenz, einen einzigartigen, honorierbaren Kundennutzen stiften, insofern eine höhere wettbewerbsbezogene Substanz besitzen und zur Bestimmung von Marktpriorităten herangezogen werden können (Schiller 2000, S. 110).

429 Vgl. Schiller (2000), S. 123

430 Die Stichprobe der Hauptuntersuchung setzt sich aus 24 Unternehmen des Anlagenbaus zusammen, die im Rahmen teilstandardisierter Interviews befragt wurden. Aufgrund der geringen Große der Stichprobe können keine statistisch repräsentativen Schlüsse gezogen werden (Schiller 2000, S. 130 ff.).
} 
Ansatzpunkte für die in Teil IV dieser Arbeit verfolgte Operationalisierung organisationaler Kompetenzen.

\section{Der Ansatz von Heyse und Erpenbeck}

Die Arbeit von Heyse und Erpenbeck hat zum Ziel, vorhandene Kompetenzbarrieren durch eine Stärkung von Kernkompetenzen zu überwinden. In Verfolgung dieser Zielstellung wird ein Ansatz zur Kompetenzentwicklung von ,lernenden Unternehmen', worunter Organisationen zu verstehen sind, die den Lernprozess aller ihrer Mitglieder fördern und sich gleichzeitig selbst kontinuierlich wandeln, erarbeitet. ${ }^{431}$ Seine Relevanz für die vorliegende Arbeit zeigt sich in der in diesem Zusammenhang gestellten Frage, ob es , ,[...] verallgemeinerte Gesichtspunkte [gibt, Anm. d. Verf.], die Unternehmenskompetenzen zu ordnen." ${ }^{\text {432 Auf }}$ Basis handlungstheoretischer Erkenntnisse wird ein Operationalisierungsvorschlag zur Identifikation organisationaler (Kern-)Kompetenzen erarbeitet, der sowohl objektive, als auch subjektive Kenngrößen lernender Unternehmen umfasst. ${ }^{433}$ Unter dem Vorbehalt ihrer ausstehenden empirischen Prüfung werden in der nachfolgenden Tabelle jene Kenngrößen aufgelistet, die Rückschlüsse auf das Vorliegen organisationaler Kompetenzen eines Unternehmens geben. Nicht aufgelistet werden hingegen jene Kenngrößen, die sich vorrangig auf die Kompetenzentwicklung eines, lernenden Unternehmens' beziehen.

\begin{tabular}{|c|c|}
\hline Kenngrößen & $\begin{array}{l}\text { relevante Indikatoren für die Operationalisierung } \\
\text { organisationaler Kompetenzen }\end{array}$ \\
\hline $\begin{array}{l}\text { objektive } \\
\text { Kenngrößen } \\
\text { als } \\
\text { betriebswirtschaftliche } \\
\text { Parameter }\end{array}$ & $\begin{array}{l}\text { - Kapitalstock/Kapitalstruktur, technische Ausstattung } \\
\text { - } \text { Betriebsmittelausstattung/Technologie/Investitionen } \\
\text { - Personalausstattung und -qualifikation } \\
\text { - Personalkosten } \\
\text { - } \text { Organisationsstruktur und Arbeitsorganisation } \\
\text { - Planungs- und Controllingsystem } \\
\text { - } \text { Absatzmenge und -stabilität } \\
\text { - Produktionskapazität/Produktionsgestaltung/Flexibilität } \\
\text { - Produktionskosten und Verkaufspreise } \\
\text { - Führungsstruktur }\end{array}$ \\
\hline $\begin{array}{l}\text { subjektive } \\
\text { Kenngrößen } \\
\text { als immaterielle } \\
\text { Ressourcen }\end{array}$ & $\begin{array}{l}\text { - Einschätzung von Unternehmenserfolg, Ergebnis- und } \\
\text { Leistungsorientierung des Unternehmens } \\
\text { - Einschätzung der Produktivität und Preisakzeptanz, der } \\
\text { Kosten-Nutzen-Orientierung des Unternehmens am } \\
\text { Markt }\end{array}$ \\
\hline
\end{tabular}

$431 \quad$ Vgl. Heyse/Erpenbeck (1997), S. 68

432 Heyse/Erpenbeck (1997), S. 74

433 Vgl. Heyse/Erpenbeck (1997), S. 78 ff. 


\begin{tabular}{|l|ll|}
\hline$\bullet$ & Einschätzung der Innovations- und Anpassungsfähigkeit \\
& des Unternehmens \\
$\bullet$ & Einschätzung der Perspektivität \\
$\bullet$ & Einschätzung der Lieferflexibilität \\
$\bullet$ & Einschätzung der Produktqualität und \\
& Technologieorientierung \\
$\bullet$ & Einschätzung der Führungstätigkeit und -kompetenz \\
$\bullet$ & Einschätzung des Betriebsklimas \\
$\bullet$ & Einschätzung der Informationsdistribution und \\
& Kommunikationsorientierung \\
$\bullet$ & Einschätzung der Kundenorientierung \\
$\bullet$ & Einschätzung der Öffentlichkeitsarbeit und \\
& Öffentlichkeitswirkung \\
$\bullet$ & Einschätzung des Unternehmensleitbilds aus \\
& Kundensicht \\
$\bullet$ & Problemlösungspotential des Unternehmens
\end{tabular}

Tabelle 7: Kenngrößen zur Identifikation organisationaler Kompetenzen

Quelle: In Anlehnung an Heyse/Erpenbeck (1997, S. 78 f.)

Die aufgelisteten Kenngrößen finden im Rahmen der Operationalisierung organisationaler Kompetenzen in Teil IV der vorliegenden Arbeit Berücksichtigung.

\section{Der Ansatz von Faix und Krupp}

Die Arbeit von Faix und Krupp zielt darauf ab, einen Ansatz zur Identifikation von Kernkompetenzen zu erarbeiten. Mithilfe einer dimensionalen Analyse $\mathrm{e}^{434}$ wird ein Vorschlag zur Operationalisierung erarbeitet, der von der wettbewerbspolitischen Bedeutung des Kernkompetenzkonstrukts ausgeht und dementsprechend drei Merkmalsdimensionen unterscheidet: Nicht-Imitierbarkeit, NichtSubstituierbarkeit und Potential zur Stiftung von Kundennutzen. ${ }^{435}$ Unter Bezugnahme auf transaktionskostentheoretische Erkenntnisse und auf Überlegungen des marktorientierten Managementansatzes wird ein Indikatorensystem ent-

434 Dabei handelt es sich um eine Methode, um hypothetische, selbst nicht direkt messbare Konstrukte im Zuge eines mehrstufigen Prozesses möglichst genau zu erfassen. Dazu werden direkt wahmehmbare Phänomene gesucht, die Rückschlüsse auf das Konstrukt ermöglichen bzw. es einer Operationalisierung zugänglich machen (Faix/Krupp 1999, S. 11).

435 Vgl. Faix/Krupp (2002), S. 62 ff. Unter Substituierbarkeit ist die Ersetzung eines Gutes oder Produktionsfaktors durch ein anderes Gut oder einen anderen Produktionsfaktor zu verstehen. Güter sind dann substitutiv, wenn sie die gleiche Nutzenkomponente aufweisen. Da solche Güter dem Kunden keinen einzigartigen Nutzen stiften können, verlieren sie an Wert. Kernkompetenzen müssen sich daher gegen Substitutionsgefahren behaupten können (Faix/Krupp 2002, S. 64; sowie die dort zit. Literatur). Das von Prahalad und Hamel (1990) genannte Kernkompetenzmerkmal der Transferierbarkeit findet im Rahmen des Ansatzes von Faix/Krupp keine Beachtung, da letztere der Ansicht sind, dass zum einen die Beschränkung auf einen Markt sinnvoll oder zwingend sein kann und zum anderen die Einsetzbarkeit einer Kompetenz wesentlich von der Făhigkeit des Managements, neue Märkte zu erkennen und zu schaffen, abhängt. 
wickelt, das zentrale Ansatzpunkte für die in Teil IV dieser Arbeit verfolgte Operationalisierung organisationaler Kompetenzen liefert. Die nachstehende Graphik bildet den Operationalisierungsvorschlag von Faix und Krupp zur Identifikation organisationaler Kompetenzen ab.

Theoretisches

Konstrukt

Merkmalsdimension

Indikatoren

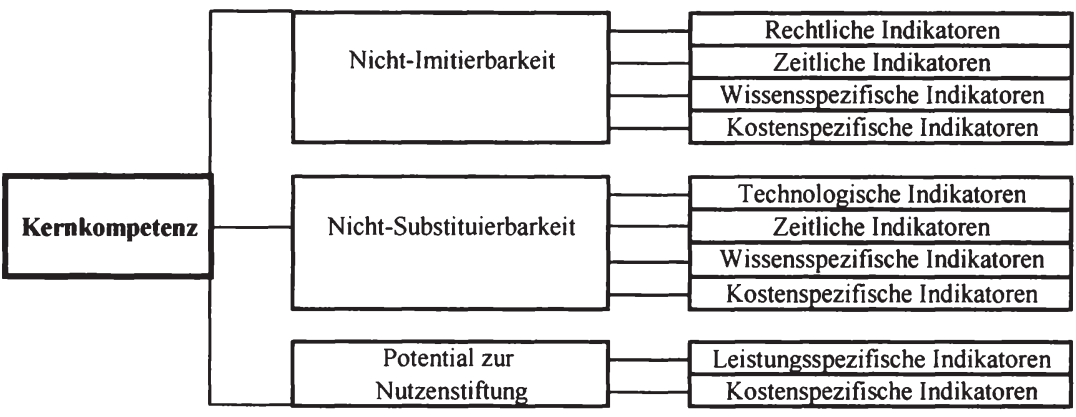

Abbildung 11: Die Operationalisierung des Kernkompetenzkonstrukts

Quelle: In Anlehnung an Faix/Krupp (2002, S. 79)

Bezugnehmend auf die Merkmalsdimension der Nicht-Imitierbarkeit zählen zu den rechtlichen Indikatoren Patente und Arbeitsverträge, unter die zeitliche Indikatorengruppe fällt der Zeitaufwand für die Kompetenzentwicklung, während es sich bei den wissensspezifischen Indikatoren um den Grad der Personengebundenheit/der organisatorischen Eingebundenheit des Wissens, sowie um das implizite Wissen handelt. Die kostenspezifischen Indikatoren bezeichnen die Kosten der Imitation für die Konkurrenz. Bezüglich der Merkmalsdimension der Nicht-Substituierbarkeit zeigt sich, dass die Zunahme aktueller spezifischer Veröffentlichungen, die Zahl der Patentmeldungen der Konkurrenz in einzelnen Patentklassen, sowie die Zitierhäufigkeit von Patenten als Einzelindikatoren der technologischen Indikatorgruppe aufzufassen sind. $\mathrm{Zu}$ den zeitlichen Indikatoren zählen die Entwicklungsdauer und der Grad der Übernahme der Kompetenz durch die Konkurrenz. Unter die wissensspezifische Indikatorengruppe fallen Informationsredundanzen, während $\mathrm{zu}$ den kostenspezifischen Indikatoren der Grad der Komplexität und der Grad der Eingebundenheit gezählt werden können. Bezugnehmend auf die Merkmalsdimension der Fähigkeit zur Nutzenstiftung handelt es sich bei exklusivem Zugang zu Ressourcen, loyalen Zuliefern, Kundenbindung und spezialisierter Herstellungstechnik um kostenspezifische Indikatoren. Bekanntheitsgrad, Kompetenz als Kaufgrund und der Grad des Beitrags zu kaufentscheidungsrelevanten Eigenschaften stellen leistungsspezifische Indikatoren dar. ${ }^{436}$ 
Mit Blick auf die im Rahmen dieser Arbeit verfolgte Modellentwicklung (vgl. Teil IV) lässt sich resümieren, dass die erläuterten Ansätze (vgl. Tabelle 8) aufgrund ihrer organisationalen Untersuchungsperspektive inhaltliche Ansatzpunkte zur Identifikation organisationaler Kompetenzen liefern. Um die Ableitung konsensfähiger und gültiger Indikatoren, die als empirische Äquivalente der Basisund Kernkompetenzen fungieren, sekundäranalytisch abzusichern, wird im Zuge der in Teil IV verfolgten Operationalisierung Bezug auf weitere für diese Arbeit relevante Beiträge im Bereich der organisationalen Kompetenzforschung genommen.

\begin{tabular}{|c|c|c|c|}
\hline Autor & $\begin{array}{c}\text { Ziel der } \\
\text { Untersuchung }\end{array}$ & $\begin{array}{l}\text { Relevanz für die } \\
\text { Operationalisierung } \\
\text { organisationaler } \\
\text { Kompetenzen }\end{array}$ & $\begin{array}{l}\text { Auswertung } \\
\text { (Datengrund- } \\
\text { lage) }\end{array}$ \\
\hline Weiss (1992) & $\begin{array}{l}\text { Messung der } \\
\text { Kompetenz- } \\
\text { wahrnehmung }\end{array}$ & $\begin{array}{l}6 \text { Kompetenzdimensionen; } \\
44 \text { Kompetenzindikatoren }\end{array}$ & $\begin{array}{l}\text { Conjoint- } \\
\text { Analyse, kon- } \\
\text { firmatorische } \\
\text { Faktorenanalyse } \\
(\mathrm{N}=93)\end{array}$ \\
\hline $\begin{array}{l}\text { Möhringer } \\
\text { (1998) }\end{array}$ & $\begin{array}{l}\text { Erklärungsansatz } \\
\text { für die } \\
\text { Kompetenz- } \\
\text { kommunikation }\end{array}$ & $\begin{array}{l}\text { Überprüfung der (phasen- } \\
\text { bezogenen) Relevanz der } 6 \\
\text { Kompetenzdimensionen von } \\
\text { Weiss }\end{array}$ & $\begin{array}{l}\text { deskriptive } \\
\text { Auswertungs- } \\
\text { verfahren, } \\
\text { Diskrepanz- } \\
\text { analyse }(\mathrm{N}=62)\end{array}$ \\
\hline $\begin{array}{l}\text { Bouncken } \\
(2003)\end{array}$ & $\begin{array}{l}\text { Theorie } \\
\text { organisationaler } \\
\text { Kompetenzen }\end{array}$ & $\begin{array}{l}7 \text { Kompetenzdimensionen: } \\
\text { verbale Ausdifferenzierung }\end{array}$ & $\begin{array}{l}\text { ausstehende } \\
\text { empirische } \\
\text { Überprüfung }\end{array}$ \\
\hline Schiller (2000) & $\begin{array}{l}\text { Kompetenz- } \\
\text { Management- } \\
\text { Ansatz }\end{array}$ & $\begin{array}{l}9 \text { primäre Kompetenzfelder; } \\
4 \text { sekundäre Kompetenz- } \\
\text { felder; verbale } \\
\text { Ausdifferenzierung }\end{array}$ & $\begin{array}{l}\text { deskriptive } \\
\text { Auswertungs- } \\
\text { verfahren. } \\
\text { Clusteranalyse } \\
(\mathrm{N}=24)\end{array}$ \\
\hline $\begin{array}{l}\text { Heyse/ } \\
\text { Erpenbeck } \\
\text { (1997) }\end{array}$ & $\begin{array}{l}\text { Kompetenz- } \\
\text { entwicklung von } \\
\text {,lernenden Unter- } \\
\text { nehmen' zur } \\
\text { Stärkung von } \\
\text { Kernkompetenzen }\end{array}$ & $\begin{array}{l}\text { Ableitung von } 41 \text { Kom- } \\
\text { petenzindikatoren aus } \\
\text { objektiven und subjektiven } \\
\text { Kenngrößen .lernender } \\
\text { Unternehmen' }\end{array}$ & $\begin{array}{l}\text { ausstehende } \\
\text { empirische } \\
\text { Überprüfung }\end{array}$ \\
\hline $\begin{array}{l}\text { Faix/Krupp } \\
\text { (2002) }\end{array}$ & $\begin{array}{l}\text { Operationali- } \\
\text { sierung des Kern- } \\
\text { kompetenz- } \\
\text { Konstrukts }\end{array}$ & $\begin{array}{l}3 \text { Kompetenzdimensionen: } \\
10 \text { Kompetenzindikatoren }\end{array}$ & $\begin{array}{l}\text { ausstehende } \\
\text { empirische } \\
\text { Überprüfung }\end{array}$ \\
\hline
\end{tabular}

Tabelle 8: Ansătze zur Identifikation organisationaler Kompetenzen 


\subsection{Ansätze zur Identifikation individueller Kompetenzen}

Die Ansätze zur Identifikation individueller Kompetenzen lassen sich grundsätzlich danach unterscheiden, ob es sich um eine prozessuale oder punktuelle Analyse handelt. Ebenso variieren die Ansätze nach der Perspektive der Analyse, d.h. ob eine Selbsteinschätzung durch den Kompetenzinhaber und/oder eine Fremdeinschätzung durch andere Akteure erfolgt. Weiters können die Ansätze danach differenziert werden, ob die individuellen Kompetenzen quantitativ oder qualitativ erfasst werden.

Im Folgenden werden das Selbstkonzept beruflicher Kompetenz, die Kompetenzbiographie und das Kasseler-Kompetenz-Raster zur Identifikation individueller Kompetenzen erläutert.

\begin{tabular}{|l|c|c|c|}
\hline & \multicolumn{1}{|c|}{$\begin{array}{c}\text { Selbstkonsätze } \\
\text { beruflicher } \\
\text { Kompetenz }\end{array}$} & $\begin{array}{c}\text { Kompetenz- } \\
\text { biographie }\end{array}$ & $\begin{array}{c}\text { Kasseler } \\
\text { Kompetenz- } \\
\text { Raster }\end{array}$ \\
\hline Forschungsperspektive & individualzentriert & individualzentriert & individualzentriert \\
\hline Messmethode & quantitativ & qualitativ & qualitativ \\
\hline Beurteilerperspektive & Selbsteinschätzung & Selbsteinschätzung & $\begin{array}{c}\text { Fremdeinschätzung } \\
\text { (Beobachtung) }\end{array}$ \\
\hline zeitliche Dimension & Bestandsanalyse & $\begin{array}{c}\text { biographische } \\
\text { Prozessanalyse }\end{array}$ & $\begin{array}{c}\text { verlaufsorientierte } \\
\text { Bestandsanalyse }\end{array}$ \\
\hline
\end{tabular}

Tabelle 9: Überblick über die Ansätze zur Identifikation individueller Kompetenzen

Die Auswahl dieser Ansätze aus der Fülle von Kompetenzmessverfahren ${ }^{437}$ beruht auf ihrer Eignung für die im Rahmen dieser Arbeit verfolge Modellentwicklung. Diese ergibt sich einerseits daraus, dass die Ansätze die in Kapitel 2.2 vorgestellte Grundstruktur personenbezogener Kompetenzen widerspiegeln. Andererseits lassen ihre jeweiligen Zielsetzungen, theoretischen Grundlagen, methodologischen Einordnungen und verfahrensbezogenen Beurteilungen der Gütekriterien darauf schließen, dass ihre Erkenntnisse die in Teil IV dieser Arbeit verfolgte Operationalisierung individueller Kompetenzen unterstützen.

\section{Das Selbstkonzept beruflicher Kompetenz}

Im Rahmen dieses Ansatzes wird die individuelle Kompetenz als Kapazität einer Person, Aufgaben erfolgreich zu bewältigen, verstanden. Für ihre Erfassung sieht der Ansatz einen subjektiven Messzugang vor. Mit Hilfe standardisierter Analyseinstrumente ${ }^{438}$ werden die Einschätzungen der Kompetenzinhaber bezüglich ver-

437 Vgl. dazu die Sammelbände zu Kompetenzmessverfahren von Erpenbeck/Rosenstiel (2007), sowie Erpenbeck (2004).

438 Vgl. zu derartigen standardisierten subjektiven Analyseinstrumenten z.B. das Selbstkonzept beruflicher Kompetenz von Bergmann (2007), sowie Sonntag/Schäfer-Rauser (1993), den Kompetenz- 
schiedener Facetten des Selbstkonzepts als individuell strukturierte Gesamtheit kognitiver Repräsentationen von Gedanken und Emotionen über sich selbst ${ }^{439}$ erfasst. Dieser Ansatz zur Identifikation individueller Kompetenzen beruht auf der Annahme, dass Individuen als Experten ihrer beruflichen Tätigkeit, deren Bedingungen, Ziele und Ergebnisse einschätzen können. Dementsprechend können sie sowohl die Funktion des Analytikers ihrer beruflichen Tätigkeit, als auch die des Analytikers ihres individuellen Kompetenzprofils übernehmen. ${ }^{40}$ Die jeweils zeitpunktbezogene Offenlegung und Reflexion der eigenen Kompetenzen stellen dann jene Informationsgrundlage dar, die für die Evaluation des in der Berufsund Wirtschaftspädagogik und in der Personalentwicklung interessierenden Kompetenzentwicklungsbedarfs von Relevanz ist. Frei et al. betonen die praktische Bedeutung des Selbstkonzepts, indem sie darauf hinweisen, dass die Entwicklung von Kompetenzen ein ,[...] bewusstes Reflektieren einschließt und es erforderlich macht, dass Qualifizierungsprozesse von den Handelnden als solche kogniziert werden, eine Veränderung der eigenen Kompetenzen wahrgenommen und diese in das jeweilige Selbstkonzept integriert wird." ${ }^{441}$ Die empirische Verwertbarkeit der kompetenzbezogenen Selbsteinschätzung setzt allerdings voraus, dass die Anonymität der Angaben gewährleistet wird, dimensionsorientierte anstelle globaler Kompetenzurteile erhoben werden und von einer Evaluation spezifischer Persönlichkeitsmerkmale als Indizes für die Persönlichkeitskompetenz abgesehen wird. $^{442}$

Im Folgenden wird das Selbstkonzept von Sonntag und Schäfer-Rauser (1993) erläutert, da es explizit den beruflichen Kontext fokussiert ${ }^{443}$ und über eine Evaluation der Methodenkompetenz $z^{444}$ hinausreicht. Das im Rahmen dieses Ansatzes entwickelte standardisierte Analyseinstrument berücksichtigt nämlich auch die Selbsteinschätzung der Fach- und Sozialkompetenz. Für seine Eignung im vorliegenden Untersuchungskontext spricht daher vor allem, dass er die in Kapitel 2.2 vorgestellte Grundstruktur individueller Kompetenzen annähernd widerspiegelt. Ein weiterer Vorteil des Ansatzes liegt in der Operationalisierung der einzelnen Kompetenzarten: Es werden 27 Kompetenzindikatoren identifiziert und nach einer faktorenanalytisch bestätigten Struktur kategorisiert. ${ }^{445}$ Unter der An-

fragebogen von Stäudel (1988), die Frankfurter Selbstkonzeptskalen von Deusinger (1986), sowie den Problemlösefragebogen von Holling et al. (1980).

439 Vgl. Thomas (1992), S. 22

440 Vgl. Hacker (1992); Ulich (1998); Bergmann (2007), S. 195

441 Frei et al. (1984), S. $103 \mathrm{f}$.

442 Vgl. Bergmann (2007), S. 195

443 Allgemeinerer Art sind hingegen die Frankfurter Selbstkonzeptskalen von Deusinger (1986), die auf die Erfassung der Selbsteinschätzung von zehn Handlungs- und Lebensbereichen abzielen.

444 Vgl. dazu Holling et al. (1980), sowie Stäudel (1988).

445 Vgl. Sonntag/Schäfer-Rauser (1993), S. 165 f. Die empirische Überprüfung des Analyseinstruments zur Erfassung des Selbstkonzepts beruflicher Kompetenz erfolgte im Rahmen einer explorativen Feldstudie $(\mathrm{N}=152)$, wobei neben der Faktorenstruktur auch die interne Konsistenz der Kompetenz- 
nahme einer grundsätzlich notwendigen Kongruenz zwischen den Kompetenzanforderungen des Unternehmens A an seine Mitarbeiter bzw. Kooperationsbeauftragten und den Kompetenzerwartungen des potentiellen Kooperationspartners B an die Akteure des Unternehmens A im Rahmen von dessen Vertrauensentscheidung werden die tabellarisch erfassten Kompetenzdimensionen und -indikatoren ${ }^{446}$ im Zuge der in Teil IV dieser Arbeit verfolgten Operationalisierung der individuellen Kompetenzen berücksichtigt.

\begin{tabular}{|c|c|c|}
\hline Fachkompetenz & Methodenkompetenz & Sozialkompetenz \\
\hline $\begin{array}{l}\text { Fertigkeiten } \\
\text { - } \quad \text { berufspraktische } \\
\text { Fertigkeiten } \\
\text { - Handhabung von } \\
\text { Arbeitsgeräten } \\
\text { - Genauigkeit, Sorg- } \\
\text { falt und Geschick- } \\
\text { lichkeit bei der } \\
\text { Arbeitsausführung }\end{array}$ & $\begin{array}{l}\text { Denken und Problemlösen } \\
\text { - } \quad \text { abstraktes Denken } \\
\text { - Diagnostizieren, Fehler- } \\
\text { suche } \\
\text { - Problemlösen, Fehler- } \\
\text { beseitigung } \\
\text { - Informationsbeschaffung } \\
\text { - Planung, Kontrolle, } \\
\text { Bewertung }\end{array}$ & $\begin{array}{l}\text { Kommunikation } \\
\text { - } \quad \text { Kontaktfähigkeit } \\
\text { - } \text { Fähigkeit } \\
\text { Unterstützung/Hilfe zu } \\
\text { beantragen } \\
\text { - Vertretung des eigenen } \\
\text { Standpunktes, } \\
\text { Meinungsäußerung } \\
\text { - Informationsweitergabe }\end{array}$ \\
\hline \multirow[t]{2}{*}{$\begin{array}{l}\text { Kenntnisse } \\
\text { - } \quad \text { berufsspezifische } \\
\text { Kenntnisse } \\
\text { Wissen über } \\
\text { Arbeitsabläufe, } \\
\text { Geräte und } \\
\text { Maschinen } \\
\text { - Fachtermini } \\
\text { - Kenntnis der } \\
\text { Arbeitsschutzmaß- } \\
\text { nahmen }\end{array}$} & $\begin{array}{l}\text { Kreativität } \\
\text { - } \quad \text { Einfallsreichtum } \\
\text { - } \quad \text { Flexibilität } \\
\text { - } \quad \text { Interesse, Neues aus- } \\
\quad \text { zuprobieren }\end{array}$ & $\begin{array}{l}\text { Kooperation } \\
\text { - Fähigkeit zur Zusam- } \\
\text { menarbeit und } \\
\text { Gruppenarbeit } \\
\text { - } \text { aktive Mitarbeit und } \\
\text { Durchsetzungsfähigkeit } \\
\text { in Gruppen } \\
\text { - Konfliktfähigkeit } \\
\text { - } \quad \text { Hilfsbereitschaft, } \\
\text { Kollegialität }\end{array}$ \\
\hline & $\begin{array}{l}\text { Lernfähigkeit } \\
\text { - } \quad \text { Allgemeine Lernfähigkeit } \\
\text { - } \quad \text { Gedächtnis, Merkfähigkeit } \\
\text { - } \quad \text { Arbeits- und Lerntechniken } \\
\text { - } \quad \text { Auffassungsgabe }\end{array}$ & \\
\hline
\end{tabular}

Tabelle 10: Indikatoren individueller Kompetenz nach Sonntag/Schäfer-Rauser

Quelle: In Anlehnung an Sonntag/Schäfer-Rauser (1993, S. 165)

dimensionen bestätigt wurde. Folgende Gütekriterien würde dabei erhoben: Mittelwerte, Standardabweichungen, Schwierigkeitsindizes, Trennschärfeindizes und Reliabilitätskoeffizienten.

446 Die tabellarisch aufgelisteten 27 Kompetenzindikatoren stellen eine Grobklassifikation der dem Analyseinstrument zugrundeliegenden 57 Items dar. Die Selbsteinschätzung der Kompetenzen anhand ihrer Indikatoren, erfolgte auf einer 6-stufigen Ratingskala. Im Zuge der in dieser Arbeit verfolgten Operationalisierung der individuellen Kompetenzen wird auf die Grobklassifikation Bezug genommen. 


\section{Die Kompetenzbiographie}

Im Rahmen dieses Ansatzes von Erpenbeck und Heyse (2007) wird Kompetenz als Disposition zur Selbstorganisation menschlichen Handelns verstanden. ${ }^{447}$ Folglich stellen die individuellen Kompetenzarten innerpsychische Voraussetzungen dar, um bei der Erfüllung von Aufgaben geistig und physisch (Fachkompetenz), aktiv und gesamtheitlich (Methodenkompetenz), kommunikativ und kooperativ (Sozialkompetenz) und reflexiv (Personalkompetenz) selbstorganisiert handeln zu können. ${ }^{448}$

Bezugnehmend auf ihre Messung lassen sich individuelle Kompetenzen, im Gegensatz zu Fertigkeiten und Qualifikationen, die direkt prüfbar sind, nur aus der Realisierung der Dispositionen, d.h. anhand des Grades der Aufgabenerfüllung, rückblickend erschließen. Sie können Erfahrungen, Willenskomponenten, Fähigkeiten, Wissen und Werte beinhalten, lassen sich aber auf diese Konstrukte nicht reduzieren, sondern schließen sie in verfügungs- und handlungsrelevante Beziehungen ein. Zusammenfassend werden Kompetenzen von Wissen fundiert, durch Werte konstituiert, als Fähigkeiten disponiert, durch Erfahrungen konsolidiert und auf Grund von Willen realisiert. Zielpunkt der Kompetenzentwicklung ist daher selbstorganisierte Handlungsfähigkeit. ${ }^{449}$

Zur Erfassung der individuellen Kompetenzen sieht der Ansatz von Erpenbeck und Heyse (2007) einen subjektiven Messzugang vor. Mit Hilfe einer qualitativen biographischen Analysemethode werden die Einschätzungen der Kompetenzinhaber bezüglich ihrer Kompetenzen erhoben. Das Ziel dieser Vorgehensweise besteht in der Erfassung einer individuellen Kompetenzbiographie, die ,[...] die qualitative und quantitative Entfaltung menschlicher Handlungskompetenz als komplexes, selbstorganisiertes Netzwerk fachlicher, methodischer, sozialer und personaler Einzelkompetenzen in der stets einzigartigen, lebenslangen real-biographischen Entwicklung “450 darstellt. Dieser Ansatz zur Identifikation individueller Kompetenzen beruht auf der Annahme, dass Individuen in komplexen, nicht vollständig vorhersagbaren Situationen sich selbst Ziele setzen, Pläne und Strategien zu ihrer Erreichung entwerfen und erproben, aus den daraus resultierenden Erfahrungen lernen und dementsprechend als Produzenten ihrer Kompetenzbiographie diese analysieren können. ${ }^{451}$ Vor diesem Hintergrund wird die Relevanz der Kompetenzbiographie-Forschung damit begründet, dass die in der Berufs- und Wirtschaftspädagogik interessierende Evaluation und prospektive Beurteilung des Kompetenzentwicklungsbedarfs stets an einer retrospektiven Beur-

\footnotetext{
447 Vgl. Erpenbeck (2007), S. 489; Erpenbeck/Rosenstiel (2007), S. XIX

$448 \quad$ Vgl. Baitsch (1996), S. 102 f.; Erpenbeck/Rosenstiel (2007), S. XXIV

449 Vgl. Erpenbeck (2007), S. 489 f.; Erpenbeck/Heyse (2007), S. 163

450 Erpenbeck/Heyse (2007), S. 228

451 Vgl. Erpenbeck/Heyse (2007), S. 164. Ähnlich dazu z.B. Hacker (1992); Ulich (1998); Bergmann (2007)
} 
teilung ansetzen muss, d.h. an einer Rekonstruktion der biographischen Ereignisse, im Rahmen derer Individuen ihre Kompetenzen erworben haben. ${ }^{452}$

Bei dem Verfahren zur Erfassung der individuellen Kompetenzbiographie handelt es sich um ein narratives Interview, das Elemente des problemzentrierten und des fokussierten Interviews einbezieht, allerdings in Form von Selbstzentrierung und Selbstfokussierung durch den interviewten Kompetenzinhaber. ${ }^{453}$ Im Rahmen dieses Interviews werden die persönlichen Stärken hinsichtlich der beruflichen Tätigkeit, sowie deren biographischer Entwicklung erfasst. Der qualitativen Auswertung dieser individuellen Stärkenprofile liegt die Unterteilung in die vier Arten Fach-, Methoden-, Sozial- und Persönlichkeitskompetenz zugrunde. Für die in Teil IV dieser Arbeit verfolgte Operationalisierung individueller Kompetenzen liefern die in der empirischen Studie von Erpenbeck und Heyse erfassten Kompetenzprofile $^{454}$ - unter der Annahme der grundsätzlich notwendigen Kongruenz zwischen den Kompetenzanforderungen des Unternehmens A an seine Mitarbeiter bzw. Kooperationsbeauftragten und den Kompetenzerwartungen des potentiellen Kooperationspartners B an die Akteure des Unternehmens A im Rahmen seiner Vertrauensentscheidung - wichtige inhaltliche Ansatzpunkte. Selbiges gilt für den im Zuge der qualitativen Folgeerhebung eingesetzten Kompetenzfragebogen, der der Vervollständigung der Ergebnisse des narrativen Interviews und der Selbsteinschätzung der Kompetenzinhaber unter Berücksichtigung eines Zeitintervalls dient. Für seine Eignung im vorliegenden Untersuchungskontext spricht vor allem, dass er die in Kapitel 2.2 vorgestellte Grundstruktur individueller Kompetenzen widerspiegelt. Ein weiterer Vorteil liegt in der Operationalisierung der einzelnen Kompetenzarten: Es wird eine Vielzahl an Indikatoren aus unterschiedlichen Formen selbstorganisierten biographischen Handelns abgeleitet und den Kompetenzarten zugeordnet. ${ }^{455}$ Unter dem Vorbehalt der testkritischen Prüfung des Erhebungsinstruments werden die tabellarisch erfassten Kompetenzindikatoren im Zuge der in Teil IV dieser Arbeit verfolgten Operationalisierung berücksichtigt.

$452 \mathrm{Zu}$ den biographischen Ereignissen zählen beispielsweise Konflikte und Krisensituationen, die Individuen durchlebt haben (Erpenbeck/Heyse 2007, S. 201).

453 Vgl. Erpenbeck/Heyse (2007), S. 228

454 Die Stichprobe umfasste Unternehmensgründer $(\mathrm{N}=20)$, die so ausgewählt wurden, dass ihre Kompetenzen durch den persönlichen Erfolg als Unternehmer und durch den Unternehmenserfolg objektiv dokumentiert sind (Erpenbeck/Heyse 2007, S. 229 ff.). Die empirisch erfassten Kompetenzprofile weisen demnach einen idealtypischen Charakter auf, was ihre Eignung für den vorliegenden Untersuchungskontext unterstreicht. Auf ihre Darstellung wird an dieser Stelle verzichtet. Die im Zuge der Modellentwicklung berücksichtigten Einzelkompetenzen finden sich als Indikatornachweise in Teil IV dieser Arbeit.

455 Vgl. Erpenbeck/Heyse (2007), S. 158 ff., sowie S. 240 ff. Die Selbsteinschätzung der Kompetenzen erfolgt anhand einer 10-stufigen Ratingskala. 


\section{Fachkompetenzen}

selbstorganisativ-dispositionell verfugbar

- technisches Wissen

- kaufmännisches Wissen

- fachübergreifendes Wissen

- Anwendungserfahrung

- flexible Informationsverarbeitung

- Abstraktionsfähigkeit

- Vermittlungs- und Darstellungsfähigkeit

\section{Sozialkompetenzen}

selbstorganisativ-dispositionell verfugbar

- sozial akzeptierter Umgang mit sich selbst; Selbststeuerung und Rollendistanz

- Verantwortungsbewusstsein gegenüber Kollegen, dem Unternehmen, der Gesellschaft

- Verantwortungsgefühl gegenüber Moral und Wertvorstellungen

- Kooperationsfähigkeit, Kommunikationsfähigkeit, Integrationsfähigkeit, Solidarität, Einfühlungsvermögen

- Verhaltens-, Konflikt- und Rollenflexibilität, Mitteilungsfähigkeit von Gefühlen und Gedanken

- Überzeugungsfähigkeit, Charisma ${ }^{456}$. Wirkung

- Führungsfähigkeit von Gruppenprozessen. Ausschöpfung der Fähigkeiten aller Beteiligten

\section{Methodenkompetenzen}

selbstorgansiativ-dispositionell verfuggbar

- Methoden der Informationsstrukturierung und -darstellung

- Management und Leistungsmethoden; Zielanalysen

- Prioritätensetzung, Kontrolle und Planung

- Problemlösungs-, Lern- und Kreativmethoden

- Führungsmethoden, Umgang mit Mitarbeitern, Gruppensteuerung und Konsensbildung

- Methoden des Zeit- und des Selbstmanagements sowie der Selbstreflexion

\section{Personale Kompetenzen}

selbstorganisativ-dispositionell verfügbar

- ein zutreffendes Selbstkonzept, Selbstvertrauen, Selbstwertgefühl, ein gesundes Dominanzstreben

- kritische Selbstwahrnehmung in Wechselwirkung mit dem sozialen Umfeld

- konstruktiver Umgang mit Unsicherheiten, Ängsten, irritierenden Emotionen und Zielkonflikten

- authentische Werte. Interessen, Pflicht- und Sinnvorstellungen. Problembetrachtung von höherer Warte aus

- Selbstdisziplin, geistige Konzentration, produktive Begeisterungsfähigkeit

- Tatkraft, Ehrgeiz, Zielstrebigkeit. Experimentierfreudigkeit, Risikoverhalten, Effizienzstreben, persönliche Energie

Tabelle 11: Indikatoren individueller Kompetenz nach Erpenbeck/Heyse Quelle: In Anlehnung an Erpenbeck/Heyse (2007, S. 161 und 214 f.) 
Die weiteren, im Rahmen der Folgestudie eingesetzten Erhebungsinstrumente (Wertefragebogen, LIFO®-Fragebogen, sowie der Fragebogen zum organisierten und selbstorganisierten Lernen auf die Kompetenzentwicklung ${ }^{457}$ ) liefern vorrangig Erkenntnisse bezüglich der Kompetenzentwicklung, weshalb sie im Rahmen dieser Arbeit nicht berücksichtigt werden.

\section{Das Kasseler-Kompetenz-Raster}

Im Rahmen dieses Ansatzes von Kauffeld, Grote und Frieling (2007) wird Kompetenz als ein differenziertes System von Fähigkeiten, Fertigkeiten und Wissensbeständen verstanden, die eine Person, eine Gruppe oder eine Organisation dazu befähigen, bei der Bewältigung von konkreten Arbeitsaufgaben erfolgreich zu agieren und zu reagieren. Wie auch Erpenbeck und Heyse im zuvor beschriebenen Ansatz, gehen Kauffeld, Grote und Frieling davon aus, dass sich individuelle Kompetenzen als Handlungsvoraussetzungen erst im Handlungsprozess offenbaren. Bezogen auf den beruflichen Kontext zeigen sie sich in Form beobachtbarer, aufgabengebundener Verhaltensweisen zur effektiven Bewältigung spezifischer beruflicher Aufgaben. ${ }^{458}$

Folglich setzt der Ansatz von Kauffeld, Grote und Frieling, im Gegensatz zu den zuvor erläuterten Kompetenzmessverfahren, nicht an einer Selbsteinschätzung der Kompetenzinhaber an, sondern zielt darauf ab, die individuellen Kompetenzen im jeweiligen Handlungskontext zu erfassen. Da das organisationale Geschehen in einem engen Zusammenhang mit der Arbeit in Gruppen steht, wird mit dem Kasseler-Kompetenz-Raster die Gruppe als Analyseebene zur Kompetenzmessung fokussiert. Das Interesse gilt hierbei der Bewältigung von Optimierungsaufgaben: Nach einer detaillierte Beobachtung der Gruppenmitglieder bei der Bewältigung dieser Optimierungsaufgaben werden deren verbale Verhaltensäußerungen nach den Kriterien des Kasseler-Kompetenz-Rasters, das sich auf die Bereiche der Fach-, Methoden-, Sozial- und Selbstkompetenz aufteilen lassen, ausgewertet. ${ }^{459}$ Für die Eignung des Kasseler-Kompetenz-Rasters im vorliegenden Untersuchungskontext spricht, dass es die in Kapitel 2.2 vorgestellte Grundstruktur individueller Kompetenzen, die zwischen Fach-, Methoden-, Sozial- und Persönlichkeits- (hier: Selbstkompetenz) Kompetenz widerspiegelt. Ein weiterer Vorteil liegt in der Operationalisierung der einzelnen Kompetenzarten: Es werden ca. 50

457 Vgl. Erpenbeck/Heyse (2007), S. 226 f. Da Kompetenzen durch Werte konstituiert werden, wird der Wertefragebogen eingesetzt, um ein Polaritätsprofil zu Werthaltungen und Wertentwicklungen zu generieren. Der LIFO®-Fragebogen, der später durch die Kode®-Methode ersetzt wird (Erpenbeck 2007, S. 491 f.), gibt Aufschluss über das individuelle Kompetenzmanagement und über die Kompetenzentwicklung. Abschließend wird mit dem Fragebogen zum Einfluss von organisiertem und selbstorganisiertem Lernen die Art und Weise des Kompetenzerwerbs eruiert. Im Zentrum stehen dabei die Formen des Kompetenzlernens (Erpenbeck/Heyse 2007, S. 226).

$458 \quad$ Vgl. Kauffeld et al. (2007), S. 224

459 Vgl. Kauffeld et al. (2007), S. 225 f. 
prozess- bzw. verlaufsorientierte Indikatoren, die zur Bewältigung von Aufgaben, z.B. zur Aufrechterhaltung einer zwischenbetrieblichen Kooperationsbeziehung, erforderlich sind, identifiziert und den Kompetenzarten zugeordnet.

\begin{tabular}{|c|c|c|}
\hline Kompetenzarten & $\begin{array}{l}\text { kompetenzrelevante } \\
\text { Verhaltensäußerungen }\end{array}$ & Kompetenzindikatoren \\
\hline Fachkompetenz & $\begin{array}{l}\text { Äußerungen zu Lösungen } \\
\text { Äußerungen zu } \\
\text { Organisation } \\
\text { Äußerungen zu Wissens- } \\
\text { management }\end{array}$ & $\begin{array}{l}\text { zur Differenziertheit des Problems: } \\
\text { (Teil-) Problem benennen; Problem } \\
\text { erläutern und veranschaulichen } \\
\text { zur Vernetztheit des Problems: } \\
\text { Ursachen und Folgen des Problems } \\
\text { bei der Problemanalyse veranschau- } \\
\text { lichen } \\
\text { zur Differenziertheit der Lösung: } \\
\text { Visionen und Anforderungen be- } \\
\text { schreiben (Sollentwurf); (Teil-) } \\
\text { Lösungen benennen (Lösungs- } \\
\text { vorschlag); Lösung veranschau- } \\
\text { lichen (Lösungserläuterung) } \\
\text { zur Vernetztheit der Lösung: } \\
\text { Einwände gegen Lösung erheben: } \\
\text { Vorteile der Lösung benennen } \\
\text { Wissen über Organisation und } \\
\text { Abläufe } \\
\text { Verweis auf Spezialisten; Fragen } \\
\text { nach Meinung, Inhalt und Erfahrung }\end{array}$ \\
\hline $\begin{array}{l}\text { Methoden- } \\
\text { kompetenz }\end{array}$ & $\begin{array}{l}\text { positive Äußerungen zur } \\
\text { Strukturierung } \\
\text { negative Äußerungen zur } \\
\text { Strukturierung }\end{array}$ & $\begin{array}{l}\text { Zielorientierung; Klärung und } \\
\text { Konkretisierung von Beiträgen; } \\
\text { Verfahrensvorschlag; Priorisierung; } \\
\text { Zeitmanagement; Vornahme Auf- } \\
\text { gabenverteilung; Visualisierung; } \\
\text { Kosten-Nutzen-Abwägung; Zu- } \\
\text { sammenfassung der Ergebnisse } \\
\text { Verlieren in Details und Beispielen } \\
\text { (z.B. Monologe, nicht zielführende } \\
\text { Beispiele) }\end{array}$ \\
\hline Sozialkompetenz & $\begin{array}{l}\text { positive, wertende } \\
\text { Äußerungen gegenüber } \\
\text { Personen oder ihren } \\
\text { Handlungen }\end{array}$ & $\begin{array}{l}\text { ermunternde Ansprache; Unter- } \\
\text { stützung durch Zustimmung; aktives } \\
\text { Zuhören; sachliche Ablehnung; } \\
\text { Rückmeldung; atmosphärische Auf- } \\
\text { lockerung; Ich-Botschaft zur Kenn- }\end{array}$ \\
\hline
\end{tabular}




\begin{tabular}{|l|l|l|}
\hline & & $\begin{array}{l}\text { zeichnung der eigenen Meinung; } \\
\text { Aussprechen der Gefühle; positive } \\
\text { Äußerungen gegenüber Anderen } \\
\text { Abwertung von Anderen; } \\
\text { Unterbrechung anderer; Seiten- } \\
\text { gespräche; Verweis auf die eigene } \\
\text { Erfahrung zur autoritären Beein- } \\
\text { flussung }\end{array}$ \\
\hline $\begin{array}{l}\text { negative, wertende } \\
\text { Äußerungen gegenüber } \\
\text { Personen ihren } \\
\text { Handlungen }\end{array}$ & $\begin{array}{l}\text { Interesse an Veränderungen; Über- } \\
\text { nahme von Eigenverantwortung; } \\
\text { Vereinbarung von Aufgaben zur } \\
\text { Umsetzung }\end{array}$ \\
& $\begin{array}{l}\text { positive Äußerungen zur } \\
\text { Mitwirkung }\end{array}$ & $\begin{array}{l}\text { kein Interesse an Veränderungen; } \\
\text { Betonung des negativen Ist- } \\
\text { Zustandes (Schwarzmalerei); } \\
\text { inhaltsloses Gerede; Person- } \\
\text { alisierung von Problemen; Betonung } \\
\text { autoritärer Elemente; vorzeitiger } \\
\text { Abbruch von Diskussionen }\end{array}$ \\
\hline
\end{tabular}

Tabelle 12: Indikatoren individueller Kompetenz nach Kauffeld/Grote/Freiling Quelle: In Anlehnung an Kauffeld et al. (2007, S. 231)

Für eine Berücksichtigung des Kasseler-Kompetenz-Rasters im Rahmen der in Teil IV dieser Arbeit verfolgten Operationalisierung individueller Kompetenzen spricht auch, dass die von Kauffeld, Grote und Freiling identifizierten Indikatoren empirisch bestätigt wurden. ${ }^{460}$

Mit Blick auf die im Rahmen dieser Arbeit verfolgte Modellentwicklung (vgl. Teil IV) lässt sich resümieren, dass die erläuterten Ansätze (vgl. Tabelle 13) aufgrund ihrer individualzentrierten Untersuchungsperspektive wichtige inhaltliche Ansatzpunkte zur Identifikation individueller Kompetenzen liefern. Um die Ableitung konsensfähiger und gültiger Indikatoren, die als empirische Äquivalente der Fach-, Methoden-, Sozial- und Persönlichkeitskompetenz fungieren, sekundäranalytisch abzusichern, wird im Zuge der Operationalisierung Bezug auf

460 Die Praktikabilităt des Kompetenzmessverfahrens wurde im Zuge einer empirischen Studie zur „Bewältigung von Optimierungsaufgaben“" in drei kommunalen Versorgungsunternehmen $(\mathrm{N}=37)$ und in zehn Industrieuntemehmen $(\mathrm{N}=145)$ bestätigt. Die Reliabilität des Instruments wird durch die für Beobachtungsverfahren hohen Inter-Rate-Übereinstimmungen von Cohens Kappa $=.60$ für wenig geschulte Beurteiler bis Cohens Kappa $=.90$ für Experten in der Anwendung des KasselerKompetenz-Rasters (KKR) belegt. Dementsprechend kann davon ausgegangen werden, dass die zur Kodierung dienenden Kompetenzindikatoren des KKR wenig Interpretationsspielraum zulassen. Die Validität des Verfahrens wird firmenübergreifend durch eine Vielzahl empirischer Kontrastgruppenvergleiche abgesichert (Kauffeld et al. 2000, S. 213; Kauffeld et al. 2007, S. 226). 
weitere für diese Arbeit relevante Beiträge im Bereich der individuellen Kompetenzforschung genommen.

\begin{tabular}{|c|c|c|c|}
\hline Autor & $\begin{array}{c}\text { Ziel der } \\
\text { Untersuchung }\end{array}$ & $\begin{array}{c}\text { Relevanz für die } \\
\text { Operationalisierung } \\
\text { individueller Kompetenz }\end{array}$ & $\begin{array}{c}\text { Auswertung } \\
\text { (Datengrundlage } \\
\text { ) }\end{array}$ \\
\hline $\begin{array}{l}\text { Sonntag/ } \\
\text { Schăfer- } \\
\text { Rauser (1993) }\end{array}$ & $\begin{array}{l}\text { Ermittlung eines } \\
\text { Selbstkonzepts } \\
\text { beruflicher } \\
\text { Kompetenz }\end{array}$ & $\begin{array}{l}3 \text { Kompetenzdimensionen; } \\
27 \text { Kompetenzindikatoren }\end{array}$ & $\begin{array}{l}\text { Faktoren-, Skalen- } \\
\text { und Itemanalyse } \\
\text { zur Überprüfung } \\
\text { des standar- } \\
\text { disierten Analyse- } \\
\text { instruments SBK } \\
(\mathrm{N}=152)\end{array}$ \\
\hline $\begin{array}{l}\text { Erpenbeck/ } \\
\text { Heyse (2007) }\end{array}$ & $\begin{array}{l}\text { Ermittlung einer } \\
\text { individuellen } \\
\text { Kompetenz- } \\
\text { biographie }\end{array}$ & $\begin{array}{l}4 \text { Kompetenzdimensionen: } \\
\text { ca. } 26 \text { Kompetenz- } \\
\text { indikatoren }\end{array}$ & $\begin{array}{l}\text { qualitative Aus- } \\
\text { wertung der } \\
\text { narrativen Inter- } \\
\text { views; deskriptive } \\
\text { Auswertung des } \\
\text { Kompetenz- } \\
\text { fragebogens } \\
(\mathrm{N}=40)\end{array}$ \\
\hline $\begin{array}{l}\text { Kauffeld/ } \\
\text { Grote/ } \\
\text { Freiling } \\
(\mathbf{2 0 0 7 )}\end{array}$ & $\begin{array}{l}\text { verlaufsorientierte } \\
\text { Ermittlung } \\
\text { individueller } \\
\text { Kompetenz im } \\
\text { Kontext der } \\
\text { Handlungssituation }\end{array}$ & $\begin{array}{l}4 \text { Kompetenzdimensionen; } \\
\text { ca. } 50 \text { Kompetenz- } \\
\text { indikatoren }\end{array}$ & $\begin{array}{l}\text { qualitative Aus- } \\
\text { wertung der kom- } \\
\text { petenzrelevanten } \\
\text { Verhaltens- } \\
\text { äußerungen mit } \\
\text { Hilfe des Kas- } \\
\text { seler-Kompetenz- } \\
\text { Rasters }(\mathrm{N}=182)\end{array}$ \\
\hline
\end{tabular}

Tabelle 13: Ansătze zur Identifikation individueller Kompetenz

\section{Die Bedeutung Von Kompetenz AUS VERSCHIEDENEN PERSPEKTIVEN}

In den vorangegangen Kapiteln wurde Kompetenz begrifflich definiert und von verwandten Konstrukten abgegrenzt. Weiters wurden die Kompetenzarten auf organisationaler und individueller Ebene verrastert und erläutert, sowie einer Operationalisierung zugänglich gemacht. Da bei der Erarbeitung des Wirkungsmodells (vgl. Teil IV), d.h. bei der Entwicklung des Bezugsrahmens der Wirkung von Kompetenz auf die Entstehung von Vertrauen, ein Rückgriff auf Erkenntnisse der Soziologie und der Neuen Institutionenökonomie erfolgt, wird nachfolgend das Kompetenzkonstrukt aus der Sicht der soziologischen Handlungstheorie und aus der Perspektive der Principal-Agent-Theorie betrachtet. 


\subsection{Kompetenz aus Sicht der soziologischen Handlungstheorie}

Da sich die persönlichkeitstheoretischen Ansätze vorrangig mit der Entstehung und Messung von Kompetenz beschäftigen, dies aber nicht zu einer Erklärung ihres Einflusses im Rahmen der Entstehung von Vertrauensbeziehungen und ihrer Bedeutung beiträgt, ist eine Konzentration auf Konzepte, die sich mit der sozialen Ebene befassen, erforderlich. Soziale Phänomene sind die Domäne der Soziologie. Insofern steht nachfolgend die soziologische Perspektive auf das Kompetenzkonstrukt, die sich stark an philosophischen, anthropologischen und ethischen Kompetenzverständnissen orientiert, im Vordergrund. Aus soziologischer Sicht kann Kompetenz als sozial zugeschriebene Qualität verstanden werden, die sich über Kommunikation und Interaktion manifestiert bzw. als sich manifestierend dem Akteur attestiert wird. Dementsprechend ist die soziologische Kompetenzforschung unmittelbar mit Überlegungen zu Theatralität, Performanz und Inszenierung verbunden. Das Kompetenzkonstrukt wird aber nicht bloß von Inszenierungstheoretikern wie Goffman und Kommunikationstheoretikern wie Habermas erläutert, es ist vielmehr auch Gegenstand der Organisationsüberlegungen von Weber und Luhmann, sowie der Professionstheorie von Parsons und der Analysen des Verhältnisses von Bildung und sozialer Ungleichheit von Bourdieu. Aufgrunddessen, dass Kompetenz im Rahmen der vorliegenden Arbeit als handlungstheoretisches Konstrukt verstanden wird, das nur über das Handeln, d.h. im Zuge der Aufgabenerfüllung, erschlossen werden kann, bietet sich die Option, die Bedeutung von Kompetenz aus handlungstheoretischer Perspektive zu untersuchen.

Ausgangspunkt der Handlungstheorie ist die Überlegung, dass dem Handeln sozialisierter Akteure allgemeine Regeln, Werte und Normen zugrunde liegen, die auch in anderen Handlungsbereichen von Relevanz sind. Zentral ist daher die Auseinandersetzung mit sinnorientiertem Handeln interagierender Akteure. Durch die Verbindung mit der Sinnorientierung lässt sich das Handlungskonstrukt vom Verhaltenskonstrukt abgrenzen. Während das Verhalten instinktive, durch Reize ausgelöste Aktivitäten bezeichnet, erfolgt das Handeln intentional und zielgerichtet. Es unterscheidet sich vom Verhalten durch subjektiven Sinn, Intentionalität des Vorgehens und die Reflexion eintretender Handlungskonsequenzen. Aufgrund der handlungstheoretischen Annahme, dass das Handeln eines Akteurs mit dem Handeln anderer Akteure verbunden ist, wird im Rahmen dieser Theorie das Handeln eines Akteurs im Hinblick auf die Beziehung zu anderen Akteuren analysiert. Werden beispielsweise Kooperationen aus Sicht der Handlungstheorie untersucht, so werden diese nicht als isolierte Systeme, sondern als integrierte Teilbereiche der gesamten Gesellschaft verstanden. Kooperative Prozesse stellen daher auch immer gesellschaftliche Prozesse dar. Gemäß dieser Perspektive erscheinen Kooperationen als Handlungsfelder, die durch soziale Mechanismen re- 
guliert werden, welche gleichzeitig auch auf andere soziale Bereiche wirken ${ }^{461}$ Aufgrund der zentralen Bedeutung des Kriteriums der Interaktion im Rahmen dieser Theorie werden nachfolgend die interaktionistisch orientierten Überlegungen der Soziologen Weber, Parsons und Habermas zu sinnorientiertem Handeln einer näheren Betrachtung unterzogen. Die Auseinandersetzung mit den Kategorien Handeln und Sinn ist notwendig, um die zentralen Bezugspunkte zu Kompetenz, von der ausgegangen wird, dass sie sich im Handeln zeigt, und ihre Bedeutung im Rahmen von zwischenbetrieblichen Kooperationen herausstellen zu können.

\section{Der Ansatz von Weber}

Handeln wird je nach Handlungstheorie unterschiedlich definiert. Ausgehend davon, dass sich Kompetenz im Handeln zeigt, ist es von hoher Relevanz, zu eruieren, wie Handeln zustande kommt. Nach Weber, dem Begründer der klassischen soziologischen Theorie des Handelns, kennzeichnet sich das Handlungskonstrukt durch seine Verbindung mit Sinn ${ }^{462}$ Ausgehend von der Prämisse, dass Handelnde mit ihrem Tun oder Unterlassen einen Sinn verbinden, der sich aus kulturellen Vorgaben wie Tradition, Brauch oder Sitte erschlie $\beta \mathrm{At}^{463}$, kann Handeln nur im Zusammenhang mit Sinn erklärt werden. Generell ist zwischen Handeln und sozialem Handeln zu differenzieren, wobei soziales Handeln als solches Handeln, ,welches seinem von dem oder den Handelnden gemeinten Sinn nach auf das Verhalten anderer bezogen wird und daran in seinem Ablauf orientiert ist ${ }^{\text {" } 464}$ zu verstehen ist. Soziales Handeln orientiert sich demnach an vergangenem, gegenwärtigem oder zukünftigem Handeln anderer Akteure. Bezugnehmend auf die Gründe, die soziales Handeln bestimmen, unterscheidet Weber vier Typen. ${ }^{465}$ So ist Handeln zweckrational, wenn ihm eine Orientierung an Zweck, Mittel und Nebenfolgen zugrunde liegt, im Rahmen derer „sowohl die Mittel gegen die Zwecke, wie die Zwecke gegen die Nebenfolgen, wie endlich auch die verschiedenen möglichen Zwecke gegeneinander “466 abgewogen werden. Weitere Kennzeichen dieses Handlungstyps sind, dass das Handeln rein aus dem Nutzenkalkül einer individuellen Interessenslage erfolgt, und dass es von Akteuren abstrahiert, d.h. es setzt unpersönliche Beziehungen voraus, sowie dass alle Rationalitätskriterien operationalisierbar sind. ${ }^{467}$ Bezugnehmend auf Kooperationsbeziehungen zeigt sich zweckrationales Handeln darin, dass die Beziehungen der be-

\footnotetext{
$461 \quad$ Vgl. Kaufhold (2006), S. 77; Buß (2008), S. 136

462 Weber (1980, S. 1) definiert Handeln als „ein menschliches Verhalten (einerlei ob äußeres oder innerliches Tun, Unterlassen oder Dulden) [...], wenn und insofern als der oder die Handelnden mit ihm einen subjektiven Sinn verbinden".

463 Vgl. Abels (2007b), S. $134 \mathrm{f}$.

464 Weber (1980), S. 1

465 Vgl. Abels (2007b), S. 145

466 Weber (1972), S. 13

467 Vgl. Buß (2008), S. 136
} 
teiligten Unternehmen nicht auf moralischem Engagement und sozialen Verpflichtungen beruhen, sondern auf den Bedingungen des unpersönlichen und emotionsfreien, objektiven Vergleichs von Leistungen und Gegenleistungen, womit auf organisationaler Ebene sowohl die Basis- als auch die Kernkompetenzen, sowie auf individueller Ebene vorrangig die fachlichen und methodischen Kompetenzen der Kooperationspartner von Relevanz sind. Handeln ist wertrational, wenn unbedingte Eigenwerte unabhängig vom Handlungserfolg leitend sind. Wertrationales Handeln kann damit als Handeln aus einem Gesinnungsprinzip oder aus einem absoluten Pflichtgefühl aufgefasst werden. ${ }^{468}$ Bezugnehmend auf Kooperationsbeziehungen liegt wertrationales Verhalten vor, wenn sich die beteiligten Unternehmen selbst dazu verpflichten, auf das Ausnützen von Opportunismusspielräumen zu verzichten. Eine hohe Bedeutung kann in diesem Zusammenhang den sozialen und persönlichen Kompetenzen der Kooperationspartner zugeschrieben werden. Handeln ist traditionell, wenn es einer überlieferten Einstellung oder Routine folgt. Sowohl die Handlungsziele, als auch der Handlungsverlauf sind an traditionelle Gewohnheitsnormen gebunden und kennzeichnen sich durch einen niedrigen Reflexionsgrad. ${ }^{469}$ Bezugnehmend auf Kooperationsbeziehungen liegt traditionelles Handeln vor, wenn das Ziel der Bestandssicherung handlungsleitend ist und die Handlungsmaßstäbe der Kooperationspartner an traditionelle Gewohnheitsnormen gebunden sind. In diesem $\mathrm{Zu}-$ sammenhang sind auf organisationaler Ebene vorrangig Basiskompetenzen von Bedeutung. Affektuelles Handeln liegt vor, wenn eine unreflektierte Reaktion auf besondere Reize oder Situationsumstände erfolgt. Dieses emotionale Handeln ist durch gegenwärtige Gefühlslagen bestimmt. ${ }^{470}$ Bezugnehmend auf Kooperationsbeziehungen würde affektuelles Handeln vorliegen, wenn sich Kooperationspartner aufgrund ihrer Gefühle, und nicht aufgrund von Kosten-Nutzen-Überlegungen für das Annehmen eines Auftrags entscheiden. In diesem Zusammenhang sind vor allem die soziale und persönliche Kompetenz des Auftraggebers von Bedeutung.

Aufgrund Webers Auffassung, dass sowohl traditionelles, als auch affektuelles Handeln an der Grenze dessen stehen, was bewusst sinnhaftes Handeln darstellt, kann im Rahmen dieses Ansatzes von einer Dominanz zweck- und wertrationalen Handelns ausgegangen werden. Zusammenfassend wird das Handeln nach Weber durch normative Sinnelemente, in Form von kulturellen Vorgaben wie Tradition, Brauch oder Sitte bestimmt. Unter der Annahme, dass Handelnde bei ihrem Tun oder Unterlassen von kulturellen Werten und gesellschaftlichen Normen geleitet und reguliert werden, können diese Faktoren als Kriterien bei der Kompetenzerfassung herangezogen werden. Durch das Einbeziehen normativer Gesichts- 
punkte „kann eine „Vorstellung“ von ,richtigem“ Handeln angenommen werden. Eine Person, die „richtig“ handelt, könnte demnach als kompetent eingestuft werden." ${ }^{471}$

\section{Der Ansatz von Parsons}

Nach Parsons erfolgen Handlungen im Kontext institutioneller Vorgaben, mit der Funktion die gesellschaftliche Ordnung aufrecht zu erhalten. ${ }^{472}$ Im Zuge dieses struktur-funktionalistischen Verständnisses ergeben sich Handlungen aus den gesellschaftlich vorgegebenen sozialen Rollen der Handelnden und den entsprechenden Handlungsmustern, die für die Handelnden verbindlich sind. Die Verbindlichkeit dieser Handlungsmuster resultiert aus der sinnvermittelnden Struktur der Gesellschaftsordnung. Jede Form von Ordnung weist eine spezifische Struktur von Werten, Regeln und Normen auf. Aufgrund der bindenden Kraft dieser Normen fügen sich die Mitglieder in die bestehende Ordnung (bzw. in das kulturelle System in der Terminologie Parsons) ein und, bedingt durch ihre sinnvermittelnde Kraft, passen die Akteure ihre Bedürfnisse, Erwartungen und Fähigkeiten kontinuierlich an die Ordnung an. ${ }^{473}$ Dementsprechend erfolgen Handlungen nicht zufällig, sondern in Abhängigkeit von der bestehenden Ordnung. Im Verständnis von Parsons ist Handeln daher einerseits als Anpassung an ein institutionalisiertes Rollenmuster, und andererseits als Ausdruck verinnerlichter Werthaltungen aufzufassen. ${ }^{474}$ Individuelle Handlungsentscheidungen, die in Abhängigkeit von kulturellen Werten, Regeln, sozialen Normen und persönlichen Motiven getroffen werden, sind durch kulturelle Orientierungsalternativen begrenzt, die ihrerseits wiederum durch die gesellschaftlich vorgegebenen sozialen Rollen und die entsprechenden Handlungsmuster, die für die Akteure verbindlich sind, bestimmt werden. Nach Parsons können die Gründe, die dem Handeln zugrunde liegen, in einem Modell kultureller Orientierungsalternativen dargestellt werden. Im Zuge dieses idealtypisierenden Modells differenziert Parsons zwischen fünf Variablenpaaren mit je zwei gegensätzlichen Orientierungsmustern: ${ }^{475}$

1. Affektivität vs. affektive Neutralität: Handeln ist affektiv, wenn Gefühle und persönliche Beziehungen handlungsleitend sind. Es ist affektiv neutral, wenn nach rein sachlichen Gesichtspunkten gehandelt wird. Bezugnehmend auf Kooperationsbeziehungen liegt affektiv neutrales Handeln vor, wenn organisations- und aufgabenbezogene Aspekte in den Kooperationsentscheidungen dominieren. In diesem Zusammenhang sind auf organi-

471 Kaufhold (2006), S. 80

$472 \quad$ Vgl. Abels (2007a), S. 128

473 Vgl. Parsons (1986), S. 46 ff., sowie S. 218 ff; Abels (2007a), S. 128; Buß (2008), S. 142

$474 \quad$ Vgl. Miebach (1991), S. 21

$475 \mathrm{Vgl}$. Buß (2008), S. $140 \mathrm{f}$. 
sationaler Ebene sowohl Basis- als auch Kernkompetenzen, und auf individueller Ebene vor allem Fach- und Methodenkompetenzen von Bedeutung.

2. Spezifität vs. Diffusität: Handeln ist spezifisch, wenn ein einziges, klar abgegrenztes Interesse bzw. Ziel handlungsleitend ist. Es ist diffus, wenn es auf mehreren miteinander vermischten Interessen beruht. Bezugnehmend auf Kooperationsbeziehungen handeln die Kooperationspartner spezifisch, wenn sie klar abgegrenzte Aufgaben im Bereich ihrer Zuständigkeiten wahrnehmen und die Erfüllung von Aufgaben, denen keine eindeutige Funktionsbeschreibung zugrunde liegt, ablehnen. Von hoher Bedeutung in diesem Zusammenhang sind auf organisationaler Ebene sowohl Basis- als auch Kernkompetenzen, und auf individueller Ebene vor allem Fach- und Methodenkompetenzen.

3. Universalismus vs. Partikularismus: Handeln ist universalistisch, wenn allgemeine Grundsätze handlungsleitend sind. Es ist partikularistisch, wenn auf Grundlage besonderer persönlicher Beziehungen gehandelt wird. So sind Kooperationsbeziehungen durch Partikularismus gekennzeichnet, wenn die Kooperationspartner nach Maßgabe dieser kooperativen Beziehung handeln (z.B. Zusammenarbeit von Unternehmen A und Unternehmen B im Rahmen eines Auftrags $X$ aufgrund der Angehörigkeit zur selben Kooperation $Y$ ). In diesem Zusammenhang sind vor allem auf individueller Ebene Sozial- und Persönlichkeitskompetenzen von hoher Relevanz.

4. Zuschreibung versus Leistung: Handeln basiert auf dem Prinzip der $\mathrm{Zu}$ schreibung, wenn der einem anderen Akteur zugeschriebene Status handlungsleitend ist. Es basiert auf dem Prinzip der Leistung, wenn auf der Grundlage der Leistung des anderen Akteurs gehandelt wird. Bezugnehmend auf Kooperationsbeziehungen ist das Prinzip der Leistung handlungsleitend, wenn sich kooperierende Unternehmen aufgrund der Kern-, sowie der Fach- und Methodenkompetenzen eines potentiellen Kooperationspartners für dessen Aufnahme in die Kooperation entscheiden.

5. Kollektivorientierung versus Selbstorientierung: Handeln ist kollektivistisch orientiert, wenn übergeordnete Gemeinschaftsnormen handlungsleitend sind. Es kennzeichnet sich durch Selbstorientierung, wenn nach individualistischen Kalkülen und Interessen gehandelt wird. Bezugnehmend auf Kooperationsbeziehungen handeln die Kooperationspartner kollektivistisch orientiert, wenn sie ihre Ressourcen einsetzen, um die unter den Partnern vereinbarten Kooperationsziele zu erreichen und zugunsten gemeinsamer langfristiger Vorteile auf die Realisierung kurzfristiger Vorteile verzichten. In diesem Zusammenhang sind auf individueller Ebene vor allem Sozial- und Persönlichkeitskompetenzen von Relevanz.

Indem Parsons davon ausgeht, dass die Handlungsgründe von den gesellschaftlich vorgeschriebenen Rollen und den entsprechenden Handlungsmustern bestimmt 
werden, kann diesem Ansatz eine Dominanz funktionalen Handelns zugeschrieben werden. Zusammenfassend ist das Handeln nach Parsons im Kontext institutioneller Vorgaben (Rollen) zu analysieren und anhand kultureller Wertmuster zu interpretieren. Parsons zentraler Bezug zu Rollen, die von den in der Gesellschaft geltenden Normen bestimmt werden, ergibt sich aus seinem Verständnis, dass die sozialen Rollen der Interaktion zwischen Akteuren dienen, diese bestimmen und eine Erklärung der Handlungsgründe ermöglichen. Indem er sie als soziale Erwartungen an das Handeln versteht, kann im Rahmen dieses Ansatzes davon ausgegangen werden, dass sich Kompetenz anhand der Wahrnehmung bestimmter Funktionen und Rollen erfassen und anhand idealisierter Rollenerwartungen beurteilen lässt. ${ }^{476}$

\section{Der Ansatz von Habermas}

Nach Habermas, dem Begründer der Theorie des kommunikativen Handelns, kennzeichnet sich das Handlungskonstrukt durch seine Verbindung mit Interaktion. ${ }^{477}$ Bezugnehmend auf die Gründe, die Handeln bestimmen, differenziert Habermas grundsätzlich vier Typen: ${ }^{478}$ So ist Handeln teleologisch, wenn ihm eine Orientierung an Zweck, Mittel und Folgen zugrunde liegt, d.h. wenn es sich auf die objektive Welt ${ }^{479}$ bezieht und den Geltungsanspruch der Wahrheit verfolgt. Damit knüpft Habermas an den Handlungsbegriff von Weber an, dessen klassische soziologische Theorie des Handelns eine Dominanz zweckrationalen Handelns impliziert. Bezugnehmend auf Kooperationsbeziehungen zeigt sich teleologisches Handeln darin, dass die Beziehungen der beteiligten Unternehmen nicht auf moralischem Engagement und sozialen Verpflichtungen beruhen, sondern auf den Bedingungen des unpersönlichen und emotionsfreien, objektiven Vergleichs von Leistungen und Gegenleistungen, womit auf organisationaler Ebene sowohl die Basis- als auch die Kernkompetenzen, sowie auf individueller Ebene vorrangig die fachlichen und methodischen Kompetenzen der Kooperationspartner von Relevanz sind. Handeln ist normativ, wenn ihm eine Orientierung an gemeinsamen Werten zugrunde liegt, d.h. wenn es sich auf die soziale Welt ${ }^{480}$ bezieht und den Geltungsanspruch der Richtigkeit verfolgt. Damit knüpft Habermas an den Handlungsbegriff von Parsons an, dessen Rollentheorie eine Dominanz normengebundenen Handelns impliziert. Bezugnehmend auf Ko-

\footnotetext{
476 Vgl. Parsons (1978); Miebach (1991), S. 19; Kaufhold (2006), S. 91 f.; Abels (2007a)

$477 \quad$ Vgl. Treibel (1994), S. 153

$478 \mathrm{Vgl}$. Habermas (1988a), S. $126 \mathrm{ff}$.

479 Die objektive Welt stellt die Welt der Dinge und Sachverhalte dar. Sie bezeichnet die sog. äußere Natur, die die handelnden Akteure vorfinden, die „Gesamtheit der Tatsachen“(Treibel 1994, S. 156 f.).

480 „Eine soziale Welt besteht aus einem normativen Kontext, der festlegt, welche Interaktionen zur Gesamtheit berechtigter interpersonaler Beziehungen gehören“. Ihr gehören alle Akteure an, „für die entsprechende Normen gelten“ und „von denen sie als gültig akzeptiert werden" (Habermas 1988a, S. 132)
} 
operationsbeziehungen zeigt sich normatives Handeln darin, dass die Kooperationspartner entsprechend ihrer gesellschaftlich vorgegebenen Rollen und den entsprechenden Handlungsmustern handeln. Indem mit zwischenbetrieblicher Kooperation die in der Gesellschaft als verbindlich anerkannte Regel des Verzichts auf die Realisierung kurzfristiger Vorteile zugunsten langfristiger Vorteile seitens der Kooperationspartner einhergeht, kann davon ausgegangen werden, dass die Kooperationspartner normativ handeln, wenn sie auf die Ausnützung kurzfristiger Opportunismusspielräume verzichten. Eine hohe Bedeutung kann in diesem $\mathrm{Zu}$ sammenhang den sozialen und persönlichen Kompetenzen der Kooperationspartner zugeschrieben werden. Handeln ist dramaturgisch, wenn es auf der Selbstdarstellung der handelnden Akteure beruht, d.h. wenn es sich auf die subjektive Welt $^{481}$ bezieht und den Geltungsanspruch der Wahrhaftigkeit verfolgt. Diese Selbstrepräsentation der Interaktionsteilnehmer, „die füreinander ein Publikum bilden, vor dessen Augen sie sich darstellen“, versteht Habermas nicht als spontanes Ausdrucksverhalten, sondern als „zuschauerbezogene Stilisierung des Ausdrucks eigener Erlebnisse“482. Damit knüpft Habermas an den Handlungsbegriff von Goffman an, dessen Theorie eine Dominanz dramaturgischen Handelns impliziert. ${ }^{483}$ Die erläuterten Typen des teleologischen, normativen und dramaturgischen Handelns stellen Formen zweckrationalen Handelns dar, die, bedingt durch ihren Bezug auf jeweils nur eine Welt, bloß einzelne Aspekte der Wirklichkeit handelnder Akteure abbilden. ${ }^{484}$ Um die gesamte Wirklichkeit handelnder Akteure erfassen zu können, identifiziert Habermas einen vierten Handlungstyp. So bezieht sich kommunikatives Handeln auf die Interaktion von mindestens zwei sprach- und handlungsfähigen Akteuren, die eine Verständigung - im Sinne eines kooperativen Deutungsprozesses - über ihre Handlungssituation suchen, um so ihre Handlungspläne und damit ihre Handlungen zu koordinieren. ${ }^{485}$ Die Gültigkeit von Sinnzusammenhängen im Verständigungsprozess wird im Rahmen des kommunikativen Handelns vorausgesetzt. ${ }^{486}$ Dieser Handlungstyp kennzeichnet sich weiters durch seinen Bezug auf alle drei Welten - die objektive, subjektive und soziale Welt - und durch seinen Geltungsanspruch der Verständlichkeit. ${ }^{487}$ Damit knüpft Habermas an die Theorie des Symbolischen Interaktionismus (Mead) und die Ethnomethodologie (Garfinkel) an.

\footnotetext{
481 Die subjektive Welt wird als innere Welt der Wünsche, Gefühle und Erlebnisse der handelnden Akteure charakterisiert. Der Zugang zur subjektiven Welt ist dem Akteur selbst vorbehalten (Maas 2001, S. 31)

482 Habermas $(1988 \mathrm{a})$, S. 128

483 Vgl. hierzu Goffman (2006)

484 Vgl. Treibel (1994), S. 160

485 Vgl. Habermas (1988a), S. 128, sowie S. 151

486 Vgl. Habermas (1988b), S. 41

487 Vgl. Habermas (1988a), S. 143 f.; Abels (2007a)
} 
Indem sich Habermas im Rahmen seiner Theorie auf die zwischen den handelnden Akteuren stattfindenden Interaktionen konzentriert und sich dabei von einer strengen normengeleiteten Zweckrationalität distanziert, kann im Rahmen dieses Ansatzes von einer Dominanz kommunikativen Handelns ausgegangen werden. Im Zuge dieses Handelns verständigen sich die Akteure über ihre Handlungssituationen, um auf diese Weise ihre Handlungsziele abzustimmen, und in weiterer Folge ihre Handlungspläne und Handlungen zu koordinieren. Bei dem Mechanismus zur Handlungskoordination handelt es sich um die Interpretationsleistung - im Sinne von kooperativen Deutungsprozessen - der handelnden Akteure. Bezugnehmend auf das Kompetenzkonstrukt implizieren die wechselseitige Verständigung über und die Koordination von Handlungen als Charakteristika kommunikativen Handelns eine hohe Bedeutung von Basis- und Kernkompetenzen auf organisationaler Ebene, sowie von Fach-, Methoden-, Sozialund Persönlichkeitskompetenzen auf individueller Ebene.

Zusammenfassend zeigt sich die Gemeinsamkeit der erläuterten Handlungstheorien darin, dass sie Handeln als mit Sinn verbunden definieren. Unterschiede bestehen hinsichtlich der Annahmen, wie Handeln zustande kommt. Ausgehend von den Merkmalen der dargestellten Handlungstypen lassen sich unter analytischen Gesichtspunkten zwei Betrachtungsweisen zur Klassifizierung von Handeln ableiten. So können das zweckrationale Handeln nach Weber und das funktionale Handeln nach Parsons dem Handlungstyp des normengeleiteten Handelns zugeordnet werden. Handeln ist normengeleitet, wenn es sich an kulturellen Vorgaben wie Tradition, Brauch und Sitte bzw. gesellschaftlichen Normen orientiert und durch diese reguliert wird. ${ }^{488}$ Zur Erfassung und Beurteilung von normengeleitetem Handeln und damit von Kompetenz, von der angenommen wird, dass sie sich im Handeln zeigt, können die in der Gesellschaft geltenden Normen als Kriterien herangezogen werden. Ein Akteur, der im Zuge seines Handelns die gesellschaftlich vorgegebenen Kriterien erfüllt, könnte demnach als kompetent eingestuft werden.

Dagegen ist das Konzept des kommunikativen Handelns von Habermas abzugrenzen, das, wie die Konzepte von Mead und Schütz, dem Handlungstyp des sinnbezogenen Handelns zugeordnet werden kann. Im Vergleich zum Handlungstyp des normengeleiteten Handelns sind im Rahmen dieses Konzepts die handelnden Akteure, sowie die zwischen den Akteuren stattfindenden Interaktionen von höherer Relevanz. Es erfolgt eine Distanzierung von dem Prinzip der strengen normengeleiteten Zweckrationalität. Stattdessen wird den handelnden Akteuren durch die Annahme wechselseitiger Interaktionen die Entwicklung individueller Zielsetzungen und eigener Handlungsspielräume gewährt. Situationsbe- 
zogene Sinnkonstruktionen und damit auch individuelle Entwicklungsprozesse der Akteure sind in ein solches Verständnis integriert. Indem Handeln im Rahmen dieser Betrachtungsweise an Vielfalt gewinnt, kann es für die Erfassung und Beurteilung von Kompetenz, von der angenommen wird, dass sie sich im Handeln zeigt, nicht - wie im Falle normengeleiteten Handelns - als Moment des Erreichens vorab festgelegter Ziele begriffen werden, da Zieldefinitionen im Zuge dieser prozessorientierten Perspektive eher als situationsspezifische, von den handelnden Akteuren erzeugte Sinnsysteme zu verstehen sind. Zur Erfassung von Kompetenz im Zuge sinnorientierten Handelns sind die Handlungs- und Gestaltungsspielräume der handelnden Akteure zu berücksichtigen. ${ }^{489}$

Zusammenfassend zeigt sich, dass Handeln je nach Handlungstheorie unterschiedlich definiert wird. Ausgehend davon, dass sich Kompetenz im Handeln zeigt, ist es von hoher Relevanz zu eruieren, wie Handeln zustande kommt. Je nach handlungstheoretischem Konzept sind unterschiedliche Kompetenzarten im Rahmen zwischenbetrieblicher Kooperationen von Bedeutung. Grundsätzlich ist nach Kaufhold eine stärker am Akteur orientierte Erklärung des Handlungskonzepts - wie beispielsweise jene von Habermas - zu präferieren, da sie „eher in Zusammenhang zu dem [stünde, Anm. d. Verf.], was Kompetenz in Abgrenzung zu Qualifikation eigentlich ausmacht [...] Allerdings ist anzunehmen, dass vor allem im Kontext des betrieblichen Handelns eine normengeleitete Erklärung von Handeln herangezogen wird. Hier erscheint sie nützlich, um die betrieblichen Abläufe entsprechend steuern und regulieren zu können. Eine Erfassung von Kompetenz, die sich an solchem Handeln orientiert, scheint vor diesem Hintergrund angemessen." ${ }^{490}$

Abschließend zeigen die erläuterten handlungstheoretischen Ansätze folgende Gemeinsamkeiten in Bezug auf das Kompetenzkonstrukt: Kompetenz als Handlungskategorie ist dynamisch und wird im Handlungsprozess entwickelt. Dadurch, dass sie nicht direkt beobachtbar ist, lässt sie sich nur indirekt im Zuge des Handelns erschließen. Ihre Erfassung anhand vorab festgelegter Kriterien eignet sich vor allem unter der Annahme zweckrationalen und funktionalen Handelns. ${ }^{491}$

\subsection{Kompetenz aus Sicht der Principal-Agent-Theorie}

Nachdem die Principal-Agent-Theorie bereits in Teil II dieser Arbeit ausführlich erläutert wurde, erfolgt in diesem Kapitel eine Auseinandersetzung mit den Unsicherheiten des Prinzipals in Bezug auf das Verhalten des Agenten, um sodann die Bedeutung von Kompetenz als Zusatzinformation im Rahmen dieser Theorie,

\footnotetext{
489 Vgl. Kaufhold (2006), S. 93

$490 \quad$ Kaufhold (2006), S. 80

491 Vgl. Kaufhold (2006), S. 94
} 
und ihre Relevanz im Bereich zwischenbetriebliche Kooperationen herausstellen zu können.

Grundsätzlich sieht sich der Prinzipal mit drei Arten von Unsicherheiten bezüglich des Agenten konfrontiert, die sich aus den zwischen ihm und dem Agenten bestehenden Informationsasymmetrien ergeben. Erstens besteht seitens des Prinzipals Unsicherheit über die tatsächlichen Eigenschaften (z.B. Qualifikationen) des Agenten (,Qualitätsunsicherheit'). Zweitens ist der Prinzipal unsicher in Bezug auf die Absichten des Agenten (,Entscheidungsunsicherheit'). Und drittens besteht Unsicherheit im Hinblick auf die Anstrengungen des Agenten (,Ergebnisunsicherheit'), da der Prinzipal nicht beurteilen kann, welchen Anteil die Anstrengungen des Agenten und welchen Anteil Umwelteinflüsse an dem zustande gekommenen Ergebnis nehmen. Aus dieser Tatsache kann ein Informationsvorsprung für den Agenten abgeleitet werden, der ihm Raum für opportunistisches Handeln und damit die Verfolgung persönlicher Ziele lässt. Aus dieser Informationsasymmetrie zwischen Prinzipal und Agenten erwachsen dem Prinzipal spezifische Risiken. Um diese abzuwenden, hat der Prinzipal zwei Möglichkeiten: einerseits kann er die Unsicherheiten in Bezug auf das Verhalten des Agenten akzeptieren und die damit zusammenhängenden Risikokosten hinnehmen. Andererseits kann er Aktionen (z.B. explizite Verträge) zur Verringerung dieser Unsicherheiten setzen und die damit korrelierenden Kosten akzeptieren. ${ }^{4}$

Eine Entscheidung für die Akzeptanz der Unsicherheiten seitens des Prinzipals kann nur vor dem Hintergrund einer tragfähigen Vertrauensbeziehung erfolgen, weshalb sie mit einer Entscheidung für Vertrauen gleichzusetzen ist. Grundsätzlich kennzeichnet sich eine Vertrauensentscheidung durch die Vertrauenserwartung und die Vertrauenshandlung. Voraussetzung für die Vertrauenshandlung im Sinne der freiwilligen Erbringung einer riskanten Vorleistung unter Verzicht auf explizite vertragliche Sicherungs- und Kontrollmaßnahmen zur Begrenzung der Opportunismusspielräume des Agenten ist die vertrauensvolle Erwartung, dass sich der Agent, trotz Fehlens dieser Schutzmaßnahmen, vertrauenswürdig verhalten wird. Bei der Bildung seiner Vertrauenserwartung sieht sich der Prinzipal also mit einem Adverse Selection-Problem konfrontiert, da er in seiner Rolle als Vertrauensgeber einen vertrauenswürdigen Agenten auswählen muss bzw. entscheiden muss, ob der potentielle Vertrauensnehmer vertrauenswürdig ist, ihm jedoch diese Eigenschaft vor Eingehen der Vertrauensbeziehung weitgehend verborgen bleibt. Die Vertrauenserwartung basiert damit auf der zugeschriebenen Motivation des Handelns des Agenten, genauer auf der Einschätzung der Intensität und Stabilität der Motivation des Vertrauensnehmers und damit der Antizipation von dessen Handlungsabsicht seitens des Prinzipals. Bei der Einschätzung des Agenten sieht sich der Prinzipal mit subjektiver Unsicherheit im Hinblick auf 
dessen Absichten und Eigenschaften, sowie mit objektiver Unsicherheit in Bezug auf das Eintreten möglicher Umweltzustände und der sich daraus ergebenden Handlungsrestriktionen konfrontiert. ${ }^{493}$ Im Zuge der Bildung der spezifischen Vertrauenserwartung, die auf der subjektiven Einschätzung der Vertrauenswürdigkeit eines spezifischen Agenten in einer spezifischen Situation beruht, wird der Prinzipal daher bestrebt sein, seine subjektive Unsicherheit durch zusätzliche Informationen zu reduzieren. ${ }^{494}$

In diesem Zusammenhang wird die Bedeutung des Kompetenzkonstrukts aus Sicht der Principal-Agent-Theorie deutlich: Die Bildung einer vertrauensvollen Erwartung seitens des Prinzipals bezüglich der Absichten des Agenten und damit der bewusste Verzicht auf Sicherungs- und Kontrollmaßnahmen können nur antizipiert werden, wenn Zusatzinformationen wahrgenommen werden, die den Prinzipal dazu veranlassen, auf eine höhere Eintrittswahrscheinlichkeit der Vertrauenserwartung zu schließen. Bei den vom Prinzipal wahrgenommenen Kompetenzen des Agenten handelt es sich um eine solche Zusatzinformation, durch die die subjektive Unsicherheit von ersterem in Bezug auf das Verhalten von letzterem reduziert werden $\operatorname{kann}^{495}$, was die Entstehung einer vertrauensvollen Erwartung begünstigt.

Die Erfüllung der Erwartungen des Prinzipals ist grundsätzlich von der Existenz zweier Größen abhängig: dem Können und dem Wollen des Agenten. Aufgrund der zwischen Prinzipal und Agenten herrschenden Informationsasymmetrien besteht seitens des Prinzipals einerseits Unsicherheit darüber, ob der Agent die potentielle Vertrauensentscheidung honorieren kann (,Qualitätsunsicherheit') und andererseits ist der Prinzipal unsicher, ob sich der Agent vertrauenswürdig verhalten will (,Entscheidungsunsicherheit ${ }^{\circ}$ ). Die auf organisationaler Ebene wahrgenommenen Basis- und Kernkompetenzen, sowie auf individueller Ebene wahrgenommenen Fach- und Methodenkompetenzen des Agenten begünstigen die Entstehung von Vertrauen seitens des Prinzipals nun insofern, als dass sie seine Unsicherheit bezüglich des Könnens des Agenten reduzieren. Verfügt der Prinzipal also über die Einschätzung, dass der Agent die genannten Kompetenzen zur Ho-

493 Vgl. hierzu die Ausführungen in Teil II Kap. 1.2.2 und Kap. 5.1, sowie die dort zit. Literatur.

494 Da diese Zusatzinformationen auf einer subjektiven Wahmehmung seitens des Prinzipals beruhen, kollidiert ihr Heranziehen nicht mit der Entscheidung des Prinzipals, die Unsicherheiten in Bezug auf das Verhalten des Agenten vor dem Hintergrund einer tragfähigen Vertrauensbeziehung zu akzeptieren. Dieses Verständnis begründet sich auch dadurch, dass es sich bei dem Heranziehen von Zusatzinformationen nicht um eine explizite vertragliche Sicherungs- und Kontrollmaßnahme zur Begrenzung von Opportunismusspielräumen handelt. Vielmehr wirken Zusatzinformationen bei der subjektiven Einschätzung der Vertrauenswürdigkeit des Agenten unterstützend und können erforderlich sein, um eine vertrauensvolle Erwartung im Hinblick auf das Verhalten des Agenten bilden zu können.

495 Vgl. Weiss (1992), S. 63; Stahl (1996), S. 219; Möhringer (1998), S. 28; Morrow et al. (1999), S. 7; Deckow (2006), S. 116; 
norierung der Vertrauensentscheidung besitzt, dann kann er aufgrund seiner $\mathrm{Zu}$ trauenserwartung eher geneigt sein, eine Vertrauenshandlung $\mathrm{zu}$ setzen ${ }^{496}$, und dem Agenten sein Vertrauen zu schenken. ${ }^{497}$ Die auf individueller Ebene wahrgenommenen Sozial- und Persönlichkeitskompetenzen des Agenten können die Entstehung von Vertrauen seitens des Prinzipals insofern begünstigen, als dass sie seine Unsicherheit bezüglich des Willens des Agenten, sich vertrauenswürdig zu verhalten, reduzieren. ${ }^{498}$ Nimmt der Prinzipal Zusatzinformationen wie Kommunikations-, Kooperations-, Konfliktfähigkeit, sowie Empathie, Ambiguitätstoleranz und Selbstöffnung als Komponenten der sozialen Kompetenz, sowie realitätsnahe Einstellungen, Tatkraft, Zielstrebigkeit, und Kritikfähigkeit als Komponenten der persönlichen Kompetenz ${ }^{499}$ seitens des Agenten wahr, so reduziert sich seine subjektive Unsicherheit in Bezug auf die vertrauenswürdigen Absichten des Agenten. Die kompetenzbezogenen Zusatzinformationen, die den Prinzipal auf eine höhere Eintrittswahrscheinlichkeit seiner Erwartungen bezüglich des Könnens (,Zutrauenserwartung') und des Wollens (,Vertrauenserwartung') des Agenten schließen lassen, können ihn zur Vornahme einer Vertrauenshandlung im Sinne der freiwilligen Erbringung einer riskanten Vorleistung, in Abhängigkeit von der Höhe seiner situationsspezifischen Risikowahrnehmung und seiner prinzipalspezifischen Risikobereitschaft, motivieren. ${ }^{500}$

Zusammenfassend zeigt sich die Bedeutung von Kompetenz aus Sicht der Principal-Agent-Theorie darin, dass sie die Entstehung von Vertrauensbeziehungen begünstigen kann. Indem sie als Zusatzinformation die Entscheidungsunsicherheit des Prinzipals bezüglich der Absichten des Agenten reduziert, determiniert sie die Entstehung der vertrauensvollen Erwartung des Prinzipals, dass der Agent freiwillig auf opportunistisches Verhalten verzichtet. Diese Vertrauenserwartung motiviert in Folge die Vornahme der Vertrauenshandlung, d.h. die freiwillige Erbringung einer riskanten Vorleistung. Indem Kompetenz als Zusatzinformation auch die Qualitätsunsicherheit des Prinzipals bezüglich der Eigenschaften (z.B. Qualifikationen) des Agenten reduziert, kann von ihrem positiven Einfluss auf die Vornahme einer Vertrauenshandlung, über das Konstrukt des $\mathrm{Zu}$ trauens, ausgegangen werden. Dementsprechend ist die wahrgenommene Kompetenz des Agenten als Zusatzinformation zur Reduktion der Unsicherheiten des

\footnotetext{
496 Vgl. hierzu die Ausführungen in Teil II Kap. 6, sowie die dort zit. Literatur.

497 Vgl. dazu Shaw (1997, S. 30): , [...] even if people's motives are characterized by goodwill, they will not retain our trust if they are incompetent or powerless to fulfil the expectations we have of them." Ähnlich auch Butler/Cantrell (1984), S. 24; Mishra (1996), S. 266; Bittl (1997), S. 163 f: Möhringer (1998), S. 29 und S. 35; Seifert (2001), S. 63; Pirson (2007), S. 65; Sichtmann (2007), S. 1007

498 Vgl. Hinterhuber/Stahl (1996), S. 108; Mishra (1996), S. 267 f.; Stahl (1996), S. 229 ff; Bittl (1997), S. 164; Shaw (1997), S. 32 f.; Hänggi (1998), S. 179 f.: Möhringer (1998), S. 35; Seifert (2001), S. 53 ff.; Sichtmann (2007), S. 1002

499 Vgl. hierzu die Ausführungen in Teil III Kap. 2.2.3 und 2.2.4, sowie die dort zit. Literatur.

$500 \mathrm{Vgl}$. hierzu die Ausführungen in Teil II Kap. 6, sowie die dort zit. Literatur.
} 
Prinzipals, und damit als Determinante der Entstehung von Principal-Agent-Beziehungen auf der Grundlage von Vertrauen aufzufassen. Die experimentelle Überprüfung dieser Annahmen ist Gegenstand des empirischen Teils dieser Arbeit. 


\section{TEIL IV: KOMPETENZ ALS DETERMINANTE DER ENTSTEHUNG VON VERTRAUEN IN ZWISCHENBETRIEBLICHEN KOOPERATIONEN}

Nach der begrifflichen Klärung und der inhaltlichen Auseinandersetzung mit den Konstrukten Vertrauen und Kompetenz, erfolgt in diesem Teil die Modellierung des Einflusses von Kompetenz auf die Entstehung von Vertrauen. Dass es sich bei der Analyse der Rolle von Kompetenzen im Vertrauenskonstrukt und auf die Vertrauensevolution um ein bestenfalls ansatzweise erschlossenes Forschungsfeld handelt, wird anhand des gegenwärtigen Forschungsstandes aufgezeigt (Kap. 1). Ausgangspunkt der Modellentwicklung stellt die Erläuterung der methodischen Grundlagen und Vorgehensweisen im Zuge der Modellgenerierung dar (Kap. 2). Um die interdisziplinären Erkenntnisse aus der Vertrauens- und Kompetenzforschung modelltheoretisch integrieren zu können, ist eine Operationalisierung von Kompetenz (Kap. 3) und Vertrauen (Kap. 4) auf Basis der in dieser Arbeit analysierten Ansätze erforderlich. Den Abschluss dieses Teils bildet das Modell, das den Einfluss von Kompetenz auf die Entstehung von Vertrauen hypothetisiert (Kap. 5).

\section{DiE FORSCHUNG ZUR WIRKUNG VON KOMPETENZ ALS VERTRAUENSDETERMINANTE}

Aufgrund seiner Relevanz für die Modellentwicklung zielt dieses Kapitel darauf $\mathrm{ab}$, einen Überblick über die Bemühungen zur Erforschung der Rolle individueller und organisationaler Kompetenzen im Vertrauenskonstrukt und auf die Vertrauensentstehung zu generieren. Die im Zuge dieser Überblicksgenerierung analysierten Studien operieren allerdings nicht mit den Begriffen Fach-, Methoden-, Sozial- und Persönlichkeitskompetenz, sowie Basis- und Kernkompetenz. ${ }^{501}$ Trotz dieses Unterschiedes in Bezug auf die vorliegende Arbeit handelt es sich bei der vorgenommenen Systematik um keine den Konzepten von außen oktroyierte Ordnung, sondern sie gibt inhaltliche Übereinstimmungen wider.

\subsection{Der Einfluss individueller Kompetenzen auf Vertrauen}

Die zur Erfüllung einer Aufgabe benötigten fachlichen Fertigkeiten und Fähigkeiten, sowie die Summe der persönlichen Problemlösungsverfahren des potentiellen Vertrauensnehmers werden vielfach als Determinanten der Entstehung von Vertrauen seitens des Vertrauensgebers angesehen. ${ }^{502}$ So stellen auch Sitkin und

501 Eine Diskussion der Gründe, die eine Untergliederung der sekundäranalytisch ermittelten Kompetenz in die einzelnen Kompetenzarten erforderlich macht, erfolgt am Ende dieses Kapitels. Die Untergliederung erfolgt auf Basis der begrifflichen Klärung (Teil III Kap. 1) und der inhaltlichen Auseinandersetzung mit dem Kompetenzkonstrukt (Teil III Kap. 2).

502 Vgl. Barber (1983), S. 14; Butler (1991), S. 648; Shapiro et al. (1992), S. 371; Moorman et al (1993); Bentele (1994), S. 145; Mayer et al. (1995), S. 717 f.; Mishra (1996), S. 266; Bittl (1997), 
Roth die vom Vertrauensgeber wahrgenommene Fach- und Methodenkompetenz des Vertrauensnehmers als zentrale und unverzichtbare Vertrauensbedingung heraus: ,[...] trust rests on a foundation of expectations about an employee's ability to complete task assignments reliably [...].“503 Andererseits stellt gemäß einer Reihe von Vertrauensforschern die Problem- bzw. „Leistungsfähigkeit [...] - anders als der Leistungswille - kein konstitutives Element des Vertrauenskonstrukt dar. “504 Der Grund hierfür liegt in der Annahme, dass die wahrgenommene Fach- und Methodenkompetenz einen direkten Einfluss auf die Zutrauenserwartung in den Vertrauensnehmer hat, nicht jedoch auf das Vertrauen. ${ }^{505}$ Diese Hypothese wurde von Davis et al. empirisch bestätigt: Im Gegensatz zur Sozial- und Persönlichkeitskompetenz, die bei separater Betrachtung einen positiven Einfluss auf die Entstehung von Vertrauen ausübt, besteht kein signifikanter Zusammenhang zwischen Fach- und Methodenkompetenz einerseits und Vertrauen andererseits. ${ }^{506}$ Hingegen ergaben die Experimente von Butler und Cantrell, dass aus Sicht der Probanden die fachliche und methodische Kompetenz des Vertrauensnehmers von zentraler Bedeutung für die Entstehung von Vertrauen seitens des Vertrauensgebers sind. ${ }^{507}$ Aufgrund dieser widersprüchlichen Befunde stellt sich im Rahmen dieser Arbeit die Frage, ob die Fachund Methodenkompetenz als Determinante der Entstehung von Vertrauen ausgeschlossen werden soll. Da die Vertrauensentscheidung aber nicht bloß die Einschätzung der Vertrauenswürdigkeit des Vertrauensnehmers seitens des Vertrauensgebers, sondern auch die Vornahme einer Vertrauenshandlung umfasst, besteht die Möglichkeit, dass die Wahrnehmung der Fach- und Methodenkompetenz über die Dimension der Zutrauenserwartung die Vornahme einer Vertrauenshandlung, und damit die Vertrauensentscheidung positiv beeinflusst. Davis et al. halten fest: „If a manager is perceived as able to get something done about a particular problem, he or she is more likely to be trusted than a manager who is perceived as impotent in the situation." ${ }^{\text {508 }}$ Die Wirkung der wahrgenommenen Fach- und Methodenkompetenz auf die Entstehung von Vertrauen wird im empirischen Teil dieser Arbeit untersucht.

Die zum konstruktiven Umgang mit sich und anderen, und damit zum Erhalt zwischenmenschlicher Interaktionen benötigten Fähigkeiten des potentiellen Vertrauensnehmers werden vielfach als Determinanten der Entstehung von Vertrauen

\footnotetext{
S. 164; Doney/Cannon (1997); Shaw (1997), S. 30; Seifert (2001), S. 63 f.; Abrams et al. (2003), S. 65 ff.; Deckow (2006), S. 117 f.; Sichtmann (2007), S. 1002; Bahlmann et al. (2008), S. 139

503 Sitkin/Roth (1993), S. 367 f.

504 Plötner (1995), S. 42

505 Vgl. Dasgupta (1988), S. 52; Ripperger (1998), S. 39 f.; Klaus (2002), S. 117; Fink (2004), S. 37

506 Vgl. Davis et al. (2000)

$507 \quad$ Vgl. Butler/Cantrell (1984)

508 Davis et al. (2000), S. 97
} 
seitens des Vertrauensgebers verstanden. ${ }^{509}$ Ausgehend von der Annahme, dass sich Vertrauen auf Verhaltensrisiken bezieht, wird der Vertrauensgeber bestrebt sein, seine Unsicherheit über den Leistungswillen des potentiellen Vertrauensnehmers zu reduzieren. Nimmt er in diesem Zusammenhang Kommunikations-, Kooperations- und Koordinations- bzw. Team-, sowie Konfliktfähigkeiten ${ }^{510}$ als Komponenten der Sozialkompetenz des Vertrauensnehmers wahr, d.h. Fähigkeiten, die ihm suggerieren, dass der Vertrauensnehmer ihm keinen Schaden zufügt, auch wenn der Anreiz dazu bestünde ${ }^{511}$, so beeinflusst diese Wahrnehmung von Wohlwollen implizierenden Fähigkeiten ${ }^{512}$ die Vornahme einer Vertrauensentscheidung über die Dimension der Vertrauenserwartung positiv. ${ }^{513}$ Diese Annahme, dass Vertrauen durch soziale Kompetenz determiniert wird, wurde von Davis et al. in einer Feldstudie empirisch bestätigt. ${ }^{514}$ Auch die Experimente von Butler und Cantrell ergaben, dass die soziale Kompetenz des Vertrauensnehmers aus Sicht der Probanden von zentraler Bedeutung für die Entstehung von Vertrauen seitens des Vertrauensgebers ist. ${ }^{515}$ Die Rolle der Sozialkompetenz im Vertrauensentstehungsprozess wird im empirischen Teil dieser Arbeit überprüft.

Auch die Persönlichkeitsmerkmale und Handlungsdispositionen eines potentiellen Vertrauensnehmers, die es ihm ermöglichen, sein Handeln in der verschiedensten Situationen zu steuern, zu motivieren und zu initiieren, werden vielfach als Determinanten der Entstehung von Vertrauen seitens des Vertrauensgebers aufgefasst. ${ }^{516}$ Ausgehend von der Annahme, dass sich Vertrauen auf Verhaltensrisiken bezieht, wird der Vertrauensgeber bestrebt sein, seine Unsicherheit über den Leistungswillen des potentiellen Vertrauensnehmers zu reduzieren. Nimmt er in diesem Zusammenhang, basierend auf eigenen Erfahrungen mit dem potentiellen Vertrauensnehmer oder anhand der Informationen Dritter ${ }^{517}$, energetische Persön-

509 Vgl. Barber (1983), S. 16; Butler (1991), S. 648; Shapiro et al. (1992), S. 366 ff.; Bentele (1994), S 145; Mayer et al. (1995), S. 718 f.; Mishra (1996), S. 267 f.; Bittl (1997), S. 164; Shaw (1997), S. 32 f.; Seifert (2001), S. 68 ff.; Abrams et al. (2003), S. 65 ff.; Deckow (2006), S. 118 f.; Sichtmann (2007), S. 1002; Bahlmann et al. (2008), S. 139

510 Als explizite Beispiele für die aufgezählten Fähigkeiten können Loyalităt, Offenheit, Zugänglichkeit und Erreichbarkeit genannt werden, da sie einen positiven Einfluss auf die Initiierung und Erhaltung sozialer Austauschbeziehungen haben (Butler 1991, S. 648); vgl. dazu auch die Ausführungen in Teil III Kap. 2.2.3 und Kap. 3.2, sowie die dort zit. Literatur.

511 Vgl. Gambetta (1988), S. 217

512 Nooteboom (2002, S. 51) versteht unter Wohlwollen den direkten Gegensatz zu Opportunismus.

513 Vgl. Gabarro (1978), S. 296

$514 \quad$ Vgl. Davis et al. (2000)

$515 \quad \mathrm{Vgl}$. Butler/Cantrell (1984)

516 Vgl. Gabarro (1978), S. 295 f.; Butler (1991), S. 648; Shapiro et al. (1992), S. 366; Moorman et al (1993); Bentele (1994), S. 145; Mayer et al. (1995), S. 718 f.; Mishra (1996), S. 266 ff; Bittl (1997), S. 169; Shaw (1997), S. 31; Seifert (2001), S. 64 f.; Abrams et al. (2003), S. 65 ff.; Deckow (2006), S. $119 \mathrm{f}$.

517 Vgl. Mayer et al. (1995), S. 719; Bühner (2004), S. 30 
lichkeitsmerkmale wie Tatkraft, Zielstrebigkeit, Ausdauer, Neugier und Belastbarkeit, sowie realitätsnahe Einstellungen, Motive, Werthaltungen und persönliche Begabungen ${ }^{518}$ als Aspekte der Persönlichkeitskompetenz des Vertrauensnehmers wahr, so beeinflusst die Wahrnehmung dieser Integrität implizierenden Faktoren die Entstehung von Vertrauen über die Dimension der Vertrauenserwartung positiv. Diese Annahme, dass die persönlichkeitskompetenzspezifische Vertrauenserwartung die Vertrauensentscheidung determiniert, wurde mehrfach empirisch bestätigt ${ }^{519}$, und wird auch im Zuge des in dieser Arbeit vorgenommenen Experiments überprüft.

Nachfolgende Tabelle fasst die synoptische Sicht auf Ansätze, die auf eine Erforschung der Vertrauensdeterminanten abzielen, in Bezug auf die für die Vertrauensentstehung relevanten individuellen Kompetenzen zusammen. In diesem Zusammenhang bleibt festzuhalten, dass die erläuterten Studien nicht mit den Begriffen Fach-, Methoden-, Sozial- und Persönlichkeitskompetenz operieren. Aufgrund gewisser inhaltlicher Übereinstimmungen zwischen diesen Ansätzen und dem im Zuge der begrifflichen Klärung und Abgrenzung des Kompetenzkonstrukts entwickelten Kompetenzverständnis dieser Arbeit, handelt es sich bei der Zuordnung der, von den genannten Autoren identifizierten Vertrauensdeterminanten $\mathrm{zu}$ den individuellen Kompetenzarten um keine willkürliche Systematisierung. Allerdings wird durch diese sekundäranalytisch begründete $\mathrm{Zu}-$ ordnung auch deutlich, dass es an Hypothesen zum Einfluss individueller Kompetenzarten auf die Entstehung von Vertrauen, sowie ihrer empirischen Überprüfung mangelt. Diese Lücke ${ }^{520}$ soll im Rahmen der vorliegenden Arbeit durch eine experimentaltechnische Vorgehensweise, sowie durch ex ante und im Zuge des Experiments erfolgende Befragungen von Probanden geschlossen werden.

\begin{tabular}{|l|l|l|l|l|}
\cline { 3 - 4 } \multicolumn{2}{c|}{} & \multicolumn{3}{c|}{$\begin{array}{c}\text { Individuelle Kompetenzen als } \\
\text { Vertrauensdeterminanten }\end{array}$} \\
\hline Autor & $\begin{array}{c}\text { Auswertung } \\
\text { (Daten- } \\
\text { grundlage) }\end{array}$ & $\begin{array}{c}\text { Fach- und } \\
\text { Methoden- } \\
\text { kompetenz }\end{array}$ & $\begin{array}{c}\text { Sozial- } \\
\text { kompetenz }\end{array}$ & $\begin{array}{l}\text { Persönlichkeits- } \\
\text { kompetenz }\end{array}$ \\
\hline $\begin{array}{l}\text { Gabarro } \\
\mathbf{( 1 9 7 8 )}\end{array}$ & $\begin{array}{l}\text { Inhalts- } \\
\text { analytische } \\
\text { Auswertung }\end{array}$ & $\begin{array}{l}\text { funktionale/spezifi } \\
\text { sche Kompetenz, } \\
\text { allgemeine } \\
\text { Geschäftsfähigkeit }\end{array}$ & $\begin{array}{l}\text { interpersonale } \\
\text { Kompetenz, } \\
\text { Offenheit }\end{array}$ & $\begin{array}{l}\text { Motive, Charakter } \\
\text { (Integrität, Diskre- } \\
\text { tion, Konsistenz im } \\
\text { Verhalten) }\end{array}$ \\
\hline
\end{tabular}

\footnotetext{
518 Als explizite Beispiele für die aufgezählten Aspekte können Diskretion, Fairness, Verlässlichkeit und Konsistenz genannt werden, da sie einen positiven Einfluss auf die Initiierung und Erhaltung sozialer Austauschbeziehungen haben (Butler 1991, S. 648); vgl. dazu auch die Ausführungen in Teil III Kap. 2.2.4 und Kap. 3.2, sowie die dort zit. Literatur.

519 Vgl. Butler/Cantrell (1984); Konovsky/Pugh (1994); Korsgaard et al. (1995); Podsakoff et al. (1996); Abrams et al. (2003); De Cremer et al. (2006)

520 Vgl. dazu auch Gillespie (2003), S. 39
} 


\begin{tabular}{|c|c|c|c|c|}
\hline $\begin{array}{l}\text { Barber } \\
\text { (1983) }\end{array}$ & $\begin{array}{l}\text { Plausibilitäts- } \\
\text { überlegungen }\end{array}$ & $\begin{array}{l}\text { technische } \\
\text { Kompetenz }\end{array}$ & $\begin{array}{l}\text { Loyalität } \\
\text { (,fiduciary } \\
\text { obligation') }\end{array}$ & Verlässlichkeit \\
\hline $\begin{array}{l}\text { Butler } \\
\text { (1991) }\end{array}$ & $\begin{array}{l}\text { Faktoren- } \\
\text { analyse } \\
(\mathrm{N}=380)\end{array}$ & $\begin{array}{l}\text { Kompetenz (i.S.v. } \\
\text { Fachkompetenz) }\end{array}$ & $\begin{array}{l}\text { Loyalität, Offen- } \\
\text { heit, Zugäng- } \\
\text { lichkeit, Erreich- } \\
\text { barkeit }\end{array}$ & $\begin{array}{l}\text { Integrität, } \\
\text { Diskretion, Fair- } \\
\text { ness, Verlässlich- } \\
\text { keit, Konsistenz }\end{array}$ \\
\hline $\begin{array}{l}\text { Bentele } \\
\text { (1994) }\end{array}$ & $\begin{array}{l}\text { Plausibilitäts- } \\
\text { überlegungen }\end{array}$ & $\begin{array}{l}\text { Sachkompetenz, } \\
\text { Problemlösungs- } \\
\text { kompetenz }\end{array}$ & $\begin{array}{l}\text { Kommunikative } \\
\text { Konsistenz, } \\
\text { Transparenz, } \\
\text { Offenheit }\end{array}$ & Verantwortung \\
\hline $\begin{array}{l}\text { Mayer et } \\
\text { al. (1995) }\end{array}$ & $\begin{array}{l}\text { Plausibilitäts- } \\
\text { überlegungen }\end{array}$ & $\begin{array}{l}\text { Fähigkeit (i.S.v. } \\
\text { Fachkompetenz) }\end{array}$ & Wohlwollen & Integrität \\
\hline $\begin{array}{l}\text { Mishra } \\
\text { (1996) }\end{array}$ & $\begin{array}{l}\text { Inhalts- } \\
\text { analytische } \\
\text { Auswertung } \\
(\mathrm{N}=33)\end{array}$ & $\begin{array}{l}\text { Kompetenz (i.S.v. } \\
\text { Fachkompetenz) }\end{array}$ & $\begin{array}{l}\text { Offenheit, } \\
\text { Loyalität, } \\
\text { Zugänglichkeit } \\
\text { (,concern') }\end{array}$ & Verlässlichkeit \\
\hline $\begin{array}{l}\text { Shaw } \\
\text { (1997) }\end{array}$ & $\begin{array}{l}\text { Plausibilitäts- } \\
\text { überlegungen }\end{array}$ & $\begin{array}{l}\text { Fähigkeit zum } \\
\text { Erzielen von } \\
\text { Geschäfts- } \\
\text { ergebnissen }\end{array}$ & $\begin{array}{l}\text { Wohlwollen, } \\
\text { Verständnis und } \\
\text { Respekt der } \\
\text { Bedürfnisse } \\
\text { anderer Akteure, } \\
\text { Loyalität }\end{array}$ & $\begin{array}{l}\text { Integrität } \\
\text { (Ehrlichkeit, } \\
\text { Konsistenz und } \\
\text { Stetigkeit im } \\
\text { Handeln) }\end{array}$ \\
\hline $\begin{array}{l}\text { Bittl } \\
(1997)\end{array}$ & $\begin{array}{l}\text { Plausibilitäts- } \\
\text { überlegungen }\end{array}$ & $\begin{array}{l}\text { Problemlösungs- } \\
\text { fähigkeit }\end{array}$ & $\begin{array}{l}\text { Problemlösungs- } \\
\text { wille }\end{array}$ & $\begin{array}{l}\text { Problemlösungs- } \\
\text { wille }\end{array}$ \\
\hline $\begin{array}{l}\text { Seifert } \\
(2001)\end{array}$ & $\begin{array}{l}\text { Inhalts- } \\
\text { analytische } \\
\text { Auswertung } \\
(\mathrm{N}=42)\end{array}$ & $\begin{array}{l}\text { Kompetenz (i.S.v. } \\
\text { Fach- und } \\
\text { Methoden- } \\
\text { kompetenz): } \\
\text { Expertenwissen, } \\
\text { Problemlösungs- } \\
\text { fähigkeit, kom- } \\
\text { petente Aus- } \\
\text { führung all- } \\
\text { täglicher Routine- } \\
\text { handlungen }\end{array}$ & $\begin{array}{l}\text { Gesinnung } \\
\text { (Loyalität, } \\
\text { Zugänglichkeit, } \\
\text { Erreichbarkeit) }\end{array}$ & $\begin{array}{l}\text { Integrität } \\
\text { (Zuverlässigkeit. } \\
\text { Wahrheit/ } \\
\text { Ehrlichkeit, } \\
\text { Diskretion) }\end{array}$ \\
\hline $\begin{array}{l}\text { Späth } \\
(2008)\end{array}$ & $\begin{array}{l}\text { Regressions- } \\
\text { analyse } \\
(\mathrm{N}=90)\end{array}$ & & Wohlwollen & \\
\hline
\end{tabular}

Tabelle 14: Individuelle Kompetenzen als Vertrauensdeterminanten

\subsection{Der Einfluss organisationaler Kompetenzen auf Vertrauen}

Die zur Sicherstellung der Wettbewerbsfähigkeit benötigen Basiskompetenzen eines Unternehmens werden vielfach als Determinanten der Entstehung von Ver- 
trauen seitens des Vertrauensgebers aufgefasst. ${ }^{521}$ So stellt auch Sichtmann die zentrale Bedeutung der ,necessary competencies', über die ein Unternehmen verfügen muss, um am Markt agieren zu können, für die Entstehung von Vertrauen seitens des Vertrauensgebers heraus: „Without being competent, a supplier cannot deliver good quality. Therefore, a consumer will only trust a supplier if $s /$ he is convinced that the supplier is competent enough to fulfill her/his demands." ${ }^{\text {(522 }}$ Auch die zur Durchsetzung eines langfristigen Wettbewerbsvorteils benötigten Kernkompetenzen eines Unternehmens werden vielfach als Determinanten der Entstehung von Vertrauen seitens des Vertrauensgebers aufgefasst. ${ }^{523}$ So stellt auch Ichijo das vom Vertrauensgeber wahrgenommene Bündel verschiedener Technologien und Fähigkeiten, das einen besonderen Kundennutzen generiert und den Zugang zu einer Vielzahl von Märkten eröffnet, als zentrale und unverzichtbare Vertrauensbedingung heraus: ,[...] core competence [...] should generate mutual unterstanding and trust. ${ }^{\text {" } 524}$ Allerdings ist, ausgehend von der dieser Arbeit zugrunde liegenden Annahme, dass sich Vertrauen auf Verhaltensrisiken bezieht, der direkte positive Einfluss wahrgenommener Basis- und Kernkompetenzen auf die Entstehung von Vertrauen, über die Dimension der Vertrauenserwartung, fraglich. Auch gibt die in dieser Arbeit vertretene Auffassung, dass Vertrauen zwischen Unternehmen immer durch die diesen Unternehmen angehörenden Individuen begründet und zerstört wird, Anlass zum Zweifel darüber, ob ein Vertrauensgeber die Vertrauenswürdigkeit eines Unternehmens einschätzen kann. ${ }^{525}$ Vielmehr ist davon auszugehen, dass die Wahrnehmung organisationaler Kompetenzen die Vornahme einer Vertrauenshandlung, und damit die Platzierung von Vertrauen seitens des Vertrauensgebers über das Konstrukt des Zutrauens positiv beeinflusst.

Nachfolgende Tabelle fasst die synoptische Sicht auf Ansätze, die auf eine Erforschung der Vertrauensdeterminanten abzielen, in Bezug auf die für die Vertrauensentstehung relevanten organisationalen Kompetenzen zusammen. In diesem Zusammenhang bleibt festzuhalten, dass die erläuterten Studien nicht mit den Begriffen Basis- und Kernkompetenz operieren. Aufgrund gewisser inhaltlicher Übereinstimmungen zwischen diesen Ansätzen und dem im Zuge der begrifflichen Klärung und Abgrenzung des Kompetenzkonstrukts entwickelten Kompetenzverständnis dieser Arbeit, handelt es sich bei der Zuordnung der, von den genannten Autoren identifizierten Vertrauensdeterminanten zu den organisationalen Kompetenzarten aber um keine willkürliche Systematisierung. Allerdings

\footnotetext{
521 Vgl. Bittl (1997), S. 163 f.; Doney/Cannon (1997), S. 44 f.; Sako/Helper (1998), S. 406; Apelt (1999), S. 88 f.; Seifert (2001), S. 84 ff.; Sichtmann (2007), S. 1009

522 Sichtmann (2007), S. 1002

523 Vgl. Bittl (1997), S. 162; Doney/Cannon (1997), S. 44 f.; Sako/Helper (1998), S. 406; Apelt (1999), S. 88 f.; Deckow (2006), S. 124; Sichtmann (2007), S. 1002

$524 \quad$ Ichijo (2007), S. 88

525 Vgl. zu einer diesbezüglichen Diskussion Janowicz/Noorderhaven (2006).
} 
wird durch diese sekundäranalytisch begründete Zuordnung auch deutlich, dass es an Hypothesen zum direkten und indirekten Einfluss organisationaler Kompetenzarten auf die Entstehung von Vertrauen, sowie ihrer empirischen Überprüfung mangelt.

\begin{tabular}{|c|c|c|c|}
\hline \multirow[b]{2}{*}{ Autor } & \multirow[b]{2}{*}{$\begin{array}{c}\text { Auswertung } \\
\text { (Datengrundlage) }\end{array}$} & \multicolumn{2}{|c|}{$\begin{array}{l}\text { Organisationale Kompetenzen als } \\
\text { Vertrauensdeterminanten }\end{array}$} \\
\hline & & Basiskompetenz & Kernkompetenz \\
\hline Bittl (1997) & Plausibilitätsüberlegungen & $\begin{array}{l}\text { Fähigkeit zur } \\
\text { Bedarfsdeckung } \\
\text { durch Produkte und } \\
\text { Dienstleistungen }\end{array}$ & $\begin{array}{l}\text { technische } \\
\text { Problemlösungs- } \\
\text { fähigkeit zur } \\
\text { Bedarfsdeckung }\end{array}$ \\
\hline $\begin{array}{l}\text { Doney/Cannon } \\
\text { (1997) }\end{array}$ & $\begin{array}{l}\text { Logistische Regression } \\
(\mathrm{N}=210)\end{array}$ & $\begin{array}{l}\text { entsprechende } \\
\text { Unternehmens- } \\
\text { größe zur Sicher- } \\
\text { stellung der } \\
\text { branchendurch- } \\
\text { schnittlichen Wett- } \\
\text { bewerbsfähigkeit }\end{array}$ & $\begin{array}{l}\text { Fähigkeit zur Vor- } \\
\text { nahme kunden- } \\
\text { spezifischer An- } \\
\text { passungen }\end{array}$ \\
\hline $\begin{array}{l}\text { Sako/Helper } \\
\text { (1998) }\end{array}$ & $\begin{array}{l}\text { Faktor- und Regressions- } \\
\text { analyse }(\mathrm{N}=1147)\end{array}$ & Informationstausch & $\begin{array}{l}\text { Technologie- } \\
\text { kompetenz, } \\
\text { Kundenreputation, } \\
\text { langfristige } \\
\text { Verpflichtung } \\
\end{array}$ \\
\hline Apelt (1999) & $\begin{array}{l}\text { Faktor- und Regressions- } \\
\text { analyse }(\mathrm{N}=262)\end{array}$ & $\begin{array}{l}\text { Informationstausch, } \\
\text { Sicherheiten, ange- } \\
\text { messenes Preis- } \\
\text { Leistungsverhältnis, } \\
\text { Bestand und Größe } \\
\text { des Unternehmens }\end{array}$ & $\begin{array}{l}\text { Qualitätssicherung, } \\
\text { Entwicklungs- } \\
\text { kooperationen }\end{array}$ \\
\hline Seifert (2001) & $\begin{array}{l}\text { Inhaltsanalytische } \\
\text { Auswertung }(\mathrm{N}=42)\end{array}$ & $\begin{array}{l}\text { Management- } \\
\text { kompetenz, Erfolg } \\
\text { (Umsatz, Gewinn) }\end{array}$ & $\begin{array}{l}\text { besonderer } \\
\text { Kundennutzen }\end{array}$ \\
\hline $\begin{array}{l}\text { Sichtmann } \\
(2007)\end{array}$ & LISREL $8(N=308)$ & $\begin{array}{l}\text { Management- } \\
\text { kompetenz }\end{array}$ & $\begin{array}{l}\text { Qualitätssicherung, } \\
\text { Innovationen }\end{array}$ \\
\hline
\end{tabular}

Tabelle 15: Organisationale Kompetenzen als Vertrauensdeterminanten

Zusammenfassend zeigt sich, dass die Forschung zur Wirkung von Kompetenz als Determinante der Entstehung von Vertrauen am Anfang steht. Das gilt vor allem für Kompetenzarten, die sich nicht auf die Übereinstimmung von fachlichen Fähigkeiten und den mit einer Aufgabe verbundenen fachlichen Anforderungen 
beziehen. So nennen einige Studien ${ }^{526}$ Kompetenz als Vertrauensdeterminante, unterlassen es aber zu erläutern, welche Arten von Kompetenz auf die Entstehung von Vertrauen Einfluss nehmen, und wie sie auf die Vertrauensentwicklung wirken. Gründe hierfür mögen in der mangelnden Konzeptualisierung und Operationalisierung sowohl des Kompetenz-, als auch des Vertrauenskonstrukts liegen. So besteht in Bezug auf das Vertrauenskonstrukt nicht bloß Uneinigkeit hinsichtlich der Vorbedingungen, direkten Determinanten, Dimensionen, Manifestationen und Konsequenzen von Vertrauen, sondern auch Unklarheit darüber, ob es sich bei gewissen Faktoren um Ursache, Wirkung oder Vertrauen per se handelt. ${ }^{527}$ Diese Probleme zeigen sich besonders bei der Analyse der Wirkung von Kompetenz als Determinante der Entstehung von Vertrauen: Aufgrund der mangelnden Differenzierung zwischen den verschiedenen Kompetenzarten wird Kompetenz vielfach als direkte Determinante auf die Vertrauensentstehung aufgefasst, ohne allerdings zu berücksichtigen, dass bestimmte Kompetenzarten erst über das Konstrukt des Zutrauens die Platzierung von Vertrauen motivieren können. Auch wird vielfach außer Acht gelassen, dass sich die Vertrauensentscheidung aus Vertrauenserwartung und Vertrauenshandlung zusammensetzt. Einerseits ist nämlich anzunehmen, dass bestimmte Kompetenzarten einen direkten Einfluss auf die Vertrauenserwartung, d.h. die Einschätzung der Vertrauenswürdigkeit des Vertrauensnehmers seitens des Vertrauensgebers, nehmen, und dadurch die Vornahme einer Vertrauenshandlung, die auch von dem wahrgenommenen situationsspezifischen Risiko und der vertrauensgeberspezifischen Risikobereitschaft beeinflusst wird, motivieren, was sodann in einer Vertrauensentscheidung mündet. Andererseits besteht Grund zur Annahme, dass bestimmte Kompetenzarten einen positiven Einfluss auf die Vornahme einer Vertrauenshandlung nehmen, und zwar indem sie über das Konstrukt des Zutrauens die Unsicherheit über das Können des Vertrauensnehmers reduzieren.

\section{DiE METHODISCHEN GRUNDLAGEN DER MODELLIERUNG VON KOMPETENZ ALS DETERMINANTE DER VERTRAUENSENTSTEHUNG}

Die Modellierung des Einflusses von Kompetenz auf die Vertrauensentstehung seitens des Vertrauensgebers ist mit besonderen Anforderungen verbunden. Diese ergeben sich dadurch, dass Kompetenz und Vertrauen als latente Konstrukte, d.h. als ,[...] abstract entity which represents the true, nonobservable state or nature of a phenomenon ${ }^{\text {“ } 528}$, über keine unmittelbar empirisch erfassbaren Korrelate verfügen, sodass eine direkte Erfassung unmöglich ist. Um also den Einfluss von

526 Als Beispiele können die Studien von Kee/Knox (1970), S. 361; Butler (1991), S. 648; Sitkin/Roth (1993), S. 373; McKnight et al. (1998), S. 476 ff.; Adler (2001), S. 218 genannt werden.

527 Vgl. zu den Defiziten der Studien zur Erforschung des Vertrauenskonstrukts auch Gillespie (2003); Späth (2008).

528 Bagozzi/Fornell (1982), S. 24 
Kompetenz auf Vertrauen modelltheoretisch abbilden und empirisch abtesten zu können, ist es daher erforderlich, die latenten Konstrukte in ihre theoretisch postulierten Teilmerkmale (Dimensionen) zu zerlegen. Auf Basis dieser Dimensionen sind sodann direkt wahrnehmbare Phänomene (Indikatoren) zu identifizieren, die Rückschlüsse auf die theoretischen Konstrukte zulassen, und diese einer Operationalisierung ${ }^{529}$ zugänglich machen. ${ }^{530}$

Die in dieser Arbeit verfolgte Modellierung des Einflusses von Kompetenz auf die Entstehung von Vertrauen beruht auf einer dimensionalen Analyse. Als Technik zur Operationalisierung theoretischer Konstrukte setzt sie einen mehrstufigen Prozess voraus, im Zuge dessen eine Bestimmung von Merkmalsdimensionen, Faktoren und Indikatoren, die Rückschlüsse auf die Konstrukte zulassen, erfolgt (nachfolgende Graphik stellt eine vereinfachte Abbildung des Prozesses der Operationalisierung des latenten Kompetenzkonstrukts dar). ${ }^{531}$

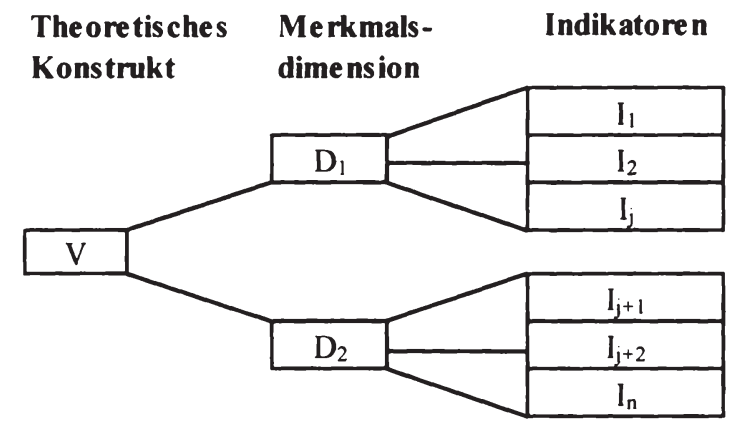

Abbildung 12: Der Prozess der Modellierung

Quelle: In Anlehnung an Faix/Krupp (2002, S. 62)

Der erste Schritt der dimensionalen Analyse besteht in der Klärung, welche theoretischen Merkmale eines festgelegten Untersuchungsgegenstandes durch das theoretische Konstrukt bezeichnet werden. Hierbei sind gültige, voneinander unabhängige Merkmalsdimensionen zu identifizieren, die Rückschlüsse auf das Konstrukt zulassen und vor dem Hintergrund der Untersuchung als relevant erachtet werden. Die Entscheidung für die Merkmalsdimensionen des theoretischen Kompetenz- und des Vertrauenskonstrukts beruht auf den Ergebnissen der vorangegangenen Kapitel. Bezugnehmend auf das Vertrauenskonstrukt wird in dieser Arbeit die Auffassung vertreten, dass sich die Vertrauensentscheidung aus Ver-

529 Friedrichs (1990, S. 79) versteht unter Operationalisierung „die Angabe von beobachtbaren Designaten (Indikatoren) und deren Kombination". Sie dient der Schaffung der Verbindung von begrifflicher Ebene und Beobachtungsebene, wobei die Korrespondenz der Ebenen durch die identifizierten Indikatoren erreicht wird.

530 Vgl. Faix/Krupp (2002), S. 60; Späth (2008), S. 132

531 Vgl. Laatz (1993), S. 323: Schnell et al. (1999), S. $121 \mathrm{ff}$ 
trauenserwartung und Vertrauenshandlung zusammensetzt ${ }^{532}$ - als Merkmalsdimensionen ${ }^{533}$, die Rückschlüsse auf das Vertrauenskonstrukt zulassen, sollen die Vertrauenserwartung einerseits, und die Vertrauenshandlung andererseits, daher im Zuge der Modellentwicklung berücksichtigt werden. Bezugnehmend auf das Kompetenzkonstrukt spiegelt die in dieser Arbeit getroffene Entscheidung für die Merkmalsdimensionen die Erkenntnisse der Kompetenzforschung wider, wonach entsprechend der Besitzverhältnisse von Kompetenzen zwischen organisationalen und individuellen Kompetenzen zu differenzieren ist. ${ }^{534}$

Um den Einfluss organisationaler und individueller Kompetenzen auf die Entstehung von Vertrauen hinreichend modelltheoretisch abbilden und empirisch abtesten zu können, ist es in einem weiteren Schritt der dimensionalen Analyse erforderlich, die identifizierten Merkmalsdimensionen in gültige und voneinander unabhängige Faktoren aufzuspalten. ${ }^{535}$ Wie auch die Entscheidung für die Merkmalsdimensionen, reflektiert die Bestimmung der Faktoren den zum Teil resümierenden Charakter der Modellierung des Kompetenzeinflusses auf die Vertrauensentstehung, da auch im Zuge dieses zweiten Schrittes der Analyse ein Rückgriff auf die Ergebnisse der in den vorangegangenen Kapiteln dieser Arbeit angestellten Überlegungen erfolgt. So finden sowohl die Aufspaltung der organisationalen Kompetenzen in Basis- und Kernkompetenzen, als auch die Aufspaltung der individuellen Kompetenzen in Fach-, Methoden, Sozial- und Persönlichkeitskompetenzen ihre begriffliche und inhaltliche Fundierung in der Kompetenzforschung. ${ }^{536}$

In einem dritten Schritt sind konsensfähige und gültige Indikatoren für die festgelegten Merkmalsdimensionen bzw. Faktoren zu bestimmen, die als deren empirische Äquivalente Rückschlüsse auf die Merkmalsdimensionen bzw. Faktoren, und damit auf die theoretischen Konstrukte Vertrauen und Kompetenz ermöglichen. ${ }^{537}$ Ebenso wie die vorangegangenen Schritte der dimensionalen Analyse,

Vgl. hierzu die Ausführungen in Teil Il Kap. 5.1, sowie die dort zit. Literatur.

Die Unabhăngigkeit der Merkmalsdimensionen ergibt sich durch Einbeziehung des situationsspezifischen Faktors Risiko: So kann eine positive Vertrauenserwartung gegeben sein, aber aufgrund der Höhe des wahrgenommenen Risikos erfolgt dennoch keine Vertrauenshandlung. Andererseits kann eine Vertrauenshandlung gesetzt werden, der, aufgrund eines als niedrig wahrgenommenen Risikos, keine hohe Vertrauenserwartung vorangeht. Eine Vertrauensentscheidung im Sinne eines Schenkens von Vertrauen durch den Vertrauensgeber liegt allerdings nur dann vor, wenn eine Vertrauenshandlung vorgenommen, und diese durch eine vertrauensvolle Erwartung motiviert wurde (vgl. hierzu die Ausführungen in Teil II Kap. 5.1.1, sowie die dort zit. Literatur (v.a. Ripperger 1998)).

Vgl. Turner/Crawford (1994), S. 242, und die Ausführungen in Teil III Kap. 2, sowie die dort zit. Literatur.

Vgl. Faix/Krupp (2002), S. 61

Vgl. hierzu die Ausführungen in Teil III Kap. 2.2, sowie die dort zit. Literatur.

Vgl. Schweer/Thies (2003), S. 24. Im Falle der vorliegenden Indikatoren handelt es sich um sog. reflektive Indikatoren. Sie werden durch eine Merkmalsdimension bzw. einen Faktor verursacht 
beruht die Indikatorenbestimmung auf subjektiven Relevanzüberlegungen ${ }^{538}$, die sich ihrerseits auf die Ergebnisse erfolgter Untersuchungen in den Bereichen Kompetenz- und Vertrauensforschung stützen. Um dem Anspruch einer fundierten Modellierung des Kompetenzeinflusses auf die Vertrauensentstehung gerecht zu werden, wird sich im Zuge der Indikatorenbestimmung an folgender Vorgehensweise orientiert: Unter Berücksichtigung des Untersuchungskontexts erfolgt in Kapitel 3 bzw. Kapitel 4 eine Auswahl konsensfähiger Kompetenzindikatoren bzw. Vertrauensindikatoren, die anhand sekundäranalytischer Nachweise aus der Kompetenzforschung bzw. aus der Vertrauensforschung bestätigt wird. Um die empirische Überprüfung des in Kapitel 5 zu entwickelnden Modells erleichtern zu können, beispielsweise im Hinblick auf die Itemgenerierung, erfolgt im Anschluss an die Indikatorenauswahl, eine Beschreibung der identifizierten Kompetenzindikatoren bzw. Vertrauensindikatoren am vorliegenden Untersuchungsgegenstand, d.h. mit Bezug auf zwischenbetriebliche Kooperationen (vgl. Kapitel 3 bzw. 4).

\section{DiE INDIKATOREN ZUR OPERATIONALISIERUNG DES KOMPETENZ- KONSTRUKTS}

Nachdem das Kompetenzkonstrukt in Merkmalsdimensionen und Faktoren aufgespalten wurde, zielt das vorliegende Kapitel darauf ab, auf Basis der in Kapitel 3 des Teils III dieser Arbeit erläuterten Kompetenzmessansätze, konsensfähige und gültige Indikatoren, die als empirische Äquivalente der einzelnen Kompetenzarten fungieren, zu bestimmen, und am Fokus ,zwischenbetriebliche Kooperationen' zu erläutern. Die identifizierten und sekundäranalytisch abgesicherten Indikatoren dienen der fundierten Modellierung des Einflusses von Kompetenz auf die Entstehung von Vertrauen, sowie der Generierung von Items für die empirische Überprüfung der hypothetisierten Kausalbeziehung zwischen Kompetenz und Vertrauen in zwischenbetrieblichen Kooperationen.

\subsection{Die Indikatoren zur Operationalisierung individueller Kompetenzen}

Das Ziel der Operationalisierung individueller Kompetenzen setzt - entsprechend der in Kapitel 2 erfolgten Faktorenbestimmung - die Identifikation fachlicher,

und deshalb als Messungen dieser Merkmalsdimension bzw. dieses Faktors verstanden (Homburg/ Giering 1996, S. 6). Demnach handelt es sich auch bei der modelltheoretischen Abbildung des Kompetenzeinflusses auf das Vertrauenskonstrukt um ein reflektives Messmodell, da angenommen wird, dass sich die zugeordneten empirischen Variablen aus den latenten Konstrukten ergeben, d.h. dass die Indikatoren Konsequenzen der hinter ihnen stehenden theoretischen Konstrukte sind (Fink et al. 2009, S. 116). 
methodischer, sozialer und personaler Kompetenzindikatoren voraus. Nachfolgende Tabelle zeigt daher eine tabellarische Auflistung der Indikatoren, die als empirische Äquivalente der individuellen Kompetenzarten fungieren, sowie ihre Erläuterung am vorliegenden Untersuchungsgegenstand, wobei unter dem ,Kooperationspartner' das für die Kooperation zuständige Individuum, im Sinne eines Kooperationsbeauftragten, verstanden wird.

\begin{tabular}{|c|c|c|}
\hline $\begin{array}{c}\text { fachliche Kompetenz- } \\
\text { indikatoren }\end{array}$ & $\begin{array}{l}\text { sekundäranalytische Nach- } \\
\text { weise der Kompetenz- } \\
\text { forschung }\end{array}$ & $\begin{array}{l}\text { Erläuterung der fachlichen } \\
\text { Kompetenzindikatoren am } \\
\text { vorliegenden Untersuchungs- } \\
\text { gegenstand }\end{array}$ \\
\hline Fachwissen & $\begin{array}{l}\text { Sonntag/Schäfer-Rauser } \\
\text { (1993); Stahl (1996); } \\
\text { Hänggi (1998); Belz (1999); } \\
\text { Edwards/Ewen (2000); } \\
\text { Erpenbeck/Heyse (2007); } \\
\text { Kauffeld et al. (2007) }\end{array}$ & $\begin{array}{l}\text { Der Kooperationspartner verfügt } \\
\text { über das technische Wissen, um } \\
\text { im Rahmen der Kooperations- } \\
\text { beziehung sachverständig und } \\
\text { effizient handeln zu können. }\end{array}$ \\
\hline $\begin{array}{c}\text { fachübergreifendes } \\
\text { Wissen }\end{array}$ & $\begin{array}{l}\text { Hänggi (1998); } \\
\text { Edwards/Ewen (2000); } \\
\text { Erpenbeck/Heyse (2007) }\end{array}$ & $\begin{array}{l}\text { Der Kooperationspartner verfügt } \\
\text { über ein breites Allgemein- und } \\
\text { Geschäftswissen, um im Rahmen } \\
\text { von Kooperationsbeziehungen } \\
\text { flexibel handeln zu können. }\end{array}$ \\
\hline kaufmännisches Wissen & $\begin{array}{l}\text { Belz (1999); Edwards/Ewen } \\
\text { (2000); Erpenbeck/Heyse } \\
\text { (2007); Kauffeld et al. } \\
\text { (2007) }\end{array}$ & $\begin{array}{l}\text { Der Kooperationspartner verfügt } \\
\text { über betriebswirtschaftliche Ken- } \\
\text { ntnisse, um im Rahmen von Ko- } \\
\text { operationsbeziehungen Ressour- } \\
\text { cen effizient einsetzen zu } \\
\text { können. }\end{array}$ \\
\hline Erfahrungswissen & $\begin{array}{l}\text { Sonntag/Schäfer-Rauser } \\
\text { (1993); Stahl (1996); } \\
\text { Hänggi (1998); } \\
\text { Erpenbeck/Heyse (2007) }\end{array}$ & $\begin{array}{l}\text { Der Kooperationspartner besitzt } \\
\text { berufspraktische Grund- und } \\
\text { Fachfertigkeiten, sodass er im } \\
\text { Rahmen von Kooperationsbe- } \\
\text { ziehungen effektiv und effizient } \\
\text { handeln kann. }\end{array}$ \\
\hline Abstraktionsfähigkeit & $\begin{array}{l}\text { Sonntag/Schäfer-Rauser } \\
\text { (1993); Erpenbeck/Heyse } \\
\text { (2007); Kauffeld et al. } \\
\text { (2007) }\end{array}$ & $\begin{array}{l}\text { Der Kooperationspartner verfügt } \\
\text { über ein auf Zusammenhänge } \\
\text { und Überblick ausgerichtetes } \\
\text { Denken, sodass er die Ver- } \\
\text { netztheit von Problemen im } \\
\text { Rahmen von Kooperations- } \\
\text { beziehungen erkennen kann. }\end{array}$ \\
\hline $\begin{array}{l}\text { Vermittlungs- und } \\
\text { Darstellungsfähigkeit }\end{array}$ & $\begin{array}{l}\text { Hänggi (1998); Belz (1999); } \\
\text { Edwards/Ewen (2000); } \\
\text { Erpenbeck/Heyse (2007); } \\
\text { Kauffeld et al. (2007) }\end{array}$ & $\begin{array}{l}\text { Der Kooperationspartner verfügt } \\
\text { über die Fähigkeit, die Differen- } \\
\text { ziertheit und Vernetztheit von } \\
\text { Problemen und Lösungen im } \\
\text { Rahmen von Kooperationsbe- } \\
\text { ziehungen zu erläutern und zu } \\
\text { veranschaulichen. }\end{array}$ \\
\hline
\end{tabular}




\begin{tabular}{|c|c|c|}
\hline $\begin{array}{c}\text { methodische Kompetenz- } \\
\text { indikatoren }\end{array}$ & $\begin{array}{l}\text { sekundäranalytische Nach- } \\
\text { weise der Kompetenz- } \\
\text { forschung }\end{array}$ & $\begin{array}{l}\text { Erläuterung der methodischen } \\
\text { Kompetenzindikatoren am } \\
\text { vorliegenden Untersuchungs- } \\
\text { gegenstand }\end{array}$ \\
\hline $\begin{array}{c}\text { Methoden zur } \\
\text { Informationsverarbeitung }\end{array}$ & $\begin{array}{l}\text { Sonntag/Schäfer-Rauser } \\
\text { (1993); Erpenbeck/Heyse } \\
\text { (2007); Kauffeld et al. } \\
\text { (2007) }\end{array}$ & $\begin{array}{l}\text { Der Kooperationspartner } \\
\text { beherrscht Methoden zur } \\
\text { Informationsbeschaffung (z.B. } \\
\text { Anwendungstechniken im } \\
\text { Umgang mit Datenbanken), - } \\
\text { strukturierung (z.B. Techniken } \\
\text { der Prioritätensetzung), - } \\
\text { bewertung (z.B. statistische } \\
\text { Auswertungsverfahren) und - } \\
\text { darstellung (z.B. } \\
\text { Präsentationstechniken). }\end{array}$ \\
\hline $\begin{array}{l}\text { Management- und } \\
\text { Leistungsmethoden }\end{array}$ & $\begin{array}{l}\text { Hänggi (1998); } \\
\text { Erpenbeck/Heyse (2007); } \\
\text { Kauffeld et al. (2007) }\end{array}$ & $\begin{array}{l}\text { Der Kooperationspartner } \\
\text { beherrscht Methoden, um } \\
\text { Kooperationsbeziehungen ziel- } \\
\text { und konsensorientiert managen } \\
\text { zu können (z.B. Zielanalysen). }\end{array}$ \\
\hline $\begin{array}{c}\text { Planungs- und } \\
\text { Kontrollmethoden }\end{array}$ & $\begin{array}{l}\text { Sonntag/Schäfer-Rauser } \\
\text { (1993); Erpenbeck/Heyse } \\
\text { (2007); Kauffeld et al. } \\
\text { (2007) }\end{array}$ & $\begin{array}{l}\text { Der Kooperationspartner } \\
\text { beherrscht Methoden zur } \\
\text { Planung und Kontrolle, um Ko- } \\
\text { operationsaufgaben planen, ko- } \\
\text { ordinieren, delegieren und } \\
\text { kontrollieren zu können. }\end{array}$ \\
\hline $\begin{array}{l}\text { Problemlösung-, Lern- } \\
\text { und Kreativmethoden }\end{array}$ & $\begin{array}{l}\text { Sonntag/Schäfer-Rauser } \\
\text { (1993); Stahl (1996); } \\
\text { Hänggi (1998); Belz (1999); } \\
\text { Edwards/Ewen (2000); } \\
\text { Erpenbeck/Heyse (2007); } \\
\text { Kauffeld et al. (2007) }\end{array}$ & $\begin{array}{l}\text { Der Kooperationspartner } \\
\text { beherrscht Methoden, um seinen } \\
\text { fachspezifischen und fachüber- } \\
\text { greifenden Wissensstand konti- } \\
\text { nuierlich den Erfordernissen der } \\
\text { Kooperationsbeziehung anzu- } \\
\text { passen, sowie Methoden zur Be- } \\
\text { schreibung, Analyse und Lösung } \\
\text { von Problemen im Rahmen von } \\
\text { Kooperationsbeziehungen. }\end{array}$ \\
\hline $\begin{array}{l}\text { soziale Kompetenz- } \\
\text { indikatoren }\end{array}$ & $\begin{array}{c}\text { sekundäranalytische Nach- } \\
\text { weise der Kompetenz- } \\
\text { forschung }\end{array}$ & $\begin{array}{l}\text { Erläuterung der sozialen } \\
\text { Kompetenzindikatoren am } \\
\text { vorliegenden Untersuchungs- } \\
\text { gegenstand }\end{array}$ \\
\hline $\begin{array}{c}\text { Einfühlungsvermögen } \\
\text { und Solidarität } \\
\text { (Personenwahrnehmung) }\end{array}$ & $\begin{array}{l}\text { Stahl (1996); Belz (1999); } \\
\text { Henning-Thurau/Thurau } \\
\text { (1999); Kanning (2005); } \\
\text { Edwards/Ewen (2000); } \\
\text { Erpenbeck/Heyse (2007) }\end{array}$ & $\begin{array}{l}\text { Der Kooperationspartner verfügt } \\
\text { über Einfühlungsvermögen und } \\
\text { Menschenkenntnis, um die } \\
\text { Bedürfnisse und Verhaltens- } \\
\text { weisen seines Partners im } \\
\text { Rahmen der Kooperation wahr- } \\
\text { nehmen, beurteilen und } \\
\text { berücksichtigen zu können. }\end{array}$ \\
\hline
\end{tabular}




\begin{tabular}{|c|c|c|}
\hline $\begin{array}{l}\text { Kontaktfähigkeit } \\
\text { (Selbstőffnung) }\end{array}$ & $\begin{array}{l}\text { Sonntag/Schäfer-Rauser } \\
\text { (1993); Stahl (1996); Belz } \\
\text { (1999); Edwards/Ewen } \\
\text { (2000); Erpenbeck/Heyse } \\
\text { (2007); Kauffeld et al. } \\
\text { (2007); }\end{array}$ & $\begin{array}{l}\text { Der Kooperationspartner verfügt } \\
\text { über die Fähigkeit, die Be- } \\
\text { ziehung zu seinem Partner im } \\
\text { Rahmen der Kooperation zu } \\
\text { initiieren und zu pflegen, indem } \\
\text { er sich selbst öffnet. }\end{array}$ \\
\hline $\begin{array}{c}\text { Überzeugungsfăhigkeit, } \\
\text { Charisma }\end{array}$ & $\begin{array}{l}\text { Sonntag/Schäfer-Rauser } \\
\text { (1993); Belz (1999); } \\
\text { Henning-Thurau/Thurau } \\
\text { (1999); Erpenbeck/Heyse } \\
\text { (2007); Kauffeld et al. } \\
\text { (2007) }\end{array}$ & $\begin{array}{l}\text { Der Kooperationspartner verfügt } \\
\text { über die Fähigkeit, seinem } \\
\text { Partner im Rahmen der } \\
\text { Kooperation kooperations- } \\
\text { relevante Vorgehensweisen } \\
\text { überzeugend darzustellen, sodass } \\
\text { dessen Zustimmung auf Einsicht } \\
\text { beruht. }\end{array}$ \\
\hline $\begin{array}{c}\text { Bereitschaft zur } \\
\text { Informationsweitergabe }\end{array}$ & $\begin{array}{l}\text { Sonntag/Schäfer-Rauser } \\
\text { (1993); Belz (1999); } \\
\text { Edwards/Ewen (2000); } \\
\text { Erpenbeck/Heyse (2007); } \\
\text { Kauffeld et al. (2007) }\end{array}$ & $\begin{array}{l}\text { Der Kooperationspartner ist } \\
\text { bereit, kooperationsrelevante } \\
\text { Informationen, sowie sein fach- } \\
\text { liches Wissen mit seinem Partner } \\
\text { im Rahmen der Kooperation zu } \\
\text { teilen (iSv Know-How-Transfer). }\end{array}$ \\
\hline soziale Gesinnung & $\begin{array}{l}\text { Hänggi (1998); Belz (1999); } \\
\text { Henning-Thurau/Thurau } \\
\text { (1999); Edwards/Ewen } \\
\text { (2000); Erpenbeck/Heyse } \\
\text { (2007); Kauffeld et al. } \\
\text { (2007) }\end{array}$ & $\begin{array}{l}\text { Der Kooperationspartner ist } \\
\text { seinem Partner gegenüber wohl- } \\
\text { wollend gesinnt, d.h. er ist auf- } \\
\text { richtig, offen, zugänglich und } \\
\text { erreichbar und erwartet nicht für } \\
\text { jede Leistung eine Gegenleistung } \\
\text { (iSv Orientierung an sozialen } \\
\text { Regeln und Verpflichtungen). }\end{array}$ \\
\hline $\begin{array}{l}\text { Kommunikations- } \\
\text { fahigkeit }\end{array}$ & $\begin{array}{l}\text { Sonntag/Schäfer-Rauser } \\
\text { (1993); Hänggi (1998); Belz } \\
\text { (1999); Henning- } \\
\text { Thurau/Thurau (1999); } \\
\text { Edwards/Ewen (2000); } \\
\text { Erpenbeck/Heyse (2007) }\end{array}$ & $\begin{array}{l}\text { Der Kooperationspartner verfügt } \\
\text { über die Fähigkeit zur syste- } \\
\text { matischen Planung der Kom- } \\
\text { munikation (z.B. Ziele, } \\
\text { Strategien, Instrumente), sowie } \\
\text { die verbalen Fähigkeiten (z.B. } \\
\text { Rhetorik) zur Gestaltung der } \\
\text { Kommunikation mit seinem } \\
\text { Partner. }\end{array}$ \\
\hline Kooperationsfähigkeit & $\begin{array}{l}\text { Sonntag/Schäfer-Rauser } \\
\text { (1993); Hänggi (1998); Belz } \\
\text { (1999); Edwards/Ewen } \\
\text { (2000); Erpenbeck/Heyse } \\
\text { (2007); Kauffeld et al. } \\
\text { (2007) }\end{array}$ & $\begin{array}{l}\text { Der Kooperationspartner ist } \\
\text { fähig, mit seinem Partner } \\
\text { zusammenzuarbeiten. Dies zeigt } \\
\text { sich in der Nicht-Ausnützung } \\
\text { von Opportunismusspielräumen, } \\
\text { sowie in der Bereitschaft zur } \\
\text { Unterstützung und Hilfestellung } \\
\text { seines Partners }\end{array}$ \\
\hline Konfliktfähigkeit & $\begin{array}{l}\text { Sonntag/ Schäfer-Rauser } \\
\text { (1993); Hänggi (1998); } \\
\text { Edwards/Ewen (2000); } \\
\text { Erpenbeck/ Heyse (2007); }\end{array}$ & $\begin{array}{l}\text { Der Kooperationspartner ist } \\
\text { fähig, kooperationsbezogene } \\
\text { Konflikte zu erkennen } \\
\text { (Integrationsfähigkeit), }\end{array}$ \\
\hline
\end{tabular}




\begin{tabular}{|c|c|c|}
\hline & Kauffeld et al. (2007) & $\begin{array}{l}\text { konstruktiv darauf zu reagieren } \\
\text { (Toleranz) und sie konsens- } \\
\text { orientiert zu lösen. }\end{array}$ \\
\hline $\begin{array}{c}\text { personale Kompetenz- } \\
\text { indikatoren }\end{array}$ & $\begin{array}{l}\text { sekundäranalytische Nach- } \\
\text { weise der Kompetenz- } \\
\text { forschung }\end{array}$ & $\begin{array}{l}\text { Erläuterung der personalen } \\
\text { Kompetenzindikatoren am } \\
\text { vorliegenden Untersuchungs- } \\
\text { gegenstand }\end{array}$ \\
\hline Aufgeschlossenheit & $\begin{array}{l}\text { Stahl (1996); Hänggi } \\
\text { (1998); Brandstătter (2000); } \\
\text { Edwards/Ewen (2000); } \\
\text { Erpenbeck/ Heyse (2007); } \\
\text { Kauffeld et al. (2007) }\end{array}$ & $\begin{array}{l}\text { Der Kooperationspartner ist } \\
\text { sozial aufgeschlossen, was sich } \\
\text { in seinem Interesse an seinem } \\
\text { Partner zeigt. }\end{array}$ \\
\hline Fairness & $\begin{array}{l}\text { Hănggi (1998); } \\
\text { Edwards/Ewen (2000); } \\
\text { Erpenbeck/Heyse (2007) }\end{array}$ & $\begin{array}{l}\text { Der Kooperationspartner verhălt } \\
\text { sich seinem Partner gegenüber } \\
\text { fair. }\end{array}$ \\
\hline $\begin{array}{l}\text { Selbstvertrauen, } \\
\text { Selbstwertgefühl } \\
\text { (Selbstwahrnehmung) }\end{array}$ & $\begin{array}{l}\text { Sonntag/Schăfer-Rauser } \\
\text { (1993); Hänggi ( } 1998) ; \\
\text { Brandstätter (2000); } \\
\text { Erpenbeck/Heyse (2007) }\end{array}$ & $\begin{array}{l}\text { Der Kooperationspartner ist } \\
\text { făhig, seine Persönlichkeit } \\
\text { realitătsnah einzuschätzen, } \\
\text { wodurch es ihm möglich ist, } \\
\text { Handlungen im Rahmen der } \\
\text { Kooperation zu initiieren. }\end{array}$ \\
\hline $\begin{array}{c}\text { produktive } \\
\text { Begeisterungsfähigkeit }\end{array}$ & $\begin{array}{l}\text { Hänggi (1998); Henning- } \\
\text { Thurau/Thurau (1999); } \\
\text { Erpenbeck/Heyse (2007) }\end{array}$ & $\begin{array}{l}\text { Der Kooperationspartner ist } \\
\text { făhig, sich für die Aufgaben im } \\
\text { Rahmen der Kooperation zu } \\
\text { begeistern, und damit sein ko- } \\
\text { operationsbezogenes Handeln zu } \\
\text { motivieren. }\end{array}$ \\
\hline $\begin{array}{l}\text { konstruktiver Umgang } \\
\text { mit endogenen Reizen } \\
\text { (Fähigkeit zur Selbst- } \\
\text { kritik und Annahme } \\
\text { Kritik Dritter) }\end{array}$ & $\begin{array}{l}\text { Sonntag/Schäfer-Rauser } \\
\text { (1993); Hänggi ( } 1998) ; \\
\text { Edwards/Ewen (2000); } \\
\text { Erpenbeck/Heyse (2007) }\end{array}$ & $\begin{array}{l}\text { Der Kooperationspartner ist } \\
\text { belastbar und zu einem kon- } \\
\text { struktiven Umgang mit Unsicher- } \\
\text { heiten, Ängsten, irritierenden } \\
\text { Emotionen und Zielkonflikten } \\
\text { fähig, um Koopera- } \\
\text { tionsbeziehungen zu erhalten. }\end{array}$ \\
\hline $\begin{array}{l}\text { Zielstrebigkeit, Ehrgeiz, } \\
\text { Efrizienzstreben } \\
\text { (Selbstdisziplin) }\end{array}$ & $\begin{array}{l}\text { Hänggi (1998); Brandstätter } \\
(2000) ; \text { Edwards/Ewen } \\
(2000) \text {; Erpenbeck/Heyse } \\
(2007) ; \text { Kauffeld et al. } \\
(2007)\end{array}$ & $\begin{array}{l}\text { Der Kooperationspartner ist ehr- } \\
\text { geizig, zielstrebig und effizienz- } \\
\text { orientiert, sodass er sich zur } \\
\text { Setzung von nutzenstiftenden } \\
\text { Handlungen im Rahmen von } \\
\text { Kooperationsbeziehungen selbst } \\
\text { motivieren kann. }\end{array}$ \\
\hline $\begin{array}{l}\text { Experimentierfreudig- } \\
\text { keit, Neugier }\end{array}$ & $\begin{array}{l}\text { Sonntag/Schäfer-Rauser } \\
\text { (1993); Belz (1999); } \\
\text { Edwards/Ewen (2000); } \\
\text { Erpenbeck/Heyse (2007); } \\
\text { Kauffeld et al. (2007) }\end{array}$ & $\begin{array}{l}\text { Der Kooperationspartner ist Ver- } \\
\text { änderungen gegenüber positiv } \\
\text { eingestellt, sodass er Probleme } \\
\text { im Rahmen der Kooperations- } \\
\text { beziehung flexibel bewaltigen } \\
\text { kann. }\end{array}$ \\
\hline Verlässlichkeit, & Hănggi (1998); Brandstătter & Der Kooperationspartner ist \\
\hline
\end{tabular}




\begin{tabular}{|l|l|l|}
\hline Konsistenz & $\begin{array}{l}(2000) ; \text { Edwards/Ewen } \\
(2000) ; \text { Erpenbeck/Heyse } \\
(2007)\end{array}$ & $\begin{array}{l}\text { Prinzipien treu, die sein Partner } \\
\text { im Rahmen der Kooperations- } \\
\text { beziehung akzeptiert. Diese } \\
\text { Prinzipientreue zeigt sich in der } \\
\text { Einhaltung seiner Versprechen, } \\
\text { sowie in der Konsistenz seiner } \\
\text { vergangenen Handlungen. }\end{array}$ \\
\hline
\end{tabular}

Tabelle 16: Indikatoren individueller Kompetenzen

\subsection{Die Indikatoren zur Operationalisierung organisationaler Kompetenzen}

Das Ziel der Operationalisierung organisationaler Kompetenzen setzt - entsprechend der in Kapitel 2 erfolgten Faktorenbestimmung - die Identifikation von Basis- und Kernkompetenzen voraus. Nachfolgende Tabelle zeigt daher eine tabellarische Auflistung der Indikatoren, die als empirische Äquivalente der organisationalen Kompetenzarten fungieren, sowie ihre Erläuterung am vorliegenden Untersuchungsgegenstand, wobei unter dem ,Kooperationspartner das Unternehmen, mit dem eine Kooperation unterhalten wird, verstanden wird.

\begin{tabular}{|c|c|c|}
\hline Basiskompetenzindikatoren & $\begin{array}{c}\text { sekundäranalytische Nach- } \\
\text { weise der Kompetenz- } \\
\text { forschung }\end{array}$ & $\begin{array}{l}\text { Erläuterung der Basiskom- } \\
\text { petenzindikatoren am vor- } \\
\text { liegenden Untersuchungs- } \\
\text { gegenstand }\end{array}$ \\
\hline $\begin{array}{l}\text { Unternehmensgröße, } \\
\text { Kapitalstock/Kapitalstruktur, } \\
\text { Betriebsmittelausstattung, } \\
\text { Personalausstattung, } \\
\text { Produktionskapazität }\end{array}$ & $\begin{array}{l}\text { Weiss (1992); } \\
\text { Heyse/Erpenbeck (1997); } \\
\text { Möhringer (1998); } \\
\text { Rockenhäuser (1998); } \\
\text { Schiller (2000) }\end{array}$ & $\begin{array}{l}\text { Der Kooperationspartner } \\
\text { weist eine Größe (auch im } \\
\text { Hinblick auf den Kapital- } \\
\text { stock, die Ausstattung mit } \\
\text { Betriebsmitteln und } \\
\text { Personal, die Produktions- } \\
\text { kapazität) auf, bei der das } \\
\text { ökonomische Risiko einer } \\
\text { Insolvenz, sowie das } \\
\text { organisatorische Risiko } \\
\text { einer Kapazitätsgrenze im } \\
\text { Zuge der Auftragsabwick- } \\
\text { lung geringer zu bewerten } \\
\text { ist, d.h. er verfügt über die } \\
\text { notwendige Größe zur } \\
\text { Sicherstellung der branchen- } \\
\text { durchschnittlichen Wett- } \\
\text { bewerbsfähigkeit und damit } \\
\text { zur Unterhaltung von } \\
\text { Kooperationsbeziehungen. }\end{array}$ \\
\hline Marktstellung & $\begin{array}{l}\text { Weiss (1992); Belz (1999); } \\
\text { Heyse/Erpenbeck (1997); } \\
\text { Möhringer (1998) }\end{array}$ & $\begin{array}{l}\text { Die quantitativen Erfolgs- } \\
\text { größen, wie Marktanteil, } \\
\text { Gewinn, Umsatz des Ko- }\end{array}$ \\
\hline
\end{tabular}




\begin{tabular}{|c|c|c|}
\hline & & $\begin{array}{l}\text { operationspartners lassen als } \\
\text { objektive Indizien des } \\
\text { Unternehmenserfolgs darauf } \\
\text { schließen, dass die Auf- } \\
\text { gaben im Rahmen der Ko- } \\
\text { operationsbeziehung } \\
\text { effizient bewältigen werden } \\
\text { können. }\end{array}$ \\
\hline $\begin{array}{l}\text { Investitions- und Finanzkraft, } \\
\text { Erfolgsgeschichte und } \\
\text { Tradition (zukünftige } \\
\text { Marktpräsenz) }\end{array}$ & $\begin{array}{l}\text { Weiss (1992); } \\
\text { Heyse/Erpenbeck (1997); } \\
\text { Möhringer (1998); Riess } \\
\text { (1998); Rockenhăuser } \\
\text { (1998); Bouncken (2003) }\end{array}$ & $\begin{array}{l}\text { Die quantitativen Kenn- } \\
\text { größen Finanz- und } \\
\text { Investitionskraft, Verfüg- } \\
\text { barkeit von Eigen- und } \\
\text { Fremdkapital, sowie die } \\
\text { qualitativen Kenngrößen } \\
\text { Erfolgsgeschichte und } \\
\text { Tradition des Unternehmens } \\
\text { lassen darauf schließen, dass } \\
\text { der Kooperationspartner die } \\
\text { branchendurchschnittliche } \\
\text { Wettbewerbsfahigkeit auch } \\
\text { im Rahmen einer Koopera- } \\
\text { tionsbeziehung sicherstellen } \\
\text { kann. }\end{array}$ \\
\hline Kostenmanagement & $\begin{array}{l}\text { Conant et al. (1990); } \\
\text { Buchholz/Olemotz (1995); } \\
\text { Heyse/Erpenbeck (1997); } \\
\text { Möhringer (1998); Belz } \\
\text { (1999); Schiller (2000); } \\
\text { Thomson (2000); Bouncken } \\
\text { (2003) }\end{array}$ & $\begin{array}{l}\text { Die vom Partner akzep- } \\
\text { tierten Leistungspreise als } \\
\text { Indiz einer adäquaten } \\
\text { Kosten-Nutzen-Orientierung } \\
\text { des Unternehmens am } \\
\text { Markt lassen darauf } \\
\text { schließen, dass der Ko- } \\
\text { operationspartner die } \\
\text { Kosten im Rahmen der Ko- } \\
\text { operationsbeziehung } \\
\text { managen kann. }\end{array}$ \\
\hline Zielfestlegung und Planung & $\begin{array}{l}\text { Buchholz/Olemotz (1995); } \\
\text { Heyse/Erpenbeck (1997); } \\
\text { Belz (1999); Schiller (2000); } \\
\text { Jacob (2003) }\end{array}$ & $\begin{array}{l}\text { Der Kooperationspartner } \\
\text { verfügt über Făhigkeiten zur } \\
\text { Festlegung von Zielen, } \\
\text { sowie zur Beherrschung von } \\
\text { Planungsprozessen, um auf } \\
\text { zufriedenstellendem Niveau } \\
\text { die Erfüllung einer Vielzahl } \\
\text { notwendiger betrieblicher } \\
\text { und zwischenbetrieblicher } \\
\text { Ablăufe gewährleisten zu } \\
\text { können. }\end{array}$ \\
\hline $\begin{array}{l}\text { Organisationsfahigkeit, } \\
\text { kooperationsorientierte } \\
\text { Organisationsstruktur }\end{array}$ & $\begin{array}{l}\text { Dosi et al. (1992); } \\
\text { Buchholz/Olemotz (1995); } \\
\text { Zahn (1996); } \\
\text { Heyse/Erpenbeck (1997); }\end{array}$ & $\begin{array}{l}\text { Der Kooperationspartner } \\
\text { verfügt über Fähigkeiten zur } \\
\text { Beherrschung der Orga- } \\
\text { nisations-, Kontroll- und }\end{array}$ \\
\hline
\end{tabular}




\begin{tabular}{|c|c|c|}
\hline & $\begin{array}{l}\text { Krüger/Homp (1997); } \\
\text { Schiller (2000); Jacob (2003) }\end{array}$ & $\begin{array}{l}\text { Koordinationsprozesse, } \\
\text { sowie über kooperations- } \\
\text { orientierte Organisations- } \\
\text { strukturen, um betriebliche } \\
\text { Abläufe und kooperations- } \\
\text { bezogene Aufgaben auf } \\
\text { zufriedenstellendem Niveau } \\
\text { erfüllen zu können. }\end{array}$ \\
\hline Termintreue & $\begin{array}{l}\text { Buchholz/Olemotz (1995); } \\
\text { Thomson (2000) }\end{array}$ & $\begin{array}{l}\text { Der Kooperationspartner } \\
\text { erstellt bzw. liefert seine } \\
\text { Leistungen im Rahmen der } \\
\text { Kooperationsbeziehung } \\
\text { termingetreu. }\end{array}$ \\
\hline Kooperationsfähigkeit & $\begin{array}{l}\text { Schiller (2000); Harmsen et } \\
\text { al. (2002); Bouncken (2003); } \\
\text { Jacob (2003) }\end{array}$ & $\begin{array}{l}\text { Der Kooperationspartner ist } \\
\text { fähig, mit seinen Partnern } \\
\text { zusammenzuarbeiten und } \\
\text { gemeinsam am Markt zu } \\
\text { agieren. }\end{array}$ \\
\hline Kernkompetenzindikatoren & $\begin{array}{l}\text { sekundäranalytische Nach- } \\
\text { weise der Kompetenz- } \\
\text { forschung }\end{array}$ & $\begin{array}{l}\text { Erläuterung der Kernkom- } \\
\text { petenzindikatoren am vor- } \\
\text { liegenden Untersuchungs- } \\
\text { gegenstand }\end{array}$ \\
\hline Bekanntheit & $\begin{array}{l}\text { Weiss (1992); Möhringer } \\
\text { (1998); Faix/Krupp (2002) }\end{array}$ & $\begin{array}{l}\text { Der Kooperationspartner } \\
\text { verfügt über einen hohen } \\
\text { Bekanntheitsgrad am Markt, } \\
\text { was auf sein hohes } \\
\text { Potential zur Stiftung von } \\
\text { Kundennutzen schließen } \\
\text { lässt. }\end{array}$ \\
\hline $\begin{array}{c}\text { Know-How der } \\
\text { Unternehmensmitglieder }\end{array}$ & $\begin{array}{l}\text { Weiss (1992); Hall (1993); } \\
\text { Heyse/Erpenbeck (1997); } \\
\text { Möhringer (1998); } \\
\text { Rockenhäuser (1998); Belz } \\
\text { (1999); Magali (1999); } \\
\text { Schiller (2000); Faix/Krupp } \\
\text { (2002); Bouncken (2003); } \\
\text { Jacob (2003) }\end{array}$ & $\begin{array}{l}\text { Das implizite und explizite } \\
\text { Wissen der Organisations- } \\
\text { mitglieder, sowie der Grad } \\
\text { der Personengebundenheit } \\
\text { bzw. organisatorischen Ein- } \\
\text { gebundenheit des Wissens - } \\
\text { als Indikatoren des Kern- } \\
\text { kompetenzmerkmals der } \\
\text { Nicht-Imitierbarkeit - er- } \\
\text { möglichen es dem Kooper- } \\
\text { ationspartner, betriebliche } \\
\text { und zwischenbetriebliche } \\
\text { Aufgaben auf überdurch- } \\
\text { schnittlichem Niveau zu } \\
\text { erfullen. }\end{array}$ \\
\hline $\begin{array}{l}\text { bedarfsgerechtes Produkt- } \\
\text { und Leistungsangebot }\end{array}$ & $\begin{array}{l}\text { Weiss (1992); Hamel (1994); } \\
\text { Möhringer (1998); Belz } \\
\text { (1999); Bouncken (2003) }\end{array}$ & $\begin{array}{l}\text { Der Kooperationspartner } \\
\text { bietet bedarfsgerechte } \\
\text { Fertigung von Produkten } \\
\text { bzw. Erstellung von }\end{array}$ \\
\hline
\end{tabular}




\begin{tabular}{|c|c|c|}
\hline & & $\begin{array}{l}\text { Leistungen, d.h. verfügt } \\
\text { über } \\
\text { funktionalitătsbezogene } \\
\text { Fähigkeiten zur Generierung } \\
\text { von Produkten mit hohem } \\
\text { Kundennutzen im Rahmen } \\
\text { der Kooperationsbeziehung. }\end{array}$ \\
\hline Vermarktungsfähigkeiten & $\begin{array}{l}\text { Weiss (1992); Hamel (1994); } \\
\text { Heyse/Erpenbeck (1997); } \\
\text { Möhringer (1998); Belz } \\
\text { (1999); Bouncken (2003) }\end{array}$ & $\begin{array}{l}\text { Der Kooperationspartner } \\
\text { verfügt über Marketing- und } \\
\text { Vertriebsfăhigkeiten zur } \\
\text { Forcierung von Kundennähe } \\
\text { im Rahmen der Koopera- } \\
\text { tionsbeziehung. }\end{array}$ \\
\hline Unternehmensimage & $\begin{array}{l}\text { Hall (1993); } \\
\text { Hinterhuber/Stahl (1996); } \\
\text { Heyse/Erpenbeck (1997); } \\
\text { Riess (1998); Rockenhäuser } \\
\text { (1998); Belz (1999); } \\
\text { Faix/Krupp (2002); } \\
\text { Bouncken (2003) }\end{array}$ & $\begin{array}{l}\text { Der Kooperationspartner } \\
\text { verfügt über ein gutes } \\
\text { Unternehmens- und } \\
\text { Produktimage am Markt, } \\
\text { was auf sein hohes Potential } \\
\text { zur Stiftung von } \\
\text { Kundennutzen schließen } \\
\text { lässt. }\end{array}$ \\
\hline exklusiver Ressourcenzugang & $\begin{array}{l}\text { Hamel (1994); Schiller } \\
(2000) ; \text { Faix/Krupp (2002) }\end{array}$ & $\begin{array}{l}\text { Der Kooperationspartner } \\
\text { verfügt über einen } \\
\text { exklusiven Ressourcen- } \\
\text { zugang, sowie loyale } \\
\text { Zulieferer. Diese kosten- } \\
\text { spezifischen Indikatoren des } \\
\text { Kernkompetenzmerkmals } \\
\text { der Kundennutzenstiftung } \\
\text { tragen maßgeblich zur } \\
\text { Durchsetzung eines lang- } \\
\text { fristigen Wettbewerbsvor- } \\
\text { teils im Rahmen der Ko- } \\
\text { operationsbeziehung bei. }\end{array}$ \\
\hline Just-In-Time Management & $\begin{array}{l}\text { Hamel (1994); } \\
\text { Heyse/Erpenbeck (1997); } \\
\text { Hănggi (1998) }\end{array}$ & $\begin{array}{l}\text { Der Kooperationspartner } \\
\text { verfügt über integrităts- } \\
\text { bezogene Făhigkeiten (z.B. } \\
\text { Just-In-Time-Management, } \\
\text { Simultaneous Engineering), } \\
\text { um im Vergleich zu } \\
\text { Konkurrenzunternehmen } \\
\text { schneller und flexibler } \\
\text { operieren zu können. }\end{array}$ \\
\hline Kundenbindung & $\begin{array}{l}\text { Lado et al. (1992); } \\
\text { Buchholz/Olemotz (1995); } \\
\text { Belz (1999); Schiller (2000); } \\
\text { Faix/Krupp (2002); Jacob } \\
\text { (2003); Bouncken (2003) }\end{array}$ & $\begin{array}{l}\text { Der Kooperationspartner } \\
\text { verfügt über die Făhigkeit, } \\
\text { Kunden an sich zu binden. } \\
\text { Dieser kostenspezifische } \\
\text { Indikator des Kernkom- } \\
\text { petenzmerkmals der }\end{array}$ \\
\hline
\end{tabular}




\begin{tabular}{|c|c|c|}
\hline & & $\begin{array}{l}\text { Kundennutzenstiftung trägt } \\
\text { maßgeblich zur Durch- } \\
\text { setzung eines langfristigen } \\
\text { Wettbewerbsvorteils im } \\
\text { Rahmen der } \\
\text { Kooperationsbeziehung bei. }\end{array}$ \\
\hline Servicefähigkeiten & $\begin{array}{l}\text { Weiss (1992); Hamel (1994); } \\
\text { Möhringer (1998); Belz } \\
\text { (1999); Schiller (2000); Zins } \\
\text { (2001); Bouncken (2003) }\end{array}$ & $\begin{array}{l}\text { Der Kooperationspartner } \\
\text { verfügt über ein umfang- } \\
\text { reiches Pre-Sales- und } \\
\text { After-Sales-Dienstleistungs- } \\
\text { angebot, um im Rahmen } \\
\text { von Kooperationsbe- } \\
\text { ziehungen Kundennähe zu } \\
\text { forcieren. }\end{array}$ \\
\hline Qualitätsmanagement & $\begin{array}{l}\text { Conant et al. (1990); Lado et } \\
\text { al. (1992); Weiss (1992); } \\
\text { Hamel (1994); } \\
\text { Heyse/Erpenbeck (1997); } \\
\text { Möhringer (1998); Schiller } \\
\text { (2000); Bouncken (2003) }\end{array}$ & $\begin{array}{l}\text { Der Kooperationspartner ist } \\
\text { fahig, neue Qualitäts- } \\
\text { prozesse und -prozeduren zu } \\
\text { entwickeln und zu managen, } \\
\text { um im Vergleich zu } \\
\text { Konkurrenzunternehmen } \\
\text { schneller und verlässlicher } \\
\text { zu operieren. }\end{array}$ \\
\hline $\begin{array}{l}\text { Produkt- und Prozess-Know- } \\
\text { How }\end{array}$ & $\begin{array}{l}\text { Tampoe (1994); } \\
\text { Heyse/Erpenbeck (1997); } \\
\text { Rockenhäuser (1998); Belz } \\
\text { (1999); Schiller (2000); } \\
\text { Thomson (2000); } \\
\text { Faix/Krupp (2002); } \\
\text { Bouncken (2003); Jacob } \\
\text { (2003) }\end{array}$ & $\begin{array}{l}\text { Der Kooperationspartner be- } \\
\text { herrscht spezialisierte Her- } \\
\text { stellungstechniken, die als } \\
\text { kostenspezifische } \\
\text { Indikatoren des Kernkom- } \\
\text { petenzmerkmals der } \\
\text { Kundennutzenstiftung } \\
\text { maßgeblich an der Durch- } \\
\text { setzung eines langfristigen } \\
\text { Wettbewerbsvorteils im } \\
\text { Rahmen der Kooperations- } \\
\text { beziehung beteiligt sind. }\end{array}$ \\
\hline $\begin{array}{l}\text { Innovationsfähigkeit, } \\
\text { Zeitmanagement }\end{array}$ & $\begin{array}{l}\text { Dosi et al. (1992); Weiss } \\
\text { (1992); Hamel (1994); } \\
\text { Heyse/Erpenbeck (1997); } \\
\text { Möhringer (1998); Belz } \\
\text { (1999); Schiller (2000); } \\
\text { Faix/Krupp (2002); } \\
\text { Bouncken (2003) }\end{array}$ & $\begin{array}{l}\text { Der Kooperationspartner } \\
\text { verfügt über Innovations- } \\
\text { fähigkeit, was sich in der } \\
\text { Zahl der Patentmeldungen } \\
\text { (als rechtlicher Indikator des } \\
\text { Kernkompetenzmerkmals } \\
\text { der Nicht-Imitierbarkeit) } \\
\text { und der aktuellen spezi- } \\
\text { fischen Veröffentlichungen } \\
\text { (als technologischer Indi- } \\
\text { kator des Kernkompetenz- } \\
\text { merkmals der Nicht- } \\
\text { Substituierbarkeit) zeigt. }\end{array}$ \\
\hline Kundenorientierung & $\begin{array}{l}\text { Weiss (1992); Hamel (1994); } \\
\text { Heyse/Erpenbeck (1997); }\end{array}$ & $\begin{array}{l}\text { Der Kooperationspartner } \\
\text { orientiert sich im Zuge der }\end{array}$ \\
\hline
\end{tabular}




\begin{tabular}{|c|l|l|}
\hline & $\begin{array}{l}\text { Möhringer (1998); Belz } \\
(1999) ; \text { Schiller (2000); } \\
\text { Faix/Krupp (2002); } \\
\text { Bouncken (2003); Jacob } \\
(2003)\end{array}$ & $\begin{array}{l}\text { Leistungserstellung an den } \\
\text { Kunden (z.B. individuelle } \\
\text { Problemlösungen), um } \\
\text { Produkte mit hohem } \\
\text { Kundennutzen im Rahmen } \\
\text { der Kooperationsbeziehung } \\
\text { zu generieren. }\end{array}$ \\
\hline $\begin{array}{c}\text { Flexpassungsfähigkeit, } \\
\text { Veränderungen }\end{array}$ & $\begin{array}{l}\text { Weiss (1992); } \\
\text { Heyse/Erpenbeck (1997); } \\
\text { Möhringer (1998); Schiller } \\
(2000) ; \text { Faix/Krupp (2002); } \\
\text { Bouncken (2003) }\end{array}$ & $\begin{array}{l}\text { Der Kooperationspartner ist } \\
\text { zur dynamischen Anpas- } \\
\text { sung, Integration und } \\
\text { Konfiguration von Wissens- } \\
\text { und Fähigkeitsbasen im } \\
\text { Unternehmen făhig, um sich } \\
\text { und seinem Partner im } \\
\text { Rahmen der Kooperation } \\
\text { Zugang zu verschiedenen } \\
\text { Märkten zu eröffnen. }\end{array}$ \\
\hline
\end{tabular}

Tabelle 17: Indikatoren organisationaler Kompetenzen

\section{DIE INDIKATOREN ZUR OPERATIONALISIERUNG DES VERTRAUENS- KONSTRUKTS}

Nachdem das Vertrauenskonstrukt in Merkmalsdimensionen aufgespalten wurde, zielt das vorliegende Kapitel darauf ab, auf Basis der in Kapitel 7 des Teils II dieser Arbeit erläuterten Vertrauensmessansätze, konsensfähige und gültige Indikatoren, die als empirische Äquivalente der Merkmalsdimension, Vertrauenshandlung' fungieren ${ }^{539}$, zu bestimmen, und am Fokus, zwischenbetriebliche Kooperationen' ${ }^{\circ} \mathrm{u}$ erläutern. Die identifizierten und sekundäranalytisch abgesicherten Indikatoren dienen der fundierten Modellierung des Einflusses von Kompetenz auf die Entstehung von Vertrauen, sowie der Identifikation beobachtbarer Verhaltensweisen im Rahmen der empirischen Überprüfung der hypothetisierten Kausalbeziehung zwischen Kompetenz und Vertrauen in zwischenbetrieblichen Kooperationen.

Als Indikatoren der Merkmalsdimension ,Vertrauenserwartung ' werden in der Vertrauensliteratur vielfach Eigenschaften des Vertrauensnehmers genannt (vgl. hierzu die in Teil II Kap. 7 und Teil IV Kap. 1 angeführten Kritikpunkte). Hierbei handelt es sich allerdings um keine Manifestationen der vertrauensvollen Erwartung des Vertrauensgebers in die Absichten des Kooperationspartners, sondern vielmehr um Determinanten. Hingegen kann sich die Vertrauenserwartung in der Vornahme einer Vertrauenshandlung manifestieren, bzw. die Höhe der Vertrauenserwartung kann sich daran erkennen lassen, dass trotz geringer Vertrauensbereitschaft und hoher Risikowahmehmung, freiwillig eine riskante Vorleistung erbracht wird. Daher wird im Rahmen dieser Arbeit auf eine Ableitung von Indikatoren, die als empirische Äquivalente der Merkmalsdimension ,Vertrauenserwartung' fungieren, verzichtet. Das Vorliegen der Erwartung, dass sich der Kooperationspartner vertrauenswürdig verhält, und der Einfluss bestimmter Kompetenzarten auf die Entstehung der Vertrauenserwartung werden daher im empirischen Teil dieser Arbeit über die Merkmalsdimension ,Vertrauenshandlung' erfasst. 
Entsprechend des dieser Arbeit zugrunde liegenden Vertrauensverständnisses zeigt sich die Vornahme einer Vertrauenshandlung in der freiwilligen Erbringung einer riskanten Vorleistung, sowie in dem Verzicht auf explizite Kontroll- und Sicherungsinstrumente zur Begrenzung der Opportunismusspielräume des Kooperationspartners, und damit in Handlungen, die die Verwundbarkeit des Vertrauensgebers gegenüber dem Vertrauensnehmer erhöhen. Das Eingehen und Aufrechterhalten einer Kooperationsbeziehung mit einem anderen Unternehmen A stellen daher nicht notwendigerweise Manifestationen einer Vertrauenshandlung dar, wie bereits in Teil II Kapitel 5.1.1 erläutert wurde. Dies ergibt sich dadurch, dass das Eingehen und Aufrechterhalten einer kooperativen Beziehung durch Zwang, das Fehlen von Alternativen oder externe Kontrollinstrumente, die unkooperatives Verhalten sanktionieren, begründet sein kann. ${ }^{540}$ In diesen Fällen liegt geringe bis keine Verwundbarkeit des kooperierenden Unternehmens B vor, denn "cooperation does not necessarily put a party at risk. “541 Sofern sich das Unternehmen B jedoch durch seine Handlung im Rahmen der Kooperationsbeziehung freiwillig gegenüber seinem Kooperationspartner $\mathrm{A}$ verwundbar macht ${ }^{542}$, ist davon auszugehen, dass es sich hierbei um eine Vertrauenshandlung handelt.

Demnach können nur jene kooperativen Handlungen als empirische Äquivalente der Vertrauenshandlung fungieren, die darauf schließen lassen, dass sich der Vertrauensgeber durch ihre Vornahme freiwillig einem Risiko aussetzt. Von zentraler Bedeutung im Rahmen einer Kooperationsbeziehung sind kommunikative Handlungen zwischen den Kooperationspartnern: ,a cooperative process is characterized by open and honest communication of relevant information between the participants. Each is interested in informing, and being informed by the other. In contrast, a competitive process is characterized by either lack of information or misleading information. ${ }^{, 43}$ Kommunikation kann in diesem Zusammenhang als Informationsaustausch verstanden werden, wobei Informationen als "zweckgerichtetes, speziell entscheidungssteuerndes Wissen"544 aufzufassen sind. Bezugnehmend auf Kooperationsbeziehungen, zeigt sich die Bedeutung der Quantität und Qualität des Informationsaustausches vor allem dann, wenn sich der Kooperationspartner B durch die Offenlegung von akkuraten Informationen gegenüber seinem Partner A verwundbar macht. ${ }^{545}$ Dies begründet sich dadurch, dass A die von B erhaltenen Informationen zum Nachteil von B nutzen kann, womit es sich bei offener und ehrlicher Informationsabgabe um Indikatoren der freiwilligen Erbringung einer riskanten Vorleistung handelt. Da auch der Verzicht auf explizite

\footnotetext{
540 Vgl. Ripperger (1998), S. 93; Späth (2008), S. 65

$541 \quad$ Mayer et al. (1995), S. 712

542 Vgl. Zand (1972), S. 230; Lindskold (1981), S. 245

543 Deutsch (1990), S. 241; vgl. dazu auch Ring/Van de Ven (1992); Teece (1998)

544 Bronner (1999), S. 29

545 Vgl. Currall/Judge (1995), S. 153 f; Madhok (2006), S. 110
} 
Kontroll- und Sicherungsinstrumente zur Begrenzung der Opportunismusspielräume von $A$ ein konstitutives Merkmal der Vertrauenshandlung darstellt, fungiert die reduzierte Kontrolle der Informationshandlungen von A durch B als empirisches Äquivalent für die Vornahme einer Vertrauenshandlung. Nachfolgende Tabelle zeigt eine tabellarische Auflistung der Indikatoren, die als empirische Äquivalente der Vertrauenshandlung fungieren, sowie ihre Erläuterung am vorliegenden Untersuchungsgegenstand, wobei unter dem ,Kooperationspartner' das für die Kooperation zuständige Individuum, im Sinne eines Kooperationsbeauftragten, verstanden wird.

\begin{tabular}{|c|c|c|}
\hline $\begin{array}{l}\text { Vertrauenshandlungs- } \\
\text { indikatoren }\end{array}$ & $\begin{array}{c}\text { sekundïranalytische Nach- } \\
\text { weise der } \\
\text { Vertrauensforschung }\end{array}$ & $\begin{array}{l}\text { Erlüuterung der } \\
\text { Vertrauenshandlungs- } \\
\text { indikatoren am } \\
\text { vorliegenden } \\
\text { Untersuchungsgegenstand }\end{array}$ \\
\hline $\begin{array}{c}\text { offene } \\
\text { Informationsabgabe }\end{array}$ & $\begin{array}{l}\text { Zand (1972); O'Reilly } \\
\text { III/Roberts (1974); Gaines } \\
\text { (1980); Currall/Judge (1995); } \\
\text { Butler (1999); Gillespie } \\
\text { (2003); Späth (2008) }\end{array}$ & $\begin{array}{l}\text { Der Kooperationspartner } \\
\text { erbringt freiwillig eine } \\
\text { riskante Vorleistung, indem } \\
\text { er seinem Partner im } \\
\text { Rahmen der Kooperations- } \\
\text { beziehung Informationen } \\
\text { offen abgibt. }\end{array}$ \\
\hline $\begin{array}{c}\text { ehrliche } \\
\text { Informationsabgabe }\end{array}$ & $\begin{array}{l}\text { Zand (1972); O'Reilly } \\
\text { III/Roberts (1974); } \\
\text { Currall/Judge (1995); } \\
\text { Cummings/Bromiley (1996); } \\
\text { Gillespie (2003) }\end{array}$ & $\begin{array}{l}\text { Der Kooperationspartner } \\
\text { erbringt freiwillig eine } \\
\text { riskante Vorleistung, indem } \\
\text { er seinem Partner im } \\
\text { Rahmen der Kooperations- } \\
\text { beziehung Informationen un- } \\
\text { verzerrt abgibt. }\end{array}$ \\
\hline $\begin{array}{c}\text { reduzierte } \\
\text { Kontrollintensität }\end{array}$ & $\begin{array}{l}\text { Strickland (1958); Zand } \\
\text { (1972); Currall/Judge (1995); } \\
\text { Das/Teng (1998); } \\
\text { Mayer/Davis (1999); } \\
\text { Gillespie (2003) }\end{array}$ & $\begin{array}{l}\text { Der Kooperationspartner } \\
\text { verzichtet auf die Kontrolle } \\
\text { der von seinem Partner im } \\
\text { Rahmen der Kooperations- } \\
\text { beziehung erhaltenen } \\
\text { Informationen. }\end{array}$ \\
\hline
\end{tabular}

Tabelle 18: Indikatoren der Vertrauenshandlung

\section{Die MOdellierung VON KOMPETENZ ALS DETERMINANTE DER ENTSTEHUNG VON VERTRAUEN}

Die Zielsetzung des vorliegenden Kapitels besteht darin, anhand der in den vorangegangenen Teilen erläuterten Erklärungsansätze und empirischen Befunde, ein Modell zu präsentieren, das die Konstrukte Kompetenz und Vertrauen in zwischenbetrieblichen Kooperationen zusammenführt, und das anschließend einer empirischen Prüfung unterzogen werden kann. 
Aufgrund des zeitlichen Auseinanderfallens der Handlungen der Kooperationsakteure $^{546}$ liegt die besondere Problematik zwischenbetrieblicher Kooperationen darin, dass nicht alle Handlungen des Partners überwacht oder erzwungen werden können, obwohl gerade diese Handlungen einen nachhaltigen Einfluss auf die Erreichung der Kooperationsziele haben und die Kooperationspartner im Hinblick auf diese erwarteten Handlungen kooperative Vorleistungen erbringen. ${ }^{547}$ Somit liegt das Problem kooperativer Beziehungen in der Gefahr, im Rahmen einer Kooperation vom Partner übervorteilt zu werden. ${ }^{548}$ Das ist dann der Fall, wenn sich dieser mit seiner gewählten Verhaltensoption ausschließlich an eigenen kurzfristigen Interessen orientiert. ${ }^{549}$

Aufgrund der Opportunismusspielräume des Kooperationspartners erwachsen dem kooperationswilligen Unternehmen entsprechende Risiken (Adverse Selection, Hold Up, Moral Hazard). Das Unternehmen hat einerseits die Möglichkeit, die Opportunismusneigung des Kooperationspartners zu reduzieren und die, aufgrund der unbeschränkten Opportunismusspielräume bestehen bleibenden Unsicherheiten in Bezug auf das Verhalten des Kooperationspartners und die damit zusammenhängenden Risikokosten zu akzeptieren. Andererseits kann es sich dazu entschließen, Aktionen zur Verringerung der Opportunismusspielräume und der damit korrelierenden Unsicherheiten zu setzen, und die mit diesen Maßnahmen verbundenen Agency-Kosten (z.B. Vereinbarungs- und Überwachungskosten, Kosten des Aufbaus von Sanktionspotential) zu akzeptieren. ${ }^{550}$ Eine Akzeptanz der Unsicherheiten ist allerdings nur vor dem Hintergrund einer tragfähigen Vertrauensbeziehung möglich, da Vertrauen die in einer Kooperationsbeziehung immanenten Verhaltensrisiken absorbieren kann. Um Austauschbeziehungen aufzubauen und aufrechtzuerhalten, können zwischenbetriebliche Kooperationen demnach auf der Grundlage von Vertrauen funktionieren und müssen dies sogar, wenn andere Koordinationsmechanismen nicht verfügbar oder relativ, in Bezug auf die reduzierten Risiken, teuer sind. ${ }^{551}$

Um das Vertrauenskonstrukt in Form eines integrativ theoretischen Modells erfassen und erklären zu können, und damit eine Identifikation jener Variablen, die das

\footnotetext{
546 Vgl. Emerson (1962) aus der Power-Dependence-Perspektive; Macneil (1980) in der Vertragstheorie

547 Kooperatives Verhalten zeigt sich darin, dass der kooperationswillige Akteur von der kurzfristigen Vorteilhaftigkeitsorientierung zugunsten einer Verhaltensweise abweicht, deren langfristige Vorteile vom unsicheren zukünftigen Verhalten des Interaktionspartners abhängen (Rößl 1994, S. 52 f;; Pleitner/Röß1 1995, S. 672).

548 Die gegenseitige wirtschaftliche Abhängigkeit bei gleichzeitiger wechselseitiger Verhaltensunsicherheit (doppelte Kontingenz, vgl. Luhmann 2000) macht Kooperationen zwischen Unternehmen zu komplexen Arrangements, die durch soziale Dilemmata (vgl. Le/Boyd 2006) bedroht sind.

549 Vgl. Wurche (1994), S. 144 f.

550 Vgl. Rößl (2006), S. 39

551 Vgl. Rößl (1994), S. 381
} 
Entscheidungskalkül des kooperationswilligen Unternehmens in seiner Rolle als Vertrauensgeber ${ }^{552}$ beeinflussen, $\mathrm{zu}$ ermöglichen, ist eine Differenzierung zwischen subjektiver Erwartungshaltung und objektiv beobachtbarem Verhalten unerlässlich: Eine Vertrauensentscheidung im Sinne eines Schenkens von Vertrauen durch den Vertrauensgeber liegt demnach nur dann vor, wenn eine Vertrauenshandlung vorgenommen, und diese Handlung durch eine Vertrauenserwartung motiviert wurde. Allerdings muss nicht jedes kooperative Verhalten auf einer vertrauensvollen Erwartung basieren und nicht immer resultiert eine Vertrauenserwartung in einem vertrauensvollen Verhalten. Damit ist Kooperation nicht notwendigerweise ein Zeichen für Vertrauen und Vertrauen alleine nicht immer eine ausreichende Bedingung für Kooperation. ${ }^{553}$

Bezugnehmend auf die Vertrauenshandlung lässt sich zusammenfassen, dass sie sich in der freiwilligen Erbringung einer riskanten Vorleistung und in dem Verzicht auf explizite vertragliche Kontroll- und Sicherungsmaßnahmen zur Begrenzung der Opportunismusspielräume des Kooperationspartners in seiner Rolle als Vertrauensnehmer zeigt: Der Vertrauensgeber überantwortet dem Vertrauensnehmer Ressourcen in Form des Anvertrauten, die dieser zum Nutzen oder Schaden des Vertrauensgebers einsetzen kann, womit er sich gegenüber dem Vertrauensnehmer in eine Position der Verwundbarkeit begibt. Dieser ,Einsatz' begründet als spezifische Investition eine einseitige Abhängigkeit des Vertrauensgebers vom Vertrauensnehmer, die dieser zum Schaden des ersteren ausnutzen kann. Die Möglichkeit des Schadens durch den Vertrauensnehmer und dementsprechend das Vertrauensrisiko, werden durch die Wahlfreiheit des Vertrauensnehmers zwischen der Honorierung und Enttäuschung des in ihn gesetzten Vertrauens begründet. Die Entscheidung des Vertrauensgebers, sich dem Hold-Up Risiko ungeschützt auszusetzen, beruht unter anderem auf der vertrauensvollen Erwartung, dass der Vertrauensnehmer freiwillig auf opportunistisches Verhalten, also auf die Veruntreuung des Einsatzes verzichtet. ${ }^{54}$

Bezugnehmend auf die Vertrauenserwartung sieht sich das kooperationswillige Unternehmen mit einem Adverse Selection-Problem konfrontiert, da es in seiner Rolle als Vertrauensgeber entscheiden muss, ob der potentielle Vertrauensnehmer vertrauenswürdig ist, ihm jedoch diese Eigenschaft vor Eingehen der Vertrauensbeziehung weitgehend verborgen bleibt. Die Vertrauenserwartung basiert

552 Aufgrunddessen, dass jede Vertrauenspartei über verschiedene Informationen und Handlungsalternativen verfügt, andere Interessen verfolgt und unterschiedlichen Anreiz- und Sanktionsmechanismen ausgesetzt ist, ist die vorgenommene Rollentrennung aus analytischen Gründen unverzichtbar. Grundsätzlich, und vor allem im Hinblick auf Kooperationsbeziehungen ist davon auszugehen, dass die Kooperationspartner abwechselnd die Rolle von Vertrauensgeber und Vertrauensnehmer einnehmen.

$553 \quad V g l$. hierzu die Ausführungen in Teil Il Kap. 5.1.1, sowie die dort zit. Literatur.

554 Vgl. hierzu die Ausführungen in Teil II Kap. 1.1.1 und Kap. 5.1.3, sowie die dort zit. Literatur 
damit auf der zugeschriebenen Motivation des Handelns des Vertrauensnehmers, genauer auf der Einschätzung der Intensität und Stabilität der Motivation des Vertrauensnehmers und damit der Antizipation von dessen Handlungsabsicht seitens des Vertrauensgebers. Bei der Einschätzung der Handlungsabsicht des Vertrauensnehmers sieht sich der Vertrauensgeber mit subjektiver Unsicherheit im Hinblick auf dessen wahre Präferenzen, sowie mit objektiver Unsicherheit in Bezug auf das Eintreten möglicher Umweltzustände und der sich daraus ergebenden Handlungsrestriktionen konfrontiert. Im Zuge der Bildung seiner Vertrauenserwartung, die auf der subjektiven Einschätzung der Vertrauenswürdigkeit eines bestimmten Vertrauensnehmers in einer spezifischen Situation beruht, wird der Vertrauensgeber daher bestrebt sein, seine subjektive Unsicherheit durch zusätzliche Informationen zu reduzieren. Da Informationslücken aber nicht vollständig ausgefüllt werden können, erfordert Vertrauen grundsätzlich die Extrapolation von vorhandenen Informationen aus der Vergangenheit in die Zukunft. ${ }^{555}$

Das nachfolgende Modell bildet die Vertrauensentscheidung als Prozessergebnis ab. Darauf aufbauend wird anschließend der modellierte Einfluss der individuellen Kompetenzarten auf die Vertrauensentstehung diskutiert und hypothetisiert, sowie auf die Rolle der organisationalen Kompetenzen und der vertrauensgeber- und sitatuionsspezifischen Variablen Bezug genommen.

555 Vgl. hierzu die Ausführungen in Teil II Kap. 5.1.2, sowie die dort zit. Literatur. 
Individuelle Kompetenzen

\begin{tabular}{|l|}
\hline \multicolumn{1}{|l|}{ Fachkompetenzen } \\
\hline Fachwissen \\
\hline fachübergreifendes Wissen \\
\hline kaufmannisches Wissen \\
\hline Erfahrungswissen \\
\hline Abstraktionstahigkeit \\
\hline Vermittlungs- und \\
\hline Darstellungstahigkeit \\
\hline
\end{tabular}

Methodenkompetenzen

Methoden zur Informations.

verarbeitung

Management- und Leistungs-

Planungs- und Kontroll-

methoden

Problemlosungs- Lern- und

Darstellungsfahigke
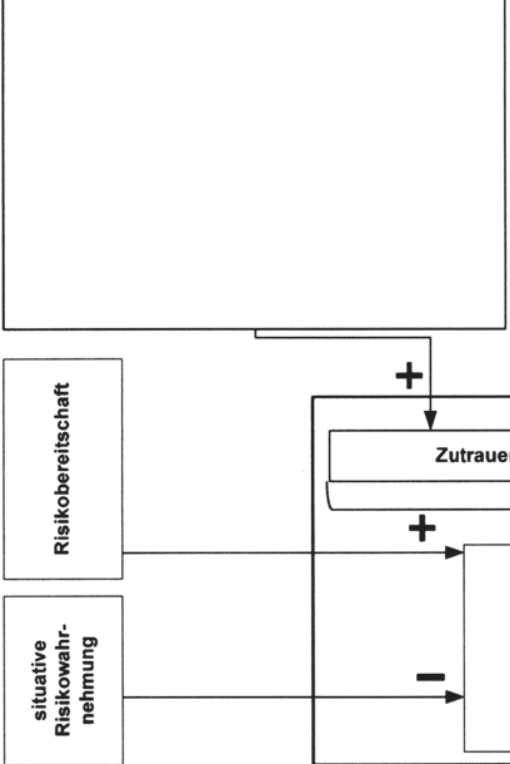

Organisationale Kompetenzen

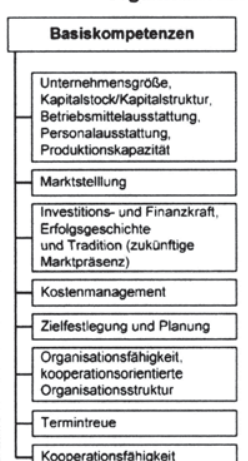

Kernkompetenzen

\section{Bekanntheil}

Know-How der

Know-How der
Unternehmensmitglieder

bedartsgerechtes Produkt-

Vermarktungsfanigkeiten

Unternehmensimage

exklusiver Ressourcenzugang

Just-In-Time Management

Kundenbindung

Servicefahigkeiten

Qualitatsmanagement

Produkt- und Prozess-

Innovationstanigken

Zeitmanagement

Kundenorientierung

Anpassungsfanigkeit,
Flexibilitat in Umgang Veranderungen

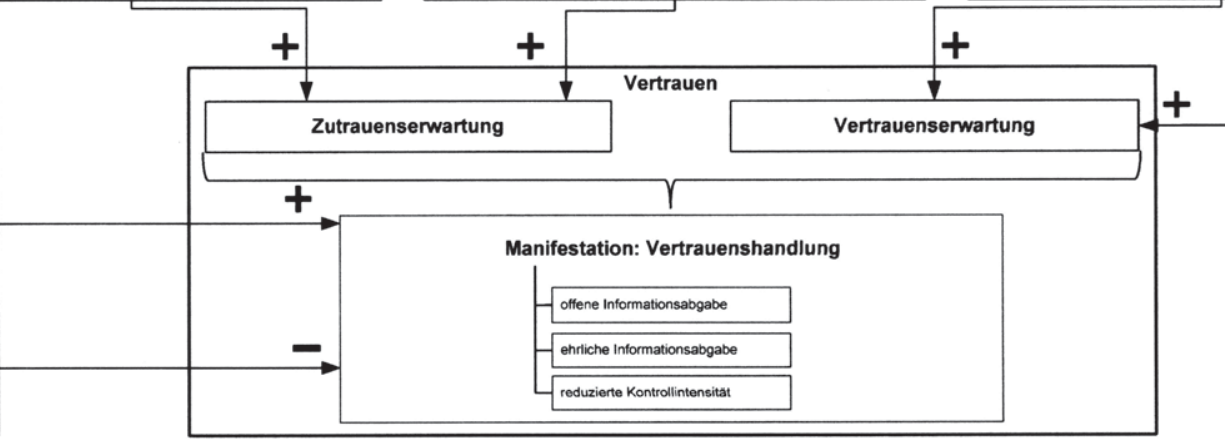

Abbildung 13: Die Modellierung von Kompetenz als Determinante der Entstehung von Vertrauen

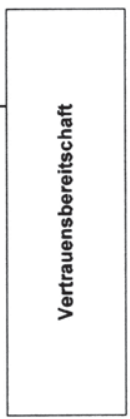


In dem im Rahmen der Bildung der spezifischen Vertrauenserwartung angesprochenen Bestreben des Vertrauensgebers, seine subjektive Unsicherheit durch zusätzliche Informationen zu reduzieren, wird die Bedeutung des Kompetenzkonstrukts deutlich: Die Bildung einer vertrauensvollen Erwartung seitens des Vertrauensgebers kann nur antizipiert werden, wenn Zusatzinformationen wahrgenommen werden, die den Vertrauensgeber darauf schließen lassen, dass der Vertrauensnehmer der Vertrauenserwartung entsprechen wird. Da die subjektive Einschätzung der Vertrauenswürdigkeit eines bestimmten Vertrauensnehmers in einer spezifischen Situation vorrangig von der Persönlichkeit des Vertrauensnehmers beeinflusst wird, ist anzunehmen, dass es sich bei der vom Vertrauensgeber wahrgenommenen Kompetenz des Vertrauensnehmers um eine solche Zusatzinformation zur Reduktion der subjektiven Unsicherheit des Vertrauensgebers in Bezug auf das Verhalten des Vertrauensnehmers handelt, wodurch sie die Bildung einer vertrauensvollen Erwartung begünstigt. ${ }^{556}$

Die Erfüllung der Erwartungen des Vertrauensgebers ist grundsätzlich von der Existenz zweier Größen abhängig: dem Können und dem Wollen des Vertrauensnehmers. Aufgrund der zwischen den Kooperationspartnern herrschenden Informationsasymmetrien besteht seitens des Vertrauensgebers einerseits Unsicherheit darüber, ob der Vertrauensnehmer die potentielle Vertrauensentscheidung honorieren kann und andererseits ist der Vertrauensgeber unsicher, ob sich der Vertrauensnehmer vertrauenswürdig verhalten will. ${ }^{557} \mathrm{Die}$ auf individueller Ebene wahrgenommene Fachkompetenz des potentiellen Vertrauensnehmers, verstanden als die zur Erfüllung einer Kooperationsaufgabe benötigten fachlichen Fertigkeiten und Fähigkeiten, kann nun dazu beitragen, die Unsicherheit des Vertrauensgebers über das Können des Vertrauensnehmers zu reduzieren. Einhergehend mit dieser reduzierten Qualitätsunsicherheit erwartet der Vertrauensgeber von dem Vertrauensnehmer, dass letzterer die potentielle Vertrauensentscheidung honorieren kann. Es wird daher angenommen, dass die wahrgenommene Fachkompetenz über die Dimension der Zutrauenserwartung die Vornahme einer Vertrauenshandlung begünstigt. Die zur Rolle der Fachkompetenz im Vertrauensentstehungsprozess angestellten Überlegungen werden gleichermaßen für die Wirkung der wahrgenommenen Methodenkompetenz des potentiellen Vertrauensnehmers, verstanden als Summe der persönlichen Problemlösungsverfahren, in Bezug auf das Vertrauenskonstrukt angenommen. Diese Annahme ergibt sich aus der Auffassung, dass die Methodenkompetenz die Fachkompetenz ergänzt. ${ }^{558}$ Die auf individueller Ebene wahrgenommene Sozialkompetenz des potentiellen Vertrauensnehmers, verstanden als die zum konstruktiven Umgang mit anderen, und damit zum Erhalt zwischenmenschlicher Interaktionen be-

556 Vgl. hierzu die Ausführungen in Teil III Kap. 3.1 und 4.2, sowie die dort zit. Literatur.

557 Vgl. hierzu die Ausführungen in Teil II Kap. 2.3, sowie die dort zit. Literatur.

558 Vgl. hierzu die Ausführungen in Teil III Kap. 2.2.2, sowie die dort zit. Literatur. 
nötigten Fähigkeiten, kann im Gegensatz zu wahrgenommenen Fach- und Methodenkompetenzen dazu beitragen, die Unsicherheit des Vertrauensgebers über das Wollen des Vertrauensnehmers zu reduzieren. Bedingt durch diese reduzierte Entscheidungsunsicherheit erwartet der Vertrauensgeber von dem Vertrauensnehmer, dass letzterer die potentielle Vertrauensentscheidung honorieren will. Es wird daher angenommen, dass die wahrgenommene Sozialkompetenz über die Dimension der Vertrauenserwartung die Vornahme einer Vertrauenshandlung begünstigt. Die zur Rolle der Sozialkompetenz im Vertrauensentstehungsprozess angestellten Überlegungen werden gleichermaßen für die Wirkung der Persönlichkeitskompetenz des potentiellen Vertrauensnehmers, verstanden als Persönlichkeitsmerkmale und Handlungsdispositionen, die es dem potentiellen Vertrauensnehmer ermöglichen, sein Handeln in den verschiedensten Situationen zu steuern, zu motivieren und zu initiieren, im Rahmen der Entstehung von Vertrauen angenommen. Diese Annahme ergibt sich durch die Auffassung, dass die beiden Konstrukte eng miteinander verwoben sind. ${ }^{559}$ Zusammenfassend gibt nachfolgende Tabelle einen Überblick über die Annahmen bezüglich des Einflusses individueller Kompetenzen auf die Entstehung von Vertrauen in zwischenbetrieblichen Kooperationen. Während die Haupthypothesen $\mathrm{H}_{1}, \mathrm{H}_{2}, \mathrm{H}_{3}$ und $\mathrm{H}_{4}$ im Rahmen dieser Arbeit einer empirischen Überprüfung unterzogen werden, fassen die Unterhypothesen $\mathrm{H}_{1 \mathrm{~A}}, \mathrm{H}_{1 \mathrm{~B}}, \mathrm{H}_{2 \mathrm{~A}}{ }^{560}$ etc. die angestellten Überlegungen zusammen.

$\mathrm{H}_{1}$ : Die wahrgenommene Fachkompetenz des Vertrauensnehmers hat einen positiven Einfluss auf die Vornahme einer Vertrauenshandlung durch den Vertrauensgeber.

4) $\mathrm{H}_{\mathrm{IA}}$ : Die wahrgenommene Fachkompetenz des Vertrauensnehmers erhöht die Sicherheit des Vertrauensgebers über das Können des Vertrauensnehmers.

4 $\mathrm{H}_{\mathrm{IB}}$ : Die Sicherheit des Vertrauensgebers über das Können des Vertrauensnehmers hat einen positiven Einfluss auf die Bildung der Zutrauenserwartung seitens des Vertrauensgebers.

(4) $\mathrm{H}_{1 \mathrm{C}}$ : Die Zutrauenserwartung des Vertrauensgebers hat einen positiven Einfluss auf die Vornahme einer Vertrauenshandlung.

559 Vgl. hierzu die Ausführungen in Teil III Kap. 2.2.4, sowie die dort zit. Literatur.

560 Aufgrunddessen, dass sowohl die Bildung einer Vertrauenserwartung, als auch die Bildung einer Zutrauenserwartung seitens des Vertrauensgebers im Zuge der Anwendung eines Experimentaldesigns keiner direkten Beobachtung zugänglich sind, wird auf eine Überprüfung der Unterhypothesen im Rahmen dieser Arbeit verzichtet. 
$\mathrm{H}_{2}$ : Die wahrgenommene Methodenkompetenz des Vertrauensnehmers hat einen positiven Einfluss auf die Vornahme einer Vertrauenshandlung durch den Vertrauensgeber.

4) $\mathrm{H}_{2 \mathrm{~A}}$ : Die wahrgenommene Methodenkompetenz des Vertrauensnehmers erhöht die Sicherheit des Vertrauensgebers über das Können des Vertrauensnehmers.

4 $\mathrm{H}_{2 \mathrm{~B}}$ : Die Sicherheit des Vertrauensgebers über das Können des Vertrauensnehmers hat einen positiven Einfluss auf die Bildung der Zutrauenserwartung seitens des Vertrauensgebers.

$\Leftrightarrow \mathrm{H}_{2 \mathrm{C}}$ : Die Zutrauenserwartung des Vertrauensgebers hat einen positiven Einfluss auf die Vornahme einer Vertrauenshandlung.

$\mathrm{H}_{3}$ : Die wahrgenommene Sozialkompetenz des Vertrauensnehmers hat einen positiven Einfluss auf die Vornahme einer Vertrauenshandlung durch den Vertrauensgeber.

4) $\mathrm{H}_{3 \mathrm{~A}}$ : Die wahrgenommene Sozialkompetenz des Vertrauensnehmers erhöht die Sicherheit des Vertrauensgebers über das Wollen des Vertrauensnehmers.

4 $\mathrm{H}_{3 \mathrm{~B}}:$ Die Sicherheit des Vertrauensgebers über das Wollen des Vertrauensnehmers hat einen positiven Einfluss auf die Bildung der Vertrauenserwartung seitens des Vertrauensgebers.

4 $\mathrm{H}_{3 \mathrm{C}}$ : Die Vertrauenserwartung des Vertrauensgebers hat einen positiven Einfluss auf die Vornahme einer Vertrauenshandlung durch den Vertrauensgeber.

$\mathrm{H}_{4}$ : Die wahrgenommene Persönlichkeitskompetenz des Vertrauensnehmers hat einen positiven Einfluss auf die Vornahme einer Vertrauenshandlung durch den Vertrauensgeber.

4) $\mathrm{H}_{4 \mathrm{~A}}$ : Die wahrgenommene Persönlichkeitskompetenz des Vertrauensnehmers erhöht die Sicherheit des Vertrauensgebers über das Wollen des Vertrauensnehmers.

(4) $\mathrm{H}_{4 \mathrm{~B}}$ : Die Sicherheit des Vertrauensgebers über das Wollen des Vertrauensnehmers hat einen positiven Einfluss auf die Bildung der Vertrauenserwartung seitens des Vertrauensgebers.

(4) $\mathrm{H}_{4 \mathrm{C}}$ : Die Vertrauenserwartung des Vertrauensgebers hat einen positiven Einfluss auf die Vornahme einer Vertrauenshandlung durch den Vertrauensgeber.

Tabelle 19: Hypothesen zum Einfluss individueller Kompetenzen auf Vertrauen

Die auf organisationaler Ebene wahrgenommenen Basiskompetenzen des Kooperationspartners, verstanden als ,necessary competencies', über die ein Unter- 
nehmen verfügen muss, um am Markt agieren zu können, können ebenso dazu beitragen, die Unsicherheit des Vertrauensgebers über das Können des Vertrauensnehmers zu reduzieren. Einhergehend mit dieser reduzierten Qualitätsunsicherheit erwartet der Vertrauensgeber von dem kooperierenden Unternehmen in dessen Rolle als Vertrauensnehmer, dass letzterer die potentielle Vertrauensentscheidung honorieren kann. Es wird daher angenommen, dass die wahrgenommenen Basiskompetenzen über das Konstrukt des Zutrauens die Vornahme einer Vertrauenshandlung begünstigen. Die zur Rolle der Basiskompetenzen im Vertrauensentstehungsprozess angestellten Überlegungen werden gleichermaßen für die Wirkung der wahrgenommenen Kernkompetenzen des potentiellen Vertrauensnehmers, verstanden als Bündel verschiedener Technologien und Fähigkeiten, das einen besonderen Kundennutzen generiert und den Zugang zu einer Vielzahl von Märkten eröffnet, in Bezug auf das Vertrauenskonstrukt angenommen. ${ }^{561}$ Aus forschungspragmatischer Sicht und aufgrund der in dieser Arbeit vertretenen Auffassung, dass Vertrauen zwischen Unternehmen immer durch die diesen Unternehmen angehörenden Individuen begründet und zerstört wird, wird im Zuge der hypothesentestenden Forschung in dieser Arbeit auf die Überprüfung der Annahmen bezüglich des Einflusses organisationaler Kompetenzen auf die Entstehung von Vertrauen verzichtet. ${ }^{562}$

Abschließend wird auf vertrauensgeberbedingte und situationsbedingte Faktoren, die die Wirkung der erläuterten individuellen Kompetenzen des Vertrauensnehmers auf die Entstehung von Vertrauen seitens des Vertrauensgebers beeinflussen können, eingegangen.

So ist anzunehmen, dass die generelle Vertrauensbereitschaft, als stabiles Merkmal der Persönlichkeit des Vertrauensgebers, die Erwartung, dass der Kooperationspartner die Vertrauensentscheidung honorieren will, positiv beeinflusst: Aufgrunddessen, dass der Vertrauensgeber bei der Bildung seiner Vertrauenserwartung in einer spezifischen Situation mit einem Informationsmangel konfrontiert ist, wird er auf Basis vergangener Erfahrungen mit Dritten generalisierte Erwartungen entwickeln. Diese können sodann als Informationssubstitute in Form der generellen Vertrauensbereitschaft die Vertrauenserwartung in einer spezifischen Situation, die wiederum Einfluss auf die Wirkung der wahrgenommenen Sozial- und Persönlichkeitskompetenz des Vertrauensnehmers auf die Vornahme einer Vertrauenshandlung durch den Vertrauensgeber nimmt, beeinflussen. ${ }^{563}$

561 Vgl. hierzu die Ausführungen in Teil III Kap. 3.1 und 4.2 und Teil IV Kap. 1.2, sowie die dort zit. Literatur.

562 Weiters würde die experimentelle Überprüfung der angenommenen Wirkung von organisationalen Kompetenzen auf die Entstehung von Vertrauen ein völlig anderes Setting als die Überprüfung des hypothetisierten Einflusses individueller Kompetenzen auf die Entstehung von Vertrauen erfordem. Vgl. hierzu die Ausführungen in Teil II Kap. 5.1.2, sowie die dort zit. Literatur. 
Hingegen ist es nicht erforderlich, eine Variable ,generelle Zutrauensbereitschaft" für die Bildung der Erwartung, dass der Kooperationspartner die Vertrauensentscheidung honorieren kann, zu konstruieren: Zutrauen bezieht sich auf technische Aspekte von Kooperationen. Das heißt, nimmt der Vertrauensgeber seitens des Vertrauensnehmers Fach- und Methodenkompetenzen wahr, die ihn erwarten lassen, dass der Vertrauensnehmer die Vertrauensentscheidung honorieren kann, dann traut er ihm die Honorierung der Vertrauensentscheidung zu. Allerdings besteht die Gefahr, dass der Vertrauensnehmer die Vertrauensentscheidung aufgrund widriger Umstände letztendlich doch nicht honorieren kann. Diesbezüglich hilft dem Vertrauensgeber eine Zutrauensbereitschaft nicht weiter er ist der Gefahr ohnehin ausgeliefert. ${ }^{564} \mathrm{Ob}$ der Vertrauensgeber also über die Einschätzung verfügt, dass der Kooperationspartner die Vertrauensentscheidung honorieren kann, ist vielmehr eine Frage vorliegender technischer kompetenzbezogener Zusatzinformationen. Hingegen wird die potentielle Vertrauenserwartungsenttäuschung als vom Verhalten des Vertrauensnehmers abhängig betrachtet. $\mathrm{Ob}$ der Vertrauensgeber über die Einschätzung verfügt, dass der Kooperationspartner die Vertrauensentscheidung honorieren will, ist also eine Frage motivationaler kompetenzbezogener Zusatzinformationen und damit eine Frage, ob der Vertrauensgeber bereit ist, dem Vertrauensnehmer sein Vertrauen zu schenken. ${ }^{565}$

Bezugnehmend auf die Vertrauenshandlung selbst ist anzunehmen, dass sie, neben der Höhe der Vertrauenserwartung, auch von der vertrauensgeberspezifischen Risikobereitschaft, verstanden als Bereitschaft, ein gegebenes Risiko zu akzeptieren, positiv beeinflusst wird. Im Gegensatz zur generellen Vertrauensbereitschaft, die ausschließlich die subjektive Neigung des Vertrauensgebers, dem potentiellen Vertrauensnehmer vertrauenswürdiges Verhalten zu unterstellen, betrifft, zeigt sich die Risikobereitschaft erst als Komponente des Entscheidens und Handelns und damit in ihrem Einfluss auf die konkrete Vertrauenshandlung des Vertrauensgebers. Während sich die Vertrauensbereitschaft ausschließlich auf die Bereitschaft, durch das unsichere Verhalten des Vertrauensnehmers begründete Risiken in der Vertrauenserwartung zu absorbieren, bezieht, umfasst die Risikobereitschaft sowohl Verhaltens- als auch Umweltrisiken. ${ }^{566}$

Weiters kann davon ausgegangen werden, dass die Vertrauenshandlung negativ von der Höhe des wahrgenommenen situationsspezifischen Risikos, verstanden als Einschätzung des Vertrauensgebers, wie riskant eine Situation bezüglich der Unsicherheit der Ereignisse und deren Konsequenzen ist ${ }^{567}$, beeinflusst wird. Aufgrunddessen, dass die Entscheidung des Vertrauensgebers für oder gegen die Vornahme einer Vertrauenshandlung zu unsicheren Ereignissen mit unterschiedlichen

564 Vgl. hierzu die Ausführungen in Teil II Kap. 1.1.1, sowie die dort zit. Literatur.

565 Vgl. Luhmann (1988), 97 ff.; Mayer et al. (1995); Eberl (2004)

566 Vgl. hierzu die Ausführungen in Teil II Kap. 5.1.3, sowie die dort zit. Literatur.

567 Vgl. Sitkin/Weingart (1995), S. 1575 
Auswirkungen auf seinen Nutzen führt, befindet er sich in einer durch Risiko gekennzeichneten Situation. Die situationsspezifische Risikowahrnehmung des Vertrauensgebers umfasst also seine den Vertrauensnehmer weitgehend ausblendende Einschätzung der Eintrittswahrscheinlichkeiten der mit der Vornahme der Vertrauenshandlung verbundenen Kontingenzen und der dadurch bedingten ökonomischen Folgen in einer spezifischen Situation. ${ }^{568}$ Aufgrund des in der Risikoverhaltensforschung empirisch bestätigten Zusammenhangs zwischen der situationsspezifischen Risikowahrnehmung und dem Verhalten in risikobehafteten Entscheidungssituationen ${ }^{569}$ kann davon ausgegangen werden, dass die Höhe des vom Vertrauensgeber wahrgenommenen situationsspezifischen Risikos als Möglichkeit eines Schadens die Vertrauenshandlung negativ beeinflusst.

Zusammenfassend ist daher anzunehmen, dass neben den individuellen Kompetenzen sowohl die vertrauensgeberspezifische Risikobereitschaft, als auch die generelle Vertrauensbereitschaft und die situationsspezifische Risikowahrnehmung des Vertrauensgebers Einfluss auf die Vornahme einer Vertrauenshandlung durch den Vertrauensgeber nehmen. Die Überprüfung dieser Annahmen ist Gegenstand des empirischen Teils dieser Arbeit.

568 Vgl. Mayer et al. (1995, S. 726) , ,[...] the perception of risk involves the trustor's beliefs about likelihoods of gains and losses outside of considerations that involve the relationship with the particular trustee.". Ähnlich auch Köszegi (2001, S. 69): "[...] Konsequenzen von vertrauensvollem Verhalten sind in der Regel situationsbedingt und kaum durch das Vertrauensobjekt beeinflussbar." Vgl. Sitkin/Pablo (1992); Sitkin/Weingart (1995); Pablo (1997); Wiemann (1998) 
Isabella Hatak - 978-3-631-74970-8 Downloaded from PubFactory at 01/11/2019 09:36:38AM via free access 
Nach der Modellierung des Einflusses von Kompetenz auf die Entstehung von Vertrauen, erfolgt in diesem Teil die Konzeption der Untersuchung zur Überprüfung der hypothetisierten Kausalbeziehung zwischen den Dimensionen ,individuelle Kompetenzen' und ,Vertrauenshandlung'. Die Analyse der Möglichkeiten hinsichtlich der Untersuchungsumgebung (Kap. 1.1) und der Gestaltung der Untersuchungssituation (Kap. 1.2) begründet die Entscheidung für eine experimentelle Variation der unabhängigen Variable mit psychometrischen Tests zur Erhebung von Dispositionen und Wahrnehmungen im Labor als Untersuchungsstrategie (Kap. 1.3). Im Anschluss an die Darstellung der betriebswirtschaftlichen Entscheidungsaufgabe, die von den Probanden zu bearbeiten ist (Kap. 2.1), wird die Manipulation der unabhängigen Variable erläutert (Kap. 2.2). Nach einem Überblick über Verfahren, die im Rahmen dieser Untersuchung zur Datengewinnung angewendet werden (Kap. 3.1), erfolgt die Entscheidung für Skalen zur Messung der Vertrauensbereitschaft (Kap. 3.2), Risikobereitschaft und Risikowahrnehmung (Kap. 3.3), sowie die Beschreibung der Messung der Vertrauenshandlung der Probanden (Kap. 3.4). Im Anschluss an die Darstellung der Ergebnisse des Pretests (Kap. 4.1), erfolgt die Beschreibung der zu untersuchenden Stichprobe (Kap. 4.2) und des Untersuchungsablaufs (Kap. 4.3). Den Abschluss dieses Teils bilden die Qualitätsanalyse der erhobenen Daten (Kap. 5.1) und die Beschreibung der statistischen Auswertungsmethoden zur Hypothesenprüfung (Kap. 5.2).

\section{DiE METHOdisChEN GRUNDLAGEN}

Nachdem in Teil IV festgelegt wurde, welche Operationen als indikativ für die zu erfassenden Faktoren der Dimension, individuelle Kompetenzen' und der Dimension ,Vertrauenshandlung" angesehen werden, fokussiert das vorliegende Kapitel die Erfassung dieser Operationen. Nach Opp handelt es sich bei der Operationalisierung um einen Prozess, der spezifiziert ,wie man vorgehen könnte, um das, was man ermitteln will, herauszufinden “570. Im weitesten Sinne ${ }^{571}$ bezeichnet die Operationalisierung also die Umsetzung der Forschungsfrage in Vorgehensweisen, die eine Beschaffung von Daten zur Überprüfung des hypothetisierten Kompetenzeinflusses auf die Vornahme einer Vertrauenshandlung, ermöglichen. ${ }^{572} \mathrm{Zu}$ diesem Zweck erfolgt in diesem Kapitel die Umsetzung des Untersuchungsziels in ein Untersuchungsdesign.

$570 \quad$ Opp (1999b), S. 57

571 Als Beispiel für eine engere Definition des Operationalisierungsbegriffs vgl. die Ausführungen in Teil IV Kap. 2 und 3, im Rahmen dessen die Begriffe Vertrauen und Kompetenz in Forschungsoperationen übersetzt werden (ähnlich dazu auch Friedrichs 1990, S. 79). 


\subsection{Die Untersuchungsumgebung}

In Bezug auf die Umgebung, in der die Daten für eine Überprüfung von Hypothesen gewonnen werden, ist generell - unabhängig von der Untersuchungsstrategie und der Datengewinnungsmethode ${ }^{573}$ - zwischen Labor- und Felduntersuchung zu differenzieren. ${ }^{574}$ Während die Felduntersuchung in der natürlichen Umgebung der Untersuchungsobjekte stattfindet, wird die Laboruntersuchung in einem künstlichen, speziell für die Untersuchungszwecke geschaffenen Milieu durchgeführt. Dadurch ergeben sich zentrale Unterschiede im Hinblick auf die Kontrollierbarkeit störender Einflussgrößen ${ }^{575}$, womit die Entscheidung für oder gegen Feld- bzw. Laborstudien zu unterschiedlichen Auswirkungen auf die eindeutige Interpretierbarkeit und Generalisierbarkeit der Untersuchungsergebnisse führt. Einerseits wird die Entscheidung für eine Untersuchungsumgebung also von der angestrebten Gültigkeit bzw. der Eindeutigkeit der mit den Untersuchungsergebnissen verbundenen Aussagen, andererseits vom Stand der Forschung bestimmt. $^{576}$

\section{Die Gültigkeit der Untersuchungsbefunde}

Als Kriterium bei der Entscheidung für die Untersuchungsumgebung fungiert im Rahmen dieser Arbeit die Validität, die als Gütekriterium der Untersuchung das Ausmaß der Gültigkeit bzw. die Aussagekraft der Untersuchungsergebnisse beurteilt. ${ }^{577}$ Insbesondere wird zwischen interner und externer Validität empirischer Untersuchungen differenziert: Interne Validität liegt vor, wenn Variationen der abhängigen Variablen eindeutig auf den Einfluss der unabhängigen Variablen zurückgeführt werden können bzw. wenn neben der Untersuchungshypothese keine besseren Alternativerklärungen vorliegen. ${ }^{578}$ Das Ausmaß der inneren Gültigkeit wird im Wesentlichen von der Adäquanz der Messebene, d.h. wenn die Messungen an den richtigen Untersuchungsobjekten vorgenommen werden, und von der Kontrolle der Störvariablen zur Vermeidung einer Kofundierung verschiedener Zusammenhänge bestimmt. ${ }^{579}$ Externe Validität hingegen liegt vor, wenn die aus einer Stichprobenuntersuchung gezogenen Schlussfolgerungen hinsichtlich anderer Situationen, Personen und Zeitpunkte generalisiert werden

573 Vgl. hierzu die Ausführungen in Teil V Kap. 1.3 und Kap. 3.1.

574 Vgl. Fromkin/Steufert (1976), S. 417. Da Laborstudien nicht zwangsläufig mit Laborexperimenten gleichzusetzen sind, ist die Untersuchungsumgebung terminologisch von der Untersuchungsstrategie zu unterscheiden. Stapf (1999, S. 228) schreibt dazu: „Den begrifflichen Gegensatz zur Laborforschung (Laborbeobachtung, Laborexperiment) bildet (in den Sozialwissenschaften) die Feldforschung (Feldbeobachtung, Feldexperiment)."

575 Vgl. Stapf (1999), S. 228; Bortz/Döring (2006), S. 57

576 Vgl. Bortz/Döring (2006), S. 49 f.

577 Vgl. Laatz (1993), S. 32. Zu den Aspekten der Validität vgl. ausführlich Cook/Campbell (1979).

578 Vgl. Gachowetz (1999), S. 253; Bortz/Döring (2006), S. 53

$579 \quad$ Vgl. Laatz (1993), S. 33 
können. ${ }^{580}$ Das Ausmaß der äußeren Gültigkeit wird also im Wesentlichen von der Realitätsadäquanz, d.h. wenn die Untersuchungsergebnisse über die besonderen Bedingungen der Untersuchungssituation und über die untersuchten Objekte hinaus auf andere reale Problemfelder übertragbar sind, bestimmt. ${ }^{581}$

Bezugnehmend auf das Verhältnis von interner und externer Validität merken Campell und Stanley an, dass beide Kriterien von hoher Relevanz für die Güte einer Untersuchung sind: „While internal validity is the sine qua non, and while the question of external validity, like the question of inductive inference, is never completely answerable, the selection of designs strong in both types of validity is obviously our ideal.“582 Der internen Validität ist demnach eine höhere Bedeutung einzuräumen, da sie eine notwendige Voraussetzung für die externe Validität darstellt - allerdings ist das Vorliegen der inneren Gültigkeit für die äußere Gültigkeit oft nicht hinreichend, da die Merkmale, die das eine Gültigkeitskriterium verbessern häufig das andere verschlechtern. ${ }^{583}$ So kennzeichnet sich die Felduntersuchung durch eine hohe externe Validität: Da sie in einer natürlichen Umgebung stattfindet, wird ihren Ergebnissen die Übertragbarkeit auf andere Situationen, Personen oder Zeitpunkte zugeschrieben ${ }^{584}$ Allerdings geht dieser Vorteil zu Lasten der internen Validität, da die Natürlichkeit der Untersuchungsumgebung und die damit verbundene eingeschränkte Kontrolle von Störvariablen häufig mehrere gleichwertige Erklärungsalternativen der Ergebnisse zulässt. Da im Zuge einer in einer planmäßig hergestellten Labor-Umwelt stattfindenden Untersuchung Störvariablen, die Einfluss auf die abhängige Variable nehmen können, weitgehend ausgeschaltet oder kontrolliert werden können ${ }^{585}$ liegt der Vorteil der Laboruntersuchung in ihrer hohen internen Validität. Allerdings sieht sich diese Untersuchungsumgebung mit dem Vorwurf der Unnatürlichkeit, bedingt durch die aktive Gestaltbarkeit der Szenerie, und der damit verbundenen eingeschränkten Generalisierbarkeit der Untersuchungsergebnisse konfrontiert. ${ }^{586}$

\section{Stand der empirischen Forschung}

Aufgrunddessen, dass die Entscheidung für eine Untersuchungsumgebung mit unterschiedlichen Vor- und Nachteilen bezüglich der Güte der Untersuchung ver-

\footnotetext{
$580 \quad$ Vgl. Gachowetz (1999), S. 253; Stapf (1999), S. 237; Bortz/Döring (2006), S. 53

$581 \quad$ Vgl. Laatz(1993), S. 33

582 Campbell/Stanley (1963), S. 175

583 Vgl. Stapf (1999), S. 237

584 Die Annahme, dass Felduntersuchungen eine höhere externe Validität als Laboruntersuchungen aufweisen, findet allerdings keine uneingeschränkte Zustimmung, vgl. zu diesbezüglicher Kritik z.B. Schuler (1980), S. 153; Oser (1982), S. 137; Westmeyer (1982), S. 75, die im Rahmen ihrer Arbeiten davon ausgehen, dass sich die Ergebnisse von Felduntersuchungen nur auf klar abgegrenzte, ähnliche Felder übertragen lassen.

585 Vgl. Laatz (1993), S. 460

586 Vgl. Stapf (1999), S. 228; Bortz/Döring (2006), S. 57. Zu einer kritischen Gegenüberstellung der Feld- und Laboruntersuchung vgl. Gachowetz (1999), S. 252-257.
} 
bunden ist, fungiert der Stand der empirischen Vertrauensforschung als ausschlaggebendes Entscheidungskriterium: Unter Berücksichtigung des Anspruchs dieser Arbeit, einen Beitrag zur Weiterentwicklung der Vertrauensforschung zu leisten, stellt sich die Frage, ob es von höherer Bedeutung ist, die interne Validität des im Rahmen dieser Arbeit generierten Modells zu prüfen, oder die hypothetisierte Beziehung zwischen individuellen Kompetenzen und der Vertrauenshandlung extern $\mathrm{zu}$ validieren. ${ }^{587}$ Für den Fall, dass in einem Untersuchungsgebiet Laboruntersuchungen dominieren und damit ein geringerer Zweifel bezüglich der internen Validität der Untersuchungsergebnisse besteht, bietet es sich an, die gewonnenen Erkenntnisse mit Felduntersuchungen auf ihre externe Validität hin zu überprüfen. Überwiegen in einem Untersuchungsgebiet allerdings realitätsnahe Felduntersuchungen, deren interne Validität nicht ausreichend dokumentiert erscheint, so sind Überlegungen zur Umsetzung der Forschungsfrage in Laboruntersuchungen anzustellen.

Die Ausführungen in Teil II Kap. 7 und Teil IV Kap. 1 lassen darauf schließen, dass Vertrauen als Untersuchungsgebiet elaboriert ist. Aufgrund uneinheitlicher Konzeptualisierungen und Operationalisierungen des Vertrauenskonstrukts mangelt es allerdings an einer Vergleichbarkeit und Integration der Ergebnisse. ${ }^{589}$ Zwar implizieren die in großer Zahl vorgenommenen Felduntersuchungen eine gewisse Breite des Untersuchungsgebiets und eine hohe Relevanz des Vertrauensphänomens in der Realität, doch lassen ihre Ergebnisse auch darauf schließen, dass Grundlagenforschung und eine detaillierte Analyse der Zusammenhänge notwendig sind ${ }^{590}$ Die Unklarheiten sowohl bezüglich der Vorbedingungen, direkten Determinanten, Dimensionen, Manifestationen und Konsequenzen von Vertrauen, als auch darüber, ob es sich bei gewissen Faktoren um Ursachen, Wirkungen oder Vertrauen per se handelt ${ }^{591}$, begründen unter anderem die zunehmende Forderung nach Untersuchungen, die einen Beitrag zur Erklärung des Konstrukts leisten. ${ }^{592}$

Abschließend ergibt sich die Entscheidung für die Untersuchungsumgebung aus dem Erklärungsinteresse der vorliegenden Arbeit. Aufgrunddessen, dass in den vergangenen Jahrzehnten in verschiedenen Fachdisziplinen bedeutende theoretische Vertrauensmodelle entwickelt wurden, die interdisziplinäre Vertrauensforschung sich aber erst im Anfangsstadium ihrer Entwicklung befindet ${ }^{593}$, wird im Rahmen dieser Arbeit das Ziel verfolgt, bereits entwickelte theoretische An-

\footnotetext{
587 Vgl. Späth (2008), S. 115

$588 \quad$ Vgl. Bortz/Döring (2006), S. 57

$589 \quad$ Vgl. Raimondo (2000); Lewicki et al. (2006), S. 1014

590 Vgl. Stein (1990), S. 21; Schweer/Thies (2003), S. 36; Späth (2008), S. 115

591 Vgl. hierzu die Ausführungen in Teil Il Kap. 7 und Teil IV Kap. 1, sowie die dort zit. Literatur.

592 Vgl. hierzu insbesondere Raimondo (2000); Gillespie (2003), S. 7 und 39; Möllering (2006), S. 140 ff.; Späth (2008), S. 116

593 Vgl. Späth (2008), S. 116
} 
sätze und empirische Forschungsergebnisse zusammenzuführen und grundlegende Zusammenhänge zu überprüfen. Der internen Validität der vorliegenden Untersuchung wird daher eine erhöhte Bedeutung beigemessen, weshalb das in Teil IV Kap. 5 generierte Modell im Labor überprüft wird.

\subsection{Die Untersuchungssituation}

Die betriebswirtschaftlichen Laborforschung differenziert zwischen drei Methoden zur Gestaltung der Untersuchungssituation, die darauf abzielen, ein beobachtbares Ereignis herbeizuführen: Fallstudie, Simulation und Fallsimulation. Die Entscheidung für eine Methode nimmt Einfluss auf die Wahrnehmung der Untersuchungssituation durch die Versuchspersonen und demnach auch auf deren Verhalten. ${ }^{594}$

Unter einer Fallstudie ist die wirklichkeitsgetreue Darstellung eines Entscheidungsproblems aus der betriebswirtschaftlichen Praxis zu verstehen. ${ }^{595}$ Während sich die Statik als Charakteristikum der Fallstudie aus der höchst spezifischen Beschreibung einer singulären Entscheidungssituation ergibt $^{596}$, kennzeichnet sich die Simulation durch Dynamik: „Simulation is the exercise of a flexible imitation of processes and outcomes for the purpose of clarifying or explaining the underlying mechanisms involved." ${ }^{\text {"597 }}$ Mit Hilfe der Simulationsmethode wird durch Nachbildung realer Zusammenhänge eine Ersatzrealität geschaffen, die unter anderem eine Vorabbildung antizipierter Konsequenzen erlaubt. ${ }^{598}$ Die Fallsimulation hingegen charakterisiert sich durch ein ausgewogenes Verhältnis von Statik und Dynamik: In einer kontrollierten Umgebung wird mit Hilfe einer realitätsnahen, komplexen Problemstellung bei den Versuchspersonen Dynamik erzeugt, sodass Interaktionsprozesse einer Untersuchung zugänglich werden. ${ }^{599}$ Als erprobte Methode der Gestaltung des laboratorischen Interaktionsfeldes ${ }^{600}$ wird der Fallsimulation in dieser Untersuchung der Vorzug gegenüber Fallstudie und Simulation gegeben. Zur Realisierung des Potentials der Fallsimulation, die externe Validität von in Laboruntersuchungen gewonnenen Ergebnissen zu erhöhen, sind im Zuge der Gestaltung der Untersuchungssituation eine Vergleichbarkeit der Entscheidungsaufgabe im Labor mit realen zwischenbetrieblichen Aufgaben, und

\footnotetext{
594 Vgl. Stein (1989); Stein (1990); Stapf (1999), S. 233 ff.; Spăth (2008), S. 116

595 Vgl. Kaiser/Kaminski (1999), S. 154

596 Vgl. Späth (2008), S. 116

597 Abelson (1968), S. 275

598 Vgl. Stapf (1999), S. 234

$599 \quad$ Vgl. Stein (1989), S. 531

600 Vgl. zu Studien, die im weitesten Sinne Fallsimulationen zur Situationsgestaltung (z. B. Rollenspiele zur Beobachtung des Verhaltens bei der Bearbeitung von Verhandlungs-, Planungs-, und Problemlösungsaufgaben) einsetzen: Berg et al. (1995); Glaeser et al. (2000); Köszegi (2001); Ostrom/Walker (2002); Gachter et al. (2004); Kosfeld et al. (2005); Späth (2008).
} 
bei der Gestaltung der Interaktionsstruktur eine Annäherung an realitätsnahe Interorganisationsprinzipien (z.B. asymmetrische Informationen, Arbeitsteilung, Rollenzuweisung etc.) sicherzustellen. ${ }^{601}$ Abgesehen von diesen grundsätzlichen Anforderungen an die Fallsimulation sind im Zuge der Erforschung des Vertrauenskonstrukts besondere Punkte bei der Gestaltung der Untersuchungssituation zu berücksichtigen: Vertrauen wird in Situationen relevant, die durch Abhängigkeit des Vertrauensgebers vom Verhalten des Vertrauensnehmers gekennzeichnet sind und in denen sich der Vertrauensgeber durch seine Vertrauensentscheidung freiwillig einem Risiko (z.B. keine Schutzmaßnahmen zur Begrenzung von Opportunismusspielräumen) aussetzt. Damit eine Überprüfung des hypothetisierten Einflusses individueller Kompetenzen auf die Vornahme einer Vertrauenshandlung im Labor möglich ist, muss die Fallsimulation daher folgende Elemente beinhalten:

- Interaktionsbeziehung zwischen Vertrauensgeber und Vertrauensnehmer

- Unsicherheit über das Verhalten des Interaktionspartners und potentieller Schaden

- Verhaltensoptionen, die die Vornahme einer Vertrauenshandlung reflektieren

Die Umsetzung der Anforderungen an die Untersuchungssituation in eine Fallsimulation wird in Teil V Kap. 2.1 beschrieben.

\subsection{Die Untersuchungsstrategie}

Im Hinblick auf die Untersuchungsmethoden im Labor ist grundsätzlich zwischen Beobachtung und Experiment zu differenzieren. Zwar beruht im weitesten Sinne jede Datenerhebung auf einer Beobachtung ${ }^{602}$, doch lässt sich die systematische Laborbeobachtung ${ }^{63}$ vom Laborexperiment insofern abgrenzen, als dass manipulative Eingriffe mit dem Ziel isolierender Bedingungsvariationen nur im Rahmen von Laborexperimenten erfolgen. ${ }^{604}$ Gemeinsam ist diesen Untersuchungsmethoden die künstlich generierte Untersuchungssituation zur Kontrolle von Störvariablen. ${ }^{605}$

Bezugnehmend auf die Vertrauensforschung dient die Beobachtung als zentrale psychologische Untersuchungsmethode der Erfassung spezifischer, klar um-

601 Vgl. Picot (1980); Stein (1989, S. 531) schreibt hierzu: „Zusammenfassend betrachtet vermag die Fallsimulation durch die Zerlegung eines abstrakten Gesamtentscheidungsproblems in inhaltlich zwar eigenständige, aber sachlich zusammenhängende Teilaufgaben, verbunden mit einer problemadäquaten Gestaltung der Aufgabenumgebung, einen validen laborexperimentellen Handlungsrahmen zu schaffen."

602 Vgl. Laatz (1993), S. 169

603 Zur systematischen Beobachtung vgl. Bortz/Döring (2006), S. 263 ff.

604 Vgl. Stapf (1999), S. 233

605 Vgl. Späth (2008), S. 118 
schriebener Vertrauenshandlungen in Interaktionsbeziehungen. ${ }^{606}$ Da die systematische Beobachtung der Vornahme einer Vertrauenshandlung aber keine Variation einer oder mehrerer unabhängiger Variablen (z.B. Kompetenz) unter gleichzeitiger Kontrolle anderer Variablen zulässt, erlaubt dieses Verfahren keine intern valide Klärung der Fragen bezüglich der Determinanten der Vertrauensentstehung. In der betriebswirtschaftlichen Vertrauensforschung finden sich daher nur vereinzelt Studien, im Rahmen derer eine systematische Beobachtung unter kontrollierten Bedingungen eingesetzt wurde. ${ }^{607}$ Hingegen stellt das Experiment die zentrale Untersuchungsmethode betriebswirtschaftlicher Entscheidungsforschung dar, dessen einzigartige Stärke in der Überprüfung von Ursache-WirkungBeziehungen besteht: „Das Experiment läßt sich insofern als die vornehmste aller Forschungsmethoden bezeichnen, als nur dieses Kausalbeziehungen festzustellen erlaubt. “608 Durch Variation einer oder mehrerer unabhängiger Variablen und der Feststellung der Veränderung nachgelagerter Variablen, wie zum Beispiel der Vertrauenshandlung, wird unter kontrollierten Bedingungen eine Analyse kausaler Zusammenhänge ${ }^{609}$ möglich. Deshalb kann davon ausgegangen werden, dass sich das Experiment vor allem zur Prüfung von aus der Theorie abgleiteten Kausalhypothesen eignet. ${ }^{610}$ Charakteristisch für das Laborexperiment ist das ,Prinzip der isolierenden Bedingungsvariation', d.h. unter Konstanthaltung anderer unabhängiger Variablen wird eine unabhängige Variable isoliert variiert und im Anschluss an diese isolierte Variation wird die Veränderung in der abhängigen Variable gemessen. Nur so kann der Einfluss der variierten Variable auf die abhängige Variable eindeutig festgestellt werden. ${ }^{611}$ Auch wird es durch die Einhaltung dieses Prinzips möglich, Störvariablen, d.h. Variablen, die außer den planmäßig variierten unabhängigen Variablen noch Einfluss auf die abhängige Variable nehmen könnten ${ }^{612}$, zu kontrollieren bzw. konstant zu halten, sodass Veränderungen in der abhängigen Variable eindeutig auf die Variation der unab-

\footnotetext{
606 Vgl. Schweer/Thies (2003), S. 37

607 Vgl. hierzu die Studien von O'Reilly/Roberts (1974) und Dirks (1999); bzw. als Beispiel für eine Studie, die der psychologischen Vertrauensforschung zuzuordnen ist, vgl. Petermann (1996), der die Vertrauensbeziehung zwischen Kinderarzt und Patient systematisch beobachtet.

608 Mayntz et al. (1972), S. 168. Dazu äußert sich Bredenkamp (1980, S. 1) folgendermaßen: „Die Interpretation einer gerichteten Relation zwischen zwei oder mehreren Variablen ist allerdings nicht nur in Experimenten möglich [...] Festzuhalten ist jedoch, daß im Experiment auf eine bestimmte charakteristische Weise (Manipulation und Randomisierung), die es gegen andere Methoden abhebt, versucht wird, die Relation zwischen verschiedenen Variablen eindeutig interpretierbar zu machen und daß der Grad an Eindeutigkeit der Interpretation bei dem Experiment am größten ist."

609 Nach Mayntz et al. (1972, S. 168) sind Variablen .,kausal verbunden, wenn sie in einem empirisch nicht umkehrbaren, asymmetrischen Zusammenhang stehen. $\mathrm{X}$ erzeugt $\mathrm{Y}$, aber nicht umgekehrt. $\mathrm{X}$ ist hierbei die unabhängige, $Y$ die abhängige Variable."

610 Vgl. Stein (1990), S. 15; Kittner (1994), S. 1; Stapf (1999), S. 236

611 Vgl. Stapf (1999), S. 232

612 Vgl. Stein (1990), S. 23
} 
hängigen Variable zurückzuführen sind. ${ }^{613}$ Stelzl merkt abschließend hierzu an: „Auch wenn bei der Definition des Experiments die Akzente bisweilen unterschiedlich gesetzt werden [...], so besteht doch allgemein Einigkeit darin, daß die aktive Manipulation der Bedingungen durch den Experimentator und damit die Möglichkeit, Ursache und Wirkung zu unterscheiden, das Wesentliche am Experiment ausmacht. “614

Anstatt einer aktiven Manipulation der unabhängigen Variablen können auch natürliche Variationen der Versuchspersonen (z.B. Vergleiche zwischen Personen mit hoher und geringer Risikobereitschaft) herangezogen werden, um Veränderungen in der abhängigen Variable im Labor zu messen. Gemäß Stapf handelt es sich hierbei allerdings nicht um Experimente, sondern um psychometrische Tests als Form differentieller Methoden, die darauf abzielen, Differenzen in der individuellen Merkmalsstruktur der Testpersonen, unabhängig von den Bedingungen der Untersuchungssituation, zu erfassen. ${ }^{615}$

Im Zentrum der vorliegenden Untersuchung steht die Überprüfung von Kausalzusammenhängen. Die gewählte Untersuchungsstrategie verbindet daher eine laborexperimentelle Variation der unabhängigen Variable mit psychometrischen Tests zur Erfassung der Dispositionen und der Wahrnehmung der Versuchspersonen. ${ }^{616}$ Ein großer Vorteil des Einsatzes psychometrischer Tests liegt in der Vergleichbarkeit der Testresultate. ${ }^{617}$ Auch eignet sich diese differentielle Methode, um unter kontrollierten Bedingungen Ursache-Wirkung-Beziehungen zu untersuchen, da Variablen wie Persönlichkeitsdispositionen und situative Risikowahrnehmung durch die Erhebung ihrer Ausprägungen im Rahmen psychometrischer Tests zu Kontrollvariablen transformiert werden. ${ }^{618}$

Abschließend ist auf Barrieren bezüglich der Untersuchung des Kompetenzeinflusses auf Vertrauen im Labor, die sich aus den theoretischen Ausführungen dieser Arbeit ableiten und zu überwinden sind, hinzuweisen: Die Vertrauensentscheidung ist das Ergebnis eines Prozesses, der mit der Vertrauenserwartung beginnt und mit der Vertrauenshandlung enden kann, aber nicht muss. Da Vertrauen auch künstlich nicht ad hoc erzwungen werden kann, besteht die Möglichkeit, dass die Probanden im Zuge der Bearbeitung der Fallsimulation keine Vertrauenshandlung vornehmen. Weiters ist anzumerken, dass es sich bei Kompetenz um ein dynamisches Konzept handelt, dessen Erfassung grundsätzlich erschwert ist. Allerdings besteht im Labor die Möglichkeit, durch die Variation der unabhängigen Variable unterschiedliche Kompetenzarten der Interaktionspartner in

\footnotetext{
$613 \quad$ Vgl. Späth (2008), S. 119

614 Stelzl (1999), S. 108

615 Vgl. Stapf (1999), S. 233

616 Vgl. zu den Vorteilen der Kombination von Befragung und Experiment z.B. Glaeser et al. (2000)

617 Vgl. Stapf (1999), S. 233

618 Vgl. Bortz/Döring (2006), S. 3
} 
einem dynamischen Kontext darzustellen. Diese Eingriffsmöglichkeit wird in der vorliegenden Untersuchung insofern umgesetzt, als dass eine Versuchsgruppe bei der Bearbeitung der Aufgabe Hinweise über die ,guten' Fach- und Methodenkompetenzen bei Konstanthaltung der Sozial- und Persönlichkeitskompetenzen ihres Interaktionspartners erhält, während die zweite Versuchsgruppe die gleiche Aufgabe, allerdings mit Hinweisen über die ,guten' Sozial- und Persönlichkeitskompetenzen bei Konstanthaltung der Fach- und Methodenkompetenzen ihres Interaktionspartners bearbeitet, und die Kontrollgruppe schließlich die gleiche Aufgabe unter Konstanthaltung der Fach-, Methoden-, Sozial- und Persönlichkeitskompetenzen des Interaktionspartners löst. Demnach setzt sich die vorliegende Untersuchung aus zwei Experimenten zusammen. Aufgrunddessen, dass die individuellen Kompetenzen der Interaktionspartner einerseits nicht ad hoc durch den Versuchsleiter, und andererseits deren Wahrnehmung durch die Probanden nicht im Zuge einer einmaligen Untersuchung erfassbar sind, werden die Probanden in der vorliegenden Untersuchung eine Rolle einnehmen - um allerdings Realitätsnähe zu gewährleisten, gehen die Probanden im Zuge der ,Vor-Bearbeitung ' der Fallsimulation von einer anschließenden Begegnung mit ihrem ,fiktiven“ Interaktionspartner aus (vgl. Kap. 2.1).

\section{DAS EXPERIMENT}

Nach der Begründung der Entscheidungen für die Untersuchungsumgebung, Gestaltung der Untersuchungssituation und Untersuchungsstrategie, erfolgt in diesem Kapitel die Erläuterung des Experiments, das in der vorliegenden Arbeit zur Überprüfung der hypothetisierten Kausalbeziehung zwischen individuellen Kompetenzen und Vertrauen durchgeführt wird. Im Anschluss an die Darstellung der betriebswirtschaftlichen Entscheidungsaufgabe, die von den Probanden zu bearbeiten ist (Kap. 2.1), wird die Manipulation der unabhängigen Variable (Kap. 2.2) erläutert.

\subsection{Die Integration der Vertrauenselemente in eine Fallsimulation}

Um die Vertrauenshandlung im Zuge des Experiments erheben zu können, ist es erforderlich, den Probanden die Möglichkeit zum Setzen von Handlungen zu geben, die einer Erfassung zugänglich und nachvollziehbar sind. Das vorliegende Kapitel beschreibt die Entwicklung der Fallsimulation ${ }^{619}$, die die Probanden zur Arbeit an einer Problemstellung veranlasst und messbare Reaktionsmöglichkeiten bietet. Durch Konfrontation mit einer interdependenten Entscheidungssituation

619 Bei der Entwicklung der vorliegenden Fallsimulation zur Untersuchung des Kompetenzeinflusses auf die Vertrauensentstehung erfolgt eine Orientierung am Versuchsaufbau von Späth (2008). 
müssen die Probanden Erwartungen bezüglich des Könnens und Wollens ihres Interaktionspartners bilden und können sich in unterschiedlichem Ausmaß freiwillig einem Risiko aussetzen. Dabei fungiert der Hinweis auf die Teilnahme an einem Assessment-Center, sowie eine spieltheoretisch-basierte Dilemma-Situation als situativer Rahmen ${ }^{620}$ und durch asymmetrische Informationsverteilung werden beobachtbare Reaktionen initiiert. Die Probanden und ihr Interaktionspartner sind gleichzeitig Vertrauensgeber und Vertrauensnehmer, da beide Akteure die Ziele einer Drittpartei zu berücksichtigen haben, wodurch Realitätsnähe impliziert wird. ${ }^{621}$ Als zwischenbetrieblicher Anwendungsbereich der Fallsimulation wird die Verteilung knapper Ressourcen gewählt. Bevor nun auf die Rolleneinnahme, Problemstellung und Informationsbasis, sowie die Handlungsmöglichkeiten eingegangen wird, erfolgt ein Überblick über die Aufgabenstellungen.

Im Zuge der Vorarbeiten für eine Verhandlung zur Kooperationskonstituierung mit einem potentiellen Kooperationspartner und dem Abnehmer ihres Kooperationsprodukts bzw. ihrer eigenen Produkte müssen die Probanden strategische und taktische Entscheidungen treffen: Erstens müssen sie sich entscheiden, ob sie in der Verhandlung zur Kooperationskonstituierung entweder eine Kooperationsoder eine Wettbewerbsstrategie verfolgen. Den Probanden werden im Rahmen dieser Aufgabenstellung Anreize geboten, auf Kosten des potentiellen Kooperationspartners Gewinne zu erzielen. Zweitens erhalten die Probanden die Option, dem potentiellen Kooperationspartner ausgewählte Informationen vor der Verhandlung offen und ehrlich abzugeben. Drittens haben die Probanden die Möglichkeit, die Informationshandlungen des potentiellen Kooperationspartners in unterschiedlichem Ausmaß zu kontrollieren, indem sie formale Berichte über die Ehrlichkeit von dessen Informationsabgabe anfordern. ${ }^{622}$

In der Beschreibung der Situation, die in die Aufgabenstellungen mündet, wird den Probanden suggeriert, dass ein persönliches Treffen - in Form einer Verhandlung - mit dem potentiellen Kooperationspartner und dem Abnehmer des Ko-

620 Vgl. Axelrod (1991); James (2002). Spieltheoretische Versuchsanordnungen im Labor sind vor allem dann geeignet, wenn die Untersuchung kooperativen Verhaltens im Zentrum des Interesses steht. Insbesondere das Gefangenendilemma bietet einen erprobten Rahmen, um simultan vorzunehmende Kooperationsentscheidungen darzustellen, weshalb es in der vorliegenden Untersuchung zur Situationsgestaltung herangezogen wird. Auf dem Grundprinzip des Gefangenendilemmas lassen sich nämlich betriebswirtschaftliche Fallsimulationen entwickeln, die den vereinfachten und artifiziellen reinen spieltheoretischen Simulationen im Hinblick auf ihre Komplexität, Dynamik und Realitätsnähe überlegen sind. Von hoher Relevanz bei einem Rückgriff auf spieltheoretische Versuchsanordnungen ist allerdings, dass die Kooperationsentscheidung nicht mit einer Vertrauensentscheidung gleichzusetzen ist.

$621 \mathrm{Vgl} \mathrm{hierzu} \mathrm{Teil} \mathrm{II} \mathrm{Kap.} \mathrm{4.3.2.3,} \mathrm{sowie} \mathrm{die} \mathrm{dort} \mathrm{zit.} \mathrm{Literatur.} \mathrm{Übertragen} \mathrm{auf} \mathrm{die} \mathrm{Realität} \mathrm{würde} \mathrm{es}$ sich hier um Vertrauen zwischen Kooperationspartnern, die beispielsweise einem Abnehmer des Produkts ihrer Kooperation verpflichtet sind, handeln.

622 Vgl. hierzu im Detail die nachfolgenden Erläuterungen der Handlungsmöglichkeiten in der Fallsimulation. 
operationsprodukts bzw. ihrer eigenen Produkte im Anschluss an die Vorarbeiten stattfindet. Dadurch soll bei den Probanden das Bewusstsein erzeugt werden, dass ihre Entscheidungen und die Konsequenzen ihrer Handlungen in einer realen Interaktion zu vertreten und zu tragen sind. In der Realität kommt dieses in Aussicht gestellte persönliche Treffen allerdings nicht zustande, da die Untersuchung mit der Erfüllung der für die Verhandlung notwendigen Vorarbeiten endet. Die Angemessenheit dieser Täuschung lässt sich anhand von Ergebnissen der spieltheoretisch-basierten Vertrauensforschung begründen: Bei einmaligen Interaktionen, aber auch in der letzten Runde wiederholter Interaktionen ist die Versuchung groß, das Vertrauen des Interaktionspartners nicht zu rechtfertigen. ${ }^{623}$ Um diese sogenannten ,Endrundeneffekte $\mathrm{zu}$ vermeiden, ist eine Suggestion hinsichtlich des Bevorstehens einer weiteren Interaktion erforderlich. ${ }^{624}$ Dieses so geschaffene Bewusstsein, das zukünftige Interaktionen von gegenwärtigen Interaktionen determiniert werden können, nimmt Einfluss auf das Verhalten der Probanden. Für die Probanden besteht dann nämlich Grund zur Annahme, dass es zumindest in der gegenwärtigen Interaktion - von Vorteil ist, auf Gewinne zu verzichten, anstatt den aktuellen Opportunismusspielraum einmalig auszunutzen. Dieses Phänomen, auch bekannt als ,Shadow of the Future ${ }^{625}$ wird in diese Untersuchung bewusst als Stimulus eingearbeitet.

\section{Rolleneinnahme}

Der Proband nimmt im Zuge der Fallsimulation die Rolle eines Weißweinproduzenten ein, der einen Wein speziell für Geschenksituationen auf den Markt bringen will. Um den Markt für Geschenkwein vollständig zu bearbeiten, möchte er mit einem österreichischen Rotweinproduzenten zusammenzuarbeiten. Der Geschäftsführer eines Weinhandels hat bereits sein Interesse an der Idee des Weißweinproduzenten signalisiert und könnte sich vorstellen, das Ergebnis der Zusammenarbeit in einer Geschenkbox, bestehend aus einer Flasche Weißwein des Weißweinproduzenten und einer Flasche Rotwein des Rotweinproduzenten, exklusiv anzubieten. Er stellt ein Budget von 100.000,- Euro für 5.000 Geschenkboxen, bestehend aus je zwei Flaschen Wein, in Aussicht, behält sich jedoch eine genaue Prüfung der Eignung der Produkte zum Absatz auf dem Weingeschenkmarkt vor, auf deren Basis entschieden wird, ob $100 \%, 50 \%$ oder $0 \%$ der Abnahmemenge (10.000 Flaschen) bzw. des Budgets (100.000,- Euro) an den Weißweinproduzenten vergeben werden.

\footnotetext{
623 Vgl. Möllering (2003), S. 33; Wiese (2002), S. $243 \mathrm{ff}$.

624 Vgl. Späth (2008), S. 123

$625 \quad$ Vgl. Axelrod (2002), S. 112
} 


\section{Problemstellung}

Die Abnahmemenge von 10.000 Flaschen (bzw. das Budget von 100.000,- Euro) soll zwischen dem Weißwein- und dem Rotweinproduzenten aufgeteilt werden. Um das Potential der beiden Weinproduzenten zu prüfen, soll eine Verhandlung mit dem Weißwein- und dem Rotweinproduzenten, d.h. dem Probanden als Weißweinproduzent und dem ,fiktiven' Interaktionspartner als Rotweinproduzent, sowie dem ,fiktiven' Geschäftsführer des Weinhandels stattfinden. Der Geschäftsführer des Weinhandels hat bereits bekannt gegeben, dass die Weine beider Weinproduzenten gut in sein Sortiment passen, sodass er sich eine 50:50-Aufteilung des Budgets vorstellen kann. Andererseits ist für ihn auch denkbar, nur den Winzer, der über das größere Potential verfügt, mit $100 \%$ des Budgets zu unterstützen. Allerdings behält sich der Geschäftsführer des Weinhandels vor, die Gelder zurückzuziehen, wenn in der Verhandlung zur Kooperationskonstituierung offensichtlich wird, dass sich die Produkte beider Weinproduzenten nicht zum Absatz auf dem Weingeschenkmarkt eignen.

\section{Informationsbasis}

Die Probanden erhalten allgemeine Informationen über die beiden Weingüter und die Produkte: Betriebsgröße, Rebsorten, Produktionsmenge, Absatzmenge, Konsumentenpreise / Flasche. Neben diesen allgemeinen Informationen werden ihnen 16 spezifische Informationen über das eigene Weingut, die Produkte und die Studienergebnisse vorgelegt ${ }^{626}$ :

- Einnahmen-Ausgaben-Rechnung 2009: Umsatz Gastronomie, Umsatz AbHof-Verkauf, ,Schwarz'-Umsatz, Gewinn, zusätzlicher Gewinn (aus unversteuerten Verkäufen)

- Bewirtschaftung des Weinguts: Weingartenumstellung, Bewässerung, Böschungs- und Mauerterassen

- Produkte des Weinguts: Auszeichnungen, Exportländer, Besonderheiten

- Ergebnisse der vom Weißweinproduzenten bezahlten Studie über den Weingeschenkmarkt hinsichtlich Flaschenform, Verschluss, Etikette, Rebsorten, Alkoholgehalt, sowie die Umsetzbarkeit der Anforderungen für den Weißweinproduzenten ${ }^{627}$

Diese Daten sind dem Rotweinproduzenten unbekannt. Es wird in der schriftlichen Instruktion herausgestellt, dass diese Informationen ohne Referenzwerte wertlos sind, da sie nur im Vergleich mit den Daten des Rotweinproduzenten interpretiert werden können. Es wird weiters darauf hingewiesen, dass je besser der

626 Die Informationsbasis ist im Anhang dieser Arbeit dokumentiert, S. 239.

627 Diese Daten orientieren sich an den Ergebnissen der empirischen Untersuchung des Weingeschenkmarktes von Hatak (2008). 
Weißweinproduzent über die Stärken und Schwächen seines Betriebes im Vergleich zum Weingut des Rotweinproduzenten Bescheid weiß, desto eher kann er in der Verhandlung den Geschäftsführer des Weinhandels von seinem Zuteilungsvorschlag bezüglich der Abnahmemenge überzeugen.

\section{Handlungsmöglichkeiten}

Im Zuge der Vorbereitung auf die Verhandlung zur Kooperationskonstituierung müssen die Probanden die nachfolgenden drei Aufgaben ${ }^{628}$ bearbeiten:

(1) Wahl der Strategie

Bei der ersten Aufgabe müssen sich die Probanden in ihrer Rolle als Weißweinproduzent auf Basis der gegebenen Informationen für eine Strategie entscheiden, die sie in der anschließenden Verhandlung verfolgen. Zu diesem Zeitpunkt ist ihnen nicht bekannt, welche Strategie der Rotweinproduzent verfolgt. Zur Wahl stehen zwei Strategien: Einerseits können sich die Probanden für eine Kooperation mit dem Rotweinproduzenten, d.h. für eine Teilung der Abnahmemenge und des Budgets $\mathrm{zu} 50 \%$ mit dem Interaktionspartner (Strategie 1), entscheiden. Andererseits können die Probanden mit dem Rotweinproduzenten in Wettbewerb treten und auf einen Erhalt von $100 \%$ der Abnahmemenge und des Budgets abzielen (Strategie 2). Im Aufgabenblatt wird schriftlich und graphisch darauf hingewiesen, dass die Konsequenzen der Strategiewahl von der Strategiewahl des Rotweinproduzenten abhängen.

\begin{tabular}{|c|c|c|}
\hline & \multicolumn{2}{|c|}{ der Rotwe inproduze nt wählt } \\
& Strategie 1 & Strategie 2 \\
\hline \multirow{2}{*}{ Strategie 1 } & $\begin{array}{c}\text { Abnahmemenge- und Budgetaufteilung } \\
\text { zu je 50\% für beide } \\
\text { (Nutzen für Sie: } 50.000,- \text { Euro) }\end{array}$ & $\begin{array}{c}\text { 0 \% für Sie, } \\
100 \% \text { für den Rotweinproduzenten } \\
\text { (Nutzen für Sie: 0 Euro) }\end{array}$ \\
\hline \multirow{2}{*}{ Strategie 2 } & $\begin{array}{c}100 \% \text { für Sie, } \\
\text { 0\% für den Rotweinproduzenten } \\
\text { (Nutzen für Sie: } 100.000,- \text { Euro) }\end{array}$ & $\begin{array}{c}\text { Geschäftsführer des Weinhandels zieht } \\
\text { sein Angebot zurück: 0 \% für beide } \\
\text { (Nutzen für Sie: 0 Euro) }\end{array}$ \\
\hline
\end{tabular}

Abbildung 14: Ergebnismatrix zur Strategiewahl (allgemein)

Aus Sicht der Spieltheorie stellt diese Simultanentscheidung ein Zwei-PersonenDilemma $^{629}$ mit den Handlungsalternativen ,Kooperation ${ }^{\circ}$ und ,Wettbewerb“ dar. ${ }^{630}$ Einerseits besteht Unsicherheit hinsichtlich des Verhaltens des Rotwein-

628 Das Aufgabenblatt ist im Anhang dieser Arbeit dokumentiert, S. 240.

629 Für einen Überblick über Experimente auf Basis des Gefangenendilemmas vgl. Roth (1995), S. 26.

630 Die von Deutsch (1958) identifizierten, für die Untersuchung von Vertrauen im Labor notwendigen Situationsparameter wurden in der vorliegenden Variation des Gefangendilemmas (durch Integration essentieller Elemente einer Vertrauenssituation) berücksichtigt: (1) Konfrontation mit einem ambiguenten Weg, der entweder zu einem Ereignis führt, das als nutzenstiftend angesehen wird, d.h. über eine positive $\mathrm{Valenz}(\mathrm{Va}+)$ verfügt, oder in einem Ereignis, das als nachteilig wahrgenommen wird, d.h. eine negative Valenz (Va-) besitzt, resultiert, (2) Wahrnehmung, dass das Ein- 
produzenten, d.h. ob er die Strategie ,Kooperation“ oder ,Wettbewerb' wählt. Andererseits ist anzunehmen, dass beide Interaktionspartner vermeiden möchten, dass der jeweils andere die gesamte Abnahmemenge (bzw. das gesamte Budget) erhält während sie selbst leer ausgehen. Dies wäre dann der Fall, wenn sich einer der Interaktionspartner für die Kooperationsstrategie entscheidet, der andere aber die Wettbewerbsstrategie verfolgt. Bei der Wahl der Wettbewerbsstrategie besteht die Möglichkeit, dass einer der Partner im günstigsten Fall die gesamte Abnahmemenge zugeteilt bekommt. Im schlechtesten Fall wird beiden Interaktionspartner kein Budget zugeteilt. Unter der Annahme gleicher Eintrittswahrscheinlichkeiten für beide Umweltzustände, d.h. für die jeweilige Strategiewahl des Rotweinproduzenten, und bei Heranziehen der Nutzensumme der alternativen Aktionen über beide Umweltzustände als Entscheidungskriterium sollten Selbstinteresse und Ratio zu einer Entscheidung für die Wettbewerbsstrategie führen. ${ }^{631}$

Um Realitätsnähe zu gewährleisten (langfristige wechselseitige Abhängigkeit der Winzer und des Weinhändlers von den Charakteristika (z.B. Fach- und Methodenkompetenz, sowie Sozial- und Persönlichkeitskompetenz) der Partner ${ }^{632}$ ), sowie zur methodischen Vermeidung sogenannter ,Endrundeneffekte' (bei einmaligen Interaktionen ist der Anreiz groß, Opportunismusspielräume auszunutzen) wird im Zuge der schriftlichen Instruktionen darauf hingewiesen, dass, sofern eine Geschäftsbeziehung mit dem Weißweinproduzenten, dem Rotweinproduzenten, oder beiden Winzern zustande kommt, der Geschäftsführer des Weinhandels kontinuierlich die Qualität der Produkte und Anpassung an Kundenwünsche bewerten wird. Nachfolgende Graphik zeigt die Konsequenzen der Bewertung: Wenn der Weißweinproduzent $(+)$ beispielsweise mit dem Rotweinproduzenten zusammenarbeitet, dieser aber Wein schlechter Qualität (-) abfüllt, wird die 50:50Lösung zu einer 0:0-Lösung, da der Geschäftsführer beiden Winzern die Zusammenarbeit aufkündigt (der Anteil des Weißweinproduzenten ist fett markiert).

treten von Va+ oder Va- von den Handlungen des Vertrauensnehmers abhängt, und (3) Wahrnehmung, dass Va- größer ist als $\mathrm{Va}+$

631 Vgl. Späth (2008), S. $126 \mathrm{f}$.

632 In Situationen, in denen alle alles wissen (z.B. Eintrittswahrscheinlichkeiten der jeweiligen Umweltzustände) kann das Verhalten aller Akteure vorhergesagt werden - Vertrauen ist hier überflüssig. Unter den realitätsnäheren Verhaltensannahmen der begrenzten Rationalität, unvollständigen Informationen (einhergehend mit Interdependenzen) und des durch langfristige Perspektiven begrenzten kurzfristigen Opportunismus sind hingegen die Handlungen der Akteure unsicher. 


\begin{tabular}{|c|c|c|c|}
\hline & \multicolumn{2}{|c|}{ Qualităt \& Anpassung des Rotwe inproduze nten an Kundenwünsche } \\
\hline & & + & - \\
\hline \multirow{2}{*}{ 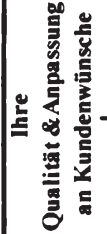 } & + & $\begin{array}{c}\mathbf{5 0}: 50 \rightarrow \mathbf{5 0 : 5 0}(50.000 \text {,- Euro für Sie }) \\
100: 0 \rightarrow 100: 0(100.000,- \text { Euro für Sie }) \\
\text { 0:100 } \rightarrow \text { 0:100 (0 Euro für Sie })\end{array}$ & $\begin{array}{c}\mathbf{5 0}: 50 \rightarrow \mathbf{0}: 0(0 \text { Euro fur Sie }) \\
100: 0 \rightarrow 100: 0(100.000,- \text { Euro furr Sie }) \\
\mathbf{0}: 100 \rightarrow \mathbf{0}: 0(0 \text { Euro fur Sie })\end{array}$ \\
\hline & - & 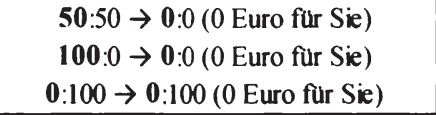 & 0:0 $\rightarrow$ 0:0 (0 Euro fur Sie $)$ \\
\hline
\end{tabular}

Abbildung 15: Ergebnismatrix zur Strategiewahl (weiterfahrend)

Im Gegensatz zu Experimenten, bei denen die Strategiewahl an eine Auszahlung von Geldbeträgen gekoppelt ist, findet im Anschluss an die im Zuge dieser Arbeit vorgenommenen Erhebungen eine Verlosung statt. Hierbei können jene Probanden, die alle drei Aufgaben gewissenhaft bearbeitet haben, eine Flasche Wein (Gesamtmenge: 150 Flaschen Weißwein) oder einen Büchergutschein (Gesamtwert: 100 Euro) gewinnen. Durch diese Stimuli soll einerseits das Entscheidungsverhalten der Probanden an persönlicher Relevanz gewinnen. Andererseits wird durch die Verlosung von Wein bzw. Büchergutscheinen anstelle einer Auszahlung von Geldbeträgen vermieden, dass monetäre Anreize zu stark in den Vordergrund $^{633}$ treten.

(2) Informationsabgabe

Im Anschluss an die Wahl der Strategie erhalten die Probanden die Möglichkeit, mit dem Rotweinproduzenten vor der Verhandlung in Kontakt zu treten. Dazu liegen den Unterlagen ein leeres Informationsformular und ein Umschlag bei, der an den Rotweinproduzenten adressiert ist. Die Probanden können in jedes Feld des Informationsformulars die richtigen Informationen über Ihr Weingut (Einnahmen-Ausgaben-Rechnung, Bewirtschaftung, Produkte) und den im Rahmen der Kooperation zu bearbeitenden Markt (Ergebnisse der Studie über den Weingeschenkmarkt) oder falsche Informationen eintragen. Sie können aber auch beliebig viele, also auch alle Felder freilassen. Die Probanden werden darauf hingewiesen, dass der Umschlag dem Rotweinproduzenten vor der anschließenden Verhandlung übergeben wird und dass sie reziprok auch einen Umschlag vom Rotweinproduzenten erhalten.

(3) Kontrollintensität

Nach richtiger, falscher oder keiner Informationsabgabe erhalten die Probanden die Option, bestimmte Informationen, die der Rotweinproduzent an sie weiterleitet, zu überprüfen. Bei Wahrnehmung dieser Möglichkeit können maximal vier

633 Nach Späth (2009, S. 127) wird .,Studien, die auf monetäre Anreize aufbauen, [...] vielfach vorgeworfen, dass diese zu Verzerrungen führen, da intrinsisch motivierte Personen weniger stark auf extrinsische Anreize reagieren." 
Berichte angefordert werden, die offenlegen, wie viel Prozent der Informationen, die der Rotweinproduzent übermittelt hat, im jeweiligen Bericht (EinnahmenAusgaben-Rechnung, Bewirtschaftung des Weinguts, Produkte des Weinguts, Bereitschaft und Fähigkeit des Rotweinproduzenten zur Umsetzung der Studienergebnisse) falsch sind. Es wird darauf hingewiesen, dass sich der Beginn der anschließenden Verhandlung pro angefordertem Bericht verzögert und dass der Rotweinproduzent darüber in Kenntnis gesetzt wird, welche Berichte die Probanden überprüfen lassen.

Zusammenfassend resultieren die dargelegten Aufgabenelemente in einer Entscheidungssituation, die durch Unsicherheit gekennzeichnet ist. Den Probanden sind die Konsequenzen ihrer Handlungen bewusst. Mit welcher Wahrscheinlichkeit die verschiedenen Zustände eintreten, ist jedoch unbekannt. Mit der Aufgabe der Strategiewahl, deren Bearbeitung der Vorbereitung auf die Verhandlung zur Kooperationskonstituierung dient, wird den Probanden die bereits verbal umschriebene Situation in kompakter Form sowie die Konsequenzen alternativer Handlungen in übersichtlicher Form näher gebracht. Von weitaus höherer Relevanz für die vorliegende Untersuchung sind jedoch die taktischen Informationsund Kontrollhandlungen der Probanden. Die Bearbeitung der zweiten und dritten Aufgabe in dieser Fallsimulation ermöglicht die Prüfung von Hypothesen zur Intensität bzw. zum Ausmaß vertrauensvoller Handlungen in einer Entscheidungssituation. ${ }^{634}$

\subsection{Die experimentelle Manipulation der unabhängigen Variable}

Da im Zuge dieser Arbeit die Frage beantwortet werden soll, welche individuellen Kompetenzen Einfluss auf die Vornahme einer Vertrauenshandlung nehmen, muss im Zuge des Laborexperiments die unabhängige Variable ,Kompetenz“ (bzw. Fach- und Methoden-, sowie Sozial- und Persönlichkeitskompetenz) isoliert variiert werden. Aufgrunddessen, dass eine tatsächliche Beziehung zwischen den Probanden zu Störeffekten bei der Wahrnehmung einer Versuchssituation führen kann $^{635}$, wird im Zuge der vorliegenden Arbeit auf reale Interaktionen zwischen den Probanden verzichtet. Stattdessen werden den Versuchspersonen die Kompetenzen ihres Interaktionspartners indirekt schriftlich erläutert.

Unter Bezugnahme auf die Indikatoren individueller Kompetenzarten (vgl. Teil IV Kap. 3.1) werden drei unterschiedliche Beschreibungen des Rotweinproduzenten in die Situationsbeschreibung der Fallsimulation eingearbeitet. Durch diese Eingriffsmöglichkeit können drei Gruppen gebildet werden:

634 Vgl. dazu auch Späth (2008), S. 128

635 Vgl. Stein (1990), S. 170 
(1) die Versuchsgruppe 1 (VG1) erhält Hinweise zu den ,guten' Fach- und Methodenkompetenzen des Rotweinproduzenten bei Konstanthaltung der Sozial- und Persönlichkeitskompetenzen:

Nachdem Sie sich nun entschieden haben, mit einem österreichischen Rotweinproduzenten zusammenzuarbeiten, besuchen Sie die Weinmesse ,VieVinum' in der Wiener Hofburg. Dort kommen Sie mit einem österreichischen Weinproduzenten ins Gespräch, der sich auf die Produktion von Rotwein spezialisiert hat.

- Fachwissen

Während des Gesprächs bemerken Sie eine Vielzahl von internationalen und nationalen Auszeichnungen an seinem Stand. Den Gütekriterien nach zu urteilen, die seine Weine erhalten haben, scheint vor allem der ,Zweigelt' bei den Testern und Konsumenten beliebt zu sein.

- Abstraktionsfähigkeit

Der Rotweinproduzent merkt an, dass er sich aufgrund mangelnder Nachfrage vor einigen Jahren dazu entschlossen hat. sein Weingut biodynamisch zu bewirtschaften.

fähigkeit

- Problemlösungs-

Lern- und Kreativmethoden

- Methoden zur Informationsverarbeitung

Nachdem er in Fachjournalen von dieser Methode der Bewirtschaftung gelesen hat, hat er mehr Informationen darüber gesammelt.

- Kaufmännisches Wissen Nach einer Kosten-Nutzen-Analyse hat er ...

- Planungs- und Kontrollmethoden

- Managementund Leistungsmethoden

... die Umsetzung der biodynamischen Bewirtschaftung in seinem Betrieb geplant, koordiniert und kontrolliert.

- Erfahrungswissen

- Vermittlungsund Darstellungsfähigkeit
Dieser Entschluss hat dem Betrieb entsprechenden Erfolg gebracht, da es der Rotweinproduzent - als Experte für biodynamischen Weinbau - Jahr für Jahr schafft, die sehr hohe Qualität zu halten. Inzwischen ist er so bekannt, dass er laufend als Referent von Weinakademien eingeladen wird. 
konstante Sozialund Persönlichkeitskompetenzen
Auf Ihr Nachfragen hin, räumt der Rotweinproduzent ein, dass diese biodynamische Bewirtschaftung mit einem hohen Aufwand verbunden ist. Sie erzählen ihm von Ihrem Weingut und Ihren Weißweinen. Nachdem Sie den ,Zweigelt' probiert haben, fällt Ihnen die traditionelle Etikettengestaltung des Rotweinproduzenten auf. Auf Ihren Vorschlag hinsichtlich einer möglichen Modernisierung reagiert der Rotweinproduzent zurückhaltend.

(2) die Versuchsgruppe 2 (VG2) erhält Hinweise zu den ,guten' Sozial- und Persönlichkeitskompetenzen des Rotweinproduzenten bei Konstanthaltung der Fachund Methodenkompetenzen:

Nachdem Sie sich nun entschieden haben, mit einem österreichischen Rotweinproduzenten zusammenzuarbeiten, besuchen Sie die Weinmesse ,VieVinum" in der Wiener Hofburg. Dort kommen Sie mit einem österreichischen Weinproduzenten ins Gespräch, der sich auf die Produktion von Rotwein spezialisiert hat.

konstante Fachund Methodenkompetenzen

Während des Gesprächs bemerken Sie eine Plakette an seinem Stand. Dieser Plakette nach zu urteilen, scheint der ,Zweigelt ${ }^{`}$ bei den Testern anzukommen. Der Rotweinproduzent gibt an, dass er sein Weingut biodynamisch bewirtschaftet. Dadurch schafft er es die Qualität zu halten und seine Produkte abzusetzen.

(Selbstöffnung) $-\div:$ Auch aus persönlicher Sicht war diese Vorgehensweise in seinen

- Selbstvertrauen

Augen erfolgreich, da er nun ...

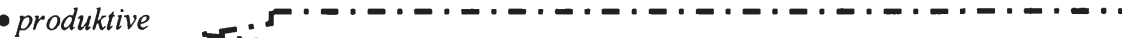
Begeisterungs- - ... viel mehr Freude bei der Arbeit auf seinem Weingut hat. i Fähigkeit

- soziale Gesinnung

- Fairness

- Zielstrebigkeit, Der Rotweinproduzent erzählt Ihnen von den Entwicklungen bei Ehrgeiz, Effizienz- i ehrlich auf alle Rückschläge und Erfolge ein. streben (Selbstdisziplin)

- Neugier $\quad-\quad$ Auf sein Nachfragen hin, erzählen Sie ihm von Ihrem Weingut.

Kommunikations - Der Rotweinproduzent kennt Ihre Weißweine und äußert sich!' fähigkeit $\quad \therefore$; begeistert über deren hohe Qualität. Er wirkt auch sehr - Aufgeschlossenheit i interessiert an Ihrem Betrieb ... 
- soziale Gesinnung

- Aufgeschlossen-

- Voller Begeisterung lädt er auch Sie mitsamt Ihrer Familie auf : heit

sein Weingut ein.

- Charisma (Überzeugungsfähigkeit)

- Bereitschaft zur Informationsweitergabe $<$ -

- Kooperations- ' fähigkeit

- Einfuihlungsvermögen und Solidarität

- Konfliktfähigkeit

- Konstruktiver $\therefore$ - -

Umgang mit endogenen Reizen

1 - - - - - - - - - - - - - - - - - - - - - - - - - -

(3) die Kontrollgruppe (KG) erhält Hinweise zu den konstant gehaltenen Fach-, Methoden, Sozial- und Persönlichkeitskompetenzen:

Nachdem Sie sich nun entschieden haben, mit einem österreichischen Rotweinproduzenten zusammenzuarbeiten, besuchen Sie die Weinmesse ,VieVinum' in der Wiener Hofburg. Dort kommen Sie mit einem österreichischen Weinproduzenten ins Gespräch, der sich auf die Produktion von Rotwein spezialisiert hat.

konstante Fach-, Methoden-,

Sozial- und

Persönlichkeits-

kompetenzen
Der Rotweinproduzent erzăhlt, dass sich sein ,Zweigelt' gut verkauft. Sie erzăhlen ihm von Ihrem Weingut und Ihren Weißweinen. Nachdem Sie den ,Zweigelt' probiert haben, fállt Ihnen die traditionelle Etikettengestaltung des Rotweinproduzenten auf. Auf Ihren Vorschlag hinsichtlich einer möglichen Modernisierung reagiert der Rotweinproduzent zurückhaltend.

Abgesehen von der variierenden Beschreibung des Rotweinproduzenten gibt es keinen Unterschied in der Situationsbeschreibung zwischen den Gruppen - auch differieren sie nicht in den Bereichen Informationsbasis, Aufgabenstellungen und psychometrische Tests.

\section{Die Messung Der VARIABLEN}

Um den Einfluss individueller Kompetenzen auf die Vornahme einer Vertrauenshandlung unter kontrollierten Bedingungen analysieren zu können, ist es von hoher Relevanz, potentielle Störvariablen in Kontrollvariablen zu transformieren. 
Da diese Variablen aber nicht im Forschungsinteresse der vorliegenden Untersuchung stehen, wurden im theoretischen Teil dieser Arbeit keine Hypothesen zum Zusammenhang zwischen Vertrauensbereitschaft, Risikobereitschaft und Risikowahrnehmung einerseits und der Vornahme einer Vertrauenshandlung andererseits abgeleitet. Um aber auszuschließen, dass Unterschiede in diesen Dispositionen und in der Wahrnehmung der Probanden die Ergebnisse der Untersuchung verzerren, werden sie als Kontrollvariablen miterfasst. Im Anschluss an die Darstellung der Verfahren zur Datengewinnung, die im Rahmen dieser Arbeit zur Anwendung kommen, erfolgt daher eine Erläuterung der Skalen zur Erhebung der Ausprägungen der Persönlichkeitsdispositionen und der situativen Risikowahrnehmung der Probanden in deren Rolle als Vertrauensgeber. Abschließend wird auf die Erhebung der Vertrauenshandlung als abhängige Variable des Kausalmodells eingegangen.

\subsection{Die Verfahren zur Datengewinnung}

Im Rahmen der vorliegenden Untersuchung kommen verschiedene Verfahren zur Datengewinnung zur Anwendung: So bietet es sich an, die Persönlichkeitsdispositionen und die situative Risikowahrnehmung der Probanden in ihrer Rolle als Vertrauensgeber im Zuge einer Befragung zu erfassen. Grundsätzlich ist zwischen mündlicher Befragung in Form von Interviews und schriftlicher Befragung über Fragebögen $\mathrm{zu}$ differenzieren. ${ }^{636} \mathrm{Im}$ Rahmen der vorliegenden Untersuchung erfolgt aus forschungsökonomischen Gründen eine standardisierte schriftliche Befragung. Durch den Einsatz von Ratingskalen vor der Bearbeitung der Fallsimulation wird es möglich, das subjektive Empfinden der Probanden bezüglich der Ausprägungen ihrer Persönlichkeitsdispositionen und ihrer situativen Risikowahrnehmung auf einem Merkmalskontinuum zu erfassen und intervallskaliert zu interpretieren. ${ }^{637}$ Bei der Messung dieser Variablen wird daher auf bewährte und standardisierte Instrumente zurückgegriffen. Aufgrunddessen, dass diese Skalen aus dem anglo-amerikanischem Raum stammen und die Akkuratheit der Übersetzung zu gewährleisten ist, erfolgt eine Rückübersetzung. ${ }^{638}$ Die Vorstellung der Skalen, d.h. die Offenlegung der Itembatterien der Messinstrumente, in der in dieser Untersuchung verwendeten deutschen Übersetzung erfolgt sodann in den nachfolgenden Unterkapiteln (vgl. Kap. 3.2 und Kap. 3.3). Der Grad der Messgenauigkeit (Präzision) der einzelnen Instrumente, d.h. die Reliabilität (Zuverlässig-

636 Vgl. zur Befragung als Datenerhebungsmethode z.B. Laatz (1993), S. 103 ff.; Bortz/Döring (2006), S. $236 \mathrm{ff}$; Diekmann (2007), S. $434 \mathrm{ff}$.

637 Vgl. Bortz/Döring (2006), S. $176 \mathrm{f}$.

638 Vgl. Brislin (1980); Späth (2008). Die einzelnen Fragen werden von einer deutschsprachigen Anglistikprofessorin ins Deutsche übersetzt und von einer englischsprachigen Person rückübersetzt. Im Anschluss daran wird die so generierte ,neue' englische Version mit der englischen Originalfassung verglichen und die deutsche Version gegebenenfalls adaptiert. 
keit) der einzelnen Skalen, wird in Kap. 5.1 in tabellarischer Form anhand von Cronbach's Alpha als Maß der internen Konsistenz dokumentiert. ${ }^{639}$

Zur Erfassung der Vornahme einer Vertrauenshandlung im Rahmen der vorliegenden Untersuchung bietet sich eine Dokumentenanalyse an. Bei Dokumenten handelt es sich grundsätzlich um gegenständliche Zeugnisse (z.B. Schriftstücke, Lösungsblätter), hervorgebracht von den Probanden, die als Vergegenständlichung ihrer Psyche zur Erklärung individuellen Verhaltens dienen. ${ }^{640}$ Mit Hilfe dieser speziellen Form der quantitativen Inhaltsanalyse, die sich besonders für Laboruntersuchungen eignet ${ }^{641}$, können Daten gewonnen werden, die durch klassische Datengewinnungsverfahren wie Befragung oder Verhaltensbeobachtung nicht zugänglich sind. Bezugnehmend auf die vorliegende Untersuchung werden die Daten über die Vornahme einer Vertrauenshandlung daher inhaltsanalytisch gewonnen - d.h. einerseits werden die Informationshandlungen der Probanden durch Analyse der an die Interaktionspartner adressierten Briefumschläge erhoben, andererseits werden die Kontrollhandlungen der Probanden durch Analyse der an die Versuchsleitung adressierten Briefumschläge erfasst (vgl. Kap. 3.4).

\subsection{Die Messung der Dispositionen des Vertrauensgebers}

Dispositionen stellen situationsübergreifende Persönlichkeitsmerkmale dar, von denen angenommen wird, dass sie Einfluss auf das Verhalten in spezifischen Situationen nehmen. ${ }^{642} \mathrm{Im}$ Zuge der Modellierung des Kompetenzeinflusses auf die Vertrauensentstehung (vgl. Teil IV Kap. 5) wurde davon ausgegangen, dass es sich bei der generellen Vertrauensbereitschaft und der vertrauensgeberspezifischen Risikobereitschaft um Variablen handelt, die die Vornahme einer Vertrauenshandlung beeinflussen. Um auszuschließen, dass Unterschiede in den Dispositionen der Probanden die Ergebnisse der Untersuchung verzerren, werden sie im Rahmen der vorliegenden Untersuchung kontrolliert miterhoben. Aufgrund der in Teil II Kap. 7 herausgestellten Vorteile des Instruments von Costa wird die generelle Vertrauensbereitschaft in der vorliegenden Untersuchung auf einer RatingSkala mit den Polen ,stimme voll zu' und ,stimme gar nicht zu` erfasst. Das Instrument besteht aus den folgenden sieben Items, die auf der theoretisch fundierten und empirisch geprüften RPHNS von Wrightsman basieren ${ }^{643}$ :

\footnotetext{
$639 \mathrm{Vgl}$. Bortz/Döring (2006), S. $198 \mathrm{f}$.

640 Vgl. zur Dokumentenanalyse als Datenerhebungsmethode z.B. Laatz (1993), S. 207 f:; Bortz Döring (2006), S. 149 ff.; Diekmann (2007), S. 576 ff.

641 Vgl. Bortz/Döring (2006), S. 151

642 Vgl. Asendorpf (2009), S. 2

643 Vgl. Costa (2000), S. 182, sowie die Ausführungen in Teil II Kap. 7 und die dort zit. Literatur.
} 
Bitte geben Sie die Antwort an, die am ehesten auf Sie zutrifft.

1. Die meisten Menschen helfen jemandem in Not ohne zu zögern.

2. Die meisten Menschen folgen dem Motto, Verhalte dich anderen gegenüber so, wie du willst, dass sie sich dir gegenüber verhalten'.

3. Die meisten Menschen sagen was sie denken.

4. Die meisten Menschen sagen im Grunde die Wahrheit; sogar dann, wenn sie wissen, dass sie besser dran wären, wenn sie lügen würden.

5. Menschen sind normalerweise aufrichtig um die Probleme anderer besorgt.

6. Die meisten Menschen stehen zu ihrer Überzeugung.

7. Menschen bleiben normalerweise bei ihrer Meinung, wenn sie denken, dass sie Recht haben, auch wenn die anderen nicht ihrer Meinung sind.

Tabelle 20: Items zur Messung der personenspezifischen Vertrauensbereitschaft

Quelle: In Anlehnung an Costa (2000)

Die Probanden beantworten vor dem Lesen der Situationsbeschreibung und der Informationsbasis, sowie vor der Bearbeitung der Aufgaben einen Fragebogen, der die Skala von Costa zur Erfassung der generellen Vertrauensbereitschaft enthält. Auch werden mit Hilfe dieses Fragebogens die Ausprägungen der Variable ,vertrauensgeberspezifische Risikobereitschaft' erhoben. Hierbei kommt die von Sitkin und Weingart entwickelte 5-Item-Skala, die sich speziell auf die Bereitschaft, Geschäftsrisiken zu akzeptieren, bezieht, zum Einsatz, da sie die Herstellung eines Bezugs zur Unternehmerrolle der Probanden in der Fallsimulation ermöglicht. Auf einer 5-stufigen Rating-Skala geben die Probanden an, ob sie als Unternehmer grundsätzlich eine starke Neigung empfinden, Risiko zu akzeptieren oder eher eine starke Neigung verspüren, das Risiko zu vermeiden. ${ }^{644}$ Der Wortlaut in der Einleitung der Fragestellung von Sitkin und Weingart wird für die vorliegende Untersuchung adaptiert:

Als Unternehmer sehen Sie sich immer wieder Entscheidungen gegenübergestellt. Wie würden Sie Ihre Bereitschaft einschätzen...

(1) Ich bin bereit, eine risikoreiche Entscheidung zu treffen, die auf der Empfehlung einer anderen Person beruht, auf die ich mich verlassen muss.

(2) Ich bin bereit, eine risikoreiche Entscheidung zu treffen, die auf methodisch hochkomplexen Analysen beruht.

(3) Ich bin bereit, eine risikoreiche Entscheidung zu treffen, die die strategische Ausrichtung meines Betriebs nachhaltig ändern könnte.

(4) Ich bin bereit, eine Entscheidung umzusetzen, auch wenn sie die Gefahr eines Fehlschlags in sich birgt.

(5) Ich bin bereit, eine Entscheidung zu unterstützen, bei der ich weiß, dass, obwohl teilweise Daten vorliegen, Informationen fehlen.

Tabelle 21: Items zur Messung der personenspezifischen Risikobereitschaft

Quelle: In Anlehnung an Sitkin/Weingart (1995) 


\subsection{Die Messung der Wahrnehmung des Vertrauensgebers}

Aufgrund des in der Risikoforschung empirisch bestätigten Zusammenhangs zwischen der situationsspezifischen Risikowahrnehmung und dem Verhalten in risikobehafteten Entscheidungssituationen wird die den Vertrauensnehmer weitgehend ausblendende Einschätzung der Eintrittswahrscheinlichkeiten der mit der Vornahme einer Vertrauenshandlung verbundenen Kontingenzen und der dadurch bedingten ökonomischen Folgen in einer spezifischen Situation im Zuge der vorliegenden Untersuchung als Kontrollvariable erfasst. Hierfür wird eine 4-ItemSkala von Sitkin und Weingart eingesetzt, die sich speziell an die in der Fallsimulation erzeugte Situation anpassen lässt. Die vier Items reflektieren Antworten auf einer 5-stufigen Rating-Skala zu folgenden Fragen ${ }^{645}$ :

Wie würden Sie Ihre aktuelle Situation bezïglich der Geschenkbox-Idee charakterisieren?

1. günstige Gelegenheit (1) ... starke Bedrohung (5)

2. Möglichkeit eines Verlusts (1) ... Möglichkeit eines Gewinns (5) [reverse scored]

3. positive Situation (1) ... negative Situation (5)

Wie hoch schätzen Sie Ihre Erfolgsaussichten ein, Ihre Vorstellungen bezüglich der Zuteilung der Abnahmemenge in der folgenden Verhandlung durchzusetzen?

4. sehr niedrig (1) ... sehr hoch (5) [reverse scored]

Tabelle 22: Items zur Messung der situativen Risikowahrnehmung

Quelle: In Anlehnung an Sitkin/Weingart (1995)

Im Gegensatz zur Erhebung der generellen Vertrauensbereitschaft und der vertrauensgeberspezifischen Risikobereitschaft erfordert der Einsatz dieses Instruments die Kenntnis der Situation. Die Probanden beantworten im Anschluss an die Bearbeitung der Aufgaben einen Fragebogen, der die Skala von Sitkin und Weingart zur Erfassung der situativen Risikowahrnehmung enthält.

\subsection{Die Messung der Handlungen des Vertrauensgebers}

Die Handlungen des Vertrauensgebers werden mit Hilfe einer Dokumentenanalyse erhoben. Im Zentrum des Interesses stehen die Informations- und Kontrollhandlungen als Indikatoren der abhängigen Variable ,Vertrauenshandlung'. Zur Überprüfung der methodischen Wirksamkeit des Versuchsaufbaus werden die Mittelwerte der Indikatorvariablen für jede Gruppe (KG, VG1, VG2) berechnet und miteinander verglichen (vgl. Teil VI Kap. 1.1).

Die Handlungsalternativen resultieren aus der Rolle, die im Rahmen der Fallsimulation vorgegeben wird. Im Zuge der Fallsimulation sollen drei Aufgaben zur Vorbereitung auf die anstehende Verhandlung zur Kooperationskonstituierung bearbeitet werden. Nachfolgend werden die Reaktionsmöglichkeiten im Hinblick 
auf Strategiewahl, Informationsabgabe und Kontrollintensität sowie deren Erfassung erläutert.

\section{Die StrategiewahI}

Die Strategiewahl fungiert (neben dem Hinweis auf die Teilnahme an einem Assessment-Center) als situativer Rahmen. Indem sich die Probanden für eine Strategie entscheiden müssen, wird Ihnen suggeriert, dass die Studie auf die Untersuchung des Verhaltens in einer spieltheoretisch-basierten Dilemma-Situation anstatt auf die Untersuchung des Kompetenzeinflusses auf die Vertrauensentstehung abzielt. Zwar kann die Strategiewahl Aufschluss darüber geben, ob die Manipulation der unabhängigen Variable die Probanden dazu veranlasst, sich in einer Situation, die durch Abhängigkeit vom Verhalten des Interaktionspartners gekennzeichnet ist, freiwillig einem Risiko auszusetzen und die Strategie der Kooperation zu wählen. Allerdings wurde in der vorliegenden Arbeit auf die Ableitung einer Hypothese zum Einfluss von Kompetenz auf die Strategiewahl in Verbindung mit Vertrauen verzichtet, da diese Entscheidung keine Aussagen zur Intensität vertrauensvoller Handlungen, die in der vorliegenden Untersuchung im Zentrum des Interesses steht, erlaubt.

Den Probanden wird die Aufgabe gestellt, sich für eine generelle Strategie, die sie in der anschließenden Verhandlung zur Kooperationskonstituierung verfolgen werden, zu entscheiden, d.h. die Abnahmemenge und das Budget zu 50\% mit dem Interaktionspartner zu teilen oder die Zuteilung von 100\% der Abnahmemenge und des Budgets anzustreben. Auf dem Aufgabenblatt, das vor der Verhandlung bei der Aufsicht abgegeben wird, kreuzen die Probanden die Wahl ihrer Strategie an.

Strategie 1: Sie versuchen, den Geschäftsführer des Weinhandels zu überzeugen, dass die Abnahmemenge gleichmäßig (50:50-Lösung) zwischen Ihnen und dem Rotweinproduzenten verteilt wird. Sie können dann Ihre Idee der Geschenkbox, bestehend aus einer Flasche Ihres Weißweines und einer Flasche des Rotweinproduzenten, mit einem zugeteilten Budget von 50.000,- Euro für 5.000 Flaschen Ihres Weißweines realisieren.

Strategie 2: Wie der Geschäftsführer des Weinhandels im E-Mail angedeutet hat, wären auch Geschenkboxen, bestehend aus ausschließlich Weißwein, für Ihn vorstellbar. Sie versuchen, den maximalen Anteil an der Abnahmemenge zu erhalten (100:0Lösung) und stellen in der Verhandlung die besonders gute Eignung Ihres Weißweines für die Geschenk-Box im Sortiment des Weinhändlers und für den Weingeschenkmarkt heraus. Sie können dann Geschenkboxen, bestehend aus zwei Flaschen Ihres Weißweines, mit einem zugeteilten Budget von 100.000,- Euro für 10.000 Flaschen Ihres Weißweines produzieren.

Für welche Strategie entscheiden Sie sich?

$\square$ Strategie 1 (50:50) $\quad \square$ Strategie 2 (100:0)

Bedenken Sie, wie sich der Rotweinproduzent verhalten wird. Dem Rotweinproduzenten wird Ihre Strategiewahl nicht mitgeteilt.

Tabelle 23: Aufgabenstellung zur Strategiewahl 


\section{Die Informationsabgabe}

Um den Einfluss der individuellen Kompetenzen als unabhängige Variablen auf die offene und ehrliche Informationsabgabe als Indikatoren der freiwilligen Erbringung einer riskanten Vorleistung zu überprüfen, ist eine Operationalisierung der Informationshandlungen in der Fallsimulation erforderlich. Die Probanden bekommen die Möglichkeit, ihrem Interaktionspartner vor der Verhandlung zur Kooperationskonstituierung maximal 16 Informationen über ihr Unternehmen und den im Rahmen der Kooperation zu bearbeitenden Markt verdeckt in einem an den Interaktionspartner adressierten Umschlag zu übermitteln. Den Angaben zur Aufgabenstellung liegt ein Formular bei, in das die Probanden richtige und falsche Informationen eintragen können. ${ }^{646}$

Welche Informationen über Ihr Weingut (Einnahmen-Ausgaben-Rechnung, Bewirtschaftung, Produkte) und den im Rahmen der Kooperation zu bearbeitenden Markt (Ergebnisse der Studie über den Weingeschenkmarkt) übermitteln Sie an den Rotweinproduzenten?

Überlegen Sie, wie viel Wissen Sie dem Rotweinproduzenten preisgeben möchten. Sie können in jedes Feld die richtigen Informationen laut Angabe (siehe Blatt ,Informationsbasis") oder falsche Informationen eintragen. Sie können aber auch beliebig viele, also auch alle Felder freilassen. Bitte bearbeiten Sie jetzt das blaue Formular und legen Sie es nach Bearbeitung in den beiliegenden Umschlag, der an den Rotweinproduzenten adressiert ist.

\section{Tabelle 24: Aufgabenstellung zur Informationsabgabe}

Nach O'Reilly III und Roberts ist die Art der Informationsabgabe durch Auszählen der einzelnen Informationen, die an den Interaktionspartner übermitteln werden, zu messen: Es wird die Summe offener und ehrlicher Informationen eruiert. ${ }^{647}$ Während die Summe offener Informationen durch Addition der Anzahl aller abgegebenen Informationen (richtige und falsche Informationen) berechnet wird, ergibt sich die Summe ehrlicher Informationen aus dem Anteil der richtigen Informationen an allen abgegebenen Informationen (multipliziert mit 10). Aufgrunddessen, dass maximal 16 Informationen übermittelt werden können, liegt der Wertbereich für die Variable ,Offene Informationsabgabe‘ zwischen 0 und 16. Damit die Variable ,Ehrliche Informationsabgabe' zu gleichen Anteilen wie die Variable ,Offene Informationsabgabe in die abhängige Variable ,Vertrauenshandlung ' eingeht, wird sie mit 1,6 gewichtet, womit ihr Wertbereich ebenfalls zwischen 0 und 16 liegt. Werden beispielsweise alle 16 Informationen korrekt übermittelt, so handelt es sich um eine offene und ehrliche Informationsabgabe. In diesem Fall werden die Variablen ,Offene Informationsabgabe " und ,Ehrliche Informationsabgabe' mit dem Wert ,16' kodiert. Erhält der Interaktionspartner ein leeres Informationsformular, so beträgt die Summe offener und ehrlicher Informationen 0 und die Variablen ,Offene Informationsabgabe ' und ,Ehrliche Informa- 
tionsabgabe" werden mit dem Wert, $0^{`}$ kodiert. Für den Fall, dass das Informationsformular 6 korrekte und 6 falsche Informationen enthält, d.h. 4 Informationen werden verschwiegen, so wird die Variable ,Offene Informationsabgabe' mit dem Wert ,12' und die Variable ,Ehrliche Informationsabgabe' mit dem Wert ,8‘ $(=(6 / 12) * 10 * 1,6)$ kodiert.

\section{Die Kontrollintensität}

Um den Einfluss der individuellen Kompetenzen als unabhängige Variablen auf die reduzierte Kontrollintensität als weiteres empirisches Äquivalent - neben der offenen und ehrlichen Informationsabgabe - der Vertrauenshandlung zu überprüfen, ist eine Erfassung der Kontrollhandlungen in der Fallsimulation erforderlich. Da den Probanden nicht bekannt ist, ob ihnen ihr Interaktionspartner richtige oder falsche Informationen über sein Unternehmen übermittelt, können sie um eine objektive Überprüfung der an sie abgegebenen Informationen ansuchen.

Auf dem Aufgabenblatt, das vor der Verhandlung bei der Aufsicht abgegeben wird, tragen die Probanden ein, wie viele der maximal 4 Berichte, die Auskunft über den prozentuellen Anteil falscher Informationen in den einzelnen Informationsbereichen geben, sie anfordern möchten. Dieses Formular wird der Versuchsleitung mit einem an sie adressierten Umschlag übermittelt. Indem betont wird, dass pro angefordertem Bericht eine gewisse Wartezeit vor der Verhandlung zur Kooperationskonstituierung anfällt, wird auf die mit Kontrollmaßnahmen verbundenen realen Kosten hingewiesen. Allerdings besteht für die Probanden auch die Möglichkeit des Verzichts auf eine externe Verifizierung der von dem Interaktionspartner übermittelten Informationen. ${ }^{648}$

Welche Berichte wünschen Sie, die zeigen wie viel Prozent der Informationen, die der Rotweinproduzent an sie übermittelt hat, falsch sind? Beachten Sie, dass sich der Beginn der anschließenden Verhandlung pro Bericht verzögert!

Bericht 1: Anteil falscher Informationen in der Einnahmen-Ausgaben-Rechnung

Bericht 2: Anteil falscher Informationen zur Bewirtschaftung

Bericht 3: Anteil falscher Informationen zu den Produkten

Bericht 4: Anteil falscher Informationen zur Bereitschaft und Fähigkeit zur Umsetzung der Studienergebnisse

Tabelle 25: Aufgabenstellung zur Kontrollintensität

Die Intensität der Kontrolle wird durch Auszählen der angeforderten Berichte zur Überprüfung der Informationshandlungen des Interaktionspartners gemessen. Es können maximal 4 Berichte angefordert werden. Damit die Variable ,Kontrollintensität' zu gleichen Anteilen wie die Variablen ,Ehrliche Informationsabgabe“ und ,Offene Informationsabgabe ' in die abhängige Variable ,Vertrauenshandlung ‘ eingeht, wird sie mit 4 gewichtet. Dadurch liegt ihr Wertbereich ebenfalls

Vgl. Späth (2008), S. 144 
zwischen 0 und 16 , wobei der Wert , $0^{\star}$ eine ausgeprägte Kontrollintensität und der Wert , 16' einen Kontrollverzicht bezeichnet.

Im Anschluss an die Bearbeitung der Fallsimulation werden also zwei Umschläge eingesammelt, wobei in dem an die Versuchsleitung adressierten Umschlag die Basisdaten jedes einzelnen Probanden, die Antworten auf die Fragen zur Vertrauensbereitschaft, Risikobereitschaft und Risikowahrnehmung sowie das Aufgabenblatt mit der Strategiewahl und den angeforderten Kontrollberichten enthalten sind. In dem an den Interaktionspartner adressierten Umschlag befindet sich das Formular mit den an diesen übermittelten Informationen. Dieser Umschlag wird allerdings nicht - wie den Probanden in Aussicht gestellt - an den Interaktionspartner weitergeleitet, sondern zur Auswertung einbehalten.

\section{DiE UNTERSUCHUNG}

\subsection{Der Pretest}

Zur Prüfung des Designs zur Untersuchung des modellierten Einflusses von Kompetenz auf die Entstehung von Vertrauen wurden zwei Pretests durchgeführt. Diese dienten in erster Linie dazu ,das erstellte Erhebungsinstrument auf seine Tauglichkeit hin zu testen und zu prüfen, inwieweit sich die beabsichtigten Hypothesenprüfungen durchführen lassen. “649

Die erste Voruntersuchung fand am 21. Jänner 2010 mit 20 Studierenden statt. Die Rückmeldungen der Studierenden im Zuge der Prüfung der Eignung der Skalen zur Messung der Vertrauens-, und Risikobereitschaft, sowie der situativen Risikowahrnehmung für den Einsatz in der Hauptuntersuchung zeigten, dass die ins Deutsche übersetzten Items gut verständlich waren. Auch die Analysen der Reliabilität der Skalen führten zu zufriedenstellenden Werten. Die Prüfung der Wirksamkeit der Fallsimulation hatte zum Ziel, sowohl die Verständlichkeit des Aufgabentextes als auch die wahrgenommene Glaubwürdigkeit der Aufgabenstellungen zu eruieren. Darüber hinaus ermöglichte diese Voruntersuchung eine Abschätzung des zeitlichen Aufwands für die Planung der Hauptuntersuchung und gab Aufschluss über die Belastung der Probanden. Die Rückmeldungen der Teilnehmer zeigten, dass die Aufgabenstellungen klar formuliert und realistisch waren. Weiters erforderte die Fallsimulation eine adäquate, aber nicht überhöhte Beanspruchung der Studierenden. Allerdings stellte sich in einer ausführlichen abschließenden Diskussion heraus, dass eine Zusammenführung der gesonderten Blätter ,Aufgabenblatt` und ,Aufgabenstellung ' der besseren Übersichtlichkeit dienen würde. Auch wurde von Teilnehmern angemerkt, dass sie ihre Entscheidungen im Zuge der Bearbeitung der Aufgaben gerne begründen würden. Diese 
Vorschläge wurden durch die Integration des ,Aufgabenblatts' in das Formular ,Aufgabenstellung' (und Benennung dieses zusammengeführten Formulars als ,Aufgabenblatt'), sowie durch die Erweiterung dieses zusammengeführten ,Aufgabenblatts' um ein freies Textfeld zur freiwilligen Begründung der Entscheidungen umgesetzt. Im Anschluss an diese Umsetzung fand am 25. Jänner 2010 die zweite Voruntersuchung mit 9 Studierenden statt, im Rahmen derer keine Probleme hinsichtlich Verständlichkeit der Skalen und des Aufgabentextes, Glaubwürdigkeit der Aufgabenstellungen und Übersichtlichkeit auftraten.

\subsection{Die Stichprobe}

Die Hauptuntersuchung wurde mit Studierenden der Wirtschaftsuniversität Wien, des Betriebswirtschaftlichen Zentrums der Universität Wien, sowie von verschiedenen österreichischen Fachhochschulstudiengängen durchgeführt. In Summe nahmen 427 Personen an dem Experiment teil. Die Zusammensetzung der Stichprobe wird in den nachfolgenden Tabellen dokumentiert.

\begin{tabular}{|c|c|c|c|}
\hline Merkmal & Aus prägung & Häufigkeit & Prozent \\
\hline \multirow{4}{*}{ Geschlecht } & männlich & 200 & $46,8 \%$ \\
\cline { 2 - 4 } & weiblich & 227 & $53,2 \%$ \\
\hline \multirow{4}{*}{ Alter } & 18-19 Jahre & 25 & $5,9 \%$ \\
\cline { 2 - 4 } & 20-21 Jahre & 112 & $26,2 \%$ \\
\cline { 2 - 4 } & 22-23 Jahre & 132 & $30,9 \%$ \\
\cline { 2 - 4 } & 24-25 Jahre & 75 & $17,6 \%$ \\
\cline { 2 - 4 } & 26-27 Jahre & 28 & $6,6 \%$ \\
\cline { 2 - 4 } & 28-29 Jahre & 21 & $4,9 \%$ \\
\cline { 2 - 4 } & 30-34 Jahre & 28 & $6,6 \%$ \\
\hline 35-55 Jahre & 6 & $1,4 \%$ \\
\cline { 2 - 4 } & Betriebswirtschaft & 156 & $36,5 \%$ \\
\cline { 2 - 4 } & Internationale Betriebswirtschaft & 87 & $20,4 \%$ \\
\cline { 2 - 4 } & Tourismus & 70 & $16,4 \%$ \\
\cline { 2 - 4 } & Miliärische Führung & 63 & $14,8 \%$ \\
\cline { 2 - 4 } & Wirtschaftsrecht & 19 & $4,4 \%$ \\
\cline { 2 - 4 } & Wirtschaftsinformatik & 12 & $2,8 \%$ \\
\cline { 2 - 4 } & Doktorat der Sozial und & 12 & $2,8 \%$ \\
\cline { 2 - 4 } & Wirtschaftswissenschaften & & $1,6 \%$ \\
\cline { 2 - 4 } & Volkswirtschaft \& Sozioökonomie & 7 & $0,2 \%$ \\
\cline { 2 - 4 } & Wirtschaftspädagogik & 1 & \\
\hline
\end{tabular}

Tabelle 26: Demographische Merkmale der Stichprobe 
Die folgende Tabelle zeigt die Zusammensetzung der Gruppen (VGl (erhält eine Situationsbeschreibung, die auf ausgeprägte Fach- und Methodenkompetenzen (FMK) des Rotweinproduzenten schließen lässt), KG (erhält eine Situationsbeschreibung, die durchschnittliche Fach-, Methoden-, Sozial- und Persönlichkeitskompetenzen des Rotweinproduzenten impliziert), VG2 (erhält eine Situationsbeschreibung, die darüber Auskunft gibt, dass der Rotweinproduzent über ausgeprägte Sozial- und Persönlichkeitskompetenzen (SPK) verfügt)), differenziert nach dem Merkmal Geschlecht.

\begin{tabular}{|c|c|c|c|c|c|c|}
\cline { 2 - 7 } \multicolumn{1}{c|}{} & \multicolumn{2}{c|}{ VG1 (FMK) } & \multicolumn{2}{c|}{ KG } & \multicolumn{2}{c|}{ VG2 (SPK) } \\
\hline Geschlecht & Häufigkeit & Prozent & Häufigkei & Prozent & Häufigkeit & Prozent \\
\hline männlich & 64 & $15 \%$ & 64 & $15 \%$ & 72 & $16,9 \%$ \\
\hline weiblich & 77 & $18 \%$ & 78 & $18,3 \%$ & 72 & $16,9 \%$ \\
\hline Summe & 141 & $33,0 \%$ & 142 & $33,3 \%$ & 144 & $33,7 \%$ \\
\hline
\end{tabular}

Tabelle 27: Geschlechterverteilung der Gruppenzusammensetzung

Im Rahmen von Laborexperimenten werden vorrangig Convenience Samples, bestehend aus Studierenden, gezogen. ${ }^{650}$ Ein zentraler Kritikpunkt, mit dem sich diese Art von Experimenten und daher auch die vorliegende Arbeit konfrontiert sehen, ist, dass - basierend auf der Annahme von Unterschieden im Verhalten zwischen Studierenden und Nicht-Studierenden ${ }^{651}$ - die ausschließliche Untersuchung von Studierendengruppen zu falschen Schlüssen führen kann. ${ }^{652}$ So implizieren beispielsweise die Ergebnisse von Carpenter et al. ${ }^{653}$ Feldexperiment Unterschiede zwischen Studierenden und Nicht-Studierenden: Nicht-Studierende spendeten 72 von 100 Dollar an Hilfsorganisationen, während die Spende der Studierenden um 17 Dollar geringer war. Andererseits lassen die repräsentativen Ergebnisse eines Ultimatum-Spiels auf keine Handlungsdifferenzen zwischen Studierenden und Nicht-Studierenden schließen. ${ }^{654}$ Grundsätzlich hat die ausschließliche Untersuchung von Studierenden den Vorteil, dass die Arbeit mit homogenen Versuchspersonen mit einer besseren Kontrolle der Störvariablen verbunden ist, was wiederum in einer verbesserten Teststärke des Experiments resultieren kann. ${ }^{655}$ Neben experimentellen Erfordernissen (isolierte Variation der unabhängigen Variable ,Kompetenz') und forschungspragmatischen Gründen (eingeschränkter Zugang zu kooperierenden Unternehmern und deren Kooperationspartnern) wird daher die im Zuge dieser Arbeit erfolgte Entscheidung für die Untersuchung des Verhaltens von Studierenden (in ihrer Rolle als mit einer Koopera-

$650 \quad$ Vgl. Naef/Schupp (2009), S. 20

651 Vgl. Levitt/List (2007)

652 Vgl. Bortz/Döring (2006), S. 75

653 Vgl. Carpenter et al. (2007)

654 Vgl. Fu et al. (2007)

655 Vgl. Stelzl (1999), S. 122 
tionsaufgabe konfrontierte Unternehmer) durch die Vorteile, die sich aus der Homogenität des zu untersuchenden Samples ergeben, begründet.

\subsection{Der Untersuchungsablauf}

Eine Standardisierung des Untersuchungsablaufs war von hoher Relevanz für die Erhöhung der Durchführungsobjektivität, da sich die Hauptuntersuchung aus forschungstechnischen Gründen über einige Monate (Sommersemester 2010) erstreckte. Der zeitliche Ablauf der gesamten Untersuchung orientierte sich an den für die Studie erforderlichen personellen und räumlichen Kapazitäten. Die Bearbeitung der Aufgabenstellungen beanspruchte in etwa eine Stunde pro Proband.

$\mathrm{Zu}$ jedem Erhebungstermin wurden die Probanden gemeinsam begrüßt und ihnen wurde das weitere Vorgehen erläutert. Die Versuchsleitung stellte heraus, dass unter jenen Teilnehmern, die die Aufgaben im Zuge des Assessment-Centers gewissenhaft bearbeiten, im Anschluss eine Verlosung von Wein (Gesamtmenge: 150 Flaschen der Sorte ,Grüner Veltliner') bzw. von Büchergutscheinen (Gesamtwert: 100 Euro) stattfindet. Auch wurde darauf hingewiesen, dass es keine richtigen oder falschen Antworten gibt, sondern die Entscheidungen so zu treffen sind, dass sie aus den Unterlagen heraus begründet werden können. Danach wurden die Probanden jedes Erhebungstermins in drei Gruppen (VG 1, VG 2 und KG) aufgeteilt, allerdings ohne dies zu bemerken: Jeder Proband erhielt einen DIN A4-Umschlag, der die Fragebögen, Situationsbeschreibung, Informationsbasis, sowie das Aufgabenblatt und das leere blaue Informationsformular enthielt. Weiters wurden den Teilnehmern an den Rotweinproduzenten adressierte DIN A5-Umschläge zur Übermittlung des ausgefüllten blauen Informationsformulars ausgehändigt. Auf beiden Umschlägen befand sich eine Nummer, sodass z.B. ein Proband die DIN A4- und DIN A5-Umschläge mit der Nummer 1 erhielt (diese Nummerierung diente auch der Probandenidentifikation im Zuge der Auswertung). Waren bei einem Erhebungstermin beispielsweise 30 Probanden, so wurden die DIN A4-Umschläge (inkl. den dazugehörigen DIN A5-Umschlägen), die eine Situationsbeschreibung, in der der Rotweinproduzent als fach- und methodenkompetent dargestellt wird, enthielten, von 1 bis 10 nummeriert (= VG 1). Die Umschläge der Kontrollgruppe erhielten die Nummern 11 bis 20 und jene der Versuchsgruppe 2 (hohe Sozial- und Persönlichkeitskompetenz des Rotweinproduzenten) wurden mit 21 bis 30 nummeriert.

Für eine bestmögliche Neutralisierung von personenbezogenen Störvariablen erfolgte eine zufällige Zuweisung der Teilnehmer zu den Gruppen, sodass im Anschluss an diese Randomisierung von probabilistisch äquivalenten Gruppen aus- 
gegangen werden konnte. ${ }^{656}$ Die Vorarbeiten für die Zufallszuweisung erfolgten vor dem Erhebungstermin mit Hilfe eines Online-Randomisierungstools, das eine zufällige Reihenfolge ausgewählter Zahlen (z.B. 1 bis 30) auswirft. Vor Beginn des Experiments wurden die Umschläge entsprechend dieser Reihenfolge (z.B. 29, 2, 16) sortiert und den Teilnehmern im Zuge der Erhebung ausgehändigt, womit die Probanden den Gruppen zufällig zugewiesen wurden.

Nach Bearbeitung der Aufgaben und Abgabe der Unterlagen bei der Versuchsleitung, wurden die Probanden gebeten, sich wieder auf ihren Platz zu begeben. Nachdem alle Teilnehmer eines Erhebungstermins ihre Arbeiten beendet hatten, wurden sie darüber informiert, dass die Aufgabe hiermit beendet sei, da aufgrund begrenzter Personal- und Raumkapazitäten nicht alle Probanden an der Verhandlung zur Kooperationskonstituierung teilnehmen könnten. ${ }^{657}$ Die Täuschung der Teilnehmer hinsichtlich einer anstehenden Kooperationsverhandlung war für die Aufrechterhaltung des Stimulus, die Entscheidungen in naher Zukunft vor realen Personen verantworten zu müssen, notwendig: „Minor deciption is sometimes necessary (or cost-effective) for achieving the desired level of experimental control over stimuli or feedback. “658 Von hoher Bedeutung für eine Studie, deren Datenerhebung sich über einen längeren Zeitraum erstreckt, ist, dass das Untersuchungsziel, die Aufgaben und der Aufbau des Versuchs verdeckt bleiben. ${ }^{659}$ Mit wiederholter Durchführung muss die Fallsimulation zu vergleichbaren Ergebnissen führen. ${ }^{660}$ Abschließend wurden die Probanden daher darum gebeten, nicht mit Kollegen über Inhalte und Ablauf des Assessment-Centers zu sprechen, um verzerrende Reaktionen bei zukünftigen Teilnehmern zu vermeiden.

656 Durch die Randomisierung (zufällige Aufteilung) werden Unterschiede zwischen den Gruppen, die in quasiexperimentellen Untersuchungen durch den Vergleich natürlicher Gruppen nicht nur hinsichtlich der unabhängigen Variable, sondem zusätzlich hinsichtlich vieler weiterer Variablen bestehen können, in experimentellen Untersuchungen minimiert. Das der Randomisierung zugrunde liegende Prinzip des statistischen Fehlerausgleichs besagt, dass sich die Besonderheiten von Probanden in der einen Gruppe durch die Besonderheiten von Probanden in der anderen Gruppe ausgleichen. Die Äquivalenz beider Gruppen wird bei der Randomisienung statistisch erzielt, sodass als Ergebnis alle für die Untersuchung potentiell relevanten Variablen im Durchschnitt in beiden Gruppen annähernd gleich ausgeprägt sind, $d . h$. Gruppenunterschiede hinsichtlich der abhängigen Variable gehen mit hoher Wahrscheinlichkeit auf die unabhängige Variable zurück (Laatz 1993, S. 465; Stelzl 1999, S. 109; Bortz/Döring 2006, S. 54).

657 Den persönlichen Gesprächen mit Teilnehmern nach zu schließen, waren einige Probanden durchaus enttäuscht, ihre Entscheidungen in der Verhandlung nicht rechtfertigen zu können.

658 Scharlemann et al. (2001), S. 628

659 Vgl. Späth (2008), S. 150

660 Zur Wiederholbarkeit als zentrales Gütekriterium für Laboruntersuchungen vgl. Stein (1991), S. 109 
Das vorliegende Kapitel zum Design der Untersuchung der modellierten Wirkung von Kompetenz auf die Vertrauensentstehung schließt den Teil V mit einer Erläuterung der Qualität der erhobenen quantitativen Daten und einer anschlieBenden Beschreibung der statistischen Analysemethoden zur Hypothesenprüfung ab.

\subsection{Die Gütekriterien der Messung}

Die Qualität einer Messung lässt sich anhand von drei zentralen Kriterien Gütekriterien bestimmen: Objektivität, Reliabilität und Validität. Der Grad der Objektivität eines Messinstruments gibt an, in welchem Ausmaß die Ergebnisse unabhängig vom Versuchsleiter, der das Messinstrument einsetzt, sind. Vollständige Objektivität liegt demnach vor, wenn verschiedene Versuchsleiter mit demselben Messinstrument bei denselben Probanden zu den gleichen Ergebnissen gelangen. ${ }^{661}$ Die Forderung nach Objektivität richtet sich sowohl auf die Durchführung der Untersuchung, als auch auf die Auswertung der erhobenen Daten und die Interpretation der Ergebnisse. ${ }^{662}$ Um dem Anspruch nach einer hohen Durchführungsobjektivität im Rahmen der vorliegenden Untersuchung gerecht zu werden, erfolgte eine Standardisierung des Vorgehens, beginnend bei der Begrüßung der Probanden, über die Bearbeitungsanweisungen bis hin zur Verabschiedung. $\mathrm{Zu}-$ dem werden die individuellen Spielräume der Teilnehmer im Zuge der Bearbeitung der Aufgabenstellungen dadurch beschränkt, dass die Probanden anhand einheitlicher schriftlicher Instruktionen durch die Veranstaltung geleitet werden. Zur Wahrung der Auswertungs- und Interpretationsobjektivität umfassen die eingesetzten Ratingskalen ausschließlich Items mit Antwortvorgaben. Auch die Auswertung und Interpretation der Dokumente zur Erfassung der abhängigen Variable erfolgt nach einem standardisierten Verfahren und lässt keine individuellen Deutungen zu.

Die Reliabilität eines Messinstruments gibt den Grad der Genauigkeit (Präzision), mit dem ein geprüftes Merkmal gemessen wird, an ${ }^{663}$ Als Maß für die Reproduzierbarkeit von Messergebnissen liegt Reliabilität vor, wenn wiederholte Messungen unter denselben Umständen zu den gleichen Ergebnissen führen. ${ }^{64}$ Zur Berechnung der Zuverlässigkeit von Messinstrumenten können unterschiedliche Verfahren herangezogen werden, wie beispielsweise Test-Retest-, Paralleltest- und Split-Half-Methode. ${ }^{665}$ Eines der gängigsten Verfahren in diesem $\mathrm{Zu}$ -

661 Vgl. Diekmann (2007), S. $247 \mathrm{ff}$.

662 Vgl. Bortz/Döring (2006), S. 195

663 Vgl. Bortz/Döring (2006), S. 196

664 Vgl. Laatz (1993), S. 32; Diekmann (2007), S. 250

665 Für eine detaillierte Erlăuterung dieser Verfahren vgl. Bortz/Döring (2006) und Diekmann (2007). 
sammenhang ist die Prüfung der internen Konsistenz anhand von Cronbach's Alphakoeffizienten. Da die Berechnung der internen Konsistenz zu stabileren Reliabilitätsschätzungen als die Testhalbierungsmethode führt ${ }^{666}$, wird dieser Methoden im Rahmen der vorliegenden Untersuchung der Vorzug gegeben. Hinsichtlich der Relabilitätsanforderungen wird davon ausgegangen, dass sich eine zufriedenstellende Skala durch einen Reliabilitätskoeffizienten von 0,70 oder höher charakterisiert. ${ }^{667}$ Ein Alphakoeffizient von über 0,90 wird als hoch angesehen. ${ }^{668}$ Die nachfolgende Tabelle dokumentiert die Reliabilitätskoeffizienten der im Zuge der vorliegenden Untersuchung verwendeten psychometrischen Tests.

\begin{tabular}{|c|c|c|}
\hline Autor & Skala & Cronbachs Alpha \\
\hline Costa (2000) & personenspezifische Vertrauensbereitschaft & 0,934 \\
\hline Sitkin/Weingart (1995) & personenspezifische Risikobereitschaft & 0,861 \\
\hline Sitkin/Weingart (1995) & situationsspezifische Risikowahrnehmung & 0,796 \\
\hline
\end{tabular}

Tabelle 28: Reliabilitătskoefízienten der Skalen

Die geschätzten Reliabilitätskoeffizienten lassen auf eine hohe interne Konsistenz der drei psychometrischen Tests schließen. Ein herausragendes Alpha von 0,934 weist die Skala von Costa zur Messung der generellen Vertrauensbereitschaft auf. Auch impliziert das $\alpha$ für die Skala von Sitkin und Weingart zur Erfassung der personenspezifischen Risikobereitschaft eine hochgradige Reproduzierbarkeit der Messergebnisse. Die Reliabilität des Instruments von Sitkin und Weingart zur Messung der situationsspezifischen Risikowahrnehmung kann als zufriedenstellend eingestuft werden - besonders auch unter dem Aspekt, dass die Autoren in ihrer Studie eine Reliabilität von 0,75 erzielten. ${ }^{669}$ Wie nachfolgende Tabelle zeigt, würde auch eine Entfernung von Items ${ }^{670}$ zu keinem höheren $\alpha$ führen.

\begin{tabular}{|c|c|}
\hline $\begin{array}{c}\text { Items zur Erfass ung der } \\
\text { situativen Risikowahmehmung }\end{array}$ & $\begin{array}{c}\text { Cronbach's Alpha } \\
\text { bei Entfernung des Ite ms }\end{array}$ \\
\hline Item 1 & 0,741 \\
\hline Item 2 & 0,785 \\
\hline Item 3 & 0,720 \\
\hline Item 4 & 0,734 \\
\hline
\end{tabular}

Tabelle 29: Cronbach's Alpha bei der Entfernung von Items (situative Risikowahrnehmung)

Die Validität als drittes Gütekriterium bezeichnet die Gültigkeit einer Messung. Sie gibt an, ob ein Messinstrument das misst, was es messen soll bzw. was es zu

666 Vgl. Bortz/Döring (2006), S. 198

667 Vgl. Nunnally (1978), S. 245; Diekmann (2007), S. 253

668 Vgl. Weise (1975), S. 219

669 Vgl. Sitkin/Weingart (1995)

670 Für den genauen Wortlaut der Items vgl. Teil V Kap. 3.3 
messen vorgibt. ${ }^{671}$ Grundsätzlich kann zwischen drei Arten der Validität differenzieren werden: Inhaltsvalidität, Kriteriumsvalidität und Konstruktvalidität. Inhaltsvalidität bezieht sich auf die Vollständigkeit und Repräsentativität der Testitems zur Messung der zentralen Aspekte eines Konstrukts, d.h. ein Messinstrument ist inhaltsvalide, wenn die Auswahl von Items das zu messende Merkmal in hohem Grad repräsentiert. ${ }^{672}$ Die inhaltliche Validität gilt bei einem Rückgriff auf bereits erprobte Messinstrumente, wie in der vorliegenden Untersuchung, als gesichert. ${ }^{673}$ Die Kriteriumsvalidität gibt an, in welchem Grad die Ergebnisse eines Tests zur Messung eines theoretischen Konstrukts mit Messungen eines korrespondierenden manifesten Merkmals, d.h. einem Außenkriterium, übereinstimmen. ${ }^{674}$ Bei der Erfassung latenter Konstrukte, wie beispielsweise Vertrauen oder Kompetenz, gestaltet sich die Angabe einer beobachtbaren Kriteriumsvariable, die mit dem relevanten Merkmal in Beziehung steht und zudem reliabel und valide operationalisiert werden muss, jedoch als problematisch. ${ }^{675}$ Auf eine Korrelation zwischen den Testwerten und den Kriteriumswerten einer Stichprobe muss daher in der vorliegenden Untersuchung verzichtet werden. Die dritte Validität, die Konstruktvalidität eines Messinstruments, wird durch Formulierung eines Netzes „von Hypothesen über das Konstrukt und seine Relationen zu anderen manifesten und latenten Variablen “676 überprüft. Je mehr der aus dem zu messenden Zielkonstrukt abgeleiteten Hypothesen anhand der Testwerte bestätigt werden können, desto überzeugender ist die Konstruktvalidierung. ${ }^{677}$ Ihre Prüfung stellt einen komplexen Prozess dar, der nach einer Vielzahl an Verfahren, Techniken und methodischen Gültigkeitstests verlangt und damit über den Rahmen dieser Arbeit hinausgeht. ${ }^{678}$ Abschließend ist darauf hinzuweisen, dass die Validität in der Vertrauensforschung das am schwierigsten $\mathrm{zu}$ prüfende Gütekriterium und ein Kardinalproblem darstellt. ${ }^{679}$ Aufgrunddessen, dass die empirische Vertrauensforschung noch relativ jung ist, ist die Anwendung üblicher Methoden, wie beispielsweise die Korrelation mit bereits anerkannten Messinstrumenten, zur Prüfung der Validität erschwert. ${ }^{680}$

Vgl. Bortz/Döring (2006), S. 200. Dieses testtheoretische Kriterium der Validität, das Auskunft über die Qualität des Messinstruments gibt, ist von den Kriterien der internen und externen Validität zu differenzieren (vgl. dazu Teil V Kap. 1.1).

Vgl. Diekmann (2007), S. 258

Vgl. Bortz/Döring (2006), S. 200 ff.; Späth (2008), S. 154

Vgl. Diekmann (2007), S. 258

Vgl. Späth (2008), S. 154; Zimbardo/Gerrig (2008), S. 329

Bortz/Döring (2006), S. 201

Vgl. Bortz/Döring (2006), S. 201

Für eine schrittweise Erklärung und praktische Umsetzung der Konstruktvalidierung des BTI (siehe Teil III Kap. 7) vgl. Gillespie (2003), S. 13-34.

679 Vgl. Schweer/Thies (2003), S. 30

680 Vgl. Lewicki et al. (2006); Späth (2008), S. 154 


\subsection{Die statistischen Analysemethoden}

\subsubsection{Die statistische Modellierung}

Die Erfassung und Auswertung der Daten erfolgt mit dem Statistikprogramm SPSS für Windows in der Version 16.0. Im Folgenden wird auf die Analysemethoden, die im Zuge der Hypothesenprüfung eingesetzt werden, eingegangen. Besonderes Augenmerk wird hierbei auf die Interpretation der generierten Werte gelegt.

Die Zufallsabhängigkeit der Ergebnisse statistischer Analysen wird anhand von Signifikanztests beurteilt, die formal zwei sich ausschließende Hypothesen überprüfen: In der Nullhypothese $\left(\mathrm{H}_{0}\right)$ wird behauptet, dass es keinen Zusammenhang zwischen den untersuchten Merkmalen in der Population, der die Stichprobe entnommen wurde, gibt. Als Ausgangspunkt aller inferenzstatistischen Analysen wird diese $\mathrm{H}_{0}$ als richtig unterstellt. Die entsprechende Gegenbehauptung liefert die Alternativhypothese $\left(\mathrm{H}_{\mathrm{A}}\right)$, die zwischen den untersuchten Merkmalen einen Zusammenhang von ungleich 0 postuliert. ${ }^{681}$ Nach dem indirekten Prinzip des Falsifikationismus ist es nicht möglich, eine Hypothese empirisch zu verifizieren. Sie kann nur widerlegt werden. Aus diesem Grund wird versucht, die Falschheit von $\mathrm{H}_{0}$ anstelle der Richtigkeit von $\mathrm{H}_{\mathrm{A}}$ nachzuweisen. Die Wahrscheinlichkeit, mit der eine richtige Nullhypothese fälschlicherweise zurückgewiesen wird, wird als Irrtumswahrscheinlichkeit bezeichnet. Das Ausmaß dieser Irrtumswahrscheinlichkeit wird durch das Signifikanzniveau eines Tests indiziert. Wird beispielsweise ein Signifikanzniveau von $\alpha=0,05$ angesetzt, so bedeutet das, dass die richtige Nullhypothese in $5 \%$ aller (hypothetisch unterstellten) Schätzungen fälschlicherweise zugunsten der Annahme der Alternativhypothese verworfen wird. In der vorliegenden Untersuchung werden als Schwellenwerte für die Irrtumswahrscheinlichkeit geschätzter Koeffizienten $\alpha<0,05$ (signifikant), $\alpha<0,01$ (hoch signifikant) und $\alpha<0,001$ (höchst signifikant) festgelegt, die in der Forschung allgemein üblich sind. ${ }^{682}$ Allerdings handelt es sich bei signifikanten Ergebnissen bloß um „Aussagen darüber, dass es eine akzeptable Wahrscheinlichkeit für die Nicht-Zufälligkeit statistisch produzierter Zusammenhänge gibt.“683

Die im Rahmen der Modellierung des Kompetenzeinflusses auf die Vertrauensentstehung aufgestellten Hypothesen werden anhand klassischer Regressionsanalysen mit der Kleinst-Quadrat-Schätzmethode (OLS-Regression) geprüft. „Deren Anwendung ist vor allem dann angemessen, wenn (lineare und linearisierbare) Effekte zwischen mindestens zwei Variablen vermutet werden, und die Werte der abhängigen Variablen zumindest annäherungsweise kontinuierlich ver-

681 Vgl. Bortz/Döring (2006), S. 492

682 Vgl. Urban/Mayerl (2006), S. $134 \mathrm{f}$.

683 Urban/Mayerl (2006), S. 137 
teilt sind.“684 Grundsätzlich sollten sowohl die abhängige, als auch die unabhängigen Variablen ein metrisches Messniveau aufweisen. ${ }^{685}$ Allerdings hat sich in der Forschungspraxis gezeigt, dass auch dichotom gemessene unabhängige Variablen, d.h. jene Variablen, die ein binomiales Messniveau aufweisen ${ }^{686}$, in die OLS-Regression einbezogen werden können. ${ }^{687}$

Ausgangspunkt der Regressionsanalyse ist die Transformation eines theoretischen Modells in ein statistisches Modell, im Zuge derer die Modellgrößen mit einer mathematisch formulierten Modelltechnik geschätzt werden. ${ }^{688}$ Bei multivariaten oder multiplen Modellen, die sich durch mehrere unabhängige Variablen charakterisieren, erfolgt diese Umwandlung indem für jede erklärende Variable ein Regressionskoeffizient geschätzt wird, der deren Einflussstärke unter Kontrolle im Sinne eines statistisch hergestellten Nicht-Einflusses aller anderen im Modell berücksichtigen unabhängigen Variablen ausdrückt. ${ }^{689}$ Die Güte der Anpassung des Modells an die Daten lässt sich anhand des Bestimmtheitsmaßes $\mathbf{R}^{2}$ eruieren, das angibt, wieviel Prozent der Varianz durch das Modell erklärt wird. ${ }^{690}$ Im Extremfall, wenn die gesamte Streuung erklärt wird, d.h. wenn ein perfekter Zusammenhang zwischen der abhängigen und den unabhängigen Variablen besteht, nimmt $\mathrm{R}^{2}$ einen Wert von 1 an. Je kleiner $\mathrm{R}^{2}$ jedoch ist, desto schlechter ist auch die Anpassung der Regressionsgeraden an die beobachteten Werte. ${ }^{691}$ Da die Höhe des Bestimmtheitsmaßes mit der Zahl der Regressoren monoton zunimmt, jedoch nach dem Prinzip der Parsimonität ein hoher Erklärungsgrad mit möglichst wenig erklärenden Variablen erzielt werden sollte, verfügt $\mathbf{R}^{2}$ korr über weitaus mehr Aussagekraft im Zuge der Beurteilung des Erklärungsgrads des Modells: Durch Korrektur des einfachen Bestimmtheitsmaßes unter Berücksichtigung der Anzahl der Regressoren, kann $\mathrm{R}^{2}$ kor im Gegensatz zu $\mathrm{R}^{2}$ mit der Aufnahme weiterer Regressoren nicht zunehmen. ${ }^{692}$ Zur Überprüfung der Abhängigkeit der Gesamtschätzung eines Regressionsmodells von zufälligen Verzerrungen, d.h. der nicht

684

685

686

687

688

689

690

691

692

Urban/Mayerl (2006), S. 12

Vgl. Backhaus (2003), S. 9. Die Erfassung von Merkmalsausprägungen anhand von Rating-Skalen wird im Allgemeinen als eine Messung auf metrischem Niveau angesehen (vgl. Backhaus (2003), S. 5).

In der vorliegenden Untersuchung handelt es sich bei der Variable ,Kompetenzwahrnehmung ${ }^{\text {um }}$ eine Dummy-Variable, die nur die Werte 0 (keine Kompetenzwahrnehmung; dieser Wert gilt für die Kontrollgruppe) und 1 (Kompetenzwahrnehmung; dieser Wert gilt für die Versuchsgruppe 1 [hohe Fach- und Methodenkompetenzwahmehmung] und die Versuchsgruppe 2 [hohe Sozial- und Persönlichkeitskompetenzwahrnehmung]) annehmen kann.

Vgl. Backhaus et al. (2003), S. 9

Vgl. Urban/Mayerl (2006), S. 16

Vgl. Schendera (2008), S. 102

Vgl. Cohen et al. (2003), S. 70

Vgl. Fahrmeir et al. (2009). Urban und Mayerl (2006, S. 56) halten fest: „In der sozialwissenschaftlichen Forschungspraxis ,erklärt“ X [die abhängige Variable, Anm. d. Verf.] nur einen gewissen Anteil der Varianz, vielleicht $40 \%$ oder auch nur $10 \%$.“

Vgl. Backhaus et al. (2003), S. 67 f. 
durch Zufall bestimmten Erklärungsleistung des Regressionsmodells, wird als Prüfgröße der F-Wert bestimmt. Die empirische Irrtumswahrscheinlichkeit „sollte kleiner oder gleich 0,05 sein, um bei einem Signifikanzniveau von $5 \%$ von einer „signifikanten“ Varianzausschöpfung des Gesamtmodells sprechen zu können."693

Zusätzlich zum Erklärungsgrad des Gesamtmodells interessiert in der vorliegenden Untersuchung der Effekt der Änderung einer unabhängigen Variable auf die abhängige Variable. Die Richtung (positiv oder negativ) und die Stärke des Einflusses dieses Regressors auf die abhängige Variable wird durch den Regressionskoeffizienten beschrieben. Im Gegensatz zu den Ergebnissen der unstandardisierten Regressionsschätzung sind die standardisierten Regressionskoeffizienten unabhängig von linearen Transformationen der entsprechenden Messskala, wodurch ein Vergleich der Werte verschiedener Regressionskoeffizienten möglich wird. Diese Beta-Werte bezeichnen also das Ausmaß der Veränderung der abhängigen Variable auf einer Standardskala (Mittelwert $=0$, Standardabweichung $=1$ ), wobei diese Veränderung dadurch ausgelöst wird, dass der Wert der dazugehörigen unabhängigen Variable um eine Standardeinheit ansteigt während gleichzeitig alle anderen erklärenden Variablen im Regressionsmodell auf statistische Weise konstant gehalten werden (= Auspartialisierung von X-Anteilen $^{694}$ ). Da es in multiplen Regressionsmodellen wahrscheinlich ist, dass sich die Regressoren wechselseitig beeinflussen, werden durch dieses Verfahren der Bereinigung einer unabhängigen Variable um die Effekte weiterer unabhängiger Variablen partielle, standardisierte Regressionskoeffizienten generiert. Durch Standardisierung der Regressionskoeffizienten werden die Ergebnisse der unstandardisierte Regressionsschätzung allerdings nicht überflüssig: Aufgrund ihrer Varianzabhängigkeit sind die standardisierten Koeffizienten nicht dazu geeignet, Variableneffekte zwischen mehreren Modellen zu vergleichen. Um dem hohen Risiko einer Fehlinterpretation zu entgehen, werden daher in der vorliegenden Arbeit beim Vergleich der Einflüsse unabhängiger Variablen auf die abhängige Variable innerhalb eines Modells stets standardisierte und zwischen mehreren Modellen stets unstandardisierte Koeffizienten interpretiert. ${ }^{695}$

Die Überprüfung der Regressionskoeffizienten erfolgt anhand der t-Statistik, die dem Test der Nullhypothese, dass ein bestimmter Koeffizient $\beta_{i}$ der Grundgesamtheit gleich 0 ist, d.h. die entsprechende unabhängige Variable keinen Beitrag zur Erklärung der abhängigen Variable leistet, dient. Zur Überprüfung der Signifikanz des empirischen $t$-Wertes werden die per Konvention festgelegten und bereits dargelegten Vertrauenswahrscheinlichkeiten von $90 \%$, $95 \%$ und $99 \%$

693 Vgl. Urban/Mayerl (2006), S. 173

694 Vgl. Cohen et al. (2003), S. 80

695 Vgl. Backhaus et al. (2003), S. 61 f.; Urban/Mayerl (2006), S. $103 \mathrm{ff}$. 
herangezogen. ${ }^{696}$ Die Nullhypothese wird verworfen, wenn der empirische t-Wert absolut größer als das $\alpha / 2$-Quantil der Student t-Verteilung bei n-k-1 Freiheitsgraden ist.

Zusammenfassend lässt sich festhalten, die Regressionsanalyse „yields measures of the magnitude of the total effect of a factor on the dependent variable [...] is a major tool in the methods of causal (path, structural, equation) analysis [...] is a versatile, all-purpose system of analyzing data over a wide range of sciences and technologies." ${ }^{697}$ Abschließend ist darauf hinzuweisen, dass die Ergebnisse einer Regressionsanalyse nicht als 'richtig' oder 'falsch' deklariert werden können, sie sind allein angemessen oder unangemessen. ${ }^{698}$

\subsubsection{Die Prüfung der Modellprämissen}

Die Beurteilung der Güte der Schätzung für die Regressionsparameter, sowie der Anwendbarkeit der Tests zur Überprüfung der Güte, und damit der Eignung eines Regressionsmodells beruhen auf gewissen Annahmen betreffend der Residuen: 1) die Residuen (= Differenz zwischen dem beobachteten Wert der abhängigen Variable und dem dazugehörigen vorhergesagten Wert auf der Regressionsgerade) sind für jedes $t$ normalverteilt mit Erwartungswert 0 und konstanter Varianz $\sigma^{2}$ (keine Heteroskedastizität), 2) die Residuen und die Regressoren sind unkorreliert (keine fehlenden erklärenden Variablen), 3) die Residuen sind unkorreliert (keine Autokorrelation), und 4) zwischen den Regressoren besteht keine lineare Abhängigkeit (keine perfekte Multikollinearität). Wenn diese Annahmen erfüllt sind, dann liefert die Kleinst-Quadrat-Methode (KQ) lineare Schätzfunktionen für die Regressionskoeffizienten, die alle wünschenswerten Eigenschaften von Schätzern besitzen, d.h. sie sind unverzerrt (erwartungstreu), effizient (kleinstmögliche Varianz unter allen linearen und unverzerrten Schätzern) sowie konsistent, und damit BLUE (Best Linear Unbiased Estimators). ${ }^{699}$

Alle im Zuge der vorliegenden Arbeit geschätzten Regressionsmodelle (vgl. Teil VI) wurden auf Prämissenverstöße geprüft. Im Folgenden wird diese Überprüfung exemplarisch anhand zweier Modelle erläutert (Modell A-II: abhängige Variable ,Vertrauenshandlung', unabhängige Variable ,Kompetenzwahrnehmung' (DummyVariable, wobei hohe Fach- und Methodenkompetenzwahrnehmung mit , 1' und konstante Kompetenzwahrnehmung mit , $0^{\star}$ kodiert ist), Kontrollvariablen ,Vertrauensbereitschaft', ,Risikobereitschaft' und ,situative Risikowahrnehmung'; Modell B-II: abhängige Variable ,Vertrauenshandlung،, unabhängige Variable ,Kompetenzwahrnehmung' (Dummy-Variable, wobei hohe Sozial- und Persönlichkeits-

696 Vgl. Backhaus et al. (2003), S. $73 \mathrm{ff}$.; Cohen et al. (2003), S. $47 \mathrm{ff}$.

697 Cohen et al. (2003), S. 2

698 Vgl. Urban/Mayerl (2006), S. 21

699 Vgl. dazu z. B. Backhaus et al. (2003), S. $78 \mathrm{f}$. 
kompetenzwahrnehmung mit , $1^{\text {' }}$ und konstante Kompetenzwahrnehmung mit , 0 “ kodiert ist), Kontrollvariablen ,Vertrauensbereitschaft', ,Risikobereitschaft" und ,situative Risikowahrnehmung'). Die Erläuterung der Ergebnisse der multiplen Regressionsanalysen zur Hypothesenprüfung, inklusive der tabellarischen Darstellung der standardisierten und unstandardisierten Regressionskoeffizienten, des $\mathrm{R}^{2}$ und $\mathrm{R}^{2}$ korr, sowie des F-Werts des jeweiligen Modells, erfolgt in Teil VI.

Zunächst erfolgt der Test auf Normalverteilung der Residuen (A1), im Rahmen dessen die Häufigkeitsverteilung der aufgetretenen Residuen einer Normalverteilung gegenübergestellt wird: Liegt keine Normalverteilung vor, dann sind die KQSchätzwerte zwar unverzerrt und konsistent, aber nicht effizient, d.h. es gibt andere Schätzfunktionen mit größerer Präzision. ${ }^{700}$ Außerdem besteht dann die Möglichkeit, dass die t-Statistiken für die Signifikanzbeurteilungen nicht mehr adäquat sind. ${ }^{701}$ Gründe hierfür können in einer Fehlspezifikation des Modells liegen (z.B. fehlende unabhängige Variablen). Die Histogramme lassen darauf schließen, dass die standardisierten Residuen der abhängigen Variable der eingezeichneten Normalverteilung folgen. Die P-P-Diagramme zeigen, dass die standardisierten Residuen der abhängigen Variable annähernd ${ }^{702}$ auf der eingezeichneten Linie der Referenzverteilung (Normalverteilung) liegen. Damit kann das Vorliegen einer Normalverteilung der standardisierten Residuen als Voraussetzung einer linearen Regression für Modell A-II und B-II (sowie für alle im Rahmen dieser Arbeit durchgeführten Regressionsanalysen) als erfüllt betrachtet werden.

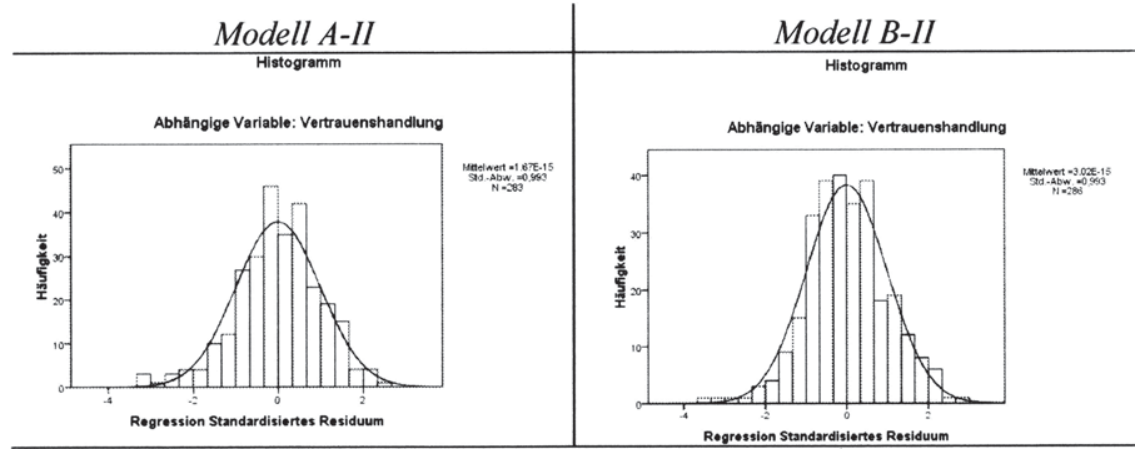

$700 \quad$ Vgl. Kmenta (1971), S. 248

701 Vgl. Backhaus (2003), S. 92

702 Geringfügige Abweichungen der Häufigkeitsverteilung der Residuen von der Normalverteilung sind nicht ungewöhnlich und durchaus zu tolerieren. Eine vollkommene Übereinstimmung der beiden Verteilungen wäre demnach ein großer Zufall, der in der Praxis selten eintritt (Brosius 2002, S. 558). 

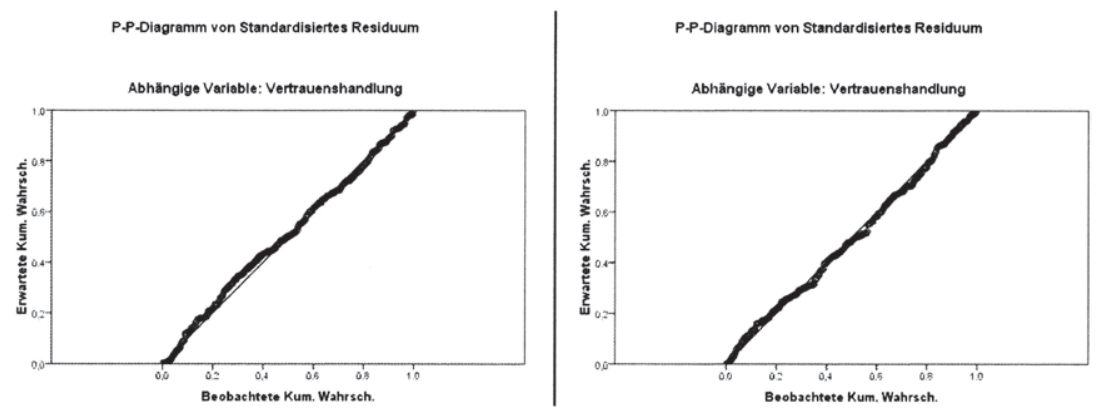

Abbildung 16: Die Prüfung der Normalverteilung der Residuen

Heteroskedastizität (A1) heißt, dass die Streuung von $Y_{t}$ um den Erwartungswert $E\left[Y_{t} \mid X_{t}\right]$ nicht für jedes $X_{t}$ gleich ist, da die Varianz der Residuen $\epsilon_{t}$ nicht konstant ist. Wenn Heteroskedastizität gegeben ist, dann sind die KQ-Schätzwerte nicht effizient, aber unverzerrt und konsistent. ${ }^{703}$ Die Standardfehler der geschätzten Regressionskoeffizienten ( $\mathrm{s}_{\mathrm{bi}}$ sind hingegen verzerrt. Diese Verzerrung ist negativ, wenn die Varianz der Residuen $e_{t}$ und die unabhängige Variable $x_{t}$ positiv korrelieren. In diesem Fall sind die Standardfehler zu klein und die tWerte zu groß. Dies hat zur Folge, dass $\mathrm{H}_{0}$ bei gegebenem Signifikanzniveau zu oft verworfen wird und damit Koeffizienten fälschlicherweise als signifikant beurteilt werden. Gründe hierfür können in Fehlspezifikationen des Regressionsmodells liegen. ${ }^{704}$ Eine graphische Methoden zur Aufdeckung von Heteroskedastizität besteht beispielsweise darin, die standardisierten Residuen der abhängigen Variable gegen die prognostizierten (geschätzten) Werte der abhängigen Variable aufzutragen. ${ }^{705}$ Eine Änderung der Streuung der Residuen mit den vorhergesagten Werten der abhängigen Variable weist auf Heteroskedastizität hin. Genauer wäre Varianzungleichheit in den folgenden Diagrammen daran zu erkennen, dass sich die Werte etwa in Gestalt einer von links nach rechts sich öffnenden Schere bzw. trichterförmig verteilen, wobei mit zunehmenden vorhergesagten Werten der Streubereich der Residuen immer größer wird. ${ }^{706} \mathrm{Die}$ Ergebnisse der visuellen Inspektion der Residuen lassen aber darauf schließen, dass die Werte ausgewogen und zufällig streuen, d.h. dass sich die Werte gleichmäßig über und unter der Nulllinie verteilen. Damit kann das Vorliegen homoskedastischer Residuen als Voraussetzung einer linearen Regression für Modell A-II und B-II (sowie für alle im Rahmen dieser Arbeit durchgeführten Regressionsanalysen) als erfüllt ${ }^{707}$ betrachtet werden.

703 Vgl. Kmenta (1971), S. 250

704 Vgl. Geyer (2008), S. 28 ff.

705 Vgl. Backhaus et al. (2003), S. 85; Schendera (2008), S. 53

$706 \mathrm{Vgl}$. Urban/Mayerl (2006), S. $244 \mathrm{ff}$.

707 Ein eindeutiges Vorliegen von Homoskedastizität kann durch Anwendung hochkomplexer, statistischer Tests (z.B. White-Test, Goldfeld/Quandt-Test) gewährleistet werden. Da Hetero- 


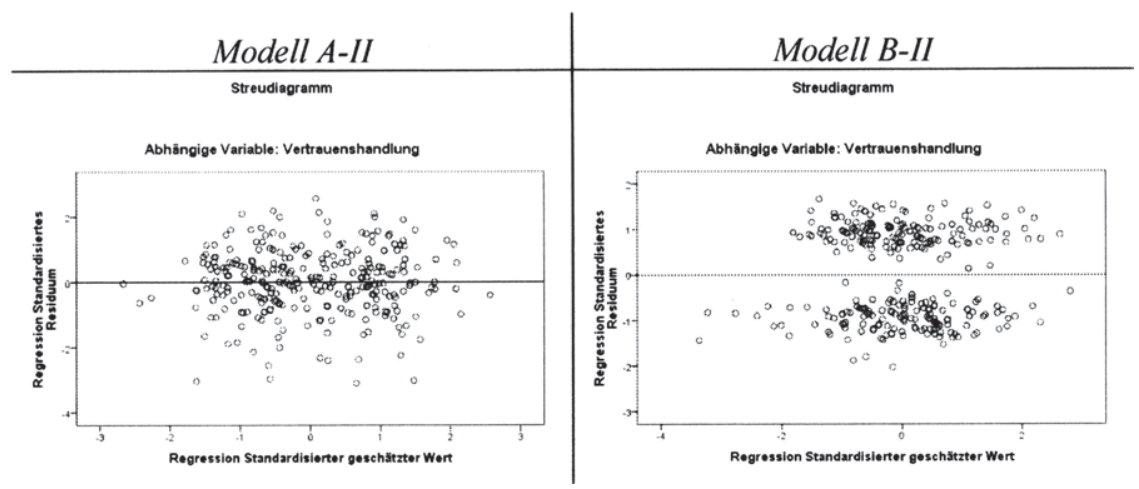

Abbildung 17: Die Prüfung der Homoskedastizităt der Residuen

Die Annahme der Unabhängigkeit zwischen Regressoren und Residuen ist von Relevanz für die Eigenschaften der KQ-Schätzung (A2). Korrelation zwischen unabhängigen Variablen und Residuen resultieren nämlich in verzerrten und inkonsistenten Schätzwerten. Gründe hierfür können beispielsweise fehlende erklärende Variablen, aber auch Messfehler in den Daten sein. ${ }^{708}$ Das schwer zu erkennende Problem fehlender erklärender Variablen (underfitting) kann durch die auf sachlichen Überlegungen basierende Aufnahme zusätzlicher Regressoren in das Modell gelöst werden. In diesem Zusammenhang ist jedoch darauf hinzuweisen, dass die Berücksichtigung irrelevanter Variablen (overfitting) im Modell (,kitchen sink models') zwar zu unverzerrten und konsistenten, aber ineffizienten Schätzwerten führt. ${ }^{709} \mathrm{Da}$ die Korrelationen zwischen den Residuen der KQ-Regression und den Regressoren aufgrund der Eigenschaften der KQ-Schätzung immer gleich 0 sind, können sie nicht dazu verwendet werden, um die Annahme der Unabhängigkeit zwischen den Residuen und den Regressoren zu bestätigen. ${ }^{710}$ $\mathrm{Da}$ die in Teil VI erläuterten Modelle aber keine widersprüchlichen Ergebnisse (z.B. ein signifikanter Koeffizient hat ein anderes Vorzeichen als angenommen) liefern, wodurch impliziert wird, dass vor der statistischen Analyse kausaler $\mathrm{Zu}$ sammenhänge theoretische Überlegungen hinsichtlich der Anzahl und Wirkung der einbezogenen erklärenden Variablen angestellt wurden ${ }^{711}$, wird angenommen, dass die Residuen und die Regressoren aller im Rahmen dieser Arbeit generierten Regressionsmodelle unkorreliert sind.

skedastizität meist aber ein Problem von Nichtlinearität ist (Backhaus et al. 2006, S. 87), die entsprechend der vorliegenden Ergebnisse der Prüfung der Annahmen nicht vorliegt, wird auf den Einsatz statistischer Tests zur Aufdeckung von Heteroskedastizität im Rahmen der vorliegenden Arbeit verzichtet.

$708 \quad$ Vgl. Thomas (1997), S. 211; Geyer (2008), S. 32

709 Vgl. Backhaus et al. (2003), S. 84

$710 \quad$ Vgl. Geyer (2008), S. 33

711 Vgl. Backhaus et al. (2003), S. 84 
Das lineare Regressionsmodell basiert auch auf der Annahme, dass die Residuen in der Grundgesamtheit unkorreliert sind (A3). Liegt hingegen Autokorrelation vor, dann sind die KQ-Schätzwerte zwar unverzerrt und konsistent, jedoch nicht effizient. ${ }^{712}$ Weiters sind die Standardfehler der Regressionskoeffizenten verzerrt (z.B. zu klein bei positiver Autokorrelation) und lassen zu häufig darauf schließen lässt, dass signifikante Abhängigkeiten ${ }^{713}$ vorliegen (,spurious regression prob$l^{l e m}{ }^{، 714}$ ). Die Prüfung der Residuen auf Autokorrelation erster Ordnung, d.h. der Unabhängigkeit eines Residuums vom direkten Vorgänger innerhalb einer Wertereihe, erfolgt in der vorliegenden Arbeit anhand des Durbin-Watson-Tests. ${ }^{715}$ Hinsichtlich der zu berechnenden Durbin-Watson-Koeffizienten lässt sich festhalten, dass ihr Wertbereich zwischen 0 und 4 liegt. Je näher der Koeffizient an dem Wert 2 ist, desto geringer ist das Ausmaß der Autokorrelation der Residuen. Ist der Koeffizient allerdings deutlich kleiner (bzw. größer) als 2, so ist von einer positiven (bzw. negativen) Autokorrelation auszugehen. Als Faustregel gilt, dass bei Werten zwischen 1,5 und 2,5 der Schluss der vernachlässigbaren Autokorrelation zulässig ist. ${ }^{716}$ Wird diese Faustregel wegen Ungenauigkeit außer Acht gelassen, so erlauben die ermittelten Werte von z.B. 1,551 für Modell A-II und 1,579 für Modell B-II (ähnliche Werte für alle anderen im Rahmen dieser Arbeit generierten Modelle) keinen direkten Schluss auf die Signifikanz der Unabhängigkeit der Residuen. Erst anhand von Durbin-Watson Tabellenbänden (z.B. näherungsweise $\mathrm{T}=430, \mathrm{~K}=4$, Alpha $=0,05$ ) kann festgestellt werden, dass die ermittelten Werte außerhalb des unteren Indifferenzbereichs $\left(\mathrm{L}_{U}=1,82767, \mathrm{~L}_{O}=1,85576\right)$ liegen und damit auf die Signifikanz positiver Autokorrelation hinweisen. Dementsprechend ist die Unabhängigkeit der Residuen nicht gewährleistet, womit Autokorrelation nicht ausgeschlossen werden kann. Die explorativ eingesetzte Pearson-Korrelation des Ausmaßes der direkten (zeitlichen, räumlichen) Versetzung der Residuen in der Datenreihe mit den Residuen ${ }^{717}$ zeigt jedoch, dass zwar der Zusammenhang signifikant ist, jedoch vom Betrag her marginal (Modell A-II: 0,216; Modell B-II: $0,211)$ und auf die Größe der Stichprobe $(\mathrm{N}=427)$ zurückgeführt werden kann. Auch bei der graphischen Darstellung der standardisierten Residuen und des ermittelten Ausmaßes der versetzten Abhängigkeit in einem Streudiagramm zeigt sich kein linearer Zusammenhang im Sinne einer Abhängigkeit der Residuen von den direkt (zeitlich, räumlich) vorangehenden Werten, womit die ermittelte Signifikanz des Durbin-Watson-Tests vor allem durch die Größe der Stichprobe verursacht wurde. Von Autokorrelation im Sinne einer (zeitlich, räumlichen) Abhängigkeit der Residuen von den direkt vorangehenden Werten ist demnach nicht

\footnotetext{
712 Vgl. Geyer (2008), S. 35

713 Vgl. Kmenta (1971), S. 273

714 Vgl. dazu z.B. Granger/Newbold (1974)

715 Vgl. Backhaus et al. (2003), S. 88

716 Vgl. Brosius (2002), S. 559

$717 \mathrm{Vgl}$. zur Eignung dieses Vorgehens z.B. Schendera (2008), S. 56 f.
} 
auszugehen, weshalb das Vorliegen von White-Noise als Voraussetzung einer linearen Regression für Modell A-II und B-II (sowie für alle im Rahmen dieser Arbeit durchgeführten Regressionsanalysen) als erfüllt betrachtet werden.

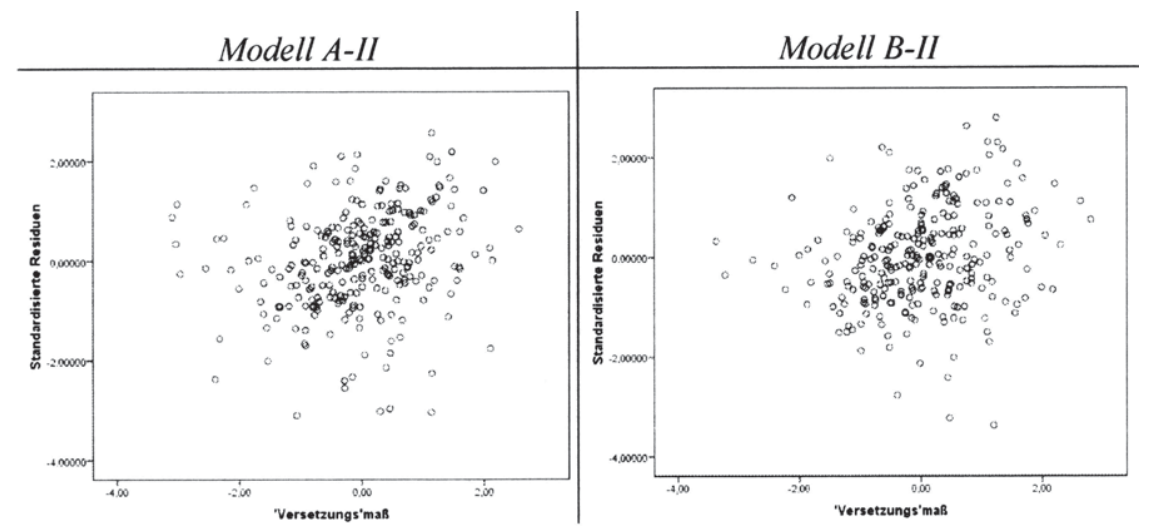

Abbildung 18: Die Prüfung der Unabhängigkeit der Residuen

Das lineare Regressionsmodell basiert auch auf der Prämisse, dass die erklärenden Variablen nicht exakt linear abhängig sind (A4). Als Folge von Fehlspezifikationen liegt perfekte Multikollinearität vor, wenn sich ein Regressor als lineare Funktion der übrigen Regressoren darstellen lässt. Grundsätzlich besteht bei empirischen Daten immer ein gewisser Grad an Multikollinearität. Eine zu starke Korrelation zwischen den erklärenden Variablen ist aber problematisch, da mit zunehmender Multikollinearität die Schätzungen der Regressionskoeffizienten unzuverlässiger werden. Die sich überschneidenden Streuungen der unabhängigen Variablen bedeuten einerseits Redundanz in den Daten und damit weniger Informationsgehalt und andererseits, dass sich die vorhandenen Informationen nicht mehr eindeutig den Variablen zuordnen lassen. ${ }^{718}$ Zur Diagnose der Multikollinearität wird in der vorliegenden Arbeit der Variance Inflation Factor (VIF) und die Tolerance berechnet. Besteht kein linearer Zusammenhang zwischen den erklärenden Variablen, d.h. sind alle Variablen orthogonal zueinander, ist VIF gleich 1. VIF größer als 10 sind in der Regel als Hinweise auf Multikollinearität zu verstehen. Tolerance, der direkte Kehrwert von VIF, ist als der Varianzanteil einer Variable zu verstehen, der nicht durch die anderen Variablen im Modell erklärt wird. ${ }^{719}$ Die Ergebnisse der Berechnung von VIF und Tolerance zeigen, dass alle VIF der Prädiktoren in Modell A-II und B-II, sowie in den weiteren im Zuge dieser Arbeit generierten Modellen weit unter 10 liegen. Damit kann das Vorliegen von nicht zu stark korrelierten Regressoren als Voraussetzung einer line-

718 Vgl. Backhaus et al. (2003), S. $88 \mathrm{f}$.

719 Vgl. Schendera (2008), S. 105 
aren Regression für Modell A-II und B-II (sowie für alle im Rahmen dieser Arbeit durchgeführten Regressionsanalysen) als erfüllt betrachtet werden.

\begin{tabular}{|l|c|c|c|c|}
\cline { 2 - 5 } \multicolumn{1}{c|}{} & \multicolumn{2}{c|}{ Modell A-II } & \multicolumn{2}{c|}{ Modell B-II } \\
\cline { 2 - 5 } \multicolumn{1}{c|}{} & Toleranz & VIF & Toleranz & VIF \\
\hline Kompetenz & 0,964 & 1,037 & 0,995 & 1,005 \\
\hline Vertrauensbereitschaft & 0,934 & 1,070 & 0,953 & 1,049 \\
\hline Risikobereitschaft & 0,860 & 1,163 & 0,912 & 1,096 \\
\hline Risikowahrnehmung & 0,884 & 1,132 & 0,951 & 1,051 \\
\hline
\end{tabular}

Tabelle 30: Die Prüfung auf Multikollinearität

Zusammenfassend lässt sich festhalten, dass alle im Zuge dieser Arbeit generierten Regressionsmodelle die Annahmen 1-4 erfüllen, sodass die KQ-Methode lineare Schätzfunktionen für die Regressionsparameter liefert, die alle wünschenswerten Eigenschaften von Schätzern besitzen, d.h. sie sind unverzerrt, effizient und konstant und damit BLUE, und inferenzstatistische Schlüsse ermöglicht. 


\section{TEIL VI: ERGEBNISSE DER UNTERSUCHUNG VON KOMPETENZ ALS VERTRAUENSDETERMINANTE}

Nach der Konzeption der Untersuchung zur Überprüfung der hypothetisierten Kausalbeziehung zwischen den Dimensionen ,individuelle Kompetenzen' und ,Vertrauenshandlung' erfolgt in diesem Teil die Erläuterung der Untersuchungsergebnisse. Im Anschluss an die Evaluierung der Eignung der Fallsimulation zur Untersuchung der hypothetisierten Kausalbeziehung (Kap. 1.1) wird die Manipulationswirkung der unabhängigen Variable kontrolliert, indem die den Gruppen VG1 FMK, VG2 SPK und KG angehörigen Probanden auf Unterschiede hinsichtlich ihrer Dispositionen und ihrer Risikowahrnehmung getestet werden (Kap. 1.2). Die Ergebnisse der regressionsanalytischen Prüfung der in Teil IV Kap. 5 abgeleiteten Hypothesen, sowie hinsichtlich der Wirkung der vertrauensgeberund situationsspezifischen Variablen (Kap. 2) werden abschließend anhand eines empirisch bestätigten Modells dargestellt (Kap. 3).

\section{DiE PRÜFUNG DER METHODISCHEN EFFEKTIVITÄT DES EXPERIMENTS}

Vor dem Einsatz regressionsanalytischer Verfahren muss geprüft werden, ob der Versuchsaufbau eine Testung der hypothetisierten Kausalbeziehung zwischen Kompetenz und Vertrauen ermöglicht. Anhand der Ergebnisse der Bearbeitung der zwischenbetrieblichen Entscheidungsaufgaben wird ermittelt, ob aufgrund der experimentellen Manipulation der unabhängigen Variable Differenzen in den Werten zwischen den Versuchsgruppen und der Kontrollgruppe ${ }^{720}$ bestehen. Anschließend erfolgt eine Analyse der Effekte dieses manipulativen Eingriffs hinsichtlich der Dispositionen und der Risikowahrnehmung der Probanden, um eine mögliche Einschränkung der Gültigkeit der Ergebnisse zu vermeiden.

\subsection{Die Eignung der Fallsimulation}

Im Folgenden wird geprüft, ob sich die Fallsimulation dazu eignet, messbare Reaktionen der Probanden zu erzeugen. Im Zentrum des Interesses stehen hierbei die offene und ehrliche Informationsabgabe und die Kontrollintensität, sowie die Strategiewahl als Elemente der Fallsimulation.

720 VG1_FMK erhält eine Situationsbeschreibung, die auf ausgeprägte Fach- und Methodenkompetenzen (FMK) des Rotweinproduzenten schließen lässt, während die KG eine Situationsbeschreibung, die durchschnittliche Fach-, Methoden-, Sozial- und Persönlichkeitskompetenzen des Rotweinproduzenten impliziert, bekommt. VG2_SPK erhält eine Situationsbeschreibung, die darüber Auskunft gibt, dass der Rotweinproduzent über ausgeprägte Sozial- und Persönlichkeitskompetenzen (SPK) verfügt. 
Vertrauen wird in Situationen relevant, die durch Unsicherheit im Hinblick auf das und durch Abhängigkeit vom Verhalten des Vertrauensnehmers gekennzeichnet sind. Im Rahmen der Fallsimulation wurde den Probanden daher, sowohl schriftlich als auch graphisch, verdeutlicht, dass der Erfolg ihres Verhaltens von dem unsicheren Verhalten ihres Interaktionspartners abhängt. Darüber hinaus liegt eine Vertrauensentscheidung nur dann vor, wenn der Vertrauensgeber freiwillig eine riskante Vorleistung unter Verzicht auf Schutzmaßnahmen zur Begrenzung von Opportunismusspielräumen erbringt. Die Fallsimulation bot den Probanden daher die Möglichkeit, Informationen an ihren Interaktionspartner offen und ehrlich abzugeben, wobei herausgestellt wurde, dass letzterer diese zu seinem eigenen Vorteil nutzen kann. Auch war es den Probanden möglich, sich durch Kontrolle der Handlungen ihres Interaktionspartners vor dessen möglicher Ausnutzung von Opportunismusspielräumen zu schützen. Die nachfolgende Tabelle dokumentiert die Eignung der Fallsimulation zur Analyse der Informations- und Kontrollhandlungen (als Indikatoren der Vertrauenshandlung) der Probanden, sowie die Effektivität der Manipulation der unabhängigen Variable im Zuge des vorliegenden Experiments.

\begin{tabular}{|c|c|c|c|c|}
\hline $\begin{array}{c}\text { Indikatoren der } \\
\text { Vertrauenshandlung }\end{array}$ & Gruppen & $\mathrm{N}$ & Mittelwert & $\begin{array}{l}\text { Standard- } \\
\text { abweichung }\end{array}$ \\
\hline \multirow{3}{*}{$\begin{array}{c}\text { offene } \\
\text { Informationsabgabe }\end{array}$} & $V G 1 F M K$ & 141 & 9,369 & 3,163 \\
\hline & $K G$ & 142 & 8,866 & 3,158 \\
\hline & $V G 2 \_S P K$ & 144 & 9,896 & 3,427 \\
\hline \multirow{3}{*}{$\begin{array}{c}\text { ehrliche } \\
\text { Informationsabgabe }\end{array}$} & $V G 1 F M K$ & 141 & 7,823 & 2,142 \\
\hline & $K G$ & 142 & 5,645 & 2,574 \\
\hline & $V G 2 \_S P K$ & 144 & 9,501 & 0,851 \\
\hline \multirow{3}{*}{$\begin{array}{c}\text { reduzierte } \\
\text { Kontrollintensität }\end{array}$} & $V G 1 F M K$ & 141 & 2,461 & 0,841 \\
\hline & $K G$ & 142 & 2,021 & 0,949 \\
\hline & $V G 2 \_S P K$ & 144 & 2,813 & 0,793 \\
\hline
\end{tabular}

Tabelle 31: Deskriptive Ergebnisse zu Informations- und Kontrollhandlungen

Die Ergebnisse zeigen deutlich, dass die Probanden sowohl die Möglichkeit der Informationsabgabe als auch der Kontrolle aktiv wahrgenommen haben. Auch lassen die Gruppenunterschiede hinsichtlich der Informations- und Kontrollhandlungen auf eine effektive Manipulation der unabhängigen Variable schließen. Damit kann das Experiment als für die Testung der abgeleiteten Hypothesen zur Vornahme einer Vertrauenshandlung (= Summe der Indikatorwerte) geeignet beurteilt werden. Die Probanden, die eine Beschreibung des Rotweinproduzenten mit ausgeprägten Sozial- und Persönlichkeitskompetenzen erhielten (VG2_SPK), haben im Durchschnitt 9,90 Informationen offen (= Anzahl der richtig und falsch abgegebenen Informationen) und 9,50 Informationen ehrlich (= Anzahl der richtigen 
Informationen an allen abgegebenen Informationen) abgegeben. Während der Mittelwert der offenen Informationsabgabe mit 9,40 für die Gruppe, die eine Beschreibung des Rotweinproduzenten mit ausgeprägten Fach- und Methodenkompetenzen erhielt (VG1_FMK), jenem Wert der VG2_SPK ähnlich ist, haben die Probanden dieser Gruppe im Durchschnitt weit weniger Informationen ehrlich abgegeben $(7,82)$ als die Versuchspersonen der Gruppe VG2_SPK. Mit Blick auf die Kontrollgruppe wird dieser Unterschied noch deutlicher: So haben die Probanden dieser Gruppe weniger Informationen offen $(8,87)$ und auch weit weniger $(5,65)$ Informationen ehrlich abgegeben, als die Probanden der VG2_SPK und der VG1_FMK. Bezugnehmend auf die Kontrollintensität liegt der Wertbereich zwischen 0 und 4 , wobei der Wert , $4^{6}$ einen kompletten Kontrollverzicht ( 0 angeforderte Berichte) und der Wert , $0^{\circ}$ (4 angeforderte Berichte) eine hohe Kontrollintensität impliziert (vgl. Teil V Kap. 3). Demnach kontrollieren die Probanden der VG2_SPK das Verhalten ihres Interaktionspartners im Mittel in einem geringerem Ausmaß (angeforderte Berichte: 1,19) als die Probanden der VG_FMK (angeforderte Berichte: 1,54), die wiederum durchschnittlich weniger Kontrollmaßnahmen setzen als die Probanden der KG (angeforderte Berichte: 1,98).

Zur Überprüfung der Effektivität der Manipulation der unabhängigen Variable und damit des Experiments kann auch die Strategiewahl in Abhängigkeit von den Kompetenzbeschreibungen des Rotweinproduzenten analysiert werden. Die differenzierte Betrachtung der Strategiewahl lässt auf höchst signifikante Zusammenhänge schließen, die durch die Ergebnisse eines $\chi^{2}$-Tests ${ }^{721}$ bestätigt werden können: Alle Probanden der VG2_SPK wählten die Kooperationsstrategie (100\%). Von den Probanden, die mit einem methodisch- und fachkompetenten Interaktionspartner konfrontiert wurden, entschieden sich $\mathbf{7 8 , 7 \%}$ für die Strategie der Kooperation. Dagegen wählten $51,4 \%$ der Versuchspersonen der KG die Wettbewerbsstrategie.

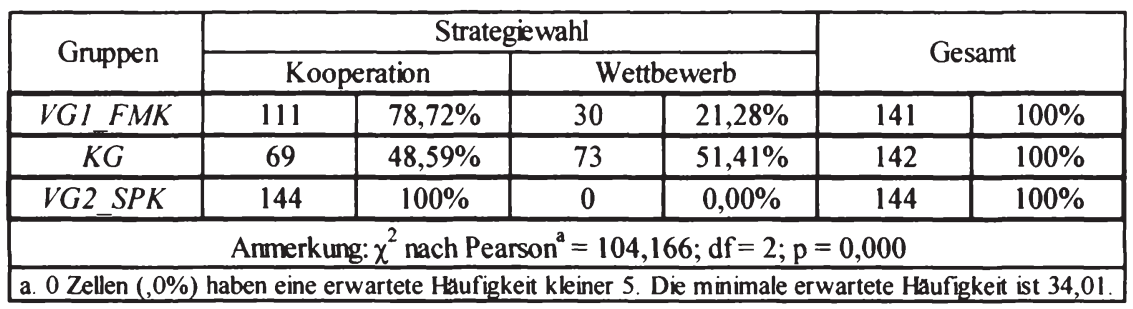

Tabelle 32: Befund zum Zusammenhang zwischen Kompetenzwahrnehmung und Strategiewahl

Da die Teststatistik $(104,166)$ den dem Signifikanzniveau von $\alpha=0,001$ bei 2 Freiheitsgraden entsprechenden Wert der $\chi^{2}$-Tabelle $(13,82)$ überschreitet, ist die

721 Der $\chi 2$-Test dient der Überprüfung der unterstellten Nullhypothese der Unabhängigkeit zweier Merkmale (Backhaus et al. 2003, S. 241). 
Nullhypothese der Unabhängigkeit der Merkmale mit einer Irrtumswahrscheinlichkeit von unter $0,1 \%$ zu verwerfen. Der höchst signifikante Zusammenhang zwischen der Kompetenzwahrnehmung, d.h. den Gruppen mit unterschiedlichen Kompetenzbeschreibungen (VG1_FMK, KG, VG2_SPK) des Rotweinproduzenten, und der Strategiewahl weist also darauf hin, dass die Manipulation der unabhängigen Variable die Handlungen der Probanden nachhaltig beeinflusste. Die Gegenüberstellung des Strategiewahlverhaltens zeigt, dass besonders die Probanden, die mit einem mit Sozial- und Persönlichkeitskompetenzen ausgestatteten Rotweinproduzenten konfrontiert waren, aber auch jene, deren Partner über Fachund Methodenkompetenzen verfügte, bereit waren, auf eigene kurzfristige Vorteile zugunsten gemeinsamer langfristiger Vorteile zu verzichten. Zwar ist Kooperation nicht mit Vertrauen gleichzusetzen, womit die Strategiewahl nicht ausreicht, um auf eine methodische Wirksamkeit der Fallsimulation zur Testung von Vertrauen schließen zu können, aber aufgrund der auch hinsichtlich der Informations- und Kontrollhandlungen bestehenden Unterschiede zwischen den Gruppen kann der Versuchsaufbau grundsätzlich als geeignet angesehen werden, um den Einfluss individueller Kompetenzarten auf die Entstehung von Vertrauen zu untersuchen.

\subsection{Die Kontrolle der Manipulationseffekte der unabhängigen Variable}

Die unabhängige Variable wurde zur Untersuchung von Kompetenz als Vertrauensdeterminante, sowie zum Vergleich der Einflussstärke personen- und situationsspezifischer Variablen auf Vertrauen bei der Wahrnehmung unterschiedlicher Kompetenzarten manipuliert. Die Effekte dieses manipulativen Eingriffs hinsichtlich der Dispositionen und der Risikowahrnehmung der Probandanden müssen überprüft werden, um eine Einschränkung der Gültigkeit der Ergebnisse zu vermeiden: Erstens muss sichergestellt werden, dass bei den Probanden der VG1_FMK und der KG, sowie bei den Versuchsteilnehmern der VG2_SPK und der KG keine Unterschiede im Hinblick auf relevante handlungsleitende Dispositionen, d.h. der Ausprägungen der personenspezifischen Vertrauens- und Risikobereitschaft, bestehen. Zweitens muss geprüft werden, ob die Manipulation der unabhängigen Variable zu Unterschieden bei der situativen Risikowahrnehmung zwischen den Probanden der VGl_FMK und der KG, sowie den Versuchspersonen der VG2_SPK und der KG führt. Die statistische Prüfung der Manipulationseffekte erfolgt anhand von t-Tests. Die nachfolgenden Tabellen geben Aufschluss über die Mittelwerte und Standardabweichungen der interessierenden Variablen für die drei Gruppen, sowie über das Signifikanzniveau, zu dem die Nullhypothese, dass die Stichproben aus derselben Grundgesamtheit mit demselben 
arithmetischen Mittel $\mu$ und derselben Varianz $\sigma^{2}$ stammen $^{722}$, abgelehnt wird. Unterschreitet die Wahrscheinlichkeit für das Eintreten des berechneten $t$-Wertes die Irrtumswahrscheinlichkeit von $5 \%$, so ist davon auszugehen, dass ein statistisch bedeutender Unterschied im Hinblick auf die Ausprägung der Variablen zwischen den Gruppen besteht ${ }^{723}$, was zu einer Zurückweisung der Nullhypothese führt.

\section{Prüfung auf Gruppenunterschiede hinsichtlich der Dispositionen}

Im Zuge der Hypothesengenerierung wurde angenommen, dass generalisierte Erwartungen als Informationssubstitute in Form der generellen Vertrauensbereitschaft die Vertrauenserwartung in einer spezifischen Situation beeinflussen. Nun wird geprüft, ob signifikante Unterschiede bezüglich der generellen Vertrauensbereitschaft zwischen den Gruppen bestehen. Um auszuschließen, dass Unterschiede in den Dispositionen der Probanden die Ergebnisse der Untersuchung verzerren, wird zudem getestet, ob sich die Gruppen hinsichtlich der personenspezifischen Risikobereitschaft, die wie die Vertrauensbereitschaft als Kontrollvariable miterfasst wurde, unterscheiden.

\begin{tabular}{|c|l|c|c|c|c|}
\hline \multirow{2}{*}{ Variable } & \multicolumn{1}{|c|}{ Gruppe } & $\mathrm{N}$ & Mittelwert & $\begin{array}{c}\text { Standard- } \\
\text { abweichung }\end{array}$ & $\begin{array}{c}\text { Sig. } \\
\text { (2-seitig) }\end{array}$ \\
\hline \multirow{5}{*}{ Vertrauensbereitschaft } & $V G 1 F M K$ & 141 & 18,84 & 6,109 & \multirow{2}{*}{0,138} \\
\cline { 2 - 5 } & $K G$ & 142 & 17,84 & 5,162 & \\
\cline { 2 - 5 } & $V G 2 S P K$ & 144 & 18,22 & 4,996 & \multirow{2}{*}{0,523} \\
\hline \multirow{5}{*}{ Risikobereitschaft } & 142 & 17,84 & 5,162 & \\
\cline { 2 - 5 } & $V G 1 F M K$ & 141 & 16,65 & 2,950 & \multirow{2}{*}{0,107} \\
\cline { 2 - 5 } & $K G$ & 142 & 16,04 & 3,320 & \\
\cline { 2 - 5 } & $V G 2 S P K$ & 144 & 16,03 & 3,174 & \multirow{2}{*}{0,984} \\
\cline { 2 - 5 } & $K G$ & 142 & 16,04 & 3,320 & \\
\hline
\end{tabular}

Tabelle 33: Ergebnisse der Prüfung auf Gruppenunterschiede bezüglich der Dispositionen

Wenn die zu testende Hypothese, die besagt, dass der Unterschied zwischen den Mittelwerten für VG1_FMK und KG, sowie für VG2_SPK und KG in der Grundgesamtheit gleich 0 ist, wahr ist, ergeben sich die für die Stichproben berechneten $\mathrm{t}$-Werte mit hohen Wahrscheinlichkeiten. Die vorliegenden hohen Wahrscheinlichkeiten (alle Werte in der Spalte ,Sig. (2-seitig)' sind größer als 0,05) lassen darauf schließen, dass die Nullhypothesen richtig sind. Die Annahme voneinander verschiedener Mittelwerte in der Grundgesamtheit $\left(\mathrm{H}_{\mathrm{A}}\right)$ kann also zu Gunsten der Annahme, auch in der Grundgesamtheit seien die Mittelwerte hinsichtlich Vertrauens- und Risikobereitschaft gleich $\left(\mathrm{H}_{0}\right)$, aufgegeben werden. Entsprechend der

722 Vgl. Janssen/Laatz (2007), S. 349

723 Vgl. Brosius (2002), S. 463 
t-Tests bestehen also keine signifikanten Unterschiede im Hinblick auf die Ausprägungen der personenspezifischen Vertrauensbereitschaft und der personenspezifischen Risikobereitschaft zwischen VG1_FMK und KG einerseits, und VG2_SPK und KG andererseits.

\section{Prüfung auf Gruppenunterschiede hinsichtlich der Risikowahrnehmung}

Im Zuge der Hypothesengenerierung wurde angenommen, dass die Entscheidungssituation in der Wahrnehmung des Vertrauensgebers ein Risiko beinhaltet, da die Entscheidung für oder gegen die Vornahme einer Vertrauenshandlung zu unsicheren Ereignissen mit unterschiedlichen Konsequenzen auf seinen Nutzen führen kann. Die Situationsspezifität der Risikowahrnehmung ergibt sich aus der Annahme, dass die Art und die Höhe der Konsequenzen der Vertrauenshandlung als gewählte Alternative vorwiegend situationsbedingt und kaum vom Vertrauensnehmer beeinflussbar sind. Die Ergebnisse der t-Tests implizieren, dass die Unterschiede zwischen den Mittelwerten hinsichtlich der Risikowahrnehmung für VG1 FMK und KG, sowie für VG2_SPK und KG in der Grundgesamtheit gleich 0 ist.

\begin{tabular}{|c|l|c|c|c|c|}
\hline \multirow{2}{*}{ Variable } & \multicolumn{1}{|c|}{ Gruppe } & $\mathrm{N}$ & Mittelwert & $\begin{array}{c}\text { Standard- } \\
\text { abweichung }\end{array}$ & $\begin{array}{c}\text { Sig. } \\
\text { (2-seitig) }\end{array}$ \\
\hline \multirow{4}{*}{ Risikowahrnehmung } & $V G 1 F M K$ & 141 & 8,97 & 2,330 & \multirow{2}{*}{0,072} \\
\cline { 2 - 5 } & $K G$ & 142 & 8,51 & 1,919 & \\
\cline { 2 - 5 } & $V G 2 S P K$ & 144 & 8,31 & 2,130 & \multirow{2}{*}{0,385} \\
\cline { 2 - 5 } & $K G$ & 142 & 8,51 & 1,919 & \\
\hline
\end{tabular}

Tabelle 34: Ergebnisse der Prüfung auf Gruppenunterschiede bezïglich der Risikowahrnehmung

Da entsprechend der t-Tests keine signifikanten Unterschiede im Hinblick auf die Ausprägung der Risikowahrnehmung bestehen, womit die Einschätzung der Eintrittswahrscheinlichkeiten der mit der Vornahme einer Vertrauenshandlung verbundenen Kontingenzen und der dadurch bedingten ökonomischen Folgen in einer spezifischen Situation vom Vertrauensnehmer weitgehend unabhängig ist, kann von einem methodisch wirksamen Experiment ausgegangen werden.

Zusammenfassend weisen die Stichprobenvergleiche darauf hin, dass die Manipulation der unabhängigen Variable nicht durch die Ausprägungen der als Kontrollvariablen miterfassten personenspezifischen Vertrauens- und Risikobereitschaft verzerrt wird. Auch implizieren die Ergebnisse, dass durch die Manipulation der erklärenden Variable kein verzerrender Effekt auf die situationsspezifische Risikowahrnehmung ausgeübt wird. Durch den Versuchsaufbau wurde somit eine Situation gestaltet, die eine Prüfung der aufgestellten Hypothesen ermöglicht. 


\section{Die Prüfung des Einflusses Von KompetenZ Auf die ENTSTEHUNG VON VERTRAUEN}

Die Prüfung der Wirkung von Kompetenz auf Vertrauen erfolgt anhand regressionsanalytischer Verfahren. ${ }^{724}$ Im Anschluss an die Untersuchung des Einflusses allgemeiner Kompetenzwahrnehmung auf die Vertrauenshandlung wird die Wirkung von Kompetenz auf die Informations- und Kontrollhandlungen geprüft (Kap. 2.1). Kapitel 2.2 hat zum Ziel, den Einfluss wahrgenommener Fach- und Methodenkompetenz, sowie der Sozial- und Persönlichkeitskompetenzwahrnehmung auf die Vornahme einer Vertrauenshandlung, und auf die offene und ehrliche Informationsabgabe, sowie die Kontrollintensität zu untersuchen. Es schließt sich eine Multigruppenanalyse an, die es ermöglicht, die Einflussstärke der unterschiedlichen Kompetenzarten auf die abhängige Variable zu testen. Nach der Untersuchung der Annahmen zum Einfluss der kontrolliert miterhobenen Variablen Vertrauensbereitschaft, Risikobereitschaft und Risikowahrnehmung auf die Vertrauenshandlung, sowohl in Verbindung mit Fach- und Methodenkompetenz, als auch mit Sozial- und Persönlichkeitskompetenz, werden die Differenzen hinsichtlich der Wirkung der Kontrollvariablen auf die Vertrauenshandlung bei Wahrnehmung unterschiedlicher Kompetenzarten geprüft (Kap. 2.3). Den Abschluss dieses Kapitels bildet die Analyse der Korrelationen zwischen den interessierenden Variablen (Kap. 2.4).

\subsection{Der Einfluss von Kompetenz auf Vertrauen}

In einem ersten Schritt wird geprüft, ob die Wahrnehmung von Kompetenz den Vertrauensgeber grundsätzlich zur Vornahme einer Vertrauenshandlung im Rahmen von zwischenbetrieblichen Kooperationen veranlasst. Die Ergebnisse der Regressionsanalyse lassen darauf schließen, dass Kompetenz, unabhängig davon, ob es sich um technische oder motivationale Kompetenzarten handelt, einen höchst signifikanten Einfluss ( $\beta$-Wert: 0,577) auf die Entstehung von Vertrauen nimmt. Auch das Regressionsmodell ist mit einem korrigierten $\mathrm{R}^{2}$ von 0,331 höchst signifikant: Da der empirische F-Wert stark von Null abweicht $(212,036)$ und den kritischen Wert von 15,43 bei 425 Freiheitsgraden überschreitet, kann die Nullhypothese, derzufolge in der Grundgesamtheit kein kausaler Zusammenhang zwischen Kompetenzwahrnehmung und Vertrauenshandlung besteht, mit einer Irrtumswahrscheinlichkeit von $0,01 \%$ verworfen werden.

$724 \mathrm{Vgl}$. zur Erläuterung der Methode und zur Interpretation der Werte die Ausführungen in Teil V Kap. 5.2.1 


\begin{tabular}{|l|c|}
\hline Unabhängige Variable & $\begin{array}{c}\text { Modell } \\
\text { Abhängige Variable:Vertrauenshandlung }\end{array}$ \\
\hline Kompetenzwahrnehmung & $0,577^{* * *}$ \\
\hline $\mathrm{R}^{2}$ & 0,333 \\
\hline $\mathrm{R}_{\text {kor }}^{2}$ & 0,331 \\
\hline $\mathrm{F}$-Wert & $212,036^{* * *}$ \\
\hline $\mathrm{N}$ & 427 \\
\hline${ }^{*} \mathrm{p}<0,05 ;{ }^{* *} \mathrm{p}<0,01 ;{ }^{* * *} \mathrm{p}<0,001$ \\
\hline
\end{tabular}

Tabelle 35: Ergebnisse der Prüfung des Einflusses von Kompetenz auf Vertrauen

In einem zweiten Schritt wird untersucht, ob die Wahrnehmung von Kompetenz den Vertrauensgeber zu offener (OI) und ehrlicher (EI) Informationsabgabe im Sinne der freiwilligen Erbringung einer riskanten Vorleistung, sowie zu reduzierter Kontrolle im Sinne eines Verzichts auf Maßnahmen zur Begrenzung von Opportunismusspielräumen veranlasst. Die Ergebnisse der Regressionsanalyse lassen darauf schließen, dass einem kompetenten Kooperationspartner signifikant weniger Informationen verschwiegen werden als einem Partner, dessen Kompetenz als durchschnittlich eingestuft wird. Das Regressionsmodell ist zwar signifikant, erklärt jedoch nur 1\% der Varianz der offenen Informationsabgabe.

\begin{tabular}{|l|c|}
\hline Unabhängige Variable & $\begin{array}{c}\text { Modell a } \\
\text { Abhängige Variable: Offene Informationsabgabe }\end{array}$ \\
\hline Kompetenzwahrnehmung & $0,111^{*}$ \\
\hline $\mathrm{R}^{2}$ & 0,012 \\
\hline $\mathrm{R}^{2}$ kor & 0,010 \\
\hline $\mathrm{F}-$ Wert & $5,284^{*}$ \\
\hline $\mathrm{N}$ & 427 \\
\hline$* \mathrm{p}<0,05 ; * * \mathrm{p}<0,01 ; * * * \mathrm{p}<0,001$ \\
\hline
\end{tabular}

Tabelle 36: Ergebnisse der Prüfung des Einflusses von Kompetenz auf OI

Die zweite Handlungsdimension, die im Interesse dieser Untersuchung steht, ist das Ausmaß der ehrlichen Informationsabgabe (EI) durch den Vertrauensgeber. Das Modell b erklärt mehr als $31 \%$ der Varianz und ist höchst signifikant (FWert: 196,126). Es zeigt, dass die ehrliche Informationsabgabe durch die Kompetenz, die der Kooperationspartner dem Vertrauensgeber vermittelt, determiniert wird ( $\beta$-Wert: 0,562). Einem kompetenten Kooperationspartner werden höchst signifikant mehr Informationen ehrlich abgegeben als einem Partner, der als durchschnittlich kompetent eingeschätzt wird. 


\begin{tabular}{|l|c|}
\hline Unabhängige Variable & $\begin{array}{c}\text { Modell b } \\
\text { Abhängige Variable: Ehrliche Informationsabgabe }\end{array}$ \\
\hline Kompetenzwahrnehmung & $0,562^{* * *}$ \\
\hline $\mathrm{R}^{2}$ & 0,316 \\
\hline $\mathrm{R}_{\text {kor }}^{2}$ & 0,314 \\
\hline $\mathrm{F}-$ Wert & $196,126^{* * *}$ \\
\hline $\mathrm{N}$ & 427 \\
\hline${ }^{*} \mathrm{p}<0,05 ;{ }^{* *} \mathrm{p}<0,01 ;{ }^{* * *} \mathrm{p}<0,001$ \\
\hline
\end{tabular}

Tabelle 37: Ergebnisse der Prüfung des Einflusses von Kompetenz auf EI

Modell c, das knapp 10\% der Varianz erklärt, gibt Aufschluss darüber, dass die Kontrollhandlungen des Vertrauensgebers höchst signifikant von der Kompetenz des Kooperationspartners determiniert werden ( $\beta$-Wert: 0,316). Bei einem Kooperationspartner, der als kompetent wahrgenommen wird, werden weniger Schutz- und Kontrollmaßnahmen zur Begrenzung von dessen Opportunismusspielräumen gesetzt, als bei einem Partner, der über ein durchschnittliches Aus$\mathrm{maß}$ an Kompetenz verfügt. Da der empirische F-Wert stark von Null abweicht $(47,296)$ und den kritischen Wert von 15,43 bei 425 Freiheitsgraden überschreitet, kann die Nullhypothese, derzufolge in der Grundgesamtheit kein kausaler Zusammenhang zwischen Kompetenzwahrnehmung und reduzierter Kontrollintensität besteht, mit einer Irrtumswahrscheinlichkeit von $0,01 \%$ verworfen werden.

\begin{tabular}{|l|c|}
\hline Unabhängige Variable & $\begin{array}{c}\text { Modell c } \\
\text { Abhängige Variable: Reduzierte Kontrollintensiät }\end{array}$ \\
\hline Kompetenzwahrnehmung & $0,316^{* * *}$ \\
\hline $\mathrm{R}^{2}$ & 0,100 \\
\hline $\mathrm{R}_{\text {korr }}^{2}$ & 0,098 \\
\hline $\mathrm{F}-$ Wert & $47,296^{* * *}$ \\
\hline $\mathrm{N}$ & 427 \\
\hline${ }^{*} \mathrm{p}<0,05 ;{ }^{* *} \mathrm{p}<0,01 ;{ }^{* * *} \mathrm{p}<0,001$ \\
\hline
\end{tabular}

Tabelle 38: Ergebnisse der Prüfung des Einflusses von Kompetenz auf KI

Zusammenfassend bestätigen die Ergebnisse der Regressionsanalysen die Annahme, dass Kompetenz die Entstehung von Vertrauen im Kontext zwischenbetriebliche Kooperation determiniert. So werden einem Kooperationspartner, der einen kompetenten Eindruck vermittelt, Informationen offener und vor allem ehrlicher abgegeben, als einem Partner, dessen Kompetenz als durchschnittlich oder nicht vorhanden wahrgenommen wird. Auch beeinflusst die Kompetenzwahrneh- 
mung durch den Vertrauensgeber positiv den Verzicht auf Kontrollmaßnahmen zur Beschränkung von Opportunismusspielräumen des Kooperationspartners.

\subsection{Der Einfluss von individuellen Kompetenzen auf Vertrauen}

Nachdem gezeigt wurde, dass die Wahrnehmung von Kompetenz die Entstehung von Vertrauen determiniert, wird nun geprüft, welche der theoretisch postulierten individuellen Kompetenzen die Entstehung von Vertrauen begünstigen. Dazu wurden in Teil V Kap. 5 der vorliegenden Arbeit folgende Hypothesen aufgestellt:

Hypothese 1: Die wahrgenommene Fachkompetenz des Vertrauensnehmers hat einen positiven Einfluss auf die Vornahme einer Vertrauenshandlung durch den Vertrauensgeber.

Hypothese 2: Die wahrgenommene Methodenkompetenz des Vertrauensnehmers hat einen positiven Einfluss auf die Vornahme einer Vertrauenshandlung durch den Vertrauensgeber.

Hypothese 3: Die wahrgenommene Sozialkompetenz des Vertrauensnehmers hat einen positiven Einfluss auf die Vornahme einer Vertrauenshandlung durch den Vertrauensgeber.

Hypothese 4: Die wahrgenommene Persönlichkeitskompetenz des Vertrauensnehmers hat einen positiven Einfluss auf die Vornahme einer Vertrauenshandlung durch den Vertrauensgeber.

\subsubsection{Der Einfluss von Fach- und Methodenkompetenz auf Vertrauen}

Es wurde angenommen, dass die auf individueller Ebene wahrgenommene Fachkompetenz und die Methodenkompetenz (FMK) des potentiellen Kooperationspartners als Zusatzinformationen dazu beitragen, die Unsicherheit des Vertrauensgebers über das Können des Partners zu reduzieren, womit sie die Vornahme einer Vertrauenshandlung über die Dimension der Zutrauenserwartung positiv beeinflussen. Die Ergebnisse der Regressionsanalyse lassen darauf schließen, dass die Wahrnehmung von Fach- und Methodenkompetenz einen höchst signifikanten Einfluss $(\beta$-Wert: 0,475$)$ auf die Vornahme einer Vertrauenshandlung hat. Auch das Regressionsmodell ist mit einem korrigierten $R^{2}$ von 0,223 höchst signifikant: Da der empirische F-Wert stark von Null abweicht $(82,080)$ und den kritischen Wert von 15,58 bei 281 Freiheitsgraden überschreitet, kann die Nullhypothese, derzufolge in der Grundgesamtheit kein kausaler Zusammenhang zwischen Fachund Methodenkompetenzwahrnehmung und Vertrauenshandlung besteht, mit einer Irrtumswahrscheinlichkeit von $0,01 \%$ verworfen werden. 


\begin{tabular}{|l|c|}
\hline Unabhängige Variable & $\begin{array}{c}\text { Modell A-I } \\
\text { Abhängige Variable: Vertrauenshand hung }\end{array}$ \\
\hline Kompetenzwahrnehmung & $0,475^{* * *}$ \\
\hline $\mathrm{R}^{2}$ & 0,226 \\
\hline $\mathrm{R}_{\text {korr }}^{2}$ & 0,223 \\
\hline $\mathrm{F}-$ Wert & $82,080^{* * *}$ \\
\hline $\mathrm{N}$ & 283 \\
\hline${ }^{*} \mathrm{p}<0,05 ;{ }^{* *} \mathrm{p}<0,01 ;{ }^{* * *} \mathrm{p}<0,001$ \\
\hline
\end{tabular}

Tabelle 39: Ergebnisse der Prüfung des Einflusses von FMK auf Vertrauen

Hinsichtlich des Einflusses der Fach- und Methodenkompetenzwahrnehmung auf die Informationshandlungen zeigt Modell A-Ia, dass einem fach- und methodenkompetenten Kooperationspartner nicht mehr Informationen abgegeben werden, als einem Partner, der als durchschnittlich kompetent eingeschätzt wird. Auch erklärt das Modell nur 3\% der Varianz der offenen Informationsabgabe und ist nicht signifikant. Ein Grund hierfür mag darin liegen, dass die Wahrnehmung von Fach- und Methodenkompetenz nur die Unsicherheit hinsichtlich des Könnens des potentiellen Kooperationspartners reduziert. Da die Unsicherheit bezüglich des Wollens weiterhin bestehen bleibt, sieht sich der Vertrauensgeber mit dem Risiko konfrontiert, dass der fach- und methodenkompetente Kooperationspartner ebenso wie ein durchschnittlich kompetenter Partner - die an ihn abgegebenen Informationen zum Nachteil des Vertrauensgebers nutzen kann. Unter der Annahme, dass die Höhe dieses Risikos mit der Anzahl der offen abgegebenen Informationen steigt - da mehr Informationen größere Opportunismusspielräume implizieren - erscheint es logisch, dass der Vertrauensgeber einem fach- und methodenkompetenten Partner nicht mehr Informationen abgibt als einem durchschnittlich kompetenten Kooperationspartner. Ein weiterer Grund für den mangelnden Einfluss der Fach- und Methodenkompetenzwahrnehmung auf die offene Informationsabgabe mag darin bestehen, dass von einem fach- und methodenkompetenten Partner, der nur durchschnittlich hohe Sozial- und Persönlichkeitskompetenz zeigt, eher erwartet wird, dass er die an ihn abgegebenen Informationen zum Nachteil des Vertrauensgebers nützt, da ihn die Summe seiner fachlichen Fertigkeiten und Fähigkeiten, sowie der persönlichen Problemlösungsverfahren dazu ermächtigt. Dagegen macht es wenig Sinn, einem durchschnittlich kompetenten Interaktionspartner viele Informationen abzugeben, da dieser womöglich nicht über die notwendige Kapazität der Verarbeitung verfügt und dadurch von vornherein nicht für eine Kooperation in Frage kommt. 


\begin{tabular}{|l|c|}
\hline Unabhängige Variable & $\begin{array}{c}\text { Modell A-Ia } \\
\text { Abhängige Variable: Offene Informationsabgabe }\end{array}$ \\
\hline Kompetenzwahrnehmung & 0,080 \\
\hline $\mathrm{R}^{2}$ & 0,006 \\
\hline $\mathrm{R}_{\text {korr }}^{2}$ & 0,003 \\
\hline $\mathrm{F}-$ Wert & 1,789 \\
\hline $\mathrm{N}$ & 283 \\
\hline$* \mathrm{p}<0,05 ; * * \mathrm{p}<0,01 ; * * * \mathrm{p}<0,001$ \\
\hline
\end{tabular}

Tabelle 40: Ergebnisse der Prüfung des Einflusses von FMK auf OI

Allerdings zeigt das höchst signifikante Modell A-Ib (F-Wert: 59,839), dass einem fach- und methodenkompetenten Partner höchst signifikant mehr Informationen ( $\beta$-Wert: 0,419$)$ ehrlich abgegeben werden, als einem potentiellen Kooperationspartner, der als durchschnittlich kompetent wahrgenommen wird. Der Grund hierfür mag darin bestehen, dass die Wahrnehmung von Fach- und Methodenkompetenz den Vertrauensgeber dazu veranlasst, dem Partner die Verarbeitung der Informationen und die Aufrechterhaltung einer Kooperationsbeziehung zuzutrauen, wodurch wiederum das Wollen des Vertrauensgebers hinsichtlich des Eingehens bzw. Aufrechterhaltens einer Kooperationsbeziehung positiv beeinflusst wird. Will der Vertrauensgeber die fachlichen Fertigkeiten, Fähigkeiten und Problemlösungsverfahren des potentiellen Partners im Rahmen einer Kooperation also nützen, so wird er dem fach- und methodenkompetenten Interaktionspartner zwar weniger Informationen (vgl. Modell A-Ia), aber diese dafür ehrlich abgeben, um den Grundstein für eine erfolgreiche Zusammenarbeit zu legen. Hinzu kommt, dass der Partner aufgrund seiner Informationsverarbeitungskapazität erkennen kann, ob die an ihn abgegeben Informationen richtig oder falsch sind, sodass bei unehrlicher Informationsabgabe Probleme hinsichtlich der zukünftigen oder bereits bestehenden Zusammenarbeit auftreten können.

\begin{tabular}{|l|c|}
\hline Unabhängige Variable & $\begin{array}{c}\text { Modell A-Ib } \\
\text { Abhängige Variable: Ehrliche Informationsabgabe }\end{array}$ \\
\hline Kompetenzwahrnehmung & $0,419^{* * *}$ \\
\hline $\mathrm{R}^{2}$ & 0,176 \\
\hline $\mathrm{R}_{\text {korr }}^{2}$ & 0,173 \\
\hline $\mathrm{F}-$ Wert & $59,839^{* * *}$ \\
\hline $\mathrm{N}$ & 283 \\
\hline${ }^{*} \mathrm{p}<0,05 ; * * \mathrm{p}<0,01 ; * * * \mathrm{p}<0,001$ \\
\hline
\end{tabular}

Tabelle 41: Ergebnisse der Prüfung des Einflusses von FMK auf EI 
Entsprechend der Analyse der Regression der reduzierten Kontrollintensität auf die Kompetenzwahrnehmung werden bei einem fach- und methodenkompetenten Partner höchst signifikant weniger Kontrollmaßnahmen $(\beta$-Wert: 0,239$)$ gesetzt als bei einem durchschnittlich kompetenten Interaktionspartner. Das Regressionsmodell ist mit einem korrigierten $\mathrm{R}^{2}$ von $5,4 \%$ höchst signifikant (F-Wert: 17,022). Dies kann sich dadurch begründen, dass der Vertrauensgeber aufgrund seiner Zutrauenserwartung mit dem Partner zusammenarbeiten will und ihm daher sein Wollen durch eine vertrauensvolle Handlung in Form des Kontrollverzichts signalisiert.

\begin{tabular}{|l|c|}
\hline Unabhängige Variable & $\begin{array}{c}\text { Modell A-Ic } \\
\text { Abhängige Variable: Reduzierte Kontrollintensität }\end{array}$ \\
\hline Kompetenzwahrnehmung & $0,239^{* * *}$ \\
\hline $\mathrm{R}^{2}$ & 0,057 \\
\hline $\mathrm{R}_{\text {korr }}^{2}$ & 0,054 \\
\hline $\mathrm{F}-$ Wert & $17,022^{* * *}$ \\
\hline $\mathrm{N}$ & 283 \\
\hline${ }^{*} \mathrm{p}<0,05 ; * * \mathrm{p}<0,01 ; * * * \mathrm{p}<0,001$ \\
\hline
\end{tabular}

Tabelle 42: Ergebnisse der Prüfung des Einflusses von FMK auf KI

Zusammenfassend bestätigen die Ergebnisse der Regressionsanalysen die Hypothesen 1 und 2, wonach Fachkompetenz und Methodenkompetenz die Entstehung von Vertrauen im Kontext zwischenbetriebliche Kooperation determinieren. So werden einem potentiellen Kooperationspartner, der Fach- und Methodenkompetenz vermittelt, Informationen ehrlicher abgegeben, als einem Partner, dessen Kompetenz als durchschnittlich oder nicht vorhanden wahrgenommen wird. Auch beeinflusst die Fach- und Methodenkompetenzwahrnehmung den Verzicht auf Kontrollmaßnahmen zur Beschränkung von Opportunismusspielräumen positiv. Hingegen besteht kein signifikanter Einfluss der Fach- und Methodenkompetenz auf die Anzahl der abgegebenen Informationen.

\subsubsection{Der Einfluss von Sozial- und Persönlichkeitskompetenz auf Vertrauen}

Es wurde angenommen, dass die auf individueller Ebene wahrgenommene Sozialkompetenz und die Persönlichkeitskompetenz des potentiellen Kooperationspartners als Zusatzinformationen dazu beitragen, die Unsicherheit des Vertrauensgebers über das Wollen des Partners zu reduzieren, womit sie die Vornahme einer Vertrauenshandlung über die Dimension der Vertrauenserwartung positiv beeinflussen. Die Ergebnisse der Regressionsanalyse lassen darauf schließen, dass die Wahrnehmung von Sozial- und Persönlichkeitskompetenz einen höchst signifikanten Einfluss ( $\beta$-Wert: 0,733 ) auf die Vornahme einer Vertrauenshandlung hat. 
Auch das Regressionsmodell ist mit einem korrigierten $\mathrm{R}^{2}$ von 0,535 höchst signifikant: Da der empirische F-Wert stark von Null abweicht $(329,429)$ und den kritischen Wert von 15,58 bei 284 Freiheitsgraden überschreitet, kann die Nullhypothese, derzufolge in der Grundgesamtheit kein kausaler Zusammenhang zwischen Sozial- und Persönlichkeitskompetenzwahrnehmung und Vertrauenshandlung besteht, mit einer Irrtumswahrscheinlichkeit von $0,01 \%$ verworfen werden.

\begin{tabular}{|l|c|}
\hline Unabhängige Variable & $\begin{array}{c}\text { Modell B-I } \\
\text { Abhängige Variable:Vertrauenshandlung }\end{array}$ \\
\hline Kompetenzwahrnehmung & $0,733^{* * *}$ \\
\hline $\mathrm{R}^{2}$ & 0,537 \\
\hline $\mathrm{R}^{2}{ }_{\text {orr }}$ & 0,535 \\
\hline $\mathrm{F}-$ Wert & $329,429 * * *$ \\
\hline $\mathrm{N}$ & 286 \\
\hline${ }^{*} \mathrm{p}<0,05 ; * * \mathrm{p}<0,01 ;{ }^{* * *} \mathrm{p}<0,001$ \\
\hline
\end{tabular}

Tabelle 43: Ergebnisse der Prüfung des Einflusses von SPK auf Vertrauen

Modell B-Ia ist hoch signifikant, erklärt jedoch nur etwas mehr als $2 \%$ der Varianz der offenen Informationsabgabe. Es zeigt, dass das Verschweigen von Informationen durch die Wahrnehmung von Sozial- und Persönlichkeitskompetenz determiniert wird ( $\beta$-Wert: 0,155$)$. Einem sozial- und persönlichkeitskompetenten Kooperationspartner werden hoch signifikant mehr Informationen abgegeben als einem Partner, der als durchschnittlich kompetent wahrgenommen wird.

\begin{tabular}{|l|c|}
\hline Unabhängige Variable & Modell B-Ia \\
\hline Kompetenzwahrnehmung & $0,155^{* *}$ \\
\hline $\mathrm{R}^{2}$ & 0,024 \\
\hline $\mathrm{R}_{\text {korr }}^{2}$ & 0,021 \\
\hline $\mathrm{F}-$ Wert & $6,976^{* *}$ \\
\hline $\mathrm{N}$ & 286 \\
\hline$* \mathrm{p}<0,05 ; * * \mathrm{p}<0,01 ; * * * \mathrm{p}<0,001$ \\
\hline
\end{tabular}

Tabelle 44: Ergebnisse der Prüfung des Einflusses von SPK auf OI

Einem sozial- und persönlichkeitskompetenten Kooperationspartner werden auch höchst signifikant mehr Informationen ehrlich abgegeben ( $\beta$-Wert: 0,711$)$ als einem Partner, dessen Kompetenz als durchschnittlich oder nicht vorhanden wahrgenommen wird. Das Regressionsmodell B-Ib ist höchst signifikant (F-Wert: $290,947)$ und kann mit einer erklärten Varianz von $R^{2}{ }_{\text {korr }}=0,504$ als sehr zufriedenstellend eingestuft werden. 


\begin{tabular}{|c|c|}
\hline Unabhängige Variable & $\begin{array}{l}\text { Modell B-Ib } \\
\text { Abhängige Variable: Ehrliche Informationsabgabe }\end{array}$ \\
\hline Kompetenzwahrnehmung & $0,711^{* * *}$ \\
\hline $\mathrm{R}^{2}$ & 0,506 \\
\hline $\mathrm{R}_{\text {korr }}^{2}$ & 0,504 \\
\hline F-Wert & $290,947^{* * *}$ \\
\hline $\mathrm{N}$ & 286 \\
\hline \multicolumn{2}{|c|}{${ }^{*} \mathrm{p}<0,05 ;{ }^{* *} \mathrm{p}<0,01 ;{ }^{* * *} \mathrm{p}<0,001$} \\
\hline
\end{tabular}

Tabelle 45: Ergebnisse der Prüfung des Einflusses von SPK auf EI

Entsprechend der Analyse der Regression der reduzierten Kontrollintensität auf die Kompetenzwahrnehmung werden bei einem sozial- und persönlichkeitskompetenten Partner höchst signifikant weniger Kontrollmaßnahmen ( $\beta$-Wert: 0,414) als bei einem durchschnittlich kompetenten Interaktionspartner gesetzt. Das Regressionsmodell ist mit einem korrigierten $\mathrm{R}^{2}$ von knapp $17 \%$ höchst signifikant (F-Wert: 58,635).

\begin{tabular}{|l|c|}
\hline Unabhängige Variable & $\begin{array}{c}\text { Modell B-Ic } \\
\text { Abhängige Variable: Reduzierte Kontrollintensität }\end{array}$ \\
\hline Kompetenzwahrnehmung & $0,414^{* * *}$ \\
\hline $\mathrm{R}^{2}$ & 0,171 \\
\hline $\mathrm{R}_{\text {korr }}^{2}$ & 0,168 \\
\hline $\mathrm{F}-$ Wert & $58,635^{* * *}$ \\
\hline $\mathrm{N}$ & 286 \\
\hline${ }^{*} \mathrm{p}<0,05 ;{ }^{* *} \mathrm{p}<0,01 ;{ }^{* * *} \mathrm{p}<0,001$ \\
\hline
\end{tabular}

Tabelle 46: Ergebnisse der Prüfung des Einflusses von SPK auf KI

Zusammenfassend bestätigen die Ergebnisse der Regressionsanalysen die Hypothesen 3 und 4, wonach Sozialkompetenz und Persönlichkeitskompetenz die Entstehung von Vertrauen im Kontext zwischenbetriebliche Kooperation determinieren. Dies zeigt sich auch darin, dass einem potentiellen Kooperationspartner, der Sozial- und Persönlichkeitskompetenz vermittelt, Informationen offener und vor allem ehrlicher abgegeben werden, als einem Partner, dessen Kompetenz als durchschnittlich oder nicht vorhanden wahrgenommen wird. Auch beeinflusst die Sozial- und Persönlichkeitskompetenzwahrnehmung den Verzicht auf Kontrollmaßnahmen zur Beschränkung von Opportunismusspielräumen positiv. 


\subsubsection{Der Vergleich der Einflussstärke der individuellen Kompetenzen}

Zur Analyse, ob sich der Einfluss der Fach- und Methodenkompetenz von jenem der Sozial- und Persönlichkeitskompetenz auf die Entstehung von Vertrauen unterscheidet, wird eine Multigruppenanalyse durchgeführt. Dieses Analyseverfahren ist vor allem dann geeignet, wenn, wie in der vorliegenden Arbeit, durch Einbeziehung einer Dummy-Variable überprüft werden soll, ob für verschiedene Sample-Gruppen der gleiche lineare Zusammenhang zwischen unabhängiger und abhängiger Variable besteht. ${ }^{725}$ Bei einem Vergleich der Einflussstärken zwischen Regressionsmodellen müssen anstelle der in den vorangegangenen Modellen erörterten standardisierten Regressionskoeffizienten die unstandardisierten Koeffizienten interpretiert werden, da die $\beta$-Werte anfällig für Varianzunterschiede in den Gruppen sind. ${ }^{726}$ Die Prüfung des Vorliegens statistisch bedeutender Unterschiede zwischen dem Einfluss von Fach- und Methodenkompetenz und Sozialund Persönlichkeitskompetenz erfolgt anhand von t-Tests. ${ }^{727}$

Die Gegenüberstellung der Modelle bestätigt den Befund, dass Vertrauen von der Kompetenzwahrnehmung, unabhängig davon, um welche individuelle Kompetenzart es sich handelt, beeinflusst wird. Der Anteil der durch die Sozial- und Persönlichkeitswahrnehmung erklärten Varianz $\left(\mathrm{R}_{\text {korr }}^{2}=0,535\right)$ der Vertrauenshandlung ist allerdings höher als der Varianzanteil der durch die Fach- und Methodenkompetenz $\left(\mathrm{R}_{\text {korr }}^{2}=0,223\right)$ erklärt wird. Auch der Signifikanzwert des Gesamtmodells (F-Wert: 329,429) ist bei Sozial- und Persönlichkeitskompetenzwahrnehmung höher als bei Fach- und Methodenkompetenzwahrnehmung (FWert: 82,080). Der visuelle Vergleich der B-Werte der beiden Regressionsmodelle zeigt, dass der positive Einfluss von Kompetenz auf Vertrauen größer ist, wenn der Vertrauensgeber sein Gegenüber als sozial- und persönlichkeitskompetent wahrnimmt. Dieser Unterschied hinsichtlich der Einflussstärke von Fachund Methodenkompetenz und Sozial- und Persönlichkeitskompetenz ist gemäß des $t$-Tests $\left(t_{e m p}=5,41\right)$ statistisch signifikant. Demnach wird einem Akteur, der Sozial- und Persönlichkeitskompetenz vermittelt, in höherem Maße vertraut, als einem potentiellen Kooperationspartner, der Fach- und Methodenkompetenz zeigt. Es kann daher davon ausgegangen werden, dass motivationale kompetenzbezogene Zusatzinformationen zur Reduktion der Entscheidungsunsicherheit für den Vertrauensgeber im Zuge der Bildung seiner Vertrauensentscheidung von

$725 \quad$ Vgl. Urban/Mayerl (2006), S. 289

726 Unstandardisierte Regressionskoeffizienten sind zwar von den empirischen Messeinheiten unabhängiger Variablen, aber nicht von deren Varianzen in verschiedenen Populationen abhängig. „Sie können deshalb zum Vergleich der Einflussstärke einer einzigen Variable in verschiedenen Stichproben benutzt werden [...]" (Urban/Mayerl 2006, S. 79).

727 Bei einem Signifikanzniveau von $\alpha=0,05$ muss der empirische $t$-Wert den theoretischen $t$-Wert von $|1,96|$ überschreiten, um davon ausgehen zu können, dass ein statistisch bedeutender Unterschied hinsichtlich der Einflussstärke der unabhängigen Variable auf die abhängige Variable zwischen den Gruppen besteht (Urban/Mayerl 2006, S. 302). 
höherer Bedeutung sind als technische kompetenzbezogene Zusatzinformationen zur Reduktion der Qualitätsunsicherheit.

\begin{tabular}{|c|c|c|}
\hline Unabhängige Variable & $\begin{array}{c}\text { Modell A-I } \\
\text { Abhängige Variable: } \\
\text { Vertrauenshandlung }\end{array}$ & $\begin{array}{c}\text { Modell B-I } \\
\text { Abhängige Variable: } \\
\text { Vertrauenshandlung }\end{array}$ \\
\hline Kompetenzwahrnehmung & $5,748 * * *$ & $10,365^{* * *}$ \\
\hline $\mathrm{R}^{2}$ & 0,226 & 0,537 \\
\hline $\mathbf{R}_{\text {korr }}^{2}$ & 0,223 & 0,535 \\
\hline F-Wert & $82,080 * * *$ & $329,429 * * *$ \\
\hline $\mathrm{N}$ & 283 & 286 \\
\hline
\end{tabular}

Tabelle 47: Ergebnisse des Vergleichs der Einflussstärke individueller Kompetenzen auf Vertrauen

Allerdings besteht kein statistisch bedeutsamer Unterschied zwischen dem Einfluss von Fach- und Methodenkompetenz und Sozial- und Persönlichkeitskompetenz auf die offene Informationsabgabe $\left(t_{e m p}=0,97\right)$. Unter Berücksichtigung der Ergebnisse der vorangegangenen Regressionsanalysen ist die Überlegung anzustellen, ob es sich bei der Abgabe von Informationen (richtige und falsche Informationen) eher um eine notwendige Kooperationsvoraussetzung, denn um einen Indikator der Vertrauenshandlung ${ }^{728}$ handelt.

\begin{tabular}{|l|c|c|}
\hline Unabhängige Variable & $\begin{array}{c}\text { Modell A-la } \\
\text { Abhängige Variable: } \\
\text { Offene Informationsabgabe }\end{array}$ & $\begin{array}{c}\text { Modell B-Ia } \\
\text { Abhängige Variable: } \\
\text { Offene Informationsabgabe }\end{array}$ \\
\hline Kompetenzwahrnehmung & $\mathbf{0 , 5 0 3}$ & $\mathbf{1 , 0 3 0 * *}$ \\
\hline $\mathrm{R}^{2}$ & 0,006 & 0,024 \\
\hline $\mathrm{R}_{\text {korr }}^{2}$ & 0,003 & 0,021 \\
\hline $\mathrm{F}$-Wert & 1,789 & $6,976^{* *}$ \\
\hline $\mathrm{N}$ & 283 & 286 \\
\hline${ }^{*} \mathrm{p}<0,05 ;{ }^{* *} \mathrm{p}<0,01 ; * * * \mathrm{p}<0,001$ & \\
\hline
\end{tabular}

Tabelle 48: Ergebnisse des Vergleichs der Einflussstärke individueller Kompetenzen auf OI

Hingegen ist der Anteil der durch die Sozial- und Persönlichkeitswahrnehmung erklärten Varianz $\left(R_{\text {korr }}^{2}=0,504\right)$ der ehrlichen Informationsabgabe höher als der

728 Zwar kann ein hoch signifikanter Einfluss von Sozial- und Persönlichkeitskompetenzwahrnehmung auf die offene Informationsabgabe festgestellt werden, jedoch ist das Gesamtmodell nicht höchst signifikant ( F-Wert: 6,976) 
Varianzanteil, der durch die Fach- und Methodenkompetenz $\left(\mathrm{R}_{\text {korr }}^{2}=0,173\right)$ erklärt wird. Auch der Signifikanzwert des Gesamtmodells (F-Wert: 290,947) ist bei Sozial- und Persönlichkeitskompetenzwahrnehmung höher als bei Fach- und Methodenkompetenzwahrnehmung (F-Wert: 59,839). Der visuelle Vergleich der BWerte der beiden Regressionsmodelle zeigt, dass der positive Einfluss von Kompetenz auf die ehrliche Informationsabgabe größer ist, wenn der Vertrauensgeber sein Gegenüber als sozial- und persönlichkeitskompetent wahrnimmt. Dieser Unterschied hinsichtlich der Einflussstärke von Fach- und Methodenkompetenz und Sozial- und Persönlichkeitskompetenz ist gemäß des $t$-Tests $\left(t_{e m p}=4,64\right)$ statistisch signifikant. Demnach werden einem potentiellen Kooperationspartner, der sehr sozial- und persönlichkeitskompetent ist, mehr Informationen ehrlich abgegeben, als einem Akteur, der zwar über Fach- und Methodenkompetenz verfügt, aber nur durchschnittliche Sozial- und Persönlichkeitskompetenz zeigt.

\begin{tabular}{|c|c|c|}
\hline Unabhängige Variable & $\begin{array}{c}\text { Modell A-Ib } \\
\text { Abhängige Variable: } \\
\text { Ehrliche Informationsabgabe }\end{array}$ & $\begin{array}{c}\text { Modell B-Ib } \\
\text { Abhängige Variable: } \\
\text { Ehrliche Informationsabgabe }\end{array}$ \\
\hline Kompetenzwahrnehmung & $2,179^{* * *}$ & $3,856^{* * *}$ \\
\hline $\mathrm{R}^{2}$ & 0,176 & 0,506 \\
\hline $\mathrm{R}_{\text {korr }}^{2}$ & 0,173 & 0,504 \\
\hline F-Wert & $59,839 * * *$ & $290,947 * * *$ \\
\hline $\mathrm{N}$ & 283 & 286 \\
\hline
\end{tabular}

Tabelle 49: Ergebnisse des Vergleichs der Einflussstärke individueller Kompetenzen auf EI

Auch wird bei einem Partner, der als sozial- und persönlichkeitskompetent wahrgenommen wird, in höherem Ausmaß auf den Einsatz von Kontrollmaßnahmen zur Begrenzung von Opportunismusspielräumen verzichtet. Der Unterschied zwischen den unstandardisierten Regressionskoeffizienten ist gemäß des t-Tests $\left(t_{\mathrm{emp}}=2,36\right)$ statistisch signifikant. Auch der Signifikanzwert des Gesamtmodells ist bei Sozial- und Persönlichkeitskompetenzwahrnehmung (F-Wert: 58,635) mit einem korrigierten $\mathrm{R}^{2}$ von 0,168 höher als bei Fach- und Methodenkompetenzwahrnehmung mit einem korrigierten $R^{2}$ von 0,054 (F-Wert: 17,022). 


\begin{tabular}{|l|c|c|}
\hline Unabhängige Variable & $\begin{array}{c}\text { Modell A-Ic } \\
\text { Abhängige Variable: } \\
\text { Reduzierte Kontrollintensität }\end{array}$ & $\begin{array}{c}\text { Modell B-Ic } \\
\text { Abhängige Variable: } \\
\text { Reduzierte Kontrollintensität }\end{array}$ \\
\hline Kompetenzwahrnehmung & $\mathbf{0 , 4 4 0 * * *}$ & $\mathbf{0 , 7 9 1 * * *}$ \\
\hline $\mathrm{R}^{2}$ & 0,057 & 0,171 \\
\hline $\mathrm{R}_{\text {korr }}^{2}$ & 0,054 & 0,168 \\
\hline $\mathrm{F}$-Wert & $17,022^{* * *}$ & $58,635^{* * *}$ \\
\hline $\mathrm{N}$ & 283 & 286 \\
\hline${ }^{*} \mathrm{p}<0,05 ;{ }^{* *} \mathrm{p}<0,01 ;{ }^{* *} \mathrm{p}<0,001$ & \\
\hline
\end{tabular}

Tabelle 50: Ergebnisse des Vergleichs der Einflussstårke individueller Kompetenzen auf KI

Die Ergebnisse dieser Multigruppenanalyse implizieren, dass die Sozialkompetenz und die Persönlichkeitskompetenz die Vornahme einer Vertrauenshandlung im Kontext zwischenbetriebliche Kooperation stärker determinieren als die Fach- und Methodenkompetenz. Schätzt der Vertrauensgeber den potentiellen Kooperationspartner als sozial- und persönlichkeitskompetent ein, wird er ihm höchst signifikant mehr Informationen ehrlich abgeben und ihn in geringerem Ausmaß kontrollieren, als einem bzw. einen Interaktionspartner, der ausgeprägte Fach- und Methodenkompetenz zeigt, aber bloß durchschnittlich sozial- und persönlichkeitskompetent ist. Hingegen besteht kein statistischer Unterschied hinsichtlich der Einflussstärke individueller Kompetenzarten auf das Ausmaß der offenen Informationsabgabe.

\subsection{Der Einfluss von individuellen Kompetenzen auf Vertrauen unter Berücksichtigung von Dispositionen und situativer Wahrnehmung}

Nachdem gezeigt wurde, dass Fach-, Methoden-, Sozial- und Persönlichkeitskompetenz die Vornahme einer Vertrauenshandlung begünstigen, wird nun der Einfluss der Dispositionen und der situativen Wahrnehmung, die als potentielle Störvariablen kontrolliert miterhoben wurden, auf die Vornahme einer Vertrauenshandlung im Zusammenhang mit Fach- und Methodenkompetenzwahrnehmung einerseits und der Wahrnehmung von Sozial- und Persönlichkeitskompetenz andererseits analysiert. Dazu wurden in Teil V Kap. 5 der vorliegenden Arbeit folgende Annahmen getroffen: Erstens wurde angenommen, dass die generelle Vertrauensbereitschaft als Informationssubstitut die Vertrauenserwartung in einer spezifischen Situation beeinflusst. Zweitens wurde davon ausgegangen, dass die vertrauensgeberspezifische Risikobereitschaft, die sich darauf bezieht, sowohl Verhaltens- als auch Umweltrisiken zu absorbieren, und sich im Gegensatz zur Vertrauensbereitschaft erst als Komponente des Entscheidens und Handelns zeigt, 
einen positiven Einfluss auf die Vornahme einer Vertrauenshandlung nimmt. Drittens wurde angenommen, dass die Vertrauenshandlung negativ von der Höhe des wahrgenommenen situationsspezifischen Risikos beeinflusst wird.

\subsubsection{Der Einfluss von Fach- und Methodenkompetenz auf Vertrauen unter Berücksichtigung von Dispositionen und situativer Wahrnehmung}

Die Annahme, dass die Wahrnehmung von Fach- und Methodenkompetenz die Sicherheit über das Können des Vertrauensnehmers $\left(\mathrm{H}_{1 \mathrm{~A}}\right.$ und $\left.\mathrm{H}_{2 \mathrm{~A}}\right)$ erhöht und damit über die Bildung einer Zutrauenserwartung $\left(\mathrm{H}_{1 \mathrm{~B}}\right.$ und $\left.\mathrm{H}_{2 \mathrm{~B}}\right)$ einen positiven Einfluss auf die Vertrauenshandlung hat $\left(\mathrm{H}_{1 \mathrm{C}}\right.$ und $\left.\mathrm{H}_{2 \mathrm{C}}\right)$, kann durch die Ergebnisse der nachfolgenden Regressionsanalyse bestätigt werden. Da die Vertrauensbereitschaft bei Wahrnehmung von Fach- und Methodenkompetenz keinen signifikanten Einfluss auf die Vertrauenshandlung ( $\beta$-Wert: 0,079) nimmt, kann davon ausgegangen werden, dass zwischen den Konstrukten Vertrauenserwartung und Zutrauenserwartung zu differenzieren ist: Die Wahrnehmung technischer kompetenzbezogener Zusatzinformationen kann durch Reduktion von Qualitätsunsicherheit nur die Bildung einer zutrauensvollen Erwartung begünstigen, da die Unsicherheit über das Wollen, d.h. hinsichtlich motivationaler Aspekte, des Vertrauensnehmers bestehen bleibt. Bezugnehmend auf die Ergebnisse der Schätzung beeinflusst die Wahrnehmung von Fach- und Methodenkompetenz die Vornahme einer Vertrauenshandlung daher über die Dimension der Zutrauenserwartung höchst signifikant ( $\beta$-Wert: 0,467). Auch die Annahmen hinsichtlich der Wirkung der vertrauensgeberspezifischen Risikobereitschaft und der situativen Risikowahrnehmung werden durch die Ergebnisse der Modellschätzung bestätigt: Während die Vertrauenshandlung höchst signifikant positiv von der vertrauensgeberspezifischen Risikobereitschaft ( $\beta$-Wert: 0,195 ) beeinflusst wird, übt die situative Risikowahrnehmung einen hoch signifikanten negativen Einfluss ( $\beta$-Wert: $-0,158$ ) auf die Vertrauenshandlung aus. 


\begin{tabular}{|l|c|}
\hline Unabhängige Variable & $\begin{array}{c}\text { Modell A-II } \\
\text { Abhängige Variable:Vertrauenshandlung }\end{array}$ \\
\hline Kompetenzwahrnehmung & $0,467^{* * *}$ \\
\hline Vertrauensbereitschaft & 0,079 \\
\hline Risikobereitschaft & $0,195^{* * *}$ \\
\hline Risikowahrnehmung & $-0,158^{* *}$ \\
\hline $\mathrm{R}^{2}$ & 0,324 \\
\hline $\mathrm{R}_{\text {korr }}^{2}$ & 0,315 \\
\hline $\mathrm{F}-$ Wert & $33,367^{* * *}$ \\
\hline $\mathrm{N}$ & 283 \\
\hline${ }^{*} \mathrm{p}<0,05 ;{ }^{* *} \mathrm{p}<0,01 ;{ }^{* * *} \mathrm{p}<0,001$ \\
\hline
\end{tabular}

Tabelle 51: Ergebnisse der erweiterten Prüfung des Einflusses von FMK auf Vertrauen

Das Regressionsmodell ist höchst signifikant: Da der empirische F-Wert stark von Null abweicht $(33,367)$ und den kritischen Wert von 6,11 bei 278 Freiheitsgraden überschreitet, kann die Nullhypothese, derzufolge in der Grundgesamtheit kein kausaler Zusammenhang zwischen Fach- und Methodenkompetenzwahrnehmung, Risikobereitschaft und Risikowahrnehmung einerseits und Vertrauenshandlung andererseits besteht, mit einer Irrtumswahrscheinlichkeit von $0,01 \%$ verworfen werden. Durch Einbeziehung der Variablen Risikobereitschaft und Risikowahrnehmung erklärt das multiple Regressionsmodell knapp 10\% mehr der Varianz der Vertrauenshandlung $\left(R^{2}=0,324 ; R^{2}{ }_{\text {kor }}=0,315\right)$, als die einfache Regression der Vertrauenshandlung auf die Fach- und Methodenkompetenz $\left(R^{2}=0,226\right.$; $\mathrm{R}^{2}{ }_{\text {korr }}=0,223$ ). Der abschließende Vergleich der standardisierten Regressionskoeffizienten zeigt jedoch, dass die Vornahme einer Vertrauenshandlung, trotz der signifikanten Einflüsse von Risikobereitschaft und Risikowahrnehmung, am stärksten von der Kompetenzwahrnehmung determiniert wird. Nachfolgende Graphik bildet die Differenzen in der Vertrauenshandlung zwischen den Probanden, die ihren Partner als durchschnittlich kompetent wahrgenommen haben (KG), und jenen, die mit einem fach- und methodenkompetenten Kooperationspartner konfrontiert waren (VGI_FMK), unter Berücksichtigung der vertrauensgeberspezifischen Risikobereitschaft (RB) und der situativen Risikowahrnehmung (RW) ab. Der Vergleich der Regressionsflächen zeigt deutlich, dass sowohl bei höchster (bzw. niedrigster) Risikobereitschaft, als auch bei niedrigster (bzw. höchster) Risikowahrnehmung eine weitreichendere Vertrauenshandlung gesetzt wird, wenn der potentielle Kooperationspartner Fach- und Methodenkompetenz vermittelt, als wenn die Kompetenzen des Partners als durchschnittlich oder nicht vorhanden wahrgenommen werden. 


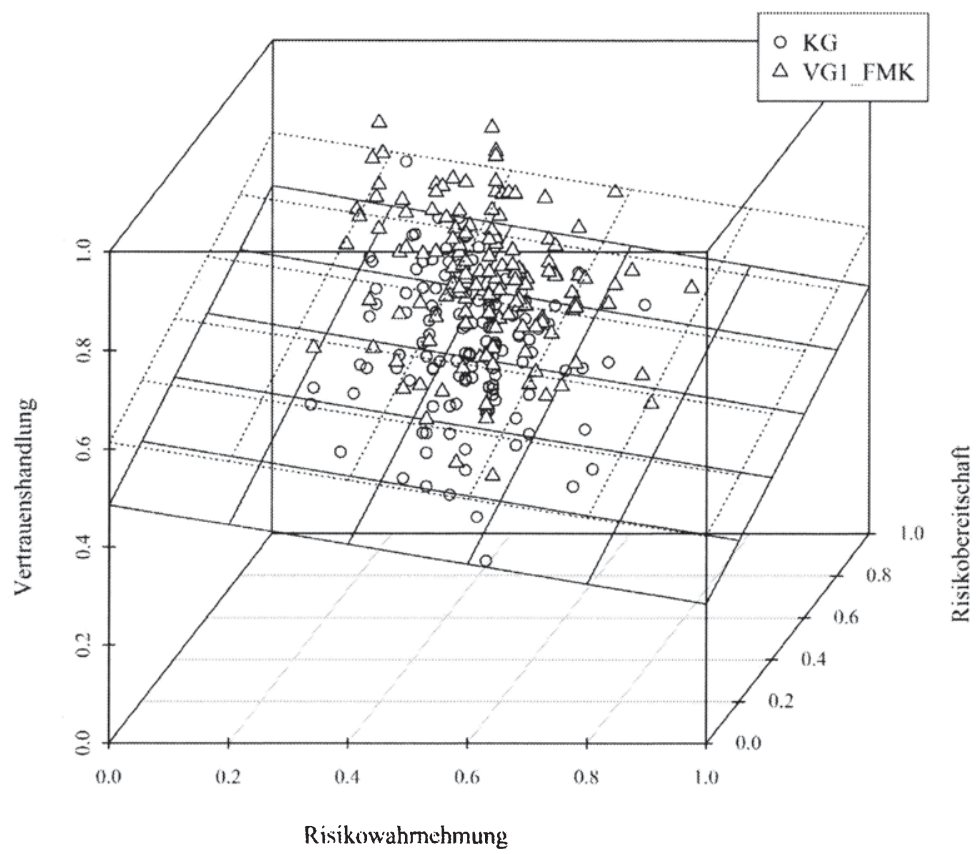

Abbildung 19: Der Zusammenhang zwischen Vertrauen, FMK, RB und RW

Auch zeigt die Abbildung deutlich, dass das Ausmaß, in dem der Vertrauensgeber eine Vertrauenshandlung setzt, grundsätzlich mit zunehmender Risikobereitschaft und sinkender Risikowahrnehmung steigt - unabhängig davon, ob der Vertrauensnehmer als sehr fach- und methodenkompetent oder bloß durchschnittlich kompetent wahrgenommen wird.

\subsubsection{Der Einfluss von Sozial- und Persönlichkeitskompetenz auf Vertrauen unter Berücksichtigung von Dispositionen und situativer Wahrnehmung}

Die Annahme, dass die Wahrnehmung von Sozial- und Persönlichkeitskompetenz die Sicherheit über das Wollen des Vertrauensnehmers $\left(\mathrm{H}_{3 \mathrm{~A}}\right.$ und $\left.\mathrm{H}_{4 \mathrm{~A}}\right)$ erhöht und damit über die Bildung einer Vertrauenserwartung $\left(\mathrm{H}_{3 \mathrm{~B}}\right.$ und $\left.\mathrm{H}_{4 \mathrm{~B}}\right)$ einen positiven Einfluss auf die Vertrauenshandlung nimmt $\left(\mathrm{H}_{3 \mathrm{C}}\right.$ und $\left.\mathrm{H}_{4 \mathrm{C}}\right)$, kann durch die Ergebnisse der nachfolgenden Regressionsanalyse bestätigt werden. Da die Vertrauensbereitschaft bei Wahrnehmung von Sozial- und Persönlichkeitskompetenz einen signifikanten Einfluss auf die Vertrauenshandlung ( $\beta$-Wert: 0,121) nimmt, kann davon ausgegangen werden, dass die Wahrnehmung motivationaler kompetenzbezogener Zusatzinformationen durch Reduktion von Entscheidungsunsicher- 
heit die Bildung einer vertrauensvollen Erwartung begünstigt. Bezugnehmend auf die Ergebnisse der Schätzung beeinflusst die Wahrnehmung von Sozial- und Persönlichkeitskompetenz die Vornahme einer Vertrauenshandlung daher über die Dimension der Vertrauenserwartung höchst signifikant ( $\beta$-Wert: 0,724). Auch die Annahmen hinsichtlich der Wirkung der vertrauensgeberspezifischen Risikobereitschaft und der situativen Risikowahrnehmung werden durch die Ergebnisse der Modellschätzung bestätigt: Während die Vertrauenshandlung höchst signifikant positiv von der vertrauensgeberspezifischen Risikobereitschaft ( $\beta$-Wert: 0,142 ) beeinflusst wird, übt die situative Risikowahrnehmung einen hoch signifikanten negativen Einfluss ( $\beta$-Wert: $-0,126)$ auf die Vertrauenshandlung aus.

\begin{tabular}{|l|c|}
\hline Unabhängige Variable & $\begin{array}{c}\text { Modell B-II } \\
\text { Abhängige Variable:Vertrauenshandlung }\end{array}$ \\
\hline Kompetenzwahrnehmung & $0,724^{* * *}$ \\
\hline Vertrauensbereitschaft & $0,121^{*}$ \\
\hline Risikobereitschaft & $0,142^{* * *}$ \\
\hline Risikowahrnehmung & $-0,126^{* *}$ \\
\hline $\mathrm{R}^{2}$ & 0,590 \\
\hline $\mathrm{R}_{\text {korr }}^{2}$ & 0,584 \\
\hline $\mathrm{F}-$ Wert & $101,131^{* * *}$ \\
\hline $\mathrm{N}$ & 286 \\
\hline${ }^{*} \mathrm{p}<0,05 ;{ }^{* *} \mathrm{p}<0,01 ;{ }^{* * *} \mathrm{p}<0,001$ \\
\hline
\end{tabular}

Tabelle 52: Ergebnisse der erweiterten Prüfung des Einflusses von SPK auf Vertrauen

Das Regressionsmodell ist höchst signifikant: Da der empirische F-Wert stark von Null abweicht $(101,131)$ und den kritischen Wert von 6,10 bei 281 Freiheitsgraden überschreitet, kann die Nullhypothese, derzufolge in der Grundgesamtheit kein kausaler Zusammenhang zwischen Sozial- und Persönlichkeitskompetenzwahrnehmung, Vertrauensbereitschaft, Risikobereitschaft und Risikowahrnehmung einerseits und Vertrauenshandlung andererseits besteht, mit einer Irrtumswahrscheinlichkeit von $0,01 \%$ verworfen werden. Durch Einbeziehung der Variablen Vertrauensbereitschaft, Risikobereitschaft und Risikowahrnehmung erklärt das multiple Regressionsmodell knapp 5\% mehr der Varianz der Vertrauenshandlung $\left(R^{2}=0,590 ; R^{2}{ }_{k o r}=0,584\right)$, als die einfache Regression der Vertrauenshandlung auf die Sozial- und Persönlichkeitskompetenz $\left(R^{2}=0,537 ; R^{2}{ }_{\text {kor }}=0,535\right)$. Der abschließende Vergleich der standardisierten Regressionskoeffizienten zeigt jedoch, dass die Vornahme einer Vertrauenshandlung, trotz der signifikanten Einflüsse von Vertrauensbereitschaft, Risikobereitschaft und Risikowahrnehmung, am stärksten von der Kompetenzwahrnehmung determiniert wird. Nachfolgende Graphik bildet die Differenzen in der Vertrauenshandlung zwischen den Probanden, die ihren Partner als durchschnittlich kompetent wahrgenommen haben 
$(\mathrm{KG})$, und jenen, die mit einem sozial- und persönlichkeitskompetenten Kooperationspartner konfrontiert waren (VG2_SPK), unter Berücksichtigung der vertrauensgeberspezifischen Risikobereitschaft (RB) und der situativen Risikowahrnehmung (RW) ab. ${ }^{729}$ Der Vergleich der Regressionsflächen zeigt deutlich, dass sowohl bei höchster (bzw. niedrigster) Risikobereitschaft, als auch bei niedrigster (bzw. höchster) Risikowahrnehmung in höherem Ausmaß vertraut wird, wenn der potentiellen Kooperationspartner Sozial- und Persönlichkeitskompetenz vermittelt, als wenn die Kompetenzen des Partners als durchschnittlich oder nicht vorhanden wahrgenommen werden.

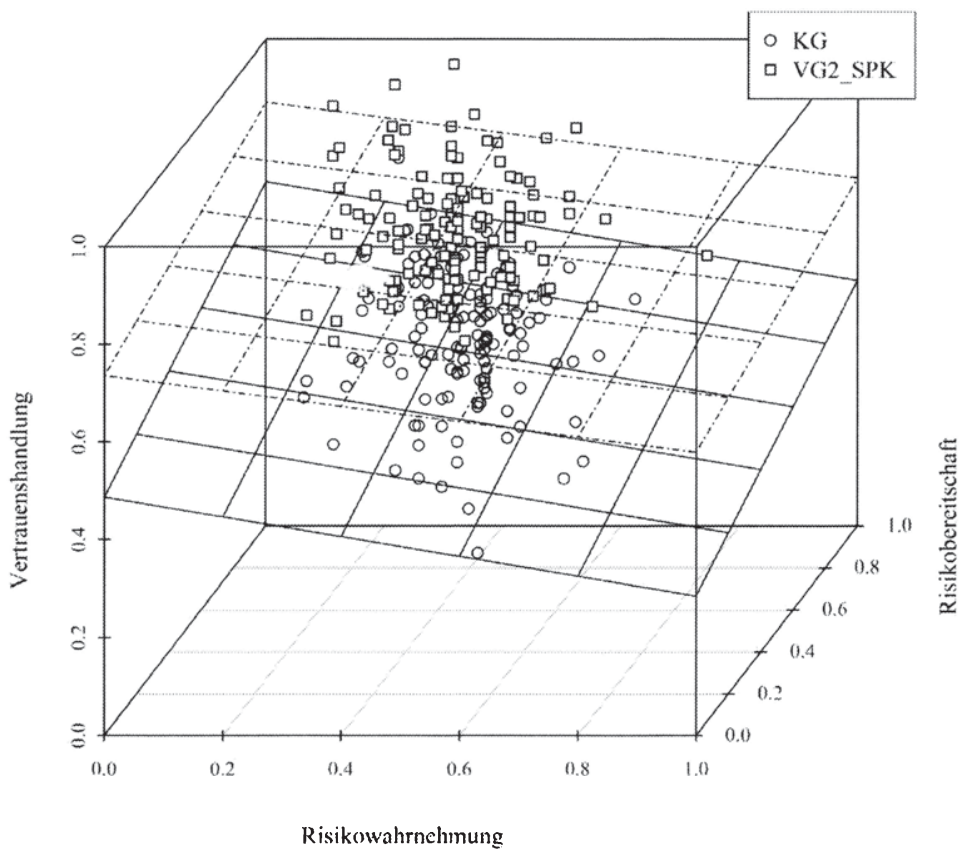

Abbildung 20: Der Zusammenhang zwischen Vertrauen, SPK, RB und RW

Auch zeigt die Abbildung deutlich, dass Vertrauen grundsätzlich mit zunehmender Risikobereitschaft und sinkender Risikowahrnehmung steigt - unab-

729 Da eine 4-dimensionale Darstellung nicht machbar ist, muss auf eine graphische Integration der Vertrauensbereitschaft zugunsten der Variablen ,Risikobereitschaft' und ,Risikowahrnehmung', die auf die Vertrauenshandlung einen signifikanteren Einfluss nehmen als die Vertrauensbereitschaft, verzichtet werden. Ein Grund für die höhere Irrtumswahrscheinlichkeit, mit der H0 (kein Kausalzusammenhang zwischen Vertrauensbereitschaft und Vertrauenshandlung) zurückgewiesen wird, mag darin bestehen, dass die Vertrauenshandlung von der Risikobereitschaft und der Risikowahrnehmung direkt beeinflusst wird, während die Vertrauensbereitschaft einen indirekten Einfluss über die Dimension der Vertrauenserwartung auf die Vornahme einer Vertrauenshandlung nimmt. 
hängig davon, ob der Vertrauensnehmer als sehr sozial- und persönlichkeitskompetent oder bloß durchschnittlich kompetent wahrgenommen wird. Allerdings impliziert der Vergleich der Regressionsflächen, dass Vertrauen mit sinkender Risikowahrnehmung und steigender Risikobereitschaft bei Konfrontation mit einem durchschnittlich kompetenten Partner eher steigt, als wenn der Partner als sozialund persönlichkeitskompetent wahrgenommen wird. Die Abbildung lässt also darauf schließen, dass die vertrauensgeberspezifische Risikobereitschaft und situative Risikowahrnehmung einen stärkeren Einfluss auf die Vertrauenshandlung nehmen, wenn der Kooperationspartner durchschnittlich kompetent ist. Wird der Kooperationspartner als sozial- und persönlichkeitskompetent wahrgenommen, so reduzieren diese motivationalen kompetenzbezogenen Zusatzinformationen die Unsicherheit über dessen Verhalten bereits in so einem hohen Ausmaß, dass die vertrauensgeberspezifische Risikobereitschaft und die situative Risikowahrnehmung bezüglich ihrer Wirkung auf die Vertrauenshandlung in den Hintergrund treten.

\subsubsection{Der Vergleich der Einflussstärke der individuellen Kompetenzen unter Berücksichtigung von Dispositionen und situativer Wahrnehmung}

Zur Analyse, ob Differenzen hinsichtlich der Wirkung der Kontrollvariablen auf die Vertrauenshandlung bei Wahrnehmung unterschiedlicher Kompetenzarten bestehen, wird eine Multigruppenanalyse durchgeführt.

Die Gegenüberstellung der Modelle bestätigt den Befund, dass Vertrauen von der Kompetenzwahrnehmung, unabhängig davon, um welche individuelle Kompetenzart es sich handelt, beeinflusst wird. Der Anteil der durch die Sozial- und Persönlichkeitswahrnehmung und die Kontrollvariablen erklärten Varianz $\left(\mathrm{R}^{2}\right.$ korr $=$ 0,584) der Vertrauenshandlung ist allerdings höher als der Varianzanteil, der durch die Fach- und Methodenkompetenz und die Kontrollvariablen $\left(\mathrm{R}^{2}{ }_{\text {korr }}=\right.$ 0,315) erklärt wird. Auch der Signifikanzwert des Gesamtmodells (F-Wert: 101,131) ist bei Sozial- und Persönlichkeitskompetenzwahrnehmung höher als bei Fach- und Methodenkompetenzwahrnehmung (F-Wert: 33,367). Der visuelle Vergleich der B-Werte der beiden Regressionsmodelle zeigt, dass der positive Einfluss von Kompetenz auf Vertrauen größer ist, wenn der Vertrauensgeber sein Gegenüber als sozial- und persönlichkeitskompetent (B-Wert: 10,236) wahrnimmt. Dieser Unterschied hinsichtlich der Einflussstärke von Fach- und Methodenkompetenz (B-Wert: 5,642) und Sozial- und Persönlichkeitskompetenz ist gemäß des $t$-Tests $\left(t_{e m p}=5,65\right)$ statistisch signifikant. Demnach wird einem potentiellen Kooperationspartner, der Sozial- und Persönlichkeitskompetenz vermittelt, in höherem Maße vertraut, als einem Akteur, der Fach- und Methodenkompetenz zeigt. Es kann daher davon ausgegangen werden, dass motivationale kompetenzbezogene Zusatzinformationen zur Reduktion der Entscheidungsun- 
sicherheit für den Vertrauensgeber grundsätzlich von höherer Bedeutung sind, als technische kompetenzbezogene Zusatzinformationen zur Reduktion der Qualitätsunsicherheit im Zuge der Bildung seiner Vertrauensentscheidung.

\begin{tabular}{|c|c|c|}
\hline Unabhängige Variable & $\begin{array}{c}\text { Modell A-II } \\
\text { Abhängige Variable: } \\
\text { Vertrauenshandhung }\end{array}$ & \begin{tabular}{|c|} 
Modell B-II \\
Abhängige Variable: \\
Vertrauenshandlung
\end{tabular} \\
\hline Kompetenzwahrnehmung & $5,642 * * *$ & $10,236^{* * *}$ \\
\hline Vertrauensbereitschaft & 0,085 & $0,169^{*}$ \\
\hline Risikobereitschaft & $0,374 * * *$ & $0,310 * * *$ \\
\hline Risikowahrnehmung & $-0,447^{* *}$ & $-0,441 * *$ \\
\hline $\mathrm{R}^{2}$ & 0,324 & 0,590 \\
\hline $\mathrm{R}_{\text {korr }}^{2}$ & 0,315 & 0,584 \\
\hline F-Wert & $33,367 * * *$ & $101,131 * * *$ \\
\hline $\mathrm{N}$ & 283 & 286 \\
\hline
\end{tabular}

Tabelle 53: Ergebnisse des Vergleichs der Einflussstärke individueller Kompetenzen und VB, RB, RW auf Vertrauen

Bezugnehmend auf die Wirkung der Dispositionen und der situativen Risikowahrnehmung auf die Vertrauenshandlung zeigt der visuelle Vergleich der B-Werte der beiden Regressionsmodelle, dass der positive Einfluss der generellen Vertrauensbereitschaft gegeben ist, wenn der Vertrauensgeber sein Gegenüber als sozial- und persönlichkeitskompetent wahrnimmt. Dieser Unterschied hinsichtlich des Einflusses von Vertrauensbereitschaft auf die Vornahme einer Vertrauenshandlung ist bei Wahrnehmung unterschiedlicher Kompetenzarten gemäß des tTests $\left(t_{e m p}=2,37\right)$ statistisch signifikant. Allerdings besteht kein statistisch bedeutsamer Unterschied hinsichtlich des Einflusses von Risikobereitschaft und Risikowahrnehmung auf die Vornahme einer Vertrauenshandlung bei Wahrnehmung unterschiedlicher Kompetenzarten. Nachfolgende Graphik bildet abschließend die Differenzen in der Vertrauenshandlung zwischen den Probanden, die ihren Partner als durchschnittlich kompetent wahrgenommen haben (KG) und jenen, die mit einem sozial- und persönlichkeitskompetenten Kooperationspartner konfrontiert waren (VG2_SPK), sowie jenen, die ihren Partner als fach- und methodenkompetent wahrgenommen haben (VG1_FMK) unter Berücksichtigung der vertrauensgeberspezifischen Risikobereitschaft (RB) und der situativen Risikowahrnehmung (RW) ab. Der Vergleich der Regressionsflächen zeigt deutlich, dass sowohl bei höchster (bzw. niedrigster) Risikobereitschaft, als auch bei niedrigster (bzw. höchster) Risikowahrnehmung eher eine Vertrauenshandlung gesetzt wird, wenn der potentiellen Kooperationspartner Sozial- und Persönlichkeitskompetenz vermittelt, als wenn der Kooperationspartner ausgeprägte Fach- und Methoden- 
kompetenz zeigt oder die Kompetenzen des Partners als durchschnittlich oder nicht vorhanden wahrgenommen werden.

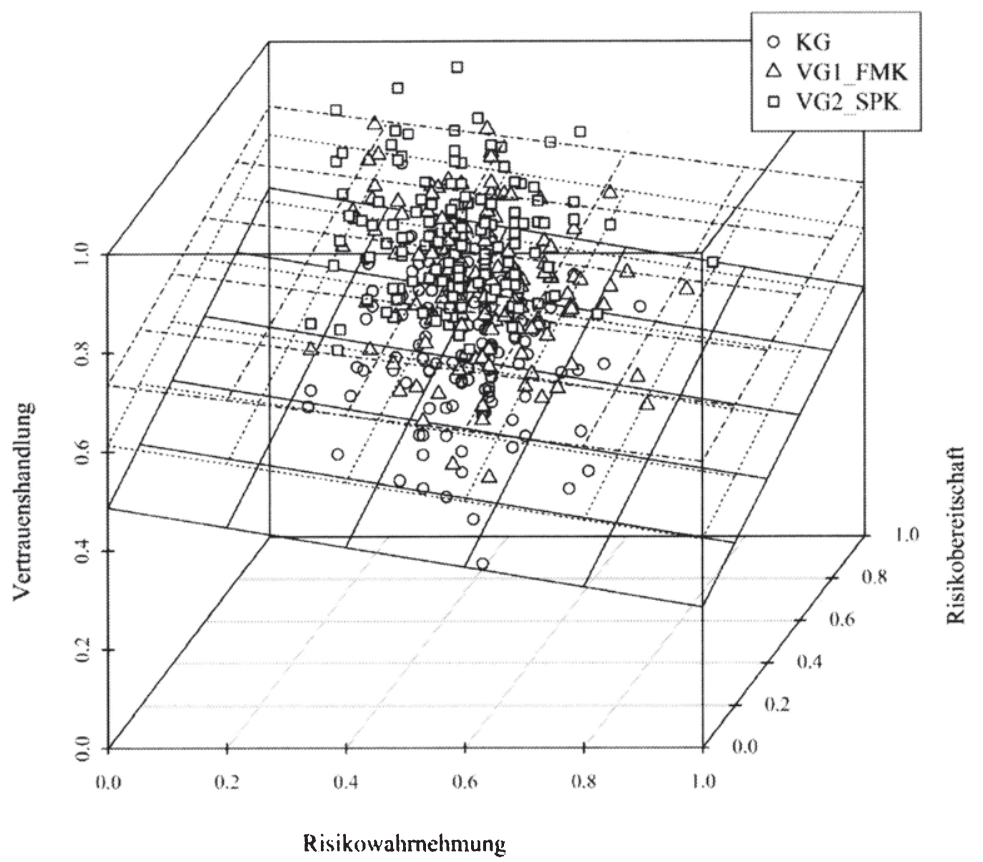

Abbildung 21: Der Zusammenhang zwischen Vertrauen, SPK, FMK, RB und RW

Die Ergebnisse dieser Multigruppenanalyse implizieren, dass die Wahrnehmung von Sozial- und Persönlichkeitskompetenz unter Berücksichtigung der Dispositionen und situativen Wahrnehmung die Vornahme einer Vertrauenshandlung stärker determiniert, als die Fach- und Methodenkompetenzwahrnehmung. Schätzt der Vertrauensgeber den potentiellen Kooperationspartner als sozial- und persönlichkeitskompetent ein, wird er ihm in höherem Maße vertrauen, als einem Interaktionspartner, der Fach- und Methodenkompetenz zeigt, aber bloß durchschnittlich sozial- und persönlichkeitskompetent ist. Weiters konnte gezeigt werden, dass die generelle Vertrauensbereitschaft des Vertrauensgebers bei Konfrontation mit einem sozial- und persönlichkeitskompetenten Partner einen Einfluss auf die Vornahme einer Vertrauenshandlung ausübt. Sie wirkt jedoch nicht auf die Vertrauenshandlung, wenn der Vertrauensgeber den Partner als fach- und methodenkompetent einschätzt. Hingegen besteht kein statistischer Unterschied hinsichtlich der Einflussstärke der vertrauensgeberspezifischen Risikobereitschaft und der situativen Risikowahrnehmung auf die Vornahme einer Vertrauenshandlung bei Wahrnehmung unterschiedlicher individueller Kompetenzarten. 


\subsection{Die Analyse der Korrelationen zwischen den interessierenden Variablen}

Im Zuge der regressionsanalytischen Hypothesenprüfung wurde gezeigt, dass die Kompetenzwahrnehmung, sowie gewisse Dispositionen und die situative Risikowahrnehmung einen signifikanten Einfluss auf die Vornahme einer Vertrauenshandlung nehmen. Dementsprechend kann auch von einer Korrelation zwischen diesen Prädiktoren und der abhängigen Variable ausgegangen werden. Während diese Korreliertheit der Prädiktoren mit der abhängigen Variable erwünscht ist (Ziel: maximale Korrelation), ist die Korreliertheit der Prädiktoren untereinander hingegen nicht erwünscht (Ziel: minimale Korrelation). ${ }^{730}$

Aus der nachfolgenden Tabelle, die die Korrelationskoeffizienten zwischen den Variablen ,Vertrauenshandlung', ,Kompetenzwahrnehmung' (Dummy-Variable, wobei hohe Fach- und Methodenkompetenz mit , 1' und durchschnittliche Kompetenzwahrnehmung mit, $0^{\star}$ kodiert ist), ,Vertrauensbereitschaft', ,Risikobereitschaft' und ,Risikowahrnehmung' abbildet, wird ersichtlich, dass zwischen der vertrauensgeberspezifischen Risikobereitschaft und der situativen Risikowahrnehmung ein schwacher linearer Zusammenhang $(-0,302)$ besteht. ${ }^{731}$ Nachdem die Nullhypothese, derzufolge in der Grundgesamtheit kein linearer Zusammenhang zwischen den Variablen Risikobereitschaft und Risikowahrnehmung bei Konfrontation mit einem fach- und methodenkompetenten oder durchschnittlich kompetenten Partner besteht, mit einer Irrtumswahrscheinlichkeit von $0,01 \%$ zu verwerfen ist, kann davon ausgegangen werden, dass Akteure, die über eine hohe (bzw. geringe) Risikobereitschaft verfügen, Situationen grundsätzlich als wenig (bzw. hoch) riskant wahrnehmen bzw. dass Akteure, die eine Situation als hoch (bzw. wenig) riskant wahrnehmen, generell über eine geringe (bzw. hohe) Risikobereitschaft verfügen.

730 Vgl. Schendera (2008), S. 120, sowie die Erläuterungen zu Multikollinearität in Teil V Kap. 5.2.

731 Die Beträge der Korrelationskoeffizienten können anhand folgender Richtwerte interpretiert werden (Brosius 2002, S. 501): $0=$ keine Korrelation, $>0$ bis 0,2 = sehr schwache Korrelation, 0,2 bis $0,4=$ schwache Korrelation, 0,4 bis $0,6=$ mittlere Korrelation, 0,6 bis $0,8=$ starke Korrelation, 0,8 bis $<1$ $=$ sehr starke Korrelation, 1 = perfekte Korrelation. 
Korrelationen nach Pearson - A

\begin{tabular}{|c|c|c|c|c|c|}
\hline \multicolumn{6}{|c|}{ Korrelationen nacn Pearson - A } \\
\hline & $\begin{array}{c}\text { Vertrauens- } \\
\text { handlung }\end{array}$ & Kompetenz & $\begin{array}{l}\text { Vertrauens- } \\
\text { bereitschaft }\end{array}$ & $\begin{array}{c}\text { Risiko- } \\
\text { bereitschaft }\end{array}$ & $\begin{array}{c}\text { Risiko- } \\
\text { wahmehmung }\end{array}$ \\
\hline $\begin{array}{c}\text { Vertrauens- } \\
\text { handlung }\end{array}$ & 1,000 &, $475^{\cdots}$ &, 104 &, $306^{\ldots \ldots}$ &,$- 178^{\circ}$ \\
\hline Kompetenz &, $475^{\cdots \cdots}$ & 1,000 & ,088 & ,096 &,- 107 \\
\hline $\begin{array}{l}\text { Vertrauens- } \\
\text { bereitschaft }\end{array}$ &, 104 & ,088 & 1,000 &, 098 &,- 106 \\
\hline $\begin{array}{c}\text { Risiko- } \\
\text { bereitschaft }\end{array}$ &, $306^{\cdots \cdots}$ &, 096 & ,098 & 1,000 &,$- 3022^{\cdots}$ \\
\hline $\begin{array}{c}\text { Risiko- } \\
\text { wahmehmung }\end{array}$ &,$- 178^{\circ}$ &,- 107 &,- 106 &,$- 302^{\cdots}$ & 1,000 \\
\hline \multicolumn{6}{|c|}{ ***. Die Komelation ist auf dem Niveau von 0,001 (2-seitig) signifikant } \\
\hline \multicolumn{6}{|c|}{ **. Die Korrelation ist auf dem Niveau von 0,01 (2-seitig) signifikant. } \\
\hline \multicolumn{6}{|c|}{ * Die Korrelation ist auf dem Niveau von 0,05 (2-seitig) signifikant. } \\
\hline
\end{tabular}

Tabelle 54: Korrelationskoeffizienten zwischen FMK, VB, RB, RW und Vertrauen

Der bereits im Zuge der vorangegangenen Regressionsanalysen bestätigte $\mathrm{Zu}$ sammenhang zwischen Sozial- und Persönlichkeitskompetenz, vertrauensgeberspezifischer Risikobereitschaft, situativer Risikowahrnehmung, sowie genereller Vertrauensbereitschaft (wodurch sich das SPK-Modell vom FMK-Modell unterscheidet) einerseits und Vertrauen andererseits, wird durch die Ergebnisse der Korrelationsanalyse bestätigt. Unabhängig von diesen signifikanten, mehr oder weniger starken linearen Zusammenhängen zwischen den Prädiktoren und der abhängigen Variable, zeigt die Analyse der Korrelation zwischen Risikobereitschaft und Risikowahrnehmung, dass ein schwacher linearer Zusammenhang $(-0,210)$ zwischen diesen beiden Kontrollvariablen besteht. Nachdem die Nullhypothese auch bei Konfrontation mit einem sozial- und persönlichkeitskompetenten oder durchschnittlich kompetenten Partner mit einer Irrtumswahrscheinlichkeit von $0,01 \%$ verworfen werden kann, ist davon auszugehen, dass Akteure, die über eine hohe (bzw. geringe) Risikobereitschaft verfügen, Situationen grundsätzlich als wenig (bzw. hoch) riskant wahrnehmen bzw. dass Akteure, die eine Situation als hoch (bzw. wenig) riskant wahrnehmen, generell über eine geringe (bzw. hohe) Risikobereitschaft verfügen. Der lineare Zusammenhang zwischen vertrauensgeberspezifischer Risikobereitschaft und situativer Risikowahrnehmung besteht demnach unabhängig von der Art der wahrgenommenen individuellen Kompetenzen. 
Korrelationen nach Pearson - B

\begin{tabular}{|c|c|c|c|c|c|}
\hline & $\begin{array}{c}\text { Vertrauens- } \\
\text { handhung }\end{array}$ & Kompetenz & $\begin{array}{l}\text { Vertrauens- } \\
\text { bereitschaft }\end{array}$ & $\begin{array}{c}\text { Risiko- } \\
\text { bereitschaft }\end{array}$ & $\begin{array}{c}\text { Risiko- } \\
\text { wahrnehmung }\end{array}$ \\
\hline $\begin{array}{c}\text { Vertrauens- } \\
\text { handhung }\end{array}$ & 1,000 &, $733^{\cdots}$ &, $130 *$ &, $183^{n *}$ &,$- 193^{\circ}$ \\
\hline Kompetenz &, $733^{\ldots \ldots}$ & 1,000 &, 038 &, 001 &,- 052 \\
\hline $\begin{array}{l}\text { Vertrauens- } \\
\text { bereitschaft }\end{array}$ &, $130^{*}$ &, 038 & 1,000 &, 106 &,- 001 \\
\hline $\begin{array}{c}\text { Risiko- } \\
\text { bereitschaft }\end{array}$ &, $183^{\star *}$ &, 001 &, 106 & 1,000 &,$- 210^{\cdots \cdots}$ \\
\hline $\begin{array}{c}\text { Risiko- } \\
\text { wahrnehmung }\end{array}$ &,$- 193^{* *}$ &,- 052 &,- 001 &,$- 210^{n \times n}$ & 1,000 \\
\hline \multicolumn{6}{|c|}{ ***. Die Korrelation ist auf dem Niveau von 0,001 (2-seitig) signifikant. } \\
\hline \multicolumn{6}{|c|}{ **. Die Korrelation ist auf dem Niveau von 0,01 (2-seitig) signifikant. } \\
\hline \multicolumn{6}{|c|}{ *. Die Korrelation ist auf dem Niveau von 0,05 (2-seitig) signifikant. } \\
\hline
\end{tabular}

Tabelle 55: Korrelationskoeffizienten zwischen SPK, VB, RB, RW und Vertrauen

Die Ergebnisse der Korrelationsanalysen lassen darauf schließen, dass die Wirkung der Wahrnehmung individueller Kompetenzen auf die Vertrauenshandlung nicht direkt durch die Variablen ,Vertrauensbereitschaft", ,Risikobereitschaft" und ,Risikowahrnehmung' beeinflusst (bzw. moderiert) wird. Dieser Schluss wird durch die Berechnung von Interaktionseffekten ${ }^{732}$ bestätigt: Die Schätzung der Modelle A-III und B-III durch Einbeziehung von drei Interaktionsvariablen (Kompetenz*VB, Kompetenz*RB, Kompetenz*RW) in Modell A-II und B-II zeigt, dass keiner der Interaktionsterme eine signifikante Wirkung auf die Vertrauenshandlung ausübt. Demnach moderieren weder die kontrolliert miterhobenen Dispositionen und die situative Wahrnehmung den Einfluss der Kompetenzwahrnehmung auf die Vornahme einer Vertrauenshandlung, noch handelt es sich bei der Kompetenzwahrnehmung um eine Moderatorvariable, die die Stärke, Signifikanz und/oder Einflussrichtung der Effekte der Vertrauensbereitschaft, Risikobereitschaft und Risikowahrnehmung auf die Vornahme einer Vertrauenshandlung beeinflusst.

\section{DIE ZUSAMMENFASSUNG DER UNTERSUCHUNGSERGEBNISSE}

Abschließend werden die im Zuge dieser Arbeit aufgestellten und empirisch geprüften Hypothesen, sowie die Annahmen zu den personen- und situationsspezifischen Variablen zusammengeführt. Entsprechend der regressionsanalytischen Ergebnisse der experimentellen Untersuchung (vgl. Teil VI Kap. 2) nimmt die Wahrnehmung individueller Kompetenzen des Vertrauensnehmers einen positiven Einfluss auf die Vornahme einer Vertrauenshandlung durch den Vertrauensgeber im Kontext zwischenbetriebliche Kooperation. Die nachfolgende Tabelle gibt

732 Zu Interaktionseffekten vgl. Urban/Mayerl (2006), S. 295 ff. 
einen Überblick über die anhand der Ergebnisse der durchgeführten Experimente bestätigten Hypothesen zum Einfluss von Fach-, Methoden-, Sozial- und Persönlichkeitskompetenz auf die Entstehung von Vertrauen in zwischenbetrieblichen Kooperationen.

\begin{tabular}{|c|c|}
\hline Hypothese & Bestätigung \\
\hline $\begin{array}{l}\mathrm{H}_{1}: \text { Die wahrgenommene Fachkompetenz des Vertrauensneh- } \\
\text { mers hat einen positiven Einfluss auf die Vornahme einer } \\
\text { Vertrauenshandlung durch den Vertrauensgeber. }\end{array}$ & $\checkmark$ \\
\hline $\begin{array}{l}\mathrm{H}_{2}: \text { Die wahrgenommene Methodenkompetenz des Vertrauens- } \\
\text { nehmers hat einen positiven Einfluss auf die Vornahme } \\
\text { einer Vertrauenshandlung durch den Vertrauensgeber. }\end{array}$ & $\checkmark$ \\
\hline $\begin{array}{l}\mathrm{H}_{3}: \text { Die wahrgenommene Sozialkompetenz des Vertrauensneh- } \\
\text { mers hat einen positiven Einfluss auf die Vornahme einer } \\
\text { Vertrauenshandlung durch den Vertrauensgeber. }\end{array}$ & $\checkmark$ \\
\hline $\begin{array}{l}\mathrm{H}_{4}: \text { Die wahrgenommene Persönlichkeitskompetenz des Ver- } \\
\text { trauensnehmers hat einen positiven Einfluss auf die Vor- } \\
\text { nahme einer Vertrauenshandlung durch den Vertrauens- } \\
\text { geber. }\end{array}$ & $\checkmark$ \\
\hline
\end{tabular}

Tabelle 56: Bestătigte Hypothesen zum Einfluss individueller Kompetenzen auf Vertrauen

Basierend auf theoretischen Annahmen und empirischen Ergebnissen wurde zum Abschluss des Teils $V$ ein Modell präsentiert, das die individuellen Kompetenzen unter Berücksichtigung der generellen Vertrauensbereitschaft, der vertrauensgeberspezifischen Risikobereitschaft und der situationsspezifischen Risikowahrnehmung mit der Vertrauenshandlung verbindet. Anhand der Ergebnisse der Regressions- und Korrelationsanalysen konnte gezeigt werden, dass die vertrauensgeberspezifische Risikobereitschaft und die situative Risikowahrnehmung - unabhängig von der Art der wahrgenommenen Kompetenzen - die Vornahme einer Vertrauenshandlung beeinflussen, während die generelle Vertrauensbereitschaft nur bei Wahrnehmung von Sozial- und Persönlichkeitskompetenz Einfluss auf die Vornahme einer Vertrauenshandlung nimmt. Das Modell (Abb. 22) fasst die empirisch bestätigten Zusammenhänge graphisch zusammen, wobei die Stärke der Pfeile die Bedeutung des Einflusses der jeweiligen Variable ausdrückt. 

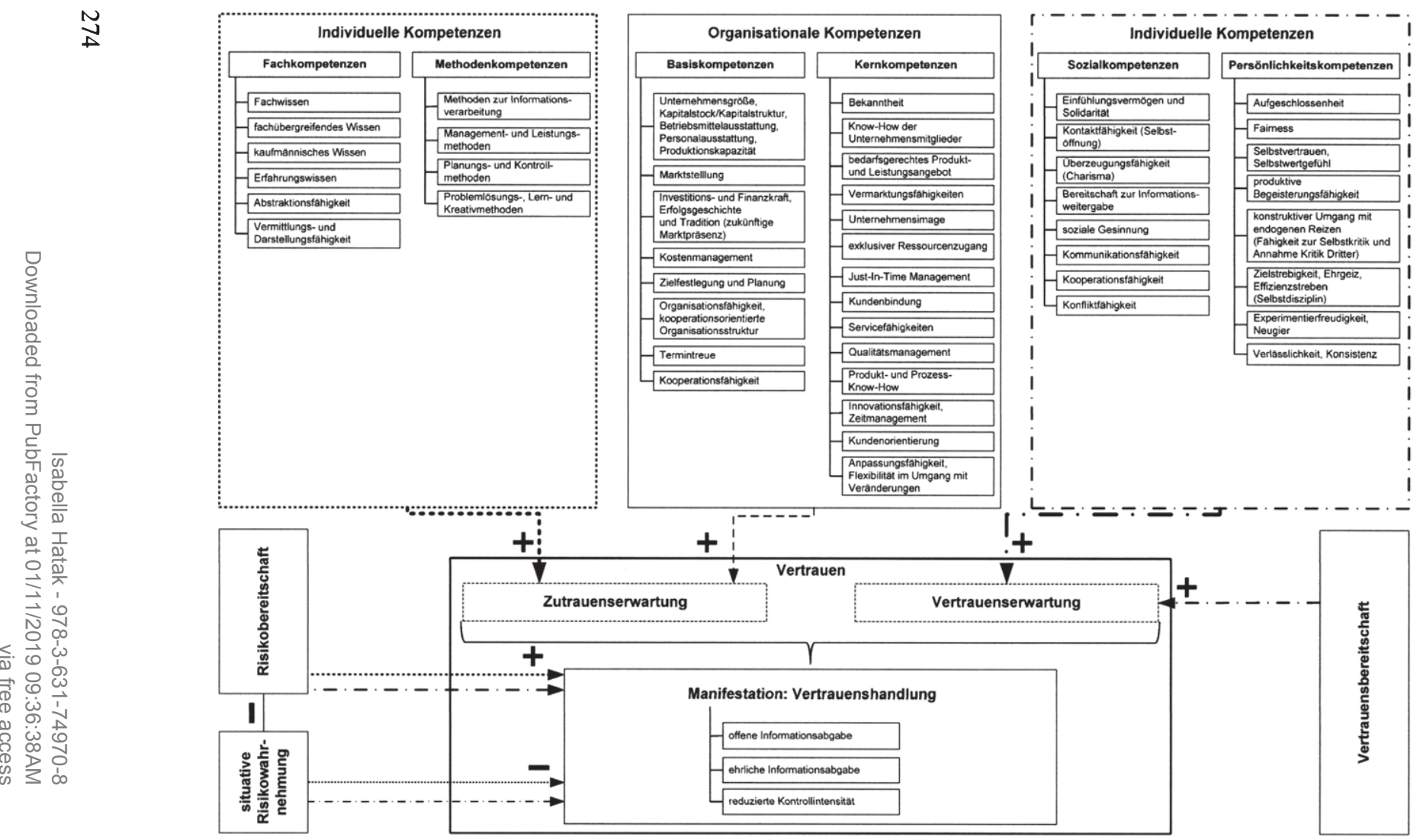

Abbildung 22: Kompetenz als Determinante der Entstehung von Vertrauen 
In diesem Zusammenhang muss darauf hingewiesen werden, dass die zwei in Abb. 22 breit strichliert umrahmten Felder und der breit strichlierte Pfeil nicht empirisch untersucht wurden. Vertrauen wurde über die Vertrauenshandlung gemessen, da die den Handlungen zugrunde liegenden Erwartungen nicht direkt erfasst werden können. Aus forschungspragmatischen Gründen und aufgrund der in dieser Arbeit vertretenen Auffassung, dass Vertrauen zwischen Unternehmen letztlich immer durch die diesen Unternehmen angehörenden Individuen begründet und zerstört wird, wurde auf eine Überprüfung der Annahmen zum Einfluss organisationaler Kompetenzen auf Vertrauen verzichtet. Die nachfolgende Abbildung (Abb. 23) gibt Aufschluss über die im Zuge des Experiments untersuchten Beziehungen und damit über die messtechnische Annäherung an das Modell.

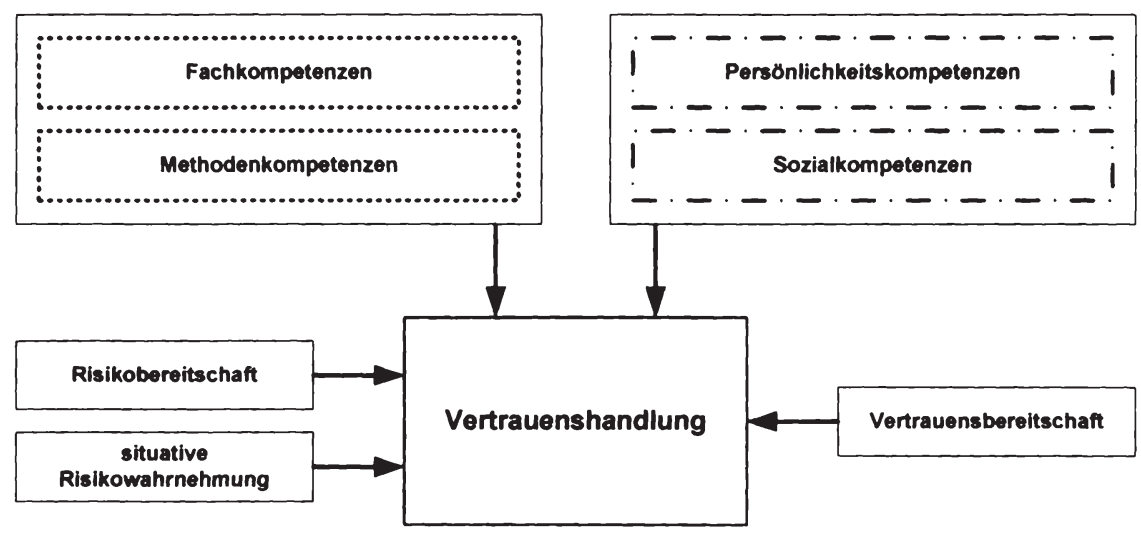

Abbildung 23: Messmodell 
Isabella Hatak - 978-3-631-74970-8 Downloaded from PubFactory at 01/11/2019 09:36:38AM via free access 


\section{TEIL VII: SCHLUSSBETRACHTUNG}

Der vorliegende Teil hat zum Ziel, die Untersuchungsbefunde vor dem Hintergrund des in der Einleitung dargelegten Erklärungsinteresses dieser Arbeit zu diskutieren. Im Anschluss an die Zusammenführung der Ergebnisse dieser Arbeit (Kap. 1), wird auf den Erklärungsbeitrag der Arbeit eingegangen (Kap. 2). Abschließend werden die inhaltlichen und methodischen Grenzen der Untersuchung erläutert und Anregungen für weiterführende Forschungsaktivitäten geliefert (Kap. 3).

\section{ZUSAMMENFASSUNG DER ERGEBNISSE}

Das Erklärungsinteresse der vorliegenden Arbeit bestand in der Generierung eines Modells, das den Einfluss unterschiedlicher Kompetenzarten auf die Entstehung von Vertrauen in zwischenbetrieblichen Kooperationen abbildet. Durch Konsolidierung interdisziplinärer Erklärungsansätze von Vertrauen und Kompetenz sollten folgende Forschungsfrage beantwortet werden:

- Wie wird Kompetenz definiert, welche Kompetenzarten gibt es und wie kann man sie operationalisieren?

- Welche Arten von Kompetenz beeinflussen die Evolution von Vertrauen?

- Wie beeinflussen die einzelnen Kompetenzarten des Vertrauensnehmers die Evolution von Vertrauen auf der Seite des Vertrauensgebers?

Um sich diesem Forschungsziel zu nähern, wurde zunächst die Bedeutung von Vertrauen aus soziologischer Perspektive ${ }^{733}$ und aus Sicht der Neuen Institutionenökonomie analysiert. Die Konsolidierung der Ansätze, sowie die Abgrenzung des Vertrauenskonstrukts von verwandten Konstrukten, machten deutlich, dass die modelltheoretische Erfassung und Erklärung der Vertrauensentscheidung als Reaktion auf die subjektive Unsicherheit über das Verhalten des Vertrauensnehmers eine Differenzierung zwischen subjektivem Entscheidungskalkül und objektiv beobachtbarem Verhalten des Vertrauensgebers erfordert: Eine Vertrauensentscheidung im Sinne eines Schenkens von Vertrauen liegt nur dann vor, wenn eine Vertrauenshandlung vorgenommen wurde, die durch eine Vertrauenserwartung motiviert wurde. Während die Vertrauenserwartung der subjektiven Einschätzung des Vertrauensgebers entspricht, dass der Vertrauensnehmer freiwillig auf opportunistisches Verhalten verzichtet, zeigt sich die Vertrauenshandlung in der freiwilligen Erbringung einer riskanten Vorleistung (z.B. offene und ehrliche Informationsabgabe an den Vertrauensnehmer) und in einem Verzicht auf explizite vertragliche Sicherungs- und Kontrollmaßnahmen (z.B. Reduktion der Kontrollintensität) zur Begrenzung von Opportunismusspielräumen. Dieser Ent- 
schluss, sich dem Hold Up-Risiko ungeschützt auszusetzen, ist neben der Höhe der Vertrauenserwartung, die ihrerseits von der generellen Vertrauensbereitschaft beeinflusst wird, zusätzlich abhängig von der Höhe des wahrgenommenen situationsspezifischen Risikos, sowie der subjektiven Risikobereitschaft des Vertrauensgebers. Während sich die generelle Vertrauensbereitschaft ausschließlich auf die Bereitschaft bezieht, durch das Verhalten des Vertrauensnehmers begründete Risiken in der Vertrauenserwartung zu absorbieren, umfasst die personenspezifische Risikobereitschaft auch jene Risiken, die nicht nur durch die Motivation des Vertrauensnehmers, sondern auch durch unsichere exogene Ereignisse begründet werden. Dagegen ergibt sich die Situationsspezifität der Risikowahrnehmung daraus, dass die Art und die Höhe der Konsequenzen der Vertrauenshandlung als gewählte Alternative vorwiegend situationsbedingt und kaum vom Vertrauensnehmer beeinflussbar sind.

Zur Konkretisierung der kausalen Beziehung zwischen Kompetenz und Vertrauen unter Berücksichtigung des Einflusses von personen- und situationsbedingten Faktoren wurde der Fokus auf interdependente Entscheidungssituationen im Bereich zwischenbetriebliche Kooperation gerichtet. Einerseits erfüllte dieser Kontext die konstitutiven Bedingungen einer Beziehung, die sich durch den Vertrauensmechanismus koordinieren lässt, d.h. durch Unsicherheit über das Verhalten des Kooperationspartners aufgrund asymmetrischer Informationsverteilung, potentiellen Schaden, etc. charakterisiert ist. Andererseits bot die aus principalagent-theoretischer Sicht analysierte Untersuchungsumgebung einen strukturellen Rahmen zur systematischen Analyse der Rolle von Kompetenz im Vertrauensentstehungsprozess. Da hier die Auffassung vertreten wird, dass Vertrauen zwischen Unternehmen letztlich immer durch Individuen, die diesen Unternehmen angehören, begründet und zerstört wird, wurde die Vertrauensbeziehung als Beziehung zwischen Individuen analysiert.

Die in dieser Arbeit verfolgte Modellierung des Einflusses von Kompetenz auf die Entstehung von Vertrauen in zwischenbetrieblichen Kooperationen erfolgte anhand einer dimensionalen Analyse nach Faix/Krupp ${ }^{734}$. Im Anschluss an die Klärung, welche theoretischen Merkmalsdimensionen durch die theoretischen Konstrukte Vertrauen und Kompetenz bezeichnet werden, wurden die Dimensionen des Kompetenzkonstrukts entsprechend der Erkenntnisse der Kompetenzforschung in gültige und voneinander unabhängige Faktoren aufgespalten. Basierend auf subjektiven Relevanzüberlegungen, die sich ihrerseits auf die Ergebnisse erfolgter Untersuchungen in den Bereichen Kompetenz- und Vertrauensforschung stützen, wurden in einem dritten Schritt konsensfähige und gültige Indikatoren für die festgelegten Merkmalsdimensionen bzw. Faktoren bestimmt, die als deren empirische Äquivalente Rückschlüsse auf die Merkmalsdimensionen bzw. Faktoren, 
und damit auf die theoretischen Konstrukte Vertrauen und Kompetenz ermöglichten. Die Zusammenführung interdisziplinärer Ansätze und empirischer Ergebnisse resultierte in einem Modell, durch das die Kausalbeziehung zwischen Kompetenz und Vertrauen einer Operationalisierung zugänglich gemacht wurde.

Die empirische Prüfung des Modells erfolgte im Zuge eines verhaltenswissenschaftlich-ökonomischen Laborexperiments. Die Entscheidung für dieses Forschungsdesign begründete sich dadurch, dass es durch Variation einer oder mehrerer unabhängiger Variablen und der Feststellung der Veränderung nachgelagerter Variablen eine Analyse kausaler Zusammenhänge unter kontrollierten Bedingungen ermöglicht: „Das Experiment lässt sich insofern als die vornehmste aller Forschungsmethoden bezeichnen, als nur dieses Kausalbeziehungen festzustellen erlaubt. ${ }^{6735}$ Die Verbindung der laborexperimentellen Variation der unabhängigen Variable mit psychometrischen Tests zur Erfassung von Dispositionen und der situativen Wahrnehmung zielte auf eine Vergleichbarkeit der Testresultate ab. Weiters erlaubte die Anwendung dieser differentiellen Methode die Untersuchung von Kausalbeziehungen unter kontrollierten Bedingungen, da potentielle Störvariablen durch die Erhebung ihrer Ausprägungen im Zuge psychometrischer Tests zu Kontrollvariablen transformiert werden konnten.

Vor dem Hintergrund der Ergebnisse der Konsolidierung der interdisziplinären Erklärungsansätze und der regressionsanalytischen Auswertung der empirischen Daten erfolgt nun die Beantwortung der in Teil I dieser Arbeit gestellten Forschungsfragen:

\section{Wie wird Kompetenz definiert, welche Kompetenzarten gibt es und wie kann man Kompetenz operationalisieren?}

Basierend auf handlungstheoretischen Überlegungen lässt sich Kompetenz als dynamisches Konzept anhand der Dimensionen ,Aufgabe', ,Fähigkeit' und ,Aufgabenerfüllung eingrenzen: „Since competence implies an intention to achieve some desired result (goal) through action, and since action-taking requires some notions of cause and effect, knowledge and the application of knowledge through action are the foundation of the concepts of skills, capabilities and (ultimately) competence. ${ }^{\text {"736 }}$ Kompetenz wird demnach als Übereinstimmung der Anforderungen der Aufgabe an den Aufgabenerfüller mit den Fähigkeiten des Aufgabenerfüllers definiert. Damit lässt sich das Kompetenzausmaß am Grad der Aufgabenerfüllung erkennen.

Grundsätzlich ist zwischen organisationalen und individuellen Kompetenzen zu differenzieren, wobei ,[...] most organizational competencies start with the 
individual.“" ${ }^{737} \mathrm{Zu}$ den organisationalen Kompetenzen, verstanden als die aufgabenbezogene Bündelung organisationaler Fähigkeiten, die in Bezug auf ihren Beitrag zur Erfüllung von Aufgaben bewertet werden, zählen die Basis- und Kernkompetenzen. Während durch Basiskompetenzen auf zufriedenstellendem Niveau übliche Unternehmensaufgaben erfüllt werden, lassen sich Kernkompetenzen vorrangig an ihrer Wettbewerbswirkung festmachen und von Basiskompetenzen durch ihre Merkmale der Nicht-Imitierbarkeit, Stiftung von Kundennutzen und Transferierbarkeit abgrenzen. Individuelle Kompetenzen unterscheiden sich von organisationalen Kompetenzen durch ihre Personenbindung. Anhand der Dimensionen Wissen, Erfahrungen, Einstellungen und Persönlichkeitsmerkmale ergibt sich eine Differenzierung zwischen den folgenden vier Arten individueller Kompetenz: Während unter Fachkompetenz die Übereinstimmung der technischen Anforderungen einer Aufgabe mit den fachlichen Fertigkeiten und Fähigkeiten des Aufgabenerfüllers zu verstehen ist, stellt die methodische Kompetenz die Summe der persönlichen Problemlösungsverfahren dar, die die Bewältigung neuartiger Situationen, für die das Fachwissen nicht mehr ausreicht, ermöglicht. Bei der Sozialkompetenz handelt es sich um die Kompetenz, soziale Austauschbeziehungen mit Hilfe von kommunikativen Mitteln zu initiieren, zu erhalten und zu beenden. Hierfür sind Komponenten der Persönlichkeitskompetenz, verstanden als Übereinstimmung motivationaler Anforderungen einer Aufgabe mit den von anderen an der Person des Aufgabenerfüllers wahrgenommenen personalen Fähigkeiten, wie Aufrichtigkeit, Kritik- und Konfliktfähigkeit, Selbstorganisation und Rollendistanz im Umgang mit sich selbst notwendig.

Als handlungstheoretisches Konstrukt ist Kompetenz nur über das Handeln, d.h. im Zuge der bzw. anhand des Grades der Aufgabenerfüllung, zu erschließen. Um das latente Kompetenzkonstrukt daher einer Operationalisierung zugänglich zu machen, müssen direkt wahrnehmbare Phänomene für die festgelegten Kompetenzarten identifiziert werden, die als deren empirische Äquivalente Rückschlüsse auf die festgelegten Kompetenzarten und damit auf das theoretische Kompetenzkonstrukt erlauben. Ziel der Identifikation sekundäranalytisch abgesicherter Indikatoren ist es also, ,,den Begriffen einer Wissenschaft größere Präzision zu geben, sie somit empirisch gehaltvoller" ${ }^{\text {“738 }}$ und einer Messung zugänglich zu machen. Nachdem, basierend auf den Erkenntnissen der Kompetenzforschung, in einem ersten Schritt nach den Besitzverhältnissen von Kompetenzen zwischen organisationalen und individuellen Kompetenzen als Merkmalsdimensionen differenziert wurde, erfolgte in einem zweiten Schritt die Aufspaltung organisationaler Kompetenzen in die Faktoren Basis- und Kernkompetenzen, sowie der individuellen Kompetenzen in die Faktoren Fach-, Methoden-, Sozial- und Persönlichkeitskompetenzen, wobei diese Aufspaltung ihre begriffliche und inhaltliche Fun-

737 Doz (1996), S. 157

$738 \quad$ Friedrichs (1990), S. 78 
dierung in der Kompetenzforschung findet. Ebenso wie die vorangegangenen Schritte der dimensionalen Analyse als Technik zur Operationalisierung theoretischer Konstrukte beruht die Bestimmung reflektiver Indikatoren als exemplarische Manifestationen einer latenten Variable ${ }^{739}$ auf subjektiven Relevanzüberlegungen, die sich ihrerseits auf die Ergebnisse erfolgter Untersuchungen im Bereich Kompetenzforschung stützen. So fungieren Marktstellung, Investitionsund Finanzkraft, Kostenmanagement, etc. als empirische Äquivalente der Basiskompetenz, sowie exklusiver Ressourcenzugang, Just-In-Time Management, Innovationsfähigkeit, etc. als Indikatoren der Kernkompetenz. Fachkompetenz kann anhand der Übereinstimmung von Abstraktionsfähigkeit, kaufmännischem Wissen, Fachwissen, etc. mit den technischen Anforderungen der Aufgabe gemessen werden. Methodenkompetenz lässt sich anhand des Einsatzes von Managementund Leistungsmethoden, Planungs- und Kontrollmethoden, etc. im Zuge der Aufgabenerfüllung bewerten. Zu den Indikatoren der Sozialkompetenz zählen Kontaktfähigkeit, Einfühlungsvermögen, Bereitschaft zur Informationsabgabe, Kommunikations-, Kooperations- und Konfliktfähigkeit, etc. Bei Aufgeschlossenheit, produktiver Begeisterungsfähigkeit, Fähigkeit zur Selbstkritik, Zielstrebigkeit, Verlässlichkeit, Experimentierfreudigkeit, etc. handelt es sich um empirische Äquivalente der Persönlichkeitskompetenz.

\section{Welche Arten von Kompetenz beeinflussen die Evolution von Vertrauen?}

Vertrauen zeigt sich in Handlungen, die darauf schließen lassen, dass sich der Vertrauensgeber durch ihre Vornahme freiwillig einem Risiko aussetzt. Unter Bezugnahme auf das Forschungsinteresse dieser Arbeit sind kommunikative Handlungen für das Entstehen und Aufrechterhalten von zwischenbetrieblichen Kooperationsbeziehungen von zentraler Bedeutung: Da der Kooperationspartner die vom Vertrauensgeber erhaltenen Informationen zum Nachteil des Vertrauensgebers nutzen kann, macht sich der Vertrauensgeber durch die Offenlegung von akkuraten Informationen gegenüber seinem Partner verwundbar. Demnach handelt es sich bei offener und ehrlicher Informationsabgabe um sekundäranalytisch abgesicherte Indikatoren der freiwilligen Erbringung einer riskanten Vorleistung. Da auch der Verzicht auf explizite Kontroll- und Sicherungsmaßnahmen zur Begrenzung der Opportunismusspielräume des Partners ein konstitutives Merkmal von Vertrauen darstellt, fungiert die reduzierte Kontrolle der Informationshandlungen des Partners durch den Vertrauensgeber als weiteres empirisches Äquivalent für die Vornahme einer Vertrauenshandlung.

739 Vgl. Eberl (2004), S. 3. Die reflektive Spezifikationsart kennzeichnet sich dadurch, dass die Ausprägungen der beobachtbaren Variablen kausal durch die latente Variable verursacht werden, womit die Unterstellung, dass Veränderungen der latenten Variable zu Veränderungen aller beobachtbaren Indikatoren gleichermaßen führen, einhergeht. Chin (1998, S. 9) schreibt dazu: ,if one of the items (assuming all coded in the same direction) were to suddenly change in a particular direction, the others will change in a similar manner". 
Bezugnehmend auf die Forschungsfrage lassen die Ergebnisse der Regressionsanalyse der im Zuge des Experiments ${ }^{740}$ generierten Daten darauf schließend, dass die auf individueller Ebene wahrgenommene Fach-, Methoden-, Sozial- und Persönlichkeitskompetenz des Kooperationspartners die Entstehung von Vertrauen seitens des Vertrauensgebers determinieren.

Nimmt der Vertrauensgeber den Kooperationspartner als fach- und methodenkompetent wahr, so begünstigt diese Wahrnehmung die Evolution von Vertrauen. Dies zeigt sich darin, dass der Vertrauensgeber einem fach- und methodenkompetenten Kooperationspartner mehr Informationen ehrlich abgibt als einem durchschnittlich kompetenten Interaktionspartner. Auch beeinflusst die Fach- und Methodenkompetenzwahrnehmung den Verzicht auf Kontrollmaßnahmen zur Beschränkung von Opportunismusspielräumen positiv. Allerdings werden einem fach- und methodenkompetenten Kooperationspartner nicht weniger Informationen verschwiegen als einem durchschnittlich kompetenten Partner. Ein Grund hierfür mag sein, dass der Entscheidungsträger beabsichtigt, seine Verwundbarkeit durch Zurückhalten von Informationen möglichst gering zu halten, da die Fach- und Methodenkompetenzwahrnehmung nicht dazu beitragen kann, seine Unsicherheit hinsichtlich des Wollens des Partners zu reduzieren. Da der Vertrauensgeber aber aufgrund der durch die Fach- und Methodenkompetenzwahrnehmung reduzierten Unsicherheit hinsichtlich des Könnens des Partner eher geneigt sein mag, mit diesem eine Kooperation einzugehen, gibt er ihm zwar gleich viele Informationen ab wie einem durchschnittlich kompetenten Interaktionspartner, diese dafür aber ehrlicher, um den Grundstein für eine vertrauensbasierte Zusammenarbeit zu legen.

Hingegen werden einem Kooperationspartner, der Sozial- und Persönlichkeitskompetenz vermittelt, Informationen sowohl offener als auch ehrlicher abgegeben, als einem Partner, dessen Kompetenz als durchschnittlich oder nicht vorhanden wahrgenommen wird. Auch beeinflusst die Sozial- und Persönlichkeitswahrnehmung den Verzicht auf Kontrollmaßnahmen zur Beschränkung von Opportunismusspielräumen positiv. Die Ergebnisse der Multigruppenanalyse implizieren, dass Sozial- und Persönlichkeitskompetenz die Entstehung von Vertrauen stärker beeinflussen, als Fach- und Methodenkompetenz. Dies zeigt sich darin, dass der Vertrauensgeber einem sozial- und persönlichkeitskompetenten Kooperationspartner höchst signifikant mehr Informationen ehrlich abgibt bzw. ihn in geringerem Ausmaß kontrolliert, als einem bzw. einen Interaktionspartner, der sehr fach- und methodenkompetent, aber bloß durchschnittlich sozial- und persön-

740 Aus forschungspragmatischer Sicht und aufgrund der in dieser Arbeit vertretenen Auffassung, dass Vertrauen zwischen Unternehmen immer durch die diesen Unternehmen angehörenden Individuen begründet und zerstört wird, wurde im Zuge der hypothesentestenden Forschung in dieser Arbeit auf die Überprüfung der Annahmen zum Einfluss organisationaler Kompetenzen auf die Vertrauensentstehung in zwischenbetrieblichen Kooperationen verzichtet. 
lichkeitskompetent ist. Während die offene und ehrliche Informationsabgabe in zwischenbetrieblichen Kooperationen im Allgemeinen und in Entscheidungssituationen im Speziellen als positive beispielhafte Manifestationen von Vertrauen $\mathrm{zu}$ werten sind, kann die Feststellung, dass sich Vertrauen in einer reduzierten Kontrollintensität des Vertrauensgebers zeigt, Kritik an den Konsequenzen dieser Handlung hervorrufen (z.B. indem sie zu einer reduzierten Leistung der an der Kooperation beteiligten Individuen führt). Trotz der Gefahr, dass erforderliche Kontrollmaßnahmen nicht gesetzt werden, darf nicht unerwähnt bleiben, dass Kontrolle die Vertrauensentstehung verhindert und bereits bestehendes Vertrauen reduziert: „Kontrolle zerstört Vertrauen insbesondere dann, wenn sie (übermäßig) versucht, Handlungsspielräume zu beschneiden und Gewissheit dort zu erzwingen, wo Verwundbarkeit und Ungewissheit schlicht nicht weiter reduzierbar und nur durch Vertrauen akzeptierbar sind." ${ }^{\text {741 }}$

Zwar ist Kooperation nicht notwendigerweise ein Zeichen für Vertrauen und Vertrauen alleine nicht immer eine ausreichende Bedingung für Kooperation, doch lassen die Ergebnisse der Regressionsanalyse in diesem Zusammenhang darauf schließen, dass nicht nur die Vertrauensentscheidung, aber auch die Kooperationsentscheidung des Vertrauensgebers am stärksten von der Sozial- und Persönlichkeitskompetenz determiniert wird. Aber auch Vertrauensgeber, die mit einem fach- und methodenkompetenten Interaktionspartner konfrontiert sind, sind eher geneigt, auf eigene kurzfristige Vorteile zugunsten gemeinsamer langfristiger Vorteile zu verzichten, als jene Vertrauensgeber, deren potentieller Partner bloß durchschnittlich fach-, methoden-, sozial- und persönlichkeitskompetent ist.

\section{Wie beeinflussen die einzelnen Kompetenzarten des Vertrauensnehmers die Evolution von Vertrauen auf der Seite des Vertrauensgebers?}

Beim Treffen seiner Vertrauensentscheidung sieht sich der Vertrauensgeber mit Unsicherheiten konfrontiert: Aufgrund der zwischen den Kooperationspartnern herrschenden Informationsasymmetrien besteht seitens des Vertrauensgebers einerseits Unsicherheit darüber, ob der Vertrauensnehmer die potentielle Vertrauensentscheidung honorieren kann und andererseits ist der Vertrauensgeber unsicher, ob sich der Vertrauensnehmer vertrauenswürdig verhalten will. In diesem Zusammenhang wird die Relevanz des Kompetenzkonstrukts deutlich: Die Bildung einer zutrauens- bzw. vertrauensvollen Erwartung seitens des Vertrauensgebers kann nur antizipiert werden, wenn Zusatzinformationen wahrgenommen werden, die den Vertrauensgeber darauf schließen lassen, dass der Vertrauensnehmer die Vertrauensentscheidung honorieren kann bzw. honorieren will.

Bei der wahrgenommenen Kompetenz des Vertrauensnehmers handelt es sich um solche Zusatzinformation: Die auf individueller Ebene wahrgenommene Fach- 
kompetenz des Vertrauensnehmers trägt dazu bei, die Unsicherheit des Vertrauensgebers über das Können des Vertrauensnehmers zu reduzieren. Einhergehend mit dieser reduzierten Qualitätsunsicherheit erwartet der Vertrauensgeber vom Vertrauensnehmer, dass letzterer die potentielle Vertrauensentscheidung honorieren kann. Die wahrgenommene Fachkompetenz des Vertrauensnehmers begünstigt daher über die Dimension der Zutrauenserwartung die Vertrauensevolution seitens des Vertrauensgebers. Da die Methodenkompetenz die Fachkompetenz ergänzt, wirkt sie im Vertrauensentstehungsprozess wie die Fachkompetenz. Die auf individueller Ebene wahrgenommene Sozialkompetenz des Vertrauensnehmers trägt im Gegensatz zur wahrgenommenen Fach- und Methodenkompetenz dazu bei, die Unsicherheit des Vertrauensgebers über das Wollen des Vertrauensnehmers zu reduzieren. Bedingt durch diese reduzierte Entscheidungsunsicherheit erwartet der Vertrauensgeber vom Vertrauensnehmer, dass letzterer die potentielle Vertrauensentscheidung honorieren will. Die wahrgenommene Sozialkompetenz begünstigt daher über die Dimension der Vertrauenserwartung die Evolution von Vertrauen auf der Seite des Vertrauensgebers. Aufgrund der engen Verwobenheit der Konstrukte Sozial- und Persönlichkeitskompetenz gelten die zur Rolle der Sozialkompetenz angestellten Überlegungen gleichermaßen für die Wirkung der Persönlichkeitskompetenz im Vertrauensentstehungsprozess.

Die Höhe der Erwartung, dass der Kooperationspartner die Vertrauensentscheidung honorieren will, wird neben der Sozial- und Persönlichkeitskompetenzwahrnehmung positiv von der generellen Vertrauensbereitschaft beeinflusst. Die Handlung des Vertrauensgebers, durch die er sich dem Hold Up-Risiko ungeschützt aussetzt, wird neben der Höhe der Vertrauenserwartung bzw. Zutrauenserwartung positiv von der Höhe der vertrauensgeberspezifischen Risikobereitschaft und negativ von der Höhe der situativen Risikowahrnehmung beeinflusst. Abschließend ist auf den linearen Zusammenhang zwischen vertrauensgeberspezifischer Risikobereitschaft und situativer Risikowahrnehmung hinzuweisen, der unabhängig von der Art der wahrgenommenen individuellen Kompetenzen besteht: Akteure, die über eine hohe (bzw. geringe) Risikobereitschaft verfügen, nehmen Situationen grundsätzlich als wenig (bzw. hoch) riskant wahr bzw. Akteure, die eine Situation als hoch (bzw. wenig) riskant wahrnehmen, verfügen generell über eine geringe (bzw. hohe) Risikobereitschaft.

\section{ERKLÄRUNGSBEITRAG UND PRAKTISCHE FOLGERUNGEN}

$\mathrm{Da}$ in den vergangenen Jahrzehnten in verschiedenen Fachdisziplinen bedeutende theoretische Vertrauensmodelle entwickelt wurden, die interdisziplinäre Vertrauensforschung sich aber erst im Anfangsstadium ihrer Entwicklung befindet, wurde im Rahmen dieser Arbeit das Ziel verfolgt, bereits entwickelte theoretische Ansätze und empirische Forschungsergebnisse zusammenzuführen und grund- 
legende Zusammenhänge zu überprüfen. Es wurde versucht, der sich durch die bestehenden Unklarheiten sowohl bezüglich der Vorbedingungen, direkten Determinanten, Dimensionen, Manifestationen und Konsequenzen von Vertrauen, als auch darüber, ob es sich bei gewissen Faktoren um Ursachen, Wirkungen oder Vertrauen per se handelt, begründenden Forderung nach einer Untersuchung, die einen Beitrag zur Erklärung der Vertrauensdeterminanten leistet ${ }^{72}$, folgendermaßen nachzukommen: Das im Zuge dieser Arbeit generierte und empirisch überprüfte Modell berücksichtigt explizit sowohl Charakteristika des Vertrauensgebers, als auch Eigenschaften des Vertrauensnehmers. Es differenziert zwischen subjektiven und objektiven Dimensionen des Vertrauenskonstrukts und grenzt Vertrauen von seinen Determinanten ab. Um der Forderung nach einer engen Übereinstimmung von Messinstrument und Vertrauensdefinition nachzukommen $^{743}$, wurde auf Basis der dieser Arbeit zugrunde liegenden Vertrauensdefinition festgelegt, welche Operationen unter Bezugnahme auf den vorliegenden Untersuchungskontext als indikativ für die Vertrauenshandlung anzusehen sind. Die Absicherung dieser Vertrauensindikatoren erfolgte anhand der validierten Ergebnisse empirischer Studien, sowie durch Ergebnisse einer weiterführenden Literaturrecherche in den Bereichen Neue Institutionenökonomie, Soziologie und Psychologie, da eine ausführliche Bedeutungsanalyse des im Fokus stehenden Konstrukts die Voraussetzung für eine erfolgreiche Operationalisierung darstellt. Um das Modell einer hinreichenden Prüfung unterziehen zu können, wurden weiters, auf Basis der dieser Arbeit zugrunde liegenden Kompetenzdefinition, Indikatoren festgelegt, die als empirische Äquivalente der einzelnen Kompetenzarten Rückschlüsse auf die individuellen Kompetenzen erlauben.

Die Ergebnisse der Arbeit bestätigen frühere konzeptionelle Studien, die die Vertrauenserwartung von der Zutrauenserwartung im weiteren Sinne als unabhängig voneinander thematisieren ${ }^{744}$ und tragen zur Schaffung konzeptioneller Klarheit hinsichtlich der unterschiedlichen Dimensionen von Vertrauen und ihren Einflussfaktoren in interorganisationalen Beziehungen bei, wie von Welter und Smallbone ${ }^{745}$ gefordert. Insgesamt weisen die Ergebnisse darauf hin, dass eine wesentlich detailliertere Sicht auf Vertrauen in zwischenbetrieblichen Kooperationen erforderlich ist, als dies bislang in einer großen Anzahl empirischer Studien der Fall war. Die Unterscheidung im Beziehungsmuster zwischen Erwartungsdimensionen und Wirkungsvariablen unterstreichen die Notwendigkeit, zukünftig nicht nur den Einfluss von Faktoren auf Vertrauen als Gesamtpaket, sondern vor allem deren dimensionsbezogenen Wirkungsweg zu untersuchen. Die Differen-

742 Vgl. dazu insbesondere Raimondo (2000); Gillespie (2003); Möllering (2006); Spăth (2008)

743 Vgl. Gillespie (2003, S. 7): „In summary, the organizational trust literature is characterized by a mismatch between the defining features of trust and its measurement."

744 Vgl. Luhmann (1988); Plötner (1995); Sichtmann (2007)

745 Vgl. Welter/Smallbone (2006) 
zierung zwischen den Dimensionen Zutrauenserwartung, Vertrauenserwartung und Vertrauenshandlung einerseits und die Unterscheidung zwischen den Dimensionen organisationale Kompetenzen und individuelle Kompetenzen in Verbindung mit deren Aufspaltung in die Faktoren Basis- und Kernkompetenzen und Fach-, Methoden-, Sozial- und Persönlichkeitskompetenzen ermöglichen die Entflechtung und das Verständnis des Einflusses von Kompetenz auf die Entstehung von Vertrauen in zwischenbetrieblichen Kooperationsbeziehungen.

Zusammenfassend leistet die vorliegende Arbeit folgenden Erklärungsbeitrag: Erstens vertiefen die Ergebnisse den Wissensstand zu den Kompetenzdimensionen und den Dimensionen des Vertrauenskonstrukts, indem die in verschiedenen Fachdisziplinen entwickelten theoretischen Ansätze und empirischen Forschungsergebnisse zusammengeführt wurden. Darüber hinaus adressiert die Arbeit durch die principal-agent-theoretische Analyse der Rolle von Kompetenz, in Verbindung mit personen- und situationsbedingten Faktoren, im Vertrauensentstehungsprozess die bislang ,weißen Flecken' auf der Forschungslandkarte der Vertrauensforschung, wie von Mayer et al. ${ }^{746}$ gefordert. Schließlich konnte durch eine intern valide laborexperimentelle Manipulation der unabhängigen Variable in Verbindung mit psychometrischen Tests die Kausalbeziehung zwischen den einzelnen Kompetenzarten und Vertrauen im Kontext zwischenbetriebliche Kooperationen eindeutig festgestellt werden

Aus der Arbeit lassen sich Implikationen für die Praxis ableiten. Die Ergebnisse machen deutlich, dass sich ein Mangel an Vertrauen in Situationen, die sich durch Unsicherheit, potentiellen Schaden, etc. aufgrund von Informationsasymmetrien kennzeichnen, in verstärkter Wettbewerbsorientierung, erhöhter Kontrollintensität und der Abnahme offener und ehrlicher Informationsabgabe zeigt. Da „Vertrauen [...] der stärkste Einflußfaktor auf menschliches Verhalten und somit auf Geschäftsbeziehungen “747 ist, ist das Ergebnis der vorliegenden Arbeit, dass die auf individueller Ebene wahrgenommenen Kompetenzen des Vertrauensnehmers die Entstehung von Vertrauen in zwischenbetrieblichen Kooperationsbeziehungen determinieren, von zentraler Relevanz. Einem sozial- und persönlichkeitskompetenten Akteur wird in höherem Ausmaß vertraut, als einem fach- und methodenkompetenten Kooperationspartner, dem wiederum stärker vertraut wird, als einem Partner, dessen Kompetenzen als durchschnittlich wahrgenommen werden. Diese Ergebnisse implizieren, dass bereits die erste Interaktion zwischen potentiellen Kooperationspartnern von großer Bedeutung für das Zustandekommen und Aufrechterhalten von Kooperationsbeziehungen ist. Schätzt der Vertrauensgeber den potentiellen Kooperationspartner als sozial- und persönlichkeitskompetent ein, wird er ihm mehr Informationen ehrlich abgegeben und ihn in geringerem

746 Vgl. Mayer et al. (1995), S. 730

747 Vgl. Bleicher (1995), S. 395 
Ausmaß kontrollieren, als einem bzw. einen Interaktionspartner, der ausgeprägte Fach- und Methodenkompetenzen zeigt, aber bloß durchschnittlich sozial- und persönlichkeitskompetent ist.

Die ehrlichere Informationsabgabe und die reduzierte Kontrollintensität als beispielhafte Manifestationen von Vertrauen können wiederum den potentiellen Kooperationspartner dazu veranlassen, selbst die Rolle des Vertrauensgebers einzunehmen und sich dem Hold up-Risiko ungeschützt auszusetzen, wodurch die Entstehung einer vertrauensbasierten Kooperationsbeziehung, in der sich die Kooperateure gegenseitig ausliefern und so die Opportunismusneigung ihres Gegenübers reduzieren, begünstigt wird.

Die Untersuchungsergebnisse betonen daher die Relevanz von Fairness, Kooperations- und Konfliktfähigkeit, produktive Begeisterungsfähigkeit, etc. als Komponenten der Sozial- und Persönlichkeitskompetenz für den Vertrauensgeber im Vertrauensentstehungsprozess. Da aber eine „direkte Einflussnahme auf Vertrauen im Sinnes eines auf Handlungsanweisungen basierenden Vertrauensmanagements ${ }^{\text {“748 }}$ als weitgehend unrealistisch einzustufen ist, können im Folgenden keine rezeptartigen Anleitungen zur Erzeugung von Vertrauen in zwischenbetrieblichen Kooperationen gegeben werden. Vielmehr ist Unternehmen zu empfehlen, bei der Besetzung der Position eines Kooperationsbeauftragten die vorliegenden Ergebnisse zu berücksichtigen und jenen Mitarbeiter mit der Aufgabe der Kooperationsinitiierung zu betrauen, der bereits in der Vergangenheit bei der Erfüllung von Aufgaben Einfühlungsvermögen, Kontakt-, Kommunikations-, und Konfliktfähigkeit, sowie Selbstvertrauen, Konsistenz und Neugier gezeigt hat.

Auch bietet es sich an, durch interne Weiterbildungsmaßnahmen das Bewusstsein der Mitarbeiter hinsichtlich der Stärkung und Kommunikation ihrer Sozial- und Persönlichkeitskompetenz zu schärfen. Mit Blick auf die inhaltliche Gestaltung der Kompetenzkommunikation sollte die Festlegung der Kommunikationsbotschaft an den zuvor identifizierten Erwartungen des Vertrauensgebers ansetzen, um tatsächlich vertrauensfördernde Kompetenzinformationen zu vermitteln. Informationsökonomisch betrachtet, handelt es sich bei gewissen, in dieser Arbeit identifizierten Kompetenzindikatoren um Signale, die vom kompetenzvermittelnden Akteur direkt beeinflussbar sind, z.B. produktive Begeisterungsfähigkeit (personale Kompetenz) oder Fachwissen (fachliche Kompetenz). Bei anderen Indikatoren, z.B. Charakter (personale Kompetenz), berufspraktische Erfahrungen (fachliche Kompetenz), handelt es sich dagegen um Indizes, die kurzfristig nicht beeinflussbar sind. Die Frage, ob Kompetenzinformationen Indizes oder Signale darstellen, ist insofern von Relevanz, als dass die Möglichkeit des Eingehens einer Kooperationsbeziehung den kooperationswilligen und kompetenzvermittelnden Akteur dazu veranlassen kann, die Signale im Hinblick auf eine vertrauenswirk- 
same Steuerung der Erwartungen des Vertrauensgebers zu verändern. Im Wissen um diese Manipulationsoption des potentiellen Kooperationspartners wird der Vertrauensgeber bei der Bildung seiner Vertrauensentscheidung daher auch auf kurzfristig nicht beeinflussbare Indizes zurückgreifen. $\mathrm{Zu}$ berücksichtigen bei der Festlegung der Kommunikationsbotschaft ist neben der Beeinflussbarkeit der Kompetenzinformationen auch die Rolle von deren Vergangenheitsbezug. In diesem Zusammenhang gewinnen Kompetenzinformationen an Bedeutung, die den Vertrauensgeber darauf schließen lassen, dass der potentielle Kooperationspartner bereits bei vergangenen Geschäftstätigkeiten seine Kompetenzen unter Beweis gestellt hat (z.B. Auszeichnungen, Preise, Zertifizierungsnachweise, Referenzen, Bilanzen, etc.).

\section{LIMITATIONEN UND AUSBLICK}

Im Zuge dieser Arbeit wurde die Auffassung vertreten, dass Vertrauen zwischen Unternehmen letztlich immer durch die Individuen, die diesen Unternehmen angehören, begründet und zerstört wird. Die Vertrauensentscheidung wurde daher als eine Entscheidung eines spezifischen Vertrauensgebers, einem spezifischen Vertrauensnehmer sein Vertrauen zu schenken, analysiert. Dementsprechend liegt die Erklärung der Entstehung von Vertrauen in sozialen Systemen nicht im Forschungsinteresse dieser Arbeit. Weiters wurde die Entstehung von Vertrauen nur in eine Richtung modelliert: seitens eines gegebenen Vertrauensgebers in einen gegebenen Vertrauensnehmer. Diese Einschränkung kann jedoch beseitigt werden, indem das Modell gespiegelt wird, sodass es den dynamischen Prozess wechselseitiger Vertrauensentstehung abbildet. Hinsichtlich der Entwicklungsstufen des Vertrauensprozesses bestand das Interesse dieser Arbeit in der Erforschung der Entstehung von Vertrauen. Das Verständnis hierfür bildet eine Grundlage für die Untersuchung der sich kausal anknüpfenden Phasen des Verlusts und Wiederaufbaus von enttäuschtem Vertrauen, der in jüngster Zeit besondere Aufmerksamkeit zuteil wird und die empirische Vertrauensforschung mit Herausforderungen konfrontiert (nicht zuletzt wegen des begrenzten Zugangs zu derart sensiblen Daten, sowohl im Feld als auch im Labor). Bezugnehmend auf die Operationalisierung der Konstrukte wurde die Identifikation von Indikatoren sekundäranalytisch abgesichert - die selektierten empirischen Äquivalente reflektierten die Konstrukte am eindeutigsten, jedoch war es aus Konsistenzgründen teilweise notwendig, die Begriffe leicht umzuformulieren. Da aber als Indikatoren der Vertrauenserwartung in der Literatur vielfach Eigenschaften des Vertrauensnehmers genannt werden, bei denen es sich nicht um Manifestationen der vertrauensvollen Erwartung des Vertrauensgebers in die Absichten des Vertrauensnehmers handelt (sondern vielmehr um Determinanten), musste im Zuge dieser Arbeit auf eine Identifikation von Indikatoren, die als empirische Äquivalente der Vertrauenserwartung fungieren, verzichtet werden. 
Neben diesen vorrangig inhaltlich begründeten Grenzen der vorliegenden Arbeit bestehen Limitationen, die mit der Untersuchungskonzeption einhergehen. Wie bereits in Teil V Kap. 1 erläutert, beeinflusst die Entscheidung für die Untersuchungsumgebung die Gültigkeit und Eindeutigkeit der mit den Untersuchungsergebnissen verbundenen Aussagen. Da durch die experimentelle Manipulation der unabhängigen Variable präzise Aussagen zur Kausalbeziehung zwischen Kompetenz und Vertrauen gewonnen werden sollten, wurde der internen Validität der Untersuchung eine besondere Bedeutung beigemessen. Um die externe Validität der Untersuchungsergebnisse zu erhöhen, wurde versucht, bei der Gestaltung der Untersuchungssituation eine Vergleichbarkeit der Entscheidungsaufgabe im Labor mit realen zwischenbetrieblichen Aufgaben und bei der Gestaltung der Interaktionsstruktur eine Annäherung an realitätsnahe Interaktionsprinzipien zu erzielen. Obwohl sich die Fallsimulation als geeignet für die Untersuchung von Kompetenz als Vertrauensdeterminante im Labor erwies, muss konstatiert werden, dass es sich um fiktive Aufgabenstellungen handelte, in die die Elemente einer Vertrauenssituation künstlich eingearbeitet wurden. Neben experimentellen Erfordernissen (isolierte Variation der unabhängigen Variable) wurde aus forschungspragmatischen Gründen (eingeschränkter Zugang zu kooperierenden Unternehmern und deren Kooperationspartnern) und aufgrund der Vorteile, die sich aus der Homogenität des untersuchten Samples ergaben, die Vornahme einer Vertrauensentscheidung durch Studierende in ihrer Rolle als Vertrauensgeber untersucht. Es besteht jedoch die Möglichkeit, dass Unterschiede im Verhalten zwischen Studierenden und Unternehmern bestehen, sodass die ausschließliche Untersuchung von Studierenden zu falschen Schlüssen führt. Allerdings kann, beispielsweise mit Blick auf die gewählten Studienrichtungen, einerseits nicht ausgeschlossen werden, dass im Sample enthaltene Studierende bereits unternehmerisch tätig sind bzw. waren und diese Erfahrungen in ihre Entscheidungen eingeflossen sind. Andererseits wurde in Teil V Kap. 4.2 bereits auf Studien verwiesen, die zeigen, dass keine Handlungsdifferenzen zwischen Studierenden und Nicht-Studierenden bestehen.

Der Vertrauensentstehungsprozess kann nur begrenzt in einer einmaligen Erhebung erfasst werden. Wie jedoch die vorliegende Untersuchung zeigt, ist die Nachbildung elementarer Entwicklungsstufen, wie die Entstehung von Vertrauen in erstmaligen Begegnungen, durch Gestaltung der Szenerie möglich. Dennoch handelt es sich hierbei um eine statische Analyse. Aufschluss über den gesamten Vertrauensentstehungsprozess können letztlich nur Längsschnittstudien geben. Die Begleitung einer Stichprobe über mehrere Monate oder Jahre erlaubt nicht nur Aussagen zur Zirkularität von Vertrauen, sondern auch die Analyse einzelner Entwicklungsphasen von Vertrauen.

Zusammenfassend müssen die Ergebnisse der vorliegenden Arbeit unter Beachtung der angeführten Limitationen interpretiert werden. Gleichzeitig bieten die in- 
haltlichen und methodischen Grenzen aber auch Anhaltspunkte für weiterführende Forschungsaktivitäten. Um das vorliegende Modell weiter zu spezifizieren, sollte in einem nächsten Schritt die Dimension der Vertrauenserwartung mittels eines Messmodells operationalisiert werden, d.h. dem interessierenden Konstrukt sollten beobachtbare Variablen zugeordnet werden, ,um so etwaige Verzerrungen in einzelnen Indikatoren aufzufangen“. ${ }^{749}$ In einem nächsten Schritt wäre es wünschenswert, dass zukünftige Studien auf dem generierten Modell aufsetzen, und anhand des entwickelten Experimentaldesigns Annahmen hinsichtlich der Wirkung von Faktoren wie Reputation, Bekanntheit, etc. auf die Vertrauensentstehung überprüfen. Von Interesse wäre es auch, das vorliegende Experiment mit ausländischen Studierenden durchzuführen, um etwaige kulturelle Unterschiede hinsichtlich des Einflusses der Wahrnehmung unterschiedlicher Kompetenzarten in Verbindung mit personen- und situationsbezogenen Faktoren auf die Entstehung von Vertrauen aufzudecken. Weiters könnte geprüft werden, ob die Wahrnehmung der Kompetenz des potentiellen Kooperationspartners reale Unternehmer zur Vornahme einer Vertrauenshandlung veranlasst. In diesem Zusammenhang ist vor allem interessant, welche Kompetenzarten die Entstehung von Vertrauen begünstigen. Einerseits könnte für diese Untersuchung das vorliegende Experimentaldesign zum Einsatz kommen, dessen Vorteil in der möglichen Vergleichbarkeit der erzielten Ergebnisse mit den vorliegenden Resultaten liegt. Andererseits würde sich für diese Untersuchung eine Modifikation des methodischen Vorgehens anbieten, und zwar insofern, als dass das Design dann auch eine Prüfung des Einflusses organisationaler Kompetenzen auf die Vertrauensentstehung seitens des kooperationswilligen Unternehmers zulässt.

749 Homburg/Dobratz (1991), S. 214 


\section{LITERATURVERZEICHNIS}

Abels, H. (2007a): Einführung in die Soziologie. Bd. 1: Der Blick auf die Gesellschaft. 3. Aufl. Wiesbaden.

Abels, H. (2007b): Einführung in die Soziologie. Bd. 2: Die Individuen in ihrer Gesellschaft. 3. Aufl. Wiesbaden.

Abelson, R.P. (1968): Simulation of Behaviour. In: Lindzey, G. / Aronson, E. (Hrsg.): The Handbook of Social Psychology. 2. Aufl. Reading, S. 274-356.

Abrams, L.C. / Cross, R. / Lesser, E. / Levin, D.Z. (2003): Nurturing interpersonal trust in knowledge-sharing networks. In: Academy of Management Executive. 17 (4), S. 64-77.

Adler, P.S. (2001): Market, Hierarchy and Trust: The Knowledge Economy and the Future of Capitalism. In: Organization Science. 12 (2), S. 215-234.

Ajzen, I. / Fishbein, M. (1980): Understanding Attitudes and Predicting Behavior. Englewood Cliffs.

Albach, H. (1980): Vertrauen in der ökonomischen Theorie. In: Zeitschrift für die gesamte Staatswissenschaft. 136, S. 2-11.

Albach, H. / Albach, R. (1989): Das Unternehmen als Institution: rechtlicher und gesellschaftlicher Rahmen - eine Einführung. Wiesbaden.

Albach, H. (1989): Organisation - mikroökonomische Theorie und ihre Anwendung. Wiesbaden.

Alchian, A. / Demsetz, H. (1973): The Property Rights Paradigm. In: The Journal of Economic History. 33 (1), S. 16-27.

Alchian, A. / Woodward, S. (1987): Reflections on the Theory of the Firm. In: Zeitschrift für die gesamte Staatswissenschaft. 143, S. 110-136.

Amelang, M. / Gold, A. / Kübel, E. (1984): Über einige Erfahrungen mit der deutschsprachigen Skala zur Erfassung zwischenmenschlichen Vertrauens (Interpersonal Trust). In: Diagnostica. 30 (3), S. 198-215.

Amit, R. / Schoemaker, P. (1993): Strategic Assets and Organizational Rent. In: Strategic Management Journal. 14 (1), S. 33-46.

Anderson, J.C. / Narus, J.A. (1990): A Model of the Distributor Firm and Manufacturer Firm Working Partnerships. In: Journal of Marketing. 54, S. 4258.

Ansoff, I. (1965): Corporate Strategy. An Analytic Approach to Business Policy for Growth and Expansion. 3. Aufl. New York.

Antfang, P. /Urban, D. (1994): „Vertrauen“ - soziologisch betrachtet. Ein Beitrag zur Analyse binärer Interaktionssysteme. In: SISS: Schriftreihe des Instituts für Sozialwissenschaften der Universität Stuttgart. No.1., Stuttgart. 
Apelt, M. (1999): Vertrauen in der zwischenbetrieblichen Kooperation.Wiesbaden.

Argyle, M. (1969): Social Interaction. London.

Arrow, K.J. (1985): The Economics of Agency. In: Pratt, J.W. / Zeckhause, R.J. (Hrsg.): Principals and Agents: The Structure of Business. Boston, S. 37-51.

Asendorpf, J.B. (2009): Persönlichkeitspsychologie. Heidelberg.

Atteslander, P. (1995): Methoden der empirischen Sozialforschung. 8. Aufl. Berlin/New York.

Axelrod, R. (1991): The Evolution of Cooperation. 2. Aufl. New York.

Axelrod, R. (2002): Die Evolution der Kooperation. 5. Aufl. München/Wien.

Backhaus, K. (1992): Investitionsgüter-Marketing - Theorieloses Konzept mit Allgemeingültigkeitsanspruch? In: Zeitschrift für betriebswirtschaftliche Forschung. 44 (9), S. 771-791.

Backhaus, K. (1995): Investitionsgütermarketing. 4. Aufl. München.

Backhaus, K. / Erichson, B. / Plinke, W. / Weiber, R. (2003): Multivariate Analysemethoden. 10. überarb. Aufl. Berlin.

Bagozzi, R. / Fornell, C. (1982): Theoretical Concepts, Measurements, and Meaning. In: Fornell, C. (Hrsg.): A Second Generation of Multivariate Analysis. New York, S. 24-38.

Bahlmann, J. / Schulze, B. / Spiller, A. (2008): Vertrauen als wettbewerbsrelevanter Faktor in der deutschen Schweinefleischproduktion: Eine empirische Untersuchung zum Vertrauen von Schweineerzeugern gegenüber Schlachtunternehmen. In: Spiller, A. / Schulze, B. (Hrsg.): Zukunftsperspektiven der Fleischwirtschaft - Verbraucher, Märkte, Geschäftsbeziehungen. Göttingen.

Baitsch, C. (1996): Kompetenz von Individuen, Gruppen und Organisationen: Psychologische Überlegungen zu einem Analyse- und Bewertungskonzept. In: Denisow, K. / Friecke, W. / Stieler-Lorenz, B. (Hrsg.): Partizipation und Produktivität. Zu einigen kulturellen Aspekten der Ökonomie. Bonn, S. 102-112.

Bakker, H. / Jones, W. / Nichols, M. (1994): Using Core Competencies to Develop New Business. In: Long Range Planning. 27 (6), S. 13-27.

Baldwin, C.Y. / Clark, K.B. (1994): Capital-Budgeting Systems and Capabilities Investments in U.S. Companies after the Second World War. In: The Business History Review. 68 (1), S. 73-109.

Bamberg, G. / Coenenberg, A.G. (1994): Betriebswirtschaftliche Entscheidungslehre. 8. Aufl. München.

Barber, B. (1983): The Logic and Limits of Trust. New Brunswick.

Barney, J. (1991): Firm Resources and Sustained Competitive Advantage. In: Journal of Management. 17 (1), S. 99-120. 
Bayon, T. (1997): Neuere Mikroökonomie und Marketing: eine wissenschaftstheoretisch geleitete Analyse. Wiesbaden.

Becerra, M. / Gupta, A.K. (2003): Perceived Trustworthiness within the Organization: The Moderating Impact of Communication Frequency on Trustor and Trustee Effects. In: Organization Science. 14 (1), S. 32-44.

Becker, M. / Rother, G. (1998): Kompetenzentwicklung. Halle/Saale.

Belz, C. (1999): Verkaufskompetenz. Chancen in umkämpften Märkten, Konzepte und Innovationen, Kunden- und Leistungskriterien, Organisation und Führung. St. Gallen.

Bentele, G. (1994): Öffentliches Vertrauen - normative und soziale Grundlage für Public Relations. In: Armbrecht, W. / Zabel, U. (Hrsg.): Normative Aspekte der Public Relations: Grundlegende Fragen und Perspektiven: Eine Einführung. Opladen, S. 131-158.

Berg, J. / Dickhaut, J. / McCabe, K. (1995): Trust, reciprocity, and social history. In: Games and Economic Behavior. 10 (1), S. 122-142.

Berggren, N. / Jordahl, H. (2006): Free to Trust: Economic Freedom and Social Capital. In: Kyklos. 59 (2), S. 141-169.

Bergmann, B. (2007): Selbstkonzept beruflicher Kompetenz. In: Erpenbeck, J. / Rosenstiel, Lutz v. (Hrsg.): Handbuch Kompetenzmessung. 2. Aufl. Stuttgart, S. 194-223.

Bhide, A. / Stevenson, H. (1990): Trust, Uncertainty, and Profit. In: Journal of Socio-Economics. 21, S. 191-208.

Bierhoff, H.W. (2000): Sozialpsychologie: Ein Lehrbuch. 5. Aufl. Stuttgart.

Bittl, A. (1997): Vertrauen durch kommunikationsintendiertes Handeln: eine grundlagentheoretische Diskussion in der Betriebswirtschaftslehre mit Gestaltungsempfehlungen für die Versicherungswirtschaft. Wiesbaden.

Bleicher, K. (1995): Vertrauen als kritischer Faktor einer Bewältigung des Wandels. In: Zeitschrift Führung + Organisation (zfo). 64 (6), S. 390-395.

Bortz, J. / Döring, N. (2006): Forschungsmethoden und Evaluation für Humanund Sozialwissenschaftler. 4. Aufl. Heidelberg.

Bouncken, R.B. (2000): Dem Kern des Erfolgs auf der Spur? State of the Art zur Identifikation von Kernkompetenzen. In: Zeitschrift für Betriebswirtschaft. 70 (7/8), S. 865-885.

Bouncken, R.B. (2003): Organisationale Metakompetenzen. Wiesbaden.

Bradach, J.L. / Eccles, R.G. (1989): Price, Authority and Trust: From Ideal Types to Plural Form. In: Annual Review of Sociology. 15, S. 97-118.

Brandstätter, H. (2000): 16-Persönlichkeits-Adjektiv-Skalen. In: Sarges, W. I Wottawa, H. (Hrsg.): Handbuch wirtschaftspsychologischer Testverfahren. Lengerich, S. 3-7. 
Bredenkamp, J. (1980): Theorie und Planung psychologischer Experimente. Darmstadt.

Brennan, G. / Buchanan, J.M. (1993): Die Begründung von Regeln. Tübingen.

Brislin, R.W. (1980): Translation and Content Analysis of Oral and Written Material. In: Triandis, H.C. / Berry, J.W. (Hrsg.): Handbook of Cross-Cultural Psychology: Social Psychology. 5. Aufl. Boston, S. 389-444.

Brockhaus (2008a): Die Enzyklopädie - Online-Ausgabe. In: http://lexika.tanto. de/artikel.php?TANTO_SID=898cfc6f91d136838171 fe06dc037279\&TANTO KID $=$ wu_wien\&TANTO_AGR $=41197 \&$ shortname $=$ b24\&artikel_id $=230280$ 3. Stand: 24. September 2008.

Brockhaus (2008b): Die Enzyklopädie - Online-Ausgabe. In: http://lexika.tanto. de/artikel.php?TANTO SID $=68 \mathrm{~d} 670376 \mathrm{c} 826 \mathrm{e} 59 \mathrm{c} 108 \mathrm{f} 2803 \mathrm{c} 8 \mathrm{f} 1076 \&$ TANT O_KID=wu_wien\&TANTTO_AGR $=41197 \&$ shortname=b24\&artikel_id $=1203$ 308. Stand: 28 . September 2008.

Brockhaus (2009a): Die Enzyklopädie - Online-Ausgabe. In: http://lexika.tanto. de/artikel.php?TANTO_SID=691dfc376c826e59c108f2803c8f1079\&TANTO KID $=$ wu_wien\&TANTO_AGR $=41197 \&$ shortname $=$ b24\&artikel_id $=120235$ 8. Stand: 26 . Februar 2009.

Brockhaus (2009b): Die Enzyklopädie - Online-Ausgabe. In: http://lexika.tanto. de/artikel.php?TANTO_SID=ff5b755077f298444c7ad74leab107c0\&TANTO KID=wu_wien\&TANTO_AGR $=41197 \&$ shortname $=$ b15\&artikel_id $=100024$ 78\#100018060. Stand: 05. März 2009.

Bronner, R. (1999): Planung und Entscheidung: Grundlagen - Methoden - Fallstudien. 3. Aufl. München.

Brosius, F. (2002): SPSS 11. Bonn.

Bruch, H. (1999): Wissens- und kompetenzorientiertes Management in virtuellen Strukturen: Konzepte-Spannungsfelder-neue Wege. In: Sattelberger, T. (Hrsg.): Wissenskapitalisten oder Söldner? Personalarbeit in Unternehmensnetzwerken des 21. Jahrhunderts. Wiesbaden, S. 97-148.

Brumagim, A. (1994): A Hierarchy of Corporate Resources. In: Advances in Strategic Management. 10A, S. 81-112.

Buchholz, W. / Olemotz, T. (1995): Markt- vs. Ressourcenbasierter Ansatz: Konkurrierende oder komplementäre Konzepte im Strategischen Management? Arbeitspapier Nr. 1/95 der Professur für Betriebswirtschaftslehre II der Justus-Liebig-Universität. Gießen.

Bullinger, H.J. / Kugel, R. / Ohlhausen, P. / Stanke, A. (1995): Integrierte Produktentwicklung. Wiesbaden.

Buß, E. (2008): Management-Soziologie. München. 
Butler, J.K. (1991): Towards Understanding and Measuring Conditions of Trust: Evolution of a Conditions of Trust Inventory. In: Journal of Management. 17 (3). S. $643-663$.

Butler, J.K. (1999): Trust Expectations, Information Sharing, Climate of Trust, and Negotiation Effectiveness and Efficiency. In: Group \& Organization Management. 24 (2), S. 217-238.

Butler, J.K. / Cantrell, R.S. (1984): A Behavioral Decision Theory Approach to Modelling Dyadic Trust in Superiors and Subordinates. In: Psychological Reports. 55, S. 19-28.

Bühner, R. (2004): Mitarbeiterkompetenzen als Qualitätsfaktor. Strategieorientierte Personalentwicklung mit dem House of Competence. München.

Campbell, D.T. / Stanley, J.C. (1963): Experimental and Quasi-Experimental Designs for Research on Teaching. In: Gage, N.L. (Hrsg.): Handbook of Research on Teaching. Chicago, S. 171-246.

Carpenter, J. / Connolly, C. / Myers, C. (2007): Altruistic behavior in a representative dictator experiment. Working Paper.

Carson, S.J. / Madhok, A. / Wu, T. (2006): Uncertainty, opportunism, governance: the effects of volatility and ambiguity on formal and relational contracting. In: Academy of Management Journal. 49 (5), S. 1058-1077.

Child, J. (1998): Trust and International Strategic Alliances: The Case of SinoForeign Joint Ventures. In: Lane, Ch. / Bachmann, R. (Hrsg.): Trust Within and Between Organizations. Oxford, S. 241-272.

Chin, W.W. (1998): Issues and Opinion on Structural Equation Modeling. In: MIS Quarterly. 22 (1), S. 7-16.

Clark, M. / Payne, R. (1995): Interpersonal Trust: A Review and Reconceptualization. Discussion Paper Nr. 9116. Sheffield.

Cohen, J. / Cohen, P. / West, St. G. / Aiken, L. S. (2003): Applied Multiple Regression / Correlation Analysis for the Behavioral Sciences. 3. Aufl. Mahwah, NJ.

Coleman, J.S. (1991): Grundlagen der Sozialtheorie - Handlungen und Handlungssysteme, Band 1. München.

Conant, J. / Mokwa, M. / Varadarajan, R. (1990): Types, Distinctive Marketing Competences and Organizational Performance: A Multiple Measures-Based Study. In: Strategic Management Journal. 17 (1), S. 365-383.

Cook, T.D. / Campbell, D.T. (1979): Quasi-Experimentation: Design and Analysis Issues for Field Setting. Chicago.

Corazzini, J.G. (1977): Trust as a complex multi-dimensional construct. In: Psychological Reports. 40, S. 75-80. 
Costa, A. (2000): A matter of trust: effects on the performance and effectiveness of teams in organizations. Tilburg.

Costa, A. (2003): Work team trust and effectiveness. In: Personnel Review. 32 (5), S. 605-622.

Costa, A. / Roe, R.A. / Taillieu, Th. (2001): Trust within teams: The relation with performance effectiveness. In: European Journal of Work and Organizational Psychology. 10 (3), S. 225-244.

Coyle-Shapiro, J. / Morrow, P.C. (2001): The Role of Individual Differences in Employee Adoption of TQM Orientation. In: Journal of Vocational Behavior. 62 , S. 320-340.

Cummings, L.L. / Bromiley, P. (1996): The Organizational Trust Inventory (OTI) - Development and Validation. In: Kramer, R.M. / Tyler, T.R. (Hrsg.): Trust in Organizations: Frontiers of Theory and Research. Thousand Oaks (CA), S. 302-330.

Currall, S.C. / Judge, T.A. (1995): Measuring Trust Between Organizational Boundary Role Persons. In: Organizational Behavior and Human Decision Processes. 64 (2), S. 151-170.

D'Aveni, R.A. (1995): Hyperwettbewerb. Strategien für die neue Dynamik der Märkte. Frankfurt.

Das, T.K. / Teng, B.S. (1998): Between Trust and Control: Developing Confidence in Partner Cooperation in Alliances. In: Academy of Management Review. 23 (3), S. 491-512.

Davis, J.H. / Schoorman, F.D. / Mayer, R.C. / Tan, H.H. (2000): The Trusted General Manager and Business Unit Performance: Empirical Evidence of a Competitive Advantage. In: Strategic Management Journal. 21 (5), S. 563576.

Dasgupta, P. (1988): Trust as a Commodity. In: Gambetta, D. (Hrsg.): Trust: Making and Breaking Cooperative Relations. Oxford, S. 49-72.

Deckow, F. (2006): Vertrauen durch Kompetenzmarketing: Ein ganzheitlicher Ansatz zur Vermarktung von Kontraktgütern. Wiesbaden.

De Cremer, D. / Van Dijke, M. / Bos, A.E.R. (2006): Leader's Procedural Justice affecting Identification and Trust. In: Leadership and Organization Development Journal. 27 (7), S. 554-565.

Deusinger, I. (1986): Die Frankfurter Selbstkonzeptskalen (FSKN). Göttingen.

Deutsch, M. (1958): Trust and Suspicion. In: The Journal of Conflict Resolution. 2(4), S. 265-279.

Deutsch, M. (1962): Cooperation and Trust: Some Theoretical Notes. In: Nebraska Symposium of Motivation. Nebraska, S. 275-319. 
Deutsch, M. (1990): Sixty Years of Conflict. In: The Journal of Conflict Management. 1 (3), S. 237-263.

Diekmann, A. (2007): Empirische Sozialforschung: Grundlagen, Methoden, Anwendungen. 18. Aufl. Reinbek bei Hamburg.

Dietl, H. (1993): Institutionen und Zeit. Tübingen.

Diller, H. / Kusterer, M. (1988): Beziehungsmanagement: Theoretische Grundlagen und empirische Befunde. In: Marketing - Zeitschrift für Forschung und Praxis. 10, S. 211-220.

Dirks, K.T. (1999): The Effects of Interpersonal Trust on Work Group Performance. In: Journal of Applied Psychology. 84 (3), S. 445-455.

Dirks, K.T. / Ferrin, D.L. (2001): The role of trust in organizational settings. In: Organization Science. 12 (4), S. 450-467.

Docherty, P. / Marking, Ch. (1997): Understanding Changing Competence Demands. In: Docherty, P. / Nyhan, B. (Hrsg.): Human Competence and Business Development. Emerging Patterns in European Companies. London, S. 19-42.

Doney, P. / Cannon, J.P. (1997): An Examination of the Nature of Trust in Buyer-Seller Relationships. In: Journal of Marketing. 61 (2), S. 35-51.

Dosi, G. / Nelson, R. / Winter, S. (2000): The Nature and Dynamics of Organizational Capabilities. Oxford.

Dosi, G. / Teese, D. / Winter, S. (1990): Toward a Theory of Corporate Coherence. In: Mimeo. 3, S. 8-16.

Doz, Y. (1996): Managing Core Competency. In: Dosi, G. / Malerba, F. (Hrsg.): Organization and Strategy in the Evolution of the Enterprise. London, S. 155178.

Dwyer, F.R. / Schurr, P.H. / Oh, S. (1987): Developing Buyer-Seller Relationships. In: Journal of Marketing. 51 (2), S. 11-27.

Eberl, M. (2004): Formative und reflektive Indikatoren im Forschungsprozess: Entscheidungsregeln und die Dominanz des reflektiven Modells. In: Schriften zur Empirischen Forschung und Quantitativen Unternehmensplanung der Ludwig-Maximilians-Universität München. Heft 19, S. 1-34.

Eberl, P. (2003): Vertrauen und Management - Studien zu einer theoretischen Fundierung des Vertrauenskonstrukts in der Managementlehre. Stuttgart.

Eberl, P. (2004): The Development of Trust and Implications for Organizational Design: A Game- and Attribution-Theoretical Framework. In: Schmalenbachs Business Review. 56, S. 258-273.

Ebers, M. / Gotsch, W. (1999): Institutionenökonomische Theorien der Organisation. In: Kieser, A. (Hrsg.): Organisationstheorien. 3. Aufl. Stuttgart, S. 199-251. 
Edelmann, W. (1993): Lernpsychologie. 3. Aufl. Weinheim.

Edge, G. / Klein, J. / Hiscocks, P. / Plasonig, G. (1995): Technologiekompetenz und Skill-basierter Wettbewerb. In: Zahn, E. (Hrsg.): Handbuch Technologiemanagement. Stuttgart, S. 185-217.

Edinsel, K. (1998): Soziale Kompetenz und Berufserfolg. Aachen.

Edwards, M.R. / Ewen, A.J. (2000): 360-Beurteilung: Klares Feedback, höhere Motivation und mehr Erfolg für alle Mitarbeiter. München.

Emerson, R.M. (1962): Power-Dependence Relations. In: American Sociological Review. 27 (1), S. 31-41.

Enke, M. / Greschnucha, L. / Geigenmüller, A. (2007): Einflussfaktoren des Vertrauensaufbaus bei der Beratung kleiner und mittlerer Unternehmen (KMU). In: Zeitschrift für Betriebswirtschaft. Special Issue 6, S. 53-73.

Erpenbeck, J. (2004): Kompetenzmessung in Unternehmen. Lernkultur- und Kompetenzanalysen im betrieblichen Umfeld. Stuttgart.

Erpenbeck, J. (2007): Kode ${ }^{\circledR}$ - Kompetenz-Diagnostik und -Entwicklung. In: Erpenbeck, J. / Rosenstiel, Lutz v. (Hrsg.): Handbuch Kompetenzmessung. 2. Aufl. Stuttgart, S. 489-503.

Erpenbeck, J. / Heyse, V. (2007): Die Kompetenzbiographie. Wege der Kompetenzentwicklung. 2. Aufl. Münster.

Erpenbeck, J. / Rosenstiel, L. von (2007): Handbuch Kompetenzmessung. Erkennen, verstehen und bewerten von Kompetenzen in der betrieblichen, pädagogischen und psychologischen Praxis. 2. Aufl. Stuttgart.

Erpenbeck, J. / Sauer, J. (2001): Das Forschungs- und Entwicklungsprogramm ,Lernkultur Kompetenzentwicklung'. In: Arbeitsgemeinschaft Betriebliche Weiterbildungsforschung e.V. (Hrsg.): Arbeiten und Lernen: Lernkultur Kompetenzentwicklung und Innovative Arbeitsgestaltung. Heft 67. Berlin, S. 9-65.

Fahrmeir, L. / Kneib, T. / Lang, S. (2009): Regression: Modelle, Methoden und Anwendungen. 2. Aufl. Heidelberg.

Faix, W. / Laier, A. (1989): Soziale Kompetenz. Beiträge zur Gesellschafts- und Bildungspolitik, Band 151. Institut der deutschen Wirtschaft. Köln.

Faix, W. / Laier, A. (1991): Soziale Kompetenz: Das Potential zum unternehmerischen und persönlichen Erfolg. Wiesbaden.

Faix, W. / Krupp, M. (1999): Die Operationalisierung von Kernkompetenzen. Arbeitspapier des Instituts für Markt- und Distributionsforschung der Universität Köln. Köln.

Faix, W. / Krupp, M. (2002): Kriterien und Indikatoren zur Operationalisierung von Kernkompetenzen. In: Bellmann, K. / Freiling, J. / Hammann, P. / Mildenberger, U. (Hrsg.): Aktionsfelder des Kompetenz-Managements. Wiesbaden, S. 59-83. 
Fearns, H. (2004): Entstehung von Kernkompetenzen - eine evolutionstheoretische Betrachtung. Wiesbaden.

Feyerabend, P. (1976): Wider den Methodenzwang: Skizze einer anarchistischen Erkenntnistheorie. Frankfurt/Main.

Feyerabend, P. (1980): Eine Lanze für Aristoteles. In: Radnitzky, G. / Andersson, G. (Hrsg.): Fortschritte und Rationalität der Wissenschaft. Tübingen, S. 157-198.

Fink, M. (2004): Erfolgsfaktor „Selbstverpflichtung“ bei vertrauensbasierten Kooperationen - Mit einem empirischen Befund unter österreichischen KMUs. Wien.

Fink, M. / Kraus, S. (2007): Mutual Trust as a Key to Internationalization of SMEs. In: Management Research News. 30, S. 674-688.

Fink, M. / Harms, R. / Kraus, S. / Schulz, A. (2009): Wirkung und Erfolgsbeitrag der Selbstverpflichtung bei nationalen und internationalen Kooperationen junger KMU. In: Zeitschrift für Betriebswirtschaft. Special Issue 1/2009, S. 107-132.

Fink, M. / Harms, R. / Möllering, G. (2010): A strategy for overcoming the definitional struggle. In: Entrepreneurship and Innovation. 11 (2), S. 101-105.

Frey, B.S. / Osterloh, M. (2002): Successful Management by Motivation: Balancing Intrinsic and Extrinsic Incentives. Berlin.

Friede, CH.K. (1994): Sozialkompetenz als Ziel der Berufserfahrung: begriffsanalytisch betrachtet. In: Zeitschrift für Berufs- und Wirtschaftspädagogik. 90 (6), S. 606-625.

Friedrich, S.A. (1995): Mit Kernkompetenzen im Wettbewerb gewinnen. In: io Management Zeitschrift. 64 (4), S. 87-91.

Friedrichs, J. (1990): Methoden empirischer Sozialforschung. 14. Aufl. Opladen.

Fromkin, H.L. / Streufert, S. (1976): Laboratory Experimentation. In: Dunette, M.D. (Hrsg.): Handbook of Industrial and Organizational Psychology. Chicago, S. 415-416.

Fu, T.T. / Kong, W.H. / Yang, C. (2007): Monetary stakes and socioeconomic characteristics in ultimatum games: An experiment with nation-wide representative subjects. Working Paper.

Fürtjes, H.J. (1982): Das Gestaltungspotential von Instrumenten der empirischen Wirtschafts- und Sozialforschung. Berlin.

Gabarro, J.J. (1978): The development of trust, influence and expectations. In: Athos, A.G. / Gabarro, J.J. (Hrsg.): Interpersonal behaviors: communication and understanding in relationships. Englewood Cliffs, S. 290-303.

Gabler-Wirtschaftslexikon (1997): CD-Rom Version, 14. Aufl. 
Gachowetz, H. (1999): Feldforschung. In: Roth, E. / Holling, H. (Hrsg.): Sozialwissenschaftliche Methoden. Lehr- und Handbuch für Forschung und Praxis. München, S. 245-266.

Gachter, S. / Herrmann, B. / Thoni, C. (2004): Trust, voluntary cooperation, and socio-economic background: survey and experimental evidence. In: Journal of Economic Behavior \& Organization. 55 (4), S. 505-531.

Gaines, J.H. (1980): Upward Communication in Industry: An Experiment. In: Human Relations. 33 (12), S. 929-942.

Gambetta, D. (1988): Can we Trust Trust? In: Gambetta, D. (Hrsg.): Trust: Making and Breaking Cooperative Relations. Oxford, S. 213-237.

Geyer, A. (2008): Ausgewählte quantitative Methoden. Wien.

Giddens, A. (1995): Konsequenzen der Moderne. Frankfurt/Main.

Gilbert, D.U. (2007): Vertrauen als Gegenstand der ökonomischen Theorie: Ausgewählte theoretische Perspektiven, empirische Einsichten und neue Erkenntnisse. In: Zeitschrift für Management. 2 (1), S. 60-107.

Gillespie, N. (2003): Measuring Trust in Working Relationships: The Behavioral Trust Inventory. Arbeitspapier der Melbourne Business School der University of Melbourne. Melbourne.

Gimeno, J. / Woo, C.Y. (1996): Hypercompetition in a Multimarket Environment: The Role of Strategic Similarity and Multimarket Contact in Competitive De-escalation. In: Organization Science. 7 (3), S. 322-341.

Glaeser, E.L. / Laibson, D.I. / Scheinkman, J.A. / Soutter, Ch.L. (2000): Measuring Trust. In: The Quarterly Journal of Economics. 115 (3), S. 811846.

Goffman, E. (2006): Wir alle spielen Theater: die Selbstdarstellung im Alltag. 4. Aufl. München.

Good, D. (1988): Individuals, Interpersonal Relations and Trust. In: Gambetta, D. (Hrsg.): Trust: Making and Breaking Cooperative Relations. Oxford, S. 30-48.

Granger, C.W. / Newbold, P. (1974): Spurious Regressions in Econometrics. In: Journal of Econometrics. 2, S. 111-120.

Grant, R.M. (1991): The Resource Based Theory of Competitive Advantage: Implications for Strategic Formulation. In: California Management Review. 33 (3), S. 114-135.

Grant, R.M. (1995): Contemporary Strategy Analysis: Concepts, Techniques, Applications. 2. Aufl. Cambridge.

Greimel-Fuhrmann, B. / Pachlinger, I. (2008): Förderung der Sozialen Kompetenz im Studium an der Wirtschaftsuniversität Wien. In: Kasper, H. / Mühlbacher, J. (Hrsg.): Wettbewerbsvorteile durch organisationales und individuelles Kompetenzmanagement. Wien, S. 65-84. 
Habermas, J. (1988a): Theorie des kommunikativen Handelns. Handlungsrationalität und gesellschaftliche Rationalisierung. Bd. 1. Frankfurt/Main.

Habermas, J. (1988b): Theorie des kommunikativen Handelns. Zur Kritik der funktionalen Vernunft. Bd. 2. Frankfurt/Main.

Hacker, W. (1992): Expertenkönnen. Bern.

Hafeez, K. / Essmail, A.E. (2007): Evaluating organisation core competences and associated personal competencies using analytical hierarchy process. In: Management Research News. 30 (8), S. 530-547.

Halin, A. (1995): Vertikale Innovationskooperation: Eine transaktionskostentheoretische Analyse. Frankfurt/Main.

Hall, R. (1993): A Framework linking intangible Resources and Capabilities to Sustainable Competitive Advantage. In: Strategic Management Journal. 14 (5), S. 607-618.

Hamel, G. (1994): The Concept of Core Competence. In: Hamel, G. / Heene, A. (Hrsg.): Competence-based Competition. Chichester, S. 11-33.

Hamel, G. / Prahalad, C.K. (1994): Competing for the Future, Boston.

Hamel, G. / Prahalad, C.K. (1995): Wettlauf um die Zukunft: Wie Sie mit bahnbrechenden Strategien die Kontrolle über ihre Branche gewinnen und die Märkte von morgen schaffen. Wien.

Hänggi, G. (1998): Die Macht der Kompetenz: Ausschöpfung der Leistungspotentiale durch zukunftsgerichtete Kompetenzentwicklung. Frechen.

Harbach, H. (1992): Altruismus und Moral. Opladen.

Hardin, R. (2002): Trust and Trustworthiness. New York.

Hargie, O. / Saunders, Ch. / Dickson, D. (1994): Social Skills in Interpersonal Communication. 3. Aufl. London.

Harmsen, H. / Grunert, K. / Bove, K. (2002): Company Competencies as a Network. In: Journal of Product Innovation Management. 17 (5), S. 194-205.

Hart, O.D. (1995): Firms, Contracts and Financial Structure. Oxford.

Harvey, M. / Novicevic, M.M. / Kiessling, T. (2001): Hypercompetition and the Future of Global Management in the Twenty-first Century. In: Thunderbird International Business Review. 43 (5), S. 599-616.

Hatak, I. (2008): Wein als Geschenk. Exogene Einflussfaktoren auf die Weinkaufentscheidung in der Geschenksituation unter Berücksichtigung relevanter persönlicher Dispositionen. Saarbrücken.

Heckhausen, H. (1980): Motivation und Handeln: Lehrbuch der Motivationspsychologie. Berlin.

Heines, F.O. (1998): Kompetenz-Netzwerke im Anlagenbau. Diss. Universität St. Gallen. St. Gallen. 
Heisig, U. (1997): Vertrauensbeziehungen in der Arbeitsorganisation. In: Schweer, M. (Hrsg.): Interpersonales Vertrauen: Theorien und empirische Befunde. Opladen, S. 121-153.

Henning-Thurau, T. / Thurau, C. (1999): Sozialkompetenz als vernachlässigter Untersuchungsgegenstand des (Dienstleistungs-)Marketing: Einsatzmöglichkeiten und Konzeptualisierung. In: Marketing - Zeitschrift für Forschung und Praxis. 4, S. 297-311.

Heyse, V. / Erpenbeck, J. (1997): Der Sprung über die Kompetenzbarriere: Kommunikation, selbstorganisiertes Lernen und Kompetenzentwicklung von und in Unternehmen. Bielefeld.

Hinterhuber, H.H. / Stahl, H.K. (1996): Untemehmensnetzwerke und Kernkompetenzen. In: Bellmann, K. / Hippe, A. (Hrsg.): Management von Unternehmensnetzwerken: Interorganisationale Konzepte und praktische Umsetzung. Wiebaden, S. 87-117.

Hinterhuber, H.H. / Handlbauer, G. / Matzler, K. (2003): Kundenzufriedenheit durch Kernkompetenzen: eigene Potentiale erkennen - entwickeln - umsetzen. 2. Aufl. München, Wien.

Holling, H. / Liepmann, D. / König, F. / Otto, J. / Schmidt, J.U. (1980): Spezifische Zusammenhänge zwischen Problemlösungsfähigkeit, Intelligenz, Temperament, Interessen und Selbsteinschätzungen. In: Schulz, W. / Hautziger, M. (Hrsg.): Klinische Psychologie und Psychotherapie. Bd. 2. Tübingen, S. 245256.

Homburg, Ch. / Dobratz, A. (1991): Iterative Modellselektion in der Kausalanalyse. In: Zeitschrift für betriebswirtschaftliche Forschung. 43 (3), S. 213237.

Homburg, Ch. / Giering, A. (1996): Konzeptualisierung und Operationalisierung komplexer Konstrukte - Ein Leitfaden für die Marketingforschung. In: Marketing - Zeitschrift für Forschung und Praxis. 18 (1), S. 5-24.

Hoyos, C.G. (1987): Attitudes and Acceptance of Uncertain Situations. In: Bayerische Rückversicherung AG (Hrsg.): Society and Uncertainty. München, S. 49-64.

Ichijo, K. (2007): Enabling Knowledge-Based Competence of a Corporation. In: Ichijo, K. / Nonaka, I. (Hrsg.): Knowledge Creation and Management: New Challenges for Managers. Oxford, S. 83-96.

Irlenbusch, B. / Schade, L. (1999): Zur Wirksamkeit nicht bindender Verträge Eine experimentelle Untersuchung. In: Zeitschrift für betriebswirtschaftliche Forschung. 51 (7/8), S. 730-752.

Jacob, F. (2003): Kundenintegrationskompetenz - Konzeptualisierung, Operationalisierung und Erfolgswirkung. In: Marketing - Zeitschrift für Forschung und Praxis. 25 (2), S. 83-98. 
Jäger, P. (2001): Der Erwerb von Kompetenzen als Konkretisierung der Schlüsselqualifikationen - eine Herausforderung an Schule und Unterricht. Passau.

James, H.S. / Sykuta, M.E. (2005): Property Right and Organizational Characteristics of Producer-Owned Firms and Organizational Trust. In: Annals of Public and Cooperative Economics. 76 (4), S. 545-580.

Janowicz, M. / Noorderhaven, N. (2006): Levels of inter-organizational trust: conceptualization and measurement. In: Bachmann, R. / Zaheer, A. (Hrsg.): Handbook of Trust Research. Cheltenham, S. 264-279.

Janssen, J. / Laatz, W. (2007): Statistische Datenanalyse mit SPSS für Windows. 6. Aufl. Berlin.

Jones, M.T. (2002): Globalization and Organizational Restructuring: A Strategic Perspective. In: Thunderbird International Business Review. 44 (3), S. 325351.

Kaas, K.P. (1992): Kontraktgütermarketing als Kooperation zwischen Prinzipalen und Agenten. In: Zeitschrift für betriebswirtschaftliche Forschung. 44, S. 884901.

Kaiser, F.-J. / Kaminski, H. (1999): Methodik des Ökonomie-Unterrichts. Grundlagen eines handlungsorientierten Lernkonzepts mit Beispielen. 3. Aufl. Bad Heilbrunn.

Kanning, U.P. (2005): Soziale Kompetenzen - Entstehung, Diagnose und Förderung. Göttingen.

Kaplan, R.M. (1973): Components of Trust: Note on the Use of Rotter's Scale. In: Psychological Reports. 33, S. 13-14.

Kauffeld, S. / Grote, S. / Frieling, E. (2007): Das Kasseler-Kompetenz-Raster. In: Erpenbeck, J. / Rosenstiel, L. (Hrsg.): Handbuch Kompetenzmessung. 2. Aufl. Stuttgart, S. 224-243.

Kaufhold, M. (2006): Kompetenz und Kompetenzerfassung: Analyse und Beurteilung von Verfahren der Kompetenzerfassung. Wiesbaden.

Kaufmann, F. (1987): Norms and Institutions as Means of Coping with Uncertainty: The Sociological Perspective. In: Bayrische Rückversicherung AG (Hrsg.): Society and Uncertainty. München, S. 37-48.

Kee, H.W. / Knox, R.E. (1970): Conceptual and Methodological Considerations in the Study of Trust and Suspicion. In: Journal of Conflict Resolution. 14, S. 357-366.

Kenning, P. (2002): Customer Trust Management: Ein Beitrag zum Vertrauensmanagement im Lebensmitteleinzelhandel. Wiesbaden.

Kittner, I. (1994): Grundbegriffe der Experimentalpsychologie. In: Guttmann, G. (Hrsg.): Allgemeine Psychologie: Experimentalpsychologische Grundlagen. 2. Aufl. Wien, S. 1-20. 
Klaus, E. (2002): Vertrauen in Unternehmensnetzwerken - Eine interdisziplinäre Analyse. Wiesbaden.

Konovsky, M.A. / Pugh, S.D. (1994): Citizenship Behavior and Social Exchange. In: Academy of Management Journal. 37 (3), S. 656-669.

Konradt, U. / Hertel, G. / Joder, K. (2003): Web-based Assessment of Call Center Agents: Development and Validation of Computerized Instrument. In: International Journal of Selection and Assessment. 11, S. 184-193.

Korsgaard, M.A. / Schweiger, D. / Sapienza, H.J. (1995): Building Commitment, Attachment, and Trust in Strategic Decision-making Teams: the Role of Procedural Justice. In: Academy of Management Journal. 38 (1), S. 60-84.

Kosfeld, M. / Heinrichs, M. / Zak, P.J. / Fischbacher, U. / Fehr, E. (2005): Oxytocin Increases Trust in Human. In: Nature. 435, S. 673-676.

Koslowski, P. (2000): Wirtschafts- und Unternehmensethik. In: Bea, F.X. / Dichtl, E. / Schweitzer, M. (Hrsg.): Allgemeine Betriebswirtschaftslehre, Bd. 1. 8. Aufl. Stuttgart, S. 411-446.

Köszegi, S. (2001): Vertrauen in virtuellen Unternehmen. Wiesbaden.

Kmenta, J. (1971): Elements of Econometrics. London.

Kramer, R.M. (1999): Trust and Distrust in Organizations: Emerging Perspectives, Enduring Questions. In: Annual Review of Psychology, S. 569598.

Krogh, G. / Roos, J. (1992): Towards a Competence Based Perspective of the Firm. Working Paper 92/15. Norwegian School of Management. Sandvika.

Krogh, G. / Roos, J. (1995): A perspective on knowledge, competence and strategy. In: Personnel Review. 21 (3), S. 56-77.

Krogh, G. I Roos, J. (1996): Five Claims on Knowing. In: European Management Journal. 14 (4), S. 423-426.

Krüger, W. / Homp, Ch. (1997): Kernkompetenz-Management. Steigerung von Flexibilität und Schlagkraft im Wettbewerb. Wiesbaden.

Krystek, U. / Zumbrock, S. (1993): Planung und Vertrauen: Die Bedeutung von Vertrauen und Misstrauen für die Qualität von Planungs- und Kontrollsystemen. Stuttgart.

Krystek, U. / Redel, W. / Reppegather, S. (1997): Grundzüge virtueller Organisationen: Elemente und Erfolgsfaktoren, Chancen und Risiken. Wiesbaden.

Laatz, W. (1993): Empirische Methoden: Ein Lehrbuch für Sozialwissenschaftler. Thun, Frankfurt/Main.

Lado, A.A. / Boyd, N.C. / Wright, P. (1992): A Competency-Based Model of Sustainable Competitive Advantage: Towards a Conceptual Interpretation. In: Journal of Management. 18 (1), S. 77-91. 
Landes, W.M. / Posner, R.A. (1978): Altruism in Law and Economics. In: American Economic Review. 68, S. 417-421.

Lane, Ch. / Bachmann, R. (1996): The Social Constitution of Trust: Supplier Relations in Britain and Germany. In: Organization Studies. 17 (3), S. 365395.

Le, S. / Boyd, R. (2006): Evolutionary dynamics of the continuous iterated prisoner's dilemma. In: Journal of Theoretical Biology. 245 (22), S. 258-267.

Leinfellner, W. (1980): Einführung in die Erkenntnis- und Wissenschaftstheorie. 3. Aufl. Mannheim.

Leonard, D. (1995): Wellsprings of Knowledge. Building and Sustaining the Sources of Innovation. Boston.

Levitt, S.D. / List, J.A. (2007): What do laboratory experiments measuring social preferences reveal about the real world? In: Journal of Economic Perspectives. 21 (2), S. 153-174.

Lewicki, R.J. / Tomlinson, E.C. / Gillespie, N. (2006): Models of Interpersonal Trust Development: Theoretical Approaches, Empirical Evidence, and Future Directions. In: Journal of Management. 32 (6), S. 991-1022.

Lewis, J.D. / Weigert, A. (1985): Trust as a Social Reality. In: Social Forces. 64, S. 967-985.

Lindskold, S. (1981): Die Entwicklung von Vertrauen, der GRIT-Ansatz und die Wirkung von konziliantem Handeln auf Konflikt und Kooperation. In: Grunwald, W. / Lilge, H.-G. (Hrsg.): Kooperation und Konkurrenz in Organisationen. Bern, S. 241-273.

Lohmann, D. (1999): Umweltpolitische Kooperation zwischen Staat und Unternehmen aus Sicht der Neuen Institutionenökonomik. Marburg.

Loose, A. / Sydow, J. (1994): Vertrauen und Ökonomie in Netzwerkbeziehungen - Strukturationstheoretische Betrachtungen. In: Sydow, J. / Windeler, A. (Hrsg.): Management interorganisationaler Beziehungen. Opladen, S. 160193.

Lorenz, E.H. (1988): Neither Friends nor Strangers: Informal Networks of Subcontracting French Industry. In: Gambetta, D. (Hrsg.): Trust: Making and Breaking Cooperative Relations. Oxford, S. 194-210.

Lorenz, E. (1999): Trust, Contract and Economic Cooperation. In: Cambridge Journal of Economics. 23 (3), S. 301-315.

Luhmann, N. (1988): Familiarity, Confidence, Trust: Problem and Alternatives. In: Gambetta, D. (Hrsg.): Trust: Making and Breaking of Cooperative Relations. Oxford, S. 94-107.

Luhmann, N. (1991): Soziologie des Risikos. Berlin. 
Luhmann, N. (2000): Vertrauen: Ein Mechanismus der Reduktion sozialer Komplexität. 4. Aufl. Stuttgart.

Maas, A. (2001): Verantwortungswahrnehmung durch Kompetenzbildung. München.

Macneil, I.R. (1980): Power, Contract, and the Economic Model. In: Journal of Economic Issues. 11, S. 909-923.

Madhok, A. (1995): „Revisiting Multinational Firms“ - Tolerance for Joint Ventures: A Trust-Based Approach. In: Journal of International Business Studies. 26 (1), S. 117-138.

Madhok, A. (2006): Opportunism, trust and knowledge: the management of firm value and the value of firm management. In: Bachmann, R. / Zaheer, A. (Hrsg.): Handbook of Trust Research. Cheltenham (UK)/ Northhampton (US), S. 107-123.

Magali, D. (1999): Exposing Strategic Assets to Create New Competencies: The Case of Technological Acquisition in the Waste Management Industry in Europe and North America. In: Oxford University Press. 8, S. 635-671.

Mayer, R.C. / Davis, J.H. / Schoorman, D.F. (1995): An Integrative Model of Organizational Trust. In: Academy of Management Review. 20 (3), S. 709 734.

Mayer, R.C. / Davis, J.H. (1999): The Effect of the Performance Appraisal System on Trust for Management: A Field-quasi Experiment. In: Journal of Applied Psychology. 84 (1), S. 123-136.

Mayntz, R. / Holm, K. / Hübner, P. (1972): Einführung in die Methoden der empirischen Soziologie. 3. Aufl. Opladen.

McAllister, D. (1995): Affect- and Cognition-Based Trust as Foundations for Interpersonal Cooperation in Organizations. In: Academy of Management Journal. 38, S. 24-58.

McGrath, R.G. / MacMillan, I. / Venkatamaran, S. (1995): Defining and Developing Competence: A Strategic Process Paradigm. In: Strategic Management Journal. 16 (4), S. 251-275.

McKnight, H.D. / Cummings, L.L. / Chervany, N.L. (1998): Initial Trust Formation in New Organizational Relationships. In: Academy of Management. The Academy of Management Review. 23 (3), S. 473-490.

Meifert, M. (2003): Vertrauensmanagement in Unternehmen: Eine empirische Studie über Vertrauen zwischen Angestellten und ihren Führungskräften. 2. Aufl. München.

Meijboom, F. / Visak, T. / Brom, F. (2006): From Trust to Trustworthiness: Why Information is not enough in the Food Sector. In: Journal of Agriculture and Environmental Ethics. 19 (5), S. 427-442. 
Merriam Webster's Collegiate Dictionary (1996). 10. Aufl. Springfield.

Miebach, B. (1991): Soziologische Handlungstheorie. Eine Einführung. Opladen.

Milgrom, P. / Roberts, J. (1992): Economics, Organization and Management. Englewood Cliffs (NJ).

Mishra, A.K. (1996): Organizational Responses to Crisis: The Centrality of Trust. In: Kramer, R.M. / Tyler, T.R. (Hrsg.): Trust in Organizations: Frontiers of Theory and Research. Thousand Oaks (CA), S. 261-287.

Möhringer, S. (1998): Kompetenzkommunikation im Anlagengeschäft: Konzeptionelle Analyse und empirische Ergebnisse. Darmstadt.

Möllering, G. (2003): Trust: Social Science Theories and their Application to Organisations. University of Cambridge.

Möllering, G. (2006): Trust: Reason, Routine, Reflexivity. Oxford.

Möllering, G. / Sydow, J. (2005): Kollektiv, kooperativ, reflexiv: Vertrauen und Glaubwürdigkeit in Unternehmungen und Unternehmungsnetzwerken. In: Dernbach, B. / Meyer, M. (Hrsg.): Vertrauen und Glaubwürdigkeit. Interdisziplinäre Perspektiven. Wiesbaden, S. 64-93.

Moorman, Ch. / Deshpandé, R. / Zaltman, G. (1993): Factors Effecting Trust in Market Research Relationships. In: Journal of Marketing. 29 (1), S. 81-101.

Morgan, R.M. / Hunt, S.D. (1994): The Commitment-Trust Theory of Relationship Marketing. In: Journal of Marketing. 58 (3), S. 20-38.

Morgeson, F.P. / Delaney-Klinger, K. A. / Mayfield, M. S. / Ferrara, P. / Campion, M. A. (2004): Self-presentation processes in job analysis: A field experiment investigating inflation in abilities, tasks, and competencies. In: Journal of Applied Psychology. 89, S. 674-686.

Morrow, J.L. / Hansen, M.H. / Batista, J.C. (1999): Measurement Issues in Trust Research: Are We Measuring What We Think We're Measuring. In: Proceedings des IFAMA Agribusiness Forum 1999. Florenz.

Mugler, J. (1995): Betriebswirtschaftslehre der Klein- und Mittelbetriebe. 2. Aufl. Wien.

Mugler, J. (2005): Sichtweisen (in) der Betriebswirtschaftslehre. In: Rößl, D. (Hrsg.): Die Diplomarbeit in der Betriebswirtschaftslehre - Ein Leitfaden. 3. Aufl. Wien, S. 1-32.

Naef, M. / Schupp, J. (2009): Measuring Trust: Experiments and Surveys in Contrast and Combination. In: Meran, G. et al. (Hrsg): SOEPpapers on Multidisciplinary Panel Data Research, Deutsches Institut für Wirtschaftsforschung. Berlin, S. 1-44.

Nelson, R.R. / Winter, S.G. (1982): An evolutionary theory of economic change. Cambridge. 
Neubauer, W. (1999): Zur Entwicklung interpersonalen, interorganisationalen und interkulturellen Vertrauens durch Führung: Empirische Ergebnisse der sozialpsychologischen Vertrauensforschung. In: Schreyögg, G. / Sydow, J. (Hrsg.): Managementforschung 9. Berlin, S. 89-116.

Nieschlag, R. / Dichtl, E. / Hörschgen, H. (1997): Marketing. 18. Aufl. Berlin.

Nooteboom, B. (2002): Trust - Forms, Foundations, Functions, Failures and Figures. Cheltenham.

Nooteboom, B. / Berger, H. / Noorderhaven, H.G. (1997): Effects of Trust and Governance on Relational Risk. In: Academy of Management Journal. 40, S. 308-338.

North, D.C. (1991): Towards a Theory of Institutional Change. In: Quarterly Review of Economics and Business. 31, S. 3-11.

Nunnally, J.C. (1978): Psychometric Theory. 2. Aufl. New York.

Opp, K.-D. (1999a): Methodologie der Sozialwissenschaften. 4. Aufl. Opladen.

Opp, K.-D. (1999b): Wissenschaftstheoretische Grundlagen der empirischen Sozialforschung. In: Roth, E. / Holling, H. (Hrsg.): Sozialwissenschaftliche Methoden. Lehr- und Handbuch für Forschung und Praxis. München, S. 4973.

O'Reilly III, C.A. / Roberts, K.H. (1974): Information Filtration in Organizations: Three Experiments. In: Organizational Behavior and Human Performance. 11, S. 253-265.

Oser, F. (1982): Entwicklungspsychologie und Feldforschung. In: Patry, J.-L. (Hrsg.): Feldforschung. Methoden und Probleme sozialwissenschaftlicher Forschung unter natürlichen Bedingungen. Bern, S. 131-152.

Osterloh, M. / Weibel, A. (2000): Ressourcensteuerung in Netzwerken: Eine Tragödie der Allmende? In: Sydow, J. / Windeler, A. (Hrsg.): Steuerung von Netzwerken - Konzepte und Praktiken. Wiesbaden, S. 88-106.

Ostrom, E. / Walker, J. (2002): Trust and Reciprocity: Interdisciplinary Lessons for Experimental Research. New York.

Pablo, A.L. (1997): Reconciling Predictions of Decision Making under Risk: Insights from a Reconceptualized Model of Risk Behavior. In: Journal of Managerial Psychology. 12 (1), S. 4-20.

Palfrey, T.R. / Rosenthal, H. (1988): Private Incentives in Social Dilemmas: The Effects of Incomplete Information and Altruism. In: Journal of Public Economics. 35, S. 309-332.

Parsons, T. (1978): Action theory and the human condition. New York.

Parsons, T. (1986): Aktor, Situation und normative Muster. Ein Essay zur Theorie sozialen Handelns. Frankfurt/Main.

Perry, S.G. (2001): Trust and Virtual Organizations. Wien. 
Peter, S.I. (1999): Kundenbindung als Marketingziel: Identifikation und Analyse zentraler Determinanten. 2. überarb. Aufl. Wiesbaden.

Petermann, F. (1996): Psychologie des Vertrauens. 3. Aufl. Göttingen.

Picot, A. (1980): Organisationsexperimente. In: Grochla, E. (Hrsg.): Handwörterbuch der Organisation. Stuttgart, Sp. 1481-1494.

Picot, A. (1981): Der Beitrag der Theorie der Verfügungsrechte zur ökonomischen Analyse von Unternehmensverfassungen. In: Bohr, K. (Hrsg.): Unternehmensverfassung als Problem der Betriebswirtschaftslehre. Berlin, S. 153197.

Picot, A. (1991): Ökonomische Theorien der Organisation: Ein Überblick über neuere Ansätze und deren betriebswirtschaftliches Anwendungspotential. In: Ordelheide, D. / Rudolph, B. / Büsselmann, E. (Hrsg.): Betriebswirtschaftslehre und Ökonomische Theorie. Stuttgart, S. 143-170.

Picot, A. (1995): Verfügungsrechtstheorie, Transaktionskosten und Führung. In: Kieser, A. (Hrsg.): Handwörterbuch der Führung. 2. Aufl. Stuttgart, S. 21062113.

Picot, A. / Dietl, H. (1990): Transaktionskostentheorie. In: WiSt. 19 (4), S. 178184.

Picot, A. / Bortenlänger, Ch. / Röhrl, H. (1997): Organization of Electronic Markets - Contributions from the New Institutional Economics. In: Information Society. 13 (1), S. 107-123.

Picot, A. / Reichwald, R. / Wigand, R. (2001): Die grenzenlose Unternehmung: Information, Organisation und Management; Lehrbuch zur Unternehmensführung im Informationszeitalter. 4. Aufl. Wiesbaden.

Pieper, J. (2000): Vertrauen in Wertschöpfungspartnerschaften: eine Analyse aus Sicht der neuen Institutionenökonomie. Wiesbaden.

Pirson, M. (2007): Facing the Trust Gap: Measuring and Building Trust in Organizations. Diss. Universität St. Gallen.

Pleitner, H.J. / RößI, D. (1995): Wirtschaftsförderung unterhalb der Wirtschaftspolitik - der Beitrag zwischenbetrieblicher Kooperation. In: Brandenberg, A. (Hrsg.): Standpunkte zwischen Theorie und Praxis - Handlungsorientierte Problemlösungen in Wirtschaft und Gesellschaft. Festschrift für Hans Schmid. Bern, S. 671-689.

Plötner, O. (1995): Das Vertrauen des Kunden. Wiesbaden.

Podsakoff, P.M. / McKenzie, S.B. / Bommer, W.H. (1996): Transformational Leader Behaviors and Substitutes for Leadership as Determinants Employee Satisfaction, Commitment, Trust and Organizational Citizenship Behaviors. In: Journal of Management. 22, S. 259-298. 
Popper, K.R. (1973): Objektive Erkenntnis: Ein evolutionärer Entwurf. Hamburg.

Porter-Liebeskind, J. / Lumerman-Oliver, A. (1998): From Handshake to Contract: Intellectual Property, Trust, and the Social Structure of Academic Research. In: Lane, Ch. / Bachmann, R. (Hrsg.): Trust Within and Between Organizations. Oxford, S. 118-145.

Prahalad, C.K. / Hamel, G. (1990): The Core Competence of the Corporation. In: Harvard Business Review. 68 (3), S. 79-91.

Preisendörfer, P. (1995): Vertrauen als soziologische Kategorie: Möglichkeiten und Grenzen einer entscheidungstheoretischen Fundierung des Vertrauenskonzepts. In: Zeitschrift für Soziologie. 24, S. 263-272.

Probst, G. / Deussen, A. / Eppler, M. / Raub, St. (2000): KompetenzManagement - Wie Individuen und Organisationen Kompetenz entwickeln. Wiesbaden.

Putnam, R. (1995): Bowling Alone: America's Declining Social Capital. In: Journal of Democracy. 6 (1), S. 65-78.

Raimondo, M.A. (2000): The Measurement of Trust in Marketing Studies: A Review of Models and Methodologies. Arbeitspapier des Instituts Strategy, Management and Quantitative Methods der Università degli Studi di Milano Biococca. Mailand.

Rasche, C. (1994): Wettbewerbsvorteile durch Kernkompetenzen: Ein ressourcenorientierter Ansatz. Wiesbaden.

Rasmusen, E. (1989): Games and Information: An Introduction to Game Theory. Oxford.

Reed, M.I. (2001): Organization, Trust and Control: A Realist Analysis. In: Organization Studies. 22 (2), S. 201-228.

Richter, R. (1990): Sichtweise und Fragestellungen der Neuen Institutionenökonomie. In: Zeitschrift für Wirtschafts- und Sozialwissenschaften. 110, S. 571-591.

Richter, R. / Furubotn, E. (1996): Neue Institutionenökonomik. Tübingen.

Riess, S. (1998): Kernkompetenzen im Vertrieb - ein ressourcenorientierter Strategieansatz. Wiesbaden.

Ring, P.S. / Van de Ven, A. (1992): Structuring cooperative relationships between organizations. In: Strategic Management Journal. 13 (7), S. 483-498.

Ring, P.S. / Van de Ven, A. (1994): Developmental Processes of Cooperative Interorganizational Relationships. In: Academy of Management Review. 19 (1), S. 90-118.

Ripperger, T. (1998): Ökonomik des Vertrauens: Analyse eines Organisationsprinzips. Tübingen. 
Robinson, S.L. / Rousseau, D.M. (1994): Violating the Psychological Contract: Not the Exception but the Norm. In: Journal of Organizational Behavior. 15 (3), S. 245-259.

Robinson, S.L. (1996): Trust and Breach of the Psychological Contract. In: Administrative Science Quarterly. 41, S. 575-599.

Rockenhäuser, J. (1998): Digitale Konvergenz und Kompetenzmanagement. Wiesbaden.

Roeder, K. (2000): Management virtueller Unternehmen unter besonderer Berücksichtigung des Vertrauensmanagements. St. Gallen.

Rößl, D. (1994): Gestaltung komplexer Austauschbeziehungen - Analyse zwischenbetrieblicher Kooperation. Wiesbaden.

RößI, D. (1996): Selbstverpflichtung als alternative Koordinationsform von komplexen Austauschbeziehungen. In: Zeitschrift für betriebswirtschaftliche Forschung. 48 (4), S. 311-334.

RößI, D. (2000): Beziehungsmanagement im Klein- und Mittelbetrieb - Gestaltung dauerhafter Kundenbeziehungen. Wien.

Rößl, D. (2006): Relationship-Management: mit Beiträgen aus Wissenschaft und Praxis. Wien.

Rößl, D. / Fink, M. (2004): Instrumentelles und maximenbasiertes Vertrauen als Erfolgsfaktor von Kooperationen - Zur besonderen Relevanz maximenbasierten Vertrauens in Kooperationen zwischen KMU. In: Meyer, J.-A. (Hrsg.): Kooperationen von KMU in Europa - Jahrbuch der KMU-Forschung und -Praxis 2004. Köln, S. 31-52.

Rößl, D. / Fink, M. (2006): Der systemtheoretische Beitrag: Die Überwindung doppelter Kontingenz durch Vertrauen. In: Rößl, D. (Hrsg.): Relationship-Management: mit Beiträgen aus Wissenschaft und Praxis. Wien, S. 58-72.

Rößl, D. / Fink, M. (2007): Koordination von Kooperationen durch Selbstverpflichtung - wenn Markt und Hierarchie versagen. In: Zeitschrift für KMU und Entrepreneurship. 55 (2), S. 118-136.

Roth, A.E. (1995): Introduction to Experimental Economics. In: Kagel, J.H. / Roth, A.E. (Hrsg.): The Handbook of Experimental Economics. New Jersey, S. 3-109.

Rotter, J.B. (1967): A New Scale for the Measurement of Interpersonal Trust. In: Journal of Personality. 35, S. 651-665.

Rotter, J.B. (1971): Generalized Expectancies for Interpersonal Trust. In: American Psychologist. 26 (5), S. 443-452.

Rotter, J.B. (1980): Interpersonal Trust, Trustworthiness and Gullibility. In: American Psychologist. 35 (1), S. 1-7. 
Rotter, J.B. (1991): The Interpersonal Trust Scale. In: Robinson, J.P. / Shaver, P.R. / Wrightsman, L.S. (Hrsg.): Measures of Personality and Social Psychological Attitudes. San Diego, S. 395-396.

Rousseau, D.M. / Sitkin, S.B. / Burt, R.S. / Camerer, C. (1998): Not so Different After All: A Cross-Discipline View of Trust. In: Academy of Management Review. 23 (3), S. 393-404.

Sako, M. / Helper, S. (1998): Determinants of Trust in Supplier Relations: Evidence from the Automotive Industry in Japan and the United States. In: Journal of Economic Behavior and Organization. 34 (3), S. 387-417.

Sanchez, R. (1996): Dynamics of Competence-Based Competition. Theory and Practice in the New Strategic Management. Oxford.

Sanchez, R. / Thomas, H. (1996): Strategic Goals. In: Sanchez, R. / Heene, A. / Thomas, H. (Hrsg.): Dynamics of Competence-Based Competition. Theory and Practice in the New Strategic Management. Oxford, S. 63-84.

Schaeper, H. / Briedis, K. (2004): Kompetenzen von Hochschulabsolventinnen und Hochschulabsolventen, berufliche Anforderungen und Forderungen für die Hochschulreform. Kurzinformation A6/2004. Hochschul-InformationsSystem $\mathrm{GmbH}$. Hannover.

Schanz, G. (2000): Wissenschaftsprogramme der Betriebswirtschaftslehre. In: Bea, F.X. / Dichtl, E. / Schweitzer, M. (Hrsg.): Allgemeine Betriebswirtschaftslehre, Bd. 1. 8. Aufl. Stuttgart, S. 80 - 161.

Scharlemann, J.P.W. / Eckel, C.C. / Kacelnik, A. / Wilson, R.K. (2001): The Value of a Smile: Game Theory of a Human Face. In: Journal of Economic Psychology. 22, S. 617-640.

Schendera, Ch. (2008): Regressionsanalyse mit SPSS. München.

Schenk, K.E. (1992): Die Neue Institutionenökonomie: Ein Überblick über wichtige Elemente und Probleme der Weiterentwicklung. In: Zeitschrift für Wirtschafts- und Sozialwissenschaften. 112, S. 337-378.

Schiller, T. (2000): Kompetenz-Management für den Anlagenbau: Ansatz, Empirie und Aufgaben. Wiesbaden.

Schmitz, G. (1997): Marketing für professionelle Dienstleistungen: Bedeutung und Dynamik der Geschäftsbeziehungen. Wiesbaden.

Schreinemakers, J.F. (1999): Knowledge management - International ISMICK Symposium. Würzburg.

Schrader, S. / Riggs, W.M. / Smith, R.P. (1993): Choice over Uncertainty and Ambiguity in Technical Problem Solving. In: Journal of Engineering and Technology Management. 10, S. 73-99.

Schuler, H. (1980): Ethische Probleme psychologischer Forschung. Göttingen. 
Schuler, H. / Barthelme, D. (1995): Soziale Kompetenz als berufliche Anforderung. In: Seyfried, B. (Hrsg.): Stolpersteine Sozialkompetenz. Was macht es so schwierig sie zu erfassen, zu fördern und zu beurteilen? Berichte zur beruflichen Bildung, Band 179. Bielefeld, S. 77-116.

Schweer, M. / Thies, B. (2003): Vertrauen als Organisationsprinzip: Perspektiven für komplexe soziale Systeme. Bern.

Seifert, M. (2001): Vertrauensmanagement in Unternehmen: Empirische Studie über Vertrauen zwischen Angestellten und ihren Führungskräften. München.

Semlinger, K. (1993): Effizienz und Autonomie in Zulieferungsnetzwerken Zum strategischen Gehalt von Kooperationen. In: Staehle, W.H. / Sydow, J. (Hrsg.): Managementforschung 3. Berlin, S. 309-354.

Shane, S. (1994): The effect of national culture on the choice between licensing and direct foreign investment. In: Strategic Management Journal. 15 (8), 627642.

Shapiro, S.P. (1987): The Social Control of Impersonal Trust. In: The American Journal of Sociology. 93 (3), S. 623-658.

Shapiro, S.P. / Sheppard, B.H. / Cheraskin, L. (1992): Business on Handshake. In: Negotiation Journal. 8 (4), S. 365-377.

Shaw, R.B. (1997): Trust in the Balance. Building Successful Organizations on Results, Integrity and Concern. San Francisco.

Sichtmann, Ch. (2007): An analysis of antecedents and consequences of trust in a corporate brand. In: European Journal of Marketing. 41 (9/10), S. 999-1015.

Siegrist, M. (2001): Die Bedeutung von Vertrauen bei der Wahrnehmung und Bewertung von Risiken. Zürich.

Simmel, G. (1968): Soziologie. 5. Aufl. Berlin.

Sitkin, S.B. / Pablo, A.L. (1992): Reconceptualizing the Determinants of Risk Behavior. In: Academy of Management Review. 17 (1), S. 9-38.

Sitkin, S.B. / Roth, N.L. (1993): Explaining the Limited Effectiveness of Legalistic "Remedies" for Trust/Distrust. In: Organization Science. 4 (3), S. $367-393$.

Sitkin, S.B. / Weingart, L.R. (1995): Determinants of Risky Decision-Making Behavior: A Test of the Mediating Role of Risk Perceptions and Propensity. In: Academy of Management Journal. 38 (6), S. 1573-1592.

Sitkin, S.B. / Rousseau, D.M. / Burt, R.S. / Camerer, C. (1998): Not so different after all: A cross-discipline view of trust. In: Academy of Management Review. 23 (3), S. 393-404.

Slangen, L.H.G. / Van Kooten, G.C. / Suchanek, P. (2003): Trust in Countries in Transition: Empirical Evidence from Agriculture. In: International Journal for Social Economics. 30 (9-10), S. 1095-1109. 
Soh, H. / Reid, L.N. / Whitehill King, K. (2009): Measuring Trust in Advertising: Development and Validation of the ADTRUST Scale. In: Journal of Advertising. 38 (2), S. 83-103.

Sonntag, K. / Schäfer-Rauser, U. (1993): Selbsteinschätzung beruflicher Kompetenzen bei der Evaluation von Bildungsmaßnahmen. In: Zeitschrift für Arbeits- und Organisationspsychologie. 37 (4), S. 163-171.

Späth, J.F. (2008): Interpersonelles Vertrauen in Organisationen: Eine Empirische Untersuchung der Einflussfaktoren und Verhaltenswirkungen. Frankfurt/Main.

Spremann, K. (1989): Agent und Principal. In: Bamberg, G. / Spremann, K. (Hrsg.): Agency theory, information and incentives. Berlin, S. 3-35.

Spremann, K. (1990): Asymmetrische Information. In: Zeitschrift für Betriebswirtschaft. 60, S. 561-586.

Stäudel, T. (1988): Der Kompetenzfragebogen. In: Diagnostica. 34 (2), S. 136148.

Stahl, H.K. (1996): Beziehungskompetenz. In: Hinterhuber, H.H. / Al-Ani, A. / Handlbauer, G. (Hrsg.): Das neue strategische Management: Elemente und Perspektiven einer zukunftsorientierten Unternehmensführung. Wiesbaden, S. 217-244.

Stahl, H.K. (2000): Die Bedeutung des mittleren Managements für den Aufbau organisationaler Kompetenzen. In: Hammann, P. / Freiling, J. (Hrsg.): Die Ressourcen- und Kompetenzperspektive des Strategischen Managements. Wiesbaden, S. 411-438.

Stahl, H.K. (2005): Schlüsselkompetenzen für die mittlere Führungsebene. Was können Krankenhäuser von „For-Profit-Unternehmen“ lernen?. In: http://www.vinzenzgruppe.at/media/download/stahl.pdf. Stand: 20. März 2009.

Stahl, Ch. (2008): Developing a Framework for Competence Assessment. Wien.

Stapf, K.H. (1999): Laboruntersuchungen. In: Roth, E. / Holling, H. (Hrsg.): Sozialwissenschaftliche Methoden. Lehr- und Handbuch für Forschung und Praxis. München, S. 228-244.

Stein, F.A. (1989): Fallsimulation. In: Die Betriebswirtschaft. 49 (4), S. 530-532.

Stein, F.A. (1990): Betriebliche Entscheidungs-Situationen im Laborexperiment. Frankfurt/Main.

Stein, F.A. (1991): Betriebswirtschaftliche Laborforschung. In: Die Betriebswirtschaft. 51 (1), S. 109-111.

Steinle, C. / Bruch, H. / Nasner, N. (1997): Kernkompetenzen - Konzepte, Ermittlung und Einsatz zur Strategieevolution. In: Zeitschrift für Planung. 8 (1), S. 1-23. 
Stelzl, I. (1999): Experiment. In: Roth, E. / Holling, H. (Hrsg.): Sozialwissenschaftliche Methoden. Lehr- und Handbuch für Forschung und Praxis. München, S. 108-125.

Steyrer, J. (1998): Charisma and the Archetypes of Leadership. In: Organization Studies. 19 (5), S. 807-828.

Stiglitz, J.E. (1974): Information and Economic Analysis. In: Parkin, M. / Nobay, A.R. (Hrsg.): Current Economic Problems. London, S. 27-52.

Stiglitz, J.E. (1989): Markets, Market Failure, and Development, in: The American Economic Review. 79 (2), S. 197-203.

Strasmann, J. / Schüller, A. (1996): Kernkompetenzen: Was Unternehmen wirklich erfolgreich macht. Stuttgart.

Strickland, L.H. (1958): Surveillance and Trust. In: Journal of Personality. 26, S. 200-215.

Sydow, J. / Windeler, A. (2000): Steuerung von und in Netzwerken Perspektiven, Konzepte, vor allem aber offene Fragen. In: Sydow, J. I Windeler, A. (Hrsg.): Steuerung von Netzwerken - Konzepte und Praktiken. Wiesbaden, S. 1-24.

Tampoe, M. (1994): Exploiting the Core Competencies of Your Organization. In: Long Range Planning. 27 (4), S. 66-77.

Teece, D.J. / Rumelt, R. / Dosi, G. / Winter, S. (1994): Understanding Corporate Coherence - Theory and Evidence. In: Journal of Economic Behavior and Organization. 23 (1), S. 1-30.

Teece, D.J. (1998): Capturing value from knowledge assets: The new economy, markets for knowhow, and intangible assets. In: Strategic Management Journal. 17 (special issue), S. 27-44.

Teraji, S. (2008): Property Rights, Trust and Economic Performance. In: The Journal of Socio-Economics. 37, S. 1584-1596.

Thomas, A. (1992): Grundriss der Sozialpsychologie. Bd. 2. Individuum-GruppeGesellschaft. Göttingen.

Thomas, R. (1997): Modern Econometrics. Harlow.

Thomson, E.H. (2000): Management von Kernkompetenzen. München.

Treibel, A. (1994): Einführung in die soziologischen Theorien der Gegenwart. Opladen.

Trivers, R.L. (1971): The Evolution of Reciprocal Altruism. In: Quarterly Review of Biology. 46, S. 35-57.

Turner, D. / Crawford, M. (1994): Managing Current and Future Competitive Performance: The Role of Competence. In: Hamel, G. / Heene, A. (Hrsg.): Competence-based Competition. Chichester, S. 241-262.

Ulich, E. (1998): Arbeitspsychologie. 4. Aufl. Zürich, Stuttgart. 
Urban, D. / Mayerl, J. (2006): Regressionsanalyse: Theorie, Technik und Anwendung. 2. überarb. Aufl. Wiesbaden.

Vanberg, V.J. (1994): Rules and Choices in Economics. London.

Vogt, J. (1997): Vertrauen und Kontrolle in Transaktionen: eine institutionenökonomische Analyse. Wiesbaden.

Volberda, H.W. (1996): Toward the Flexible Form: How to Remain Vital in Hypercompetitive Environments. In: Organization Science. 7 (4), S. 359-374.

Weber, M. (1972): Wirtschaft und Gesellschaft, 5. Aufl. Tübingen.

Weber, M. (1980): Wirtschaft und Gesellschaft, 5. überarb. Aufl. Tübingen.

Weber, J. (1993): Kostenrechnung im System der Unternehmensführung - Stand und Perspektiven der Kostenrechnung in den 90er Jahren. In: Weber, J. (Hrsg.): Zur Neuausrichtung der Kostenrechnung - Entwicklungsperspektiven für die 90er Jahre. Stuttgart, S. 1-77.

Weigelt, K. / Camerer, C. (1988): Reputation and Corporate Strategy: A Review of Recent Theory. In: Strategic Management Journal. 9 (5), S. 443-454.

Weise, G. (1975): Psychologische Leistungstests. Ein Handbuch für Studium und Praxis. Göttingen.

Weiss, P.A. (1992): Die Kompetenz von Systemanbietern - Ein neuer Ansatz im Marketing für Systemtechnologien. Berlin.

Welter, F. / Smallbone, D. (2006): Exploring the role of trust in entrepreneurial activity. In: Entrepreneurship Theory \& Practice. 30 (4), S. 465-475.

Werner, H. (1996): Relationales Beschaffungsverhalten - Ausprägungen und Determinanten. Vallendar.

Wernerfelt, B. (1984): A Resource Based View of the Firm. In: Strategic Management Journal. 5 (2), S. 171-180.

Westmeyer, H. (1982): Wissenschaftstheoretische Aspekte der Feldforschung. In: Patry, J.-L. (Hrsg.): Feldforschung. Methoden und Probleme sozialwissenschaftlicher Forschung unter natürlichen Bedingungen. Bern, S. 67-84.

Wiemann, V. (1998): Verlust-Eskalation in Management-Entscheidungen - eine empirische Untersuchung. Frankfurt/Main.

Wiese, H. (2002): Entscheidungs- und Spieltheorie. Berlin.

Williamson, O.E. (1975): Markets and Hierarchies. New York.

Williamson, O.E. (1985): The Economic Institutions of Capitalism: Firms, Markets, Relational Contracting. New York.

Williamson, O.E. (1990a): A Comparison of Alternative Approaches to Economic Organization. In: JITE. 146, S. 61-71.

Williamson, O.E. (1990b): Die ökonomischen Institutionen des Kapitalismus: Unternehmen, Märkte, Kooperationen. Tübingen. 
Williamson, O.E. (1991): Comparative Economic Organization: Vergleichende Ökonomische Organisationstheorie: Die Analyse direkter Strukturalternativen. In: Ordelheide, D. / Rudolph, B. / Büsselmann, E. (Hrsg.): Betriebswirtschaftslehre und Ökonomische Theorie. Stuttgart, S. 13-49.

Winnes, R. (1993): Neue Anforderungen an Führungsqualität und Persönlichkeitsstruktur. In: Schuppert, D. (Hrsg.): Kompetenz zur Führung. Was Führungspersönlichkeiten auszeichnet. Wiesbaden, S. 73-111.

Wood, J.A. / Boles, J.S. / Johnston, W. / Bellenger, D. (2008): Buyers' Trust of a Salesperson: An Item-Level Meta-Analysis. In: Journal of Personal Selling \& Sales Management. 28 (3), S. 263-283.

Wrightsman, L.S. (1991): Interpersonal Trust and Attitudes toward Human Nature. In: Robinson, J.P. / Shaver, P.R. / Wrightsman, L.S. (Hrsg.): Measures of Personality and Social Psychological Attitudes. San Diego, S. 373-412.

Wrightsman, L.S. (1992): Assumptions about Human Nature. Implications for Researchers and Practitioners. 2. Aufl. Newbury Park.

Wurche, S. (1994): Vertrauen und ökonomische Rationalität in kooperativen Interorganisationsbeziehungen, in: Sydow, J. / Windeler, A. (Hrsg.): Management interorganisationaler Beziehungen - Vertrauen, Kontrolle und Informationstechnik. Opladen, S. 142-159.

Young, L.C. / Wilkinson, I.F. (1989): The Role of Trust and Co-operation in Marketing Channels: A Preliminary Study. In: European Journal of Marketing. 23 (2), S. 109-122.

Zaheer, A. / McEvily, B. / Perrone, V. (1998): Does Trust Matter? Exploring the Effects of Interorganizational and Interpersonal Trust on Performance. In: Organization Science. 9 (2), S. 141-159.

Zand, D.E. (1972): Trust and Managerial Problem Solving. In: Administrative Science Quarterly. 17 (2), S. 229-239.

Zimbardo, Ph.G. / Gerrig, R. J. (2008): Psychologie. 18. Aufl. München.

Zins, A.H. (2001): Relative Attitudes and Commitment in Customer Loyalty Models. Some Experiences in the Commercial Airline Industry. In: International Journal of Service Industry. 12 (3/4), S. 269-294.

Zürn, P. (1993): Die sieben Komponenten der persönlichen Führungskompetenz. In: Schuppert, D. (Hrsg.): Kompetenz zur Führung. Was Führungspersönlichkeiten auszeichnet. Wiesbaden, S. 187-205. 
Isabella Hatak - 978-3-631-74970-8 Downloaded from PubFactory at 01/11/2019 09:36:38AM via free access 
ANHANG

Unterlagen zum Experiment (Version, Weingewinn')

Einführungstext

320

Einführungsevaluierung

321

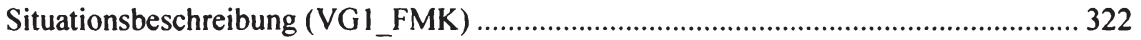

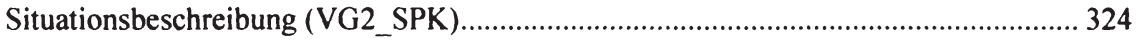

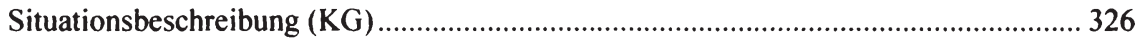

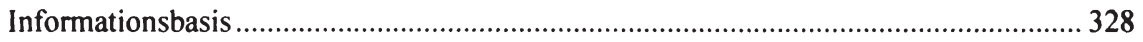

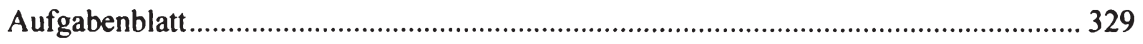

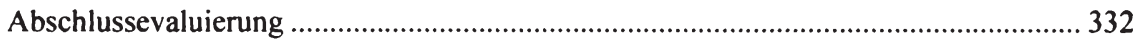




\section{EINFÜHRUNGSTEXT}

Herzlich Willkommen zu unserem Assessment-Center, im Rahmen dessen Sie bei gewissenhafter Bearbeitung der Aufgabenstellungen - als Dankeschön für Ihre Mühe Wein gewinnen können (Gesamtmenge: 150 Flaschen). Zuerst möchten wir Sie um Informationen zu Ihrer Person bitten. Bitte vergewissern Sie sich, dass Sie alle geforderten Daten bekannt geben! Vielen Dank für Ihre Teilnahme!

\section{Kontaktprotokoll}

(Bitte geben Sie die geforderten Daten zur LV und zu Ihrer Person an!)

LV-Nr./LV-Name:

Name des/der StudentIn:

Studiengang:

Alter:

Geschlecht:

$\square$ weiblich

männlich

E-Mail-Adresse: 


\section{EINFÜHRUNGSEVALUIERUNG}

\begin{tabular}{|c|c|c|}
\hline \multicolumn{3}{|c|}{$\begin{array}{l}\text { Bitte geben Sie die Antwort an, die am ehesten auf Sie zutrifft (markieren Sie durch ein Kreuz das } \\
\text { Ausmaß Ihrer Zustimmung/Ablehnung zu jeder Aussage)! }\end{array}$} \\
\hline $\begin{array}{l}\text { Die meisten Menschen helfen jemandem in Not ohne zu } \\
\text { zögern. }\end{array}$ & $\begin{array}{l}\text { stimme } \\
\text { voll zu }\end{array}$ & $\begin{array}{l}\text { stimme gar } \\
\text { nicht zu }\end{array}$ \\
\hline $\begin{array}{l}\text { Die meisten Menschen folgen dem Motto, Verhalte dich } \\
\text { anderen gegenüber so, wie du willst, dass sie sich dir } \\
\text { gegenüber verhalten`. }\end{array}$ & $\begin{array}{l}\text { stimme } \\
\text { voll } z u\end{array}$ & $\begin{array}{l}\text { stimme gar } \\
\text { nicht } z u\end{array}$ \\
\hline Die meisten Menschen sagen was sie denken. & $\begin{array}{l}\text { stimme } \\
\text { voll zu }\end{array}$ & $\begin{array}{l}\text { stimme gar } \\
\text { nicht zu }\end{array}$ \\
\hline $\begin{array}{l}\text { Die meisten Menschen sagen im Grunde die Wahrheit; } \\
\text { sogar dann, wenn sie wissen, dass sie besser dran wären, } \\
\text { wenn sie lügen würden. }\end{array}$ & $\begin{array}{l}\text { stimme } \\
\text { voll zu }\end{array}$ & $\begin{array}{l}\text { stimme gar } \\
\text { nicht zu }\end{array}$ \\
\hline $\begin{array}{l}\text { Menschen sind normalerweise aufrichtig um die Probleme } \\
\text { anderer besorgt. }\end{array}$ & $\begin{array}{l}\text { stimme } \\
\text { voll zu }\end{array}$ & $\begin{array}{l}\text { stimme gar } \\
\text { nicht } z u\end{array}$ \\
\hline Die meisten Menschen stehen zu ihrer Überzeugung. & $\begin{array}{l}\text { stimme } \\
\text { voll } z u\end{array}$ & $\begin{array}{l}\text { stimme gar } \\
\text { nicht } z u\end{array}$ \\
\hline $\begin{array}{l}\text { Menschen bleiben normalerweise bei ihrer Meinung, wenn } \\
\text { sie denken, dass sie Recht haben, auch wenn die anderen } \\
\text { nicht ihrer Meinung sind. }\end{array}$ & $\begin{array}{l}\text { stimme } \\
\text { voll zu }\end{array}$ & $\begin{array}{l}\text { stimme gar } \\
\text { nicht zu }\end{array}$ \\
\hline
\end{tabular}

Bitte kreuzen Sie pro Reihe die Antwort an, die Ihrer Meinung am ehesten entspricht (keine Mehrfachantworten möglich)!

Als Unternehmer sehen Sie sich immer wieder Entscheidungen gegenübergestellt. Wie würden Sie Ihre Bereitschaft einschätzen...

1. Ich bin bereit, eine risikoreiche Entscheidung zu treffen, die auf der Empfehlung einer anderen Person beruht, auf die ich mich verlassen muss

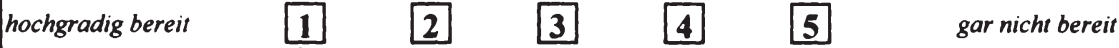

2. Ich bin bereit, eine risikoreiche Entscheidung zu treffen, die auf methodisch hochkomplexen Analysen beruht.

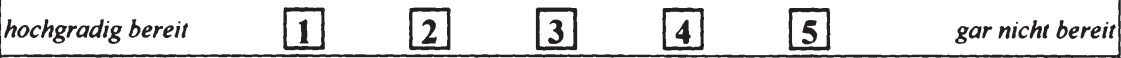

3. Ich bin bereit, eine risikoreiche Entscheidung zu treffen, die die strategische Ausrichtung meines Betriebs nachhaltig ändern könnte.

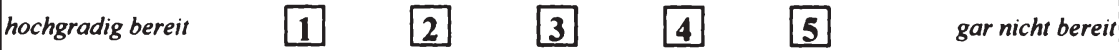

4. Ich bin bereit, eine Entscheidung umzusetzen, auch wenn sie die Gefahr eines Fehlschlags in sich birgt. $\begin{array}{lllllll}\text { hochgradig bereit } & 1 & 2 & 3 & 4 & 5 & \text { gar nicht bereit }\end{array}$

5. Ich bin bereit, eine Entscheidung zu unterstützen, bei der ich weiß, dass, obwohl teilweise Daten vorliegen, Informationen fehlen.

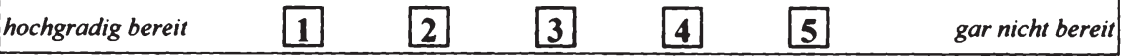

Bevor Sie nun zu der Situationsbeschreibung weiterblattern, geben Sie die zwei soeben von Ihnen ausgefallten Formulare bitte in den Briefumschlag, der an die Assessment-Center-Leitung adressiert ist. 


\section{Überblick zum Ablauf des Assessment-Centers:}

1. Lesen Sie sich jetzt sorgfältig die Situationsbeschreibung und die Informationsbasis durch (bitte nicht vorblättern!). Beachten Sie die Bearbeitungshinweise!

2. Vergewissern Sie sich, dass Sie alle 3 Aufgaben auf dem Aufgabenblatt bearbeitet haben, bevor Sie die beiden Umschläge bei der LV-Leitung abgeben.

3. Sobald Sie mit der Bearbeitung der 3 Aufgaben fertig sind und die Umschläge bei der LVLeitung abgegeben haben, begeben Sie sich bitte wieder auf Ihren Platz und warten auf die Instruktionen zum Treffen Ihres Interaktionspartners, um gemeinsam an der Aufgabenstellung weiterzuarbeiten.

\section{SITUATIONSBESCHREIBUNG}

Sie sind ein österreichischer Weißweinproduzent. Die Geschäfte laufen gut, einige Ihrer Weißweine sind bereits mit Auszeichnungen prämiert worden, doch würden Sie gerne ein neues Produkt auf den Markt bringen, das bis dato in dieser Form noch nicht angeboten wird. Und zwar haben Sie sich überlegt, einen Wein speziell für Geschenksituationen auf den Markt zu bringen. $\mathrm{Zu}$ diesem Zweck haben Sie schon vorab eine Studie über den österreichischen Weingeschenkmarkt in Auftrag gegeben, um die Präferenzen (z.B. Weinsorte, Flaschenform, Verschluss, Etikettengestaltung) der österreichischen Käufer, die Wein als Geschenk erwerben, zu erfahren. Die Ergebnisse der Studie zeigen unter anderem, dass Weinkäufer bevorzugt österreichischen Rotwein der Sorte ,Zweigelt' verschenken. Um den Markt für Geschenkwein daher vollständig zu bearbeiten, haben Sie sich überlegt, mit einem österreichischen Rotweinproduzenten zusammenzuarbeiten. Der Geschăftsführer eines Weinhandels in Wien hat bereits sein Interesse an Ihrer Idee signalisiert: Er könnte sich vorstellen, das Ergebnis Ihrer Zusammenarbeit mit dem Rotweinproduzenten in einer Geschenkbox, bestehend aus einer Flasche Ihres Weißweines und einer Flasche des Rotweines, exklusiv anzubieten.

Nachdem Sie sich nun entschieden haben, mit einem österreichischen Rotweinproduzenten zusammenzuarbeiten, besuchen Sie die Weinmesse ,VieVinum' in der Wiener Hofburg. Dort kommen Sie mit einem österreichischen Weinproduzenten ins Gespräch, der sich auf die Produktion von Rotwein spezialisiert hat. Während des Gesprächs bemerken Sie eine Vielzahl von internationalen und nationalen Auszeichnungen an seinem Stand. Den Gütekriterien nach zu urteilen, die seine Weine erhalten haben, scheint vor allem der ,Zweigelt' bei den Testern und Konsumenten beliebt zu sein. Der Rotweinproduzent merkt an, dass er sich aufgrund mangelnder Nachfrage vor einigen Jahren dazu entschlossen hat, sein Weingut biodynamisch zu bewirtschaften. Nachdem er in Fachjournalen von dieser Methode der Bewirtschaftung gelesen hat, hat er mehr Informationen darüber gesammelt. Nach einer Kosten-Nutzen-Analyse hat er die Umsetzung der biodynamischen Bewirtschaftung in seinem Betrieb geplant, koordiniert und kontrolliert. Dieser Entschluss hat dem Betrieb entsprechenden Erfolg gebracht, da es der Rotweinproduzent - als Experte für biodynamischen Weinbau - Jahr für Jahr schafft, die sehr hohe Qualität zu halten. Inzwischen ist er so bekannt, dass er laufend als Referent von Weinakademien eingeladen wird. Auf Ihr Nachfragen hin, räumt der Rotweinproduzent ein, dass diese biodynamische Bewirtschaftung mit einem hohen Aufwand verbunden ist. Sie erzählen ihm von Ihrem Weingut und Ihren Weißweinen. Nachdem Sie den ,Zweigelt' probiert haben, fallt Ihnen die traditionelle Etikettengestaltung des Rotweinproduzenten auf. Auf Ihren Vorschlag hinsichtlich einer möglichen Modernisierung reagiert der Rotweinproduzent zurückhaltend. 
Sie erzählen ihm von Ihrer Idee eine Geschenkbox, bestehend aus Ihrem Weißwein und einem Rotwein abgefüllt speziell für Geschenkzwecke, auf den Markt zu bringen. Sie geben an, bereits über Ergebnisse einer Studie über den österreichischen Weingeschenkmarkt zu verfügen und dass Sie nun auf der Suche nach einem geeigneten Partner für die Umsetzung Ihrer Idee sind. Weil der Rotweinproduzent an der Idee der gemeinsamen Geschenkbox interessiert ist, erzählen Sie ihm von Ihrem Kontakt zu einem Geschăftsführer eines Weinhandels in Wien, der die Geschenkbox exklusiv in sein Sortiment aufnehmen würde. Nach einem letzten Schluck vom ,Zweigelt verabschieden Sie sich und kontaktieren den Weinhändler, der Ihren Vorschlag bezüglich der Zusammenarbeit mit dem Rotweinproduzenten interessant findet. Er schickt Ihnen beiden folgende EMail:

\begin{abstract}
Lieber Weißweinproduzent, lieber Rotweinproduzent!
Ich freue mich, dass wir möglichweise ins Geschäft kommen. Grundsätzlich sieht die Sache so aus: Ich habe in meinem Geschäftsplan 100.000,- Euro für Geschenkboxen, bestehend aus je 2 Flaschen Wein, reserviert.

Da ich glaube, dass die Weine von Ihnen beiden gut in mein Sortiment passen, kann ich mir vorstellen, dass ich Ihnen beiden je 5.000 Flaschen Wein abnehme (5.000 Flaschen Weißwein, 5.000 Flaschen Rotwein für gemischte Geschenkboxen). Jeder von Ihnen beiden würde daher 50.000,- Euro von mir erhalten (50:50-Lösung).

Andererseits ist für mich auch denkbar, dass ich nur mit dem Winzer, der über das größere Potential verfügt, zusammenarbeite. Ich kann mir also auch vorstellen, dass ich einem von Ihnen 10.000 Flaschen abnehme (10.000 Flaschen Weißwein ODER 10.000 Flaschen Rotwein). Einer von Ihnen würde daher 100.000,- Euro von mir erhalten und der andere nichts (100:0 Lösung).

Ich lade Sie zu einem Gespräch ein, bei dem wir die Strategien gemeinsam besprechen können. An diesem Gespräch nehmen Sie beide, also der Weißweinproduzent und der Rotweinproduzent, und ich in meiner Rolle als Geschäftsführer des Weinhandels teil.

Ich freue mich und verbleibe mit den besten Grüßen,

Fritz Weinhandel, Geschäftsführer

P.S. Der guten Ordnung halber möchte ich Sie darauf hinweisen, dass ich eine mögliche Geschäftsbeziehung sofort beenden würde, sollte sich herausstellen, dass sich die Produkte von Ihnen nicht zum Absatz auf dem Weingeschenkmarkt eignen (0:0-Lösung).
\end{abstract}

\title{
Bearbeitungshinweis:
}

Ihren Anteil an der Abnahmemenge müssen Sie in dem Gespräch mit dem Rotweinproduzenten und dem Geschäftsfuhrer des Weinhandels aushandeln. In dieser Verhandlung erfolgt dann die genaue Zuteilung der Abnahmemenge bzw. des Budgets.

Um sich fur die Verhandlung vorzubereiten, verschaffen Sie sich nun durch Bearbeitung der nachfolgenden Seiten einen Überblick über Ihr Weingut und Ihre Produkte, sowie die Ergebnisse, die Sie im Rahmen der von Ihnen in Auftrag gegebenen Studie über den österreichischen Weingeschenkmarkt gewonnen haben. Je besser Sie über die Stărken und Schwăchen Ihres Betriebes im Vergleich zum Weingut des Rotweinproduzenten Bescheid wissen, desto eher können Sie in der Verhandlung den Geschăftsführer des Weinhandels von Ihrem Zuteilungsvorschlag bezüglich der Abnahmemenge äberzeugen. In der Verhandlung besteht keine Möglichkeit, mit dem Rotweinproduzenten Informationen auszutauschen, ohne dass der Geschäftsführer des Weinhandels dies bemerkt. 


\section{Überblick zum Ablauf des Assessment-Centers:}

1. Lesen Sie sich jetzt sorgfältig die Situationsbeschreibung und die Informationsbasis durch (bitte nicht vorblättern!). Beachten Sie die Bearbeitungshinweise!

2. Vergewissern Sie sich, dass Sie alle 3 Aufgaben auf dem Aufgabenblatt bearbeitet haben, bevor Sie die beiden Umschläge bei der LV-Leitung abgeben.

3. Sobald Sie mit der Bearbeitung der 3 Aufgaben fertig sind und die Umschläge bei der LVLeitung abgegeben haben, begeben Sie sich bitte wieder auf Ihren Platz und warten auf die Instruktionen zum Treffen Ihres Interaktionspartners, um gemeinsam an der Aufgabenstellung weiterzuarbeiten.

\section{SITUATIONSBESCHREIBUNG}

Sie sind ein österreichischer Weißweinproduzent. Die Geschäfte laufen gut, einige Ihrer Weißweine sind bereits mit Auszeichnungen prämiert worden, doch würden Sie gerne ein neues Produkt auf den Markt bringen, das bis dato in dieser Form noch nicht angeboten wird. Und zwar haben Sie sich überlegt, einen Wein speziell für Geschenksituationen auf den Markt zu bringen. $\mathrm{Zu}$ diesem Zweck haben Sie schon vorab eine Studie über den österreichischen Weingeschenkmarkt in Auftrag gegeben, um die Präferenzen (z.B. Weinsorte, Flaschenform, Verschluss, Etikettengestaltung) der österreichischen Käufer, die Wein als Geschenk erwerben, zu erfahren. Die Ergebnisse der Studie zeigen unter anderem, dass Weinkäufer bevorzugt österreichischen Rotwein der Sorte ,Zweigelt' verschenken. Um den Markt für Geschenkwein daher vollständig zu bearbeiten, haben Sie sich überlegt, mit einem österreichischen Rotweinproduzenten zusammenzuarbeiten. Der Geschäftsführer eines Weinhandels in Wien hat bereits sein Interesse an Ihrer Idee signalisiert: Er könnte sich vorstellen, das Ergebnis Ihrer Zusammenarbeit mit dem Rotweinproduzenten in einer Geschenkbox, bestehend aus einer Flasche Ihres Weißweines und einer Flasche des Rotweines, exklusiv anzubieten.

Nachdem Sie sich nun entschieden haben, mit einem österreichischen Rotweinproduzenten zu-
sammenzuarbeiten, besuchen Sie die Weinmesse,VieVinum ' in der Wiener Hofburg. Dort
kommen Sie mit einem österreichischen Winzer ins Gespräch, der sich auf die Produktion von
Rotwein spezialisiert hat. Während des Gesprächs bemerken Sie eine Plakette an seinem Stand.
Dieser Plakette nach zu urteilen, scheint der, Zweigelt' bei den Testern anzukommen. Der Rot-
weinproduzent gibt an, dass er sein Weingut biodynamisch bewirtschaftet. Dadurch schafft er es
die Qualität zu halten und seine Produkte abzusetzen. Auch aus persönlicher Sicht war diese
Vorgehensweise in seinen Augen erfolgreich, da er nun viel mehr Freude bei der Arbeit auf
seinem Weingut hat. Der Rotweinproduzent erzählt Ihnen von den Entwicklungen bei der bio-
dynamischen Bewirtschaftung und geht dabei offen und ehrlich auf alle Rückschläge und
Erfolge ein. Auf sein Nachfragen hin, erzählen Sie ihm von Ihrem Weingut. Der Rotweinpro-
duzent kennt Ihre Weißweine und äußert sich begeistert über deren hohe Qualität. Er wirkt auch
sehr interessiert an Ihrem Betrieb und würde Sie gerne mit seiner Familie besuchen kommen.
Voller Begeisterung lädt er auch Sie mitsamt Ihrer Familie auf sein Weingut ein. Sie tauschen
sich mit ihm über die allgemeinen Probleme, mit denen Winzer konfrontiert sind, aus und
erzählen ihm von Ihren Problemen bei der Weinlese, woraufhin er Ihnen sofort die Kontakt-
daten von Arbeitern, die ihn bei der Lese unterstützen, notiert. Nachdem Sie seinen ,Zweigelt'
probiert haben, fällt Ihnen seine traditionelle Etikettengestaltung auf. Auf Ihren Vorschlag hin-
sichtlich einer möglichen Modernisierung reagiert der Rotweinproduzent sehr neugierig und
interessiert. 
Sie erzählen ihm von Ihrer Idee eine Geschenkbox, bestehend aus Ihrem Weißwein und einem Rotwein abgefüllt speziell für Geschenkzwecke, auf den Markt zu bringen. Sie geben an, bereits über Ergebnisse einer Studie über den österreichischen Weingeschenkmarkt zu verfügen und dass Sie nun auf der Suche nach einem geeigneten Partner für die Umsetzung Ihrer Idee sind. Weil der Rotweinproduzent an der Idee der gemeinsamen Geschenkbox interessiert ist, erzählen Sie ihm von Ihrem Kontakt zu einem Geschäftsführer eines Weinhandels in Wien, der die Geschenkbox exklusiv in sein Sortiment aufnehmen würde. Nach einem letzten Schluck vom ,Zweigelt' verabschieden Sie sich und kontaktieren den Weinhändler, der Ihren Vorschlag bezüglich der Zusammenarbeit mit dem Rotweinproduzenten interessant findet. Er schickt Ihnen beiden folgende EMail:

\begin{abstract}
Lieber Weißweinproduzent, lieber Rotweinproduzent!
Ich freue mich, dass wir möglichweise ins Geschäft kommen. Grundsätzlich sieht die Sache so aus: Ich habe in meinem Geschäftsplan 100.000,- Euro für Geschenkboxen, bestehend aus je 2 Flaschen Wein, reserviert.

Da ich glaube, dass die Weine von Ihnen beiden gut in mein Sortiment passen, kann ich mir vorstellen, dass ich Ihnen beiden je 5.000 Flaschen Wein abnehme (5.000 Flaschen Weißwein, 5.000 Flaschen Rotwein für gemischte Geschenkboxen). Jeder von Ihnen beiden würde daher 50.000,- Euro von mir erhalten (50:50-Lösung).

Andererseits ist für mich auch denkbar, dass ich nur mit dem Winzer, der über das größere Potential verfügt, zusammenarbeite. Ich kann mir also auch vorstellen, dass ich einem von Ihnen 10.000 Flaschen abnehme (10.000 Flaschen Weißwein ODER 10.000 Flaschen Rotwein). Einer von Ihnen würde daher 100.000,- Euro von mir erhalten und der andere nichts (100:0 Lösung).

Ich lade Sie zu einem Gespräch ein, bei dem wir die Strategien gemeinsam besprechen können. An diesem Gespräch nehmen Sie beide, also der Weißweinproduzent und der Rotweinproduzent, und ich in meiner Rolle als Geschäftsführer des Weinhandels teil.

Ich freue mich und verbleibe mit den besten Grüßen,

Fritz Weinhandel, Geschäftsführer

P.S. Der guten Ordnung halber möchte ich Sie darauf hinweisen, dass ich eine mögliche Geschäftsbeziehung sofort beenden würde, sollte sich herausstellen, dass sich die Produkte von Ihnen nicht zum Absatz auf dem Weingeschenkmarkt eignen (0:0-Lösung).
\end{abstract}

\title{
Bearbeitungshinweis:
}

Ihren Anteil an der Abnahmemenge müssen Sie in dem Gespräch mit dem Rotweinproduzenten und dem Geschäftsführer des Weinhandels aushandeln. In dieser Verhandlung erfolgt dann die genaue Zuteilung der Abnahmemenge bzw. des Budgets.

Um sich für die Verhandlung vorzubereiten, verschaffen Sie sich nun durch Bearbeitung der nachfolgenden Seiten einen Überblick über Ihr Weingut und Ihre Produkte, sowie die Ergebnisse, die Sie im Rahmen der von Ihnen in Auftrag gegebenen Studie über den österreichischen Weingeschenkmarkt gewonnen haben. Je besser Sie über die Stärken und Schwăchen Ihres Betriebes im Vergleich zum Weingut des Rotweinproduzenten Bescheid wissen, desto eher können Sie in der Verhandlung den Geschäftsfuhrer des Weinhandels von Ihrem Zuteilungsvorschlag bezüglich der Abnahmemenge überzeugen. In der Verhandlung besteht keine Möglichkeit, mit dem Rotweinproduzenten Informationen auszutauschen. ohne dass der Geschäftsführer des Weinhandels dies bemerkt. 


\section{Überblick zum Ablauf des Assessment-Centers:}

1. Lesen Sie sich jetzt sorgfältig die Situationsbeschreibung und die Informationsbasis durch (bitte nicht vorblättern!). Beachten Sie die Bearbeitungshinweise!

2. Vergewissern Sie sich, dass Sie alle 3 Aufgaben auf dem Aufgabenblatt bearbeitet haben, bevor Sie die beiden Umschlăge bei der LV-Leitung abgeben.

3. Sobald Sie mit der Bearbeitung der 3 Aufgaben fertig sind und die Umschläge bei der LVLeitung abgegeben haben, begeben Sie sich bitte wieder auf Ihren Platz und warten auf die Instruktionen zum Treffen Ihres Interaktionspartners, um gemeinsam an der Aufgabenstellung weiterzuarbeiten.

\section{SITUATIONSBESCHREIBUNG}

Sie sind ein österreichischer Weißweinproduzent. Die Geschäfte laufen gut, einige Ihrer Weißweine sind bereits mit Auszeichnungen prämiert worden, doch würden Sie gerne ein neues Produkt auf den Markt bringen, das bis dato in dieser Form noch nicht angeboten wird. Und zwar haben Sie sich überlegt, einen Wein speziell für Geschenksituationen auf den Markt zu bringen. $\mathrm{Zu}$ diesem Zweck haben Sie schon vorab eine Studie über den österreichischen Weingeschenkmarkt in Auftrag gegeben, um die Prăferenzen (z.B. Weinsorte, Flaschenform, Verschluss, Etikettengestaltung) der österreichischen Käufer, die Wein als Geschenk erwerben, zu erfahren. Die Ergebnisse der Studie zeigen unter anderem, dass Weinkäufer bevorzugt österreichischen Rotwein der Sorte ,Zweigelt' verschenken. Um den Markt für Geschenkwein daher vollständig zu bearbeiten, haben Sie sich überlegt, mit einem österreichischen Rotweinproduzenten zusammenzuarbeiten. Der Geschäftsführer eines Weinhandels in Wien hat bereits sein Interesse an Ihrer Idee signalisiert: Er könnte sich vorstellen, das Ergebnis Ihrer Zusammenarbeit mit dem Rotweinproduzenten in einer Geschenkbox, bestehend aus einer Flasche Ihres Weißweines und einer Flasche des Rotweines, exklusiv anzubieten.

Nachdem Sie sich nun entschieden haben, mit einem österreichischen Rotweinproduzenten zusammenzuarbeiten, besuchen Sie die Weinmesse ,VieVinum' in der Wiener Hofburg. Dort kommen Sie mit einem österreichischen Winzer ins Gespräch, der sich auf die Produktion von Rotwein spezialisiert hat. Der Rotweinproduzent erzählt, dass sich sein ,Zweigelt‘ gut verkauft. Sie erzählen ihm von Ihrem Weingut und Ihren Weißweinen. Nachdem Sie den ,Zweigelt" probiert haben, fällt Ihnen die traditionelle Etikettengestaltung des Rotweinproduzenten auf. Auf Ihren Vorschlag hinsichtlich einer möglichen Modernisierung reagiert der Rotweinproduzent zurückhaltend.

Sie erzählen ihm von Ihrer Idee eine Geschenkbox, bestehend aus Ihrem Weißwein und einem Rotwein abgefüllt speziell für Geschenkzwecke, auf den Markt zu bringen. Sie geben an, bereits über Ergebnisse einer Studie über den österreichischen Weingeschenkmarkt zu verfügen und dass Sie nun auf der Suche nach einem geeigneten Partner für die Umsetzung Ihrer Idee sind. Weil der Rotweinproduzent an der Idee der gemeinsamen Geschenkbox interessiert ist, erzählen Sie ihm von Ihrem Kontakt zu einem Geschäftsfuhrer eines Weinhandels in Wien, der die Geschenkbox exklusiv in sein Sortiment aufnehmen würde. Nach einem letzten Schluck vom ,Zweigelt" verabschieden Sie sich und kontaktieren den Weinhändler, der Ihren Vorschlag bezüglich der Zusammenarbeit mit dem Rotweinproduzenten interessant findet. Er schickt Ihnen beiden folgende EMail: 


\begin{abstract}
Lieber Weißweinproduzent, lieber Rotweinproduzent!
Ich freue mich, dass wir möglichweise ins Geschäft kommen. Grundsätzlich sieht die Sache so aus: Ich habe in meinem Geschäftsplan 100.000,- Euro für Geschenkboxen, bestehend aus je 2 Flaschen Wein, reserviert.

Da ich glaube, dass die Weine von Ihnen beiden gut in mein Sortiment passen, kann ich mir vorstellen, dass ich Ihnen beiden je 5.000 Flaschen Wein abnehme (5.000 Flaschen Weißwein, 5.000 Flaschen Rotwein für gemischte Geschenkboxen). Jeder von Ihnen beiden würde daher 50.000,- Euro von mir erhalten (50:50-Lösung).

Andererseits ist für mich auch denkbar, dass ich nur mit dem Winzer, der über das größere Potential verfügt, zusammenarbeite. Ich kann mir also auch vorstellen, dass ich einem von Ihnen 10.000 Flaschen abnehme (10.000 Flaschen Weißwein ODER 10.000 Flaschen Rotwein). Einer von Ihnen würde daher 100.000,- Euro von mir erhalten und der andere nichts (100:0 Lösung).

Ich lade Sie zu einem Gespräch ein, bei dem wir die Strategien gemeinsam besprechen können. An diesem Gespräch nehmen Sie beide, also der Weißweinproduzent und der Rotweinproduzent, und ich in meiner Rolle als Geschäftsführer des Weinhandels teil.

Ich freue mich und verbleibe mit den besten Grüßen,

Fritz Weinhandel, Geschäftsführer

P.S. Der guten Ordnung halber möchte ich Sie darauf hinweisen, dass ich eine mögliche Geschäftsbeziehung sofort beenden würde, sollte sich herausstellen, dass sich die Produkte von Ihnen nicht zum Absatz auf dem Weingeschenkmarkt eignen (0:0-Lösung).
\end{abstract}

\title{
Bearbeitungshinweis:
}

Ihren Anteil an der Abnahmemenge müssen Sie in dem Gespräch mit dem Rotweinproduzenten und dem Geschäftsführer des Weinhandels aushandeln. In dieser Verhandlung erfolgt dann die genaue Zuteilung der Abnahmemenge bzw. des Budgets.

Um sich für die Verhandlung vorzubereiten, verschaffen Sie sich nun durch Bearbeitung der nachfolgenden Seiten einen Überblick über Ihr Weingut und Ihre Produkte, sowie die Ergebnisse, die Sie im Rahmen der von Ihnen in Auftrag gegebenen Studie über den österreichischen Weingeschenkmarkt gewonnen haben. Je besser Sie über die Stărken und Schwăchen Ihres Betriebes im Vergleich zum Weingut des Rotweinproduzenten Bescheid wissen, desto eher können Sie in der Verhandlung den Geschäftsfuhrer des Weinhandels von Ihrem Zuteilungsvorschlag bezüglich der Abnahmemenge überzeugen. In der Verhandlung besteht keine Möglichkeit, mit dem Rotweinproduzenten Informationen auszutauschen. ohne dass der Geschäftsführer des Weinhandels dies bemerkt. 


\section{INFORMATIONSBASIS}

Sowohl Ihnen, als auch dem Rotweinproduzenten liegen allgemeine Informationen über Ihr Weingut und Ihre Produkte, als auch allgemeine Informationen über das Weingut und die Produkte des Rotweinproduzenten vor. Für die anstehende Verhandlung ist es wichtig zu wissen, wo die Stärken und Schwächen des eigenen Weinguts liegen. Diese erkennen Sie aber nur im Vergleich mit den Zahlen des Weinguts des Rotweinproduzenten.

\begin{tabular}{|c|c|c|c|c|}
\hline & \multicolumn{2}{|c|}{$\begin{array}{c}\text { Weingut des } \\
\text { Weißweinproduzenten (Ihres) }\end{array}$} & \multicolumn{2}{|c|}{$\begin{array}{c}\text { Weingut des } \\
\text { Rotweinproduzenten }\end{array}$} \\
\hline \multicolumn{5}{|c|}{ Allgemeine Informationen zum Weingut und den Produkten: } \\
\hline Betriebsgröße & \multicolumn{2}{|c|}{5 ha (Hektar) Eigenflăche } & \multicolumn{2}{|c|}{7 ha (Hektar) Eigenflache } \\
\hline Rebsorten & \multicolumn{2}{|c|}{$\begin{array}{l}\text { Grüner Veltliner, Welschriesling, } \\
\text { Sauvignon Blanc }\end{array}$} & \multicolumn{2}{|c|}{$\begin{array}{l}\text { Zweigelt, Blaufränkisch, } \\
\text { Cabernet Sauvignon }\end{array}$} \\
\hline Produktionsmenge & \multicolumn{2}{|c|}{$54.000 \mathrm{Fl}$ (Flaschen) } & \multicolumn{2}{|c|}{ 55.100 Fl (Flaschen) } \\
\hline Absatzmenge & \multicolumn{2}{|c|}{ 43.200 Fl (Flaschen) } & \multicolumn{2}{|r|}{$44.000 \mathrm{Fl}$ (Flaschen) } \\
\hline Konsumentenpreise/FL & \multicolumn{2}{|c|}{$€ 4,00-7,00$} & \multicolumn{2}{|r|}{$€ 5,20-10,00$} \\
\hline \multicolumn{5}{|c|}{ Einnahmen-A usgaben-Rechnung 2009 (Auszüge): } \\
\hline Umsatz Gastronomie & \multicolumn{2}{|l|}{$€ 120.000$} & & $?$ \\
\hline Umsatz Ab-Hof & \multicolumn{2}{|l|}{$€ 60.000$} & & $?$ \\
\hline "Schwarz"-Umsatz & \multicolumn{2}{|c|}{$20 \%$ v. Ab-Hof-Umsatz } & & $?$ \\
\hline Gewinn & \multicolumn{2}{|c|}{$€ 50.000$} & & $?$ \\
\hline zusătzl Gewinn & \multicolumn{2}{|c|}{$€ 12.000$ (aus unversteuerten Verkäufen) } & & $?$ \\
\hline \multicolumn{5}{|c|}{ Spezifische Informationen zur Be wirtschaftung des Weinguts: } \\
\hline Weingartenumstellung & \multicolumn{2}{|c|}{ seit 2005: Spezialisierung auf Weißweimproduktion } & \multicolumn{2}{|c|}{ Spezialisierung auf Rotwein } \\
\hline Bewăsserung & \multicolumn{2}{|c|}{$\begin{array}{l}\text { derzeit: } 20 \% \text { der Eigenfläche bewässert (geplant: } \\
\text { vollstăndige Bewässerung der Qualitătsweinrebsorten) }\end{array}$} & \multicolumn{2}{|r|}{ (a) } \\
\hline $\begin{array}{l}\text { Böschungs- und } \\
\text { Mauerterassen }\end{array}$ & \multicolumn{2}{|c|}{$\begin{array}{c}\text { derzeit: } 25 \% \text { der Eigenflache terassiert (geplant: } \\
\text { Terassierung weiterer } 25 \% \text { der Eigenflache zum } \\
\text { Erosionsschutz) }\end{array}$} & & $?$ \\
\hline \multicolumn{5}{|c|}{ Spe zifische Informationen zu den Produkten des Weinguts: } \\
\hline Auszeichnungen & \multicolumn{2}{|c|}{$\begin{array}{l}\text { Goldmedaille für den Grünen Veltliner bei der Vienna } \\
\text { Wine Challenge, } 3 \text { Siegel bei der NÖ Landesweinmesse }\end{array}$} & & $?$ \\
\hline Exportlander & \multicolumn{2}{|c|}{ Slowakei Deutschland } & & $?$ \\
\hline Besonderheiten & \multicolumn{2}{|c|}{$\begin{array}{l}\text { Zusammenarbeit mit jungen Künstlern bei der } \\
\text { Gestaltung von Weinflaschenetiketten }\end{array}$} & & $?$ \\
\hline \multicolumn{2}{|c|}{$\begin{array}{l}\text { Ergebnisse der von Ihnen bezahlten Studie über den } \\
\text { Weingeschenk markt (Auszüge): }\end{array}$} & \multirow{2}{*}{\multicolumn{2}{|c|}{$\begin{array}{c}\text { Umsetzbarkeit der } \\
\text { Anforderungen fur Sie } \\
\text { (Weißweinproduzent) }\end{array}$}} & \multirow{2}{*}{$\begin{array}{c}\text { Umsetzbarkeit der } \\
\text { Anforderungen fur } \\
\text { Rotweinproduzenten }\end{array}$} \\
\hline Cues & Prafferenzen der Weinkäufer & & & \\
\hline Flaschenform & Bordeaux- oder Schlegelflasche & $\begin{array}{l}\text { Umsetzung mach } \\
\text { erfolgt bei Realisie } \\
\text { der Geschenkbox- }\end{array}$ & $\begin{array}{l}\text { ibar, } \\
\text { erung } \\
\text { Idee }\end{array}$ & $?$ \\
\hline Verschluss & Kork, Glas & $\begin{array}{l}\text { Umsetzung mach } \\
\text { erfolgt bei Realisie } \\
\text { der Geschenkbox- }\end{array}$ & $\begin{array}{l}\text { ibar, } \\
\text { erung } \\
\text { Idee }\end{array}$ & $?$ \\
\hline Etikette & moderne, künstlerische Gestaltung & Umsetzung bereits & erfolgt & $?$ \\
\hline Rebsorten & $\begin{array}{c}\text { Weißwein (Grüner Veltliner, } \\
\text { Chardonnay), Rotwein (Zweigelt, } \\
\text { Blaufränkisch) }\end{array}$ & $\begin{array}{l}\text { Umsetzung im Hin } \\
\text { auf Weißwein ( } \mathrm{Gr} \\
\text { Veltliner) bereits e } \\
\end{array}$ & $\begin{array}{l}\text { alick } \\
\text { rüner } \\
\text { ffolgt }\end{array}$ & $\begin{array}{l}\text { Umsetzung } \\
\text { bereits erfolgt }\end{array}$ \\
\hline Alkoholgehalt & $10-12 \%$ & Umsetzung bereits & erfolgt & $?$ \\
\hline
\end{tabular}




\section{AUFGABENBLATT}

\section{TEIL A: Wahl Ihrer Strategie}

Sowohl Sie als auch der Rotweinproduzent müssen sich nun vorab für eine Strategie entscheiden. die Sie in der anschließenden Verhandlung verfolgen.

Strategie 1: Sie versuchen, den Geschäftsführer des Weinhandels zu überzeugen, dass die Abnahmemenge gleichmäßig (50:50-Lösung) zwischen Ihnen und dem Rotweinproduzenten verteilt wird. Sie können dann Ihre Idee der Geschenkbox, bestehend aus einer Flasche Ihres Weißweines und einer Flasche des Rotweinproduzenten, mit einem zugeteilten Budget von 50.000,- Euro fur 5.000 Flaschen Ihres Weißweines realisieren.

Strategie 2: $\quad$ Wie der Geschäftsführer des Weinhandels im E-Mail angedeutet hat, wären auch Geschenkboxen, bestehend aus ausschließlich Weißwein, für Ihn vorstellbar. Sie versuchen, den maximalen Anteil an der Abnahmemenge zu erhalten (100:0Lösung) und stellen in der Verhandlung die besonders gute Eignung Ihres Weißweines für die Geschenk-Box im Sortiment des Weinhändlers und für den Weingeschenkmarkt heraus. Sie können dann Geschenkboxen, bestehend aus zwei Flaschen Ihres Weißweines, mit einem zugeteilten Budget von 100.000,- Euro für 10.000 Flaschen Ihres Weißweines produzieren.

Die Konsequenzen Ihrer Strategiewahl hängen von der Strategiewahl des Rotweinproduzenten ab. Dies soll an einem Beispiel verdeutlicht werden: Wenn Sie sich für Strategie 2 entscheiden. werden Sie $100 \%$ der Abnahmemenge und des Budgets erhalten, falls der Rotweinproduzent in der Verhandlung die Strategie 1 verfolgt. Die nachfolgende Graphik stellt die Konsequenzen Ihrer Entscheidung unter Berücksichtigung der Entscheidung des Rotweinproduzenten dar. Folgende Situationen könnten sich ergeben:

\begin{tabular}{|c|c|c|}
\hline & \multicolumn{2}{|c|}{ der Rotwe inproduzent wählt } \\
& Strategie 1 & Strategie 2 \\
\hline \multirow{2}{*}{ Strategie 1 } & $\begin{array}{c}\text { Abnahmemenge- und Budgetaufteilung } \\
\text { zu je } 50 \% \text { für beide } \\
\text { (Nutzen für Sie: } 50.000,- \text { Euro) }\end{array}$ & $\begin{array}{c}0 \% \text { für Sie, } \\
100 \% \text { für den Rotweinproduzenten } \\
\text { (Nutzen für Sie: 0 Euro) }\end{array}$ \\
\hline Strategie 2 & $\begin{array}{c}100 \% \text { für Sie, } \\
\text { (Nutzen für Sie: } 100.000,- \text { Euro) }\end{array}$ & $\begin{array}{c}\text { Geschäftsführer des Weinhandels zieht } \\
\text { sein Angebot zurück: 0 \% für beide } \\
\text { (Nutzen für Sie: 0 Euro) }\end{array}$ \\
\hline
\end{tabular}

Sie wissen, dass sich der Geschäftsführer des Weinhandels vorbehălt, aus der Geschăftsbeziehung auszusteigen, wenn sich Ihre Produkte nicht zum Absatz eignen. Wenn eine Zusammenarbeit mit Ihnen, dem Rotweinproduzenten oder Ihnen beiden zustande kommt, wird er kontinuierlich die Qualität der Produkte und die Anpassung an Kundenwünsche bewerten. Nachfolgende Graphik zeigt die Konsequenzen der Bewertung: Wenn Sie $(+)$ beispielsweise mit dem Rotweinproduzenten zusammenarbeiten, dieser aber Wein schlechter Qualităt (-) abfüllt. wird die 50:50Lösung zu einer 0:0-Lösung, da der Geschäftsführer Ihnen beiden die Zusammenarbeit kündigt (Ihr Anteil ist fett markiert):

\begin{tabular}{|c|c|c|c|}
\hline & \multicolumn{2}{|c|}{ Qualität \& Anpassung des Rotweinproduzenten an Kundenwünsche } \\
\hline & & + & - \\
\hline \multirow{2}{*}{ 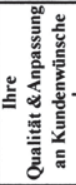 } & + & $\begin{array}{c}\mathbf{5 0}: 50 \rightarrow \mathbf{5 0}: 50(50.000 \text {,- Euro für Sie) } \\
\mathbf{1 0 0}: 0 \rightarrow \mathbf{1 0 0} 0(100.000 \text {,- Euro für Sie) } \\
\mathbf{0}: 100 \rightarrow \mathbf{0}: 100 \text { (0 Euro für Sie) }\end{array}$ & $\begin{array}{c}\mathbf{5 0}: 50 \rightarrow \mathbf{0}: 0(0 \text { Euro für Sie }) \\
\mathbf{1 0 0} 0 \rightarrow \mathbf{1 0 0}: 0(100.000,- \text { Euro für Sie }) \\
\mathbf{0}: 100 \rightarrow \mathbf{0}: 0(0 \text { Euro für } \mathrm{Sie})\end{array}$ \\
\hline & - & $\begin{array}{c}\mathbf{5 0}: 50 \rightarrow \mathbf{0}: 0(0 \text { Euro für } \mathrm{Sie}) \\
\mathbf{1 0 0 : 0} \rightarrow \mathbf{0}: 0(0 \text { Euro für } \mathrm{Sie}) \\
\mathbf{0}: 100 \rightarrow \mathbf{0}: 100(0 \text { Euro für } \mathrm{Sie})\end{array}$ & $\mathbf{0}: 0 \rightarrow \mathbf{0}: 0(0$ Euro für $\mathrm{Sie})$ \\
\hline
\end{tabular}




\section{Bearbeitungshinweis:}

- Bedenken Sie, wie sich der Rotweinproduzent verhalten könnte (lesen Sie gegebenenfalls noch einmal den grau hinterlegten Teil auf Seite 3)!

- Kreuzen Sie nun die Wahl Ihrer Strategie an (Aufgabe 1). Dem Rotweinproduzenten wird Ihre Strategie nicht mitgeteilt.

\section{Aufgabe 1:}

Für welche Strategie entscheiden Sie sich (bitte ankreuzen)?

$$
\text { Strategie } 1 \text { (50:50) } \square \text { Strategie 2 (100:0) }
$$

Bedenken Sie, wie sich der Rotweinproduzent verhalten wird. Dem Rotweinproduzenten wird Ihre Strategiewahl nicht mitgeteilt.

\section{TEIL B: Kontaktaufnahme mit dem Rotweinproduzenten}

Sowohl Sie als auch der Rotweinproduzent haben die Möglichkeit, sich gegenseitig Informationen zukommen zu lassen, die Sie in der Verhandlung sinnvoll nutzen können, z.B. um Ihren Vorschlag zur Aufteilung der Abnahmemenge vor dem Geschäftsführer des Weinhandels begründen zu können. Hierfür liegen Ihren Unterlagen ein leeres blaues Informationsformular und ein Umschlag bei, der an den Rotweinproduzenten adressiert ist. Der Umschlag wird dem Rotweinproduzenten vor der anschließenden Verhandlung übergeben.

\section{Bearbeitungshinweis:}

- Überlegen Sie, wie viel Wissen Sie dem Rotweinproduzenten preisgeben möchten. Sie können in jedes Feld die richtigen Informationen laut Angabe (siehe Blatt ,Informationsbasis') oder falsche Informationen eintragen. Sie können aber auch beliebig viele, also auch alle Felder freilassen.

- Folgen Sie dazu den Anweisungen der Aufgabe 2.

\section{Aufgabe 2:}

Welche Informationen über Ihr Weingut (Einnahmen-Ausgaben-Rechnung, Bewirtschaftung, Produkte) und den im Rahmen der Kooperation zu bearbeitenden Markt (Ergebnisse der Studie über den Weingeschenkmarkt) übermitteln Sie an den Rotweinproduzenten? Bitte bearbeiten Sie jetzt das blaue Formular und legen Sie es nach Bearbeitung in den beiliegenden Umschlag, der an den Rotweinproduzenten adressiert ist. Im Anschluss daran bearbeiten Sie bitte Teil C (siehe unten).

\section{TEIL C: Berichte zum Anteil falscher Informationen}

Auch Sie werden vor der Verhandlung einen Umschlag mit den vom Rotweinproduzenten ausgewählten Informationen erhalten. Es ist unsicher, wie viele und welche Informationen der Rotweinproduzent an Sie weiterleitet. Vor allem wissen Sie nicht, ob der Rotweinproduzent Ihnen richtige oder falsche Informationen zu seinem Weingut, seinen Produkten und seiner Bereitschaft und Fähigkeit zur Umsetzung der Studienergebnisse übermittelt. Sie haben daher die Möglichkeit, um eine Überprüfung bestimmter Informationen zu bitten, die der Rotweinproduzent an Sie weiterleitet. Sie können maximal 4 Berichte zum Anteil falscher Informationen anfordern:

- Einnahmen-Ausgaben-Rechnung des Rotweinproduzenten

- Bewirtschaftung des Weinguts des Rotweinproduzenten

- Produkte des Weinguts des Rotweinproduzenten

- Bereitschaft und Fähigkeit des Rotweinproduzenten zur Umsetzung der Studienergebnisse 
Die Berichte werden Ihnen vor Beginn der Verhandlung übergeben. Die Berichte zeigen Ihnen, wie viel Prozent der Informationen, die der Rotweinproduzent an Sie übermittelt hat, im jeweiligen Bericht falsch sind. Diese Berichte geben Ihnen jedoch keinen Aufschluss über den Inhalt oder die Ausprägungen der jeweiligen Informationen.

Konsequenzen der Berichtanforderung: Da die Prüfung der Informationen und Anfertigung der Berichte zeitaufwendig ist, wird sich der Beginn Ihrer anschließenden Verhandlung pro Bericht, den Sie anfordern, verzögem. Der Rotweinproduzent wird darüber in Kenntnis gesetzt, welche Berichte Sie überprüfen lassen.

Ebenso wie Sie hat auch der Rotweinproduzent die Möglichkeit, Berichte über den pro-zentuellen Anteil falscher Informationen anzufordern, die Sie ihm auf dem beiliegenden blauen Informationsformular übermitteln.

\section{Bearbeitungshinweis:}

- Bedenken Sie, dass die Stimmung in der Verhandlung und die Wahrscheinlichkeit einer Vereinbarung mit der Zahl der angeforderten Berichte sinken.

- Bitte kreuzen Sie nun an, welche Berichte Sie anfordern (Aufgabe 3).

\section{Aufgabe 3:}

Welche Berichte wünschen Sie, die zeigen, wie viel Prozent der Informationen, die der Rotweinproduzent an Sie übermittelt hat, falsch sind (bitte ankreuzen)? Beachten Sie, dass sich der Beginn der anschließenden Verhandlung pro Bericht verzögert!

\begin{tabular}{|l|l|l|}
\hline Anteil falscher Informationen & $\begin{array}{c}\text { Ich mőchte hie rau } \\
\text { einen Bericht }\end{array}$ & $\begin{array}{c}\text { Ich ve rzichte auf } \\
\text { einen Bericht }\end{array}$ \\
\hline - in der Einnahmen-Ausgaben-Rechnung & & \\
\hline - zur Bewirtschaftung & & \\
\hline - zu den Produkten & & \\
\hline $\begin{array}{l}\text { - zur Bereitschaft und Fahigkeit zur } \\
\text { Umsetzung der Studienergebnisse }\end{array}$ & & \\
\hline
\end{tabular}

Wenn Sie möchten, können Sie Ihre Entscheidungen bezüglich der Aufgaben 1, 2 und 3 hier begründen: 


\section{ABSCHLUSSEVALUIERUNG}

Nachdem Sie nun die Aufgabenstellungen bearbeitet haben, füllen Sie bitte diesen abschließenden Fragebogen aus!

Bitte kreuzen Sie pro Reihe die Antwort an, die Ihrer Meinung am ehesten entspricht (keine Mehrfachantworten möglich)!

Wie würden Sie Ihre aktuelle Situation bezüglich der Geschenkbox-Idee charakterisieren?

\begin{tabular}{|c|c|c|c|c|c|c|}
\hline 1. günstige Gelegenheit & 1 & 2 & 3 & 4 & 5 & starke Bedrohung \\
\hline $\begin{array}{l}\text { 2. Möglichkeit eines } \\
\text { Verlusts }\end{array}$ & 1 & 2 & 3 & 4 & 5 & $\begin{array}{r}\text { Möglichkeit eines } \\
\text { Gewinns }\end{array}$ \\
\hline 3. positive Situation & 1 & 2 & 3 & 4 & 5 & negative Situation \\
\hline \multicolumn{7}{|c|}{$\begin{array}{l}\text { Wie hoch schätzen Sie Ihre Erfolgsaussichten ein, Ihre Vorstellungen bezüglich der } \\
\text { Zuteilung der Abnahmemenge in der folgenden Verhandlung durchzusetzen? }\end{array}$} \\
\hline 4. sehr niedrig & 1 & 2 & 3 & 4 & 5 & sehr hoch \\
\hline
\end{tabular}

\section{Abschließender Bearbeitungshinweis:}

Alle Unterlagen, sowie das vorliegende von Ihnen ausgefüllte Aufgabenblatt legen Sie bitte in den Umschlag, der an die Assessment-Center-Leitung adressiert ist, und geben diesen, zusammen mit dem Umschlag, der an den Rotweinproduzenten adressiert ist und das blaue Informationsformular enthält, bei der LV-Leitung ab. Die Informationen, die Sie für die Verhandlung benötigen, erhalten Sie rechtzeitig von der LV-Leitung. 


\section{Forschungsergebnisse der Wirtschaftsuniversität Wien}

Herausgeber: Wirtschaftsuniversität Wien vertreten durch a.o. Univ. Prof. Dr. Barbara Sporn

\section{INFORMATION UND KONTAKT:}

WU (Wirtschaftsuniversităt Wien)

Department of Finance, Accounting and Statistics

Institute for Finance, Banking and Insurance

Heiligenstädter $S t r a ß e$ 46-48, 1190 Wien

Tel.: 0043-1-313 36/4556

Fax: 0043-1-313 36/904556

valentine.wendling@wu.ac.at

www.wu.ac.at/finance

Band 1 Stefan Felder: Frequenzallokation in der Telekommunikation. Ökonomische Analyse der Vergabe von Frequenzen unter besonderer Berücksichtigung der UMTS-Auktionen. 2004.

Band 2 Thomas Haller: Marketing im liberalisierten Strommarkt. Kommunikation und Produktplanung im Privatkundenmarkt. 2005.

Band 3 Alexander Stremitzer: Agency Theory: Methodology, Analysis. A Structured Approach to Writing Contracts. 2005.

Band 4 Günther Sedlacek: Analyse der Studiendauer und des Studienabbruch-Risikos. Unter Verwendung der statistischen Methoden der Ereignisanalyse. 2004.

Band 5 Monika Knassmüller: Unternehmensleitbilder im Vergleich. Sinn- und Bedeutungsrahmen deutschsprachiger Unternehmensleitbilder - Versuch einer empirischen $\left(R_{\theta-}\right)$ Konstruktion. 2005.

Band 6 Matthias Fink: Erfolgsfaktor Selbstverpflichtung bei vertrauensbasierten Kooperationen. Mit einem empirischen Befund. 2005.

Band 7 Michael Gerhard Kraft: Ökonomie zwischen Wissenschaft und Ethik. Eine dogmenhistorische Untersuchung von Léon M.E. Walras bis Milton Friedman. 2005.

Band 8 Ingrid Zechmeister: Mental Health Care Financing in the Process of Change. Challenges and Approaches for Austria. 2005.

Band 9 Sarah Meisenberger: Strukturierte Organisationen und Wissen. 2005.

Band 10 Anne-Katrin Neyer: Multinational teams in the European Commission and the European Parliament. 2005.

Band 11 Birgit Trukeschitz: Im Dienst Sozialer Dienste. Ökonomische Analyse der Beschäftigung in sozialen Dienstleistungseinrichtungen des Nonprofit Sektors. 2006

Band 12 Marcus Kölling: Interkulturelles Wissensmanagement. Deutschland Ost und West. 2006.

Band 13 Ulrich Berger: The Economics of Two-way Interconnection. 2006.

Band 14 Susanne Guth: Interoperability of DRM Systems. Exchanging and Processing XML-based Rights Expressions. 2006.

Band 15 Bernhard Klement: Ökonomische Kriterien und Anreizmechanismen für eine effiziente Förderung von industrieller Forschung und Innovation. Mit einer empirischen Quantifizierung der Hebeleffekte von F\&E-Förderinstrumenten in Österreich. 2006. 
Band 16 Markus Imgrund: Wege aus der Insolvenz. Eine Analyse der Forttührung und Sanierung insolventer Klein- und Mittelbetriebe unter besonderer Berücksichtigung des Konfigurationsansatzes. 2007.

Band 17 Nicolas Knotzer: Product Recommendations in E-Commerce Retailing Applications. 2008.

Band 18 Astrid Dickinger: Perceived Quality of Mobile Services. A Segment-Specific Analysis. 2007.

Band 19 Nadine Wiedermann-Ondrej: Hybride Finanzierungsinstrumente in der nationalen und internationalen Besteuerung der USA. 2008.

Band 20 Helmut Sorger: Entscheidungsorientiertes Risikomanagement in der Industrieunternehmung. 2008.

Band 21 Martin Rietsch: Messung und Analyse des ökonomischen Wechselkursrisikos aus Unternehmenssicht: Ein stochastischer Simulationsansatz. 2008.

Band 22 Hans Christian Mantler: Makroökonomische Effizienz des Finanzsektors. Herleitung eines theoretischen Modells und Schätzung der Wachstumsimplikationen für die Marktwirtschaften und Transformationsökonomien Europas. 2008.

Band 23 Youri Tacoun: La théorie de la valeur de Christian von Ehrenfels. 2008.

Band 24 Monika Koller: Longitudinale Betrachtung der Kognitiven Dissonanz. Eine Tagebuchstudie zur Reiseentscheidung. 2008.

Band 25 Marcus Scheiblecker: The Austrian Business Cycle in the European Context. 2008.

Band 26 Aida Numic: Multinational Teams in European and American Companies. 2008.

Band 27 Ulrike Bauernfeind: User Satisfaction with Personalised Internet Applications. 2008.

Band 28 Reinhold Schodl: Systematische Analyse und Bewertung komplexer Supply Chain Prozesse bei dynamischer Festlegung des Auftragsentkopplungspunkts. 2008.

Band 29 Bianca Gusenbauer: Öffentlich-private Finanzierung von Infrastruktur in Entwicklungsländern und deren Beitrag zur Armutsreduktion. Fallstudien in Vietnam und auf den Philippinen. 2009.

Band 30 Elisabeth Salomon: Hybrides Management in sino-österreichischen Joint Ventures in China aus österreichischer Perspektive. 2009.

Band 31 Katharina Mader: Gender Budgeting: Ein emanzipatorisches, finanzpolitisches und demokratiepolitisches Instrument. 2009.

Band 32 Michael Weber: Die Generierung von Empfehlungen für zwischenbetriebliche Transaktionen als gesamtwirtschaftliche Infrastrukturleistung. 2010.

Band 33 Lisa Gimpl-Heersink: Joint Pricing and Inventory Control under Reference Price Effects. 2009.

Band 34 Erscheint nicht.

Band 35 Dagmar Kiefer: Multicultural Work in Five United Nations Organisations. An Austrian Perspective. 2009.

Band 36 Gottfried Gruber: Multichannel Management. A Normative Model Towards Optimality. 2009.

Band 37 Rainer Quante: Management of Stochastic Demand in Make-to-Stock Manufacturing. 2009.

Band 38 Franz F. Eiffe: Auf den Spuren von Amartya Sen. Zur theoriegeschichtlichen Genese des Capability-Ansatzes und seinem Beitrag zur Armutsanalyse in der EU. 2010. 
Band 39 Astrid Haider: Die Lohnhöhe und Lohnstreuung im Nonprofit-Sektor. Eine quantitative Analyse anhand österreichischer Arbeitnehmer-Arbeitgeber-Daten. 2010.

Band 40 Maureen Lenhart: Pflegekräftemigration nach Österreich. Eine empirische Analyse. 2010.

Band 41 Oliver Schwank: Linkages in South African Economic Development. Industrialisation without Diversification? 2010.

Band 42 Judith Kast-Aigner: A Corpus-Based Analysis of the Terminology of the European Union's Development Cooperation Policy, with the African, Caribbean and Pacific Group of States. 2010.

Band 43 Emel Arikan: Single Period Inventory Control and Pricing. An Empirical and Analytical Study of a Generalized Model. 2011.

Band 44 Gerhard Wohlgenannt: Learning Ontology Relations by Combining Corpus-Based Techniques and Reasoning on Data from Semantic Web Sources. 2011.

Band 45 Thomas Peschta: Der Einfluss von Kundenzufriedenheit auf die Kundenloyalităt und die Wirkung der Wettbewerbsintensităt am Beispiel der Gemeinschaftsverpflegungsgastronomie. 2011.

Band 46 Friederike Hehle: Die Anwendung des Convenience-Konzepts auf den Betriebstyp Vending. 2011.

Band 47 Thomas Herzog: Strategisches Management von Koopetition. Eine empirisch begründete Theorie im industriellen Kontext der zivilen Luftfahrt. 2011.

Band 48 Christian Weismayer: Statische und longitudinale Zufriedenheitsmessung. 2011.

Band 49 Johannes Fichtinger: The Single-Period Inventory Model with Spectral Risk Measures. 2011.

Band 50 Isabella R. Hatak: Kompetenz, Vertrauen und Kooperation. Eine experimentelle Studie. 2011.

www.peterlang.de 


\section{Interpersonelles Vertrauen in Organisationen}

\section{Eine empirische Untersuchung der Einflussfaktoren und Verhaltenswirkungen}

Frankfurt am Main, Berlin, Bern, Bruxelles, New York, Oxford, Wien, 2008. XXVI, 242 S., 18 Abb., 34 Tab.

Schriften zur empirischen Entscheidungs- und Organisationsforschung.

Herausgegeben von Rolf Bronner. Bd. 24

ISBN 978-3-631-57261-0 · br. € 52,60*

Vertrauen beeinflusst die Leistung von Individuen und Gruppen in

Organisationen. In dieser Arbeit wird analysiert, wie sich Vertrauen auf das Kommunikations- und Kontrollverhalten in komplexen Entscheidungssituationen auswirkt und welche Faktoren dies determinieren. Vor dem Hintergrund eines interdisziplinären Vertrauensverständnisses erfolgt eine differenzierte Betrachtung der Kausalitäten zwischen vertrauensverwandten Konstrukten. Es wird ein Mediationsmodell interpersonellen Vertrauens entwickelt und empirisch geprüft. Möglichkeiten der Operationalisierung von Vertrauen werden diskutiert und reliable Messinstrumente zur Erfassung des Phänomens in Arbeitsbeziehungen präsentiert. Eine Diskussion der Befunde erfolgt aus theoretischer sowie methodologischer Perspektive und mündet in praktische Gestaltungshinweise, Vertrauensprozesse in Unternehmen positiv zu beeinflussen.

Aus dem Inhalt: Konzeptualisierung von Vertrauen - Erklärungsbeitrag zentraler Vertrauensmodelle $\cdot$ Vertrauen im Kontext verhaltenswissenschaftlicher Entscheidungsforschung: Vertrauenshandlungen, situations- und personenbedingte Vertrauensdeterminanten - Interpersonelles Vertrauen als Mediator . Operationalisierung von Vertrauen im Labor - Diskussion der Hypothesenprüfung · Gestaltungshinweise für vertrauensbewusstes Management

Frankfurt am Main - Berlin - Bern - Bruxelles - New York - Oxford - Wien

Auslieferung: Verlag Peter Lang AG

Moosstr. 1, $\mathrm{CH}-2542$ Pieterlen

Telefax 0041 (0) 32/3761727

*inklusive der in Deutschland gültigen Mehrwertsteuer

Preisänderungen vorbehalten

Homepage http://www.peterlang.de 